\title{
Aeroelastic oscillations of a pitching cantilever with structural geometric nonlinearities: theory, numerical simulation and global sensitivity analysis
}

\author{
by

\section{Brandon Robinson} \\ A thesis submitted to the \\ Faculty of Graduate and Postdoctoral Affairs \\ in partial fulfillment of the requirements for the degree of
}

Master of Applied Science in Civil Engineering

Ottawa-Carleton Institute for Civil Engineering

Department of Civil and Environmental Engineering

Carleton University

Ottawa, Ontario

August, 2018

(C)Copyright

Brandon Robinson, 2018 


\section{Abstract}

The focus of this thesis is the aeroelastic dynamics of a rectangular cantilever wing with a NACA 0012 profile, whose base is free to rotate rigidly about a longitudinal axis. The wing is analytically modelled as part of a project to simulate the dynamics of an aeroelastic wind tunnel model. Structural geometric nonlinearities are modelled to the second order to capture the essential effects of large deformation. While the derivation closely follows common approaches from the literature on the subject of the nonlinear bending and torsion of rotor blades, this aeroelastic system contains new inertial terms which stem from the fundamentally different kinematics that arise from the added rigid body degree of freedom in pitch. A system of coupled nonlinear partial differential equations (PDEs) and an ordinary differential equation (ODE) is derived using Hamilton's principle. These equations of motion describe the coupled axial-bending-bending-torsion-pitch motion of the system. A Galerkin projection scheme is employed to obtain a finite dimensional representation of the equations of motion, leading to a coupled system of ODEs. The resulting nonlinear ODEs are solved numerically using Houbolt's method. The results that are obtained are verified by comparison with the results obtained by direct integration of the equations of motion using a finite difference scheme. Using unsteady linear aerodynamics, it is observed that the system undergoes

coalescence flutter due to coupling between the rigid body pitch rotation dominated mode and the first flapwise bending dominated mode. The post-flutter behaviour is dictated by the structural geometric nonlinear terms which limit the oscillations to a limit cycle. Global sensitivity analysis is performed to study the effect of parametric uncertainty introduced by the rigid body base rotation on the flutter speed and associated frequency. It is found that the stiffness parameter has the largest influence on the variation of both the flutter speed and frequency, while the structural damping has a negligible influence. 


\section{Acknowledgments}

I would like to begin by thanking Dr. Abhijit Sarkar and Dr. Dominique Poirel for their efforts in fostering my interest in research early in my undergraduate career, and for their efforts in their continuing roles as two of my co-supervisors as I have moved on to graduate studies. I would equally like to acknowledge the support provided by my co-supervisor, Dr. Chris Pettit, as well as the involvement of Dr. Mohammad Khalil as an unofficial advisor. The breadth of their combined expertise has been instrumental in moulding the direction of my research.

I would like to thank my defense committee, Dr. Magdi Mohareb, Dr. Heng Khoo and again Dr. Mohammad Khalil for taking their time to read my thesis, and for offering insightful and thought provoking comments.

I also have to acknowledge the support of my family, who have helped ensure that I am able to live comfortably while completing my degree, as well as allowing me to claim approximately 3 square meters in the corner of the basement to call an office. My girlfriend Lauren also deserves a big thank you for enforcing some semblance of a work-life balance over the past two years and for, despite not understanding a single word, always offering a listening ear whenever I needed to practice for a conference presentation.

Finally, I would like to acknowledge the financial contributions of the Natural Science and Engineering Research Council of Canada (NSERC) Alexander Graham Bell Canada Graduate Scholarship Masters, the Ontario Graduate Scholarship, and Carleton Univsersity. 


\section{Table of Contents}

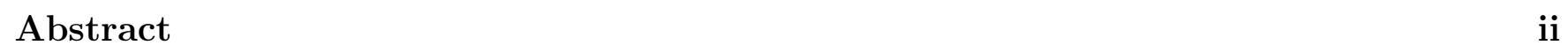

\begin{tabular}{ll}
\hline Acknowledgments & iii
\end{tabular}

$\begin{array}{ll}\text { Table of Contents } & \text { iv }\end{array}$

\begin{tabular}{ll}
\hline List of Tables & vii
\end{tabular}

\begin{tabular}{lr}
\hline List of Figures & viii
\end{tabular}

$\begin{array}{lc}\text { Nomenclature } & \text { xi }\end{array}$

\begin{tabular}{|lr|}
\hline 1 & Introduction \\
\hline
\end{tabular}

1.1 Literature Review . . . . . . . . . . . . . . . . . . . . . . 4

1.1 .1 Aeroelasticity . . . . . . . . . . . . . . . . . . . . 4

1.1 .2 Uncertainty Quantification . . . . . . . . . . . . . . . . . . . 8

1.2 Scope of the Thesis . . . . . . . . . . . . . . . . . . . . . . . . 9

\begin{tabular}{|ll|}
\hline 2 & Derivation of the Nonlinear Equations of Motion \\
\hline
\end{tabular}

2.1 Transformed Coordinate System . . . . . . . . . . . . . . . . . . . . . . . . 13

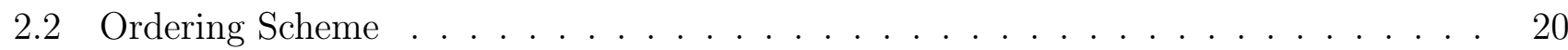

$2.3 \quad$ Strain-Displacement Relationship . . . . . . . . . . . . . . . . . . . . . . . . . . . . 21

2.3 .1 The Green Strain Tensor . . . . . . . . . . . . . . . . . . . . . . . . . . . . . 24

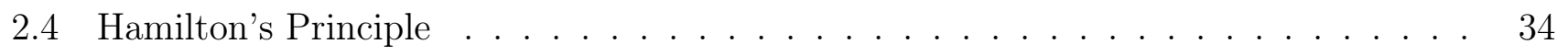

2.4 .1 Strain Energy . . . . . . . . . . . . . . . . . . . . . . . 35 
2.4 .2 Kinetic Energy $\ldots \ldots \ldots \ldots$

2.4 .3 Virtual Work Done by External Forces . . . . . . . . . . . . . . . . 60

$2.4 .4 \quad$ Set of Coupled Equations $\ldots \ldots \ldots \ldots$

2.5 Nondimensionalization . . . . . . . . . . . . . . . . . . 78

\begin{tabular}{|lll|}
\hline 3 & Numerical Solution of the Nonlinear Equations of Motion & 83 \\
\hline
\end{tabular}

$3.1 \quad$ Galerkin Projection $\ldots \ldots \ldots \ldots$. . . . . . . . . . . . . . . . 84

3.1 .1 Basis Functions . . . . . . . . . . . . . . . . . . . . . . . . . . . . . 84

3.1.2 Finite Dimensional Representation of the Nonlinear Structural Equations . . 88

3.1.3 Finite Dimensional Representation of the Unsteady Aerodynamic Model . . 93

3.1 .4 Imposing Initial Conditions $\ldots \ldots \ldots$. . . . . . . . . . . . . 97

3.2 Numerical Integration $\ldots \ldots \ldots \ldots$

3.2 .1 Houbolt's Method $\ldots \ldots \ldots$. . . . . . . . . . . . . . . . . . . . . . . 98

3.3 Numerical Results . . . . . . . . . . . . . . . . . . . . . . . . . . . . . . . . . . . 103

3.3 .1 Structural Free Vibration Simulation . . . . . . . . . . . . . . . . . 104

3.3 .2 Full Aeroelastic Simulation . . . . . . . . . . . . . . . . . . . . . . 108

3.4 Verification by Finite Difference Method $\ldots \ldots \ldots \ldots$

$3.4 .1 \quad$ Finite Differences $\ldots \ldots \ldots \ldots$

3.4 .2 Imposing Initial Conditions $\ldots \ldots \ldots \ldots$

3.4 .3 Comparison of the Performance $\ldots \ldots \ldots \ldots \ldots$

3.4 .4 Comparison of the Solutions $\ldots \ldots \ldots \ldots$

4 Variance-Based Methods for Global Sensitivity Analysis 130

4.1 Methods for Global Sensitivity Analysis . . . . . . . . . . . . . . . . . . . . 131

4.2 Sobol' Indices . . . . . . . . . . . . . . . . . . . . . . . . . . . . . . . . . . . . . . . . 132

4.3 Sensitivity Indices by Monte-Carlo Sampling . . . . . . . . . . . . . . . . . 135

4.4 Sensitivity Indices by Analytical Calculation . . . . . . . . . . . . . . . . . . 139

4.4 .1 Polynomial Chaos Representation . . . . . . . . . . . . . . . . . . . 146

4.4 .2 Polynomial Basis $\ldots \ldots \ldots \ldots$

4.4 .3 Obtaining Polynomial Chaos Coefficients . . . . . . . . . . . . . . 152 
4.4 .4 Statistical Moments. . . . . . . . . . . . . . . . . . . . . . . . . . . . . . . . 154

4.4 .5 Sensitivity Indices $\ldots \ldots \ldots$

4.5 Sensitivity of Flutter Speed and Frequency to Pitch Parameters . . . . . . . . . 159

$4.5 .1 \quad$ Sensitivity Indices . . . . . . . . . . . . . . . . . . . . . . . . . 160

$4.5 .2 \quad$ PCE Surrogate Model $\ldots \ldots$. . . . . . . . . . . . . . . . . . . 162

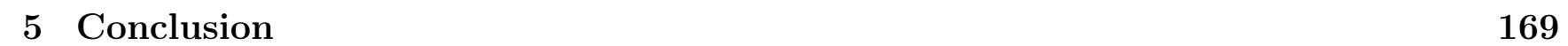

$5.1 \quad$ Concluding Remarks $\ldots \ldots \ldots \ldots . \ldots \ldots$

5.2 Future Research . . . . . . . . . . . . . . . . . . . . . . . . . . 172

$\begin{array}{ll}\text { List of References } & 174\end{array}$

\begin{tabular}{|lll}
\hline Appendix A Derivation of the Nonlinear Equations of Motion & 179
\end{tabular}

A.1 Small Angle Assumption . . . . . . . . . . . . . . . . . . . . . . . . . . . . . 179

A.2 Strain energy components . . . . . . . . . . . . . . . . . . . . . 180

A.3 Kinetic energy components . . . . . . . . . . . . . . . . . . . . . 185

\begin{tabular}{|lll}
\hline Appendix B Numerical Solution of the Nonlinear Equations of Motion & 187
\end{tabular}

B.1 Galerkin Projection . . . . . . . . . . . . . . . . . . . . . . . 187

B.1.1 Finite Dimensional Representation of the Structural Equations . . . . . . . 188

B.1.2 Finite Dimensional Representation of the Aerodynamic Loads . . . . . . . . 192

B.2 Galerkin Projection Coefficients . . . . . . . . . . . . . . . . . 196

B.3 Linear Structural and Aerodynamic M, C, K matrices . . . . . . . . . . . . . . . 199

B.4 Numerical Implementation: Galerkin Projection . . . . . . . . . . . . . . . . . 204

B.5 Numerical Implementation: Finite Difference scheme . . . . . . . . . . . . . . . 220

B.5.1 Linear Bending-Bending-Torsion-Pitch of a Cantilever . . . . . . . . . . 220

B.5.2 Nonlinear Inertial Terms . . . . . . . . . . . . . . . . . . . . . . . . . 229

B.5.3 Nonlinear Stiffness Terms . . . . . . . . . . . . . . . . . . . 234 


\section{List of Tables}

$3.1 \quad$ First 10 roots of the characteristic bending and torsion equations . . . . . . . . . 87

3.2 Parameter values used in numerical simulations . . . . . . . . . . . . . . . . 103

$4.1 \quad$ Number of terms in a PCE with increasing dimension and order . . . . . . . . . . . 148

4.2 Multi-indices and $\mathrm{PC}$ basis functions for $d=3, P=3$. . . . . . . . . . . . . . . . 149

4.3 Sobol' indices (main effect and total effect) for the current investigation . . . . . . . 161

$4.4 \quad$ PCE coefficients for the current surrogate model . . . . . . . . . . . . . . . . . . 163

B.1 Galerkin Projection Coefficents (Structural) . . . . . . . . . . . . . . . . . . . . . . 197

B.2 Galerkin Projection Coefficients (Aerodynamic) . . . . . . . . . . . . . . . . . . . 198 


\section{List of Figures}

$1.1 \quad$ Elevation view of the wind tunnel setup at RMC . . . . . . . . . . . . . . . . . . . 2

1.2 Plan view of the wind tunnel at RMC . . . . . . . . . . . . . . . . . . . . . . . 3

1.3 Streamlined body . . . . . . . . . . . . . . . . . . . . . . . . . . 4

1.4 Bluff body . . . . . . . . . . . . . . . . . . . . . . . . . . 5

$2.1 \quad$ Illustration of snapshots the aeroelastic system's motion over a portion of a cycle. . 12

2.2 Wing configurations on large, small, and unmanned aircraft . . . . . . . . . . . . . 13

2.3 The coordinate systems for rigid body pitch rotation . . . . . . . . . . . . . . . . . 14

2.4 Transformation between the coordinate systems $(x, y, z)$ and $(\mathbf{x}, \mathbf{y}, \mathbf{z})$. . . . . 15

$2.5 \quad$ Euler Angles $\gamma, \alpha, \beta$. . . . . . . . . . . . . . . . . . . . 16

$2.6 \quad$ Illustration of the reference frames as the system deforms . . . . . . . . . . . . . . . 20

2.7 Projection of the coordinate systems f . . . . . . . . . . . . . . . . . . . 23

2.8 The position vectors $r_{0}$ and $r_{1} \ldots \ldots \ldots \ldots \ldots$. . . . . . . . . . . . . . . 23

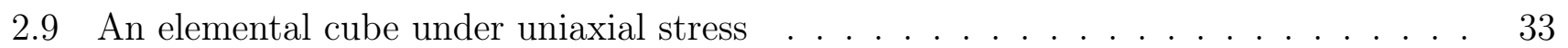

2.10 The components of the normal strains . . . . . . . . . . . . . . . . . . . . . . 37

2.11 Internal Moment, $T_{\mathbf{x}} \ldots \ldots \ldots \ldots \ldots \ldots$

2.12 Illustration of relative pressure distribution around an airfoil . . . . . . . . . . . . . 60

2.13 Aerodynamic lift and moment illustrated at two key locations along the chord . . . 61

2.14 Two degree-of-freedom airfoil in pitch and plunge . . . . . . . . . . . . . . . . . . 62

2.15 Wagner's function . . . . . . . . . . . . . . . . . . . . . . . . . 64

2.16 Illustration of streamlines, highlighting the effect of tip vortices . . . . . . . . . . 66

2.17 Lift reduction factor as a function of the normalized span . . . . . . . . . . . . . . . 66

2.18 Effects of motion in three dimensions . . . . . . . . . . . . . . . . . . . . . . . 68 
2.19 Illustration of the static lift and moment coefficients . . . . . . . . . . . . . . . 70

2.20 Surface fit to the data points . . . . . . . . . . . . . . . . . 71

2.21 Nonlinear aerodynamic model (blue) fit to the filtered data (red) . . . . . . . . . . . 71

2.22 Illustration of the simplified flexible bar system $\ldots \ldots \ldots \ldots$. . . . . . 76

2.23 Free body diagram of the bar in its deformed configuration . . . . . . . . . . 76

$3.1 \quad$ Basis functions in bending $\left(\Upsilon_{i}(x)\right.$ and $\left.\Psi_{i}(x)\right)$ and in torsion $\left(\Omega_{i}(x)\right) \ldots \ldots \ldots$

3.2 Visualization of the Modified Newton-Raphson algorithm . . . . . . . . . . . . 100

3.3 Convergence of the Newton-Raphson algorithm ～. . . . . . . . . . . . . . . . . . 101

3.4 Visualization of the convergence criteria for the Newton-Raphson algorithm. . . . . 102

3.5 Free vibration time trace . . . . . . . . . . . . . . . . . . . . . 105

$3.6 \quad$ Free vibration phase plane diagram $\ldots \ldots \ldots \ldots$

$3.7 \quad$ Free vibration power spectrum $\ldots \ldots \ldots \ldots \ldots \ldots$

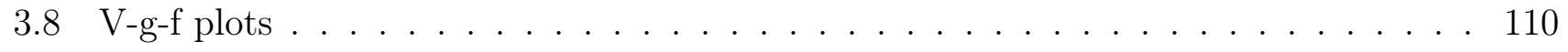

3.9 Time traces for $10 \%$ below the linear flutter speed $\ldots \ldots \ldots \ldots$

3.10 Time traces at the linear flutter speed $\ldots \ldots \ldots \ldots$

3.11 Time traces at $10 \%$ above the linear flutter speed $\ldots \ldots \ldots$. . . . . . . . 113

3.12 Limit cycle oscillations for various initial conditions . . . . . . . . . . . . . . . 114

3.13 Nodes along the span of the wing, discretized by finite difference . . . . . . . . . 115

3.14 Finite difference spanwise discretization $\ldots \ldots \ldots \ldots \ldots \ldots$

3.15 Convergence of Galerkin method with increasing number of basis functions . . . . 119

3.16 Convergence of Galerkin method with increasing number of basis functions . . . . . 120

3.17 Convergence of finite differene method with increasing spatial resolution. . . . . . . 122

3.18 Convergence of finite differene method with increasing spatial resolution. . . . . . . 123

3.19 Sparsity of the Jacobian matrices with a linear structural model . . . . . . . . . . 124

3.20 Sparsity of the Jacobian matrices for a structural model with inertial nonlinearity . 125

3.21 Sparsity of the Jacobian matrices for a structural model with stiffness nonlinearity . 125

3.22 Comparison of Galerkin projection and finite difference scheme . . . . . . . . . . . 127

3.23 Comparison of Galerkin projection and finite difference scheme . . . . . . . . . . 128

3.24 Comparison of Galerkin projection and finite difference scheme . . . . . . . . . . 129 
4.1 Unnormalized Legendre polynomials, $P_{n}(x)$ on $-1 \leq x \leq 1$, for $n=0, \ldots, 5$. . . . . 150

$4.2 \quad$ Full tensor grid weighted quadrature points in 1-D . . . . . . . . . . . . . . . . . . . 153

$4.3 \quad$ Full tensor grid weighted quadrature points in 2-D . . . . . . . . . . . . . . . . . . . 154

4.4 Full tensor grid weighted quadrature points in 3-D . . . . . . . . . . . . . . . . . . . 160

4.5 PCE basis $\left(\Psi_{\alpha}, \alpha=1, \ldots, 21\right)$ for a $5^{\text {th }}$ order expansion in two dimensions . . . . . 165

$4.6 \quad$ Flutter speed response surface . . . . . . . . . . . . . . . . . . . . . . . . . . . 166

4.7 Flutter frequency response surface . . . . . . . . . . . . . . . . . . . . . . . . . 166

4.8 Uniform input probability density functions . . . . . . . . . . . . . . . . . . . . . . 167

4.9 Output marginal and joint probability density functions . . . . . . . . . . . . . . . 167 


\title{
Nomenclature
}

\section{List of Symbols}

\author{
Derivative notation \\ ()$^{+}(), \partial() / \partial s$ \\ ()$^{\prime} \partial() / \partial x$ \\ () $\partial() / \partial t$ \\ $\delta() \quad$ Variation of () \\ $d() \quad$ Differential of ()

\section{Geometric relations} \\ $\alpha \quad$ Euler angle about the $y$-axis \\ $\beta \quad$ Euler angle about the $z$-axis \\ $\gamma \quad$ Euler angle about the $x$-axis \\ $\omega_{y} \quad$ Bending curvature about the $y$-axis \\ $\omega_{z} \quad$ Bending curvature about the $z$-axis \\ $\omega_{x} \quad$ Rate of torsional deformation about the $x$-axis \\ $\mathbf{T}$ Transformation matrix mapping between $(x, y, z)$ and $(\mathbf{x}, \mathbf{y}, \mathbf{z})$ \\ $r_{0}$ position vector to a point along the undeformed elastic axis \\ $r_{1}$ position vector to the same point along the deformed elastic axis \\ $\left[x_{1}, y_{1}, z_{1}\right]$ components of vector $r_{1}$ along $[i, j, k]$
}




\section{Coordinate systems}

$[\mathbf{I}, \mathbf{J}, \mathbf{K}]$ Unit vectors associated with the fixed reference frame

$[i, j, k]$ Unit vectors associated with the rotating reference frame

$[\mathbf{i}, \mathbf{j}, \mathbf{k}] \quad$ Unit vectors associated with the local reference frame

$(\xi, \eta, \zeta)$ Sectional coordinates associated with the local reference frame

$(\mathbf{X}, \mathbf{Y}, \mathbf{Z}) \quad$ Fixed reference frame

$(x, y, z) \quad$ Rotating reference frame

$(\mathbf{x}, \mathbf{y}, \mathbf{z}) \quad$ Local reference frame

$(\xi, \eta, \zeta) \quad$ Sectional coordinates along $(\mathbf{x}, \mathbf{y}, \mathbf{z})$

$s \quad$ Spanwise location along the deformed elastic axis

$x \quad$ Spanwise location along the undeformed elastic axis

$\lambda(\eta, \zeta) \quad$ Warping function

\section{Energy, stresses and strains}

$U \quad$ Strain Energy

$T \quad$ Kinetic Energy

$b(U), b(T) \quad$ Boundary conditions from the strain and kinetic energy

$\delta W \quad$ Virtual work of aerodynamic loads

$\varepsilon_{11}, \varepsilon_{22}, \varepsilon_{33} \quad$ Normal strains (tensor notation)

$\varepsilon_{12}, \varepsilon_{13}, \varepsilon_{23}$ Shear strains (tensor notation)

$\varepsilon_{x x} \quad$ Nonzero normal strain (engineering notation)

$\gamma_{x \eta}, \gamma_{x \zeta} \quad$ Nonzero shear strains (tensor notation)

$\sigma_{x x}, \sigma_{y y}, \sigma_{z z} \quad$ Nonzero normal stress

$\tau_{x \eta}, \tau_{x \zeta} \quad$ Nonzero shear stresses

$\nu \quad$ Poisson's ratio

$E \quad$ Elastic modulus

$G \quad$ Shear modulus

$N_{\mathrm{x}} \quad$ Normal force due to longitudinal stress

$S_{\mathbf{x}} \quad$ Moment about the $\mathbf{x}$-axis due to shear stress 
$T_{\mathbf{x}} \quad$ Moment about the $\mathbf{x}$-axis due to longitudinal stress

$P_{\mathbf{x}} \quad$ Moment about the $\mathbf{x}$-axis due to warping

$M_{\mathbf{y}} \quad$ Moment about the $\mathbf{y}$-axis

$M_{\mathbf{z}} \quad$ Moment about the $\mathbf{z}$-axis

\section{Displacements}

$u(x, t) \quad$ Axial displacement of the elastic axis in the $x$ direction

$v(x, t)$ Edgewise (in-plane) bending displacement of the elastic axis in the $y$ direction

$w(x, t) \quad$ Flapwise (out-of-plane) bending displacement of the elastic axis in the $z$ direction

$\phi(x, t) \quad$ Flexible twist displacement measuring the rotation of the cross section about the $\mathbf{x}$-axis

$\theta(t) \quad$ Rigid body pitch rotation measuring the rotation about the $\mathbf{X}$-axis

$\mathcal{V}(\mathcal{X}, \mathcal{T}) \quad$ Nondimensional edgewise bending displacement

$\mathcal{W}(\mathcal{X}, \mathcal{T}) \quad$ Nondimensional flapwise (out-of-plane) bending displacement

$\varphi(\mathcal{X}, \mathcal{T}) \quad$ Nondimensional flexible twist displacement

$\vartheta(\mathcal{T}) \quad$ Nondimensional rigid body pitch rotation

\section{Cross-sectional properties}

\section{$E A \quad$ Axial stiffness}

$E I_{\mathbf{y}} \quad$ Bending stiffness for bending about the $\mathbf{y}$-axis

$E I_{\mathbf{z}} \quad$ Bending stiffness for bending about the $\mathbf{z}$-axis

GJ Torsional stiffness for twisting about the $\mathbf{x}$-axis

$K_{\theta} \quad$ Stiffness of the torsional spring at the base of the wing

$\bar{m} \quad$ Mass of the wing per unit length

$\bar{I}_{0} \quad$ Mass moment of inertia of the wing about the elastic axis per unit length

$I_{\theta} \quad$ Mass moment of inertia of the support mechanism at the base of the wing about the pitch axis

$D_{\theta} \quad$ Structural damping coefficient in the pitch degree of freedom

$e \quad$ Distance from elastic axis to mass centroid (positive when center of gravity is ahead of elastic axis)

$e_{p} \quad$ Distance from elastic axis to pitch axis (positive when pitch axis is aft of elastic axis) 
$e_{A} \quad$ Distance from elastic axis to area centroid (positive when tensile axis is ahead of elastic axis)

$e_{c}$ Distance from elastic axis to the aerodynamic center (positive when aerodynamic center is ahead of elastic axis)

1 Nondimensional axial stiffness

$\mathcal{K}_{\mathbf{y}} \quad$ Nondimensional form of $E I_{\mathbf{y}}$

$\mathcal{K}_{\mathbf{z}} \quad$ Nondimensional form of $E I_{\mathbf{z}}$

$\mathcal{K}_{\mathbf{x}} \quad$ Nondimensional form of $G J$

$\mathcal{K}_{\theta} \quad$ Nondimensional form of $K_{\theta}$

1 Nondimensional mass of the wing per unit length

$\mathcal{I}_{0} \quad$ Nondimensional form of $\bar{I}_{0}$

$\mathcal{M}_{\theta} \quad$ Nondimensional form of $I_{\theta}$

$\mathcal{D}_{\theta} \quad$ Nondimensional form of $D_{\theta}$

$\mathcal{E}$ Nondimensional form of $e$

$\mathcal{E}_{p} \quad$ Nondimensional form of $e_{p}$

$\mathcal{E}_{A} \quad$ Nondimensional form of $e_{A}$

$\mathcal{E}_{c} \quad$ Nondimensional form of $e_{c}$

\section{Aerodynamic properties}

$x_{f} \quad$ Dimension from leading edge of wing/airfoil to axis of rotation

$\alpha$ Angle of attack or pitch (for two degree-of-freedom airfoil)

$h$ Heave or plunge (for two degree-of-freedom airfoil)

$\rho$ Density of air

$U \quad$ Free-stream air velocity

$c$ Chord length

$S \quad$ Span length

$L \quad$ Lift force

$M_{E A} \quad$ Aerodynamic moment about the elastic axis

$C_{L_{\alpha}} \quad$ Static lift coefficient

$C_{M_{A C}} \quad$ Static moment coefficient about the aerodynamic center 
$C(k)$ Theodorsen's function

$\varphi$ Wagner's function

$w_{3 c / 4}$ Downwash

$z, A_{1}, A_{2}, b_{1}, b_{2} \quad$ Unsteady aerodynamic terms

$\mathcal{P}$ Nondimensional form of $\rho$

$\mathcal{U}$ Nondimensional form of $U$

$\mathcal{C}$ Nondimensional form of $c$

1 Nondimensional form of $S$

$\tilde{w}_{3 c / 4} \quad$ Nondimensional form of $w_{3 c / 4}$

$\mathcal{Z}$ Nondimensional form of $z$

\section{Numerical Simulation}

$\Upsilon_{i}$ Basis functions for the edgewise bending displacement $(i=1, \ldots, L)$

$\Psi_{i} \quad$ Basis functions for the flapwise bending displacement $(i=1, \ldots, M)$

$\Omega_{i} \quad$ Basis functions for the flexible twist angle $(i=1, \ldots, N)$

$V_{i} \quad$ Unknown temporal coordinate for the edgewise bending displacement

$W_{i} \quad$ Unknown temporal coordinate for the flapwise bending displacement

$\Phi_{i} \quad$ Unknown temporal coordinate for the flexible twist angle

$\Theta$ Unknown temporal coordinate for the rigid body pitch rotation

$q_{i} \quad i^{\text {th }}$ element of the state vector

$\Delta x \quad$ Spatial discretization length

$\Delta t \quad$ Time step

\section{Global Sensitivity Analysis}

$X_{i} \quad i^{\text {th }}$ uncertain input parameter

$X_{\sim i}$ the set of all uncertain input parameters except $X_{i}$

$S_{i} \quad$ Sensitivity index for parameter $X_{i}$

$S_{T_{i}} \quad$ Total effect sensitivity index of parameter $X_{i}$

$V() \quad$ Variance of () 
E() Expected value of ()

$\Psi_{\alpha} \quad$ Multivariate polynomial chaos basis functions

$\psi_{\alpha_{i}} \quad$ Univariate polynomial chaos basis functions

$\alpha \quad$ Multi-indices

$c_{\alpha} \quad$ Deterministic polynomial chaos coefficients

$\xi_{i} \quad$ Random variable representing input parameter $X_{i}$

$A$ Order of polynomial expansion

$k \quad$ Number of uncertain input parameters 


\section{List of Abbreviations}

\section{Research institutions \& agencies}

NACA National Advisory Committee for Aeronautics

ONERA Office National d'Études et de Recherches Aérospatiales

RMC Royal Military College of Canada

\section{Mathematical abbreviations}

PDE Partial differential equations

ODE Ordinary differential equations

BC Boundary condition

IC Initial condition

PCE Polynomial chaos expansion

GSA Global sensitivity analysis

MC Monte Carlo

pdf Probability density function

HDMR High Dimensional Model Representation

\section{Other abbreviations}

UAV Unmanned air vehicle

SHM Simple harmonic motion

DOF Degree-of-freedom

LCO Limit cycle oscillations

V-g-f Speed, damping, frequency plot 


\section{Chapter 1}

\section{Introduction}

Aeroelasticity is the field of study that focuses on the coupled dynamics of flexible structures and airflow around them. This branch of the broader study of fluid-structure interaction considers the coupling mechanism between the structure and the airflow rather than investigating structural dynamics or aerodynamics in isolation. The most evident application of this field of research is perhaps the design and analysis of aircraft components. However, aeroelasticity has significant implications on the behaviour of constructed engineering structures such as high-rise buildings, long span bridges, wind turbines or transmission lines.

This body of work will focus on a specific aeroelastic system. A flexible cantilever wing that undergoes axial deformation, edgewise (or in-plane) bending, flapwise (or out-of-plane) bending, as well as torsion, and is physically coupled with a rigid body pitch rotation by means of a support mechanism. The flexible cantilever is rigidly connected to this nonzero mass support mechanism at the root which allow for rotation about its longitudinal axis restrained by a torsional spring, while preventing rotation and translation in all other directions as depicted in Figure 1.1 The extension of a cantilever wing to include the effects of a rigid body pitch rotation in an aeroelastic system introduces the potential for very complex and interesting dynamical behaviour. Large oscillation angles may be observed between the chord of the wing and the direction of the free stream air 
velocity, leading to nonlinearity in the aerodynamic forcing. Additionally moderate to large displacements may arise which lead to nonlinear coupling between the structural displacements as well.

Hodges and Dowell [1] provides an elegant derivation of nonlinear differential equations of motion for the elastic bending and torsional vibrations of rotor blades, which can reasonably be applied to study the coupled dynamics of the cantilever wing used in the current study. However, modelling the coupling of the flexible vibrations of the cantilever with a rigid body motion in pitch required that the equations from [1] be appropriately modified, as in da Costa [2]. This extension to the original equations of motion was subsequently refined by Robinson et al. $[3]$.

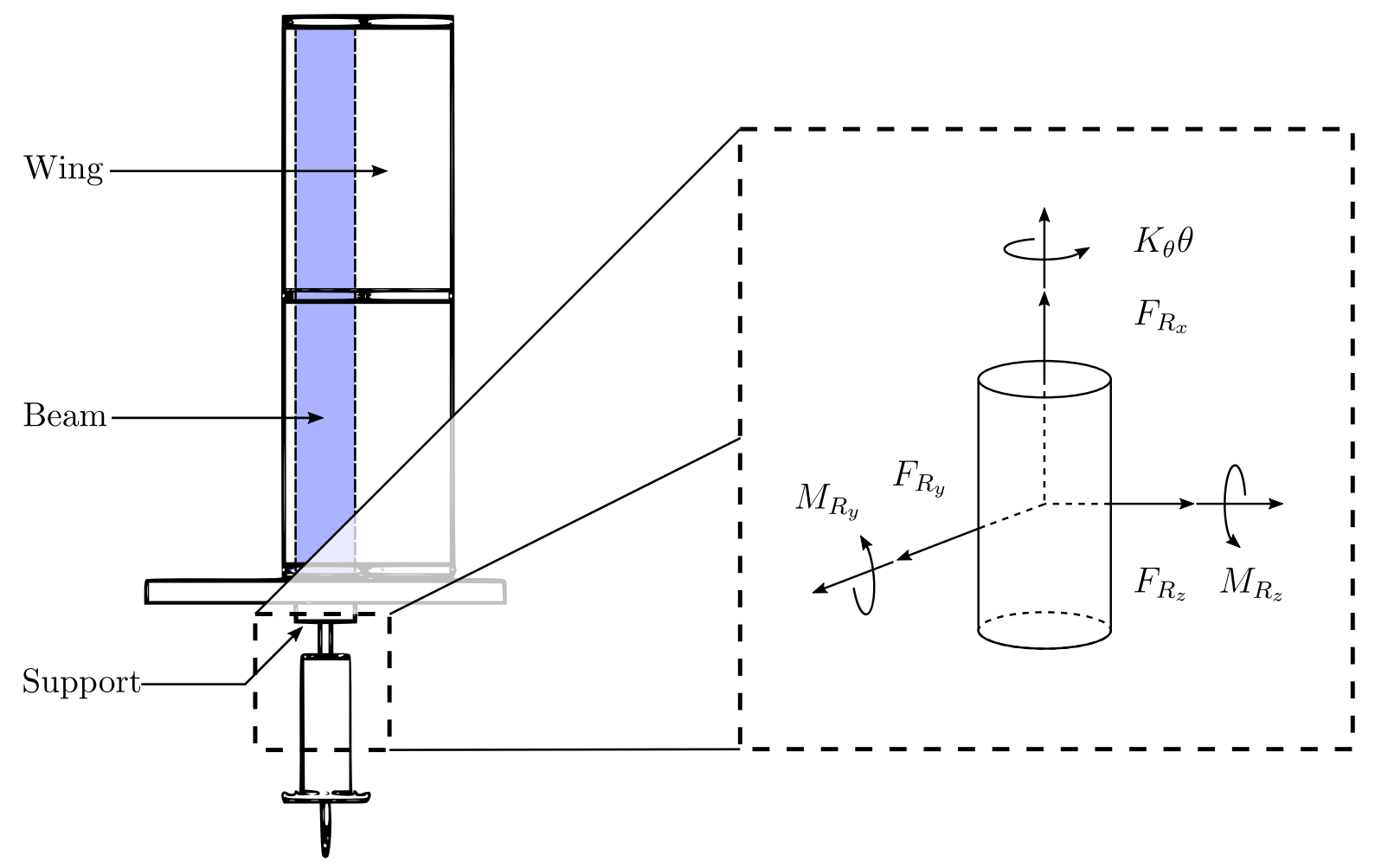

Figure 1.1: The leftmost image is a schematic of the experimental wing setup at the Royal Military College (RMC) of Canada in Kingston, ON. The rightmost image provides an illustration of the support reactions of the support mechanism. The flexible wing is rigidly connected to this support, and the support itself resists translation in all directions as well as rotation about all but one axis.

This system described above is that of a wind-tunnel setup at the Royal Military College (RMC) of Canada in Kingston, Ontario. A schematic of the wing is shown in Figure 1.1 while a plan view of the wind tunnel itself is featured in Figure 1.2 This particular wind tunnel set-up is 
instrumented with sensors (strain gauges and accelerometers) at multiple locations along the span. The strain gauge rosettes allow for flexible bending and torsional deformation to be measured, while accelerometers allow for for the analysis of modal parameters, as well as the indirect measurement of absolute displacements of the wing through double integration of the readings. Additionally, the rigid body pitch rotation is measured using a contactless potentiometer. The use of the data from experiments performed with this setup in conjunction with the simulations performed using the model derived herein, will serve as a testbed for the development of tools and algorithms for inverse uncertainty quantification relevant to highly nonlinear dynamical systems (state estimation and combined state and parameter estimation).

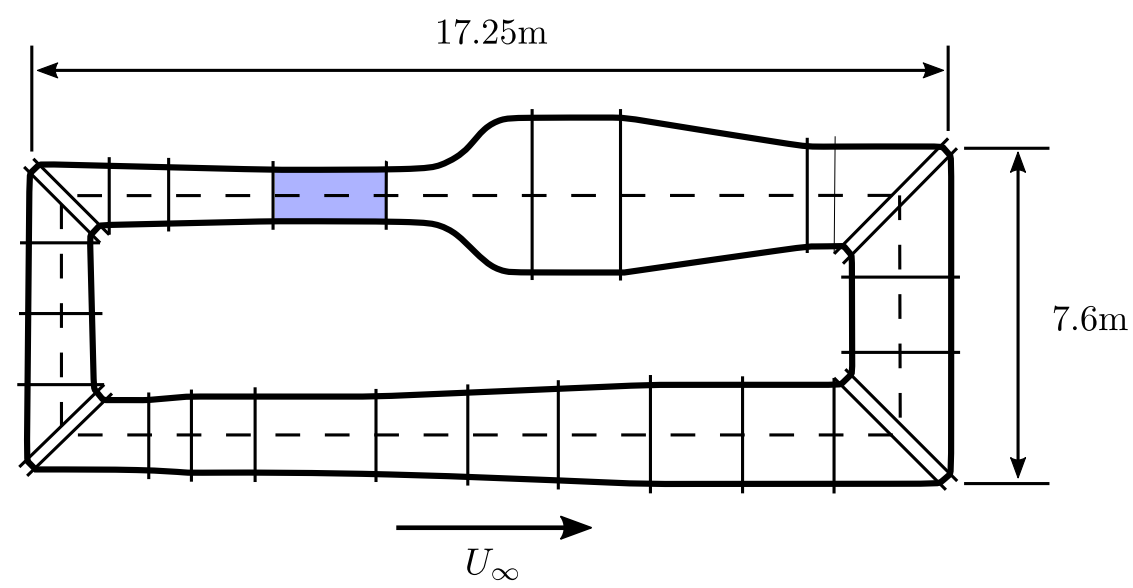

Figure 1.2: Plan view of the wind tunnel at RMC; the test section is highlighted in blue, and the airflow is indicated by the arrow (adapted from $\sqrt{4}$ ).

From previous experiments performed on similar wind tunnel setups [2], it is expected that the system is likely to become dynamically unstable at low airspeeds, in the neighbourhood of $10 \mathrm{~m} / \mathrm{s}$. Given the size of the wing used in these experiments, this corresponds to a Reynolds Number in the order of $10^{5}$ which would typically classify the flow as transitional between laminar and turbulent flow [5] [6]. Low Reynolds effects (in particular flow separation at small angles of attack) and high Reynolds number effects (turbulent boundary layer) are not explicitly accounted for here. This investigation focuses on understanding the mechanism of dynamic instability, namely, coupled flutter as explained later, as well as geometric structural nonlinearities. 


\subsection{Literature Review}

While this thesis presents some novelty through the nonlinear description of the aforementioned dynamical system, it draws from many existing sources in the literature. The derivation of the nonlinear equations of motion for the structure shows close parallels with the works of Hodges and Dowell [1] for instance, while extending these widely cited equations to suit the current wing configuration, an endeavour which was initiated by da Costa [2]. Likewise, the aerodynamic loads adopted are lifted from widely used models in the literature that are found to be consistent with the needs of the current application [7], 8], 9. Much work has been done in the development of statistical tools for studying the forward propagation of uncertainty through dynamical systems $[10],[11],[12]$. This body of work seeks to apply the existing methods of Global Sensitivity Analysis (GSA) to study the effects of uncertainty in a a subset of the system's input parameters, using a polynomial chaos expansion of the output quantity of interest 13 .

\subsubsection{Aeroelasticity}

There are two prevailing classifications of structures in the context of wind engineering; bluff bodies and streamlined bodies. While there is no strict criteria, streamlined bodies (Figure 1.3 are characterized by smooth edges and a small ratio of dimensions perpendicular and aligned with the flow, such that the flow will tend to follow its contours. The system that this document focuses on is a wing, which is a streamlined body. The cantilever wing modelled in this investigation has the cross-sectional profile of a NACA 0012 airfoil. The four digits indicate that the cross section is symmetrical and has a maximum thickness of $12 \%$ of the chord length.

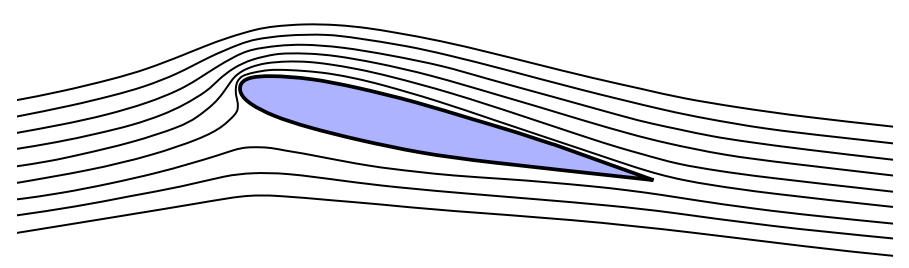

Figure 1.3: Streamlined body 
A bluff body (Figure 1.4) by contrast, is characterized by sharp corners which induce flow separation around the corners, and large dimensions perpendicular to the flow. Because of the shape, these bodies typically have large stagnation regions on the windward side. Structures in civil engineering tend to be bluff bodies, however the design of long-span bridge decks may result in them behaving more like streamlined bodies [14. These bluff bodies are susceptible to the same types of aerodynamic loading, including vortex induced vibration, galloping, divergence, aeroelastic flutter and buffeting among others [15], [14]. Wind turbines are also an interesting case, as the rotor blades themselves are streamlined, however the large cylindrical tower structures are bluff bodies. Civil engineering structures are typically subjected to wind loads which are characterized by high Reynolds numbers, indicative of turbulent flows. Note that while it is important to acknowledge these mechanisms for wind-induced vibrations and their applicability to aerospace and civil engineering structures, this body of work will focus on aeroelastic flutter.

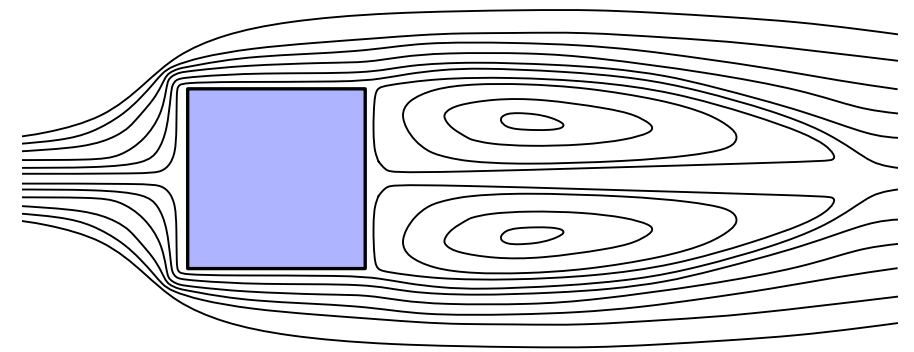

Figure 1.4: Bluff body

Aeroelasticity, and more broadly, the study of fluid-structure interaction is concerned with the feedback loop which exists between the fluid dynamics and the structural dynamics of the system (i.e. the interaction of aerodynamic forces and a structure's stiffness, damping and inertia). The fluid exerts a force on the structure as it flows around it, which will tend to cause the structure to deform. As the structure deforms, the fluid must follow a new trajectory. This in turn results in a change in the pressure distribution around the structure, and thus results in a change in the aerodynamic forces, which alters the dynamic response of the structure. The field of aeroelasticity is keenly interested in the study of the coupling between the structural dynamics and the aerodynamics [8]. Indeed, as the aerodynamic loads depend on the configuration of the structure, the aerodynamic 
forces are explicitly prescribed as a function of the structural response (displacements and velocities), hence they form part of the system's dynamics (contributing to the mass, damping and stiffness) rather than acting as an external additive forcing.

\section{Aeroelastic Flutter}

In linear systems (linear structural model coupled with a linear aerodynamic model) flutter refers to the point of dynamic instability, wherein the overall damping goes to zero, and hence the system undergoes simple harmonic motion (SHM). The flutter speed refers to the airspeed at which the structure will undergo such dynamic instability. Airspeeds below that will result in pre-flutter behaviour characterized by damped vibrations, about a stable fixed point, while airspeeds above the flutter speed will result in post-flutter behaviour, wherein the aeroelastic damping is negative, leading to vibrations with an exponentially diverging amplitude. For linear models, there are two fundamental flutter mechanisms, single degree-of-freedom flutter and two-degree of freedom flutter. Two degree of freedom flutter is often referred to as coalescence or classical flutter, as the frequencies of two coupled degrees of freedom will coalesce as the airspeed increases towards the critical flutter speed.

In the context of nonlinear aerodynamic models, there are a number of other flutter mechanisms that exist. While not the focus of the current investigation, stall flutter as it relates to nonlinearity in aerodynamics at large angles of attack imposes an important limitation on the current study. At higher angles of attack, the airflow may become separated from the wing surface leading to nonlinear behaviour 14. As this phenomenon is not accounted for in the adopted unsteady aerodynamic model, this will place limitations on the amplitude of limit cycle oscillations (LCO) that can be studied, and hold any reasonable physical meaning. The aerodynamics can largely be considered to be linear for angles of attack below $\sim 12^{\circ}[16$, [17]. In nonlinear systems, flutter will not only refer to the loss of stability, but also behaviour that occurs beyond the instability point (the flutter boundary), in the form of (self excited) LCO [18, 19. 


\section{Aeroelastic Vibrations of a Cantilever}

The rigid body pitch rotation introduced here extends the initial analytical developments of a nonlinear beam theory of non-rotating hingeless rotor blades by Houbolt and Brooks [20] and Hodges and Dowell [1] which was subsequently modified to permit forced and free rotation of the wing root in 21]. Note that this extension to include pitch rotation included a study of the system with a prescribed harmonic motion in pitch and the modelling of an imperfect hinge, hence they did not consider a point mass at the root, but rather considered the mass to be uniformly distributed along the span. Other extensions, such as 22 have included tip masses, which may be accounted for in the boundary conditions, or in the differential equations of motion. In our case, the added rigid body pitch rotation, associated with the mass moment of inertia of the base, introduces the potential for complex dynamics arising from the coupling of the rigid body pitch rotation with the flexible modes, and is accounted for by introducing an additional differential equation of motion.

The axial-bending-bending-torsion elastic equations were recently extended to account for the rigid body pitch rotation degree-of-freedom by da Costa [2] and Robinson et al. [3]. This thesis provides comprehensive account of the full derivation. Structural geometric nonlinearities are emphasized; accordingly, the aerodynamics are simply modelled based on linear unsteady theory. This is justified by small angles of attack $\left(<12^{\circ}\right)$ observed in this investigation. During investigations into the effects of free-play structural nonlinearities in aeroelastic systems, Dowell et al. [23] and O'Neil and Strganac [24] modelled the aerodynamics with a linear unsteady model and reported good agreement with wind tunnel tests.

Though a linear unsteady aerodynamic model is used in this study, a more general aerodynamic model, which accounts not only for unsteadiness but also nonlinearities such as dynamic stall, will be considered in future work. Such a model would have to account for nonlinearities arising from large angles of attack and low Reynolds number effects at small amplitudes. For instance, the semi-empirical ONERA model has been used extensively by Dowell et al. to model nonlinearities at large angels of attack subsequent to the aforementioned studies (see Tang and Dowell [21, 25] 
and Jaworski and Dowell [26]). For existing work on modelling low Reynolds number effects see Poirel and Yuan 27] and Sandhu et al. 28.

Since the formulations of the structural equations of motion by Hodges and Dowell [1], and Houbolt and Brooks [20], various refinements of the model have been proposed such as the equations used by Rosen and Friedmann [29] and Kim [30], which like their predecessors, were rooted in the nonlinear dynamics of beams.

\subsubsection{Uncertainty Quantification}

Uncertainty quantification can generally be broken into two types of problems, the forward uncertainty quantification and inverse uncertainty quantification in the context of computational mechanics [31. The former is concerned with studying the effects of uncertain inputs (defined by probability distributions) on the uncertainty in the model output of the system behaviour. The latter is concerned with using computational models and data to assess uncertainties in the model (quantifying model error) and in the parameters (obtaining joint probability distributions of the model parameters) 32 .

The nonlinear computational model of an aeroelastic cantilever is the first step to exploit the wind-tunnel data for system identification using Bayesian inference (inverse problems), extending the previous work on elastically mounted rigid airfoils (Khalil et al. [33], and Sandhu et al. [28], [34], [35]). Uncertainty quantification in aeroelasticity is important because the behaviour that is exhibited as a result of coupling of the structural dynamics and aerodynamics has the potential to be much more pronounced than in the study of either structural dynamics or aerodynamics in isolation of one another [36, 37, 38].

The current investigation seeks to use Global Sensitivity Analysis for the post processing of the forward propagation of uncertainty through the computational model as a precursor for future endeavours in inverse uncertainty quantification. In uncertainty analysis, the uncertainty in the 
input parameters is propagated forward through a model, and the uncertainty in the output is quantified. In contrast to this, in sensitivity analysis, after the uncertainty has been propagated forward through the model, the modeller seeks to apportion the uncertainty in the model output back to the uncertain input parameters. In this way, it provides a means by which the relationship between the various uncertain inputs and the output quantity of interst can be assessed [12]. Hence, it allows for the identification of parameters which are of the greatest influence on the uncertainty of the output. This can lead to the reduction of the stochastic dimensionality of the system when studying specific quantities of interest, as parameters that have a non-influential relationship with that output can be assigned fixed values. Note that the results of Global Sensitivity Analysis are dependent on the selection of the output quantity of interest, implying that parameters that are deemed non-influential for one model output are not necessarily non-influential for all potential outputs of the system.

\subsection{Scope of the Thesis}

This thesis encompasses the full derivation of the coupled nonlinear equations of motion for a cantilever undergoing flexible bending and torsion coupled with a rigid body base rotation in Chapter 2. This chapter will follow the work of Hodges and Dowell [1] closely, as well as previous extensions by da Costa [2], whereby the equations of motion are derived using Hamilton's principle. Subsequently the aerodynamic loads will be formulated using the linear theory of unsteady aerodynamics. This formulation is shown for the case of a simple two degree-of-freedom airfoil undergoing pitch and plunge motion. This aerodynamic model is then extended to the case of a flexible cantilever by accounting for motion in three dimensions.

The numerical solution of the coupled differential equations is the subject of Chapter 3 . which delves into the solution by finite dimensional representation by Galerkin projection. The implementation of the Galerkin projection into an efficient solver is investigated. Beyond this, the results obtained

by Galerkin projection are verified by comparison to the direct integration of the equations of motion by finite difference scheme for spatial discretization. This section will feature the results 
from numerical experiments for the structural free vibration as well as for the coupled aeroelastic vibrations of the structure.

Finally, Chapter 4 will investigate the forward propagation of uncertainty through the fully nonlinear model by Global Sensitivity Analysis. GSA provides a framework for modellers to evaluate the relative importance of uncertainty in the model inputs on various outputs of the model.

Finally, Chapter 5 will provide brief concluding remarks regarding the investigation, while providing an outline of future steps and considerations. 


\section{Chapter 2}

\section{Derivation of the Nonlinear Equations of Motion}

This chapter covers the derivation of the equations of motion for a pitching flexible cantilever wing. These equations of motion model the axial displacement, the edgewise (in-plane) bending, the flapwise (out-of-plane) bending and the flexible twist angle, as well as a rigid body pitch motion. This derivation follows the methods employed by Hodges and Dowell [1] closely, while introducing new terms arising from the fundamentally new kinematics of the wing. The Hodges and Dowell equations are derived for applications to rotor blades where the axis of rotation is orthogonal to the longitudinal axis of the blade and where the angular velocity is a parameter, as in the case for helicopters or wind turbines [1]. In the current investigation, the axis of rotation is aligned with the longitudinal axis of the wing, and the rotation itself is a time varying degree of freedom, rather than a prescribed fixed value. Accordingly, these equations of motion were extensively adapted by da Costa [2] to suit the current system. This includes a new definition of the system's kinematics in Eq. 2.89), as well as the inclusion of the contribution of the torsional spring at the root to the strain energy of the system in Eq. (2.56), and the inclusion of the contribution of the nonzero mass base to the kinetic energy of the system in Eq. (2.88). The current body or work presents a refinement of the efforts of da Costa [2] to couple the flexible dynamics of a continuous wing with a rigid body base rotation about the longitudinal axis. This body of work introduces generalities and relaxes some simplifying assumptions made in the previous initiative [2]. Specifically, the derivation that follows allows for an arbitrary eccentricity between the pitch axis and the elastic axis, where 
they were previously only considered to be coincident. Furthermore, this derivation includes terms relating to warping effects from the torsion of a non-circular member, and includes the effects of axial deformation in order to be applicable for larger deflections. The result is a system of equations which builds off the work in [2], but are more consistent with other applications of the Hodges and Dowell equations that have been seen elsewhere in the literature, such as in 22 , [39.

As depicted in Figure 2.1, the wing set-up being modelled is mounted vertically such that there is no sweep, and no gravitational effects. The wing's cross section is that of a NACA 0012 airfoil, which is symmetrical about the chord line. The wing has a constant chord, which is commonly referred to as a rectangular wing (as it is rectangular in elevation). While the wing being studied has a constant cross section, the derivation presented herein is applicable to wings which have a spanwise variation in the cross-sectional properties as well. However, the wing must be unswept.
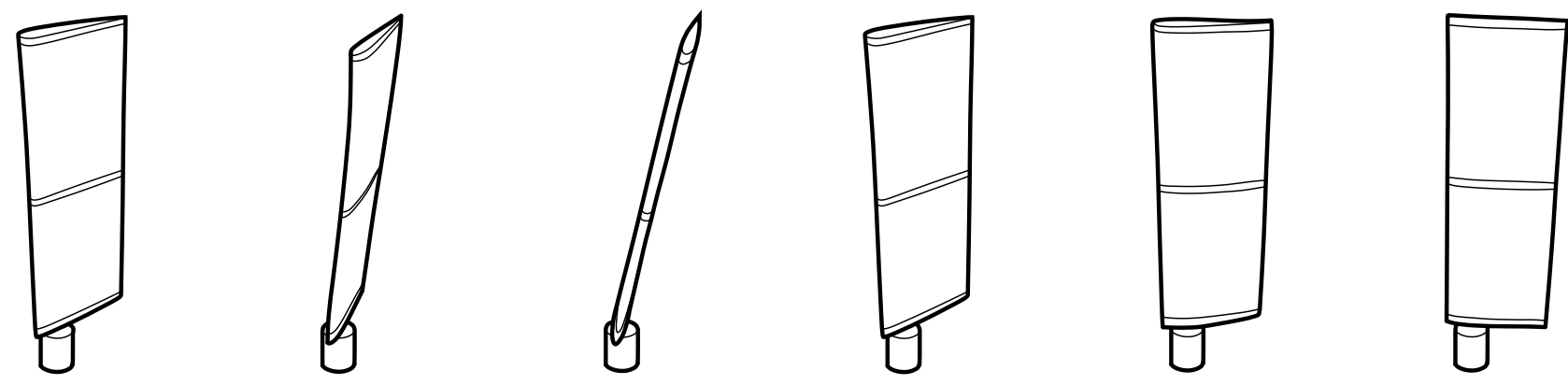

Figure 2.1: Illustration of snapshots the aeroelastic system's motion over a portion of a cycle

Figure 2.2a shows a large airplane, whose wings are swept backwards, and the chord length also changes along the span. The single engine airplane in Figure $2.2 \mathrm{~b}$ and the unmanned air vehicle in Figure 2.2c have unswept wings. The UAV has some tapering along the span from the root to the tip, while the single engine airplane has a constant chord length (rectangular). The equations of motion as derived herein are connected to the latter two aircraft configurations, as the wings are unswept. The analysis in this thesis focuses on a rectangular wing. However, the equations are defined in such a way that the cross-sectional properties can be defined to be either constant along the span (as in the case of a rectangular wing) or can be defined as functions of the position along the span (as is the case of a tapered wing). 


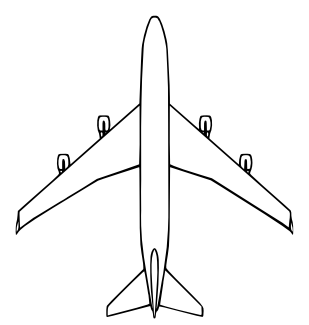

(a) Boeing 747

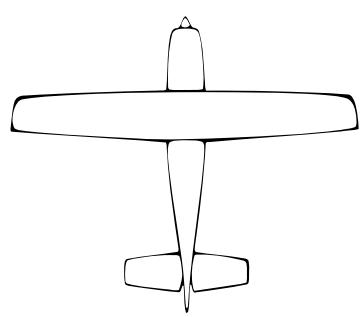

(b) Cessna 172

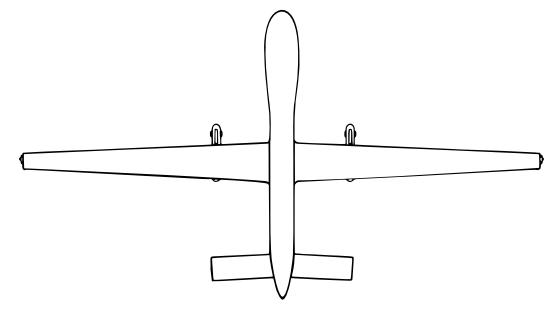

(c) UAV

Figure 2.2: Wing configurations on large, small, and unmanned aircraft

The aerodynamic loads are modelled according to established theories of linear unsteady aerodynamics. A lift force and aerodynamic moment are modelled, while the drag force is ignored in the current investigation due to the small angles of attack that are observed during full aeroelastic simulations.

\subsection{Transformed Coordinate System}

The complex dynamics of this system necessitates the use of a number of frames of reference. They consist of the fixed coordinate system $(\mathbf{X}, \mathbf{Y}, \mathbf{Z})$, a rotating frame of reference $(x, y, z)$, which follows the wing as it undergoes rigid body pitch rotation (see Figure 2.3), and a local frame of reference $(\mathbf{x}, \mathbf{y}, \mathbf{z})$ for the deformed cross section of the wing as a function of the position along the span. The use of three coordinate systems allows for a simpler account of the rigid body pitch rotation coupled with the flexible vibrations, whereby the flexible vibrations are captured within a rotating reference frame, and the rotation of this reference frame is then measured relative to a fixed global reference frame.

This first transformation relates the fixed axes $(\mathbf{X}, \mathbf{Y}, \mathbf{Z})$ to the rotating axes $(x, y, z)$ as the entire flexible structure is rotated rigidly by an angle $\theta$ about the pitch axis (defined by the 
$\mathbf{X}$ axis). This transformation captures the rigid body pitch rotation of the wing about the $\mathbf{X}$ axis (termed the pitch axis), which is a distance of $e_{p}$ aft of the $x$ axis (termed the elastic axis). The unit vectors for the coordinate systems $(\mathbf{X}, \mathbf{Y}, \mathbf{Z})$ and $(x, y, z)$ are are $[\mathbf{I} \mathbf{J} \mathbf{K}]$ and $[i j k]$, hence

$$
\left\{\begin{array}{c}
i \\
j \\
k
\end{array}\right\}=\left[\begin{array}{ccc}
1 & 0 & 0 \\
0 & \cos \theta & \sin \theta \\
0 & -\sin \theta & \cos \theta
\end{array}\right]\left\{\begin{array}{c}
\mathbf{I} \\
\mathbf{J} \\
\mathbf{K}
\end{array}\right\}+\left\{\begin{array}{c}
0 \\
e_{p} \cos \theta \\
e_{p} \sin \theta
\end{array}\right\} .
$$

In Figure 2.3, the $\mathbf{X}$ axis describes the pitch axis (denoted by $P A$ ), while the physical interpretation of the $x$ axis is that it describes the undeformed elastic axis of the wing (denoted by $E A$ ). The $\mathbf{Y}$ and $\mathbf{Z}$ axes lie in the same plane as the $y$ and $z$ axes. Since the transformation below captures a rigid body displacement, the orientation of the $y$ and $z$ axes in relation to the $\mathbf{Y}$ and $\mathbf{Z}$ axes are independent of the position along the span.

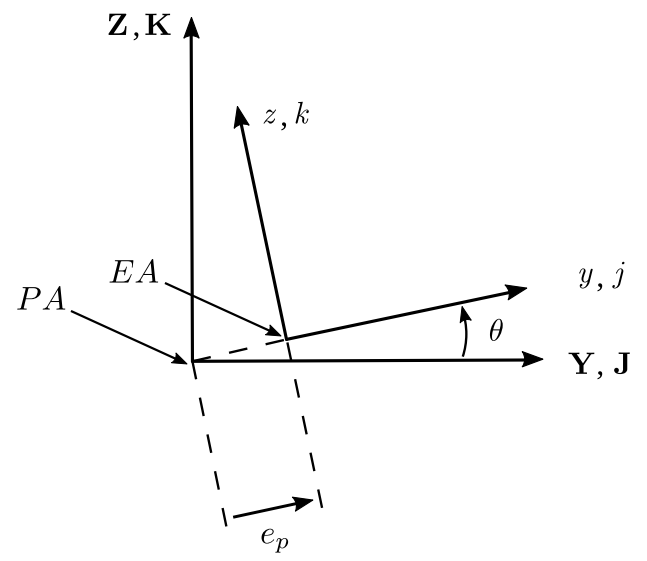

Figure 2.3: The coordinate systems for rigid body pitch rotation

The matrix $\mathbf{T}$ performs the transformation from the coordinate systems $(x, y, z)$ to $(\mathbf{x}, \mathbf{y}, \mathbf{z})$, where $\mathbf{x}$ is tangent to the deformed elastic axis (located a distance $e_{p}$ ahead of the pitch axis), $\mathbf{y}$ is aligned with the chord of the cross section such that it and $\mathbf{z}$ define the plane containing the cross section of the deformed wing. The unit vector $[\mathbf{i} \mathbf{j} \mathbf{k}]$ denotes the directions along the frame $(\mathbf{x}, \mathbf{y}, \mathbf{z})$. This transformation maps between the rotating frame of reference, to a set of axes which are local to the 
cross section of the wing along the span as it deforms. Unit vectors $[\mathbf{i} \mathbf{j} \mathbf{k}]$ and $[i j k]$ satisfy the following relation as in [1]:

$$
\left\{\begin{array}{l}
\mathbf{i} \\
\mathbf{j} \\
\mathbf{k}
\end{array}\right\}=\mathbf{T}\left\{\begin{array}{l}
i \\
j \\
k
\end{array}\right\} .
$$

where $\mathbf{T}$ is the product of the transformation matrices $\mathbf{T}_{x}, \mathbf{T}_{y}$, and $\mathbf{T}_{z}$ for the rotations about each of the coordinate axes $(x, y, z)$ respectively as in Eq. (2.6).

The transformation $\mathbf{T}$ is defined as a function of the displacements $u, v, w$, and $\phi$ which are depicted in Figure 2.4 $u, v$ and $w$ are three orthogonal displacements along $x, y$ and $z$ respectively, which capture the position of the deformed elastic axis relative to its undeformed position. Furthermore, $\phi$ captures the rotation of the cross section about the deformed elastic axis, given by $\mathbf{x}$.

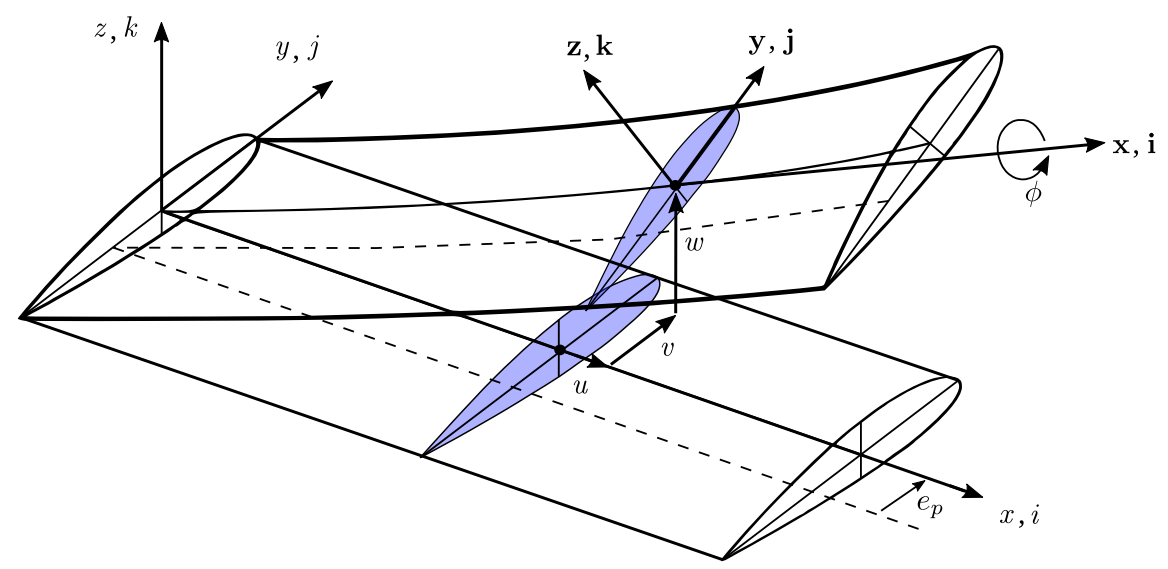

Figure 2.4: Displacements $u, v$ and $w$ are defined within the rotating reference frame $(x, y, z)$, and the flexible twist angle $\phi$ is defined about the $\mathbf{x}$ axis. The transformation matrix, $\mathbf{T}$ maps to the rotating $(x, y, z)$ coordinate system to the local $(\mathbf{x}, \mathbf{y}, \mathbf{z})$ frame of reference (adapted from [1]

The relation between the rotating reference frame and the local coordinates of the cross section is obtained from the Euler angles about the $(x, y, z)$ axes. Rotating $\gamma$ about the $x$ axis, $\alpha$ about $y$, and $\beta$ about $z$, one is able to obtain geometric relationships for the sines and cosines of $\alpha$ and $\beta$, in terms of the differential displacements $d v$ and $d w$ for a differential length $d s$. However, we are 
unable to directly obtain a relationship for the third Euler angle $\gamma[1]$.

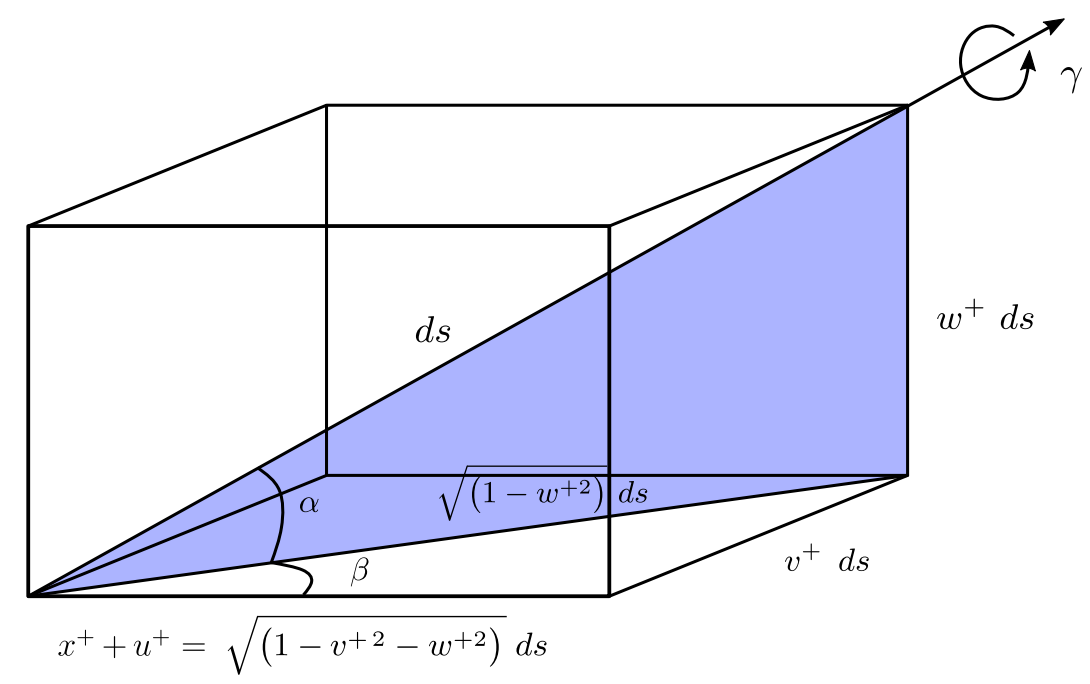

Figure 2.5: Euler Angles $\gamma, \alpha, \beta$ (adapted from [1] [2])

Referring to Figure 2.5 we can define geometrical relations for the sines and cosines of $\alpha$ and $\beta$ for the rotations about the $y$ and $z$ axes as shown below. Further, we will need to obtain an additional relationship in order to obtain some geometrical definition for the rotation about the $x$ axis, the unknown Euler angle $\gamma$. The component matrices of the transformation matrix are as follows [1]:

$$
\begin{aligned}
& \mathbf{T}_{x}=\left[\begin{array}{ccc}
1 & 0 & 0 \\
0 & \cos \gamma & \sin \gamma \\
0 & -\sin \gamma & \cos \gamma
\end{array}\right] \\
& \mathbf{T}_{y}=\left[\begin{array}{ccc}
\cos \alpha & 0 & \sin \alpha \\
0 & 1 & 0 \\
-\sin \alpha & 0 & \cos \alpha
\end{array}\right]=\left[\begin{array}{ccc}
\sqrt{1-w^{+2}} & 0 & w^{+} \\
0 & 1 & 0 \\
-w^{+} & 0 & \sqrt{1-w^{+2}}
\end{array}\right] \\
& \mathbf{T}_{z}=\left[\begin{array}{ccc}
\cos \beta & \sin \beta & 0 \\
-\sin \beta & \cos \beta & 0 \\
0 & 0 & 1
\end{array}\right]=\left[\begin{array}{ccc}
\sqrt{\frac{1-v^{+2}-w^{+2}}{1-w^{+2}}} & \frac{v^{+}}{\sqrt{1-w^{\prime 2}}} & 0 \\
-\frac{v^{+}}{\sqrt{1-w^{+2}}} & \sqrt{\frac{1-v^{+2}-w^{+2}}{1-w^{+2}}} & 0 \\
0 & 0 & 1
\end{array}\right]
\end{aligned}
$$


Having defined each of the transformation matrices pertaining to $\alpha, \beta$, and $\gamma$, as in [1], we can write

$$
\begin{gathered}
\mathbf{T}=\mathbf{T}_{x} \mathbf{T}_{y} \mathbf{T}_{z}=\left[\begin{array}{ccc}
\cos \alpha \cos \beta & \cos \alpha \sin \gamma & \sin \alpha \\
-\cos \beta \sin \gamma \sin \alpha-\sin \beta \cos \gamma & -\sin \beta \sin \gamma \sin \alpha+\cos \beta \cos \gamma & \sin \gamma \cos \alpha \\
-\cos \beta \cos \gamma \sin \alpha+\sin \beta \sin \gamma & -\sin \beta \cos \gamma \sin \alpha-\cos \beta \sin \gamma & \cos \gamma \cos \alpha
\end{array}\right] \\
=\left[\begin{array}{ccc}
\frac{v^{+}}{\sqrt{1-v^{+2}-w^{+2}}} \\
\frac{-w^{+} \sin \gamma \sqrt{1-v^{+2}-w^{+2}}-v^{+} \cos \gamma}{\sqrt{1-w^{+2}-}} \\
\frac{-v^{+} w^{+} \sin \gamma+\cos \gamma \sqrt{1-v^{+2}-w^{+2}}}{\sqrt{1-w^{+2}}} & \sin \gamma \sqrt{1-w^{+2}} \\
\frac{-w^{+} \cos \gamma \sqrt{1-v^{+2}-w^{+2}}-v^{+} \sin \gamma}{\sqrt{1-w^{+2}}} & \frac{-v^{+} w^{+} \cos \gamma-\sin \gamma \sqrt{1-v^{+2}-w^{+2}}}{\sqrt{1-w^{+2}}} & \cos \gamma \sqrt{1-w^{+2}}
\end{array}\right] .
\end{gathered}
$$

This transformation maps between the rotating coordinate system $(\mathbf{x}, \mathbf{y}, \mathbf{z})$ and the local coordinate system of the deformed cross section $(x, y, z)$. Hence, when there is no deformation, as one would expect, the transformation matrix reduces to a $3 \times 3$ identity matrix [1],

$$
\left.\mathbf{T}\right|_{v=w=\phi=0}=\left[\begin{array}{ccc}
1 & 0 & 0 \\
0 & 1 & 0 \\
0 & 0 & 1
\end{array}\right]
$$

One can construct a differential equation of the form $\mathbf{T}^{+}=\omega \mathbf{T}$ where the ()$^{+}$notation indicates the derivative with respect to $s$. This $\omega$ matrix is the cross product matrix of vector of curvatures, given by $\left\{\omega_{x} \omega_{y} \omega_{z}\right\}^{T}$. The skew-symmetric matrix of curvatures is thus 1

$$
\omega=\left[\begin{array}{rrr}
0 & -\omega_{z} & \omega_{y} \\
\omega_{z} & 0 & -\omega_{x} \\
-\omega_{y} & \omega_{x} & 0
\end{array}\right] .
$$

Noting that due to the orthonormality of the transformation matrix, the inverse of $\mathbf{T}$ is equal 
to its transpose, such that one can write $\mathbf{T}^{+} \mathbf{T}^{\mathbf{T}}=\omega^{\mathbf{T}}$. Note that because the matrix, $\omega$ is skew-symmetrix, $\omega^{\mathbf{T}}=-\omega$. Hence, the following is obtained, which once evaluated yields 9 equations, 3 of which are independent equations [1,

$$
\mathbf{T}^{+} \mathbf{T}^{\mathbf{T}}=\left[\begin{array}{rrr}
0 & \omega_{z} & -\omega_{y} \\
-\omega_{z} & 0 & \omega_{x} \\
\omega_{y} & -\omega_{x} & 0
\end{array}\right] .
$$

One should note that $\omega_{x}$, gives the rate of the rotation about the $x$ axis, which is equivalent to $\partial \phi / \partial s\left(\right.$ or $\left.\phi^{+}\right)$. Consistent to a second order approximation scheme, we can say that $d s \approx d x$ and adopt a ()$^{\prime}$ notation for the derivative with respect to $x$, replacing our ()$^{+}$convention. As in [1], solving Eq.(2.9) allows us to write each of these curvatures as follows [1]:

$$
\begin{aligned}
& \omega_{x}=\phi^{\prime}, \\
& \omega_{y}=v^{\prime \prime} \sin \phi-w^{\prime \prime} \cos \phi, \\
& \omega_{z}=v^{\prime \prime} \cos \phi+w^{\prime \prime} \sin \phi .
\end{aligned}
$$

Additionally, the solution of Eq. 2.9 gives the third Euler Angle $\gamma$ to the second order, which can be written as in $[1$

$$
\gamma=\phi-\int_{0}^{x} v^{\prime \prime} w^{\prime} d x .
$$

Substituting the expression for the Euler angle $\gamma$ from Eq.2.13 into the transformation matrix, $\mathbf{T}$ from Eq.(2.6) and simplifying the resulting expression in accordance with $[1$, gives the below in terms of the displacements $v, w$, and $\phi$ as [1], 


$$
\mathbf{T}=\left[\begin{array}{ccc}
1-\frac{v^{\prime 2}}{2}-\frac{w^{\prime 2}}{2} & v^{\prime} & w^{\prime} \\
-\left(v^{\prime} \cos \phi+w^{\prime} \sin \phi\right) & \left(1-\frac{v^{\prime 2}}{2}\right) \cos \left(\gamma+v^{\prime} w^{\prime}\right) & \left(1-\frac{w^{\prime 2}}{2}\right) \sin \left(\gamma+v^{\prime} w^{\prime}\right) \\
\left(v^{\prime} \sin \phi-w^{\prime} \cos \phi\right) & -\left(1-\frac{v^{\prime 2}}{2}\right) \sin \left(\gamma+v^{\prime} w^{\prime}\right) & \left(1-\frac{w^{\prime 2}}{2}\right) \cos \left(\gamma+v^{\prime} w^{\prime}\right)
\end{array}\right]
$$

Noting that to the second order, the products and squares of $v^{\prime}, w^{\prime}$ and their derivatives are formally negligible compared to unity, we remove them from the transformation matrix and approximate $\gamma \approx \phi$, thus we obtain a simplified form of the transformation matrix as 1

$$
\mathbf{T}=\left[\begin{array}{ccc}
1 & v^{\prime} & w^{\prime} \\
-v^{\prime} \cos \phi-w^{\prime} \sin \phi & \cos \phi & \sin \phi \\
v^{\prime} \sin \phi-w^{\prime} \cos \phi & -\sin \phi & \cos \phi
\end{array}\right]
$$

Figure 2.6 depicts the relationship of the three reference frames. The $\mathbf{Y}$ and $\mathbf{Y}$ axes are coplanar with the $y$ and $z$ axes. The projection of the $\mathbf{y}$ and $\mathbf{z}$ axes onto the plane defined by $\mathbf{Y}$ and $\mathbf{Z}$ is shown here. It can be understood that the location and orientation of the $\mathbf{y}$ and $\mathbf{z}$ axes relative to the $y$ and $z$ axes varies along the span according to the displacements $u(x, t), v(x, t), w(x, t)$, and $\phi(x, t)$. Hence, the two transformations independently capture the rigid body motion and the flexible vibrations respectively.

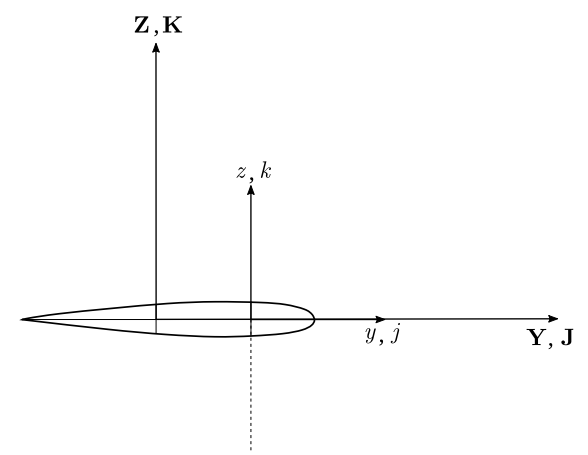

(a) No displacements

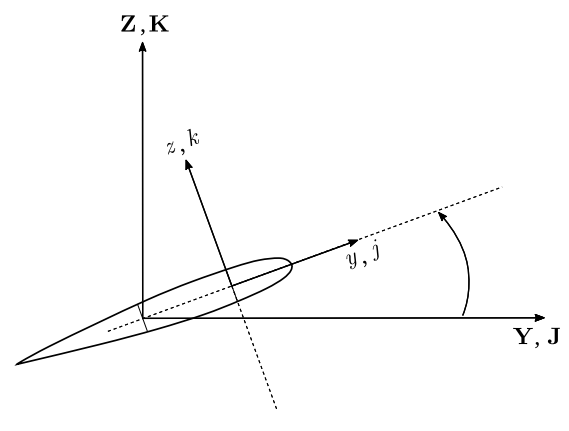

(b) $\theta(t)$

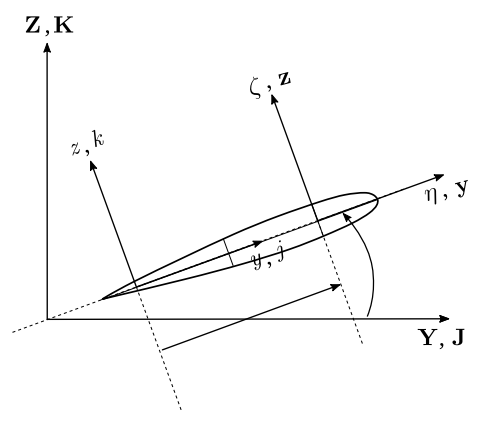

(c) $v(x, t)$ 


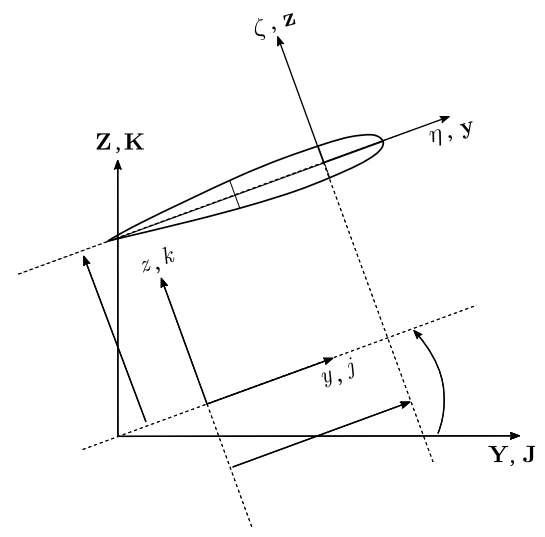

(d) $w(x, t)$

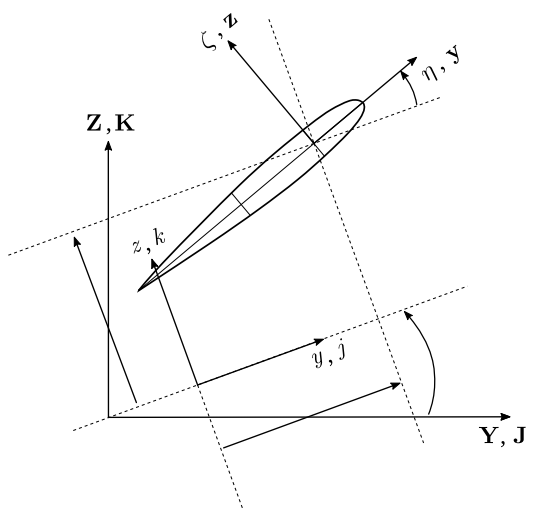

(e) $\phi(x, t)$

Figure 2.6: The three reference frames used in this study are projected onto the YZ-plane. Each subfigure highlights how these frames of reference move relative to one another as the system undergoes deformation

\subsection{Ordering Scheme}

Throughout this document, a number of second order approximations will be made, during the derivation of the equations and in the final form of the equations. This is to ensure that the equations of motion and the derivation process are not unnecessarily complicated by terms with negligible influence on the dynamics. This implies neglecting terms having an order that is greater than or equal to two or orders larger than the lowest order term. Consistent with [1], the nondimensionalized axial displacement is taken to be in the same order of magnitude as the square of the nondimensionalized bending displacements, such that it is negligible compared to unity. In view of this, $\epsilon$ is introduced as a bookkeeping measure having the same order as the nondimensinal bending displacements. Hence, the orders of the nondimensionalized displacements, $u, v, w, \phi$, and $\theta$ are:

$$
\begin{aligned}
\frac{u}{S} & =\mathcal{O}\left(\epsilon^{2}\right), \\
\frac{v}{S} & =\mathcal{O}(\epsilon), \\
\frac{w}{S} & =\mathcal{O}(\epsilon), \\
\phi & =\mathcal{O}(\epsilon), \\
\theta & =\mathcal{O}(1) .
\end{aligned}
$$


Similarly the coordinate $x$ along the elastic axis, as well as the coordinates along the deformed cross sections of the airfoil, $\eta$ and $\zeta$, will be normalized by the span $S$, and given an order. This will aid in assessing the order of terms in the later steps of the derivation, as the physical cross-sectional properties will be obtained through integral equations involving $\eta$ and $\zeta$ additionally, the sol

$$
\begin{aligned}
\frac{x}{S} & =\mathcal{O}(1), \\
\frac{\eta}{S} & =\mathcal{O}(\epsilon), \\
\frac{\zeta}{S} & =\mathcal{O}(\epsilon) .
\end{aligned}
$$

Finally the warping function and its partial derivatives with respect to $\eta$ and $\zeta$ are normalized and ordered. These orders will again be important when evaluating the order of a certain cross-sectional property.

$$
\begin{aligned}
\frac{\lambda}{S^{2}} & =\mathcal{O}\left(\epsilon^{2}\right), \\
\frac{\lambda_{\eta}}{S} & =\mathcal{O}(\epsilon), \\
\frac{\lambda_{\zeta}}{S} & =\mathcal{O}(\epsilon) .
\end{aligned}
$$

\subsection{Strain-Displacement Relationship}

This section is consistent with what is reported in [1]. A detailed account of the existing literature is presented, as the strain-displacement relationship developed in this section is integral to understanding the resulting equations of motion. Additionally, this account includes intermediate steps in the derivation that are not shown in [1]. 
We had defined our transformation matrix $\mathbf{T}$ to map a coordinate in the rotating $(x, y, z)$ defined

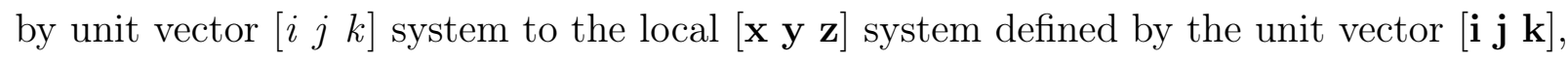

$$
\left\{\begin{array}{l}
\mathbf{i} \\
\mathbf{j} \\
\mathbf{k}
\end{array}\right\}=\mathbf{T}\left\{\begin{array}{c}
i \\
j \\
k
\end{array}\right\} \Longrightarrow\left\{\begin{array}{l}
i \\
j \\
k
\end{array}\right\}=\mathbf{T}^{-1}\left\{\begin{array}{l}
\mathbf{i} \\
\mathbf{j} \\
\mathbf{k}
\end{array}\right\}
$$

Due to the orthogonal nature of the transformation matrix, we can define the inverse of $\mathbf{T}$ as being equal to its transpose as

$$
\mathrm{T}^{-1}=\mathrm{T}^{\mathrm{T}} .
$$

Figure 2.7 provides a visualization of each of the previously defined frames of reference simultaneously. As a consequence of the transformation matrix being defined in terms of the displacements (which themselves vary along the span), the transformation is an implicit function of the position along the span $x$. This has an intuitive interpretation, because in general, the cross sections of the deformed cantilever taken at different spanwise locations in $x$ will have different orientations and positions in $(x, y, z)$. The projection of these coordinate systems on the $y$ and $z$ axis are shown, as they do not lie in the same plane except in the case that the beam is undeformed $(u=v=w=\phi=0)$.

Figure 2.7 is similar to what is presented in the works of [1] and [20, with the notable addition of the pitch axis and the rigid body motion and the associated fixed reference frame. This figure highlights four important locations along the chord of the wing; the pitch axis $(P A)$, the elastic axis $(E A)$, the centroidal axis $(C A)$ and the center of gravity $(C G)$. The elastic axis is given by $(\eta=0, \zeta=0)$, hence, each of the remaining points of interest are defined relative to the deformed elastic axis. The pitch axis located at a distance of $e_{p}$ aft of the elastic axis. The centroidal axis is at a distance of $e_{A}$ ahead of the elastic axis, and the center of gravity is at a distance of $e$ ahead of 


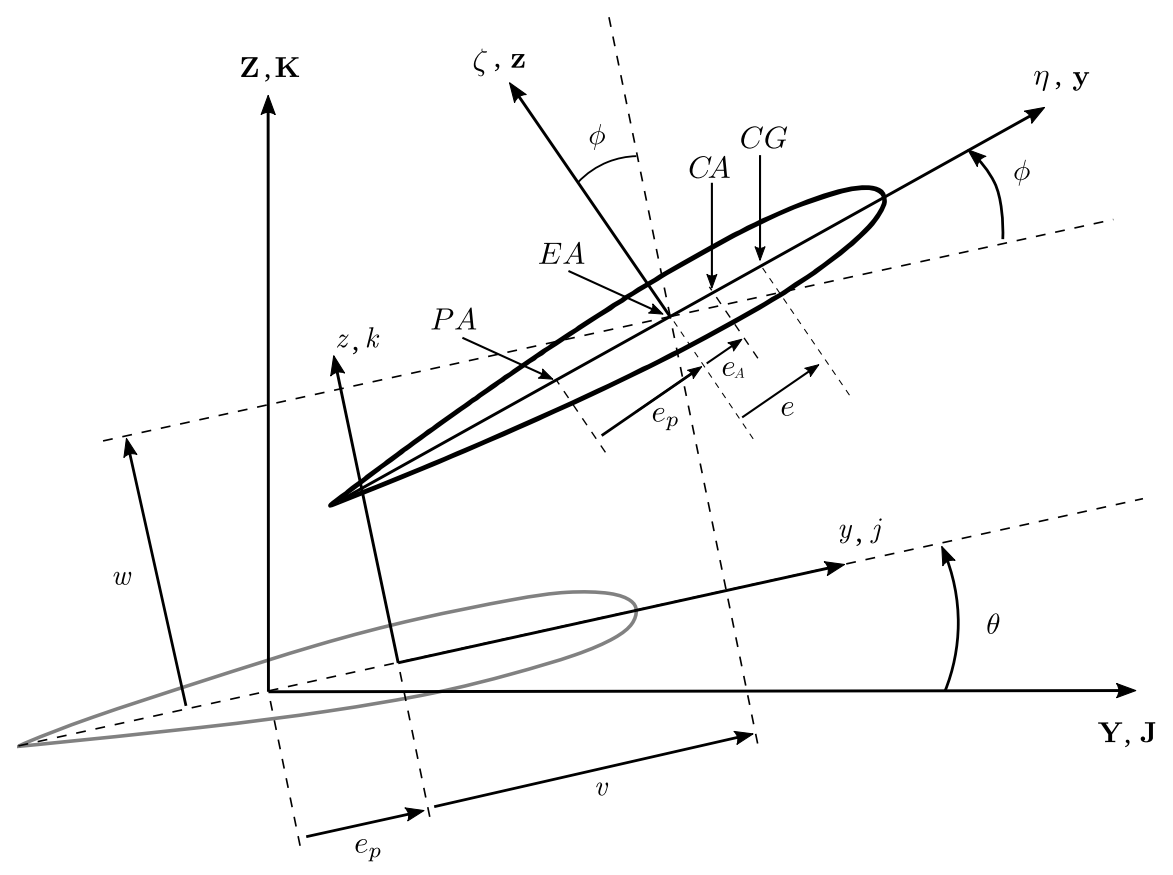

Figure 2.7: The projection of the coordinate systems onto the plane defined by the $\mathbf{Y}$, and $\mathbf{Z}$ axes (adapted from [1] [2])

the elastic axis. Note that for the case where the material density is constant over the cross section, $e$ and $e_{A}$ are coincident. Furthermore, in the case where the cross section is symmetric about the $\mathbf{z}$ axis, $e$ and $e_{A}$ vanish. The dimension $e_{p}$ is dependent on how the flexible wing is rigidly mounted to the rotating support mechanism.

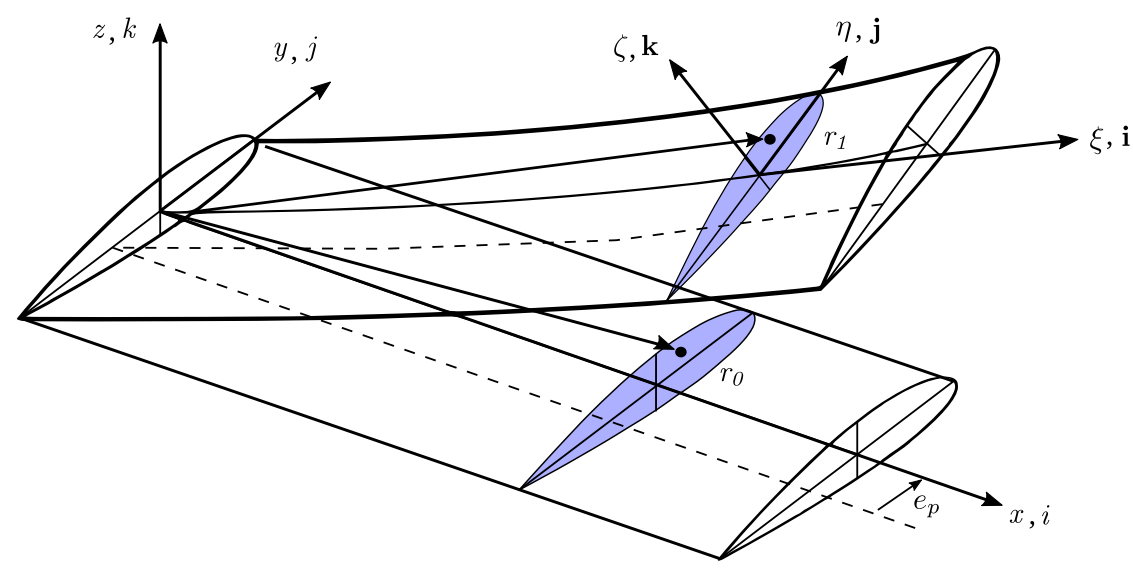

Figure 2.8: The position vectors $r_{0}$ and $r_{1}$ (adapted from $[1][2]$ )

In the rotating reference frame $(x, y, z)$, we define the position of any point on the undeformed beam by the position vector $r_{0}$, while that same point on the beam in the deformed configuration is given by the position vector $r_{1}$ as shown in Figure 2.8 . 
These vectors allow us to describe the position of the point as a funtion of its spanwise location along the undeformed elastic axis, defined by $\{x, 0,0\}^{T}$, and its coordinates, $\eta_{0}$ and $\zeta_{0}$ along the undeformed cross section. The position on the deformed beam is given by the location of the deformed elastic axis, given by $\{x+u, v, w\}^{T}$, and the deformed cross-sectional coordinates $\eta$ and $\zeta$ (defined along $\mathbf{y}$, and $\mathbf{z}$ ) which are mapped to $(x, y, z)$ by the transformation matrix, $\mathbf{T}$.

The position vectors $r_{0}$ and $r_{1}$ are thus defined as follows [1,

$$
\begin{gathered}
\left.r_{1}=\left[\begin{array}{lll}
i & j & k
\end{array}\right]\left[\begin{array}{c}
x+u \\
v \\
w
\end{array}\right\}+\mathbf{T}^{\mathbf{T}}\left\{\begin{array}{c}
-\lambda \phi^{+} \\
\eta \\
\zeta
\end{array}\right\}\right], \\
r_{0}=\left.r_{1}\right|_{u=v=w=\phi=0}=\left[\begin{array}{lll}
i & j & k
\end{array}\right]\left[\left\{\begin{array}{c}
x \\
0 \\
0
\end{array}\right\}+\left.\mathbf{T}^{\mathbf{T}}\right|_{u=v=w=\phi=0}\left\{\begin{array}{c}
0 \\
\eta_{0}=\left.\eta\right|_{u=v=w=\phi=0} \\
\zeta_{0}=\left.\zeta\right|_{u=v=w=0}
\end{array}\right\}\right] .
\end{gathered}
$$

Here, $\lambda$ represents the warping function which has dimensions of length squared, and varies along the cross section (i.e. $\lambda=\lambda(\eta, \zeta))$. The warping function is taken as zero when there is no deformation (i.e. $\lambda=\lambda_{0}\left(\eta_{0}, \zeta_{0}\right)$ ), as well as at the elastic axis (i.e. $\lambda(0,0)=0$ ) as evidenced the fact that the location of the elastic axis is intependent of $\gamma[1]$.

This definition of $r_{0}$ and $r_{1}$ will be central to the derivation of the strain energy and the kinetic energy in Section 2.4 .

\subsubsection{The Green Strain Tensor}

The Green strain tensor can be defined as follows [1]: 


$$
d r_{1} \cdot d r_{1}-d r_{0} \cdot d r_{0}=2\left[\begin{array}{lll}
d s & d \eta & d \zeta
\end{array}\right]\left[\varepsilon_{i j}\right]\left\{\begin{array}{c}
d s \\
d \eta \\
d \zeta
\end{array}\right\}
$$

where $d s, d \eta$, and $d \zeta$ are increments along the $\mathbf{x}, \mathbf{y}$, and $\mathbf{z}$ respectively.

Looking first at the vector $r_{1}$, we will obtain an expression for $d r_{1}$ by taking the differential of this position vector. Adopting the notation ()$^{+}$to represent the derivative of () with respect to $s$, as well as the subscripts ()$_{\eta}$, and ()$_{\zeta}$ to denote the partial derivatives of () with respect to $\eta$ and $\zeta$ respectively,

$$
d r_{1}=\left[\begin{array}{ccc}
i & j & k
\end{array}\right] d\left(\left[\begin{array}{c}
x+u \\
v \\
w
\end{array}\right\}+\mathbf{T}^{\mathbf{T}}\left\{\begin{array}{c}
-\lambda \phi^{+} \\
\eta \\
\zeta
\end{array}\right\}\right)
$$

Applying the product rule, this equation becomes

$$
d r_{1}=\left[\begin{array}{ccc}
i & j & k
\end{array}\right]\left[\left\{\begin{array}{c}
x^{+}+u^{+} \\
v^{+} \\
w^{+}
\end{array}\right\}+\mathbf{T}^{\mathbf{T}+} d s\left\{\begin{array}{c}
-\lambda \phi^{+} \\
\eta \\
\zeta
\end{array}\right\}+\mathbf{T}^{\mathbf{T}}\left\{\begin{array}{c}
-\left(\lambda_{\eta} d \eta+\lambda_{\zeta} d \zeta\right) \phi^{+}-\lambda \phi^{++} d s \\
\eta_{\eta} d \eta \\
\zeta_{\zeta} d \zeta
\end{array}\right\}\right.
$$

If we substitute the transpose of our relation between $(i, j, k)$ and $(\mathbf{i}, \mathbf{j}, \mathbf{k})$, we then obtain an expression for the position vector in terms of the deformed coordinate system (recalling the orthogonality property of $\mathbf{T}$ ) as given by 


$$
d r_{1}=\left[\begin{array}{lll}
\mathbf{i} & \mathbf{j} & \mathbf{k}
\end{array}\right] \mathbf{T}\left[\left\{\begin{array}{c}
x^{+}+u^{+} \\
v^{+} \\
w^{+}
\end{array}\right\}+\mathbf{T}^{\mathbf{T}+}\left\{\begin{array}{c}
-\lambda \phi^{+} \\
\eta \\
\zeta
\end{array}\right\} d s+\mathbf{T}^{\mathbf{T}}\left\{\begin{array}{c}
-\left(\lambda_{\eta} d \eta+\lambda_{\zeta} d \zeta\right) \phi^{+}-\lambda \phi^{++} d s \\
\eta_{\eta} d \eta \\
\zeta_{\zeta} d \zeta
\end{array}\right\},\right.
$$

noting the identity $(A B)^{T}=B^{T} A^{T}$

$$
\left\{\begin{array}{l}
i \\
j \\
k
\end{array}\right\}=\mathbf{T}^{-\mathbf{1}}\left\{\begin{array}{l}
\mathbf{i} \\
\mathbf{j} \\
\mathbf{k}
\end{array}\right\} \Longrightarrow\left[\begin{array}{lll}
i & j & k
\end{array}\right]=\left[\begin{array}{lll}
\mathbf{i} & \mathbf{j} & \mathbf{k}
\end{array}\right] \mathbf{T}
$$

In a subsequent step, if we distribute the transformation through the terms in brackets, and recognize that the partial derivatives of $\eta$ and $\zeta$ with respect to themselves are unity, we obtain [1]

$$
\begin{aligned}
& d r_{1}=\left[\begin{array}{lll}
\mathbf{i} & \mathbf{j} & \mathbf{k}
\end{array}\right]\left[\mathbf{T}\left\{\begin{array}{c}
x^{+}+u^{+} \\
v^{+} \\
w^{+}
\end{array}\right\} d s+\mathbf{T T}^{\mathbf{T}+}\left\{\begin{array}{c}
-\lambda \phi^{+} \\
\eta \\
\zeta
\end{array}\right\} d s\right. \\
& \left.+\mathbf{T T}^{\mathbf{T}}\left\{\begin{array}{c}
-\left(\lambda_{\eta} d \eta+\lambda_{\zeta} d \zeta\right) \phi^{+}-\lambda \phi^{++} d s \\
d \eta \\
d \zeta
\end{array}\right\}\right]
\end{aligned}
$$

We defined that due to the orthogonality of the transformation matrix, the inverse is equal to its transpose [1], 


$$
\mathbf{T}^{-\mathbf{1}}=\mathbf{T}^{\mathbf{T}} \Longrightarrow \mathbf{T T}^{\mathbf{T}}=\left[\begin{array}{ccc}
1 & 0 & 0 \\
0 & 1 & 0 \\
0 & 0 & 1
\end{array}\right]
$$

Additionally, if we then differentiate both sides with respect to $S \llbracket 1$,

$$
\mathbf{T}^{+} \mathbf{T}^{\mathbf{T}}+\mathbf{T T}^{\mathbf{T}+}=\left[\begin{array}{ccc}
0 & 0 & 0 \\
0 & 0 & 0 \\
0 & 0 & 0
\end{array}\right]
$$

Hence we can obtain an expression for $\mathbf{T}^{\mathbf{T}+}$ recalling our definition of $\mathbf{T}^{\mathbf{T}} \mathbf{T}$ that was used in determining the unknown Euler Angle $\gamma$ by establishing a relationship between the transformation matrix and the matrix of curvatures $\omega$ in Eq. 2.9 as 1

$$
\mathbf{T}^{+} \mathbf{T}^{\mathbf{T}}=-\mathbf{T} \mathbf{T}^{\mathbf{T}+} \Longrightarrow \mathbf{T} \mathbf{T}^{\mathbf{T}+}=\left[\begin{array}{rrr}
0 & -\omega_{z} & \omega_{y} \\
\omega_{z} & 0 & -\omega_{x} \\
-\omega_{y} & \omega_{x} & 0
\end{array}\right]
$$

Applying the two identities noted above, the expression for $d r_{1}$ becomes [1], as given by

$$
\begin{aligned}
d r_{1}=\left[\begin{array}{lll}
\mathbf{i} & \mathbf{j} & \mathbf{k}
\end{array}\right]\left[\begin{array}{c}
x^{+}+u^{+} \\
v^{+} \\
w^{+}
\end{array}\right\} d s+\left[\begin{array}{rrr}
0 & -\omega_{z} & \omega_{y} \\
\omega_{z} & 0 & -\omega_{x} \\
-\omega_{y} & \omega_{x} & 0
\end{array}\right]\left\{\begin{array}{c}
-\lambda \phi^{+} \\
\eta \\
\zeta
\end{array}\right\} d s \\
\left.+\left\{\begin{array}{c}
-\left(\lambda \eta t \eta+\lambda_{\zeta} d \zeta\right) \phi^{+}-\lambda \phi^{++} d s \\
d \eta
\end{array}\right\}\right] .
\end{aligned}
$$


Recalling that $r_{1}$ is the position vector to an arbitrary point under deformation, if we consider the special case where the position vector $r_{1}$ describes a point on the elastic axis, the slope of this vector $\left(d r_{1} / d s\right)$ is equal, by definition, to the $\mathbf{z}$ axis (defined by the unit vector $\mathbf{i}$ ) which is tangent to this axis (see Figure 2.8. Hence, 1

$$
\left.\frac{d r_{1}}{d s}\right|_{\eta=\zeta=0} \equiv\left[\begin{array}{lll}
\mathbf{i} & \mathbf{j} & \mathbf{k}
\end{array}\right]\left\{\begin{array}{c}
1 \\
0 \\
0
\end{array}\right\}
$$

Furthermore, taking the derivative of Eq. 2.30 with respect to $s$, evaluated at $\eta=\zeta=0$, $[1$, one obtains

$$
\frac{d r_{1}}{d s}=\left[\begin{array}{lll}
\mathbf{i} & \mathbf{j} & \mathbf{k}
\end{array}\right] \mathbf{T}\left\{\begin{array}{c}
x^{+}+u^{+} \\
v^{+} \\
w^{+}
\end{array}\right\}
$$

Hence, combining Eq. 2.31 and Eq. 2.32, gives [1]

$$
\mathbf{T}\left\{\begin{array}{c}
x^{+}+u^{+} \\
v^{+} \\
w^{+}
\end{array}\right\}=\left\{\begin{array}{l}
1 \\
0 \\
0
\end{array}\right\}
$$

If we substitute Eq.2.33 expression into our equation for $d r_{1}$ in Eq.2.30, we will have conveniently eliminated all explicit instances of the transformation matrix $\mathbf{T}$, and $x^{+}$as follows [1], 


$$
\begin{aligned}
& d r_{1}=\left[\begin{array}{lll}
\mathbf{i} & \mathbf{j} & \mathbf{k}
\end{array}\right]\left\{\begin{array}{l}
1 \\
0 \\
0
\end{array}\right\} d s+\left[\begin{array}{rrr}
0 & -\omega_{z} & \omega_{y} \\
\omega_{z} & 0 & -\omega_{x} \\
-\omega_{y} & \omega_{x} & 0
\end{array}\right]\left\{\begin{array}{c}
-\lambda \phi^{+} \\
\eta \\
\zeta
\end{array}\right\} d s \\
& \left.+\left\{\begin{array}{c}
-\left(\lambda_{\eta} d \eta+\lambda_{\zeta} d \zeta\right) \phi^{+}-\lambda \phi^{++} d s \\
d \eta \\
d \zeta
\end{array}\right\}\right]
\end{aligned}
$$

If we now combine the terms in the square brackets, we obtain the following vector equation,

$$
d r_{1}=\left[\begin{array}{lll}
\mathbf{i} & \mathbf{j} & \mathbf{k}
\end{array}\right]\left\{\begin{array}{c}
\left(1-\omega_{z} \eta+\omega_{y} \zeta+-\lambda \phi^{++}\right) d s-\left(\lambda_{\eta} \phi^{+}\right) d \eta-\left(\lambda_{\zeta} \phi^{+}\right) d \zeta \\
-\left(\omega_{z} \lambda \phi^{+}+\omega_{x} \zeta\right) d s+d \eta \\
\left(\omega_{y} \lambda \phi^{+}+\omega_{x} \eta\right) d s+d \zeta
\end{array}\right\}
$$

It follows that

$$
\begin{aligned}
d r_{1}=\{( & \left.\left.-\omega_{z} \eta+\omega_{y} \zeta-\lambda \phi^{++}\right) d s-\left(\lambda_{\eta} \phi^{+}\right) d \eta-\left(\lambda_{\zeta} \phi^{+}\right) d \zeta\right\} \mathbf{i} \\
& +\left\{-\left(\omega_{z} \lambda \phi^{+}+\omega_{x} \zeta\right) d s+d \eta\right\} \mathbf{j}+\left\{\left(\omega_{y} \lambda \phi^{+}+\omega_{x} \eta\right) d s+d \zeta\right\} \mathbf{k} .
\end{aligned}
$$

With $d r_{1}$ defined, the next step is to determine an expression for $d r_{0}$. We have previously defined $r_{0}$ as the special case where $r_{1}$ evaluated at $u, v, w$, and $\phi$ are equal to zero. Thus again, if we were to take the differential of this matrix, we obtain the following expression following from Eq.(2.23) [1],

$$
d r_{0}=\left[\begin{array}{ccc}
i & j & k
\end{array}\right]\left[\left\{\begin{array}{c}
x^{+} \\
0 \\
0
\end{array}\right\} d s+\left.\mathbf{T}^{\mathbf{T}+}\right|_{v=w=\phi=0}\left\{\begin{array}{c}
0 \\
\eta_{0} \\
\zeta_{0}
\end{array}\right\}+\left.\mathbf{T}^{\mathbf{T}}\right|_{v=w=\phi=0}\left\{\begin{array}{c}
0 \\
\eta_{0_{\eta}} d \eta \\
\zeta_{0_{\zeta}} d \zeta
\end{array}\right\}\right]
$$


If we recall from Eq. 2.7) that the transformation matrix $\mathbf{T}$ reduces to an identity matrix when the wing is undeformed, we can say $\mathbf{T}^{\mathbf{T}}=\mathbf{I}_{3 x 3}$ therefore its derivative $\mathbf{T}^{\mathbf{T}+}=\mathbf{0}_{3 x 3}$.

Additionally, evaluating the first row of Eq.2.33 using the transformation matrix from Eq.2.6, one obtains the geometric relationship $x^{+}=\sqrt{1-v^{2+}-w^{2+}}-u^{+}$(see also Figure 2.5). This leads to the following expression [1],

$$
d r_{0}=\left[\begin{array}{lll}
i & j & k
\end{array}\right]\left\{\begin{array}{c}
\left(\sqrt{\left(1-v^{+2}-w^{+2}\right)}-u^{+}\right) d s \\
\eta_{0_{\eta}} d \eta \\
\zeta_{0_{\zeta}} d \zeta
\end{array}\right\} .
$$

Clearly, note that

$$
d r_{0}=\left\{\left(\sqrt{\left(1-v^{+2}-w^{+2}\right)}-u^{+}\right) d s\right\} i+\left\{\eta_{0_{\eta}} d \eta\right\} j+\left\{\zeta_{0_{\zeta}} d \zeta\right\} k .
$$

Recalling the form of Green's strain tensor, expressed in Eq. 2.21], we will obtain expressions for the left-hand-side (LHS) and the right side (RHS) independently, and then obtain a set of equations based on the condition that the terms associated to each differential on either side must be equivalent. Hence one obtains the following expressions [1],

$$
\begin{aligned}
\text { LHS }=d r_{1} & \cdot d r_{1}-d r_{0} \cdot d r_{0} \\
=\{((1 & \left.-2 \omega_{z} \eta+2 \omega_{y} \zeta-2 \lambda \phi^{++}+\omega_{z}^{2} \eta^{2}-2 \omega_{z} \omega_{y} \eta \zeta+2 \omega_{z} \eta \lambda \phi^{++}+\omega_{y}^{2} \zeta^{2}-2 \omega_{y} \zeta \lambda \phi^{++}+\lambda^{2} \phi^{++}\right) \\
& +\left(\omega_{z}^{2} \lambda^{2} \phi^{+2}+2 \omega_{z} \lambda \phi^{+} \omega_{x} \zeta+\omega_{x}^{2} \zeta^{2}\right)+\left(\omega_{y}^{2} \lambda^{2} \phi^{+2}+2 \omega_{y} \lambda \phi^{+} \omega_{x} \eta+\omega_{x}^{2} \eta^{2}\right)-1+v^{+2}+w^{+2} \\
& \left.+2 u^{+} \sqrt{\left(1-v^{+2}-w^{+2}\right)}-u^{+2}\right) d s^{2}+\left(-2\left(\lambda_{\eta} \phi^{\prime}-\omega_{z} \eta \lambda_{\eta} \phi^{+}+\omega_{y} \zeta \lambda_{\eta} \phi^{+}-\lambda \phi^{++} \lambda_{\eta} \phi^{+}+\omega_{z} \lambda \phi^{+}\right.\right. \\
& \left.\left.+\omega_{x} \zeta\right)\right) d s d \eta+\left(-2\left(\lambda_{\zeta} \phi^{+}-\omega_{z} \eta \lambda_{\zeta} \phi^{+}+\omega_{y} \zeta \lambda_{\zeta} \phi^{+}-\lambda \phi^{++} \lambda_{\zeta} \phi^{+}-\omega_{y} \lambda \phi^{+}+-\omega_{x} \eta\right)\right) d s d \zeta+\left(\lambda_{\eta}^{2} \phi^{+2}\right. \\
& \left.+1-\eta_{0_{\eta}}^{2}\right) d \eta^{2}+\left(\lambda_{\zeta}^{2} \phi^{+2}+1-\zeta_{0_{\zeta}}^{2}\right) d \zeta^{2}+\left(\lambda_{\eta} \lambda_{\zeta} \phi^{+2}\right) d \eta d \zeta
\end{aligned}
$$


and,

$$
\begin{aligned}
\mathrm{RHS} & =2\left[\begin{array}{lll}
d s & d \eta & d \zeta
\end{array}\right]\left[\begin{array}{ccc}
\varepsilon_{11} & \varepsilon_{12} & \varepsilon_{13} \\
\varepsilon_{21} & \varepsilon_{22} & \varepsilon_{23} \\
\varepsilon_{31} & \varepsilon_{32} & \varepsilon_{33}
\end{array}\right]\left\{\begin{array}{c}
d s \\
d \eta \\
d \zeta
\end{array}\right\} \\
& =\left(2 \varepsilon_{11}\right) d s^{2}+\left(2 \varepsilon_{12}+2 \varepsilon_{21}\right) d s d \eta+\left(2 \varepsilon_{13}+2 \varepsilon_{31}\right) d s d \zeta+\left(2 \varepsilon_{22}\right) d \eta^{2}+\left(2 \varepsilon_{23}+2 \varepsilon_{32}\right) d \eta d \zeta+\left(2 \varepsilon_{33}\right) d \zeta^{2} .
\end{aligned}
$$

There are 9 elements in our $3 \times 3$ strain tensor. From Green's strain tensor, we obtained 6 equations, while the symmetry of the shear strains provide the remaining 3 equations $[1]$ :

$$
\begin{aligned}
& \varepsilon_{12}=\varepsilon_{21}, \\
& \varepsilon_{13}=\varepsilon_{31}, \\
& \varepsilon_{23}=\varepsilon_{32} .
\end{aligned}
$$

The terms associated with $d s^{2}$ in Eq. $2.40 \mathrm{a}$ and Eq. $2.40 \mathrm{~b}$ correspond to the normal strain in the longitudinal direction. Removing higher order terms, consistent with the second order approximation leaves the underlined terms. Note that we have previously stated that to the second order, $d s \approx d x$ thus $\sqrt{\left(1-v^{+2}-w^{+2}\right)} \approx 1$, and that the axial displacement $u$ is an order of magnitude smaller than the displacements $v$ and $w$ (i.e. the same order as the squares of $v$ and $w$ ). Invoking the approximation to the second order, we substitute the expressions for the curvatures $\omega_{x}, \omega_{y}$, and $\omega_{z}$, and adopt the ()$^{\prime}$ notation in lieu of the ()$^{+}$notation $[1]$, leading to

$$
\begin{aligned}
2 \varepsilon_{11}=( & \left.-\underline{2 \omega_{z} \eta}+\underline{2 \omega_{y} \zeta}-\underline{2 \lambda \phi^{++}}+\omega_{z}^{2} \eta^{2}-2 \omega_{z} \omega_{y} \eta \zeta+2 \omega_{z} \eta \lambda \phi^{++}+\omega_{y}^{2} \zeta^{2}-2 \omega_{y} \zeta \lambda \phi^{++}+\lambda^{2} \phi^{++}\right) \\
& +\left(\omega_{z}^{2} \lambda^{2} \phi^{+2}+2 \omega_{z} \lambda \phi^{+} \omega_{x} \zeta+\underline{\left.\omega_{x}^{2} \zeta^{2}\right)}+\left(\omega_{y}^{2} \lambda^{2} \phi^{+2}+2 \omega_{y} \lambda \phi^{+} \omega_{x} \eta+\underline{\omega_{x}^{2} \eta^{2}}\right)-1+\underline{v^{\prime 2}}+\underline{w^{\prime}}\right. \\
& +\underline{2 u^{\prime} \sqrt{\left(1-v^{\prime 2}-w^{\prime 2}\right)}-u^{\prime 2}}
\end{aligned}
$$




$$
\begin{aligned}
& \varepsilon_{11}=u^{\prime}+\frac{1}{2}\left(v^{\prime 2}+w^{\prime 2}\right)-\omega_{z} \eta+\omega_{y} \zeta-\lambda \phi^{\prime \prime}+\frac{1}{2}\left(\omega_{x}^{2} \zeta^{2}+\omega_{x}^{2} \eta^{2}\right), \\
& \varepsilon_{11}=u^{\prime}+\frac{v^{\prime 2}}{2}+\frac{w^{\prime 2}}{2}+\frac{\phi^{\prime 2}}{2}\left(\zeta^{2}+\eta^{2}\right)-\eta\left(v^{\prime \prime} \cos \phi+w^{\prime \prime} \sin \phi\right)+\zeta\left(v^{\prime \prime} \sin \phi-w^{\prime \prime} \cos \phi\right)-\lambda \phi^{\prime \prime} .
\end{aligned}
$$

Similarly collecting the terms associated to $d s d \eta$ and $d s d \zeta$ respectively, in in Eq.2.40 a and Eq.2.40 (b), leads to an expression for the shear stresses acting on the plane normal to the elastic axis. Removing all terms of order two or higher than the largest term in the expression and making use of the fact that $\varepsilon_{12}=\varepsilon_{21}$ and $\varepsilon_{13}=\varepsilon_{31}$ results the following expressions [1],

$$
\begin{aligned}
2 \varepsilon_{12}+2 \varepsilon_{21} & =-2\left(\underline{\lambda_{\eta} \phi^{+}}-\omega_{z} \eta \lambda_{\eta} \phi^{+}+\omega_{y} \zeta \lambda_{\eta} \phi^{+}-\lambda \phi^{++} \lambda_{\eta} \phi^{+}+\omega_{z} \lambda \phi^{+}+\underline{\omega_{x} \zeta}\right) \\
\varepsilon_{12}=\varepsilon_{21} & =-\frac{1}{2}\left(\lambda_{\eta} \phi^{\prime}+\omega_{x} \zeta\right) \\
\varepsilon_{12}=\varepsilon_{21} & =-\frac{1}{2}\left(\lambda_{\eta}+\zeta\right) \phi^{\prime} \\
2 \varepsilon_{13}+2 \varepsilon_{31} & =-2\left(\underline{\lambda_{\zeta} \phi^{+}}-\omega_{z} \eta \lambda_{\zeta} \phi^{+}+\omega_{y} \zeta \lambda_{\zeta} \phi^{+}-\lambda \phi^{++} \lambda_{\zeta} \phi^{+}-\omega_{y} \lambda \phi^{+}-\underline{\omega_{x} \eta}\right), \\
\varepsilon_{13}=\varepsilon_{31} & =\frac{1}{2}\left(-\lambda_{\zeta} \phi^{\prime}+\omega_{x} \eta\right) \\
\varepsilon_{13}=\varepsilon_{31} & =\frac{1}{2}\left(-\lambda_{\zeta}+\eta\right) \phi^{\prime} .
\end{aligned}
$$

We will impose the condition that the wing is experiencing uniaxial stress as in [1], such that we have a normal stress $\sigma_{11}$ along the longitudinal axis with associated nonzero shear stresses $\sigma_{12}$ and $\sigma_{13}$. Meanwhile $\sigma_{33}=\sigma_{23}=\sigma_{23}=0$,

$$
\left[\begin{array}{ccc}
\sigma_{11} & \sigma_{12} & \sigma_{13} \\
\sigma_{21} & \sigma_{22} & \sigma_{23} \\
\sigma_{31} & \sigma_{32} & \sigma_{33}
\end{array}\right]=\left[\begin{array}{ccc}
\sigma_{11} & \tau_{12} & \tau_{13} \\
\tau_{21} & 0 & 0 \\
\tau_{31} & 0 & 0
\end{array}\right]
$$

In addition to this, the following relationship exists for the strain tensor. The two things to note from this are the diagonal terms, which allow us to relate the normal strains in the transverse 


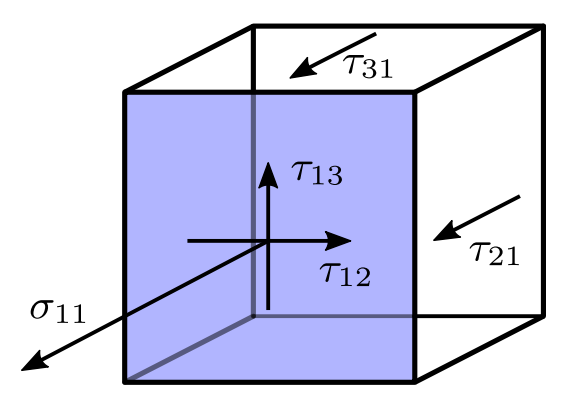

Figure 2.9: An elemental cube under uniaxial stress

directions, $\varepsilon_{22}$ and $\varepsilon_{33}$ to the normal strain in the longitudinal direction, $\varepsilon_{11}$ through Poisson's Ratio, $\nu$. What is also noteworthy is the conversion from classical strain to engineering strain, which states by definition $\varepsilon_{x x} \equiv \varepsilon_{11}, \gamma_{x \eta} \equiv 2 \varepsilon_{12}$ and $\gamma_{x \zeta} \equiv 2 \varepsilon_{13}$, leading to

$$
\left[\begin{array}{ccc}
\varepsilon_{11} & \varepsilon_{12} & \varepsilon_{13} \\
\varepsilon_{21} & \varepsilon_{22} & \varepsilon_{23} \\
\varepsilon_{31} & \varepsilon_{32} & \varepsilon_{33}
\end{array}\right]=\left[\begin{array}{ccc}
\varepsilon_{11} & \varepsilon_{12} & \varepsilon_{13} \\
\varepsilon_{12} & -\nu \varepsilon_{11} & 0 \\
\varepsilon_{13} & 0 & -\nu \varepsilon_{11}
\end{array}\right]=\left[\begin{array}{ccc}
\varepsilon_{x x} & \frac{1}{2} \gamma_{x \eta} & \frac{1}{2} \gamma_{x \zeta} \\
\frac{1}{2} \gamma_{x \eta} & -\nu \varepsilon_{x x} & 0 \\
\frac{1}{2} \gamma_{x \zeta} & 0 & -\nu \varepsilon_{x x}
\end{array}\right]
$$

Finally we obtain expressions for the strains of interest, the normal strain $\varepsilon_{x x}$, and the nonzero shear strains, $\gamma_{x \eta}$ and $\gamma_{x \zeta}[1]$ as

$$
\begin{aligned}
\varepsilon_{x x} & =\varepsilon_{11}, \\
& =u^{\prime}+\frac{v^{\prime 2}}{2}+\frac{w^{\prime 2}}{2}+\frac{\phi^{\prime 2}}{2}\left(\zeta^{2}+\eta^{2}\right)-\eta\left(v^{\prime \prime} \cos \phi+w^{\prime \prime} \sin \phi\right)+\zeta\left(v^{\prime \prime} \sin \phi-w^{\prime \prime} \cos \phi\right)-\lambda \phi^{\prime \prime} \\
\gamma_{x \eta} & =2 \varepsilon_{12}, \\
& =-\left(\lambda_{\eta}+\zeta\right) \phi^{\prime} \\
\gamma_{x \zeta} & =2 \varepsilon_{13}, \\
& =\left(-\lambda_{\zeta}+\eta\right) \phi^{\prime} .
\end{aligned}
$$

The strains $\varepsilon_{22}, \varepsilon_{33}$ and $\varepsilon_{23}$ are not explicitly required to construct the strain tensor in Eq. 2.48, 
however they will be discussed briefly below. Referring to Eq. $2.40 \mathrm{a}$ and Eq.(2.40 a), the expres-

sions for the normal strains are obtained by collecting the coefficients of $d \eta^{2}$ and $d \zeta^{2}$ respectively. Relating the normal strains $\varepsilon_{22}$, $\varepsilon_{33}$ to $\varepsilon_{11}$ using Poisson's Ratio, it can be shown that [1].

$$
\begin{aligned}
& \varepsilon_{22}=-\nu \varepsilon_{11}=\frac{1}{2}\left(\lambda_{\eta}^{2} \phi^{+2}+1-\left(\frac{d \eta_{0}}{d \eta}\right)^{2}\right) \quad \Longrightarrow \quad \frac{d \eta_{0}}{d \eta}=\left(1+\lambda_{\eta}^{2} \phi^{+2}+2 \nu \varepsilon_{11}\right)^{1 / 2} \approx 1, \\
& \varepsilon_{33}=-\nu \varepsilon_{11}=\frac{1}{2}\left(\lambda_{\zeta}^{2} \phi^{+2}+1-\left(\frac{d \zeta_{0}}{d \zeta}\right)^{2}\right) \quad \Longrightarrow \quad \frac{d \zeta_{0}}{d \zeta}=\left(1+\lambda_{\zeta}^{2} \phi^{+2}+2 \nu \varepsilon_{11}\right)^{1 / 2} \approx 1 .
\end{aligned}
$$

Note that the terms in the expression for the normal strain $\varepsilon_{11}$ in Eq. 2.44) and that the terms $\lambda_{\eta}^{2} \phi^{+2}$ and $\lambda_{\zeta}^{2} \phi^{+2}$ in the expressions above are negligible to the second order compared to unity. Hence, it can be concluded that the deformed cross-sectional coordinates $\eta$ and $\zeta$ are equivalent to the coordinates of the undeformed cross section $\eta_{0}$ and $\zeta_{0}$.

Finally, in Eq.2.48 the shear strain $\varepsilon_{23}$ were assumed to be equal to zero. Although the terms multiplying $d \eta d \zeta$ in Eq.2.40 a and Eq.2.40 b are not uniquely equal to zero, they vanish according to the second order approximation.

\subsection{Hamilton's Principle}

Hamilton's Equation has the following form for deformable bodies [1],

$$
0=\int_{t_{1}}^{t_{2}}\{\delta(U-T)-\delta W\} d t
$$

where $t_{1}$ and $t_{2}$ are two arbitrary instances in time, $U$ is the strain energy in the system, $T$ is the kinetic energy, and $\delta W$ is the virtual work done by the external forces. 


\subsubsection{Strain Energy}

In terms of engineering stresses and strains, the strain energy of the system $U$ from both the flexible and the rigid body components of the system, and the variation of the strain energy $\delta U$, are given by the following volume integrals:

$$
\begin{gathered}
U=\frac{1}{2} \int_{0}^{S} \iint_{A}\left(\sigma_{x x} \varepsilon_{x x}+\tau_{x \eta} \gamma_{x \eta}+\tau_{x \zeta} \gamma_{x \zeta}\right) d \eta d \zeta d x+\frac{1}{2} K_{\theta} \theta^{2}, \\
\delta U=\int_{0}^{S} \iint_{A}\left(\sigma_{x x} \delta \varepsilon_{x x}+\tau_{x \eta} \delta \gamma_{x \eta}+\tau_{x \zeta} \delta \gamma_{x \zeta}\right) d \eta d \zeta d x+K_{\theta} \theta \delta \theta .
\end{gathered}
$$

In Eq. 2.56), the volume integral is evaluated in the local reference frame along $\eta$ and $\zeta$, and subsequently along the span. Recall that to the second order, the coordinates of the deformed system, $\eta, \zeta$ and $s$ are equivalent to the coordinates of the undeformed system, $\eta_{0}, \zeta_{0}$ and $x$. The stresses are defined in terms of strains and the elastic and shear moduli as

$$
\begin{aligned}
& \sigma_{x x}=E \varepsilon_{x x}, \\
& \tau_{x \eta}=G \gamma_{x \eta}, \\
& \tau_{x \zeta}=G \gamma_{x \zeta} .
\end{aligned}
$$

Furthermore, taking the variation Eq.(2.49), Eq.2.50, and Eq.(2.51) as in [1] gives

$$
\begin{aligned}
\delta \varepsilon_{x x}= & \delta\left(u^{\prime}+\frac{v^{2}}{2}+\frac{w^{\prime 2}}{2}+\frac{\phi^{2}}{2}\left(\zeta^{2}+\eta^{2}\right)-\eta\left(v^{\prime \prime} \cos \phi+w^{\prime \prime} \sin \phi\right)+\zeta\left(v^{\prime \prime} \sin \phi-w^{\prime \prime} \cos \phi\right)-\lambda \phi^{\prime \prime}\right), \\
=\delta & u^{\prime}+v^{\prime} \delta v^{\prime}+w^{\prime} \delta w^{\prime}+\phi^{\prime} \delta \phi^{\prime}\left(\zeta^{2}+\eta^{2}\right)-\eta\left(\left(\delta v^{\prime \prime} \cos \phi-v^{\prime \prime} \delta \phi \sin \phi\right)+\left(\delta w^{\prime \prime} \sin \phi+w^{\prime \prime} \delta \phi \cos \phi\right)\right) \\
& \left.+\zeta\left(\left(\delta v^{\prime \prime} \sin \phi+v^{\prime \prime} \delta \phi \cos \phi\right)-\left(\delta w^{\prime \prime} \cos \phi-w^{\prime \prime} \delta \phi \sin \phi\right)\right)-\lambda \delta \phi^{\prime \prime}\right), \\
=\delta & +v^{\prime} \delta v^{\prime}+w^{\prime} \delta w^{\prime}+\phi^{\prime} \delta \phi^{\prime}\left(\zeta^{2}+\eta^{2}\right)-\eta\left(\left(\delta v^{\prime \prime}+w^{\prime \prime} \delta \phi\right) \cos \phi+\left(\delta w^{\prime \prime}-v^{\prime \prime} \delta \phi\right) \sin \phi\right) \\
& \left.+\zeta\left(\left(\delta v^{\prime \prime}+w^{\prime \prime} \delta \phi\right) \sin \phi-\left(\delta w^{\prime \prime}-v^{\prime \prime} \delta \phi\right) \cos \phi\right)\right)-\lambda \delta \phi^{\prime \prime},
\end{aligned}
$$




$$
\begin{aligned}
& \delta \gamma_{x \eta}=-\left(\lambda_{\eta}+\zeta\right) \delta \phi^{\prime}, \\
& \delta \gamma_{x \zeta}=\left(-\lambda_{\zeta}+\eta\right) \delta \phi^{\prime} .
\end{aligned}
$$

Substituting these terms into the equation for the variation of the strain energy, we obtain [1]:

$$
\begin{aligned}
\delta U=\int_{0}^{S} \iint_{A}\{ & \sigma_{x x}\left(\delta u^{\prime}+v^{\prime} \delta v^{\prime}+w^{\prime} \delta w^{\prime}+\phi^{\prime} \delta \phi^{\prime}\left(\zeta^{2}+\eta^{2}\right)-\eta\left(\left(\delta v^{\prime \prime}+w^{\prime \prime} \delta \phi\right) \cos \phi+\left(\delta w^{\prime \prime}-v^{\prime \prime} \delta \phi\right) \sin \phi\right)\right. \\
& \left.\left.+\zeta\left(\left(\delta v^{\prime \prime}+w^{\prime \prime} \delta \phi\right) \sin \phi-\left(\delta w^{\prime \prime}-v^{\prime \prime} \delta \phi\right) \cos \phi\right)\right)-\lambda \delta \phi^{\prime \prime}\right) \\
& \left.+\left(\tau_{x \eta}\left(-\left(\zeta+\lambda_{\eta}\right) \delta \phi^{\prime}\right)+\tau_{x \zeta}\left(\left(\eta-\lambda_{\zeta}\right) \delta \phi^{\prime}\right)\right)\right\} d \eta d \zeta d x+K_{\theta} \theta \delta \theta .
\end{aligned}
$$

In order to expand this expression, we introduce some new notation for stress resultants and moments such that the expression is more manageable [1]. Consequently, one obtains

$$
\begin{aligned}
\delta U=\int_{0}^{S}\{ & \left.N_{\mathbf{x}}\right\}\left(\delta u^{\prime}+v^{\prime} \delta v^{\prime}+w^{\prime} \delta w^{\prime}\right)+\left\{T_{\mathbf{x}}+S_{\mathbf{x}}\right\}\left(\delta \phi^{\prime}\right)+\left\{P_{\mathbf{x}}\right\}\left(\delta \phi^{\prime \prime}\right) \\
& -\left\{M_{\mathbf{z}}\right\}\left(\left(\delta v^{\prime \prime}+w^{\prime \prime} \delta \phi\right) \cos \phi+\left(\delta w^{\prime \prime}-v^{\prime \prime} \delta \phi\right) \sin \phi\right) \\
& \left.+\left\{M_{\mathbf{y}}\right\}\left(\left(\delta v^{\prime \prime}+w^{\prime \prime} \delta \phi\right) \sin \phi-\left(\delta w^{\prime \prime}-v^{\prime \prime} \delta \phi\right) \cos \phi\right)\right) d x+K_{\theta} \theta \delta \theta .
\end{aligned}
$$

where, $N_{\mathrm{x}}$ is the normal force due to longitudinal stress, $S_{\mathrm{x}}$ is the moment about the $\mathrm{x}$-axis due to shear stress, $T_{\mathbf{x}}$ is the moment about the $\mathbf{x}$-axis due to longitudinal stress and $P_{\mathbf{x}}$ is a bimoment relating the torsion about the $\mathbf{x}$-axis due to the warping of the section. $M_{\mathbf{y}}$ is the moment about the $\mathbf{y}$-axis, and $M_{\mathbf{z}}$ is the moment about the $\mathbf{z}$-axis.

The various components of the normal strain and their distribution along the cross section are illustrated below (adapted from Houbolt and Brooks [20]). 

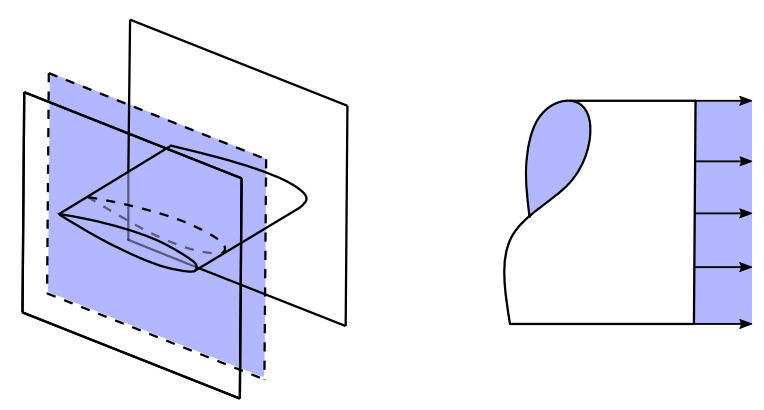

(a) $N_{\mathbf{x}}$

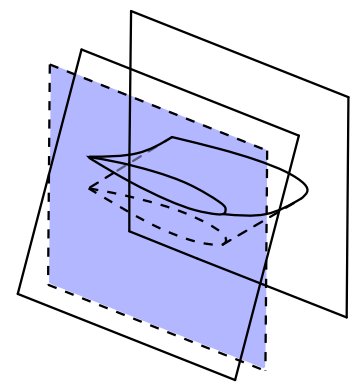

(c) $M_{\mathbf{y}}$
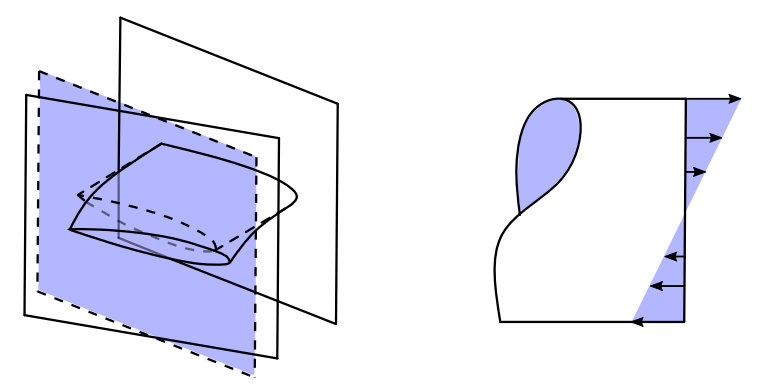

(b) $M_{\mathbf{z}}$
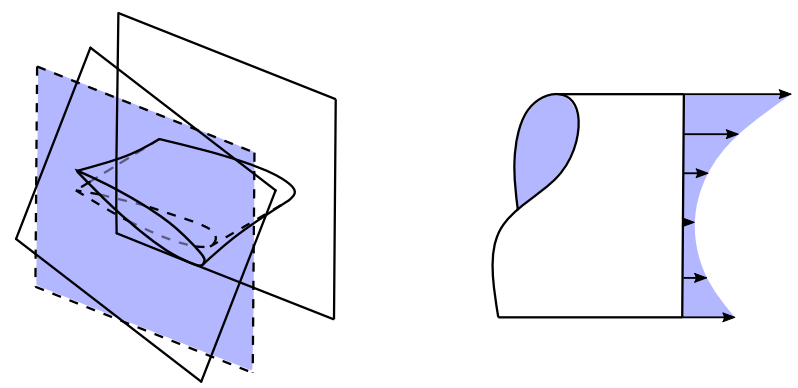

(d) $T_{\mathbf{x}}$

Figure 2.10: The components of the normal strains (adapted from 20 )

Recall that the longitudinal stress is given by

$$
\sigma_{x x}=E\left(u^{\prime}+\frac{v^{\prime 2}}{2}+\frac{w^{\prime 2}}{2}+\frac{\phi^{\prime 2}}{2}\left(\zeta^{2}+\eta^{2}\right)-\eta\left(v^{\prime \prime} \cos \phi+w^{\prime \prime} \sin \phi\right)+\zeta\left(v^{\prime \prime} \sin \phi-w^{\prime \prime} \cos \phi\right)-\lambda \phi^{\prime \prime}\right)
$$

Hence, one can begin to define the resultants introduced in Eq.(2.64) as follows:

$$
\begin{aligned}
N_{\mathbf{x}}= & \iint_{A} \sigma_{x x} d \eta d \zeta \\
= & \iint_{A} E\left(u^{\prime}+\frac{v^{\prime 2}}{2}+\frac{w^{\prime 2}}{2}+\frac{\phi^{\prime 2}}{2}\left(\zeta^{2}+\eta^{2}\right)-\eta\left(v^{\prime \prime} \cos \phi+w^{\prime \prime} \sin \phi\right)+\zeta\left(v^{\prime \prime} \sin \phi-w^{\prime \prime} \cos \phi\right)-\lambda \phi^{\prime \prime}\right) d \eta d \zeta \\
= & E\left(u^{\prime}+\frac{v^{\prime 2}}{2}+\frac{w^{\prime 2}}{2}\right) \iint_{A} d \eta d \zeta+E\left(\frac{\phi^{\prime 2}}{2}\right) \iint_{A}\left(\zeta^{2}+\eta^{2}\right) d \eta d \zeta-E\left(v^{\prime \prime} \cos \phi+w^{\prime \prime} \sin \phi\right) \iint_{A} \eta d \eta d \zeta \\
& \quad+E\left(v^{\prime \prime} \sin \phi-w^{\prime \prime} \cos \phi\right) \iint_{A} \zeta d \eta d \zeta-E\left(\phi^{\prime \prime}\right) \iint_{A} \lambda d \eta d \zeta \\
= & E A\left(u^{\prime}+\frac{v^{\prime 2}}{2}+\frac{w^{\prime 2}}{2}\right)+E A k_{A}{ }^{2}\left(\frac{\phi^{\prime 2}}{2}\right)-E A e_{A}\left(v^{\prime \prime} \cos \phi+w^{\prime \prime} \sin \phi\right)
\end{aligned}
$$




$$
\begin{aligned}
M_{\mathbf{y}}= & \iint_{A} \sigma_{x x} \zeta d \eta d \zeta \\
= & \iint_{A} E\left(u^{\prime}+\frac{v^{\prime 2}}{2}+\frac{w^{\prime 2}}{2}+\frac{\phi^{2}}{2}\left(\zeta^{2}+\eta^{2}\right)-\eta\left(v^{\prime \prime} \cos \phi+w^{\prime \prime} \sin \phi\right)+\zeta\left(v^{\prime \prime} \sin \phi-w^{\prime \prime} \cos \phi\right)-\lambda \phi^{\prime \prime}\right) \zeta d \eta d \zeta \\
= & E\left(u^{\prime}+\frac{v^{\prime 2}}{2}+\frac{w^{\prime 2}}{2}\right) \iint_{A} \zeta d \eta d \zeta+E \frac{\phi^{\prime 2}}{2} \int_{A} \zeta\left(\eta^{2}+\zeta^{2}\right) d \eta d \zeta-E\left(v^{\prime \prime} \cos \phi+w^{\prime \prime} \sin \phi\right) \int_{A} \zeta \eta d \eta d \zeta \\
& +E\left(v^{\prime \prime} \sin \phi-w^{\prime \prime} \cos \phi\right) \iint_{A} \zeta^{2} d \eta d \zeta-E\left(\phi^{\prime \prime}\right) \int_{A} \zeta \lambda d \eta d \zeta \\
= & E I_{\mathbf{y}}\left(v^{\prime \prime} \sin \phi-w^{\prime \prime} \cos \phi\right)-E C_{1}^{*} \phi^{\prime \prime} \\
M_{\mathbf{z}}= & \iint_{A} \sigma_{x x} \eta d \eta d \zeta \\
= & \iint_{A} E\left(u^{\prime}+\frac{v^{\prime 2}}{2}+\frac{w^{\prime 2}}{2}+\frac{\phi^{\prime 2}}{2}\left(\zeta^{2}+\eta^{2}\right)-\eta\left(v^{\prime \prime} \cos \phi+w^{\prime \prime} \sin \phi\right)+\zeta\left(v^{\prime \prime} \sin \phi-w^{\prime \prime} \cos \phi\right)-\lambda \phi^{\prime \prime}\right) \eta d \eta d \zeta \\
= & E\left(u^{\prime}+\frac{v^{\prime 2}}{2}+\frac{w^{\prime 2}}{2}\right) \iint_{A} \eta d \eta d \zeta+E \frac{\phi^{\prime 2}}{2} \iint_{A} \eta\left(\eta^{2}+\zeta^{2}\right) d \eta d \zeta-E\left(v^{\prime \prime} \cos \phi+w^{\prime \prime} \sin \phi\right) \iint_{A} \eta^{2} d \eta d \zeta \\
& +E\left(v^{\prime \prime} \sin \phi-w^{\prime \prime} \cos \phi\right) \iint_{A} \zeta \eta d \eta d \zeta-E\left(\phi^{\prime \prime}\right) \iint_{A} \eta \lambda d \eta d \zeta \\
= & E A e_{A}\left(u^{\prime}+\frac{v^{\prime 2}}{2}+\frac{w^{\prime 2}}{2}\right)+E B_{2}^{*}\left(\frac{\phi^{\prime 2}}{2}\right)-E I_{\mathbf{z}}\left(v^{\prime \prime} \cos \phi+w^{\prime \prime} \sin \phi\right)
\end{aligned}
$$

There are three contributions to the moment about the longitudinal axis, two of which are caused by the normal stress $\sigma_{x x}$, being $T_{\mathbf{x}}$ and $P_{\mathbf{x}}$ which arise from the component of the longitudinal stresses acting in the plane perpendicular to the elastic axis, and due to warping respectively. Then one obtains

$$
\begin{aligned}
T_{\mathbf{x}}= & \iint_{A} \sigma_{x x} \phi^{\prime}\left(\eta^{2}+\zeta^{2}\right) d \eta d \zeta \\
= & \iint_{A} E\left(u^{\prime}+\frac{v^{\prime 2}}{2}+\frac{w^{\prime 2}}{2}+\frac{\phi^{\prime 2}}{2}\left(\zeta^{2}+\eta^{2}\right)-\eta\left(v^{\prime \prime} \cos \phi+w^{\prime \prime} \sin \phi\right)+\zeta\left(v^{\prime \prime} \sin \phi-w^{\prime \prime} \cos \phi\right)-\lambda \phi^{\prime \prime}\right) \phi^{\prime}\left(\eta^{2}+\zeta^{2}\right) d \eta d \zeta \\
=E & \phi^{\prime}\left(u^{\prime}+\frac{v^{\prime 2}}{2}+\frac{w^{\prime 2}}{2}\right) \iint_{A}\left(\eta^{2}+\zeta^{2}\right) d \eta d \zeta+E \frac{\phi^{\prime 3}}{2} \int_{A}\left(\eta^{2}+\zeta^{2}\right)^{2} d \eta d \zeta \\
& \quad-E \phi^{\prime}\left(v^{\prime \prime} \cos \phi+w^{\prime \prime} \sin \phi\right) \iint_{A} \eta\left(\eta^{2}+\zeta^{2}\right) d \eta d \zeta+E \phi^{\prime}\left(v^{\prime \prime} \sin \phi-w^{\prime \prime} \cos \phi\right) \iint_{A} \zeta\left(\eta^{2}+\zeta^{2}\right) d \eta d \zeta \\
& \quad-\phi^{\prime \prime} \iint_{A} \lambda\left(\eta^{2}+\zeta^{2}\right) d \eta d \zeta \\
= & E A k_{A}{ }^{2} \phi^{\prime}\left(u^{\prime}+\frac{v^{\prime 2}}{2}+\frac{w^{\prime 2}}{2}\right)+E B_{1}^{*} \frac{\phi^{\prime 3}}{2}-E B_{2}^{*} \phi^{\prime}\left(v^{\prime \prime} \cos \phi-w^{\prime \prime} \sin \phi\right)
\end{aligned}
$$

Houbolt and Brooks [20] attempt to explain the phenomenon of the torsion caused by longitudinal 
stresses by providing the graphic in Figure 2.11. The torsion results from the slight angle between the elastic axis at $x$, and the normal vector to the plane of the cross section at $x+d x$ (shown as being equal to $\sqrt{\eta^{2}+\zeta^{2}} \phi^{\prime}$ for zero pre-twist). Applying a small angle approximation and taking the sine of this small angle as being equal to the angle itself, the component of the differential normal stress perpendicular to the elastic axis is given by $\sigma_{x x} \phi^{\prime} \sqrt{\eta^{2}+\zeta^{2}} d \eta d \zeta$ (noting of course the substitution of $d A=d \eta d \zeta)$. One final realization is that the moment arm for each of these differential stress components is given by $\sqrt{\eta^{2}+\zeta^{2}}$. Thus the moment about the elastic axis that results from this is given by $\sigma_{x x} \phi^{\prime}\left(\eta^{2}+\zeta^{2}\right) d \eta d \zeta$, which is consistent with our expression for $T_{x}$.

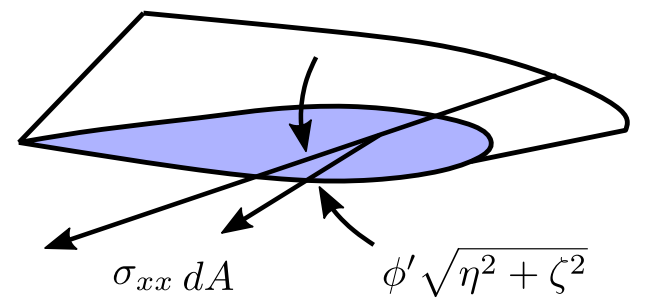

Figure 2.11: Internal Moment, $T_{\mathbf{x}}$ (adapted from 20$]$ )

The addition of component of term $P_{\mathbf{x}}$, which accounts for the torque that arises from the warping of the cross section by [1], extends the original works in [20 for the twisting action of a non-cylindrical section as given by

$$
\begin{aligned}
P_{\mathbf{x}}= & \iint_{A}-\sigma_{x x} \lambda d \eta d \zeta \\
= & \iint_{A}-E \lambda\left(u^{\prime}+\frac{v^{\prime 2}}{2}+\frac{w^{\prime 2}}{2}+\frac{\phi^{\prime 2}}{2}\left(\zeta^{2}+\eta^{2}\right)-\eta\left(v^{\prime \prime} \cos \phi+w^{\prime \prime} \sin \phi\right)+\zeta\left(v^{\prime \prime} \sin \phi-w^{\prime \prime} \cos \phi\right)-\lambda \phi^{\prime \prime}\right) d \eta d \zeta \\
= & -E\left(u^{\prime}+\frac{v^{\prime 2}}{2}+\frac{w^{\prime 2}}{2}\right) \iint_{A} \lambda d \eta d \zeta-E \frac{\phi^{\prime 2}}{2} \iint_{A} \lambda\left(\eta^{2}+\zeta^{2}\right) d \eta d \zeta+E\left(v^{\prime \prime} \cos \phi+w^{\prime \prime} \sin \phi\right) \iint_{A} \eta \lambda d \eta d \zeta \\
& \quad-E\left(v^{\prime \prime} \sin \phi-w^{\prime \prime} \cos \phi\right) \iint_{A} \zeta \lambda d \eta d \zeta+E \phi^{\prime \prime} \iint_{A} \lambda^{2} d \eta d \zeta \\
= & E C_{2}{ }^{*}\left(w^{\prime \prime} \cos \phi-v^{\prime \prime} \sin \phi\right)+E C_{1}{ }^{*} \phi^{\prime \prime}
\end{aligned}
$$

The final contribution to the moment about the $x$ axis is produced by shear stresses, $\tau_{x \eta}$ and $\tau_{x \zeta}$. Recall the shear stresses are given by $\tau_{x \eta}=-G\left(\zeta+\lambda_{\eta}\right) \phi^{\prime}$ and $\left(\eta-\lambda_{\zeta}\right)=G \tilde{\eta} \phi^{\prime}$. Evaluating this expression gives the torsion formula, hence finally we obtain, 


$$
\begin{aligned}
S_{\mathbf{x}} & =\iint_{A}\left(-\left(\zeta+\lambda_{\eta}\right) \tau_{x \eta}+\left(\eta-\lambda_{\zeta}\right) \tau_{x \zeta}\right) d \eta d \zeta \\
& =\iint_{A} G\left(-\left(\zeta+\lambda_{\eta}\right)\left(-\left(\zeta+\lambda_{\eta}\right) \phi^{\prime}\right)+\left(\eta-\lambda_{\zeta}\right)\left(\left(\eta-\lambda_{\zeta}\right) \phi^{\prime}\right)\right) d \eta d \zeta \\
& =\iint_{A} G\left(\left(\zeta+\lambda_{\eta}\right)^{2} \phi^{\prime}+\left(\eta-\lambda_{\zeta}\right)^{2} \phi^{\prime}\right) d \eta d \zeta \\
& =G \phi^{\prime} \iint_{A}\left(\left(\zeta+\lambda_{\eta}\right)^{2}+\left(\eta-\lambda_{\zeta}\right)^{2}\right) d \eta d \zeta \\
& =G J \phi^{\prime}
\end{aligned}
$$

In the equations above, the area integrals are summarized as follows:

$$
\begin{aligned}
A & =\iint_{A} d \eta d \zeta, \\
A e_{A} & =\iint_{A} \eta d \eta d \zeta, \\
I_{\mathbf{y}} & =\iint_{A} \zeta^{2} d \eta d \zeta, \\
I_{\mathbf{z}} & =\iint_{A} \eta^{2} d \eta d \zeta, \\
A{k_{A}}^{2} & =\iint_{A}\left(\eta^{2}+\zeta^{2}\right) d \eta d \zeta, \\
J & =\iint_{A}\left(\left(\zeta+\lambda_{\eta}\right)^{2}+\left(\eta-\lambda_{\zeta}\right)^{2}\right) d \eta d \zeta, \\
B_{1}{ }^{*} & =\iint_{A}\left(\eta^{2}+\zeta^{2}\right)^{2} d \eta d \zeta, \\
B_{2}{ }^{*} & =\iint_{A} \eta\left(\eta^{2}+\zeta^{2}\right) d \eta d \zeta, \\
C_{1}{ }^{*} & =\iint_{A} \lambda^{2} d \eta d \zeta, \\
C_{2}{ }^{*} & =\iint_{A} \zeta \lambda d \eta d \zeta .
\end{aligned}
$$

The area over which the integrals above are evaluated refers to the structurally effective component of the wing. Physically, this implies that these cross-sectional properties pertain to the rectangular steel beam (see Figure 1.1), which governs the stiffness of the wing. Hence, $A$ is the cross-sectional area of the wing. As indicated in Figure 2.7 $e_{A}$ is the distance from the reference point to the centroidal axis of the structurally effective area (or the tensile axis as 
in [1]). Furthermore, $I_{\mathbf{y}}$ and $I_{\mathbf{z}}$ are the moments of inertia of the beam about the $\mathbf{y}$ and $\mathbf{z}$ axes respectively. $A k_{A}^{2}$ is the polar moment of inertia, and similarly $J$ is the torsional constant (i.e. includes the effects of warping). Upon inspection, it can be seen that these two terms are identical for the case where there is no warping. $B_{1}{ }^{*}$ and $B_{2}{ }^{*}$ are higher order sectional constants introduced in [20], while the inclusion of warping result in the sectional constants $C_{1}{ }^{*}$ and $C_{2}{ }^{*}$ in [1].

Additionally due to the symmetry of the airfoil about the camber line, a number of the area integrals are equal to zero as

$$
\begin{aligned}
0 & =\iint_{A} \zeta d \eta d \zeta, \\
0 & =\iint_{A} \eta \zeta d \eta d \zeta, \\
0 & =\iint_{A} \zeta\left(\eta^{2}+\zeta^{2}\right) d \eta d \zeta, \\
0 & =\iint_{A} \lambda d \eta d \zeta, \\
0 & =\iint_{A} \lambda \eta d \eta d \zeta, \\
0 & =\iint_{A} \lambda\left(\eta^{2}+\zeta^{2}\right) d \eta d \zeta .
\end{aligned}
$$

Note that for the experimental apparatus in question, where the structurally effective cross section of the wing is a doubly symmetric rectangular section, $A e_{A}, B_{2}{ }^{*}$ and $C_{2}{ }^{*}$ become equal to zero by definition. For the sake of preserving some generality, these terms will be carried forward, as they would be nonzero if the entire cross section were structurally effective.

We seek an expression for the variation of the strain energy which is in terms of arbitrary variations of $u, v, w, \phi$ and $\theta$ as in Eq. 2.74). $\bar{U}_{u}, \bar{U}_{v}, \bar{U}_{w}, \bar{U}_{\phi}, \bar{U}_{\theta}$, are introduced for notational convenience, as derived from Eq. 2.64), given by [1].

$$
\delta U=\int_{0}^{S}\left(\bar{U}_{u} \delta u+\bar{U}_{v} \delta v+\bar{U}_{w} \delta w+\bar{U}_{\phi} \delta \phi\right) d x+\bar{U}_{\theta} \delta \theta+b(U)
$$


The variation of the strain energy in Eq. 2.64) is currently written in terms of the stress resultants and moments which are coefficients of the variations $\delta v^{\prime}, \delta v^{\prime \prime}, \delta w^{\prime}, \delta w^{\prime \prime}$, and $\delta \phi^{\prime}$ as in Eq. 2.75. In order to obtain an expression in the form of Eq. 2.74), integration by parts must be employed, leading to

$$
\delta U=\int_{0}^{S}\left(U_{u^{\prime}} \delta u^{\prime}+U_{v^{\prime \prime}} \delta v^{\prime \prime}+U_{v^{\prime}} \delta v^{\prime}+U_{w}^{\prime \prime} \delta w^{\prime \prime}+U_{w^{\prime}} \delta w^{\prime}+U_{\phi}^{\prime} \delta \phi^{\prime}+U_{\phi} \delta \phi\right) d x+\bar{U}_{\theta} \delta \theta
$$

The integration by parts is performed below, separated into five equations (one per term in Eq. 2.74) in an effort to gently guide the reader through this step. Integrating each of these equations yields a contribution to the differential equations of motion, and to the boundary conditions, leading to

$$
\begin{aligned}
u: & \int_{0}^{S}\left(U_{u^{\prime}} \delta u^{\prime}\right) d x \\
= & \int_{0}^{S}\left(\left\{N_{\mathbf{x}}\right\} \delta u^{\prime}\right) d x \\
= & \left.\left\{N_{\mathbf{x}}\right\} \delta u\right|_{0} ^{S}-\int_{0}^{S}\left\{N_{\mathbf{x}}\right\}^{\prime} \delta u d x \\
v: & \int_{0}^{S}\left(U_{v^{\prime \prime}} \delta v^{\prime \prime}+U_{v^{\prime}} \delta v^{\prime}\right) d x \\
= & \int_{0}^{S}\left(\left\{M_{\mathbf{y}} \sin \phi-M_{\mathbf{z}} \cos \phi\right\} \delta v^{\prime \prime}+\left\{N_{\mathbf{x}} v^{\prime}\right\} \delta v^{\prime}\right) d x \\
= & \int_{0}^{S}\left\{M_{\mathbf{y}} \sin \phi-M_{\mathbf{z}} \cos \phi\right\} \delta v^{\prime \prime} d x+\int_{0}^{S}\left\{N_{\mathbf{x}} v^{\prime}\right\} \delta v^{\prime} d x \\
= & \left.\left\{M_{\mathbf{y}} \sin \phi-M_{\mathbf{z}} \cos \phi\right\} \delta v^{\prime}\right|_{0} ^{S}-\int_{0}^{S}\left\{M_{\mathbf{y}} \sin \phi-M_{\mathbf{z}} \cos \phi\right\}^{\prime} \delta v^{\prime} d x+\left.\left\{N_{\mathbf{x}}\right\} v^{\prime} \delta v^{\prime}\right|_{0} ^{S}-\int_{0}^{S}\left\{N_{\mathbf{x}} v^{\prime}\right\}^{\prime} \delta v \\
= & \left.\left\{M_{\mathbf{y}} \sin \phi-M_{\mathbf{z}} \cos \phi\right\} \delta v^{\prime}\right|_{0} ^{S}-\left.\left\{M_{\mathbf{y}} \sin \phi-M_{\mathbf{z}} \cos \phi\right\}^{\prime} \delta v\right|_{0} ^{S}+\int_{0}^{S}\left\{M_{\mathbf{y}} \sin \phi-M_{\mathbf{z}} \cos \phi\right\}^{\prime \prime} \delta v d x+ \\
& \left.\quad\left\{N_{\mathbf{x}} v^{\prime}\right\} \delta v\right|_{0} ^{S}-\int_{0}^{S}\left\{N_{\mathbf{x}} v^{\prime}\right\}^{\prime} \delta v d x \\
= & \left.\left.\left.\left(\left\{M_{\mathbf{y}} \sin \phi-M_{\mathbf{z}} \cos \phi\right\} \delta v^{\prime}-\left\{M_{\mathbf{y}} \sin \phi-M_{\mathbf{z}} \cos \phi\right\}^{\prime} \delta v+\left\{N_{\mathbf{x}} v^{\prime}\right\} \delta v\right)\right|_{0} ^{S} \sin \phi-M_{\mathbf{z}} \cos \phi\right\}^{\prime \prime}-\left\{N_{\mathbf{x}} v^{\prime}\right\}^{\prime}\right) \delta v d x \\
& \left.\quad M^{S}\right)
\end{aligned}
$$




$$
\begin{aligned}
& w: \quad \int_{0}^{S}\left(U_{w^{\prime \prime}} \delta w^{\prime \prime}+U_{w^{\prime}} \delta w^{\prime}\right) d x \\
& =\int_{0}^{S}\left(-\left\{M_{\mathbf{z}} \sin \phi+M_{\mathbf{y}} \cos \phi\right\} \delta w^{\prime \prime}+\left\{N_{\mathbf{x}} w^{\prime}\right\} \delta w^{\prime}\right) d x \\
& =\int_{0}^{S}-\left\{M_{\mathbf{z}} \sin \phi+M_{\mathbf{y}} \cos \phi\right\} \delta w^{\prime \prime} d x+\int_{0}^{S}\left\{N_{\mathbf{x}} w^{\prime}\right\} \delta w^{\prime} d x \\
& =-\left.\left\{M_{\mathbf{z}} \sin \phi+M_{\mathbf{y}} \cos \phi\right\} \delta w^{\prime}\right|_{0} ^{S}+\int_{0}^{S}\left\{M_{\mathbf{z}} \sin \phi+M_{\mathbf{y}} \cos \phi\right\}^{\prime} \delta w^{\prime} d x+\left.\left\{N_{\mathbf{x}} w^{\prime}\right\} \delta w^{\prime}\right|_{0} ^{S}-\int_{0}^{S}\left\{N_{\mathbf{x}} w^{\prime}\right\}^{\prime} \delta w \\
& =-\left.\left\{M_{\mathbf{z}} \sin \phi+M_{\mathbf{y}} \cos \phi\right\} \delta w^{\prime}\right|_{0} ^{S}+\left.\left\{M_{\mathbf{z}} \sin \phi+M_{\mathbf{y}} \cos \phi\right\}^{\prime} \delta w\right|_{0} ^{S}-\int_{0}^{S}\left\{M_{\mathbf{z}} \sin \phi+M_{\mathbf{y}} \cos \phi\right\}^{\prime \prime} \delta w d x+ \\
& \left.\left\{N_{\mathbf{x}} w^{\prime}\right\} \delta w\right|_{0} ^{S}-\int_{0}^{S}\left\{N_{\mathbf{x}} w^{\prime}\right\}^{\prime} \delta w \\
& =\left.\left(-\left\{M_{\mathbf{z}} \sin \phi+M_{\mathbf{y}} \cos \phi\right\} \delta w^{\prime}+\left\{M_{\mathbf{z}} \sin \phi+M_{\mathbf{y}} \cos \phi\right\}^{\prime} \delta w+\left\{N_{\mathbf{x}} w^{\prime}\right\} \delta w\right)\right|_{0} ^{S} \\
& +\int_{0}^{S}\left(-\left\{M_{\mathbf{z}} \sin \phi+M_{\mathbf{y}} \cos \phi\right\}^{\prime \prime}-\left\{N_{\mathbf{x}} w^{\prime}\right\}^{\prime}\right) \delta w d x \\
& \phi: \quad \int_{0}^{S}\left(U_{\phi^{\prime \prime}} \delta \phi^{\prime \prime}+U_{\phi^{\prime}} \delta \phi^{\prime}+U_{\phi} \delta \phi\right) d x \\
& =\int_{0}^{S}\left(\left\{P_{\mathbf{x}}\right\} \delta \phi^{\prime \prime}+\left\{S_{\mathbf{x}}+T_{\mathbf{x}}\right\} \delta \phi^{\prime}+\left(v^{\prime \prime}\left\{M_{\mathbf{z}} \sin \phi+M_{\mathbf{y}} \cos \phi\right\}+w^{\prime \prime}\left\{-M_{\mathbf{z}} \cos \phi+M_{\mathbf{y}} \sin \phi\right\}\right) \delta \phi\right) d x \\
& =\int_{0}^{S}\left\{P_{\mathbf{x}}\right\} \delta \phi^{\prime \prime} d x+\int_{0}^{S}\left\{S_{\mathbf{x}}+T_{\mathbf{x}}\right\} \delta \phi^{\prime} d x+\int_{0}^{S}\left(v^{\prime \prime}\left\{M_{\mathbf{z}} \sin \phi+M_{\mathbf{y}} \cos \phi\right\}+w^{\prime \prime}\left\{-M_{z} \mathbf{z} \cos \phi+M_{\mathbf{y}} \sin \phi\right\}\right) \delta \phi d x \\
& =\left.\left\{P_{\mathbf{x}}\right\} \delta \phi^{\prime}\right|_{0} ^{S}-\int_{0}^{S}\left\{P_{\mathbf{x}}\right\}^{\prime} \delta \phi^{\prime} d x+\left.\left\{S_{\mathbf{x}}+T_{\mathbf{x}}\right\} \delta \phi\right|_{0} ^{S}-\int_{0}^{S}\left\{S_{\mathbf{x}}+T_{\mathbf{x}}\right\}^{\prime} \delta \phi d x+\int_{0}^{S}\left(v^{\prime \prime}\left\{M_{\mathbf{z}} \sin \phi+M_{\mathbf{y}} \cos \phi\right\}\right. \\
& \left.+w^{\prime \prime}\left\{-M_{\mathbf{z}} \cos \phi+M_{\mathbf{y}} \sin \phi\right\}\right) \delta \phi d x \\
& =\left.\left\{P_{\mathbf{x}}\right\} \delta \phi^{\prime}\right|_{0} ^{S}-\left.\left\{P_{\mathbf{x}}\right\}^{\prime} \delta \phi\right|_{0} ^{S}+\int_{0}^{S}\left\{P_{\mathbf{x}}\right\}^{\prime \prime} \delta \phi d x+\left.\left\{S_{\mathbf{x}}+T_{\mathbf{x}}\right\} \delta \phi\right|_{0} ^{S}-\int_{0}^{S}\left\{S_{\mathbf{x}}+T_{\mathbf{x}}\right\}^{\prime} \delta \phi d x \\
& +\int_{0}^{S}\left(v^{\prime \prime}\left\{M_{\mathbf{z}} \sin \phi+M_{\mathbf{y}} \cos \phi\right\}+w^{\prime \prime}\left\{-M_{\mathbf{z}} \cos \phi+M_{\mathbf{y}} \sin \phi\right\}\right) \delta \phi d x \\
& =\left.\left(\left\{P_{\mathbf{x}}\right\} \delta \phi^{\prime}-\left\{P_{\mathbf{x}}\right\}^{\prime} \delta \phi+\left\{S_{\mathbf{x}}+T_{\mathbf{x}}\right\} \delta \phi\right)\right|_{0} ^{S}+\int_{0}^{S}\left(\left\{P_{\mathbf{x}}\right\}^{\prime \prime}-\left\{S_{\mathbf{x}}+T_{\mathbf{x}}\right\}^{\prime}+v^{\prime \prime}\left\{M_{\mathbf{z}} \sin \phi+M_{\mathbf{y}} \cos \phi\right\}\right. \\
& \left.+w^{\prime \prime}\left\{-M_{\mathbf{z}} \cos \phi+M_{\mathbf{y}} \sin \phi\right\}\right) \delta \phi d x
\end{aligned}
$$

$\theta: \quad K_{\theta} \theta \delta \theta$ 
One obtains the following expressions for the strain energy contributions to the PDE and the BCs, consistent with [1], as given by

$$
\begin{aligned}
& \bar{U}_{u}=-\left\{N_{\mathbf{x}}\right\}^{\prime} \\
& \bar{U}_{v}=-\left\{N_{\mathbf{x}} v^{\prime}\right\}^{\prime}+\left\{M_{\mathbf{y}} \sin \phi-M_{\mathbf{z}} \cos \phi\right\}^{\prime \prime} \\
& =-\left(N_{\mathbf{x}}^{\prime} v^{\prime}+N_{\mathbf{x}} v^{\prime \prime}\right)+\left(M_{\mathbf{y}}^{\prime} \sin \phi+M_{\mathbf{y}} \phi^{\prime} \cos \phi-M_{\mathbf{z}}^{\prime} \cos \phi+M_{\mathbf{z}} \phi^{\prime} \sin \phi\right)^{\prime} \\
& =-\left(N_{\mathbf{x}}^{\prime} v^{\prime}+N_{\mathbf{x}} v^{\prime \prime}\right)+\left(M_{\mathbf{y}}^{\prime \prime} \sin \phi+2 M_{\mathbf{y}}^{\prime} \phi^{\prime} \cos \phi+M_{\mathbf{y}} \phi^{\prime \prime} \cos \phi-M_{\mathbf{y}} \phi^{\prime 2} \sin \phi\right. \\
& \left.-M_{\mathbf{z}}^{\prime \prime} \cos \phi+2 M_{z} \mathbf{z}^{\prime} \phi^{\prime} \sin \phi+M_{\mathbf{z}} \phi^{\prime \prime} \sin \phi+M_{\mathbf{z}} \phi^{2} \cos \phi\right) \\
& =-\left(N_{\mathbf{x}}^{\prime} v^{\prime}+N_{\mathbf{x}} v^{\prime \prime}\right)+\left(\left(M_{\mathbf{y}}^{\prime \prime}-M_{\mathbf{y}} \phi^{\prime 2}+2 M_{\mathbf{z}}^{\prime} \phi^{\prime}+M_{\mathbf{z}} \phi^{\prime \prime}\right) \sin \phi+\left(-M_{\mathbf{z}}^{\prime \prime}+M_{\mathbf{z}} \phi^{\prime 2}+2 M_{\mathbf{y}}^{\prime} \phi^{\prime}+M_{\mathbf{y}} \phi^{\prime \prime}\right) \cos \phi\right) \\
& \bar{U}_{w}=-\left\{N_{\mathbf{x}} w^{\prime}\right\}^{\prime}-\left\{M_{\mathbf{z}} \sin \phi+M_{\mathbf{y}} \cos \phi\right\}^{\prime \prime} \\
& =-\left(N_{\mathbf{x}}^{\prime} w^{\prime}+N_{\mathbf{x}} w^{\prime \prime}\right)-\left(M_{\mathbf{z}}^{\prime} \sin \phi+M_{\mathbf{z}} \phi^{\prime} \cos \phi+M_{\mathbf{y}}^{\prime} \cos \phi-M_{\mathbf{y}} \phi^{\prime} \sin \phi\right)^{\prime} \\
& =-\left(N_{\mathbf{x}}^{\prime} w^{\prime}+N_{\mathbf{x}} w^{\prime \prime}\right)-\left(M_{\mathbf{z}}^{\prime \prime} \sin \phi+2 M_{\mathbf{z}}^{\prime} \phi^{\prime} \cos \phi+M_{\mathbf{z}} \phi^{\prime \prime} \cos \phi-M_{\mathbf{z}} \phi^{2} \sin \phi\right. \\
& \left.+M_{\mathbf{y}}^{\prime \prime} \cos \phi-2 M_{\mathbf{y}}^{\prime} \phi^{\prime} \sin \phi-M_{\mathbf{y}} \phi^{\prime \prime} \sin \phi-M_{\mathbf{y}} \phi^{2} \cos \phi\right) \\
& =-\left(N_{\mathbf{x}}^{\prime} w^{\prime}+N_{\mathbf{x}} w^{\prime \prime}\right)-\left(\left(M_{\mathbf{z}}^{\prime \prime}-M_{\mathbf{z}} \phi^{2}-2 M_{\mathbf{y}}^{\prime} \phi^{\prime}-M_{\mathbf{y}} \phi^{\prime \prime}\right) \sin \phi+\left(M_{\mathbf{y}}^{\prime \prime}-M_{\mathbf{y}} \phi^{2}+2 M_{\mathbf{z}}^{\prime} \phi^{\prime}+M_{\mathbf{z}} \phi^{\prime \prime}\right) \cos \phi\right) \\
& \bar{U}_{\phi}=\left\{P_{\mathbf{x}}\right\}^{\prime \prime}-\left\{S_{\mathbf{x}}\right\}^{\prime}-\left\{T_{\mathbf{x}}\right\}^{\prime}+\left\{-M_{\mathbf{z}} \cos \phi+M_{\mathbf{y}} \sin \phi\right\} w^{\prime \prime}+\left\{M_{\mathbf{z}} \sin \phi+M_{\mathbf{y}} \cos \phi\right\} v^{\prime \prime} \\
& =\left\{P_{\mathbf{x}}\right\}^{\prime \prime}-\left\{S_{\mathbf{x}}\right\}^{\prime}-\left\{T_{\mathbf{x}}\right\}^{\prime}+\left\{M_{\mathbf{y}}\right\}\left(v^{\prime \prime} \cos \phi+w^{\prime \prime} \sin \phi\right)+\left\{M_{\mathbf{z}}\right\}\left(v^{\prime \prime} \sin \phi-w^{\prime \prime} \cos \phi\right) \\
& \bar{U}_{\theta}=K_{\theta} \theta \\
& b(U)=\left.\left\{N_{\mathbf{x}}\right\} \delta u\right|_{0} ^{S}+\left.\left(\left\{N_{\mathbf{x}}\right\} v^{\prime}+\left\{M_{\mathbf{z}} \cos \phi+M_{\mathbf{y}} \sin \phi\right\}\right) \delta v\right|_{0} ^{S}-\left.\left\{M_{\mathbf{z}} \cos \phi+M_{\mathbf{y}} \sin \phi\right\} \delta v^{\prime}\right|_{0} ^{S} \\
& +\left.\left(\left\{N_{\mathbf{x}}\right\} w^{\prime}-\left\{M_{\mathbf{z}} \sin \phi+M_{\mathbf{y}} \cos \phi\right\}\right) \delta w\right|_{0} ^{S}+\left.\left\{M_{\mathbf{z}} \sin \phi+M_{\mathbf{y}} \cos \phi\right\} \delta w^{\prime}\right|_{0} ^{S} \\
& +\left.\left\{P_{\mathbf{x}}\right\} \delta \phi^{\prime}\right|_{0} ^{S}+\left.\left(-\left\{P_{\mathbf{x}}\right\}^{\prime}+\left\{S_{\mathbf{x}}\right\}+\left\{T_{\mathbf{x}}\right\}\right) \delta \phi\right|_{0} ^{S}
\end{aligned}
$$


The stress and moment resultants as well as their relevant derivatives are presented below:

$$
\begin{aligned}
& N_{\mathbf{y}}=E A\left(u^{\prime}+\frac{v^{\prime 2}}{2}+\frac{w^{\prime 2}}{2}\right)+E A k_{a}{ }^{2}\left(\frac{\phi^{\prime 2}}{2}\right)-E A e_{A}\left(v^{\prime \prime} \cos \phi+w^{\prime \prime} \sin \phi\right) \\
& N_{\mathbf{y}}^{\prime}=E A\left(u^{\prime \prime}+\left(v^{\prime \prime} v^{\prime}\right)+\left(w^{\prime \prime} w^{\prime}\right)\right)+E A k_{a}{ }^{2}\left(\left(\phi^{\prime \prime} \phi^{\prime}\right)\right)-E A e_{A}\left(\left(v^{\prime \prime \prime}+w^{\prime \prime} \phi^{\prime}\right) \cos \phi+\left(w^{\prime \prime \prime}-v^{\prime \prime} \phi^{\prime}\right) \sin \phi\right) \\
& M_{\mathbf{y}}=E I_{\mathbf{y}}\left(v^{\prime \prime} \sin \phi-w^{\prime \prime} \cos \phi\right)-E C_{1}{ }^{*} \phi^{\prime \prime} \\
& M_{\mathbf{y}}^{\prime}=E I_{\mathbf{y}}\left(\left(v^{\prime \prime \prime}+w^{\prime \prime} \phi^{\prime}\right) \sin \phi-\left(w^{\prime \prime \prime}-v^{\prime \prime} \phi^{\prime}\right) \cos \phi\right)-E C_{1}{ }^{*} \phi^{\prime \prime \prime} \\
& M_{\mathbf{y}}^{\prime \prime}=E I_{\mathbf{y}}\left(\left(v^{\prime \prime \prime \prime}-v^{\prime \prime} \phi^{2}+2 w^{\prime \prime \prime} \phi^{\prime}+w^{\prime \prime} \phi^{\prime \prime}\right) \sin \phi+\left(-w^{\prime \prime \prime \prime}+w^{\prime \prime} \phi^{2}+2 v^{\prime \prime \prime} \phi^{\prime}+v^{\prime \prime} \phi^{\prime \prime}\right) \cos \phi\right)-E C_{1}{ }^{*} \phi^{\prime \prime \prime \prime} \\
& M_{\mathbf{z}}=E A e_{A}\left(u^{\prime}+\frac{v^{\prime 2}}{2}+\frac{w^{\prime 2}}{2}\right)+E B_{2} *\left(\frac{\phi^{\prime 2}}{2}\right)-E I_{\mathbf{z}} \operatorname{Big}\left(v^{\prime \prime} \cos \phi+w^{\prime \prime} \sin \phi\right) \\
& M_{\mathbf{z}}^{\prime}=E A e_{A}\left(u^{\prime \prime}+\left(v^{\prime \prime} v^{\prime}\right)+\left(w^{\prime \prime} w^{\prime}\right)\right)+E B_{2} *\left(\left(\phi^{\prime \prime} \phi^{\prime}\right)\right)-E I_{\mathbf{z}}\left(\left(v^{\prime \prime \prime}+w^{\prime \prime} \phi^{\prime}\right) \cos \phi+\left(w^{\prime \prime \prime}-v^{\prime \prime} \phi^{\prime}\right) \sin \phi\right) \\
& M_{\mathbf{z}}^{\prime \prime}=E A e_{A}\left(u^{\prime \prime \prime}+\left(v^{\prime \prime \prime} v^{\prime}+v^{\prime \prime 2}\right)+\left(w^{\prime \prime \prime} w^{\prime}+w^{\prime 2}\right)\right)+E B_{2} *\left(\left(\phi^{\prime \prime \prime} \phi^{\prime}+\phi^{\prime \prime 2}\right)\right) \\
& -E I_{\mathbf{z}}\left(\left(v^{\prime \prime \prime \prime}-v^{\prime \prime} \phi^{2}+2 w^{\prime \prime \prime} \phi^{\prime}+w^{\prime \prime} \phi^{\prime \prime}\right) \cos \phi+\left(w^{\prime \prime \prime \prime}-w^{\prime \prime} \phi^{2}-2 v^{\prime \prime \prime} \phi^{\prime}-v^{\prime \prime} \phi^{\prime \prime}\right) \sin \phi\right) \\
& T_{\mathbf{y}}=E A k_{A}^{2} \phi^{\prime}\left(u^{\prime}+\frac{v^{\prime 2}}{2}+\frac{w^{\prime 2}}{2}\right)+E B_{1}^{*}\left(\frac{\phi^{\prime 3}}{2}\right)+E B_{2}{ }^{*} \phi^{\prime}\left(-v^{\prime \prime} \cos \phi-w^{\prime \prime} \sin \phi\right) \\
& T_{\mathbf{y}}^{\prime}=E A k_{A}^{2}\left(\left(u^{\prime}+\frac{v^{\prime 2}}{2}+\frac{w^{\prime 2}}{2}\right) \phi^{\prime \prime}+\left(u^{\prime \prime}+\left(v^{\prime \prime} v^{\prime}\right)+\left(w^{\prime \prime} w^{\prime}\right)\right) \phi^{\prime}\right)+E B_{1}{ }^{*}\left(\frac{3}{2} \phi^{\prime \prime} \phi^{2}\right) \\
& +E B_{2}{ }^{*}\left(\left(-v^{\prime \prime} \cos \phi-w^{\prime \prime} \sin \phi\right) \phi^{\prime \prime}+\left(-\left(v^{\prime \prime \prime}+w^{\prime \prime} \phi^{\prime}\right) \cos \phi-\left(w^{\prime \prime \prime}-v^{\prime \prime} \phi^{\prime}\right) \sin \phi\right) \phi^{\prime}\right) \\
& P_{\mathbf{y}}=E C_{1}{ }^{*} \phi^{\prime \prime}+E C_{2}{ }^{*}\left(w^{\prime \prime} \cos \phi-v^{\prime \prime} \sin \phi\right) \\
& P_{\mathbf{y}}^{\prime}=E C_{1}{ }^{*} \phi^{\prime \prime \prime}+E C_{2}{ }^{*}\left(\left(w^{\prime \prime \prime}-v^{\prime \prime} \phi^{\prime}\right) \cos \phi-\left(v^{\prime \prime \prime}+w^{\prime \prime} \phi^{\prime}\right) \sin \phi\right) \\
& P_{\mathbf{y}}^{\prime \prime}=E C_{1}{ }^{*} \phi^{\prime \prime \prime \prime}+E C_{2}{ }^{*}\left(\left(w^{\prime \prime \prime \prime}-w^{\prime \prime} \phi^{2}-2 v^{\prime \prime \prime} \phi^{\prime}-v^{\prime \prime} \phi^{\prime \prime}\right) \cos \phi-\left(v^{\prime \prime \prime \prime}-v^{\prime \prime} \phi^{2}+2 w^{\prime \prime \prime} \phi^{\prime}+w^{\prime \prime} \phi^{\prime \prime}\right) \sin \phi\right) \\
& S_{\mathbf{y}}=G J \phi^{\prime} \\
& S_{\mathbf{y}}^{\prime}=G J \phi^{\prime \prime}
\end{aligned}
$$




\subsubsection{Kinetic Energy}

The expression for the kinetic energy of the system is given as:

$$
T=\frac{1}{2} \int_{0}^{S} \iint_{A} \rho(\mathbf{V} \cdot \mathbf{V}) d \eta d \zeta d x+\frac{1}{2} I_{\theta} \dot{\theta}^{2}
$$

the variation of which is

$$
\delta T=\int_{0}^{S} \iint_{A} \rho(\mathbf{V} \cdot \delta \mathbf{V}) d \eta d \zeta d x+I_{\theta} \dot{\theta} \delta \dot{\theta} .
$$

To proceed in the derivation of the kinetic energy terms, we must define the velocity vector, $\mathbf{V}$, in terms of the displacements $v, w$, and $\phi$, as in [2], with the notable addition of the eccentricity between the pitch axis and the elastic axis, $e_{p}$,

$$
\mathbf{V}=\dot{r}_{1}+\dot{\theta} \times\left(r_{1}+e_{p}\right),
$$

where

$$
\begin{aligned}
\dot{\theta} & =\dot{\theta} i, \\
e_{p} & =e_{p} j, \\
\left(r_{1}+e_{p}\right) & =\left\{x_{1} i+y_{1} j+z_{1} k\right\}, \\
\dot{r_{1}} & =\left\{\dot{x}_{1} i+\dot{y}_{1} j+\dot{z}_{1} k\right\} .
\end{aligned}
$$

We will need to define these coordinates, $x_{1}, y_{1}$, and $z_{1}$, in terms of the displacements, using the definition of the position vector $r_{1}$ from Eq. 2.19): 


$$
\left(r_{1}+e_{p}\right)=\left[\begin{array}{ccc}
i & j & k
\end{array}\right]\left[\left\{\begin{array}{c}
x+u \\
v \\
w
\end{array}\right\}+\mathbf{T}^{\mathbf{T}}\left\{\begin{array}{c}
-\lambda \phi^{\prime} \\
\eta \\
\zeta
\end{array}\right\}\right]+\left[\begin{array}{lll}
i & j & k
\end{array}\right]\left\{\begin{array}{c}
0 \\
e_{p} \\
0
\end{array}\right\}
$$

Thus subtituting the transformation matrix, $\mathbf{T}$ defined in Eq.2.15 into the above expression for $r_{1}$, the components $x_{1}, y_{1}$, and $z_{1}$ can be obtained as follows [2]:

$$
\begin{aligned}
& r_{1}=\left[\begin{array}{lll}
i & j & k
\end{array}\right]\left[\left\{\begin{array}{c}
x+u \\
v+e_{p} \\
w
\end{array}\right\}+\left[\begin{array}{ccc}
1 & -v^{\prime} \cos \phi-w^{\prime} \sin \phi & v^{\prime} \sin \phi-w^{\prime} \cos \phi \\
v^{\prime} & \cos \phi & -\sin \phi \\
w^{\prime} & \sin \phi & \cos \phi
\end{array}\right]\left\{\begin{array}{c}
-\lambda \phi^{\prime} \\
\eta \\
\zeta
\end{array}\right\}\right], \\
& =\left[\begin{array}{ccc}
i & j & k
\end{array}\right]\left[\left\{\begin{array}{c}
x+u \\
v+e_{p} \\
w
\end{array}\right\}+\left\{\begin{array}{c}
-\lambda \phi^{\prime}+\eta\left(-v^{\prime} \cos \phi-w^{\prime} \sin \phi\right)+\zeta\left(v^{\prime} \sin \phi-w^{\prime} \cos \phi\right) \\
-\lambda \phi^{\prime} v^{\prime}+\eta \cos \phi-\zeta \sin \phi \\
-\lambda \phi^{\prime} w^{\prime}+\eta \sin \phi+\zeta \cos \phi
\end{array}\right\}\right] \\
& =\left[\begin{array}{lll}
i & j & k
\end{array}\right]\left\{\begin{array}{c}
x+u-\lambda \phi^{\prime}+\eta\left(-v^{\prime} \cos \phi-w^{\prime} \sin \phi\right)+\zeta\left(v^{\prime} \sin \phi-w^{\prime} \cos \phi\right) \\
v+e_{p}-\lambda \phi^{\prime} v^{\prime}+\eta \cos \phi-\zeta \sin \phi \\
w-\lambda \phi^{\prime} w^{\prime}+\eta \sin \phi+\zeta \cos \phi
\end{array}\right\} .
\end{aligned}
$$

According to the second order approximation, the underlined terms below are formally negligible compared to the largest terms in the respective equations, and hence are eliminated from the equations. Recognizing the common terms $(\eta \cos \phi-\zeta \sin \phi)$ and $(\eta \sin \phi+\zeta \cos \phi), x_{1}$ in Eq. 2.96 a) can be rewritten in terms of $y_{1}$ from Eq. $2.96 \mathrm{~b}$ and $z_{1}$ from Eq.2.96 c). The first and second derivatives, as well as the variations of $x_{1}, y_{1}$, and $z_{1}$ are shown as they are used in subsequent steps: 


$$
\begin{aligned}
& x_{1}=x+u-\lambda \phi^{\prime}-v^{\prime}(\eta \cos \phi-\zeta \sin \phi)-w^{\prime}(\eta \sin \phi+\zeta \cos \phi), \\
& \approx x+u-\lambda \phi^{\prime}-v^{\prime}\left(y_{1}-\left(v+e_{p}\right)\right)-w^{\prime}\left(z_{1}-w\right), \\
& y_{1}=v+e_{p}-\underline{\lambda \phi^{\prime} v^{\prime}}+(\eta \cos \phi-\zeta \sin \phi), \\
& \approx v+e_{p}+\left(y_{1}-\left(v+e_{p}\right)\right) \\
& z_{1}=w-\underline{\lambda \phi^{\prime} w^{\prime}}+(\eta \sin \phi+\zeta \cos \phi), \\
& \approx w+\left(z_{1}-w\right), \\
& \dot{x}_{1}=\dot{u}-\lambda \dot{\phi}^{\prime}-\dot{v}^{\prime}(\eta \cos \phi-\zeta \sin \phi)-v^{\prime} \dot{\phi}(-\eta \sin \phi-\zeta \cos \phi)-\dot{w}^{\prime}(\eta \sin \phi+\zeta \cos \phi)-w^{\prime} \dot{\phi}(\eta \cos \phi-\zeta \sin \phi), \\
& =\dot{u}-\lambda \dot{\phi}^{\prime}-\dot{v}^{\prime}\left(y_{1}-\left(v+e_{p}\right)\right)+v^{\prime} \dot{\phi}\left(z_{1}-w\right)-\dot{w}^{\prime}\left(z_{1}-w\right)-w^{\prime} \dot{\phi}\left(y_{1}-\left(v+e_{p}\right)\right), \\
& =\dot{u}-\lambda \dot{\phi}^{\prime}-\left(\dot{v}^{\prime}+w^{\prime} \dot{\phi}\right)\left(y_{1}-\left(v+e_{p}\right)\right)-\left(\dot{w}^{\prime}-v^{\prime} \dot{\phi}\right)\left(z_{1}-w\right) \text {, } \\
& \dot{y}_{1}=\dot{v}+\dot{\phi}(-\eta \sin \phi-\zeta \cos \phi) \\
& =\dot{v}-\dot{\phi}\left(z_{1}-w\right) \\
& \dot{z}_{1}=\dot{w}+\dot{\phi}(\eta \cos \phi-\zeta \sin \phi) \\
& =\dot{w}+\dot{\phi}\left(y_{1}-\left(v+e_{p}\right)\right) \\
& \delta x_{1}=\delta u-\lambda \delta \phi^{\prime}-\left(\delta v^{\prime}+w^{\prime} \delta \phi\right)\left(y_{1}-\left(v+e_{p}\right)\right)-\left(\delta w^{\prime}-v^{\prime} \delta \phi\right)\left(z_{1}-w\right) \\
& \delta y_{1}=\delta v-\delta \phi\left(z_{1}-w\right) \\
& \delta z_{1}=\delta w+\delta \phi\left(y_{1}-\left(v+e_{p}\right)\right) \\
& \ddot{x}_{1}=\ddot{u}-\lambda \ddot{\phi}^{\prime}-\left(\ddot{v}^{\prime}+\dot{w}^{\prime} \dot{\phi}-v^{\prime} \ddot{\phi}\right)(\eta \cos \phi-\zeta \sin \phi)-\left(\dot{v}+w^{\prime} \dot{\phi}\right)(-\eta \sin \phi-\zeta \cos \phi) \dot{\phi} \\
& -\left(\ddot{w}^{\prime}-\dot{v}^{\prime} \dot{\phi}-v^{\prime} \ddot{\phi}\right)(\eta \sin \phi+\zeta \cos \phi)-\left(\dot{w}^{\prime}-v^{\prime} \dot{\phi}\right)(\eta \cos \phi-\zeta \sin \phi) \dot{\phi}, \\
& =\ddot{u}-\lambda \ddot{\phi}^{\prime}\left(-\ddot{v}^{\prime}-\dot{w}^{\prime} \dot{\phi}-w^{\prime} \ddot{\phi}-\dot{w}^{\prime} \dot{\phi}+\underline{v^{\prime}} \dot{\phi}^{2}\right)(\eta \cos \phi-\zeta \sin \phi)+\left(\dot{v}^{\prime} \dot{\phi}+v^{\prime} \ddot{\phi}-\ddot{w}^{\prime}+\dot{v}^{\prime} \dot{\phi}+\underline{w^{\prime} \dot{\phi}^{2}}\right)(\eta \sin \phi+\zeta \cos \phi),
\end{aligned}
$$




$$
\begin{aligned}
& \approx \ddot{u}-\lambda \ddot{\phi}^{\prime}-\left(\ddot{v}^{\prime}+w^{\prime} \ddot{\phi}+2 \dot{w}^{\prime} \dot{\phi}\right)(\eta \cos \phi-\zeta \sin \phi)-\left(\ddot{w}^{\prime}-v^{\prime} \ddot{\phi}-2 \dot{v}^{\prime} \dot{\phi}\right)(\eta \sin \phi+\zeta \cos \phi), \\
& \approx \ddot{u}-\lambda \ddot{\phi}^{\prime}-\left(\ddot{v}^{\prime}+w^{\prime} \ddot{\phi}+2 \dot{w}^{\prime} \dot{\phi}\right)\left(y_{1}-\left(v+e_{p}\right)\right)-\left(\ddot{w}^{\prime}-v^{\prime} \ddot{\phi}-2 \dot{v}^{\prime} \dot{\phi}\right)\left(z_{1}-w\right), \\
\ddot{y}_{1} & =\ddot{v}-\ddot{\phi}(\eta \sin \phi+\zeta \cos \phi)-\underline{\dot{\phi}^{2}}(\eta \cos \phi-\zeta \sin \phi), \\
& \approx \ddot{v}-\ddot{\phi}\left(z_{1}-w\right), \\
\ddot{z}_{1} & =\ddot{w}+\ddot{\phi}(\eta \cos \phi-\zeta \sin \phi)+\underline{\dot{\phi}^{2}}(-\eta \sin \phi-\zeta \cos \phi), \\
& \approx \ddot{w}+\ddot{\phi}\left(y_{1}-\left(v+e_{p}\right)\right) .
\end{aligned}
$$

In Eq. 2.89 we defined the velocity to be $\mathbf{V}$ as $\dot{r}_{1}+\dot{\theta} i \times\left(r_{1}+e_{p}\right)$, which can be written as:

$$
\begin{aligned}
& \mathbf{V}=\dot{x}_{1} i+\dot{y}_{1} j+\dot{z}_{1} k+\dot{\theta} i \times\left\{x_{1} i+y_{1} j+z_{1} k\right\}, \\
& \mathbf{V}=\dot{x}_{1} i+\dot{y}_{1} j+\dot{z}_{1} k+\left\{-\dot{\theta} z_{1} j+\dot{\theta} y_{1} k\right\}, \\
& \mathbf{V}=\dot{x}_{1} i+\left(\dot{y}_{1}-\dot{\theta} z_{1}\right) j+\left(\dot{z}_{1}+\dot{\theta} y_{1}\right) k .
\end{aligned}
$$

Thus the variation of the velocity is given as 2

$$
\delta \mathbf{V}=\delta \dot{x}_{1} i+\left(\delta \dot{y}_{1}-\dot{\theta} \delta z_{1}-\delta \dot{\theta} z_{1}\right) j+\left(\delta \dot{z}_{1}+\dot{\theta} \delta y_{1}+\delta \dot{\theta} y_{1}\right) k
$$

We now have an expression for the variation of the kinetic energy of the system, $\delta T$ in terms of our displacements, and coordinates $\eta$, and $\zeta$, which again will form our area integrals:

$$
\begin{aligned}
\delta T= & \int_{0}^{S} \iint_{A} \rho(\mathbf{V} \cdot \delta \mathbf{V}) d \eta d \zeta d x+I_{\theta} \dot{\theta} \delta \dot{\theta} \\
= & \int_{0}^{S} \iint_{A} \rho\left(\left\{\dot{x}_{1} i+\left(\dot{y}_{1}-\dot{\theta} z_{1}\right) j+\left(\dot{z}_{1}+\dot{\theta} y_{1}\right) k\right\} \cdot\left\{\delta \dot{x}_{1} i+\left(\delta \dot{y}_{1}-\dot{\theta} \delta z_{1}-\delta \dot{\theta} z_{1}\right) j+\left(\delta \dot{z}_{1}+\dot{\theta} \delta y_{1}+\delta \dot{\theta} y_{1}\right) k\right\}\right) d \eta d \zeta d x \\
& \quad+I_{\theta} \dot{\theta} \delta \dot{\theta}
\end{aligned}
$$




$$
\begin{gathered}
=\int_{0}^{S} \iint_{A} \rho\left(\left(\dot{x}_{1}\right)\left(\delta \dot{x}_{1}\right)+\left(\dot{y}_{1}-\dot{\theta} z_{1}\right)\left(\delta \dot{y}_{1}-\dot{\theta} \delta z_{1}-\delta \dot{\theta} z_{1}\right)+\left(\dot{z}_{1}+\dot{\theta} y_{1}\right)\left(\delta \dot{z}_{1}+\dot{\theta} \delta y_{1}+\delta \dot{\theta} y_{1}\right)\right) d \eta d \zeta d x+I_{\theta} \dot{\theta} \delta \dot{\theta} \\
=\int_{0}^{S} \iint_{A} \rho\left(\dot{x}_{1} \delta \dot{x}_{1}+\dot{y}_{1} \delta \dot{y}_{1}-\dot{y_{1}} \dot{\theta} \delta z_{1}-\dot{y}_{1} \delta \dot{\theta} z_{1}-\dot{\theta} z_{1} \delta \dot{y}_{1}+\dot{\theta} z_{1} \dot{\theta} \delta z_{1}+\theta z_{1} \delta \dot{\theta} z_{1}\right. \\
\left.\quad+\dot{z}_{1} \delta \dot{z}_{1}+\dot{z}_{1} \dot{\theta} \delta y_{1}+\dot{z}_{1} \delta \dot{\theta} y_{1}+\dot{\theta} y_{1} \delta \dot{z}_{1}+\dot{\theta} y_{1} \dot{\theta} \delta y_{1}+\dot{\theta} y_{1} \delta \dot{\theta} y_{1}\right) d \eta d \zeta d x+I_{\theta} \dot{\theta} \delta \dot{\theta} \\
=\int_{0}^{S} \iint_{A} \rho\left(\left(\dot{x}_{1}\right) \delta \dot{x}_{1}+\left(\dot{y}_{1}-\dot{\theta} z_{1}\right) \delta \dot{y}_{1}+\left(\dot{z}_{1}+\dot{\theta} y_{1}\right) \delta \dot{z}_{1}+\left(-\dot{y}_{1} z_{1}+\dot{\theta} z_{1}^{2}+\dot{z}_{1} y_{1}+\dot{\theta} y_{1}^{2}\right) \delta \dot{\theta}\right. \\
\left.\quad+\left(\dot{\theta} \dot{z}_{1}+\dot{\theta}^{2} y_{1}\right) \delta y_{1}+\left(-\dot{y}_{1} \dot{\theta}+\dot{\theta}^{2} z_{1}\right) \delta z_{1}\right) d \eta d \zeta d x+I_{\theta} \dot{\theta} \delta \dot{\theta}
\end{gathered}
$$

In its current state, we have an expression in terms of the arbitrary variations and their undefined derivatives with respect to time, however, we require an expression solely in terms of the variations themselves. We will employ integration by parts between two arbitrary points in time, $t_{1}$, and $t_{2}$. The value of the integral taken at the upper and lower bounds are zero $\left(\left.u v\right|_{t_{1}} ^{t_{2}}=0\right.$ for $\left.\int_{t_{1}}^{t_{2}} u d v\right)$, implying that this step does not yield any boundary conditions [1]:

$$
\begin{aligned}
x_{1}: & \int_{t_{1}}^{t_{2}}\left(\dot{x}_{1}\right) \delta \dot{x}_{1} d t \\
= & \left.\dot{x}_{1} \delta x_{1}\right|_{t_{1}} ^{t_{2}}-\int_{t_{1}}^{t_{2}} \ddot{x}_{1} \delta x_{1} d t \\
= & \int_{t_{1}}^{t_{2}}\left(-\ddot{x}_{1}\right) \delta x_{1} d t \\
y_{1}: & \int_{t_{1}}^{t_{2}}\left(\dot{y}_{1}-\dot{\theta} z_{1}\right) \delta \dot{y}_{1}+\left(\dot{z}_{1} \dot{\theta}+y_{1} \dot{\theta}^{2}\right) \delta y_{1} d t \\
= & \left.\left(\dot{y}_{1}-\dot{\theta}_{z_{1}}\right) \delta y_{1}\right|_{t_{1}} ^{t_{2}}-\int_{t_{1}}^{t_{2}}\left(\ddot{y}_{1}-\ddot{\theta} z_{1}-\dot{\theta} \dot{z}_{1}\right) \delta y_{1} d t+\int_{t_{1}}^{t_{2}}\left(\dot{z}_{1} \dot{\theta}+y_{1} \dot{\theta}^{2}\right) \delta y_{1} d t \\
= & \int_{t_{1}}^{t_{2}}\left(-\ddot{y}_{1}+y_{1} \dot{\theta}^{2}+\ddot{\theta} z_{1}+2 \dot{\theta} \dot{z}_{1}\right) \delta y_{1} d t \\
z_{1}: & \int_{t_{1}}^{t_{2}}\left(\dot{z}_{1}+\dot{\theta} y_{1}\right) \delta \dot{z}_{1}+\left(-\dot{y}_{1} \dot{\theta}+z_{1} \dot{\theta}^{2}\right) \delta z_{1} d t \\
= & \left.\left(\dot{z}_{1}+\dot{\theta} y_{1}\right) \delta z_{1}\right|_{t_{1}} ^{t_{2}}-\int_{t_{1}}^{t_{2}}\left(\ddot{z}_{1}+\ddot{\theta} y_{1}+\dot{\theta} \dot{y}_{1}\right) \delta z_{1} d t+\int_{t_{1}}^{t_{2}}\left(-\dot{y}_{1} \dot{\theta}+z_{1} \dot{\theta}^{2}\right) \delta z_{1} d t \\
= & \int_{t_{1}}^{t_{2}}\left(-\ddot{z}_{1}+z_{1} \dot{\theta}^{2}-\ddot{\theta} y_{1}-2 \dot{\theta} \dot{y}_{1}\right) \delta z_{1} d t \\
\theta: & \int_{t_{1}}^{t_{2}}\left(-\dot{y}_{1} z_{1}+\dot{\theta} z_{1}^{2}+\dot{z}_{1} y_{1}+\dot{\theta} y_{1}^{2}+I_{\theta} \dot{\theta}\right) \delta \dot{\theta} d t
\end{aligned}
$$




$$
\begin{aligned}
= & \left.\left(-\dot{y}_{1} z_{1}+\dot{\theta} z_{1}^{2}+\dot{z}_{1} y_{1}+\dot{\theta} y_{1}^{2}+I_{\theta} \dot{\theta}\right) \delta \theta\right|_{t_{1}} ^{t_{2}} \\
& \quad-\int_{t_{1}}^{t_{2}}\left(-\ddot{y}_{1} z_{1}-\dot{y}_{1} \dot{z}_{1}+\ddot{\theta} z_{1}^{2}+2 \dot{\theta} \dot{z}_{1} z_{1}+\ddot{z}_{1} y_{1}+\dot{z}_{1} \dot{y}_{1}+\ddot{\theta} y_{1}^{2}+2 \dot{\theta} \dot{y}_{1} y_{1}+I_{\theta} \ddot{\theta}\right) \delta \theta d t \\
= & \int_{t_{1}}^{t_{2}}\left(\ddot{y}_{1} z_{1}-\ddot{z}_{1} y_{1}-\ddot{\theta} z_{1}^{2}-\ddot{\theta} y_{1}^{2}-2 \dot{\theta} \dot{z}_{1} z_{1}-2 \dot{\theta} \dot{y}_{1} y_{1}-I_{\theta} \ddot{\theta}\right) \delta \theta d t \\
= & \int_{t_{1}}^{t_{2}}\left(\ddot{y}_{1} z_{1}-\ddot{z}_{1} y_{1}-\ddot{\theta}\left(z_{1}^{2}+y_{1}^{2}\right)-2 \dot{\theta}\left(\dot{z}_{1} z_{1}+\dot{y}_{1} y_{1}\right)-I_{\theta} \ddot{\theta}\right) \delta \theta d t
\end{aligned}
$$

We now have an expression for the variation of the kinetic energy in terms of known variations, $\delta x_{1}, \delta y_{1}, \delta z_{1}$, and $\delta \theta$, as in [1] [2],

$$
\begin{aligned}
\delta T=\int_{0}^{S} \iint_{A} \rho( & \left(\ddot{x}_{1}\right) \delta x_{1}+\left(-\ddot{y}_{1}+y_{1} \dot{\theta}^{2}+\ddot{\theta} z_{1}+2 \dot{\theta} \dot{z}_{1}\right) \delta y_{1}+\left(-\ddot{z}_{1}+z_{1} \dot{\theta}^{2}-\ddot{\theta} y_{1}-2 \dot{\theta} \dot{y}_{1}\right) \delta z_{1} \\
& \left.+\left(\ddot{y}_{1} z_{1}-\ddot{z}_{1} y_{1}-\ddot{\theta}\left(z_{1}^{2}+y_{1}^{2}\right)-2 \dot{\theta}\left(\dot{z}_{1} z_{1}+\dot{y}_{1} y_{1}\right)\right) \delta \theta\right) d \eta d \zeta d x-I_{\theta} \ddot{\theta} \delta \theta
\end{aligned}
$$

Finally, we seek an expression in the form of Eq. 2.105 in terms of the arbitrary variations of the displacements (similar to Eq. 2.74 for the strain energy). $\bar{T}_{u}, \bar{T}_{v}, \bar{T}_{w}, \bar{T}_{\phi}, \bar{T}_{\theta}$, are introduced as a concise notation to represent the coefficients of $\delta u, \delta v, \delta w, \delta \phi$, and $\delta \theta$ as [1],

$$
\delta T=\int_{0}^{S}\left(\bar{T}_{u} \delta u+\bar{T}_{v} \delta v+\bar{T}_{w} \delta w+\bar{T}_{\phi} \delta \phi\right) d x+\bar{T}_{\theta} \delta \theta+b(T)
$$

However in its current form, there are no explicit instances of these variations. Hence substituting Eq.2.96 g), Eq.2.96 h and Eq.2.96 i) for $\delta x_{1}, \delta y_{1}$, and $\delta z_{1}$ yields an expression in terms of the variations of the displacements and their derivatives:

$$
\begin{aligned}
\delta T=\int_{0}^{S} \iint_{A} \rho( & \left.-\ddot{x}_{1}\right)\left(\delta u-\lambda \delta \phi^{\prime}-\left(\delta v^{\prime}+w^{\prime} \delta \phi\right)\left(y_{1}-\left(v+e_{p}\right)\right)-\left(\delta w^{\prime}-v^{\prime} \delta \phi\right)\left(z_{1}-w\right)\right) \\
& +\left(-\ddot{y}_{1}+y_{1} \dot{\theta}^{2}+\ddot{\theta} z_{1}+2 \dot{\theta} \dot{z}_{1}\right)\left(\delta v-\delta \phi\left(z_{1}-w\right)\right) \\
& +\left(-\ddot{z}_{1}+z_{1} \dot{\theta}^{2}-\ddot{\theta} y_{1}-2 \dot{\theta} \dot{y}_{1}\right)\left(\delta w+\delta \phi\left(y_{1}-\left(v+e_{p}\right)\right)\right)
\end{aligned}
$$




$$
\left.+\left(\ddot{y}_{1} z_{1}-\ddot{z}_{1} y_{1}-\ddot{\theta}\left(z_{1}^{2}+y_{1}^{2}\right)-2 \dot{\theta}\left(\dot{z}_{1} z_{1}+\dot{y}_{1} y_{1}\right)\right) \delta \theta\right) d \eta d \zeta d x-I_{\theta} \ddot{\theta} \delta \theta
$$

Now regrouping the variations of $v, w, \phi$, and $\theta$, we obtain

$$
\begin{aligned}
\delta T=\int_{0}^{S} \iint_{A} \rho(\{ & \left.\left(-\ddot{x}_{1}\right) \delta u\right\}+\left\{\left(\ddot{x}_{1}\right)\left(y_{1}-\left(v+e_{p}\right)\right) \delta v^{\prime}+\left(-\ddot{y}_{1}+y_{1} \dot{\theta}^{2}+\ddot{\theta} z_{1}+2 \dot{\theta} \dot{z}_{1}\right) \delta v\right\} \\
+ & \left\{\left(\ddot{x}_{1}\right)(z-w) \delta w^{\prime}+\left(-\ddot{z}_{1}+z_{1} \dot{\theta}^{2}-\ddot{\theta} y_{1}-2 \dot{\theta} \dot{y}_{1}\right) \delta w\right\} \\
+ & \left\{\left(\ddot{x}_{1}\right) \lambda \delta \phi^{\prime}+\left(\left(\ddot{x}_{1}\right)\left(w^{\prime}\right)\left(y_{1}-\left(v+e_{p}\right)\right)-\left(\ddot{x}_{1}\right)\left(v^{\prime}\right)\left(z_{1}-w\right)\right.\right. \\
& \left.\quad-\left(-\ddot{y}_{1}+y_{1} \dot{\theta}^{2}+\ddot{\theta} z_{1}+2 \dot{\theta} \dot{z}_{1}\right)\left(z_{1}-w\right)+\left(-\ddot{z}_{1}+z_{1} \dot{\theta}^{2}-\ddot{\theta} y_{1}-2 \dot{\theta} \dot{y}_{1}\right)\left(y_{1}-\left(v+e_{p}\right)\right) \delta \phi\right\} \\
+ & \left.\left\{\left(\ddot{y}_{1} z_{1}-\ddot{z}_{1} y_{1}-\ddot{\theta}\left(z_{1}^{2}+y_{1}^{2}\right)-2 \dot{\theta}\left(\dot{z}_{1} z_{1}+\dot{y}_{1} y_{1}\right)\right) \delta \theta\right\}\right) d \eta d \zeta d x-I_{\theta} \ddot{\theta} \delta \theta .
\end{aligned}
$$

Again noting that we have the form given below, as with Eq. 2.75 we will integrate by parts, yielding boundary conditions and contributions to the PDE as 1

$$
\delta T=\int_{0}^{S}\left(T_{u} \delta u+T_{v^{\prime}} \delta v^{\prime}+T_{v} \delta v+T_{w}^{\prime} \delta w^{\prime}+T_{w} \delta w+T_{\phi^{\prime}} \delta \phi^{\prime}+T_{\phi} \delta \phi\right) d x+T_{\theta} \delta \theta
$$

$u: \int_{0}^{S} \iint_{A}\left(-\ddot{x}_{1}\right) \delta u d \eta d \zeta d x$

$$
\begin{aligned}
v: & \int_{0}^{S} \iint_{A}\left(\ddot{x}_{1}\right)\left(y_{1}-\left(v+e_{p}\right)\right) \delta v^{\prime}+\left(-\ddot{y}_{1}+y_{1} \dot{\theta}^{2}+\ddot{\theta} z_{1}+2 \dot{\theta} \dot{z}_{1}\right) \delta v d \eta d \zeta d x \\
= & \iint_{A}\left\{\left.\left(\ddot{x}_{1}\right)\left(y_{1}-\left(v+e_{p}\right)\right) \delta v\right|_{0} ^{S}-\int_{0}^{S}\left(\left(\ddot{x}_{1}^{\prime}\right)\left(y_{1}-\left(v+e_{p}\right)\right)+\left(\ddot{x}_{1}\right)\left(y_{1}^{\prime}-v^{\prime}\right)\right) \delta v d x\right. \\
& \left.\quad+\int_{0}^{S}\left(-\ddot{y}_{1}+y_{1} \dot{\theta}^{2}+\ddot{\theta} z_{1}+2 \dot{\theta} \dot{z}_{1}\right) \delta v d x\right\} d \eta d \zeta \\
w: & \int_{0}^{S} \iint_{A}\left(\ddot{x}_{1}\right)\left(z_{1}-w\right) \delta w^{\prime}+\left(-\ddot{z}_{1}+z_{1} \dot{\theta}^{2}-\ddot{\theta} y_{1}-2 \dot{\theta} \dot{y}_{1}\right) \delta w d \eta d \zeta d x \\
= & \iint_{A}\left\{\left.\left(\ddot{x}_{1}\right)\left(z_{1}-w\right) \delta w\right|_{0} ^{S}-\int_{0}^{S}\left(\left(\ddot{x}_{1}^{\prime}\right)\left(z_{1}-w\right)+\left(\ddot{x}_{1}\right)\left(z_{1}^{\prime}-w^{\prime}\right)\right) \delta w d x\right.
\end{aligned}
$$




$$
\begin{aligned}
&\left.+\int_{0}^{S}\left(-\ddot{z}_{1}+z_{1} \dot{\theta}^{2}-\ddot{\theta} y_{1}-2 \dot{\theta} \dot{y}_{1}\right) \delta w d x\right\} d \eta d \zeta \\
& \phi: \quad \int_{0}^{S} \int_{A}\left(\ddot{x}_{1}\right) \lambda \delta \phi^{\prime}+\left(\left(\ddot{x}_{1}\right)\left(w^{\prime}\right)\left(y_{1}-\left(v+e_{p}\right)\right)+\left(\ddot{x}_{1}\right)\left(v^{\prime}\right)\left(z_{1}-w\right)\right. \\
&\left.\quad-\left(-\ddot{y}_{1}+y_{1} \dot{\theta}^{2}+\ddot{\theta} z_{1}+2 \dot{\theta}_{z_{1}}\right)\left(z_{1}-w\right)+\left(-\ddot{z}_{1}+z_{1} \dot{\theta}^{2}-\ddot{\theta} y_{1}-2 \dot{\theta} \dot{y}_{1}\right)\left(y_{1}-\left(v+e_{p}\right)\right)\right) \delta \phi d \eta d \zeta d x \\
&=\iint_{A}\left\{\left.\left(\ddot{x}_{1}\right) \lambda \delta \phi\right|_{0} ^{S}-\int_{0}^{S}\left(\ddot{x}_{1}^{\prime}\right) \lambda \delta \phi d x+\int_{0}^{S}\left(\left(\ddot{x}_{1}\right)\left(w^{\prime}\right)\left(y_{1}-\left(v+e_{p}\right)\right)-\left(\ddot{x}_{1}\right)\left(v^{\prime}\right)\left(z_{1}-w\right)\right.\right. \\
&\left.\left.\quad-\left(-\ddot{y}_{1}+y_{1} \dot{\theta}^{2}+\ddot{\theta} z_{1}+2 \dot{\theta} \dot{z}_{1}\right)\left(z_{1}-w\right)+\left(-\ddot{z}_{1}+z_{1} \dot{\theta}^{2}-\ddot{\theta} y_{1}-2 \dot{\theta} \dot{y}_{1}\right)\left(y_{1}-\left(v+e_{p}\right)\right)\right) \delta \phi d x\right\} d \eta d \zeta \\
& \theta: \quad \int_{0}^{S} \iint_{A}\left\{\left(\ddot{y}_{1} z_{1}-\ddot{z}_{1} y_{1}-\ddot{\theta}\left(z_{1}^{2}+y_{1}^{2}\right)-2 \dot{\theta}\left(\dot{z}_{1} z_{1}+\dot{y}_{1} y_{1}\right)\right) \delta \theta d x\right\} d \eta d \zeta-I_{\theta} \ddot{\theta} \delta \theta
\end{aligned}
$$

In order to obtain the final desired form, now the expressions for $\ddot{x}_{1}, \ddot{y}_{1}, \ddot{z}_{1}, \dot{x}_{1}, \dot{y}_{1}, \dot{z}_{1}, x_{1}, y_{1}, z_{1}$ will be substituted into the expression, and then integrated over the area. In its current form, the PDE and boundary conditions are given as follows:

$$
\begin{aligned}
& \delta T=\rho \int_{0}^{S} \int_{A}\{\left\{-\left(\ddot{x}_{1}\right)\right\} \delta u+\left\{-\left(\ddot{x}_{1}^{\prime}\right)\left(y_{1}-\left(v+e_{p}\right)\right)-\left(\ddot{x}_{1}\right)\left(y_{1}^{\prime}-v^{\prime}\right)-\ddot{y}_{1}+y_{1} \dot{\theta}^{2}+\ddot{\theta} z_{1}+2 \dot{\theta} \dot{z}_{1}\right\} \delta v \\
&+\left\{-\left(\ddot{x}_{1}^{\prime}\right)\left(z_{1}-w\right)-\left(\ddot{x}_{1}\right)\left(z_{1}^{\prime}-w^{\prime}\right)-\ddot{z}_{1}+z_{1} \dot{\theta}^{2}-\ddot{\theta} y_{1}-2 \dot{\theta} \dot{y}_{1}\right\} \delta w \\
&+\left\{-\left(\ddot{x}_{1}^{\prime}\right) \lambda+\left(\left(\ddot{x}_{1}\right)\left(w^{\prime}\right)+\left(-\ddot{z}_{1}+z_{1} \dot{\theta}^{2}-\ddot{\theta} y_{1}-2 \dot{\theta} \dot{y}_{1}\right)\right)\left(y_{1}-\left(v+e_{p}\right)\right)\right. \\
&\left.+\left(-\left(\ddot{x}_{1}\right)\left(v^{\prime}\right)-\left(-\ddot{y}_{1}+y_{1} \dot{\theta}^{2}+\ddot{\theta} z_{1}+2 \dot{\theta} \dot{z}_{1}\right)\right)\left(z_{1}-w\right)\right\} \delta \phi \\
&+\left.\left\{\ddot{y}_{1} z_{1}-\ddot{z}_{1} y_{1}-\ddot{\theta}\left(z_{1}^{2}+y_{1}^{2}\right)-2 \dot{\theta}\left(\dot{z}_{1} z_{1}+\dot{y}_{1} y_{1}\right)\right\} \delta \theta\right\} d x d \eta d \zeta-I_{\theta} \ddot{\theta} \delta \theta \\
& \\
& b(T)=\rho \iint_{A}\left\{\left.\left(\ddot{x}_{1}\right)\left(y_{1}-\left(v+e_{p}\right)\right) \delta v\right|_{0} ^{S}+\left.\left(\ddot{x}_{1}\right)\left(z_{1}-w\right) \delta w\right|_{0} ^{S}+\left.\left(\ddot{x}_{1}\right) \lambda \delta \phi\right|_{0} ^{S}\right\} d \eta d \zeta
\end{aligned}
$$

Looking at the contributions to the PDE, making the appropriate substitutions and in a subsequent step integrating the terms containing $\eta$ and $\zeta$, results in the expressions $\bar{T}_{u}, \bar{T}_{v}, \bar{T}_{w}, \bar{T}_{\phi}$, and $\bar{T}_{\theta}$. The underlined terms above are retained, while all other terms can be neglected given that they are of order $O\left(\varepsilon^{2}\right)$ higher than the largest (i.e. lowest order) term, 


$$
\begin{aligned}
& u: \quad-\ddot{x}_{1} \\
& =-\left(\ddot{u}-\lambda \ddot{\phi}^{\prime}-\left(\ddot{v}^{\prime}+w^{\prime} \ddot{\phi}+2 \dot{w}^{\prime} \dot{\phi}\right)(\eta \cos \phi-\zeta \sin \phi)-\left(\ddot{w}^{\prime}-v^{\prime} \ddot{\phi}-2 \dot{v}^{\prime} \dot{\phi}\right)(\eta \sin \phi+\zeta \cos \phi)\right) \\
& v: \quad-\left(\ddot{x}_{1}^{\prime}\right)\left(y_{1}-\left(v+e_{p}\right)\right)-\left(\ddot{x}_{1}\right)\left(y_{1}^{\prime}-v^{\prime}\right)-\ddot{y}_{1}+y_{1} \dot{\theta}^{2}+\ddot{\theta} z_{1}+2 \dot{\theta} \dot{z}_{1} \\
& =-\left(\ddot{u}-\lambda \ddot{\phi}^{\prime}-\left(\ddot{v}^{\prime}+w^{\prime} \ddot{\phi}+2 \dot{w}^{\prime} \dot{\phi}\right)(\eta \cos \phi-\zeta \sin \phi)-\left(\ddot{w}^{\prime}-v^{\prime} \ddot{\phi}-2 \dot{v}^{\prime} \dot{\phi}\right)(\eta \sin \phi+\zeta \cos \phi)\right)^{\prime}(\eta \cos \phi-\zeta \sin \phi) \\
& -\left(\ddot{u}-\lambda \ddot{\phi}^{\prime}-\left(\ddot{v}^{\prime}+w^{\prime} \ddot{\phi}+2 \dot{w}^{\prime} \dot{\phi}\right)(\eta \cos \phi-\zeta \sin \phi)-\left(\ddot{w}^{\prime}-v^{\prime} \ddot{\phi}-2 \dot{v}^{\prime} \dot{\phi}\right)(\eta \sin \phi+\zeta \cos \phi)\right)(\eta \cos \phi-\zeta \sin \phi)^{\prime} \\
& +(-\ddot{v}+\ddot{\phi}(\eta \sin \phi+\zeta \cos \phi))+\left(v+e_{p}+(\eta \cos \phi-\zeta \sin \phi)\right) \dot{\theta}^{2}+(w+(\eta \sin \phi+\zeta \cos \phi)) \ddot{\theta} \\
& +2(\dot{w}+\dot{\phi}(\eta \cos \phi-\zeta \sin \phi)) \dot{\theta} \\
& \left.=(\eta \cos \phi-\zeta \sin \phi)^{2}\left(\left(\ddot{v}^{\prime \prime}+w^{\prime \prime} \ddot{\phi}+w^{\prime} \ddot{\phi}^{\prime}+2 \dot{w}^{\prime \prime} \dot{\phi}+2 \dot{w}^{\prime} \dot{\phi}^{\prime}\right)+\dot{\phi}\left(\ddot{w}-v^{\prime} \ddot{\phi}-2 \dot{v}^{\prime} \dot{\phi}\right)\right)\right) \\
& +(\eta \cos \phi-\zeta \sin \phi)(\eta \sin \phi+\zeta \cos \phi)\left(\left(1-\phi^{\prime}\right)\left(\ddot{w}^{\prime}-v^{\prime} \ddot{\phi}+2 \dot{v}^{\prime} \dot{\phi}\right)-\left(\ddot{w}^{\prime \prime}-v^{\prime \prime} \dot{\phi}-v^{\prime} \ddot{\phi}^{\prime}-2 \dot{v}^{\prime \prime} \dot{\phi}+2 \dot{v}^{\prime} \dot{\phi}^{\prime}\right)\right) \\
& +(\eta \sin \phi+\zeta \cos \phi)^{2}\left(-\phi^{\prime}\left(\ddot{w}-\dot{v}^{\prime} \ddot{\phi}-2 \dot{v}^{\prime} \dot{\theta}\right)\right)+(\eta \cos \phi-\zeta \sin \phi)\left(-\ddot{u}^{\prime}+\lambda \ddot{\phi}^{\prime \prime}+\underline{\dot{\theta}^{2}+2 \dot{\phi} \dot{\theta}}\right) \\
& +(\eta \sin \phi+\zeta \cos \phi)\left(-\ddot{u}+\lambda \ddot{\phi}^{\prime}+\underline{\ddot{\phi}+\ddot{\theta}}\right)-\underline{\ddot{v}+\left(v+e_{p}\right) \dot{\theta}^{2}+w \ddot{\theta}+2 \dot{w} \dot{\theta}}
\end{aligned}
$$

$$
\begin{aligned}
& w: \quad-\left(\ddot{x}_{1}^{\prime}\right)\left(z_{1}-w\right)-\left(\ddot{x}_{1}\right)\left(z_{1}-w\right)^{\prime}-\ddot{z}_{1}+z_{1} \dot{\theta}^{2}-\ddot{\theta} y_{1}-2 \dot{\theta} \dot{y}_{1} \\
& =-\left(\ddot{u}-\lambda \ddot{\phi}^{\prime}-\left(\ddot{v}^{\prime}+w^{\prime} \ddot{\phi}+2 \dot{w}^{\prime} \dot{\phi}\right)(\eta \cos \phi-\zeta \sin \phi)-\left(\ddot{w}^{\prime}-v^{\prime} \ddot{\phi}-2 \dot{v}^{\prime} \dot{\phi}\right)(\eta \sin \phi+\zeta \cos \phi)\right)^{\prime}(\eta \sin \phi+\zeta \cos \phi) \\
& -\left(\ddot{u}-\lambda \ddot{\phi}^{\prime}-\left(\ddot{v}^{\prime}+w^{\prime} \ddot{\phi}+2 \dot{w}^{\prime} \dot{\phi}\right)(\eta \cos \phi-\zeta \sin \phi)-\left(\ddot{w}-v^{\prime} \ddot{\phi}-2 \dot{v}^{\prime} \dot{\phi}\right)(\eta \sin \phi+\zeta \cos \phi)\right)(\eta \sin \phi+\zeta \cos \phi)^{\prime} \\
& +(-\ddot{w}-\ddot{\phi}(\eta \cos \phi-\zeta \sin \phi))+(w+(\eta \sin \phi+\zeta \cos \phi)) \dot{\theta}^{2}-\left(v+e_{p}+(\eta \cos \phi-\zeta \sin \phi)\right) \ddot{\theta} \\
& -2(\dot{v}-\dot{\phi}(\eta \sin \phi+\zeta \cos \phi)) \dot{\theta} \\
& =(\eta \cos \phi-\zeta \sin \phi)^{2}\left(\ddot{v}^{\prime}+w^{\prime} \ddot{\phi}+2 w \dot{\phi}\right)+(\eta \cos \phi-\zeta \sin \phi)(\eta \sin \phi+\zeta \cos \phi)\left(\left(1+\phi^{\prime}\right)\left(\ddot{w}^{\prime}-v^{\prime} \ddot{\phi}+2 \dot{v}^{\prime} \dot{\phi}\right)\right. \\
& \left.+\left(\ddot{v}^{\prime \prime}+w^{\prime \prime} \ddot{\phi}+w^{\prime} \ddot{\phi}^{\prime}+2 \dot{w}^{\prime \prime} \dot{\phi}+2 \dot{w}^{\prime} \dot{\phi}^{\prime}\right)\right)+(\eta \sin \phi+\zeta \cos \phi)^{2}\left(-\phi^{\prime}\left(\ddot{v}^{\prime}+w^{\prime} \ddot{\phi}+2 w \dot{\phi}\right)\right. \\
& \left.+\left(\ddot{w}^{\prime \prime}-v^{\prime \prime} \dot{\phi}-v^{\prime} \ddot{\phi}^{\prime}-2 \dot{v}^{\prime \prime} \dot{\phi}+2 \dot{v}^{\prime} \dot{\phi}^{\prime}\right)\right)+(\eta \cos \phi-\zeta \sin \phi)\left(-\ddot{u}+\lambda \ddot{\phi}^{\prime}-\ddot{\phi}-\ddot{\theta}\right) \\
& +(\eta \sin \phi+\zeta \cos \phi)\left(-\ddot{u}^{\prime}+\lambda \ddot{\phi}^{\prime \prime}+\underline{\dot{\theta}^{2}+2 \dot{\phi} \dot{\theta}}\right)-\underline{\ddot{w}+w \dot{\theta}^{2}-\left(v+e_{p}\right) \ddot{\theta}-2 \dot{v} \dot{\theta}}
\end{aligned}
$$

$$
\begin{aligned}
\phi: & -\left(\ddot{x}_{1}^{\prime}\right) \lambda+\left(y_{1}-\left(v+e_{p}\right)\right)\left(w^{\prime} \ddot{x}_{1}-\ddot{z}_{1}+z_{1} \dot{\theta}^{2}-\ddot{\theta} y_{1}-2 \dot{\theta} \dot{y}_{1}\right)+\left(z_{1}-w\right)\left(-v^{\prime} \ddot{x}_{1}+\ddot{y}_{1}-y_{1} \dot{\theta}^{2}-\ddot{\theta} z_{1}-2 \dot{\theta} \dot{z}_{1}\right) \\
= & \left(-\ddot{u}+\lambda \ddot{\phi}^{\prime}+\left(\ddot{v}^{\prime}+w^{\prime} \ddot{\phi}+2 \dot{w}^{\prime} \dot{\phi}\right)(\eta \cos \phi-\zeta \sin \phi)+\left(\ddot{w}^{\prime}-v^{\prime} \ddot{\phi}-2 \dot{v}^{\prime} \dot{\phi}\right)(\eta \sin \phi+\zeta \cos \phi)\right)^{\prime} \lambda \\
& +(\eta \cos \phi-\zeta \sin \phi)\left(w^{\prime}\left(\ddot{u}-\lambda \ddot{\phi}^{\prime}-\left(\ddot{v}^{\prime}+w^{\prime} \ddot{\phi}+2 \dot{w}^{\prime} \dot{\phi}\right)(\eta \cos \phi-\zeta \sin \phi)-\left(\ddot{w}^{\prime}-v^{\prime} \ddot{\phi}-2 \dot{\phi} \dot{v}^{\prime}\right)(\eta \sin \phi+\zeta \cos \phi)\right)\right. \\
& -(\ddot{w}+\ddot{\phi}(\eta \cos \phi-\zeta \sin \phi))+(w+(\eta \sin \phi+\zeta \cos \phi)) \dot{\theta}^{2}-\left(v+e_{p}+(\eta \cos \phi-\zeta \sin \phi)\right) \ddot{\theta} \\
& -2 \dot{\theta}(\dot{v}-\dot{\phi}(\eta \sin \phi+\zeta \cos \phi)))+(\eta \sin \phi+\zeta \cos \phi)\left(-v^{\prime}\left(\ddot{u}-\lambda \ddot{\phi}^{\prime}-\left(\ddot{v}^{\prime}+w^{\prime} \ddot{\phi}+2 \dot{w}^{\prime} \dot{\phi}\right)(\eta \cos \phi-\zeta \sin \phi)\right.\right.
\end{aligned}
$$




$$
\begin{aligned}
& \left.-\left(\ddot{w}^{\prime}-v^{\prime} \ddot{\phi}-2 \dot{\phi} \dot{v}^{\prime}\right)(\eta \sin \phi+\zeta \cos \phi)\right)+(\ddot{v}-\ddot{\phi}(\eta \sin \phi+\zeta \cos \phi))-\left(v+e_{p}+(\eta \cos \phi-\zeta \sin \phi)\right) \dot{\theta}^{2} \\
& -(w+(\eta \sin \phi+\zeta \cos \phi)) \ddot{\theta}-2(\dot{w}+\dot{\phi}(\eta \cos \phi-\zeta \sin \phi)) \dot{\theta}) \\
= & (\eta \cos \phi-\zeta \sin \phi)^{2}\left(-w^{\prime}\left(\ddot{v}^{\prime}+\ddot{\phi} w^{\prime}+2 \dot{\phi} \dot{w}^{\prime}\right) \underline{-\ddot{\phi}-\ddot{\theta}}\right)+(\eta \sin \phi+\zeta \cos \phi)^{2}\left(v^{\prime}\left(\ddot{w}^{\prime}-v^{\prime} \ddot{\phi}-2 \dot{\phi} \dot{v}^{\prime}\right)-\ddot{\phi}-\ddot{\theta}\right) \\
& +(\eta \cos \phi-\zeta \sin \phi)(\eta \sin \phi+\zeta \cos \phi)\left(-w^{\prime}\left(\ddot{w}^{\prime}-v^{\prime} \ddot{\phi}-2 \dot{\phi} \dot{v}^{\prime}\right)+v^{\prime}\left(\ddot{v}^{\prime}+w^{\prime} \ddot{\phi}+2 w^{\prime} \dot{\phi}\right)\right) \\
& +(\eta \cos \phi-\zeta \sin \phi)\left(\ddot{u} w^{\prime}-\lambda w^{\prime} \ddot{\phi}^{\prime}-\ddot{w}-\left(v+e_{p}\right) \ddot{\theta}-2 \dot{v} \dot{\theta}+w \dot{\theta}^{2}+\lambda\left(\ddot{v}^{\prime \prime}+w^{\prime \prime} \ddot{\phi}+w^{\prime} \ddot{\phi}^{\prime}+2 \dot{w}^{\prime \prime} \dot{\phi}+2 \dot{w}^{\prime} \dot{\phi}^{\prime}\right.\right. \\
& \left.+\ddot{w}^{\prime} \phi^{\prime}-v^{\prime} \ddot{\phi} \phi^{\prime}-2 \dot{v}^{\prime} \dot{\phi} \phi^{\prime}\right)+(\eta \sin \phi+\zeta \cos \phi)\left(-\ddot{u} v^{\prime}+\lambda v^{\prime} \ddot{\phi}^{\prime}+\ddot{v}-\left(v+e_{p}\right) \dot{\theta}^{2}-w \ddot{\theta}-2 \dot{w} \dot{\theta}\right. \\
& +\lambda\left(\ddot{w}^{\prime \prime}-v^{\prime \prime} \dot{\phi}-v^{\prime} \ddot{\phi}^{\prime}-2 \dot{v}^{\prime \prime} \dot{\phi}-2 \dot{v}^{\prime} \dot{\phi}^{\prime}-\ddot{v}^{\prime} \phi^{\prime}-w^{\prime} \ddot{\phi} \phi^{\prime}-2 \dot{w} \dot{\phi} \phi^{\prime}\right)-\lambda \ddot{u}+\lambda^{2} \ddot{\phi}^{\prime \prime}
\end{aligned}
$$

$\theta: \quad \ddot{y}_{1} z_{1}-\ddot{z}_{1} y_{1}-\ddot{\theta}\left(z_{1}^{2}+y_{1}^{2}\right)-2 \dot{\theta}\left(\dot{z}_{1} z_{1}+\dot{y}_{1} y_{1}\right)$

$=(\ddot{v}-\ddot{\phi}(\eta \sin \phi+\zeta \cos \phi))(w+(\eta \sin \phi+\zeta \cos \phi))-(\ddot{w}+\ddot{\phi}(\eta \cos \phi-\zeta \sin \phi))\left(v+e_{p}+(\eta \cos \phi-\zeta \sin \phi)\right)$

$-\ddot{\theta}\left((w+(\eta \sin \phi+\zeta \cos \phi))^{2}+\left(v+e_{p}+(\eta \cos \phi-\zeta \sin \phi)\right)^{2}\right)$

$-2 \dot{\theta}\left((\dot{w}+\dot{\phi}(\eta \cos \phi-\zeta \sin \phi))(w+(\eta \sin \phi+\zeta \cos \phi))+(\dot{v}-\dot{\phi}(\eta \sin \phi+\zeta \cos \phi))\left(v+e_{p}+(\eta \cos \phi-\zeta \sin \phi)\right)\right)$

$=\underline{\left((\eta \cos \phi-\zeta \sin \phi)^{2}+(\eta \sin \phi+\zeta \cos \phi)^{2}\right)(-\ddot{\phi}-\ddot{\theta})}$

$+\underline{(\eta \cos \phi-\zeta \sin \phi)\left(-\ddot{w}-\ddot{\phi}\left(v+e_{p}\right)-2 \ddot{\theta}\left(v+e_{p}\right)-2 \dot{\theta}(w \dot{\phi}+\dot{v})\right)}$

$+\underline{(\eta \sin \phi+\zeta \cos \phi)\left(\ddot{v}-w \ddot{\phi}-2 w \ddot{\theta}-2 \dot{\theta}\left(\dot{w}-\dot{\phi}\left(v+e_{p}\right)\right)\right)}$

$+\underline{\ddot{v} w-\ddot{w}\left(v+e_{p}\right)-\ddot{\theta} w^{2}-\ddot{\theta}\left(v+e_{p}\right)^{2}-2 \dot{\theta}\left(\dot{w} w+\dot{v}\left(v+e_{p}\right)\right)}$

We can write expressions for each term in Eq.2.105, associated with each of the 5 displacements.

We will rearrange these terms to isolate for $\eta$ and $\zeta$, and then integrate over the area. Through this process, we recover many of the terms presented in [2] as,

$$
\begin{aligned}
& \bar{T}_{u}=\iint_{A} \rho\{\left.-\left(\ddot{u}-\lambda \ddot{\phi}^{\prime}-\left(\ddot{v}^{\prime}+w^{\prime} \ddot{\phi}+2 \dot{w}^{\prime} \dot{\phi}\right)(\eta \cos \phi-\zeta \sin \phi)-\left(\ddot{w}^{\prime}-v^{\prime} \ddot{\phi}-2 \dot{v}^{\prime} \dot{\phi}\right)(\eta \sin \phi+\zeta \cos \phi)\right)\right\} d \eta d \zeta \\
&=\iint_{A} \rho\left\{\eta\left(\left(\ddot{v}^{\prime}+w^{\prime} \ddot{\phi}+2 \dot{w}^{\prime} \dot{\phi}\right) \cos \phi+\left(\ddot{w}^{\prime}-v^{\prime} \ddot{\phi}-2 \dot{v}^{\prime} \dot{\phi}\right) \sin \phi\right)\right. \\
&\left.\quad+\zeta\left(\left(\ddot{w}^{\prime}-v^{\prime} \ddot{\phi}-2 \dot{v}^{\prime} \dot{\phi}\right) \cos \phi-\left(\ddot{v}^{\prime}+w^{\prime} \ddot{\phi}+2 \dot{w}^{\prime} \dot{\phi}\right) \sin \phi\right)-\ddot{u}+\lambda \ddot{\phi}^{\prime}\right\} d \eta d \zeta \\
&=\left(\left(\ddot{v}^{\prime}+w^{\prime} \ddot{\phi}+2 \dot{w}^{\prime} \dot{\phi}\right) \cos \phi+\left(\ddot{w}^{\prime}-v^{\prime} \ddot{\phi}-2 \dot{v}^{\prime} \dot{\phi}\right) \sin \phi\right) \iint_{A} \rho \eta d \eta d \zeta \\
& \quad+\left(\left(\ddot{w}^{\prime}-v^{\prime} \ddot{\phi}-2 \dot{v}^{\prime} \dot{\phi}\right) \cos \phi-\left(\ddot{v}^{\prime}+w^{\prime} \ddot{\phi}+2 \dot{w}^{\prime} \dot{\phi}\right) \sin \phi\right) \iint_{A} \rho \zeta d \eta d \zeta-\ddot{u} \iint_{A} \rho d \eta d \zeta+\ddot{\phi}^{\prime} \iint_{A} \rho \lambda d \eta d \zeta \\
&=-\bar{m} \ddot{u}+\bar{m} e\left(\left(\ddot{v}^{\prime}+w^{\prime} \ddot{\phi}+2 \dot{w}^{\prime} \dot{\phi}\right) \cos \phi+\left(\ddot{w}^{\prime}-v^{\prime} \ddot{\phi}-2 \dot{v}^{\prime} \dot{\phi}\right) \sin \phi\right)
\end{aligned}
$$




$$
\begin{aligned}
& \bar{T}_{v}= \iint_{A} \rho\left\{(\eta \cos \phi-\zeta \sin \phi)\left(\dot{\theta}^{2}+2 \dot{\phi} \dot{\theta}\right)+(\eta \sin \phi+\zeta \cos \phi)(\ddot{\phi}+\ddot{\theta})-\ddot{v}+\left(v+e_{p}\right) \dot{\theta}^{2}+w \ddot{\theta}+2 \dot{w} \dot{\theta}\right\} d \eta d \zeta \\
&= \iint_{A} \rho\left\{\eta\left(\left(\dot{\theta}^{2}+2 \dot{\phi} \dot{\theta}\right) \cos \phi+(\ddot{\phi}+\ddot{\theta}) \sin \phi\right)+\zeta\left(-\left(\dot{\theta}^{2}+2 \dot{\phi} \dot{\theta}\right) \sin \phi+(\ddot{\phi}+\ddot{\theta}) \cos \phi\right)-\ddot{v}+\left(v+e_{p} \dot{\theta}^{2}\right.\right. \\
&\quad+w \ddot{\theta}+2 \dot{w} \dot{\theta}\} d \eta d \zeta \\
&=\left(\left(\dot{\theta}^{2}+2 \dot{\phi} \dot{\theta}\right) \cos \phi+(\ddot{\phi}+\ddot{\theta}) \sin \phi\right) \iint_{A} \rho \eta d \eta d \zeta+\left(-\left(\dot{\theta}^{2}+2 \dot{\phi} \dot{\theta}\right) \sin \phi+(\ddot{\phi}+\ddot{\theta}) \cos \phi\right) \iint_{A} \rho \zeta d \eta d \zeta \\
& \quad+\left(-\ddot{v}+\left(v+e_{p}\right) \dot{\theta}^{2}+w \ddot{\theta}+2 \dot{w} \dot{\theta}\right) \iint_{A} \rho d \eta d \zeta \\
&= \bar{m} e\left(\left(\dot{\theta}^{2}+2 \dot{\phi} \dot{\theta}\right) \cos \phi+(\ddot{\phi}+\ddot{\theta}) \sin \phi\right)+\bar{m}\left(-\ddot{v}+\left(v+e_{p}\right) \dot{\theta}^{2}+w \ddot{\theta}+2 \dot{w} \dot{\theta}\right) \\
&=-\bar{m} \ddot{v}+\bar{m} e \ddot{\phi} \sin \phi+\bar{m} \ddot{\theta}(e \sin \phi+w)+\bar{m} \dot{\theta}^{2}\left(\left(v+e_{p}\right)+e \cos \phi\right)+2 \bar{m} \dot{\theta}(e \dot{\phi} \cos \phi+\dot{w})
\end{aligned}
$$

$$
\begin{aligned}
& \bar{T}_{w}= \iint_{A} \rho\left\{(\eta \cos \phi-\zeta \sin \phi)(-\ddot{\phi}-\ddot{\theta})+(\eta \sin \phi+\zeta \cos \phi)\left(\dot{\theta}^{2}+2 \dot{\phi} \dot{\theta}\right)-\ddot{w}+w \dot{\theta}^{2}-\left(v+e_{p}\right) \ddot{\theta}-2 \dot{v} \dot{\theta}\right\} d \eta d \zeta \\
&=\iint_{A} \rho\left\{\eta\left(-(\ddot{\phi}+\ddot{\theta}) \cos \phi+\left(\dot{\theta}^{2}+2 \dot{\phi} \dot{\theta}\right) \sin \phi\right)+\zeta\left((\ddot{\phi}+\ddot{\theta}) \sin \phi+\left(\dot{\theta}^{2}+2 \dot{\phi} \dot{\theta}\right) \cos \phi\right)-\ddot{w}+w \dot{\theta}^{2}\right. \\
&\left.\quad-\left(v+e_{p}\right) \ddot{\theta}-2 \dot{v} \dot{\theta}\right\} d \eta d \zeta \\
&=\left(-(\ddot{\phi}+\ddot{\theta}) \cos \phi+\left(\dot{\theta}^{2}+2 \dot{\phi} \dot{\theta}\right) \sin \phi\right) \iint_{A} \rho \eta d \eta d \zeta+\left((\ddot{\phi}+\ddot{\theta}) \sin \phi+\left(\dot{\theta}^{2}+2 \dot{\phi} \dot{\theta}\right) \cos \phi\right) \iint_{A} \rho \zeta d \eta d \zeta \\
& \quad+\left(-\ddot{w}+w \dot{\theta}^{2}-\left(v+e_{p}\right) \ddot{\theta}-2 \dot{v} \dot{\theta}\right) \iint_{A} \rho d \eta d \zeta \\
&=-\bar{m} \ddot{w}-\bar{m} e \ddot{\phi} \cos \phi+\bar{m} \ddot{\theta}\left(-e \cos \phi-\left(v+e_{p}\right)\right)+\bar{m} \dot{\theta}^{2}(e \sin \phi+w)+2 \bar{m} \dot{\theta}(e \dot{\phi} \sin \phi-\dot{v})
\end{aligned}
$$

$$
\begin{aligned}
& \bar{T}_{\phi}=\iint_{A} \rho\{(\left.(\eta \cos \phi-\zeta \sin \phi)^{2}+(\eta \sin \phi+\zeta \cos \phi)^{2}\right)(-\ddot{\phi}-\ddot{\theta})+(\eta \cos \phi-\zeta \sin \phi)\left(-\ddot{w}-\left(v+e_{p}\right) \ddot{\theta}-2 \dot{v} \dot{\theta}+w \dot{\theta}^{2}\right) \\
&\left.\left.+(\eta \sin \phi+\zeta \cos \phi)\left(\ddot{v}-\left(v+e_{p}\right) \dot{\theta}^{2}-w \ddot{\theta}-2 \dot{w} \dot{\theta}\right)\right)\right\} d \eta d \zeta \\
&=\iint_{A} \rho\{(\left.\eta^{2} \cos ^{2} \phi-2 \eta \zeta \cos \phi \sin \phi+\zeta^{2} \sin ^{2} \phi+\eta^{2} \sin ^{2} \phi+2 \eta \zeta \cos \phi \sin \phi+\zeta^{2} \cos ^{2} \phi\right)(-\ddot{\phi}-\ddot{\theta}) \\
&+\eta\left(\left(-\ddot{w}-\left(v+e_{p}\right) \ddot{\theta}-2 \dot{v} \dot{\theta}+w \dot{\theta}^{2}\right) \cos \phi+\left(\ddot{v}-\left(v+e_{p}\right) \dot{\theta}^{2}-w \ddot{\theta}-2 \dot{w} \dot{\theta}\right) \sin \phi\right) \\
&\left.+\zeta\left(\left(\ddot{v}-\left(v+e_{p}\right) \dot{\theta}^{2}-w \ddot{\theta}-2 \dot{w} \dot{\theta}\right) \cos \phi-\left(-\ddot{w}-\left(v+e_{p}\right) \ddot{\theta}-2 \dot{v} \dot{\theta}+w \dot{\theta}^{2}\right) \sin \phi\right)\right\} d \eta d \zeta \\
&=\iint_{A} \rho\left(\eta^{2}\left(\cos ^{2} \phi+\sin ^{2} \phi\right)+\zeta^{2}\left(\sin ^{2} \phi+\cos ^{2} \phi\right)\right)(-\ddot{\phi}-\ddot{\theta}) \\
& \quad+\eta\left(\left(-\ddot{w}-\left(v+e_{p}\right) \ddot{\theta}-2 \dot{v} \dot{\theta}+w \dot{\theta}^{2}\right) \cos \phi+\left(\ddot{v}-\left(v+e_{p}\right) \dot{\theta}^{2}-w \ddot{\theta}-2 \dot{w} \dot{\theta}\right) \sin \phi\right) \\
&\left.\quad+\zeta\left(\left(\ddot{v}-\left(v+e_{p}\right) \dot{\theta}^{2}-w \ddot{\theta}-2 \dot{w} \dot{\theta}\right) \cos \phi-\left(-\ddot{w}-\left(v+e_{p}\right) \ddot{\theta}-2 \dot{v} \dot{\theta}+w \dot{\theta}^{2}\right) \sin \phi\right)\right\} d \eta d \zeta
\end{aligned}
$$




$$
\begin{aligned}
&=(-\ddot{\phi}-\ddot{\theta}) \iint_{A} \rho\left(\eta^{2}+\zeta^{2}\right) d \eta d \zeta \\
&+\left(\left(-\ddot{w}-\left(v+e_{p}\right) \ddot{\theta}-2 \dot{v} \dot{\theta}+w \dot{\theta}^{2}\right) \cos \phi+\left(\ddot{v}-\left(v+e_{p}\right) \dot{\theta}^{2}-w \ddot{\theta}-2 \dot{w} \dot{\theta}\right) \sin \phi\right) \iint_{A} \rho \eta d \eta d \zeta \\
&+\left(\left(\ddot{v}-\left(v+e_{p}\right) \dot{\theta}^{2}-w \ddot{\theta}-2 \dot{w} \dot{\theta}\right) \cos \phi-\left(-\ddot{w}-\left(v+e_{p}\right) \ddot{\theta}-2 \dot{v} \dot{\theta}+w \dot{\theta}^{2}\right) \sin \phi\right) \iint_{A} \rho \zeta d \eta d \zeta \\
&=-\bar{I}_{0}(\ddot{\phi}+\ddot{\theta})+\bar{m} e(\ddot{v} \sin \phi-\ddot{w} \cos \phi)-\bar{m} e \ddot{\theta}\left(\left(v+e_{p}\right) \cos \phi+w \sin \phi\right) \\
& \quad+\bar{m} e \dot{\theta}^{2}\left(w \cos \phi-\left(v+e_{p}\right) \sin \phi\right)-2 \bar{m} e \dot{\theta}(\dot{v} \cos \phi+\dot{w} \sin \phi)
\end{aligned}
$$

$$
\begin{aligned}
& \bar{T}_{\theta}=\int_{0}^{S} \iint_{A} \rho\left\{\left((\eta \cos \phi-\zeta \sin \phi)^{2}+(\eta \sin \phi+\zeta \cos \phi)^{2}\right)(-\ddot{\phi}-\ddot{\theta})\right. \\
& +(\eta \cos \phi-\zeta \sin \phi)\left(-\ddot{w}-\ddot{\phi}\left(v+e_{p}\right)-2 \ddot{\theta}\left(v+e_{p}\right)-2 \dot{\theta}(w \dot{\phi}+\dot{v})\right) \\
& +(\eta \sin \phi+\zeta \cos \phi)\left(\ddot{v}-w \ddot{\phi}-2 w \ddot{\theta}-2 \dot{\theta}\left(\dot{w}-\dot{\phi}\left(v+e_{p}\right)\right)\right) \\
& \left.+\ddot{v} w-\ddot{w}\left(v+e_{p}\right)-\ddot{\theta} w^{2}-\ddot{\theta}\left(v+e_{p}\right)^{2}-2 \dot{\theta}\left(\dot{w} w+\dot{v}\left(v+e_{p}\right)\right)\right\} d \eta d \zeta d x-I_{\theta} \ddot{\theta} \\
& =\int_{0}^{S} \iint_{A} \rho\left\{\left(\eta^{2}\left(\cos ^{2} \phi+\sin ^{2} \phi\right)+\zeta^{2}\left(\sin ^{2} \phi+\cos ^{2} \phi\right)\right)(-\ddot{\phi}-\ddot{\theta})\right. \\
& +\eta\left(\left(-\ddot{w}-\ddot{\phi}\left(v+e_{p}\right)-2 \ddot{\theta}\left(v+e_{p}\right)-2 \dot{\theta}(w \dot{\phi}+\dot{v})\right) \cos \phi+\left(\ddot{v}-w \ddot{\phi}-2 w \ddot{\theta}-2 \dot{\theta}\left(\dot{w}-\dot{\phi}\left(v+e_{p}\right)\right)\right) \sin \phi\right) \\
& +\zeta\left(\left(\ddot{v}-w \ddot{\phi}-2 w \ddot{\theta}-2 \dot{\theta}\left(\dot{w}-\dot{\phi}\left(v+e_{p}\right)\right)\right) \cos \phi-\left(-\ddot{w}-\ddot{\phi}\left(v+e_{p}\right)-2 \ddot{\theta}\left(v+e_{p}\right)-2 \dot{\theta}(w \dot{\phi}+\dot{v})\right) \sin \phi\right) \\
& \left.+\left(\ddot{v} w-\ddot{w}\left(v+e_{p}\right)-\ddot{\theta} w^{2}-\ddot{\theta}\left(v+e_{p}\right)^{2}-2 \dot{\theta}\left(\dot{w} w+\dot{v}\left(v+e_{p}\right)\right)\right)\right\} d \eta d \zeta d x-I_{\theta} \ddot{\theta} \\
& =\int_{0}^{S}\left\{(-\ddot{\phi}-\ddot{\theta}) \iint_{A} \rho\left(\eta^{2}+\zeta^{2}\right) d \eta d \zeta\right. \\
& +\left(\left(-\ddot{w}-\ddot{\phi}\left(v+e_{p}\right)-2 \ddot{\theta}\left(v+e_{p}\right)-2 \dot{\theta}(w \dot{\phi}+\dot{v})\right) \cos \phi\right. \\
& \left.+\left(\ddot{v}-w \ddot{\phi}-2 w \ddot{\theta}-2 \dot{\theta}\left(\dot{w}-\dot{\phi}\left(v+e_{p}\right)\right)\right) \sin \phi\right) \iint_{A} \rho \eta d \eta d \zeta \\
& +\left(\left(\ddot{v}-w \ddot{\phi}-2 w \ddot{\theta}-2 \dot{\theta}\left(\dot{w}-\dot{\phi}\left(v+e_{p}\right)\right)\right) \cos \phi\right. \\
& \left.-\left(-\ddot{w}-\ddot{\phi}\left(v+e_{p}\right)-2 \ddot{\theta}\left(v+e_{p}\right)-2 \dot{\theta}(w \dot{\phi}+\dot{v})\right) \sin \phi\right) \iint_{A} \rho \zeta d \eta d \zeta \\
& \left.+\left(\ddot{v} w-\ddot{w}\left(v+e_{p}\right)-\ddot{\theta} w^{2}-\ddot{\theta}\left(v+e_{p}\right)^{2}-2 \dot{\theta}\left(\dot{w} w+\dot{v}\left(v+e_{p}\right)\right)\right) \iint_{A} \rho d \eta d \zeta\right\} d x-I_{\theta} \ddot{\theta} \\
& =\int_{0}^{S}\left\{-\bar{I}_{0}(\ddot{\phi}+\ddot{\theta})+\bar{m} \ddot{v}(e \sin \phi+w)+\bar{m} \ddot{w}\left(-e \cos \phi-\left(v+e_{p}\right)\right)-\bar{m} e \ddot{\phi}\left(\left(v+e_{p}\right) \cos \phi+w \sin \phi\right)\right. \\
& -2 \bar{m} e \ddot{\theta}\left(\left(v+e_{p}\right) \cos \phi+w \sin \phi\right)-\bar{m} \ddot{\theta}\left(w^{2}+\left(v+e_{p}\right)^{2}\right) \\
& \left.-2 \bar{m} \dot{\theta}\left(\dot{v}\left(e \cos \phi+\left(v+e_{p}\right)\right)+\dot{w}(e \sin \phi+w)+e \dot{\phi}\left(w \cos \phi-\left(v+e_{p}\right) \sin \phi\right)\right)\right\} d x-I_{\theta} \ddot{\theta}
\end{aligned}
$$


The following definitions are adopted for the area integrals above, noting that these inertial prop-

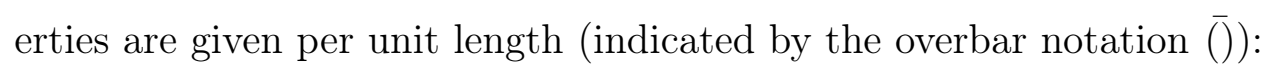

$$
\begin{aligned}
\bar{m} & =\iint_{A} \rho d \eta d \zeta, \\
\bar{m} e & =\iint_{A} \rho \eta d \eta d \zeta, \\
\bar{I}_{0} & =\iint_{A} \rho\left(\eta^{2}+\zeta^{2}\right) d \eta d \zeta, \\
0 & =\iint_{A} \rho \zeta d \eta d \zeta \\
0 & =\iint_{A} \rho \lambda d \eta d \zeta .
\end{aligned}
$$

where $\bar{m}$ is the mass of the wing assembly per unit length, $e$ is the distance between the elastic axis and the center of gravity of the entire wing assembly, and is positive when the center of gravity is ahead of the elastic axis. $\bar{I}_{0}$ is the mass moment of inertia of the wing assembly about its elastic axis. Note that unlike the section integrals for the stiffness terms which are evaluated over the structurally effective area, these integrals are performed over the entire cross section.

Following a similar procedure, we can also obtain an expression for the kinetic energy contributions to the boundary conditions, $b(T)$ :

$$
\begin{aligned}
b(T)=\rho \iint_{A}\left\{\left.\left(\ddot{x_{1}}\right)\left(y_{1}-\left(v+e_{p}\right)\right) \delta v\right|_{0} ^{S}+\left.\left(\ddot{x_{1}}\right)\left(z_{1}-w\right) \delta v\right|_{0} ^{S}+\left.\left(\ddot{x_{1}}\right) \delta \phi\right|_{0} ^{S}+\left.\left(I_{\theta} \dot{\theta}\right) \delta \theta\right|_{0} ^{S}\right\} d \eta d \zeta \\
=\rho \iint_{A}\left\{\left.\left(-\left(\ddot{v}^{\prime}+\ddot{\phi} w^{\prime}+2 \dot{\phi} \dot{w}^{\prime}\right)(\eta \cos \phi-\zeta \sin \phi)^{2}-\left(\ddot{w}^{\prime}-\ddot{\phi} v^{\prime}-2 \dot{\phi} \dot{v}^{\prime}\right)(\eta \sin \phi+\zeta \cos \phi)(\eta \cos \phi-\zeta \sin \phi)\right) \delta v\right|_{0} ^{S}\right. \\
+\left.\left(-\left(\ddot{v}^{\prime}+\ddot{\phi} w^{\prime}+2 \dot{\phi} \dot{w}^{\prime}\right)(\eta \cos \phi-\zeta \sin \phi)(\eta \sin \phi+\zeta \cos \phi)-\left(\ddot{w}^{\prime}-\ddot{\phi} v^{\prime}-2 \dot{\phi} \dot{v}^{\prime}\right)(\eta \sin \phi+\zeta \cos \phi)^{2}\right) \delta w\right|_{0} ^{S} \\
\left.+\left.\left(-\lambda\left(\left(\ddot{v}^{\prime}+\ddot{\phi} w^{\prime}+2 \dot{\phi} \dot{w}^{\prime}\right)(\eta \cos \phi-\zeta \sin \phi)-\left(\ddot{w}^{\prime}-\ddot{\phi} v^{\prime}-2 \dot{\phi} \dot{v}^{\prime}\right)(\eta \sin \phi+\zeta \cos \phi)\right)\right) \delta \phi\right|_{0} ^{S}\right\} d \eta d \zeta \\
=\rho \iint_{A}\left\{\left(-\left(\ddot{v}^{\prime}+\ddot{\phi} w^{\prime}+2 \dot{\phi} \dot{w}^{\prime}\right)\left(\eta^{2} \cos ^{2} \phi-2 \eta \zeta \cos \phi \sin \phi+\zeta^{2} \sin ^{2} \phi\right)\right.\right. \\
\left.\quad-\left(\ddot{w}^{\prime}-\ddot{\phi} v^{\prime}-2 \dot{\phi} \dot{v}^{\prime}\right)\left(\eta^{2} \cos \phi \sin \phi+\eta \zeta\left(\cos ^{2} \phi-\sin ^{2} \phi\right)-\zeta^{2} \cos \phi \sin \phi\right)\right)\left.\delta v\right|_{0} ^{S} \\
+\left(-\left(\ddot{v}^{\prime}+\ddot{\phi} w^{\prime}+2 \dot{\phi} \dot{w}^{\prime}\right)\left(\eta^{2} \cos \phi \sin \phi+\eta \zeta\left(\cos ^{2} \phi-\sin ^{2} \phi\right)+\zeta^{2} \cos \phi \sin \phi\right)\right. \\
\left.\quad-\left(\ddot{w}^{\prime}-\ddot{\phi} v^{\prime}-2 \dot{\phi} \dot{v}^{\prime}\right)\left(\eta^{2} \sin ^{2} \phi+2 \eta \zeta \cos \phi \sin \phi+\zeta^{2} \cos ^{2} \phi\right)\right)\left.\delta w\right|_{0} ^{S}
\end{aligned}
$$




$$
\begin{aligned}
+ & \left.\left.\left(-\lambda\left(\left(\ddot{v}^{\prime}+\ddot{\phi} w^{\prime}+2 \dot{\phi} \dot{w}^{\prime}\right)(\eta \cos \phi-\zeta \sin \phi)-\left(\ddot{w}^{\prime}-\ddot{\phi} v^{\prime}-2 \dot{\phi} \dot{v}^{\prime}\right)(\eta \sin \phi+\zeta \cos \phi)\right)\right) \delta \phi\right|_{0} ^{S}\right\} d \eta d \zeta \\
=\rho \iint_{A}\{ & \left(\eta^{2}\left(-\left(\ddot{v}^{\prime}+\ddot{\phi} w^{\prime}+2 \dot{\phi} \dot{w}^{\prime}\right) \cos ^{2} \phi-\left(\ddot{w}^{\prime}-\ddot{\phi} v^{\prime}-2 \dot{\phi} \dot{v}^{\prime}\right) \sin \phi \cos \phi\right)\right. \\
& +\eta \zeta\left(2\left(\ddot{v}^{\prime}+\ddot{\phi} w^{\prime}+2 \dot{\phi} \dot{w}^{\prime}\right) \sin \phi \cos \phi-\left(\ddot{w}^{\prime}-\ddot{\phi} v^{\prime}-2 \dot{\phi} \dot{v}^{\prime}\right)\left(\cos ^{2} \phi-\sin ^{2} \phi\right)\right) \\
& \left.+\zeta^{2}\left(-\left(\ddot{v}^{\prime}+\ddot{\phi} w^{\prime}+2 \dot{\phi} \dot{w}^{\prime}\right) \sin ^{2} \phi-\left(\ddot{w}^{\prime}-\ddot{\phi} v^{\prime}-2 \dot{\phi} \dot{v}^{\prime}\right) \cos ^{2} \phi\right)\right)\left.\delta v\right|_{0} ^{S} \\
+ & \eta^{2}\left(-\left(\ddot{v}^{\prime}+\ddot{\phi} w^{\prime}+2 \dot{\phi} \dot{w}^{\prime}\right) \sin \phi \cos \phi-\left(\ddot{w}^{\prime}-\ddot{\phi} v^{\prime}-2 \dot{\phi} \dot{v}^{\prime}\right) \cos ^{2} \phi\right) \\
& +\eta \zeta\left(-\left(\ddot{v}^{\prime}+\ddot{\phi} w^{\prime}+2 \dot{\phi} \dot{w}^{\prime}\right)\left(\cos { }^{2} \phi-\sin ^{2} \phi\right)+2\left(\ddot{w}^{\prime}-\ddot{\phi} v^{\prime}-2 \dot{\phi} \dot{v}^{\prime}\right) \sin \phi \cos \phi\right) \\
& \left.+\zeta^{2}\left(-\left(\ddot{v}^{\prime}+\ddot{\phi} w^{\prime}+2 \dot{\phi} \dot{w}^{\prime}\right) \sin \phi \cos \phi-\left(\ddot{w}^{\prime}-\ddot{\phi} v^{\prime}-2 \dot{\phi} \dot{v}^{\prime}\right) \sin { }^{2} \phi\right)\right)\left.\delta w\right|_{0} ^{S} \\
+ & \eta \lambda\left(-\left(\ddot{v}^{\prime}+\ddot{\phi} w^{\prime}+2 \dot{\phi} \dot{w}^{\prime}\right) \cos \phi+\left(\ddot{w}^{\prime}-\ddot{\phi} v^{\prime}-2 \dot{\phi} \dot{v}^{\prime}\right) \sin \phi\right) \\
& \left.\left.+\zeta \lambda\left(\left(\ddot{v}^{\prime}+\ddot{\phi} w^{\prime}+2 \dot{\phi} \dot{w}^{\prime}\right) \sin \phi+\left(\ddot{w}^{\prime}-\ddot{\phi} v^{\prime}-2 \dot{\phi} \dot{v}^{\prime}\right) \sin \phi\right)\right)\left.\delta \phi\right|_{0} ^{S}\right\} d \eta d \zeta \\
=\left(\bar{m} K_{m_{1}}(\right. & \left.\left(\ddot{v}^{\prime}+\ddot{\phi} w^{\prime}+2 \dot{\phi} \dot{w}^{\prime}\right) \sin { }^{2} \phi-\left(\ddot{w}^{\prime}-\ddot{\phi} v^{\prime}-2 \dot{\phi} \dot{v}^{\prime}\right) \sin \phi \cos \phi\right) \\
& \left.+\bar{m} K_{m_{2}}\left(-\left(\ddot{v}^{\prime}+\ddot{\phi} w^{\prime}+2 \dot{\phi} \dot{w}^{\prime}\right) \cos { }^{2} \phi-\left(\ddot{w}^{\prime}-\ddot{\phi} v^{\prime}-2 \dot{\phi} \dot{v}^{\prime}\right) \sin \phi \cos \phi\right)\right)\left.\delta v\right|_{0} ^{S} \\
+ & \bar{m} K_{m_{1}}\left(-\left(\ddot{v}^{\prime}+\ddot{\phi} w^{\prime}+2 \dot{\phi} \dot{w}^{\prime}\right) \sin \phi \cos \phi-\left(\ddot{w}^{\prime}-\ddot{\phi} v^{\prime}-2 \dot{\phi} \dot{v^{\prime}}\right) \sin { }^{2} \phi\right) \\
& \left.+\bar{m} K_{m_{2}}\left(-\left(\ddot{v}^{\prime}+\ddot{\phi} w^{\prime}+2 \dot{\phi} \dot{w}^{\prime}\right) \sin \phi \cos \phi-\left(\ddot{w}^{\prime}-\ddot{\phi} v^{\prime}-2 \dot{\phi} \dot{v}^{\prime}\right) \cos { }^{2} \phi\right)\right)\left.\delta w\right|_{0} ^{S} \\
+ & \left.\bar{m} D_{1}^{*}\left(-\left(\ddot{v}^{\prime}+\ddot{\phi} w^{\prime}+2 \dot{\phi} \dot{w}^{\prime}\right) \cos \phi+\left(\ddot{w}^{\prime}-\ddot{\phi} v^{\prime}-2 \dot{\phi} \dot{v}^{\prime}\right) \sin \phi\right)\right)\left.\delta \phi\right|_{0} ^{S}
\end{aligned}
$$

Making the substitutions, one obtains

$$
\begin{aligned}
\bar{m} K_{m_{1}} & =\iint_{A} \rho \zeta^{2} d \eta d \zeta, \\
\bar{m} K_{m_{2}} & =\iint_{A} \rho \eta^{2} d \eta d \zeta, \\
\bar{m} D_{1}{ }^{*} & =\iint_{A} \rho \lambda \zeta d \eta d \zeta, \\
0 & =\iint_{A} \rho \eta \zeta d \eta d \zeta, \\
0 & =\iint_{A} \rho \lambda \eta d \eta d \zeta .
\end{aligned}
$$

where $\bar{m} K_{m 1}$ and $\bar{m} K_{m 2}$ satisfy the following relation, $\bar{m} K_{m}=\sqrt{\left(\bar{m} K_{m 1}\right)^{2}+\left(\bar{m} K_{m 1}\right)^{2}}$, noting that $K_{m}$ is the radius of gyration of the wing. $D_{1}{ }^{*}$ is a higher order section integral relating to the warping function which vanishes for a doubly symmetric cross section [1]. 


\subsubsection{Virtual Work Done by External Forces}

Having derived the strain energy and the kinetic energy contributions to the equations of motion, the final remaining component in Hamilton's Principle is the virtual work done by the external forces. These forces are the nonconservative aerodynamic loads, which will be introduced here. In a similar manner as in Eq.2.74 and Eq.2.105, we seek an expression for the variation of these external loads as functions of the displacements $u, v, w, \phi$ and $\theta$ and the variations of these displacements,

$$
\delta W=\int_{0}^{S}\left(\bar{W}_{u} \delta u+\bar{W}_{v} \delta v+\bar{W}_{w} \delta w+\bar{W}_{\phi} \delta \phi\right) d x+\bar{W}_{\theta} \delta \theta
$$

where $W_{u}$ represents the aerodynamic forces acting along the longitudinal axis (which are negligible thus this term is taken as zero). $W_{v}$ represents the aerodynamic load which acts in the direction of the $y$ axis, and $W_{w}$ represents the aerodynamic forces aligned with the $z$ axis. It will be shown that these loads consist of components of the lift and drag forces (note that the aerodynamic drag is not modelled in this body of work). $W_{\phi}$ represents the aerodynamic moment acting along the elastic-axis, and $W_{\theta}$ is the total (integrated) aerodynamic moment acting along the pitch-axis.

The aerodynamic model adopted in this work is selected as it allows for the efficient implementation and computation of the aeroelastic system. Additionally, the coupling mechanism between the structural and aerodynamic forces are easily interpreted in physical terms.

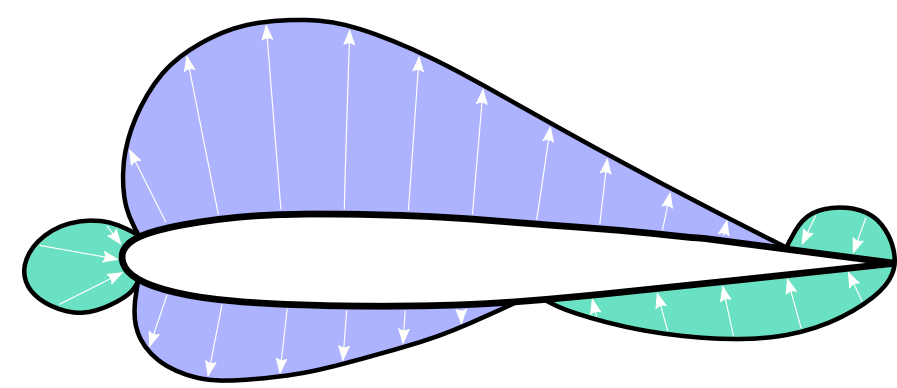

Figure 2.12: Illustration of relative pressure distribution around an airfoil, adapted from 40

The static pressure distribution across the surface as depicted in 2.12 can be concentrated as a 
single lift force, perpendicular to the direction of the flow, and a drag force parallel to the flow (not shown, and not accounted for herein). The centroid of the pressure distribution is known as the center of pressure, hence when the loads are concentrated at that point, the moment caused by the pressure distribution is equal to zero as in Figure 2.13a The other point of interest, the aerodynamic center, has the useful property that the aerodynamic moment is independent of the angle of attack [18]. For subsonic flow (as in this investigation), the aerodynamic center is located at the so-called quarter chord point, which is located one quarter of the chord length aft of the leading edge as in Figure 2.13b For supersonic flow, the aerodynamic center shifts towards the half-chord point [40].

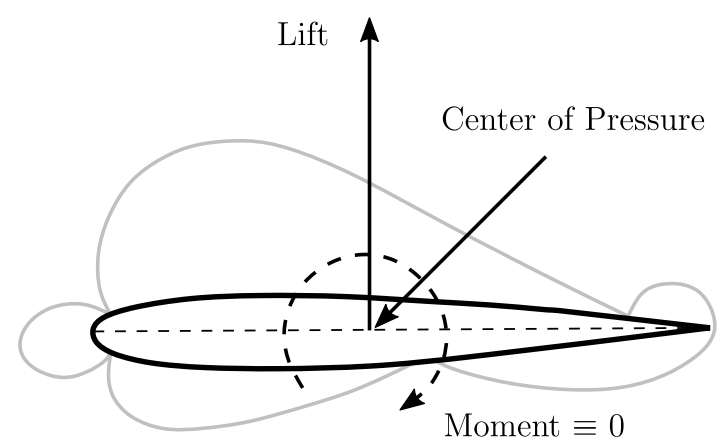

(a) Lift and moment concentrated at the center of pressure

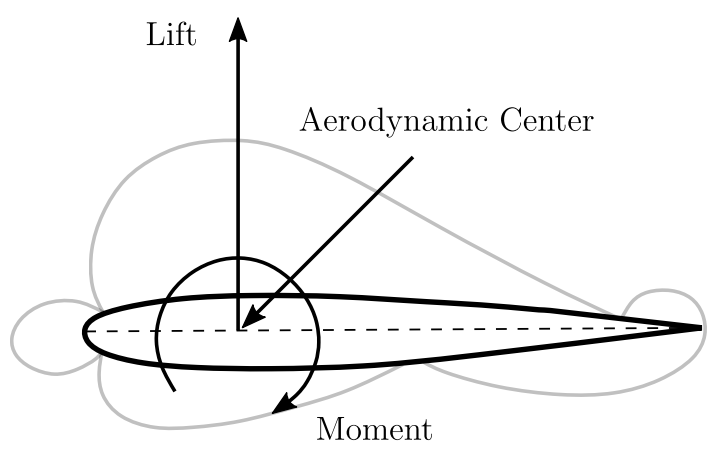

(b) Lift and moment concentrated at the aerodynamic center

Figure 2.13: Aerodynamic lift and moment illustrated at two key locations along the chord, adapted from 40

The lift force, $L$ and the aerodynamic moment about the elastic axis, $M_{E A}$ according to the theory of steady aerodynamics can be written in terms of the lift coefficient, $C_{L_{\alpha}}$ and the moment coefficient, $C_{M_{A C}}$, respectively as

$$
\begin{gathered}
L=\frac{1}{2} \rho U^{2} c S C_{L_{\alpha}} \alpha_{e f f}, \\
M_{E A}=\left(y_{f}-\frac{c}{4}\right) L+\frac{1}{2} \rho U^{2} c^{2} S C_{M_{A C}} .
\end{gathered}
$$

The effective angle of attack, $\alpha_{\text {eff }}$ should account for not only the physical rotational displacement of the wing, but also for the angle created by the relative velocities of the airflow and the wing's 
motion. The dimension $y_{f}$ represents the distance from the leading edge to the axis of rotation. Note that for the case of an airfoil with a single elastic mounting, the term $\left(y_{f}-c / 4\right)$ could simply be expressed by $e_{c}$, which is the distance between the aerodynamic center and elastic axis. With that in mind, $y_{f}$ will be used to maintain generality in the following explanation.

\section{Unsteady Aerodynamics}

There are two primary sources of unsteadiness in the aerodynamic loading; which can be grouped into circulatory and non-circulatory effects. As the name suggests, the circulatory terms relate to the aerodynamic effects caused by the circulation of the air around the airfoil, which includes the effects of vortices in the wake and the nature of their effect on the aerodynamic loads as they are convected downstream. The non-circulatory terms relate to added mass terms that arises from the inertia of the fluid around the airfoil. The descriptions of the aerodynamic loads by Wagner and Theodorsen will be discussed to the extent required to understand the application to the current system. For a more comprehensive account of these derivations refer to [4] 9 .

\section{Degree-of-Freedom Airfoil}

For simplicity, in place of a flexible wing, consider instead a 2 degree of freedom airfoil, which undergoes pitch and plunge motion. These time varying quantities are denoted in their positive sense in Figure 2.14 as $\alpha$ and $h$ respectively. The aerodynamic lift and moment follow the same positive conventions, with the lift being positive up (perpendicular to the flow indicated by $U$, which is the airspeed parameter). Likewise, the aerodynamic moment is defined about the elastic axis of the airfoil, and it is positive for a counter clockwise rotation (i.e. positive nose-up).

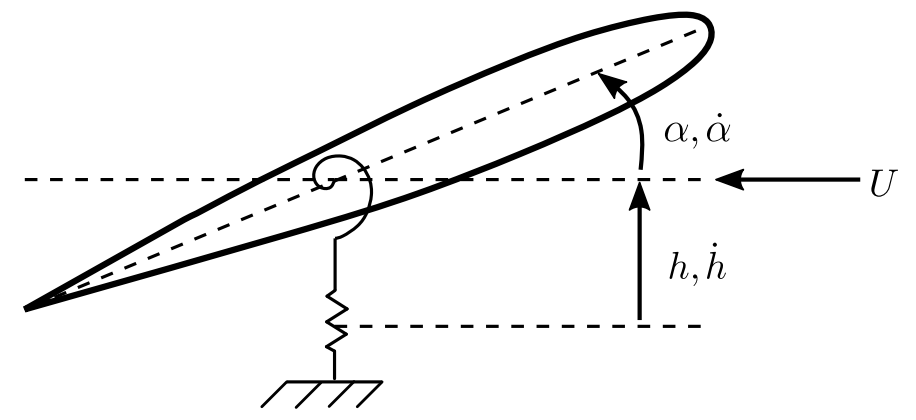

Figure 2.14: Two degree-of-freedom airfoil in pitch and plunge 8 
Theodorsen derived separate expressions for the circulatory and non-circulatory lift and moments for linear (no flow separation) and incompressible aerodynamics. The expressions of the lift force are obtained by integrating distribution of the pressure differences between the top and bottom of the wing. Likewise the expressions for the moment are obtained by integrating the product of the pressure differences and the moment arm from the elastic axis. The following expressions are obtained [8],

$$
\begin{gathered}
L_{n c}=\frac{1}{4} \rho \pi c^{2}\left(U \dot{\alpha}-\ddot{h}-\left(y_{f}-\frac{c}{2}\right) \ddot{\alpha}\right), \\
L_{c}=\rho \pi U c\left(U \alpha-\dot{h}-\left(y_{f}-\frac{3 c}{4}\right) \dot{\alpha}\right) C(k), \\
M_{n c}=\frac{1}{4} \rho \pi c^{2}\left(y_{f}-\frac{c}{2}\right)\left(-\ddot{h}-\left(y_{f}-\frac{c}{2}\right) \ddot{\alpha}\right)-\frac{1}{128} \rho \pi c^{4} \ddot{\alpha}+\frac{1}{4} \rho \pi U c^{2} \dot{h}+\frac{1}{4} \rho \pi U^{2} c^{2} \alpha, \\
M_{c}=-\frac{1}{4} \rho \pi U c^{2}\left(U \alpha-\dot{h}-\left(y_{f}-\frac{3 c}{4}\right) \dot{\alpha}\right)+\rho \pi U c^{2} e_{c}\left(U \alpha-\dot{h}+\left(y_{f}-\frac{3 c}{4}\right) \dot{\alpha}\right) C(k) .
\end{gathered}
$$

The total lift and moment are simply the sum of the circulatory and non-circulatory terms. Conveniently, some terms cancel out, leading to the following simplified form of the equations,

$$
\begin{aligned}
& L=L_{n c}+L_{c}, \\
& =\frac{1}{4} \rho \pi c^{2}\left(-\ddot{h}-\left(y_{f}-\frac{c}{2}\right) \ddot{\alpha}+U \dot{\alpha}\right)+\rho \pi U c\left(U \alpha-\dot{h}-\left(y_{f}-\frac{3 c}{4}\right) \dot{\alpha}\right) C(k), \\
& \quad \begin{aligned}
M= & M_{n c}+M_{c}, \\
= & \frac{1}{4} \rho \pi c^{2}\left(-\left(y_{f}-\frac{c}{2}\right) \ddot{h}-\left(\left(y_{f}-\frac{c}{2}\right)^{2}-\frac{c^{2}}{32}\right) \ddot{\alpha}+U\left(\left(y_{f}-\frac{3 c}{4}\right) \dot{\alpha}\right)\right) \\
\quad & \quad \rho \pi U c\left(y_{f}-\frac{3 c}{4}\right)\left(U \alpha-\dot{h}-\left(y_{f}-\frac{3 c}{4}\right) \dot{\alpha}\right) C(k) .
\end{aligned}
\end{aligned}
$$

where $C(k)$ is known as Theodorsen's function, and is defined in the frequency domain, while the 
remainder of the terms are in the time domain. Rather than dealing with the frequency domain, we will adopt the use of Wagner's function in place of Theodorsen's function in Eq. 2.130 and Eq. 2.131 above. The Wagner function, can be obtained by integrating the real and imaginary parts of Theodorsen's function as shown in 9 . Wagner's function quantifies the time dependent reduction in the aerodynamic loads caused by the circulation in the wake. In this way, one can see the diminishing effects of the trailing edge vortices as they are convected downstream. We adopt the following approximation of Wagner's function $\varphi(t)$ in this investigation 18

$$
\begin{aligned}
& \varphi(t)=1-0.165 e^{-0.0455 \frac{2 U t}{c}}-0.335 e^{-0.3 \frac{2 U t}{c}}, \\
& \varphi(t)=1-A_{1} e^{-b_{1} t}-A_{2} e^{-b_{2} t} .
\end{aligned}
$$

Figure 2.15 plots this approximation of Wagner's function in dimensionless time $\tau=\frac{2 U t}{c}$.

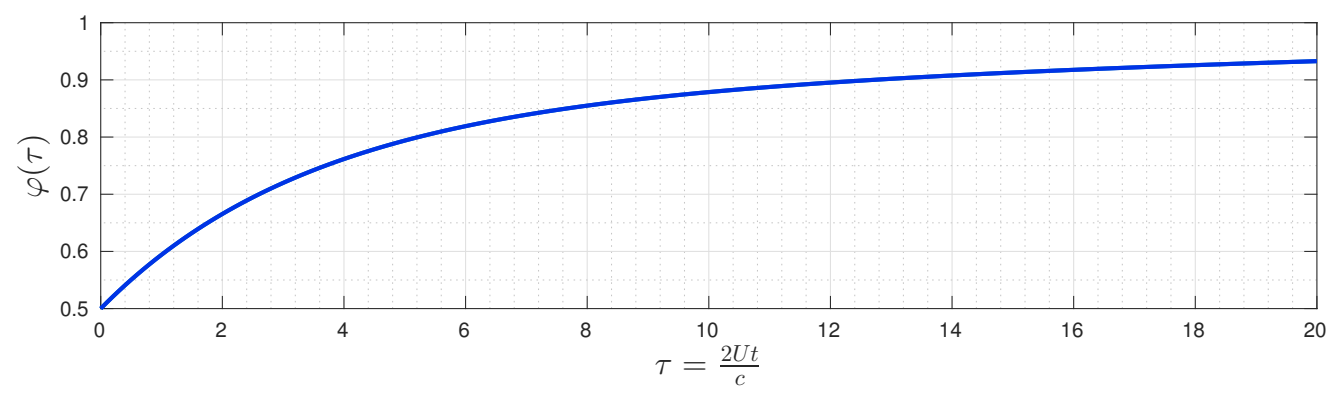

Figure 2.15: Wagner's function, illustrating the diminishing influence of the vortices in the wake in dimensionless time

This implementing the above approximation of Wagner's function using Duhamel's integral yields Eq. (2.133) and Eq. 2.134.

$$
\begin{aligned}
L=\frac{1}{4} \rho \pi c^{2}(- & \\
& \left.-\left(y_{f}-\frac{c}{2}\right) \ddot{\alpha}+U \dot{\alpha}\right) \\
& +\frac{1}{2} \rho U c 2 \pi\left(\left(1-A_{1}-A_{2}\right) w_{3 c / 4}+\dot{z}\left(A_{1} b_{1}+A_{2} b_{2}\right)+z b_{1} b_{2}\left(A_{1}+A_{2}\right)\right), \\
M_{e a}=\frac{1}{4} \rho \pi c^{2}(- & \left.\left(y_{f}-\frac{c}{2}\right) \ddot{h}-\left(\left(y_{f}-\frac{c}{2}\right)^{2}-\frac{c^{2}}{32}\right) \ddot{\alpha}+U\left(\left(y_{f}-\frac{3 c}{4}\right) \dot{\alpha}\right)\right) \\
& +\frac{1}{2} \rho U c 2 \pi\left(y_{f}-\frac{3 c}{4}\right)\left(\left(1-A_{1}-A_{2}\right) w_{3 c / 4}+\dot{z}\left(A_{1} b_{1}+A_{2} b_{2}\right)+z b_{1} b_{2}\left(A_{1}+A_{2}\right)\right) .
\end{aligned}
$$


While the equations seek to model the lift force and moment acting on the airfoil as a function of its motion, an additional state, $z(t)$ must be introduced to account for the time dependent unsteady effects. Hence in order for the system to not be underdetermined, an additional differential equation must also be introduced, where

$$
\ddot{z}+\left(b_{1}+b_{2}\right) \dot{z}+b_{1} b_{2} z=w_{3 c / 4},
$$

where

$$
w_{3 c / 4}=U \alpha-\dot{h}+\left(\frac{3 c}{4}-y_{f}\right) \dot{\alpha}
$$

\section{Continuous Wing}

Extending the derivation of aerodynamic loads for a 2-degree-of-freedom airfoil to be suitable for applications to flexible wings requires some minor manipulations to the equations. The study of a 2 degree-of-freedom airfoil is useful to understand a lot of the mechanisms and complex couplings that arise in aeroelasticity. Considering the flow around the airfoil as two-dimensional (i.e. varying only in the plane defined by the cross section of the airfoil) is justified by the assumption of infinite span in thin airfoil theory. However for applications to continuous systems in three dimensions, it fails to capture the essential effects of three dimensional flow around a wing of finite span. Particularly, the spanwise variation of the aerodynamic loads caused by vortices at the tip of the wing as depicted in Figure 2.16. These tip vortices are separate from those discussed in reference to undsteady aerodynamics, which are shed from the trailing edge of the airfoil and circulate in the wake. The boundary layer at the root of the wing would also affect the distribution of the loads along the span, although these effects are not modelled herein.

To account for three dimensional flow effects, we will introduce a lift reduction factor, which imposes 


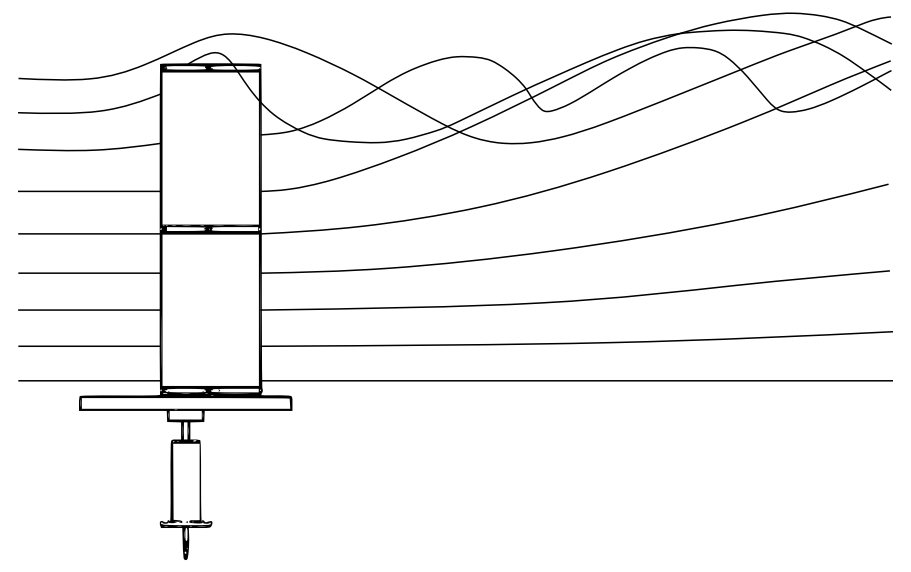

Figure 2.16: Illustration of streamlines, highlighting the effect of tip vortices

an elliptical distribution of the loads along the span of the wing, with a value of 1 at the root, and 0 at the tip. This lift reduction factor allows us to account for the effects of tip vortices, pictured in Figure 2.16. The lift reduction factor is given by Eq.(2.137), and is plotted in Figure 2.17

$$
\text { Lift Reduction Factor }=\sqrt{1-\left(\frac{x}{S}\right)^{2}} \text {. }
$$

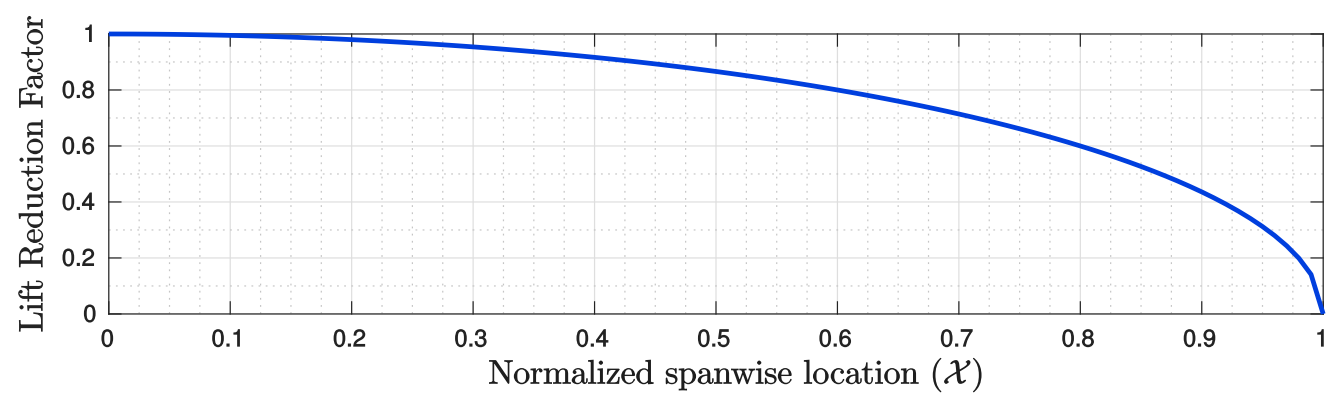

Figure 2.17: Lift reduction factor as a function of the normalized span

There exist some further modifications which are required to fully describe the unsteady aerodynamic loads on the three dimensional structure. Firstly, $\alpha$ in Eq.2.133), and Eq. 2.134), should be repalced by $(\phi+\theta)$ to reflect the fact that the flexible twist and the rigid body pitch rotation both contribute to the angle of attack. Furthermore, it is important to recall that the flexible twist angle, $\phi$ is about the elastic axis which is located $e_{c}$ aft of the aerodynamic center or $\left(c / 4+e_{c}\right)$ aft of the leading edge. Thus when concerned with $\phi$, a value of $\left(c / 4+e_{c}\right)$ shall be assigned to $y_{f}$. Meanwhile the rigid body pitch rotation, $\theta$ is about the pitch axis which is located at a distance of $e_{c}+e_{p}$ aft of the aerodynamic center. Hence when concerned with the rigid body pitch rotation, 
$y_{f}$ will instead assume a value equal to $\left(c / 4+e_{c}+e_{p}\right)$.

Furthermore, referring to Figure 2.18a, one can see that as a consequence of the rigid body base rotation, the heave (or plunge) previously denoted by $h$, consists of components of both the edgewise and flapwise bending displacements,

$$
h=w \cos \theta+v \sin \theta
$$

Additionally, the relative velocity of the airflow and the wing is given not only by the free stream velocity $U$, but also has contributions from the components of the bending displacements. However, while these terms are not formally negligible, a quick numerical experiment will reveal that the contributions of the velocity of the bending displacements to the relative velocity are inconsequential, hence,

$$
\mathbf{V}_{\text {Air } / W i n g}=U-w \sin \theta+v \cos \theta \approx U
$$

Another consequence of this rigid body pitch rotation, the lift force contributes to the aerodynamic loading not only in the flapwise bending direction but also in the edgewise bending direction, which is reflected in Eq.2.141 and Eq.2.142 respectively.

Considering the motion of the wing in three dimensions, 42$]$ and [39] state that the flexible twist angle used in determining the aerodynamic loads should be taken as $\hat{\phi}(x, t)$ as in Eq. 2.140. Given that $\phi$ is defined as the twist about the deformed elastic axis (i.e. the $\mathbf{x}$-axis), the relationship expressed in $\hat{\phi}$ seeks to obtain the projection of that twist angle on the undeformed elastic axis (i.e. the $x$-axis). However, compared to the rigid body pitch rotation, the terms in the integrand are once again formally negligible according to the second order approximation.

$$
\hat{\phi}=\phi+\int_{0}^{S} v^{\prime \prime} w^{\prime} d x \approx \phi
$$




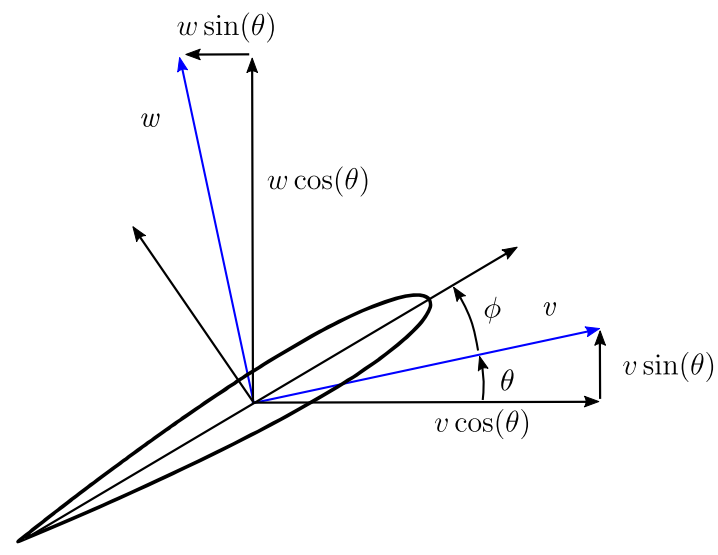

(a) Components of $v(x, t)$ and $w(x, t)$ aligned with the lift force (vertical) and the airflow (horizontal)

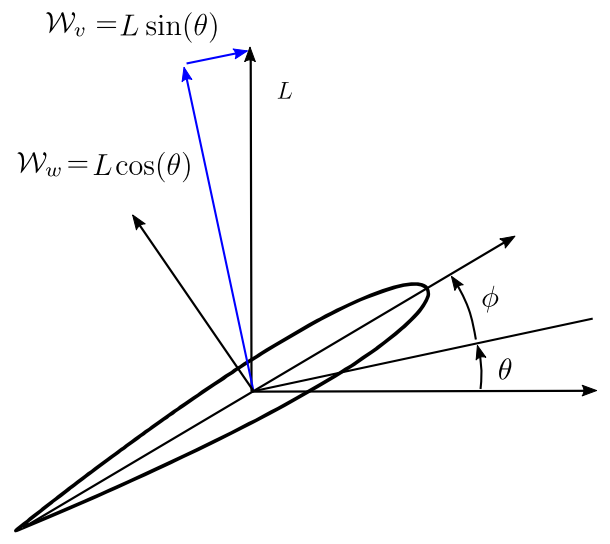

(b) Components of the lift force contributing to $\mathcal{W}_{v}=$ and $\mathcal{W}_{w}$ respectively

Figure 2.18: Effects of motion in three dimensions

This investigation will adopt a strip-theory approach to calculate the aerodynamic loads at each spanwise location $x$. Strip-theory asserts that the aerodynamic loading at location $x$ is a function of the state of the structure and its derivatives at that location only as in [18. In this sense, we are considering the continuous wing to be segmented into a series of discrete airfoils of span $d x$. Recalling that $W_{u}$ is equal to zero, extending Eq.(2.133) and Eq.(2.134) per the above discussions, one obtains

$$
\begin{aligned}
& W_{v}=\sqrt{1-\left(\frac{x}{S}\right)^{2}}\left\{\frac{1}{4} \rho c^{2} \pi\left(-\ddot{w} \cos (\theta)-\ddot{v} \sin (\theta)+U(\dot{\phi}+\dot{\theta})-\left(e_{c}-\frac{c}{4}\right) \ddot{\phi}-\left(\left(e_{c}+e_{p}\right)-\frac{c}{4}\right) \ddot{\theta}\right)\right. \\
& \left.+\frac{1}{2} \rho U c 2 \pi\left(\left(1-A_{1}-A_{2}\right) w_{3 c / 4}+\dot{z}\left(A_{1} b_{1}+A_{2} b_{2}\right)+z b_{1} b_{2}\left(A_{1}+A_{2}\right)\right)\right\} \times \sin (\theta), \\
& W_{w}=\sqrt{1-\left(\frac{x}{S}\right)^{2}}\left\{\frac{1}{4} \rho c^{2} \pi\left(-\ddot{w} \cos (\theta)-\ddot{v} \sin (\theta)+U(\dot{\phi}+\dot{\theta})-\left(e_{c}-\frac{c}{4}\right) \ddot{\phi}-\left(\left(e_{c}+e_{p}\right)-\frac{c}{4}\right) \ddot{\theta}\right)\right. \\
& \left.+\frac{1}{2} \rho U c 2 \pi\left(\left(1-A_{1}-A_{2}\right) w_{3 c / 4}+\dot{z}\left(A_{1} b_{1}+A_{2} b_{2}\right)+z b_{1} b_{2}\left(A_{1}+A_{2}\right)\right)\right\} \times \cos (\theta), \\
& W_{\phi}=\sqrt{1-\left(\frac{x}{S}\right)^{2}}\left\{\frac { 1 } { 4 } \rho c ^ { 2 } \pi \left(\left(e_{c}-\frac{c}{4}\right)(-\ddot{w} \cos (\theta)-\ddot{v} \sin (\theta))-U\left(\frac{c}{2}-e_{c}\right) \dot{\phi}-U\left(\frac{c}{2}-\left(e_{c}+e_{p}\right)\right) \dot{\theta}\right.\right. \\
& \left.-\left(\left(e_{c}-\frac{1}{4} c\right)^{2}+\frac{1}{32} c^{2}\right) \ddot{\phi}-\left(\left(\left(e_{c}+e_{p}\right)-\frac{1}{4} c\right)\left(e_{c}-\frac{1}{4} c\right)+\frac{1}{32} c^{2}\right) \ddot{\theta}\right) \\
& \left.+\frac{1}{2} \rho U c 2 \pi e_{c}\left(\left(1-A_{1}-A_{2}\right) w_{3 c / 4}+\dot{z}\left(A_{1} b_{1}+A_{2} b_{2}\right)+z b_{1} b_{2}\left(A_{1}+A_{2}\right)\right)\right\},
\end{aligned}
$$




$$
\begin{aligned}
W_{\theta}=\int_{0}^{S} \sqrt{1-\left(\frac{x}{S}\right)^{2}}\left\{\frac { 1 } { 4 } \rho c ^ { 2 } \pi \left(\left(\left(e_{c}+e_{p}\right)-\frac{c}{4}\right)(-\ddot{w} \cos (\theta)-\ddot{v} \sin (\theta))-U\left(\frac{c}{2}-e_{c}\right) \dot{\phi}-U\left(\frac{c}{2}-\left(e_{c}+e_{p}\right)\right) \dot{\theta}\right.\right. \\
\left.-\left(\left(\left(e_{c}+e_{p}\right)-\frac{1}{4} c\right)\left(e_{c}-\frac{1}{4} c\right)+\frac{1}{32} c^{2}\right) \ddot{\phi}-\left(\left(\left(e_{c}+e_{p}\right)-\frac{1}{4} c\right)^{2}+\frac{1}{32} c^{2}\right) \ddot{\theta}\right) \\
\left.+\frac{1}{2} \rho U c 2 \pi\left(e_{c}+e_{p}\right)\left(\left(1-A_{1}-A_{2}\right) w_{3 c / 4}+\dot{z}\left(A_{1} b_{1}+A_{2} b_{2}\right)+z b_{1} b_{2}\left(A_{1}+A_{2}\right)\right)\right\} d x
\end{aligned}
$$

The aerodynamic states, $z$, are given by the differential equation shown in Eq. 2.145 a 43. The effective downwash, $w_{3 c / 4}$, as it appears Eq.(2.141), Eq.(2.142), Eq.2.143), and Eq. 2.144) above and in Eq. 2.145 a below is given by Eq.2.145 b.

$$
\begin{aligned}
\ddot{z}+\dot{z}\left(b_{1}+b_{2}\right)+z\left(b_{1} b_{2}\right) & =w_{3 c / 4}, \\
w_{3 c / 4} & =U(\theta+\phi)-\dot{w} \cos (\theta)-\dot{v} \sin (\theta)+\left(\frac{1}{2} c-e_{c}\right) \dot{\phi}+\left(\frac{1}{2} c-\left(e_{c}+e_{p}\right)\right) \dot{\theta} .
\end{aligned}
$$

\section{Limitations of the Linear Aerodynamic Model}

The above described unsteady aerodynamic model asserts that the static lift and moment coefficients, $C_{L \alpha}$ and $C_{M_{A C}}$ vary linearly with the angle of attack of the airfoil relative to the free-stream velocity of air. This has been shown experimentally (as depicted in Figure 2.19) to only be a valid assumption over an approximate range of $-12^{\circ} \leq \alpha \leq 12^{\circ}$, and for high Reynolds' numbers 16 .

The inclusion of nonlinearity in the aerodynamic models to be used in future studies are necessary to generalize the model such that is capable of mimicking large angles of attack and low Reynolds' number effects that were observed in similar experiments. A potential solution could be rooted in developing an empirical model of the aerodynamic loads as illustrated in Example 2.1 below. This model captures the nonlinearity in the aerodynamics by using least-squares to fit a polynomial to a set of wind tunnel data from experiments. 


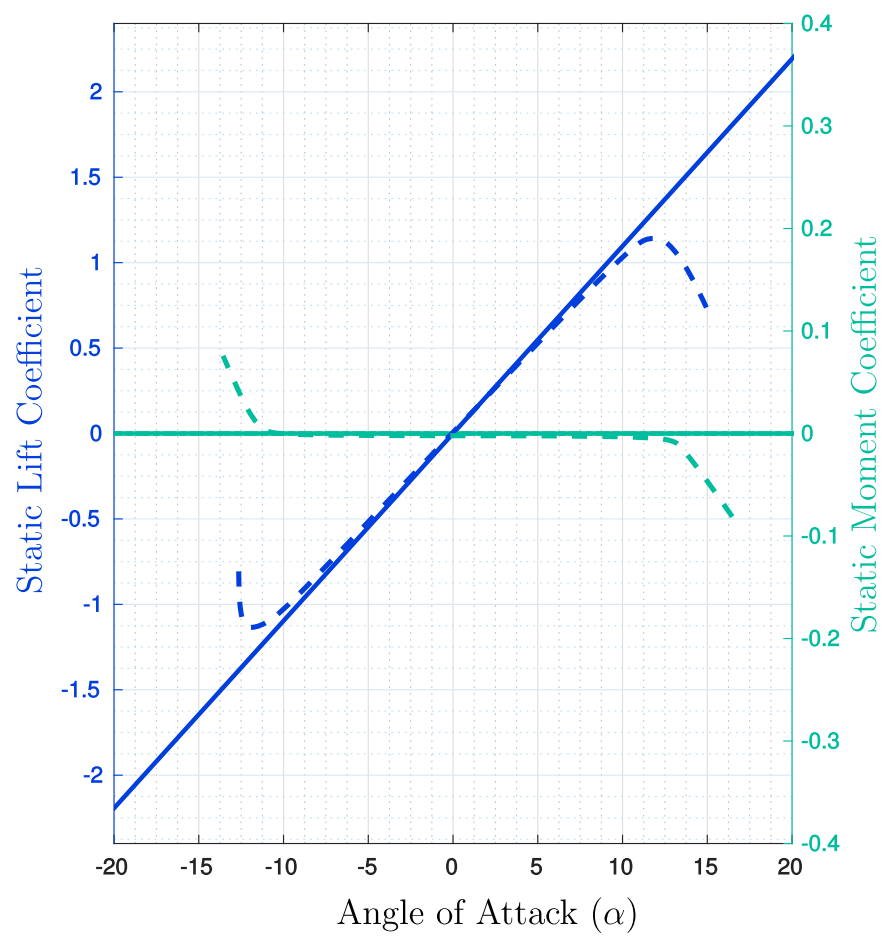

Figure 2.19: Illustration of the static lift and moment coefficients, adapted from experiments by Abbott and Doenhoff [16]. The dashed lines represent the experimental observations of the static lift coefficient, $C_{L_{\alpha}}(\square)$ and moment coefficient, $C_{M_{A C}}(\square)$ at high Reynolds numbers. The solid lines have slopes of $2 \pi$ and 0 respectively, which correspond to values of the linear static lift and moment coeffients.

\section{Example 2.1}

For a set of wind tunnel data for a pitching airfoil, one may wish to obtain a model of the nonlinear moment coefficient. Given some prior knowledge that the moment coefficient is a function of the displacement and velocity, we could attempt to fit a polynomial of the pitch displacements and velocities to the data as

$$
C_{M_{E A}}=a_{0}+a_{1} \theta+a_{2} \dot{\theta}+a_{3} \theta^{2}+a_{4} \theta \dot{\theta}+a_{5} \dot{\theta}^{2}+a_{6} \theta^{3}+a_{7} \theta^{2} \dot{\theta}+a_{8} \theta \dot{\theta}^{2}+a_{9} \dot{\theta}^{3}
$$

Adopting a simple least-squares regression model would involve the minimization of the square error between the the moment coefficient $C_{M_{E A}}$ calculated from the data, and the moment coefficient $z(\theta, \dot{\theta})$ from the polynomial, for the whole data set, shown in red in Figures 2.23 and 2.21a (i.e. all data points $i=1, \ldots, N)$ 


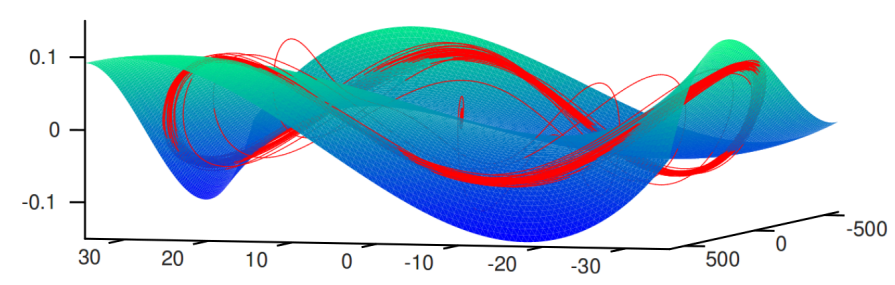

Figure 2.20: Surface fit to the data points

$$
\begin{aligned}
& E=\sum_{i=1}^{N}\left(C_{M_{E A} i}-z\left(\theta_{i}, \dot{\theta}_{i}\right)\right)^{2} \\
& E=\sum_{i=1}^{N}\left(C_{M_{E A i}}-\left(a_{0}+a_{1} \theta_{i}+a_{2} \dot{\theta}_{i}+a_{3} \theta_{i}^{2}+a_{4} \theta_{i} \dot{\theta}_{i}+a_{5} \dot{\theta}_{i}^{2}+a_{6} \theta_{i}^{3}+a_{7} \theta_{i}^{2} \dot{\theta}_{i}+a_{8} \theta_{i} \dot{\theta}_{i}^{2}+a_{9} \dot{\theta}_{i}^{3}\right)\right)^{2} .
\end{aligned}
$$

Since the quantities of interest here are the coefficients $a_{j}(j=0, \ldots, 9)$, this will involve the minimization of $E$ with respect to each of the coefficients, essentially leading to a system of 10 equations,

$$
0=\frac{\partial E}{\partial a_{j}}
$$

Fitting the data from Figure with a surface $(z(\theta, \dot{\theta}))$, pictured in Figure 2.23 by least squares allows one to obtain coefficients $a_{0}$ through $a_{9}$. Figure 2.21a shows the recreation of the pitch motion, comparing the data to the least squares model, while Figure $2.21 \mathrm{~b}$ shows the recreation of the moment coefficient in time.

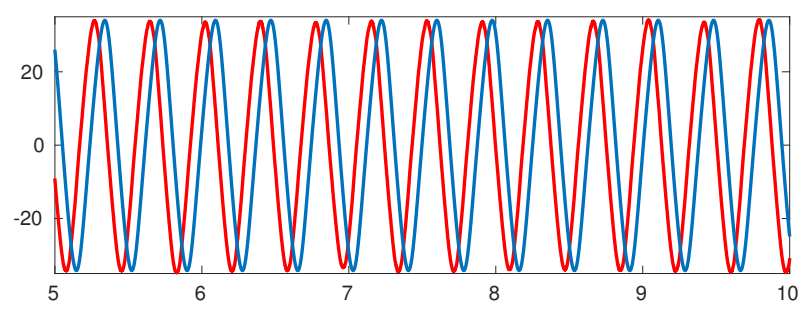

(a) Pitch motion in time

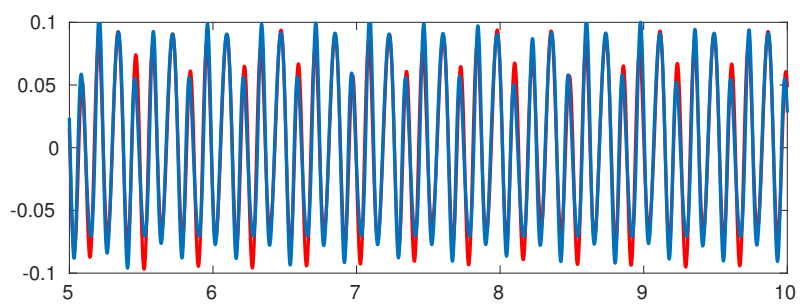

(b) Moment coefficient in time

Figure 2.21: Nonlinear aerodynamic model (blue) fit to the filtered data (red)

While this simple solution provided reasonable agreement in the steady state regime, with a slight phase lag, this kind of empirical model has several downsides. Firstly, although the form of a polynomial of the structural displacements and velocities was rooted in some understanding of the aerodynamics, the parameters in the polynomial are physically meaningless on their own, and 
secondly, the model itself cannot be reduced to any form of accepted aerodynamic theory.

A semi-empirical model could also be reasonably implemented with the current structural model. The so called ONERA model is named after the French aerospace research center (Office National d'Études et de Recherches Aérospatiales) where it was first derived for applications to dynamic stall for helicopter rotor blades. This allows for the study of the coupled aeroelastic system at larger angles of attack (which are beyond the range where the linear aerodynamic model is physically meaningful). While this model is adept at modelling the aerodynamic forces at large angles of attack, it is especially attractive as it reduces to a linear unsteady model when the amplitude of oscillations are small 44]. A drawback from this model is that as a semi-empirical model, it can often be difficult to attribute physical meaning to some of the parameters. For this reason, other candidate models, such as those discussed in 45 may be more attractive to modellers.

\subsubsection{Set of Coupled Equations}

Having defined the variation of the internal forces (strain energy $\delta U$ and kinetic energy $\delta T$ ) and of the external forces (non-conservative aerodynamic forces $\delta W$ ), one obtains

$$
\begin{aligned}
\delta U & =\int_{0}^{S}\left(\bar{U}_{u} \delta u+\bar{U}_{v} \delta v+\bar{U}_{w} \delta w+\bar{U}_{\phi} \delta \phi\right) d x+\bar{U}_{\theta} \delta \theta+b(U), \\
\delta T & =\int_{0}^{S}\left(\bar{T}_{u} \delta u+\bar{T}_{v} \delta v+\bar{T}_{w} \delta w+\bar{T}_{\phi} \delta \phi\right) d x+\bar{T}_{\theta} \delta \theta+b(T), \\
\delta W & =\int_{0}^{S}\left(\bar{W}_{v} \delta v+\bar{W}_{w} \delta w+\bar{W}_{\phi} \delta \phi+\bar{W}_{\theta} \delta \theta\right) d x .
\end{aligned}
$$

For arbitrary, admissible variations $\delta u, \delta v, \delta w, \delta \phi$, and $\delta \theta$, the coefficients of the variations must vanish in the integrand for all $x$ from 0 to $S$ and also must vanish in the remaining terms evaluated at 0 and $S$. The former condition will yield four nonlinear partial differential equations for $v, w$, $\phi$, and one ordinary differential equation for $\theta$. The latter will specify the boundary conditions at the ends of the beam. 
Referring to Appendix A, these 4 PDEs, 1 ODE and boundary conditions have been expanded, substituting the stress and moment resultants $\left(N_{x}, M_{y}, M_{z}, P_{x}, T_{x}\right.$, and $\left.S_{x}\right)$. Subsequently, applying a small angle approximation for the angle of twist, the sines and cosines of $\phi$ are linearized by taking a first order Taylor Series expansion. The resulting expressions are then simplified consistent to our second order approximation scheme. The four equations are as follows [1]:

$$
\begin{aligned}
& \delta u \\
& -\left\{N_{\mathbf{x}}\right\}^{\prime}=\bar{W}_{u} \\
& \delta v \\
& \left\{-\left\{N_{\mathbf{x}} v^{\prime}\right\}^{\prime}+\left\{M_{\mathbf{y}} \sin \phi-M_{\mathbf{z}} \cos \phi\right\}^{\prime \prime}\right\}+\left\{\bar{m} \ddot{v}-\bar{m} \ddot{\theta}(e \phi+w)-\bar{m} \dot{\theta}^{2}\left(\left(v+e_{p}\right)+e\right)-2 \bar{m} \dot{\theta}(e \dot{\phi}+\dot{w})\right\}=\bar{W}_{v}, \\
& \delta w \\
& \left\{-\left\{N_{\mathbf{x}} w^{\prime}\right\}^{\prime}-\left\{M_{\mathbf{z}} \sin \phi+M_{\mathbf{y}} \cos \phi\right\}^{\prime \prime}\right\}+\left\{\bar{m} \ddot{w}+\bar{m} e \ddot{\phi}+\bar{m} \ddot{\theta}\left(\left(v+e_{p}\right)+e\right)-\bar{m} \dot{\theta}^{2}(e \phi+w)+2 \bar{m} \dot{\theta}(\dot{v})\right\}=\bar{W}_{w}, \\
& \delta \phi \\
& \left\{\left\{P_{\mathbf{x}}\right\}^{\prime \prime}-\left\{S_{\mathbf{x}}\right\}^{\prime}-\left\{T_{\mathbf{x}}\right\}^{\prime}+\left\{-M_{\mathbf{z}} \cos \phi+M_{\mathbf{y}} \sin \phi\right\} w^{\prime \prime}+\left\{M_{\mathbf{z}} \sin \phi+M_{\mathbf{y}} \cos \phi\right\} v^{\prime \prime}\right\} \\
& +\left\{\bar{I}_{0}(\ddot{\theta}+\ddot{\phi})+\bar{m} e \ddot{w}+\bar{m} e \ddot{\theta}\left(v+e_{p}\right)-\bar{m} e \dot{\theta}^{2}(w)+2 \bar{m} e \dot{\theta}(\dot{v})\right\}=\bar{W}_{\phi}, \\
& \delta \theta \\
& I_{\theta} \ddot{\theta}+D_{\theta} \dot{\theta}+K_{\theta} \theta-\int_{0}^{S}\left\{-\bar{I}_{0}(\ddot{\theta}+\ddot{\phi})+\bar{m} \ddot{v}(e \phi+w)-\bar{m} \ddot{w}\left(\left(v+e_{p}\right)+e\right)-\bar{m} e \ddot{\phi}\left(v+e_{p}\right)-2 \bar{m} e \ddot{\theta}\left(\left(v+e_{p}\right)+w \phi\right)\right. \\
& \left.-\bar{m} \ddot{\theta}\left(w^{2}+\left(v+e_{p}\right)^{2}\right)-2 \bar{m} \dot{\theta}\left(\dot{v}\left(\left(v+e_{p}\right)+e\right)+\dot{w}(e \phi+w)+e \dot{\phi}(w)\right)\right\} d x=\bar{W}_{\theta} .
\end{aligned}
$$

In Eq.2.149, the dynamic terms are found to be formally negligible, thus removing these terms from the differential equation yields a quasi-static differential equation. Hence, given that we have previously stipulated that the aerodynamic loading in the axial direction is zero, we can further assert that the normal force, $N_{x}$, is zero on the premise that the integrated (or total) aerodynamic 
loading in that direction will also be zero. In a similar fashion to [1], we can obtain an expression for $\left(u^{\prime}+\frac{1}{2} v^{2}+\frac{1}{2} w^{\prime 2}\right)$ consistent to the second order. This term appears in $N_{x}$ (see Eq.2.66) $), M_{z}$ (see Eq.2.68) ) and $T_{x}$ (see Eq.(2.69) ), so this is convenient as it allows for a further simplification of the equations of motion. Eq.2.150), Eq.2.151) and Eq.(2.152,

$$
\begin{aligned}
-\left\{N_{\mathbf{x}}\right\}^{\prime} & =\bar{W}_{u} \equiv 0, \\
-\int_{0}^{S}\left\{N_{\mathbf{x}}\right\}^{\prime} d x & =\int_{0}^{S} \bar{W}_{u} d x \equiv 0, \\
N_{x} & =0
\end{aligned}
$$

which leads to,

$$
N_{\mathbf{x}}=E A\left(u^{\prime}+\frac{v^{\prime 2}}{2}+\frac{w^{\prime 2}}{2}\right)-E A e_{A}\left(v^{\prime \prime}+w^{\prime \prime} \phi\right)=0
$$

Thus, one obtains

$$
\left(u^{\prime}+\frac{v^{\prime 2}}{2}+\frac{w^{\prime 2}}{2}\right)=e_{A}\left(v^{\prime \prime}+w^{\prime \prime} \phi\right)
$$

Consequently, making the above substitution, the following reduced set of equations is obtained:

$\delta v$

$$
\begin{aligned}
& \left\{E I_{\mathbf{z}}\left(v^{\prime \prime \prime \prime}\right)+\left(E I_{\mathbf{z}}-E I_{\mathbf{y}}\right)\left(w^{\prime \prime \prime \prime} \phi+2 w^{\prime \prime \prime} \phi^{\prime}+w^{\prime \prime} \phi^{\prime \prime}\right)-E A e_{A}{ }^{2}\left(v^{\prime \prime \prime \prime}+w^{\prime \prime \prime \prime} \phi-2 w^{\prime \prime \prime} \phi^{\prime}+w^{\prime \prime} \phi^{\prime \prime}\right)\right\} \\
& \quad+\left\{\bar{m} \ddot{v}-\bar{m} \ddot{\theta}(e \phi+w)-\bar{m} \dot{\theta}^{2}\left(\left(v+e_{p}\right)+e\right)-2 \bar{m} \dot{\theta}(e \dot{\phi}+\dot{w})\right\}=\bar{W}_{v},
\end{aligned}
$$

$\delta w$

$$
\begin{aligned}
& \left\{E I_{\mathbf{y}}\left(w^{\prime \prime \prime \prime}\right)+\left(E I_{\mathbf{z}}-E I_{\mathbf{y}}\right)\left(v^{\prime \prime \prime \prime} \phi+2 v^{\prime \prime \prime} \phi^{\prime}+v^{\prime \prime} \phi^{\prime \prime}\right)-E A e_{A}{ }^{2}\left(v^{\prime \prime \prime \prime} \phi+2 v^{\prime \prime \prime} \phi^{\prime}+v^{\prime \prime} \phi^{\prime \prime}\right)+E C_{1}{ }^{*}\left(\phi^{\prime \prime \prime \prime}\right)\right\} \\
& \quad+\left\{\bar{m} \ddot{w}+\bar{m} e \ddot{\phi}+\bar{m} \ddot{\theta}\left(\left(v+e_{p}\right)+e\right)-\bar{m} \dot{\theta}^{2}(e \phi+w)+2 \bar{m} \dot{\theta}(\dot{v})\right\}=\bar{W}_{w},
\end{aligned}
$$


$\delta \phi$

$$
\begin{aligned}
& \left\{\left(E I_{\mathbf{z}}-E I_{\mathbf{y}}\right)\left(v^{\prime \prime} w^{\prime \prime}\right)-G J\left(\phi^{\prime \prime}\right)-E A e_{A}{ }^{2}\left(v^{\prime \prime} w^{\prime \prime}\right)+E C_{1}{ }^{*}\left(\phi^{\prime \prime \prime \prime}\right)+E C_{2}{ }^{*}\left(w^{\prime \prime \prime \prime}\right)\right\} \\
& +\left\{\bar{I}_{0}(\ddot{\theta}+\ddot{\phi})+\bar{m} e \ddot{w}+\bar{m} e \ddot{\theta}\left(v+e_{p}\right)-\bar{m} e \dot{\theta}^{2}(w)+2 \bar{m} e \dot{\theta}(\dot{v})\right\}=\bar{W}_{\phi},
\end{aligned}
$$

$\delta \theta$

$$
\begin{aligned}
I_{\theta} \ddot{\theta}+ & D_{\theta} \dot{\theta}+K_{\theta} \theta+\int_{0}^{S}\left\{\bar{I}_{0}(\ddot{\theta}+\ddot{\phi})-\bar{m} \ddot{v}(e \phi+w)+\bar{m} \ddot{w}\left(\left(v+e_{p}\right)+e\right)+\bar{m} e \ddot{\phi}\left(v+e_{p}\right)\right. \\
& \left.+2 \bar{m} e \ddot{\theta}\left(\left(v+e_{p}\right)+w \phi\right)+\bar{m} \ddot{\theta}\left(w^{2}+\left(v+e_{p}\right)^{2}\right)+2 \bar{m} \dot{\theta}\left(\dot{v}\left(\left(v+e_{p}\right)+e\right)+\dot{w}(e \phi+w)+\dot{\phi}(e w)\right)\right\} d x=\bar{W}_{\theta} .
\end{aligned}
$$

With the system of coupled differential equations established, the question arises as to why the rigid body pitch rotation, $\theta(t)$ is taken as a separate displacement from the flexible twist angle, $\phi(x, t)$ of freedom in the formulation, rather than consolidating the two into a single rotation, $\psi(x, t)$ for example. By means of a simple example, it is illustrated that the resulting system of equation are equivalent for the case where $\phi$ and $\theta$ are separated or combined. Consider the torsional vibrations of a cylindrical bar mounted to the same support mechanism as the cantilever in the current study (nonzero mass base restrained by a torsional spring). If we model the flexible torsional vibrations and the rigid body rotation as separate displacements, as in the current investigation, the result is a partial differential equation governing $\phi$, which is coupled with an ordinary equation governing $\theta$. In the case where the displacements are combined, a single partial differential equation is obtained for the combined angle $\psi$, however, the boundary condition at the root is now dynamic, given by an ordinary differential equation. It can be seen that both ways of framing the problem reduce to the same system by comparing the equations obtained in Example 2.2 with the terms concerning $\phi$ and $\theta$ Eq. (2.158) and Eq.(2.159).

\section{Example 2.2}

This example seeks to illustrate the motivation for considering the rigid body pitch rotation, $\theta$, and the flexible twist angle $\phi$ as separate degrees of freedom. Consider a flexible circular rod (having torsional rigidity, $G J$, and a mass moment of inertia about the centroid of $\bar{I}_{0}$ per unit length), attached to a nonzero mass support mechanism (having a mass moment of inertia of $I_{\theta}$ about its centroid) which is restained by a torsional spring (with stiffness $K_{\theta}$ ). 


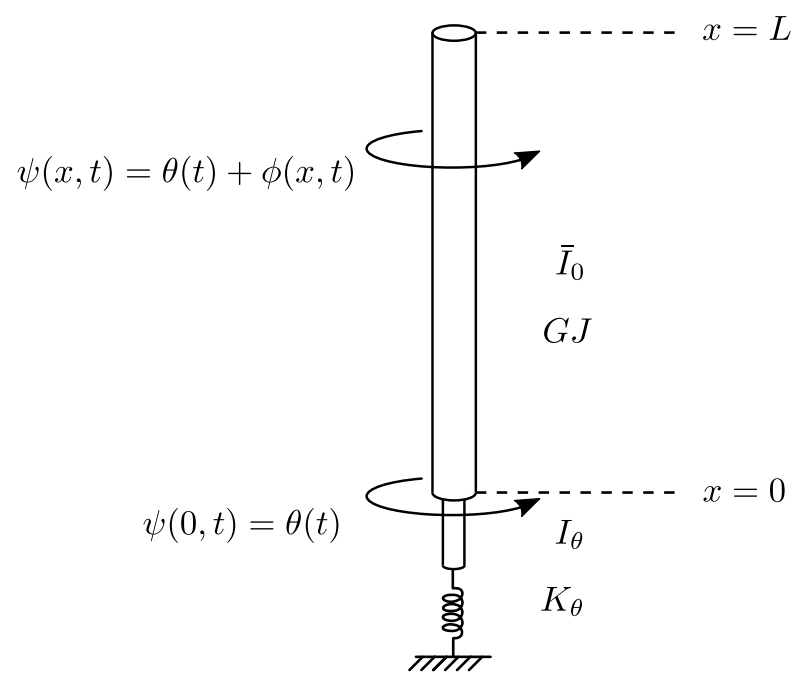

Figure 2.22: Illustration of the simplified flexible bar system

Approaching the problem from the perspective of Newton's Second Law, we draw the free body diagram of a section of the rod of length $d x$.

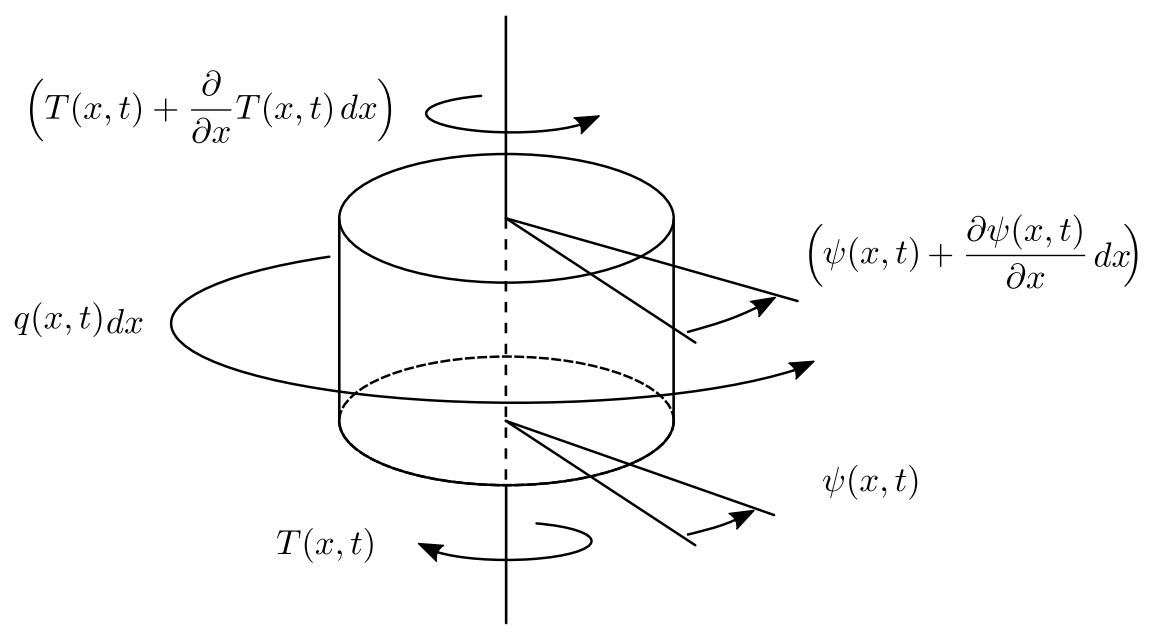

Figure 2.23: Free body diagram of the bar in its deformed configuration

The equation of equilibrium in torsion yields the following,

$$
\bar{I}_{0} d x \frac{\partial^{2} \psi(x, t)}{\partial t^{2}}=\left(T(x, t)+\frac{\partial}{\partial x} T(x, t) d x\right)-T(x, t)+q(x, t) d x
$$

which simplifies to,

$$
\bar{I}_{0} \frac{\partial^{2} \psi(x, t)}{\partial t^{2}}=\frac{\partial}{\partial x} T(x, t)+q(x, t)
$$


Applying Hooke's Law to the torsion formula, we obtain the following expression which relates the angle of twist $\psi(x, t)$ to the internal torque $T(x, t)$,

$$
T(x, t)=G J \frac{\partial \psi(x, t)}{\partial x} .
$$

Hence, differentiating Eq. 2.162 with respect to $x$, we obtain

$$
\frac{\partial}{\partial x} T(x, t)=G J \frac{\partial^{2} \phi(x, t)}{\partial x^{2}}
$$

Now substituting the expression in Eq.2.163 into Eq.2.161, we obtain

$$
\bar{I}_{0} \frac{\partial^{2} \psi(x, t)}{\partial t^{2}}=G J \frac{\partial^{2} \psi(x, t)}{\partial x^{2}}+q(x, t) .
$$

Rearranging gives,

$$
-G J \frac{\partial^{2} \psi(x, t)}{\partial x^{2}}+\bar{I}_{0} \frac{\partial^{2} \psi(x, t)}{\partial t^{2}}=q(x, t)
$$

Now we consider the boundary conditions at both ends of the rod. The bar is free at $x=L$, hence internal torque is equal to zero, allowing us to write the first boundary conditions as in Eq.2.166. The boundary condition at $x=0$ is given by the value of the internal torque at the root, which is given by the expression in Eq.2.167. Hence, we write,

$$
\begin{gathered}
0=\left.G J \frac{\partial \psi(x, t)}{\partial x}\right|_{x=L}, \\
I_{\theta} \frac{\partial^{2} \psi(0, t)}{\partial^{2} t}+K_{\theta} \psi(0, t)=\left.G J \frac{\partial \psi(x, t)}{\partial x}\right|_{x=0}
\end{gathered}
$$

If we integrate Eq.2.171, and subsequently insert the boundary conditions from Eq.2.166 and Eq.2.167 at the tip and root respectively, the following expression is obtained,

$$
\begin{gathered}
-\left.G J \frac{\partial \psi(x, t)}{\partial x}\right|_{x=0} ^{L}+\int_{0}^{L} \bar{I}_{0} \frac{\partial^{2} \psi(x, t)}{\partial t^{2}} d x=\int_{0}^{L} q(x, t) d x \\
-\left(0-\left(\left(I_{\theta}\right) \frac{\partial^{2} \psi(0, t)}{\partial^{2} t}+K_{\theta} \psi(0, t)\right)\right)+\int_{0}^{L} \bar{I}_{0} \frac{\partial^{2} \psi(x, t)}{\partial t^{2}} d x=\int_{0}^{L} q(x, t) d x,
\end{gathered}
$$




$$
I_{\theta} \frac{\partial^{2} \psi(0, t)}{\partial t^{2}}+K_{\theta} \psi(0, t)+\int_{0}^{L} \bar{I}_{0} \frac{\partial^{2} \psi(x, t)}{\partial^{2} t} d x=\int_{0}^{L} q(x, t) d x
$$

If we consider Eq.2.171 and Eq.2.170, and note that $\psi(x, t)=\theta(t)+\phi(x, t)$. Furthermore, because the bar is rigidly attached to the support mechanism, the boundary condition $\phi(0, t)=0$ is still valid. Thus, we can say that $\psi(0, t)=\theta(t)$. Note also that $\psi^{\prime \prime}(x, t)=\phi^{\prime \prime}(x, t)$ because $\theta(t)$ has no dependence on $x$. Hence,

$$
\begin{aligned}
-G J \frac{\partial^{2}(\theta(t)+\phi(x, t))}{\partial x^{2}}+\bar{I}_{0} \frac{\partial^{2}(\theta(t)+\phi(x, t))}{\partial t^{2}} & =q(x, t), \\
-G J \phi^{\prime \prime}(x, t)+\bar{I}_{0}(\ddot{\theta}(t)+\ddot{\phi}(x, t)) & =q(x, t) .
\end{aligned}
$$

$$
\begin{aligned}
I_{\theta} \frac{\partial^{2} \theta(t)}{\partial t^{2}}+K_{\theta} \theta(t)+\int_{0}^{L} \bar{I}_{0} \frac{\partial^{2}(\theta(t)+\phi(x, t))}{\partial^{2} t} d x & =\int_{0}^{L} q(x, t) d x, \\
I_{\theta} \ddot{\theta}(t)+K_{\theta} \theta(t)+\int_{0}^{L} \bar{I}_{0}(\ddot{\phi}(x, t)+\ddot{\theta}(t)) d x & =\int_{0}^{L} q(x, t) d x .
\end{aligned}
$$

\subsection{Nondimensionalization}

We introduce the following nondimensional variables for the displacements of the system. The bending displacements are nondimensionalized by the span of the wing, while the angular displacements need not be further manipulated as they are already dimensionless (radians)

$$
\begin{aligned}
\mathcal{V} & =\frac{v}{S}, \\
\mathcal{W} & =\frac{w}{S}, \\
\varphi & \equiv \phi, \\
\vartheta & \equiv \theta .
\end{aligned}
$$


We will introduce additional cross-sectional properties which have been normalized by the span of the airfoil. The following three dimensions of the cross section measured relative to the elastic axis; the center of gravity $e$, the geometric centroid $e_{A}$, and the pitch axis $e_{p}$ are nondimensionalized as follows

$$
\begin{aligned}
\mathcal{E} & =\frac{e}{S}, \\
\mathcal{E}_{A} & =\frac{e_{A}}{S}, \\
\mathcal{E}_{P} & =\frac{e_{p}}{S} .
\end{aligned}
$$

We now introduce nondimensionalized parameters associated the strain energy contributions to the equations of motion

$$
\begin{aligned}
1 & =\frac{E A}{E A}, \\
\mathcal{K}_{y} & =\frac{E I_{\mathbf{y}}}{E A S^{2}}, \\
\mathcal{K}_{z} & =\frac{E I_{\mathbf{z}}}{E A S^{2}}, \\
\mathcal{K}_{x} & =\frac{G J}{E A S^{2}}, \\
\mathcal{K}_{\theta} & =\frac{K_{\theta}}{E A S}, \\
\mathcal{K}_{\lambda 1} & =\frac{E C_{1}}{E A S^{3}}, \\
\mathcal{K}_{\lambda 2} & =\frac{E C_{2}}{E A S^{3}},
\end{aligned}
$$

as well as terms arising from the kinetic energy contributions

$$
1=\frac{\bar{m}}{\bar{m}}
$$




$$
\begin{aligned}
& \mathcal{M}_{0}=\frac{\bar{I}_{0}}{\bar{m} S^{2}}, \\
& \mathcal{M}_{\theta}=\frac{I_{\theta}}{\bar{m} S^{3}}, \\
& \mathcal{D}_{\theta}=\frac{D_{\theta}}{E A S} .
\end{aligned}
$$

Additionally, we will require that the time variable $t$, and our coordinate variable, $x$, be nondimensionalized as well. The normalization of $x$ was imposed in the derivation (as was done with the coordinates $u, v$, and $w$ ), and the corresponding scaling factor for $t$ was obtained as a byproduct of the nondimensionalization process.

$$
\begin{aligned}
& \mathcal{X}=\frac{x}{S}, \\
& \mathcal{T}=t \sqrt{\frac{E A}{\bar{m} S^{2}}} .
\end{aligned}
$$

The aerodynamic loading is also nondimensionalized through the following variables. Note that $A_{1}$, $A_{2}, b_{1}$ and $b_{2}$ from Wagner's function are already nondimensional, and hence are unchanged.

$$
\begin{aligned}
\mathcal{P} & =\rho \frac{S^{2}}{\bar{m}}, \\
\mathcal{U} & =U \sqrt{\frac{\bar{m}}{E A}}, \\
\mathcal{C} & =\frac{c}{S} \\
\mathcal{E}_{c} & =\frac{e_{c}}{S}, \\
\mathcal{Z} & =z \sqrt{\frac{E A}{\bar{m}}} .
\end{aligned}
$$

Making these substitutions, the structural components of the equations become 


$$
\begin{aligned}
& \left\{\mathcal{K}_{z}\left(\mathcal{V}^{\prime \prime \prime \prime}\right)+\left(\mathcal{K}_{z}-\mathcal{K}_{y}\right)\left(\mathcal{W}^{\prime \prime} \varphi^{\prime \prime}+2 \mathcal{W}^{\prime \prime \prime} \varphi^{\prime}+\mathcal{W}^{\prime \prime \prime \prime} \varphi\right)-\mathcal{E}_{A}^{2}\left(\mathcal{V}^{\prime \prime \prime \prime}+\mathcal{W}^{\prime \prime} \varphi^{\prime \prime}+2 \mathcal{W}^{\prime \prime \prime} \varphi^{\prime}+\mathcal{W}^{\prime \prime \prime \prime} \varphi\right)\right\} \\
& \quad+\left\{\ddot{\mathcal{V}}-\ddot{\vartheta}(\mathcal{E} \varphi+\mathcal{W})-\dot{\vartheta}^{2}\left(\left(\mathcal{V}+\mathcal{E}_{p}\right)+\mathcal{E}\right)-2 \dot{\vartheta}(\mathcal{E} \dot{\varphi}+\dot{\mathcal{W}})\right\}=\overline{\mathcal{W}}_{v}
\end{aligned}
$$

$\delta w$

$$
\begin{aligned}
& \left\{\mathcal{K}_{y}\left(\mathcal{W}^{\prime \prime \prime \prime}\right)+\left(\mathcal{K}_{z}-\mathcal{K}_{y}\right)\left(\mathcal{V}^{\prime \prime \prime \prime} \varphi+2 \mathcal{V}^{\prime \prime \prime} \varphi^{\prime}+\mathcal{V}^{\prime \prime} \varphi^{\prime \prime}\right)-\mathcal{E}_{A}^{2}\left(\mathcal{V}^{\prime \prime \prime \prime} \varphi+2 \mathcal{V}^{\prime \prime \prime} \varphi^{\prime}+\mathcal{V}^{\prime \prime} \varphi^{\prime \prime}\right)+\mathcal{K}_{\lambda 1}\left(\varphi^{\prime \prime \prime \prime}\right)\right\} \\
& +\left\{\ddot{\mathcal{W}}+\mathcal{E} \ddot{\varphi}+\ddot{\vartheta}\left(\left(\mathcal{V}+\mathcal{E}_{p}\right)+\mathcal{E}\right)-\dot{\vartheta}^{2}(\mathcal{E} \varphi+\mathcal{W})+2 \dot{\vartheta}(\dot{\mathcal{V}})\right\}=\overline{\mathcal{W}}_{w}
\end{aligned}
$$

$\delta \phi$

$$
\begin{gathered}
\left\{-\mathcal{K}_{x}\left(\varphi^{\prime \prime}\right)+\left(\mathcal{K}_{z}-\mathcal{K}_{y}\right)\left(\mathcal{V}^{\prime \prime} \mathcal{W}^{\prime \prime}\right)-\mathcal{E}_{A}^{2}\left(\mathcal{V}^{\prime \prime} \mathcal{W}^{\prime \prime}\right)+\mathcal{K}_{\lambda 1}\left(\varphi^{\prime \prime \prime \prime}\right)+\mathcal{K}_{\lambda 2}\left(\mathcal{W}^{\prime \prime \prime \prime}\right)\right\} \\
+\left\{\mathcal{M}_{0}(\ddot{\varphi}+\ddot{\vartheta})+\mathcal{E} \ddot{\mathcal{W}}+\ddot{\vartheta}\left(\mathcal{E}\left(\mathcal{V}+\mathcal{E}_{p}\right)\right)-\dot{\vartheta}^{2}(\mathcal{E} \mathcal{W})+2 \mathcal{E} \dot{\vartheta}(\dot{\mathcal{V}})\right\}=\overline{\mathcal{W}}_{\phi}
\end{gathered}
$$

$\delta \theta$

$$
\begin{aligned}
\mathcal{M}_{\theta} \ddot{\vartheta} & +\mathcal{D}_{\theta} \dot{\vartheta}+\mathcal{K}_{\vartheta} \vartheta+\int_{0}^{1}\left\{\mathcal{M}_{0}(\ddot{\varphi}+\ddot{\vartheta})-\ddot{\mathcal{V}}(\mathcal{E} \varphi+\mathcal{W})+\ddot{\mathcal{W}}\left(\left(\mathcal{V}+\mathcal{E}_{p}\right)+\mathcal{E}\right)+\mathcal{E} \ddot{\varphi}\left(\left(\mathcal{V}+\mathcal{E}_{p}\right)\right)\right. \\
& \left.+2 \mathcal{E} \ddot{\vartheta}\left(\left(\mathcal{V}+\mathcal{E}_{p}\right)+\mathcal{W} \varphi\right)+\ddot{\vartheta}\left(\mathcal{W}^{2}+\left(\mathcal{V}+\mathcal{E}_{p}\right)^{2}\right)+2 \dot{\vartheta}\left(\dot{\mathcal{V}}\left(\left(\mathcal{V}+\mathcal{E}_{p}\right)+\mathcal{E}\right)+\dot{\mathcal{W}}(\mathcal{E} \varphi+\mathcal{W})+\mathcal{E} \dot{\varphi}(\mathcal{W})\right)\right\} d \mathcal{X}=\overline{\mathcal{W}}_{\theta}
\end{aligned}
$$

Similarly, the aerodynamic loads are expressed as follows.

$$
\begin{aligned}
& \overline{\mathcal{W}}_{v}=\sqrt{1-\mathcal{X}^{2}}\left\{\frac{1}{4} \mathcal{P} \mathcal{C}^{2} \pi\left(-\ddot{\mathcal{W}} \cos (\vartheta)-\ddot{\mathcal{V}} \sin (\vartheta)+\mathcal{U}(\dot{\varphi}+\dot{\vartheta})-\left(\mathcal{E}_{c}-\frac{1}{4} \mathcal{C}\right) \ddot{\varphi}-\left(\left(\mathcal{E}_{c}+\mathcal{E}_{p}\right)-\frac{1}{4} \mathcal{C}\right) \ddot{\vartheta}\right)\right. \\
& \left.+\frac{1}{2} \mathcal{P U C} 2 \pi\left(\left(1-A_{1}-A_{2}\right) \tilde{w}_{3 c / 4}+\dot{\mathcal{Z}}\left(A_{1} b_{1}+A_{2} b_{2}\right)+\mathcal{Z} b_{1} b_{2}\left(A_{1}+A_{2}\right)\right)\right\} \times \sin (\vartheta), \\
& \overline{\mathcal{W}}_{w}=\sqrt{1-\mathcal{X}^{2}}\left\{\frac{1}{4} \mathcal{P} \mathcal{C}^{2} \pi\left(-\ddot{\mathcal{W}} \cos (\vartheta)-\ddot{\mathcal{V}} \sin (\vartheta)+\mathcal{U}(\dot{\varphi}+\dot{\vartheta})-\left(\mathcal{E}_{c}-\frac{1}{4} \mathcal{C}\right) \ddot{\varphi}-\left(\left(\mathcal{E}_{c}+\mathcal{E}_{p}\right)-\frac{1}{4} \mathcal{C}\right) \ddot{\vartheta}\right)\right. \\
& \left.+\frac{1}{2} \mathcal{P U C} 2 \pi\left(\left(1-A_{1}-A_{2}\right) \tilde{w}_{3 c / 4}+\dot{\mathcal{Z}}\left(A_{1} b_{1}+A_{2} b_{2}\right)+\mathcal{Z} b_{1} b_{2}\left(A_{1}+A_{2}\right)\right)\right\} \times \cos (\vartheta), \\
& \overline{\mathcal{W}}_{\varphi}=\sqrt{1-\mathcal{X}^{2}}\left\{\frac { 1 } { 4 } \mathcal { P } \mathcal { C } ^ { 2 } \pi \left(\left(\mathcal{E}_{c}-\frac{1}{4} \mathcal{C}\right)(-\ddot{\mathcal{W}} \cos (\vartheta)-\ddot{\mathcal{V}} \sin (\vartheta))-\mathcal{U}\left(\frac{1}{2} \mathcal{C}-\mathcal{E}_{c}\right) \dot{\varphi}-\mathcal{U}\left(\frac{1}{2} \mathcal{C}-\left(\mathcal{E}_{c}+\mathcal{E}_{p}\right)\right) \dot{\vartheta}\right.\right. \\
& \left.-\left(\left(\mathcal{E}_{c}-\frac{1}{4} \mathcal{C}\right)^{2}+\frac{1}{32} \mathcal{C}^{2}\right) \ddot{\varphi}-\left(\left(\left(\mathcal{E}_{c}+\mathcal{E}_{p}\right)-\frac{1}{4} \mathcal{C}\right)\left(\mathcal{E}_{c}-\frac{1}{4} \mathcal{C}\right)+\frac{1}{32} \mathcal{C}^{2}\right) \ddot{\vartheta}\right) \\
& \left.+\frac{1}{2} \mathcal{P U C} 2 \pi \mathcal{E}_{c}\left(\left(1-A_{1}-A_{2}\right) \tilde{w}_{3 c / 4}+\dot{\mathcal{Z}}\left(A_{1} b_{1}+A_{2} b_{2}\right)+\mathcal{Z} b_{1} b_{2}\left(A_{1}+A_{2}\right)\right)\right\},
\end{aligned}
$$




$$
\begin{aligned}
\overline{\mathcal{W}}_{\vartheta}=\int_{0}^{S} \sqrt{1-\mathcal{X}^{2}}\left\{\frac{1}{4} \mathcal{P C}^{2}\right. & \pi\left(\left(\left(\mathcal{E}_{c}+\mathcal{E}_{p}\right)-\frac{1}{4} \mathcal{C}\right)-\ddot{\mathcal{W}} \cos (\vartheta)-\ddot{\mathcal{V}} \sin (\vartheta)-\mathcal{U}\left(\frac{1}{2} \mathcal{C}-\mathcal{E}_{c}\right) \dot{\varphi}-\mathcal{U}\left(\frac{1}{2} \mathcal{C}-\left(\mathcal{E}_{c}+\mathcal{E}_{p}\right)\right) \dot{\vartheta}\right. \\
& \left.-\left(\left(\left(\mathcal{E}_{c}+\mathcal{E}_{p}\right)-\frac{1}{4} \mathcal{C}\right)\left(\mathcal{E}_{c}-\frac{1}{4} \mathcal{C}\right)+\frac{1}{32} \mathcal{C}^{2}\right) \ddot{\varphi}-\left(\left(\left(\mathcal{E}_{c}+\mathcal{E}_{p}\right)-\frac{1}{4} \mathcal{C}\right)^{2}+\frac{1}{32} \mathcal{C}^{2}\right) \ddot{\vartheta}\right) \\
& \left.+\frac{1}{2} \mathcal{P} \mathcal{U} \mathcal{C} 2 \pi\left(\mathcal{E}_{c}+\mathcal{E}_{p}\right)\left(\left(1-A_{1}-A_{2}\right) \tilde{w}_{3 c / 4}+\dot{\mathcal{Z}}\left(A_{1} b_{1}+A_{2} b_{2}\right)+\mathcal{Z} b_{1} b_{2}\left(A_{1}+A_{2}\right)\right)\right\} d \mathcal{X} .
\end{aligned}
$$

With,

$$
\begin{aligned}
\ddot{\mathcal{Z}}+\dot{\mathcal{Z}}\left(b_{1}+b_{2}\right)+\mathcal{Z}\left(b_{1} b_{2}\right) & =\tilde{w}_{3 c / 4}, \\
\tilde{w}_{3 c / 4} & =\mathcal{U}(\vartheta+\varphi)-\dot{\mathcal{W}} \cos (\vartheta)-\dot{\mathcal{V}} \sin (\vartheta) \\
& +\left(\frac{1}{2} \mathcal{C}-\mathcal{E}_{c}\right) \dot{\varphi}+\left(\frac{1}{2} \mathcal{C}-\left(\mathcal{E}_{c}+\mathcal{E}_{p}\right)\right) \dot{\vartheta}
\end{aligned}
$$




\section{Chapter 3}

\section{Numerical Solution of the Nonlinear Equations of}

\section{Motion}

This chapter outlines the methods of numerical solution of the nonlinear equations of motion as derived in Chapter 2, A finite dimensional representation of the distributed parameter system is obtained by adopting a Galerkin projection scheme, which allows us to discretize the set of coupled differential equations into a set of coupled ordinary differential equations. The set of coupled partial differential equations contains an inertial nonlinearity relating to the rigid body base rotation. This introduces complications in the numerical solution as the equations of motion cannot be tackled using popular numerical integrators used in structural dynamics 46. The formulation of an implicit Houbolt method will be described. Numerical studies including results for the pure structural vibrations will be presented. Subsequently aeroelastic simulations will be analyzed, coupling the structure with the various aerodynamic models introduced in Chapter 2 For the purpose of verification of the results obtained by the Galerkin method, a finite difference scheme is also employed to compare the results obtained by both numerical schemes. The structural vibration results are compared in the time domain as well as the frequency to demonstrate that consistent results are produced by both methods. 


\subsection{Galerkin Projection}

With there being a system of three coupled partial differential equations (Eq. 2.176), Eq. 2.177) and Eq.(2.178) ) and one ordinary differential equation (Eq.2.179), a finite dimensional representation of the system of coupled differential equations is sought. In the form of a Galerkin projection, a discretized system is obtained, adopting the normal cantilever beam modes in bending and torsion as the assumed basis functions [47].

\subsubsection{Basis Functions}

The axial displacement, edgewise bending, flapwise bending and the torsion are spatio-temporal variables, as they vary in time as well as along the length of the wing. However, the root pitch motion represents rigid body motion and thus varies only in time. Referring to Chapter 2 the system of equations consists of three PDEs and an ODE. The wing is flexible, and thus is a continuous (distributed parameter) system having infinite degrees of freedom. To obtain the solution of the PDEs, the domain is discretized using a Galerkin Projection scheme, wherein the spatio-temporal variables are given by a truncated series of unknown temporal coordinates projected on a corresponding set of spatially varying global basis functions. The basis functions: $\Upsilon_{i},(i=1,2, \ldots, L)$, $\Psi_{i},(i=1,2, \ldots, M)$, and $\Omega_{i},(i=1,2, \ldots, N)$ satisfy both the Dirichlet and the Neuman boundary conditions of the system in question. For convenience, the basis functions, $\Upsilon_{i}(\mathcal{X})$ and $\Psi_{i}(\mathcal{X})$, which correspond to the bending displacements, are taken as the first $L$, and $M$ normal vibration modes in bending given by Eq. (3.8) and Eq.(3.9). The basis function $\Omega_{i}(\mathcal{X})$ which corresponds to the flexible twist angle, is given by the first $N$ normal vibration modes in torsion, given by Eq. (3.10). The displacements $v, w, \phi$, and $\theta$ are nondimensionalized as $\mathcal{V}, \mathcal{W}, \varphi$ and $\vartheta$ respectively, as in Eq.2.175 a), Eq. 2.175 b), Eq. 2.175 c and Eq.(2.175 d). The nondimensionalized displacements are approximated by the truncated summations in Eq. 3.1), Eq.(3.2), Eq. 3.3 and Eq. 3.4 respectively. While the axial displacement, $u$, is not uniquely zero, it was shown in Chapter 2 that all explicit instances of $u$ could conveniently be removed from the system of equations. The finite dimensional approximation of the response variables are given by 25 


$$
\begin{aligned}
\mathcal{V}(\mathcal{X}, \mathcal{T}) & =\sum_{i=1}^{L} \Upsilon_{i}(\mathcal{X}) V_{i}(\mathcal{T}) \\
\mathcal{W}(\mathcal{X}, \mathcal{T}) & =\sum_{i=1}^{M} \Psi_{i}(\mathcal{X}) W_{i}(\mathcal{T}) \\
\varphi(\mathcal{X}, \mathcal{T}) & =\sum_{i=1}^{N} \Omega_{i}(\mathcal{X}) \Phi_{i}(\mathcal{T}) \\
\vartheta(\mathcal{T}) & =\Theta(\mathcal{T}) .
\end{aligned}
$$

\section{Boundary Conditions}

For a cantilever, in bending there are four boundary conditions; zero displacement and zero slope at the fixed end $(\mathcal{X}=0)$, and zero shear and zero moment at the free end $(\mathcal{X}=1)$ as given by [48].

$$
\begin{aligned}
\mathcal{V}(0) & =0, \\
\mathcal{V}^{\prime}(0) & =0, \\
\mathcal{V}^{\prime \prime}(1) & =0, \\
\mathcal{V}^{\prime \prime \prime}(1) & =0 .
\end{aligned}
$$

It follows that,

$$
\begin{aligned}
\mathcal{W}(0) & =0, \\
\mathcal{W}^{\prime}(0) & =0, \\
\mathcal{W}^{\prime \prime}(1) & =0 \\
\mathcal{W}^{\prime \prime \prime}(1) & =0
\end{aligned}
$$

Similarly, for a cantilever undergoing torsion, there are two boundary conditions, zero twisting at the fixed end, and zero slope at the free end as given by [48]. Additionally, because the rigid body 
pitch rotation and the flexible twist angle have been modelled as separate displacements, the fixed end boundary condition is valid. This separation of $\phi(x, t)$ and $\theta(t)$ is convenient for defining basis functions for the Galerkin method as,

$$
\begin{gathered}
\varphi(0)=0 \\
\varphi^{\prime}(1)=0 .
\end{gathered}
$$

As noted previously, the derivation accounts for warping of the cross section to maintain generality, however the numerical invertigations do not model any warping. One should be mindful that if warping were to be included, two additional boundary conditions would be required in torsion 1

$$
\begin{gathered}
\varphi^{\prime}(0)=0 \\
P_{\mathbf{x}}(1)=0
\end{gathered}
$$

Note that in this investigation, the linear boundary conditions mentioned above are satisfied implicitly by the selection of basis funcitons. The nonlinear boundary conditions derived in Chapter 2 however are not implemented in the model. The basis functions are taken here as the natural vibration modes of a cantilever as obtained from the characteristic equations, applying the appropriate natural (force) and geometric (displacement) boundary conditions above, as given by 48

$$
\begin{aligned}
& \Upsilon_{i}(\mathcal{X})=\cosh \left(\lambda_{i} \mathcal{X}\right)-\cos \left(\lambda_{i} \mathcal{X}\right)-\left(\frac{\cosh \left(\lambda_{i}\right)+\cos \left(\lambda_{i}\right)}{\sinh \left(\lambda_{i}\right)+\sin \left(\lambda_{i}\right)}\right)\left(\sinh \left(\lambda_{i} \mathcal{X}\right)-\sin \left(\lambda_{i} \mathcal{X}\right)\right), \\
& \Psi_{i}(\mathcal{X})=\cosh \left(\lambda_{i} \mathcal{X}\right)-\cos \left(\lambda_{i} \mathcal{X}\right)-\left(\frac{\cosh \left(\lambda_{i}\right)+\cos \left(\lambda_{i}\right)}{\sinh \left(\lambda_{i}\right)+\sin \left(\lambda_{i}\right)}\right)\left(\sinh \left(\lambda_{i} \mathcal{X}\right)-\sin \left(\lambda_{i} \mathcal{X}\right)\right),
\end{aligned}
$$




$$
\Omega_{i}(\mathcal{X})=\sin \left(\gamma_{i} \mathcal{X}\right)
$$

Above, $\lambda_{i}$ is the $i^{\text {th }}$ positive solution of the characteristic equation for pure bending given by Eq. 3.11 48

$$
0=\cos \left(\lambda_{i}\right) \cosh \left(\lambda_{i}\right)
$$

and $\gamma_{i}$ is the $i^{\text {th }}$ positive nonzero solution of the characteristic equation for pure torsion given by Eq. 3.12) 48

$$
0=\sin \left(\gamma_{i}\right)
$$

The first 10 eigenvalues $\left(\lambda_{i}\right.$ and $\left.\gamma_{i}\right)$ can be obtained by solving Eq.(3.11) and Eq.(3.12). The roots of are summarized in Table 3.1 below.

\begin{tabular}{ccccccccccc}
\hline$i$ & 1 & 2 & 3 & 4 & 5 & 6 & 7 & 8 & 9 & 10 \\
\cline { 2 - 10 }$\lambda_{i}$ & 1.875 & 4.694 & 7.854 & 10.995 & 14.137 & 17.278 & 20.420 & 23.561 & 26.703 & 29.845 \\
$\gamma_{i}$ & $\pi$ & $2 \pi$ & $3 \pi$ & $4 \pi$ & $5 \pi$ & $6 \pi$ & $7 \pi$ & $8 \pi$ & $9 \pi$ & $10 \pi$ \\
\hline
\end{tabular}

Table 3.1: First 10 roots of the characteristic bending and torsion equations

The basis functions are the normal modes of a cantilever beam 47 .

As this is an approximation based on discretizing a continuous, infinite degree of freedom system into a finite number of degrees of freedom, it is understood that there is some approximation error between the true solution (theoretically using an infinite summation) and the solution obtained by a finite summation of terms. These residuals, $R_{v}, R_{w}, R_{\phi}$, and $R_{\theta}$ are orthogonal to the solution space spanned by the basis functions. Taking the inner product of the resulting set of 


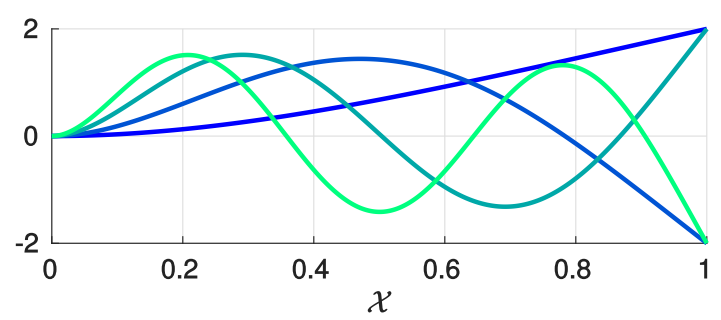

(a) Basis functions in bending $\Upsilon_{i}(\mathcal{X})$ and $\Psi_{i}(\mathcal{X})$

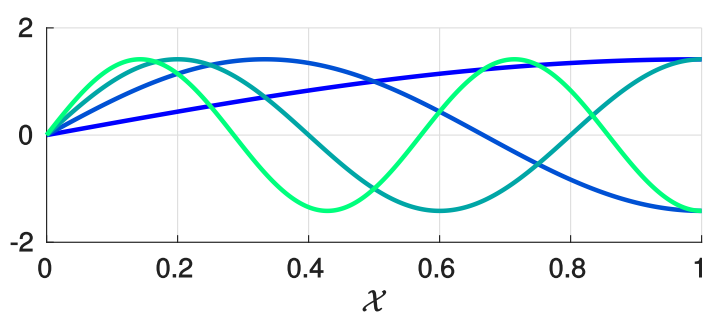

(b) Basis functions in torsion $\Omega_{i}(\mathcal{X})$

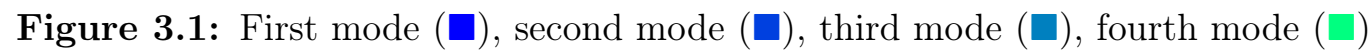

equations with a weighting function that is orthogonal to the set of basis functions will result in the integrated weighted error being equal to zero over the span 49 . Seeing as the set of shape functions that form the basis for the spatial domain readily provide a set of orthonormal functions, for convenience, $\Upsilon_{i}, \Psi_{i}$, and $\Omega_{i}$ will be used as weighting functions in the equations for $\delta v, \delta w$, and $\delta \phi$ respectively. In view of the weighting functions being equal to the assumed shape functions, this is referred to as a Galerkin projection 49 .

\subsubsection{Finite Dimensional Representation of the Nonlinear Structural Equations}

Eq.(3.1), Eq.(3.2), Eq.(3.3), and Eq.(3.4) are substituted into the nondimensionalized differential equations from Chapter 2. The resulting equations are then projected onto the space spanned by the respective basis functions by multiplying the coefficients of $\delta v, \delta w$ and $\delta \phi$ by their respective basis functions $\Upsilon_{i}(\mathcal{X}), \Psi_{i}(\mathcal{X})$ and $\Omega_{i}(\mathcal{X})$, and integrating over the span yield a finite dimensional representation of the equations of motion. The procedure for discretizing the set of coupled PDEs by Galerkin projection is shown in Appendix B.1 In the interest of conciseness, this Galerkin projection is illustrated in Example 3.1 below for the simpler case of the linear coupled bending-torsion vibrations of a cantilever beam.

\section{Example 3.1}

Consider the system of linear partial differential equations describing the free vibration of the coupled 
bending and torsion of an Euler-Bernoulli beam

$$
\begin{aligned}
& 0=E I w^{\prime \prime \prime \prime}(x, t)+m \ddot{w}(x, t)+m e \ddot{\phi}(x, t), \\
& 0=-G J \phi^{\prime \prime}(x, t)+I_{0} \ddot{\phi}(x, t)+m e \ddot{w}(x, t) .
\end{aligned}
$$

The modal approximations for both $w(x, t) \approx \sum_{j=1}^{M} \Psi_{j}(x) W(t)$ and $\phi(x, t) \approx \sum_{j=1}^{M} \Omega_{j}(x) \Phi(t)$ are substituted into the above equations. Of course, due to the truncation of the summation, these expressions will not be equal to zero, but rather some errors $R_{w}$ and $R_{\phi}$ respectively, as defined by.

$$
\begin{aligned}
& R_{w}=E I \sum_{j=1}^{M} \Psi_{j}^{\prime \prime \prime \prime}(x) W_{j}(t)+m \sum_{j=1}^{M} \Psi_{j}(x) \ddot{W}_{j}(t)+m e \sum_{k=1}^{N} \Omega_{k}(x) \ddot{\Phi}_{k}(t) \\
& R_{\phi}=-G J \sum_{k=1}^{M} \Omega_{k}^{\prime \prime}(x) \Phi_{k}(t)+I_{0} \sum_{k=1}^{M} \Omega_{k}(x) \ddot{\Phi}_{k}(t)+m e \sum_{j=1}^{M} \Psi_{j}(x) \ddot{W}_{j}(t) .
\end{aligned}
$$

These expressions should be equal to zero in some average sense 49 ; thus by multiplying the equations by a weighting function and integrating over the domain, we can obtain $\int_{0}^{S} R_{w} \mathcal{W}_{w}=0$, and $\int_{0}^{S} R_{\phi} \mathcal{W}_{\phi}=$ 0. In the Galerkin approach, the weighting function is the same as the basis function (i.e. $\mathcal{W}_{w, i}=\Psi_{i}$ and $\left.\mathcal{W}_{\phi, i}=\Omega_{i}\right)$, leading to

$$
\begin{aligned}
& 0=\int_{0}^{S}\left(E I \sum_{i=1}^{M} \sum_{j=1}^{M} \Psi_{i}(x) \Psi_{j}^{\prime \prime \prime \prime}(x) W_{j}(t)+m \sum_{i=1}^{M} \sum_{j=1}^{M} \Psi_{i}(x) \Psi_{j}(x) \ddot{W}_{j}(t)+m e \sum_{i=1}^{M} \sum_{k=1}^{N} \Psi_{i}(x) \Omega_{k}(x) \ddot{\Phi}_{k}(t)\right) d x \\
& 0=\int_{0}^{S}\left(-G J \sum_{i=1}^{N} \sum_{k=1}^{M} \Omega_{i}(x) \Omega_{k}^{\prime \prime}(x) \Phi_{k}(t)+I_{0} \sum_{i=1}^{N} \sum_{k=1}^{M} \Omega_{i}(x) \Omega_{k}(x) \ddot{\Phi}_{k}(t)+m e \sum_{i=1}^{N} \sum_{j=1}^{M} \Omega_{i}(x) \Psi_{j}(x) \ddot{W}_{j}(t)\right) d x .
\end{aligned}
$$

Clearly, integrating over the domain, eliminates the spatial varying terms (i.e. the basis functions), hence we are left with a set of $M+N$ coupled ordinary differential equations, where we once had a set of 2 coupled partial differential equations.

$$
\begin{aligned}
& 0=E I \sum_{i=1}^{M} \sum_{j=1}^{M} C_{w 1}^{i j} W_{j}(t)+m \sum_{i=1}^{M} \sum_{j=1}^{M} C_{w 2}^{i j} \ddot{W}_{j}(t)+m e \sum_{i=1}^{M} \sum_{k=1}^{N} C_{w 3}^{i k} \ddot{\Phi}_{k}(t), \\
& 0=-G J \sum_{i=1}^{N} \sum_{k=1}^{M} C_{\phi 1}^{i k} \Phi_{k}(t)+I_{0} \sum_{i=1}^{N} \sum_{k=1}^{M} C_{\phi 2}^{i k} \ddot{\Phi}_{k}(t)+m e \sum_{i=1}^{N} \sum_{j=1}^{M} C_{\phi 3}^{i j} \ddot{W}_{j}(t) .
\end{aligned}
$$

Where coefficients $C_{w 1}^{i j}, C_{w 2}^{i j}$ are $M \times M$ matrices, $C_{w 3}^{i k}$ is an $M \times N$ matrix, $C_{\phi 1}^{i k}, C_{\phi 2}^{i k}$ are $N \times N$ matrices, and $C_{\phi 3}^{i k}$ is an $N \times M$ matrix, while $W_{j}$ is an $M \times 1$ vector, and $\Phi_{k}$ is an $N \times 1$ vector. 
This procedure is performed on the full set of coupled partial differential equations derived in Chapter 2. The the full integral expressions and the matrix and tensor coefficients are summarized in Appendix B.2. Adopting the so-called Einstein Summation tensor notation, the resulting set of $L+M+N+1$ coupled ordinary differential equations are expressed in the form below:

$$
\begin{aligned}
& \delta v \\
& \left\{\mathcal{K}_{z}\left(\mathcal{V}^{\prime \prime \prime \prime}\right)+\left(\mathcal{K}_{z}-\mathcal{K}_{y}\right)\left(\mathcal{W}^{\prime \prime \prime \prime} \varphi+2 \mathcal{W}^{\prime \prime \prime} \varphi^{\prime}+\mathcal{W}^{\prime \prime} \varphi^{\prime \prime}\right)-\mathcal{E}_{A}^{2}\left(\mathcal{V}^{\prime \prime \prime \prime}+\mathcal{W}^{\prime \prime \prime \prime} \varphi+2 \mathcal{W}^{\prime \prime \prime} \varphi^{\prime}+\mathcal{W}^{\prime \prime} \varphi^{\prime \prime}\right)\right\} \\
& \quad+\left\{\ddot{\mathcal{V}}-\ddot{\vartheta}(\mathcal{E} \varphi+\mathcal{W})-\dot{\vartheta}^{2}\left(\left(\mathcal{V}+\mathcal{E}_{p}\right)+\mathcal{E}\right)-2 \dot{\vartheta}(\mathcal{E} \dot{\varphi}+\dot{\mathcal{W}})\right\}=\overline{\mathcal{W}}_{v} \\
& \mathcal{K}_{z}\left\{C_{v 1}^{i j} V_{j}\right\}+\left(\mathcal{K}_{z}-\mathcal{K}_{y}\right)\left\{C_{v 2}^{i j k} W_{j} \Phi_{k}+2 C_{v 3}^{i j k} W_{j} \Phi_{k}+C_{v 4}^{i j k} W_{j} \Phi_{k}\right\} \\
& \quad-\mathcal{E}_{A}^{2}\left\{C_{v 1}^{i j} V_{j}+C_{v 2}^{i j k} W_{j} \Phi_{k}-2 C_{v 3}^{i j k} W_{j} \Phi_{k}+C_{v 4}^{i j k} W_{j} \Phi_{k}\right\} \\
& \quad+C_{v 5}^{i j} \ddot{V}_{j}-\ddot{\Theta}\left\{\mathcal{E} C_{v 6}^{i j} \Phi_{j}+C_{v 7}^{i j} W_{j}\right\}-\dot{\Theta}^{2}\left\{C_{v 5}^{i j} V_{j}+\mathcal{E}_{p} C_{v 8}^{i}+\mathcal{E} C_{v 8}^{i}\right\}-2 \dot{\Theta}\left\{\mathcal{E} C_{v 6}^{i j k} \dot{\Phi}_{j}+C_{v 7}^{i j} \dot{W}_{j}\right\}=\mathcal{W}_{v} .
\end{aligned}
$$

$\delta w$

$$
\begin{aligned}
& \left\{\mathcal{K}_{y}\left(\mathcal{W}^{\prime \prime \prime \prime}\right)+\left(\mathcal{K}_{z}-\mathcal{K}_{y}\right)\left(\mathcal{V}^{\prime \prime \prime \prime} \varphi+2 \mathcal{V}^{\prime \prime \prime} \varphi^{\prime}+\mathcal{V}^{\prime \prime} \varphi^{\prime \prime}\right)-\mathcal{E}_{A}^{2}\left(\mathcal{V}^{\prime \prime \prime \prime} \varphi+2 \mathcal{V}^{\prime \prime \prime} \varphi^{\prime}+\mathcal{V}^{\prime \prime} \varphi^{\prime \prime}\right)+\mathcal{K}_{\lambda 1}\left(\varphi^{\prime \prime \prime \prime}\right)\right\} \\
& \quad+\left\{\ddot{\mathcal{V}}+\mathcal{E} \ddot{\varphi}+\ddot{\vartheta}\left(\left(\mathcal{V}+\mathcal{E}_{p}\right)+\mathcal{E}\right)-\dot{\vartheta}^{2}(\mathcal{E} \varphi+\mathcal{W})+2 \dot{\vartheta}(\dot{\mathcal{V}})\right\}=\overline{\mathcal{W}}_{w} \\
& \mathcal{K}_{y}\left\{C_{w 1}^{i j} W_{j}\right\}+\left(\mathcal{K}_{z}-\mathcal{K}_{y}\right)\left\{C_{w 2}^{i j k} V_{j} \Phi_{k}+2 C_{w 3}^{i j k} V_{j} \Phi_{k}+C_{w 4}^{i j k} V_{j} \Phi_{k}\right\} \\
& \quad-\mathcal{E}_{A}^{2}\left\{C_{w 2}^{i j k} V_{j} \Phi_{k}+2 C_{w 3}^{i j k} V_{j} \Phi_{k}+C_{w 4}^{i j k} V_{j} \Phi_{k}\right\}+\mathcal{K}_{\lambda 1}\left\{C_{w 5}^{i j k l} \Phi_{j}\right\} \\
& \quad+C_{w 6}^{i j} \ddot{W}_{i}+\mathcal{E} C_{w 7}^{i j} \ddot{\Phi}_{i}+\ddot{\Theta}\left\{C_{w 8}^{i j} V_{j}+\mathcal{E}_{p} C_{w 9}^{i}+\mathcal{E} C_{w 9}^{i}\right\}-\dot{\Theta}^{2}\left\{\mathcal{E} C_{w 7}^{i j} \Phi_{i}+C_{w 6}^{i j} W_{i}\right\}+2 \dot{\Theta}\left\{C_{w 8}^{i j} \dot{V}_{j}\right\}=\mathcal{W}_{w} .
\end{aligned}
$$

$\delta \phi$

$$
\begin{aligned}
& \left\{-\mathcal{K}_{x}\left(\varphi^{\prime \prime}\right)+\left(\mathcal{K}_{z}-\mathcal{K}_{y}\right)\left(\mathcal{V}^{\prime \prime} \mathcal{W}^{\prime \prime}\right)-\mathcal{E}_{A}^{2}\left(\mathcal{V}^{\prime \prime} \mathcal{W}^{\prime \prime}\right)+\mathcal{K}_{\lambda 1}\left(\varphi^{\prime \prime \prime \prime}\right)+\mathcal{K}_{\lambda 2}\left(\mathcal{W}^{\prime \prime \prime \prime}\right)\right\} \\
& \quad+\left\{\mathcal{M}_{0}(\ddot{\varphi}+\ddot{\vartheta})+\mathcal{E} \ddot{\mathcal{W}}+\ddot{\vartheta}\left(\mathcal{E}\left(\mathcal{V}+\mathcal{E}_{p}\right)\right)-\dot{\vartheta}^{2}(\mathcal{E} \mathcal{W})+2 \mathcal{E} \dot{\vartheta}(\dot{\mathcal{V}})\right\}=\overline{\mathcal{W}}_{\phi}, \\
& -\mathcal{K}_{x}\left\{C_{p 1}^{i j} \Phi_{j}\right\}+\left(\mathcal{K}_{z}-\mathcal{K}_{y}\right)\left\{C_{p 2}^{i j k} V_{j} W_{k}\right\}-\mathcal{E}_{A}^{2}\left\{C_{p 2}^{i j k l} V_{j} W_{k}\right\}+\mathcal{K}_{\lambda 1}\left\{C_{p 3}^{i j} \Omega_{j}\right\}+\mathcal{K}_{\lambda 2}\left\{C_{p 4}^{i j} W_{j}\right\}
\end{aligned}
$$




$$
\begin{aligned}
&+\mathcal{M}_{0}\left\{C_{p 5}^{i j} \ddot{\Phi}+C_{p 6}^{i} \ddot{\Theta}\right\}+\mathcal{E} C_{p 7}^{i j} \ddot{W}_{j}+\ddot{\Theta}\left\{\mathcal{E} C_{p 8}^{i j} V_{j}+\mathcal{E} \mathcal{E}_{p} C_{p 6}^{i}\right\}-\mathcal{E} \dot{\Theta}^{2}\left\{C_{p 7}^{i j} W_{j}\right\}+2 \mathcal{E} \dot{\Theta}\left\{C_{p 8}^{i j} \dot{V}_{j}\right\}=\mathcal{W}_{\phi} . \\
& \delta \\
& \mathcal{M}_{\theta} \ddot{\vartheta}+\mathcal{D}_{\theta} \dot{\vartheta}+\mathcal{K}_{\vartheta} \vartheta+\int_{0}^{1}\left\{\mathcal{M}_{0}(\ddot{\varphi}+\ddot{\vartheta})-\ddot{\mathcal{V}}(\mathcal{E} \varphi+\mathcal{W})+\mathcal{W}\left(\left(\mathcal{V}+\mathcal{E}_{p}\right)+\mathcal{E}\right)+\mathcal{E} \ddot{\varphi}\left(\left(\mathcal{V}+\mathcal{E}_{p}\right)\right)\right. \\
&+2 \mathcal{E} \ddot{\vartheta}\left(\left(\mathcal{V}+\mathcal{E}_{p}\right)+\mathcal{W} \varphi\right)+\ddot{\vartheta}\left(\mathcal{W}^{2}+\left(\mathcal{V}+\mathcal{E}_{p}\right)^{2}\right) \\
&\left.+2 \dot{\vartheta}\left(\dot{\mathcal{V}}\left(\left(\mathcal{V}+\mathcal{E}_{p}\right)+\mathcal{E}\right)+\dot{\mathcal{W}}(\mathcal{E} \varphi+\mathcal{W})+\mathcal{E} \dot{\varphi}(\mathcal{W})\right)\right\} d \mathcal{X}=\overline{\mathcal{W}}_{\theta}, \\
& \mathcal{M}_{\theta} C_{t 1} \ddot{\Theta}+\mathcal{D}_{\theta} C_{t 1} \dot{\Theta}+\mathcal{K}_{\vartheta} C_{t 1} \Theta+\mathcal{M}_{0}\left\{C_{t 2}^{j} \ddot{\Phi}_{j}+C_{t 1} \ddot{\Theta}\right\}-\mathcal{E} C_{t 3}^{j k} \ddot{V}_{j} \Phi_{k}-C_{t 4}^{j k} \ddot{V}_{j} W_{k} \\
&+C_{t 3}^{j k} \ddot{W}_{j} V_{k}+\mathcal{E}_{p} C_{t 5}^{j} \ddot{W}_{j}+\mathcal{E} C_{t 5}^{j} \ddot{W}_{j}+\mathcal{E} C_{t 3}^{j k} \ddot{\Phi}_{j} V_{k}+\mathcal{E} \mathcal{E}_{p} C_{t 2}^{j} \ddot{\Phi}_{j} \\
&+\ddot{\Theta}\left\{2 \mathcal{E} C_{t 6}^{j} V_{j}+2 \mathcal{E} \mathcal{E}_{p} C_{t 1}+2 \mathcal{E} C_{t 7}^{j k} W_{j} \Phi_{k}+C_{t 8}^{j k} W_{j} W_{k}+C_{t 9}^{j k} V_{j} V_{k}+2 \mathcal{E}_{p} C_{t 6}^{j} V_{j}+\mathcal{E}_{p}^{2} C_{t 1}\right\} \\
&+2 \dot{\Theta}\left\{C_{t 9}^{j k} V_{k} \dot{V}_{j}+\mathcal{E}_{p} C_{t 6}^{j} \dot{V}_{j}+\mathcal{E} C_{t 6}^{j} \dot{V}_{j}+\mathcal{E} C_{t 7}^{j k} \dot{W}_{j} \Phi_{k}+C_{t 8}^{j k} \dot{W}_{j} W_{k}+\mathcal{E} C_{t 7}^{j k} W_{j} \dot{\Phi}_{k}\right\}=\mathcal{W}_{\theta} .
\end{aligned}
$$

These equations are arranged into an efficient loop structure which can be implemented into numerical integration schemes for ordinary differential equations to simulate the motion. The loop structure is provided in Appendix B.4

It is important to note that the coefficients in Appendix B.2 are shown for the case of the current study which considers the vibrations of a rectangular wing, characterized by constant spanwise cross-sectional properties. Should it be necessary to model a wing which may have spanwise varying cross-sectional properties, the shape functions defining the spanwise variation of the cross-sectional properties must be included in the integral expressions of these coefficients. This is demonstrated below in Example 3.2, revisiting the coupled bending and torsional vibrations of a linear cantilever beam from Example 3.1.

\section{Example 3.2}

Reconsider the system of linear partial differential equations for the free vibration of the coupled bending and torsion of an Euler-Bernoulli beam from Example 3.1. where now the cross-sectional properties, $I, J, m, e$ and $I_{0}$ are given by generic spanwise varying functions $I(x), J(x), m(x), e(x)$ and $I_{0}(x)$. 
One must note that while it is possible to adapt the equations of motion so as to account for such variation in the cross-sectional properties, it is not as simple as subtituting the constant cross-sectional properties by the corresponding functions of $x$ in the final form of the equations as in:

$$
\begin{aligned}
& 0=E I(x) w^{\prime \prime \prime \prime}(x, t)+m(x) \ddot{w}(x, t)+m(x) e(x) \ddot{\phi}(x, t), \\
& 0=-G J(x) \phi^{\prime \prime}(x, t)+I_{0}(x) \ddot{\phi}(x, t)+m(x) e(x) \ddot{w}(x, t) .
\end{aligned}
$$

Rather it is important to make this substitution earlier in the derivation, such that the spanwise variation of the cross-sectional properties is accounted for when the stress resultants, moments and bimoment $N_{\mathbf{x}}, S_{\mathbf{x}}, T_{\mathbf{x}}, P_{\mathbf{x}}, M_{\mathbf{y}}$, and $M_{\mathbf{z}}$ are differentiated with respect to $x$. Hence, the correct form of the equations with variable cross-sectional properties is given by

$$
\begin{aligned}
& 0=\left(E I(x) w^{\prime \prime}(x, t)\right)^{\prime \prime}+m(x) \ddot{w}(x, t)+m(x) e(x) \ddot{\phi}(x, t), \\
& 0=-\left(G J(x) \phi^{\prime}(x, t)\right)^{\prime}+I_{0}(x) \ddot{\phi}(x, t)+m(x) e(x) \ddot{w}(x, t) .
\end{aligned}
$$

Making the same modal approximations for the states, $w(x, t)$ and $\phi(x, t)$, we again project the set of equations onto the subspace of the solution space formed by the assumed basis functions which yields,

$$
\begin{gathered}
0=\int_{0}^{S}\left(E I^{\prime \prime}(x) \sum_{i=1}^{M} \sum_{j=1}^{M} \Psi_{i}(x) \Psi_{j}^{\prime \prime}(x) W_{j}(t)+2 E I^{\prime}(x) \sum_{i=1}^{M} \sum_{j=1}^{M} \Psi_{i}(x) \Psi_{j}^{\prime \prime \prime}(x) W_{j}(t)+E I(x) \sum_{i=1}^{M} \sum_{j=1}^{M} \Psi_{i}(x) \Psi_{j}^{\prime \prime \prime \prime}(x) W_{j}(t)\right. \\
\left.+m(x) \sum_{i=1}^{M} \sum_{j=1}^{M} \Psi_{i}(x) \Psi_{j}(x) \ddot{W}_{j}(t)+m(x) e(x) \sum_{i=1}^{M} \sum_{k=1}^{N} \Psi_{i}(x) \Omega_{k}(x) \ddot{\Phi}_{k}(t)\right) d x \\
0=\int_{0}^{S}\left(-G J^{\prime}(x) \sum_{i=1}^{N} \sum_{k=1}^{M} \Omega_{i}(x) \Omega_{k}^{\prime}(x) \Phi_{k}(t)-G J(x) \sum_{i=1}^{N} \sum_{k=1}^{M} \Omega_{i}(x) \Omega_{k}^{\prime \prime}(x) \Phi_{k}(t)\right. \\
\left.+I_{0}(x) \sum_{i=1}^{N} \sum_{k=1}^{M} \Omega_{i}(x) \Omega_{k}(x) \ddot{\Phi}_{k}(t)+m(x) e(x) \sum_{i=1}^{N} \sum_{j=1}^{M} \Omega_{i}(x) \Psi_{j}(x) \ddot{W}_{j}(t)\right) d x
\end{gathered}
$$

Where now the variation of the cross-sectional properties form part of the integrand, whereas they were previously factored out in the case where they were constant functions of $x$. If we represent the the cross-sectional properties by their peak value multiplying a normalized shape function:

$$
\begin{array}{ll}
E I(x)=E I \times f_{E I}(x), & m(x)=m \times f_{m}(x), \\
G J(x)=G J \times f_{G J}(x), & e(x)=e \times f_{e}(x), \\
& I_{0}(x)=I_{0} \times f_{I_{0}}(x) .
\end{array}
$$


The equations maintain the same form as in the previous example, and are expressed as,

$$
\begin{aligned}
& 0=E I \sum_{i=1}^{M} \sum_{j=1}^{M}\left(C_{w 1}^{i j}+2 C_{w 2}^{i j}+C_{w 3}^{i j}\right) W_{j}(t)+m \sum_{i=1}^{M} \sum_{j=1}^{M} C_{w 4}^{i j} \ddot{W}_{j}(t)+m e \sum_{i=1}^{M} \sum_{k=1}^{N} C_{w 5}^{i k} \ddot{\Phi}_{k}(t), \\
& 0=-G J \sum_{i=1}^{N} \sum_{k=1}^{M}\left(C_{\phi 1}^{i k}+C_{\phi 2}^{i k}\right) \Phi_{k}(t)+I_{0} \sum_{i=1}^{N} \sum_{k=1}^{M} C_{\phi 3}^{i k} \ddot{\Phi}_{k}(t)+m e \sum_{i=1}^{N} \sum_{j=1}^{M} C_{\phi 4}^{i j} \ddot{W}_{j}(t) .
\end{aligned}
$$

where coefficients are now

$$
\begin{array}{lll}
C_{w 1}^{i j}=\int_{0}^{S} f_{E I}^{\prime \prime}(x) \Psi_{i}(x) \Psi_{j}^{\prime \prime}(x) d x, & C_{\phi 1}^{i k}=\int_{0}^{S} f_{G J}^{\prime}(x) \Omega_{i}(x) \Omega_{j}^{\prime}(x) d x, \\
C_{w 2}^{i j}=\int_{0}^{S} f_{E I}^{\prime}(x) \Psi_{i}(x) \Psi_{j}^{\prime \prime \prime}(x) d x, & C_{\phi 2}^{i k}=\int_{0}^{S} f_{G J}(x) \Omega_{i}(x) \Omega_{j}^{\prime \prime}(x) d x, \\
C_{w 3}^{i j}=\int_{0}^{S} f_{E I}(x) \Psi_{i}(x) \Psi_{j}^{\prime \prime \prime \prime}(x) d x, & C_{\phi 3}^{i k}=\int_{0}^{S} f_{I}(x) \Omega_{i}(x) \Omega_{j}(x) d x, \\
C_{w 4}^{i j}=\int_{0}^{S} f_{m}(x) \Psi_{i}(x) \Psi_{j}(x) d x, & C_{\phi 4}^{i k}=\int_{0}^{S} f_{m}(x) f_{e}(x) \Omega_{i}(x) \Psi_{j}(x) d x, \\
C_{w 5}^{i k}=\int_{0}^{S} f_{m}(x) f_{e}(x) \Psi_{i}(x) \Omega_{j}(x) d x . & &
\end{array}
$$

\subsubsection{Finite Dimensional Representation of the Unsteady Aerodynamic Model}

As with the structural equations of motion, a finite dimensional representation of the aerodynamic loads is also sought. The same approximations of the structural displacements from Eq.(3.1) through Eq. 3.4 are used. As was shown in Chapter 2 using an unsteady aerodynamic model requires that the state be augmented by a new term $z(x, t)$ and accordingly by of an additional differential equation. The unsteady term (nondimensionalized as $\mathcal{Z}$ ) is approximated as in Eq.(3.21), using a combination of both the basis functions in bending and in torsion to form the basis for the unsteady terms as in [50],

$$
\mathcal{Z}(\mathcal{X}, \mathcal{T})=\sum_{i=1}^{M} \Psi_{i}(\mathcal{X}) Z_{i}^{\Psi}(\mathcal{T})+\sum_{i=1}^{N} \Omega_{i}(\mathcal{X}) Z_{i}^{\Omega}(\mathcal{T})
$$


Starting from the nondimensionalized aerodynamic model from Chapter 2 a Galerkin projection scheme is adopted as follows:

$$
\begin{aligned}
& \delta v \\
& \overline{\mathcal{W}}_{v}=\sqrt{1-\mathcal{X}^{2}}\left\{\frac{1}{4} \mathcal{P} \mathcal{C}^{2} \pi\left((\ddot{\mathcal{V}} \sin \vartheta+\ddot{\mathcal{W}} \cos \vartheta)+\mathcal{U}(\dot{\varphi}+\dot{\vartheta})-\left(\mathcal{E}_{c}-\frac{1}{4} \mathcal{C}\right) \ddot{\varphi}-\left(\left(\mathcal{E}_{c}+\mathcal{E}_{p}\right)-\frac{1}{4} \mathcal{C}\right) \ddot{\vartheta}\right)\right. \\
& \left.+\frac{1}{2} \mathcal{P U C} 2 \pi\left(\left(1-A_{1}-A_{2}\right) \tilde{w}_{3 c / 4}+\left(A_{1} b_{1}+A_{2} b_{2}\right) \dot{\mathcal{Z}}+b_{1} b_{2}\left(A_{1}+A_{2}\right) \mathcal{Z}\right)\right\} \times \sin \vartheta, \\
& =\left\{\frac { 1 } { 4 } \mathcal { P } \mathcal { C } ^ { 2 } \pi \left(\left(C_{\Upsilon \Upsilon}^{i j} \ddot{V}_{j} \sin \Theta+C_{\Upsilon \Psi}^{i j} \ddot{W}_{j} \cos \Theta\right)+\mathcal{U}\left(C_{\Upsilon \Omega}^{i j} \dot{\Phi}_{j}+C_{\Upsilon}^{i} \dot{\Theta}\right)\right.\right. \\
& \left.-\left(\mathcal{E}_{c}-\frac{1}{4} \mathcal{C}\right) C_{\Upsilon \Omega}^{i j} \ddot{\Phi}_{j}-\left(\left(\mathcal{E}_{c}+\mathcal{E}_{p}\right)-\frac{1}{4} \mathcal{C}\right) C_{\Upsilon}^{i} \ddot{\Theta}\right) \\
& +\frac{1}{2} \mathcal{P U C} 2 \pi\left(\left(1-A_{1}-A_{2}\right) \tilde{w}_{3 c / 4}+\left(A_{1} b_{1}+A_{2} b_{2}\right)\left(C_{\Upsilon \Psi}^{i j} \dot{Z}_{j}^{\Psi}+C_{\Upsilon \Omega}^{i j} \dot{Z}_{j}^{\Omega}\right)\right. \\
& \left.\left.+b_{1} b_{2}\left(A_{1}+A_{2}\right)\left(C_{\Upsilon \Psi}^{i j} Z_{j}^{\Psi}+C_{\Upsilon \Omega}^{i j} Z_{j}^{\Omega}\right)\right)\right\} \times \sin \Theta
\end{aligned}
$$

$\delta w$

$$
\begin{aligned}
& \overline{\mathcal{W}}_{w}=\sqrt{1-\mathcal{X}^{2}}\left\{\frac{1}{4} \mathcal{P} \mathcal{C}^{2} \pi\left((\ddot{\mathcal{V}} \sin \vartheta+\ddot{\mathcal{W}} \cos \vartheta)+\mathcal{U}(\dot{\varphi}+\dot{\vartheta})-\left(\mathcal{E}_{c}-\frac{1}{4} \mathcal{C}\right) \ddot{\varphi}-\left(\left(\mathcal{E}_{c}+\mathcal{E}_{p}\right)-\frac{1}{4} \mathcal{C}\right) \ddot{\vartheta}\right)\right. \\
& \left.+\frac{1}{2} \mathcal{P U C} 2 \pi\left(\left(1-A_{1}-A_{2}\right) \tilde{w}_{3 c / 4}+\left(A_{1} b_{1}+A_{2} b_{2}\right) \dot{\mathcal{Z}}+b_{1} b_{2}\left(A_{1}+A_{2}\right) \mathcal{Z}\right)\right\} \times \cos \vartheta, \\
& =\left\{\frac { 1 } { 4 } \mathcal { P } \mathcal { C } ^ { 2 } \pi \left(\left(C_{\Psi \Upsilon}^{i j} \ddot{V}_{j} \sin \Theta+C_{\Psi \Psi}^{i j} \ddot{W}_{j} \cos \Theta\right)+\mathcal{U}\left(C_{\Psi \Omega}^{i j} \dot{\Phi}_{j}+C_{\Psi}^{i} \dot{\Theta}\right)\right.\right. \\
& \left.-\left(\mathcal{E}_{c}-\frac{1}{4} \mathcal{C}\right) C_{\Psi \Omega}^{i j} \ddot{\Phi}_{j}-\left(\left(\mathcal{E}_{c}+\mathcal{E}_{p}\right)-\frac{1}{4} \mathcal{C}\right) C_{\Psi}^{i} \ddot{\Theta}\right) \\
& +\frac{1}{2} \mathcal{P} \mathcal{U C} 2 \pi\left(\left(1-A_{1}-A_{2}\right) \tilde{w}_{3 c / 4}+\left(A_{1} b_{1}+A_{2} b_{2}\right)\left(C_{\Psi \Psi}^{i j} \dot{Z}_{j}^{\Psi}+C_{\Psi \Omega}^{i j} \dot{Z}_{j}^{\Omega}\right)\right. \\
& \left.\left.+b_{1} b_{2}\left(A_{1}+A_{2}\right)\left(C_{\Psi \Psi}^{i j} Z_{j}^{\Psi}+C_{\Psi \Omega}^{i j} Z_{j}^{\Omega}\right)\right)\right\} \times \cos \Theta
\end{aligned}
$$

$\delta \phi$

$$
\overline{\mathcal{W}}_{\varphi}=\sqrt{1-\mathcal{X}^{2}}\left\{\frac { 1 } { 4 } \mathcal { P } \mathcal { C } ^ { 2 } \pi \left(\left(\mathcal{E}_{c}-\frac{1}{4} \mathcal{C}\right)(\ddot{\mathcal{V}} \sin \vartheta+\ddot{\mathcal{W}} \cos \vartheta)-\mathcal{U}\left(\frac{1}{2} \mathcal{C}-\mathcal{E}_{c}\right) \dot{\varphi}-\mathcal{U}\left(\frac{1}{2} \mathcal{C}-\left(\mathcal{E}_{c}+\mathcal{E}_{p}\right)\right) \dot{\vartheta}\right.\right.
$$




$$
\begin{gathered}
\left.-\left(\left(\mathcal{E}_{c}-\frac{1}{4} \mathcal{C}\right)^{2}+\frac{1}{32} \mathcal{C}^{2}\right) \ddot{\varphi}-\left(\left(\left(\mathcal{E}_{c}+\mathcal{E}_{p}\right)-\frac{1}{4} \mathcal{C}\right)\left(\mathcal{E}_{c}-\frac{1}{4} \mathcal{C}\right)+\frac{1}{32} \mathcal{C}^{2}\right) \ddot{\vartheta}\right) \\
\left.+\frac{1}{2} \mathcal{P} \mathcal{U} \mathcal{C} 2 \pi \mathcal{E}_{c}\left(\left(1-A_{1}-A_{2}\right) \tilde{w}_{3 c / 4}+\left(A_{1} b_{1}+A_{2} b_{2}\right) \dot{\mathcal{Z}}+b_{1} b_{2}\left(A_{1}+A_{2}\right) \mathcal{Z}\right)\right\} \\
=\left\{\frac { 1 } { 4 } \mathcal { P } \mathcal { C } ^ { 2 } \pi \left(\left(\mathcal{E}_{c}-\frac{1}{4} \mathcal{C}\right)\left(C_{\Omega \Upsilon}^{i j} \ddot{V}_{j} \sin \Theta+C_{\Omega \Psi}^{i j} \ddot{W}_{j} \cos \Theta\right)-\mathcal{U}\left(\frac{1}{2} \mathcal{C}-\mathcal{E}_{c}\right) C_{\Omega \Omega}^{i j} \dot{\Phi}_{j}-\mathcal{U}\left(\frac{1}{2} \mathcal{C}-\left(\mathcal{E}_{c}+\mathcal{E}_{p}\right)\right) C_{\Omega}^{i} \dot{\Theta}\right.\right. \\
\left.-\left(\left(\mathcal{E}_{c}-\frac{1}{4} \mathcal{C}\right)^{2}+\frac{1}{32} \mathcal{C}^{2}\right) C_{\Omega \Omega}^{i j} \ddot{\Phi}_{j}-\left(\left(\left(\mathcal{E}_{c}+\mathcal{E}_{p}\right)-\frac{1}{4} \mathcal{C}\right)\left(\mathcal{E}_{c}-\frac{1}{4} \mathcal{C}\right)+\frac{1}{32} \mathcal{C}^{2}\right) C_{\Omega}^{i} \ddot{\Theta}\right) \\
+\frac{1}{2} \mathcal{P U C} 2 \pi \mathcal{E}_{c}\left(\left(1-A_{1}-A_{2}\right) \tilde{w}_{3 c / 4}+\left(A_{1} b_{1}+A_{2} b_{2}\right)\left(C_{\Omega \Psi}^{i j} \dot{Z}_{j}^{\Psi}+C_{\Omega \Omega}^{i j} \dot{Z}_{j}^{\Omega}\right)\right. \\
\left.\left.+b_{1} b_{2}\left(A_{1}+A_{2}\right)\left(C_{\Omega \Psi}^{i j} Z_{j}^{\Psi}+C_{\Omega \Omega}^{i j} Z_{j}^{\Omega}\right)\right)\right\} .
\end{gathered}
$$

$$
\begin{aligned}
& \delta \theta \\
& \overline{\mathcal{W}}_{\vartheta}=\int_{0}^{S} \sqrt{1-\mathcal{X}^{2}}\left\{\frac { 1 } { 4 } \mathcal { P } \mathcal { C } ^ { 2 } \pi \left(\left(\left(\mathcal{E}_{c}+\mathcal{E}_{p}\right)-\frac{1}{4} \mathcal{C}\right)(\ddot{\mathcal{V}} \sin \vartheta+\ddot{\mathcal{W}} \cos \vartheta)-\mathcal{U}\left(\frac{1}{2} \mathcal{C}-\mathcal{E}_{c}\right) \dot{\varphi}-\mathcal{U}\left(\frac{1}{2} \mathcal{C}-\left(\mathcal{E}_{c}+\mathcal{E}_{p}\right)\right) \dot{\vartheta}\right.\right. \\
& \left.-\left(\left(\left(\mathcal{E}_{c}+\mathcal{E}_{p}\right)-\frac{1}{4} \mathcal{C}\right)\left(\mathcal{E}_{c}-\frac{1}{4} \mathcal{C}\right)+\frac{1}{32} \mathcal{C}^{2}\right) \ddot{\varphi}-\left(\left(\left(\mathcal{E}_{c}+\mathcal{E}_{p}\right)-\frac{1}{4} \mathcal{C}\right)^{2}+\frac{1}{32} \mathcal{C}^{2}\right) \ddot{\vartheta}\right) \\
& \left.+\frac{1}{2} \mathcal{P U C} 2 \pi\left(\mathcal{E}_{c}+\mathcal{E}_{p}\right)\left(\left(1-A_{1}-A_{2}\right) \tilde{w}_{3 c / 4}+\left(A_{1} b_{1}+A_{2} b_{2}\right) \dot{\mathcal{Z}}+b_{1} b_{2}\left(A_{1}+A_{2}\right) \mathcal{Z}\right)\right\} d x, \\
& =\left\{\frac { 1 } { 4 } \mathcal { P } \mathcal { C } ^ { 2 } \pi \left(\left(\left(\mathcal{E}_{c}+\mathcal{E}_{p}\right)-\frac{1}{4} \mathcal{C}\right)\left(C_{\Upsilon}^{j} \ddot{V}_{j} \sin \Theta+C_{\Psi}^{j} \ddot{W}_{j} \cos \Theta\right)-\mathcal{U}\left(\frac{1}{2} \mathcal{C}-\mathcal{E}_{c}\right) C_{\Omega}^{j} \dot{\Phi}_{j}-\mathcal{U}\left(\frac{1}{2} \mathcal{C}-\left(\mathcal{E}_{c}+\mathcal{E}_{p}\right)\right) C^{i} \dot{\Theta}\right.\right. \\
& \left.-\left(\left(\left(\mathcal{E}_{c}+\mathcal{E}_{p}\right)-\frac{1}{4} \mathcal{C}\right)\left(\mathcal{E}_{c}-\frac{1}{4} \mathcal{C}\right)+\frac{1}{32} \mathcal{C}^{2}\right) C_{\Omega}^{j} \ddot{\Phi}_{j}-\left(\left(\left(\mathcal{E}_{c}+\mathcal{E}_{p}\right)-\frac{1}{4} \mathcal{C}\right)^{2}+\frac{1}{32} \mathcal{C}^{2}\right) C^{i} \ddot{\Theta}\right) \\
& +\frac{1}{2} \mathcal{P U C} 2 \pi\left(\mathcal{E}_{c}+\mathcal{E}_{p}\right)\left(\left(1-A_{1}-A_{2}\right) \tilde{w}_{3 c / 4}+\left(A_{1} b_{1}+A_{2} b_{2}\right)\left(C_{\Psi}^{j} \dot{Z}_{j}^{\Psi}+C_{\Omega}^{j} \dot{Z}_{j}^{\Omega}\right)\right. \\
& \left.\left.+b_{1} b_{2}\left(A_{1}+A_{2}\right)\left(C_{\Psi}^{j} Z_{j}^{\Psi}+C_{Z \Omega}^{j} Z_{j}^{\Omega}\right)\right)\right\} .
\end{aligned}
$$

Because of the way we have elected to approximate the unsteady term $z(x, t)$ in Eq. 3.21 ,,$M+N$ differential equations are introduced. There are $M$ ordinary differential equations with a basis given by $\left\{\Psi_{j}(x)\right\}_{j=1}^{M}$, and the other $N$ ordinary differential equations whose basis is given by $\left\{\Omega_{j}(x)\right\}_{j=1}^{N}$,

$$
\left\{\tilde{w}_{3 c / 4}\right\}_{i=1}^{M}=\left(C_{Z \Psi \Psi}^{i j} \ddot{Z}_{j}^{\Psi}+C_{Z \Psi \Omega}^{i j} \ddot{Z}_{j}^{\Omega}\right)+\left(C_{Z \Psi \Psi}^{i j} \dot{Z}_{j}^{\Psi}+C_{Z \Psi \Omega}^{i j} \dot{Z}_{j}^{\Omega}\right)\left(b_{1}+b_{2}\right)+\left(C_{Z \Psi \Psi}^{i j} Z_{j}^{\Psi}+C_{Z \Psi \Omega}^{i j} Z_{j}^{\Omega}\right)\left(b_{1} b_{2}\right),
$$




$$
\left\{\tilde{w}_{3 c / 4}\right\}_{i=M+1}^{M+N}=\left(C_{Z \Omega \Psi}^{i j} \ddot{Z}_{j}^{\Psi}+C_{Z \Omega \Omega}^{i j} \ddot{Z}_{j}^{\Omega}\right)+\left(C_{Z \Omega \Psi}^{i j} \dot{Z}_{j}^{\Psi}+C_{Z \Omega \Omega}^{i j} \dot{Z}_{j}^{\Omega}\right)\left(b_{1}+b_{2}\right)+\left(C_{Z \Omega \Psi}^{i j} Z_{j}^{\Psi}+C_{Z \Omega \Omega}^{i j} Z_{j}^{\Omega}\right)\left(b_{1} b_{2}\right) .
$$

In Eq. 3.23), the effective downwash, $\tilde{w}_{3 c / 4}$ is given by Eq. 3.32 a), in Eq. 3.23 , it is given by Eq. 3.32 b), for Eq. (3.27), it is given by Eq.(3.32 c), in Eq.(3.29), the form shown in Eq. 3.32 d) is used. In Eq.(3.31), Eq. $3.32 \mathrm{e}$ is used and finally in Eq. (3.30) Eq. $3.32 \mathrm{f}$ ) is used. The sole differences being the set of basis functions upon which the equation is projected, and the lift reduction factor in the first four of the aforementioned 6 differential equations. Consequently, the following equations are obtained,

$$
\begin{aligned}
& \tilde{w}_{3 c / 4}=\mathcal{U}\left(C_{\Psi}^{i} \Theta+C_{\Psi \Omega}^{i j} \Phi_{j}\right)+\left(C_{\Psi \Upsilon}^{i j} \dot{V}_{j} \sin \Theta+C_{\Psi \Psi}^{i j} \dot{W}_{j} \cos \Theta\right) \\
& +\left(\frac{1}{2} \mathcal{C}-\mathcal{E}_{c}\right) C_{\Psi \Omega}^{i j} \dot{\Phi}+\left(\frac{1}{2} \mathcal{C}-\left(\mathcal{E}_{c}+\mathcal{E}_{p}\right)\right) C_{\Psi}^{i} \dot{\Theta} \\
& \tilde{w}_{3 c / 4}=\mathcal{U}\left(C_{\Psi}^{i} \Theta+C_{\Psi \Omega}^{i j} \Phi_{j}\right)+\left(C_{\Psi \Upsilon}^{i j} \dot{V}_{j} \sin \Theta+C_{\Psi \Psi}^{i j} \dot{W}_{j} \cos \Theta\right) \\
& +\left(\frac{1}{2} \mathcal{C}-\mathcal{E}_{c}\right) C_{\Psi \Omega}^{i j} \dot{\Phi}+\left(\frac{1}{2} \mathcal{C}-\left(\mathcal{E}_{c}+\mathcal{E}_{p}\right)\right) C_{\Psi}^{i} \dot{\Theta} \\
& \tilde{w}_{3 c / 4}=\mathcal{U}\left(C_{\Omega}^{i} \Theta+C_{\Omega \Omega}^{i j} \Phi_{j}\right)+\left(C_{\Omega \Upsilon}^{i j} \dot{V}_{j} \sin \Theta+C_{\Omega \Psi}^{i j} \dot{W}_{j} \cos \Theta\right) \\
& +\left(\frac{1}{2} \mathcal{C}-\mathcal{E}_{c}\right) C_{\Omega \Omega}^{i j} \dot{\Phi}+\left(\frac{1}{2} \mathcal{C}-\left(\mathcal{E}_{c}+\mathcal{E}_{p}\right)\right) C_{\Omega}^{i} \dot{\Theta}, \\
& \tilde{w}_{3 c / 4}=\mathcal{U}\left(C^{i} \Theta+C_{\Omega}^{i j} \Phi_{j}\right)+\left(C_{\Upsilon}^{i j} \dot{V}_{j} \sin \Theta+C_{\Psi}^{i j} \dot{W}_{j} \cos \Theta\right) \\
& +\left(\frac{1}{2} \mathcal{C}-\mathcal{E}_{c}\right) C_{\Omega}^{i j} \dot{\Phi}+\left(\frac{1}{2} \mathcal{C}-\left(\mathcal{E}_{c}+\mathcal{E}_{p}\right)\right) C^{i} \dot{\Theta}, \\
& \tilde{w}_{3 c / 4}=\mathcal{U}\left(C_{Z \Psi}^{i} \Theta+C_{Z \Psi \Omega}^{i j} \Phi_{j}\right)+\left(C_{Z \Psi \Upsilon}^{i j} \dot{V}_{j} \sin \Theta+C_{Z \Psi \Psi}^{i j} \dot{W}_{j} \cos \Theta\right) \\
& +\left(\frac{1}{2} \mathcal{C}-\mathcal{E}_{c}\right) C_{Z \Psi \Omega}^{i j} \dot{\Phi}+\left(\frac{1}{2} \mathcal{C}-\left(\mathcal{E}_{c}+\mathcal{E}_{p}\right)\right) C_{Z \Psi}^{i} \dot{\Theta}, \\
& \tilde{w}_{3 c / 4}=\mathcal{U}\left(C_{Z \Omega}^{i} \Theta+C_{Z \Omega \Omega}^{i j} \Phi_{j}\right)+\left(C_{Z \Omega \Upsilon}^{i j} \dot{V}_{j} \sin \Theta+C_{Z \Omega \Psi}^{i j} \dot{W}_{j} \cos \Theta\right) \\
& +\left(\frac{1}{2} \mathcal{C}-\mathcal{E}_{c}\right) C_{Z \Omega \Omega}^{i j} \dot{\Phi}+\left(\frac{1}{2} \mathcal{C}-\left(\mathcal{E}_{c}+\mathcal{E}_{p}\right)\right) C_{Z \Omega}^{i} \dot{\Theta} .
\end{aligned}
$$




\subsubsection{Imposing Initial Conditions}

In order to properly apply the initial conditions and excite all of the modes appropriately, the deflected shape must be projected onto the set of basis functions. From there the unknown temporal coordinates can be obtained for the initial time steps. This is done simply as follows for a generic initial displacement $b\left(x, t_{0}\right)$ on $x \in(0, S)$,

$$
b\left(x, t_{0}\right) \approx \sum_{j=1}^{N} B_{j}\left(t_{0}\right) \beta_{j}(x),
$$

Leveraging the orthonormality property of the basis functions $\beta_{i}(x), i=1, \ldots, N$,

$$
\begin{aligned}
\beta_{i}(x) b\left(x, t_{0}\right) & =\sum_{j=1}^{N} B_{j}\left(t_{0}\right) \beta_{i}(x) \beta_{j}(x), \\
B_{i}\left(t_{0}\right) & =\int_{0}^{S} \beta_{i}(x) b\left(x, t_{0}\right) d x .
\end{aligned}
$$

The first three time increments must be defined in order to prescribe the initial velocity of the system. This is due to the form of the 4 point backward differences used for approximating derivatives with respect to time in Houbolt's method (see Eq. 3.38) for velocity and Eq. 3.39) for acceleration). cFor a sufficiently small timestep, as is used in the current study, one can approximately prescribe initial conditions of zero velocity and a nonzero displacement assigning the same initial displacements to each of the first three increments in time. This circumevents the need to perform an initial solve of the system to properly prescribe the initial conditions.

\subsection{Numerical Integration}

The system of coupled equations necessitates an implicit numerical integration scheme, as there is no convenient way to convert the system of equations from their implicit form into an explicit form. In state space form, a solution is sought out in the form 


$$
\dot{\mathbf{x}}=\mathbf{f}(\mathbf{x}, t) .
$$

However, given the presence of the inertial nonlinearities, this form is not easily achieved. Thus, rather than converting the system of ordinary differential equations into a state space form and solving explicitly, we must solve the following implicit problem,

$$
0=\mathbf{f}(\mathbf{x}, \dot{\mathbf{x}}, t)
$$

Houbolt's method for implicit numerical integration will be adopted in order to solve the set of coupled equations. Houbolt's method is a four point finite difference method, which necessitates the use of a root finding algorithm such as the Newton-Raphson method, 51].

\subsubsection{Houbolt's Method}

For a generic state $x_{j}$, Houbolt's method marches forward in time, solving for the values of the state at the time step $n+1$ (i.e. time $t+\Delta t$ ), in terms of the values of the state at the current and previous increments in time. Note that with the generic state $x_{j} ;(1 \leq j \leq L)$ corresponds to edgewise bending, $(L+1 \leq j \leq L+M)$ corresponds to flapwise bending, $(L+M+1 \leq j \leq L+M+N)$

corresponds to the angle of twist, and $(j=L+M+N+1)$ corresponds to the rigid body pitch rotation. Furthermore, in the case of an aeroelastic simulation with the unsteady aerodynamic model, the $x_{j}$ represents the unsteady term, $z$ for $(L+M+N+2 \leq j \leq L+2 M+2 N+1)$.

For this generic state variable $x_{j}$, we define the first and second time derivatives as [52]:

$$
\dot{x}_{j, n+1}=\frac{\left(11 x_{j, n+1}-18 x_{j, n}+9 x_{j, n-1}-2 x_{j, n-2}\right)}{(6 \Delta t)},
$$




$$
\ddot{x}_{j, n+1}=\frac{\left(2 x_{j, n+1}-5 x_{j, n}+4 x_{j, n-1}-x_{j, n-2}\right)}{(\Delta t)^{2}} .
$$

If we insert these expressions into the discretized ordinary differential equations, where $x_{j, n}, x_{j, n-1}$, $x_{j, n-2}$ are known, we now must solve a system of $(L+M+N+1)$ equations of the form,

$$
0=\mathbf{f}\left(x_{j, n+1}\right)
$$

A Newton-Raphson root finding algorithm will be adopted to solve the root finding problem, where the desitred state vector $\left\{x_{j, n+1}\right\}_{j=1}^{L+2 M+2 N+1}$, which satisfies the above equation. Simply stated, Newton-Raphson is an iterative solver which converges to the solution starting from an initial guess at the solution until some prescribed convergence criteria are satisfied. Since we are taking small time steps $\Delta t$, we will use the current value of the state as the initial guess of the future value of the state (i.e. $x_{j, n+1}(1) \approx x_{j, n}$ ), as it will provide a reasonably good starting point. In a general form, from the initial guess the i.e.gorithm encroaches on the actual solution from the initial guess by 52

$$
x_{n+1}(i+1)=x_{n+1}(i)-\mathbf{J}\left(x_{n+1}(i)\right)^{-1} \mathbf{f}\left(x_{, n+1}(i)\right), \quad i=1, \ldots, I .
$$

where $I$ represents the number of iterations required to satisfy some convergence criteria and will vary for each time step, and $x_{j, n+1}(I+1)$ from the Newton-Raphson algorithm is the value taken as the value which satisfies Eq. (3.40). After which the subscript $n$ is incremented by to $n+1$ and the process repeats over with this value, $x_{j, n}$, now being the initial guess $x_{j, n+1}(1)$.

For the aeroelastic system, $\mathbf{f}\left(x_{j, n+1}(i)\right)$ is simply an $L+2 M+2 N+1$ dimensional column vector, whose elements assume the values of the system of $L+2 M+2 N+1$ equations each evaluated at the current guess at the solution $x_{j, n+1}(i)$, 


$$
\mathbf{f}\left(x_{n+1}(i)\right)=\left\{\begin{array}{c}
\mathbf{f}\left(x_{1, n+1}(i)\right) \\
\vdots \\
\mathbf{f}\left(x_{(L+2 M+2 N+1), n+1}(i)\right)
\end{array}\right\} .
$$

Accordingly, for this $L+2 M+2 N+1$ dimensional system, the derivative term $\mathbf{J}\left(x_{j, n+1}(i)\right)$ is the following $(L+2 M+2 N+1) \times(L+2 M+2 N+1)$ Jacobian matrix, given by

$$
\mathbf{J}\left(x_{n+1}(i)\right)=\left[\begin{array}{ccc}
\frac{\partial \mathbf{f}\left(x_{1, n+1}(i)\right)}{\partial x_{1, n+1}} & \cdots & \frac{\partial \mathbf{f}\left(x_{1, n+1}(i)\right)}{\partial x_{(L+2 M+2 N+1), n+1}} \\
\vdots & \ddots & \vdots \\
\frac{\partial \mathbf{f}\left(x_{(L+2 M+2 N+1), n+1}(i)\right)}{\partial x_{1, n+1}} & \cdots & \frac{\partial \mathbf{f}\left(x_{(L+2 M+2 N+1), n+1}(i)\right)}{\partial x_{(L+2 M+2 N+1), n+1}}
\end{array}\right] .
$$

The Houbolt method and the code used to obtain the Jacobian in the Newton-Raphson algorithm are included in Appendices B.4 and B.5

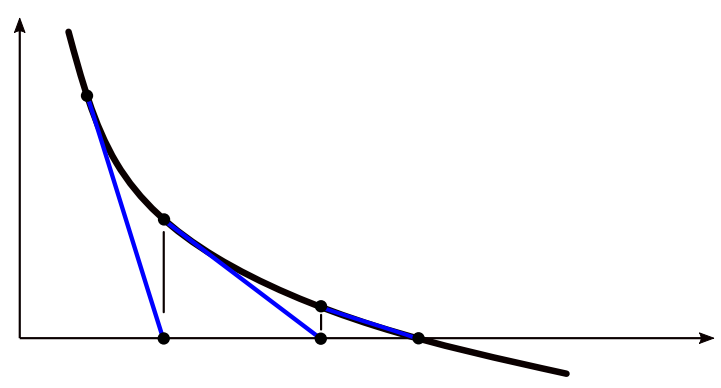

(a) Visualization of Newton-Raphson for a one dimensional problem

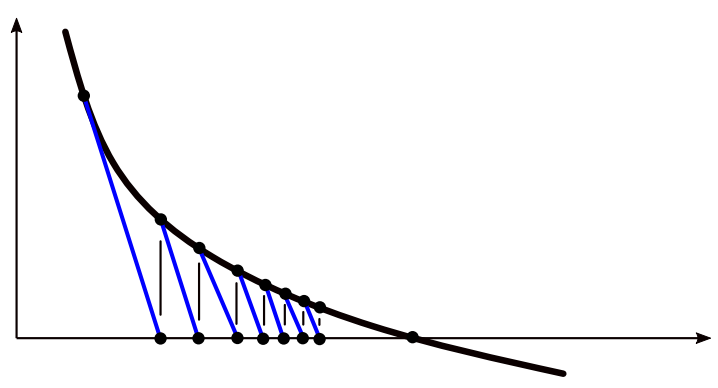

(b) Visualization of Modified NewtonRaphson for a one dimensional problem

Figure 3.2: Panel (a) takes the tangent at every iteration, whereas (b) uses the initial tangent at each step (adapted from [52]). Panel (a) converges in less iterations, but for a nonlinear problem problem, this requires that the derivative (or Jacobian matrix for multidimensional cases) be calculated more times. Meanwhile (b) only requires that the Jacobian be calculated once per time step, but may require more function evaluations to achieve convergence as depicted in the figure)

Note that for a linear system, the Jacobian matrix described above in Eq. (3.43) would be static. 
Given that the system of equations that we are interested in solving is nonlinear, the Jacobian will be a function of the state. In this case, the Jacobian changes with every time step, and even every Newton-Raphson iteration. To reduce the computational demand of re-evaluating and inverting the Jacobian at every iteration of the time integration algorithm, a Modified Newton-Raphson method is proposed 52 . This would see that the Jacobian is only updated after a certain number of iterations have been performed. It was found that for a sufficiently small time step, the number of iterations required to obtain the same level of accuracy was not significant, and were heavily outweighed by the increase in speed due to only periodically updating and inverting the Jacobian. This benefit with regards to computational time is amplified for higher dimensional problems. For a sample computation of the response of the nonlinear aeroelastic system, Figure 3.3 compares the number of iterations for each algorithm to converge based on the same criteria.

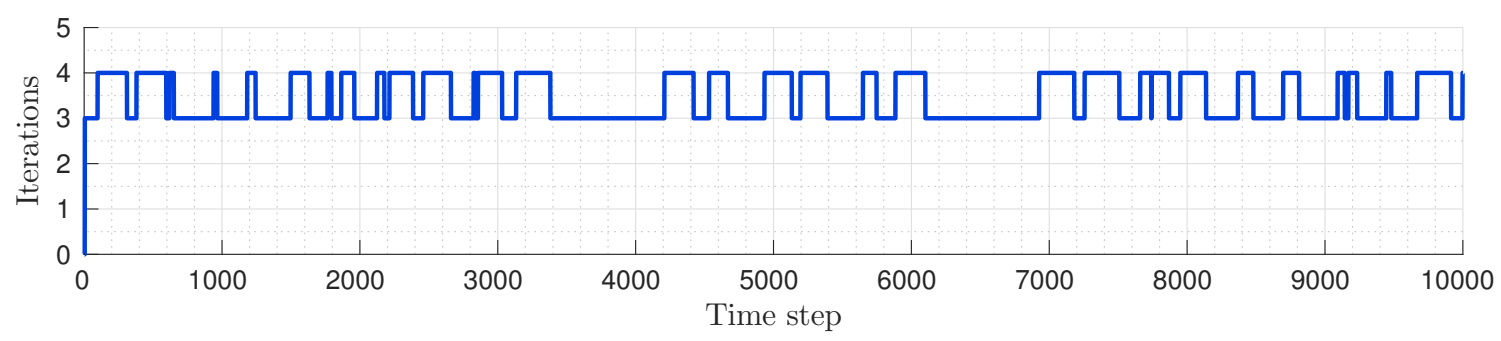

(a) Newton-Raphson

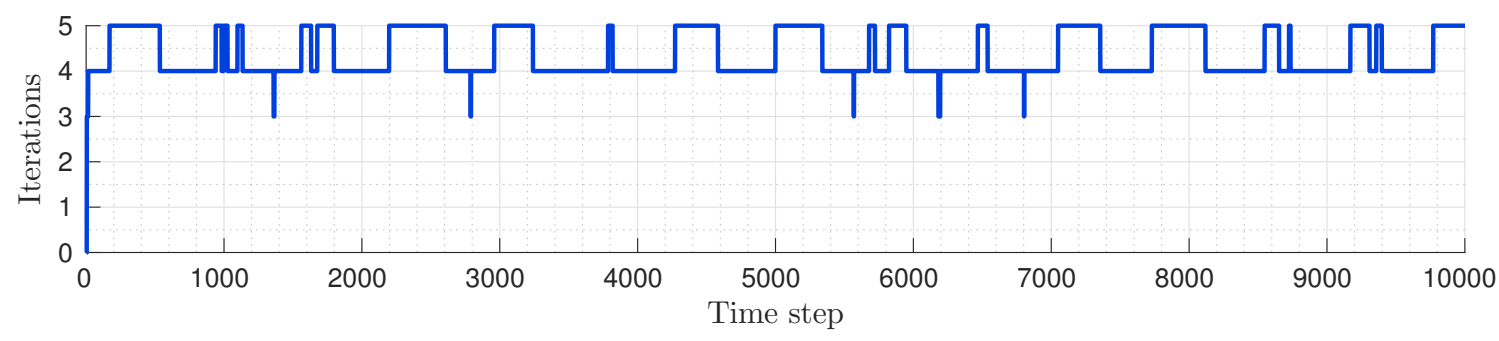

(b) Modified Newton-Raphson

Figure 3.3: Comparison of the iterations required for the Newton-Raphson and the Modified NewtonRaphson algorithms to converge

The convergence criteria is prescribed such that two conditions must be satisfied. The first condition is that from one iteration to the next for the same time step, the percent change of each element of the state vector must be less that 0.1 percent. Figure 3.4 a provides a visualization of this criterion, 


$$
\frac{\left\|x_{j, n+1}(i+1)-x_{j, n+1}(i)\right\|_{2}}{\left\|x_{j, n+1}(i)\right\|_{2}} \leq 10^{-3} .
$$

Given that the system of equations being solved are implicit ordinary differential equations (i.e. of the form $\mathbf{f}(x, \dot{x}, t)=0)$, each element of the vector output of the function evaluated at the current iteration of the state vector (the residual) must also be sufficiently small. In this case, shown in Figure $3.4 \mathrm{~b}$, this imposed criteria is that the residual must be less than $10^{-6}$. The plotted values reflect the calculated percent change and residual after a sufficient number of iterations have been performed such that convergence has been achieved,

$$
\mathbf{f}\left(x_{j, n+1}(i+1)\right) \leq 10^{-6} .
$$

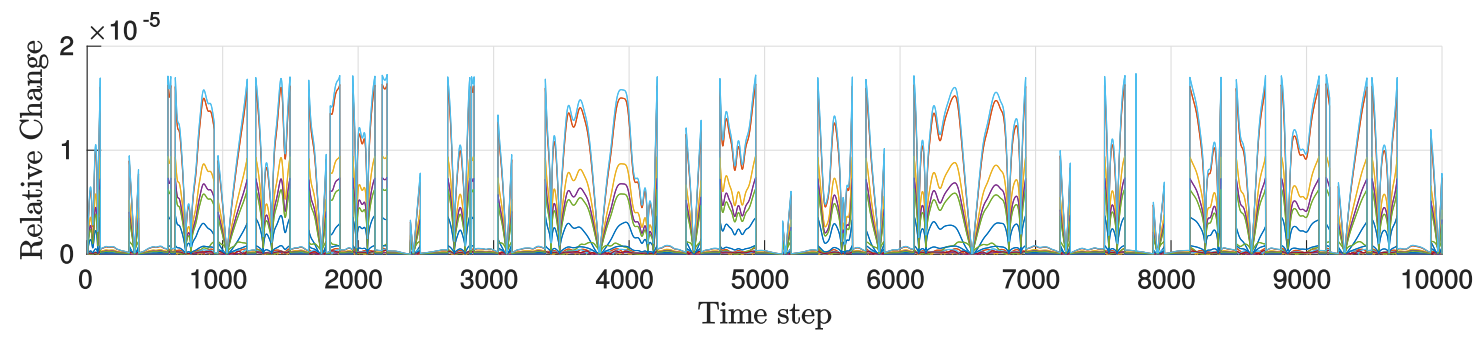

(a) Percent change

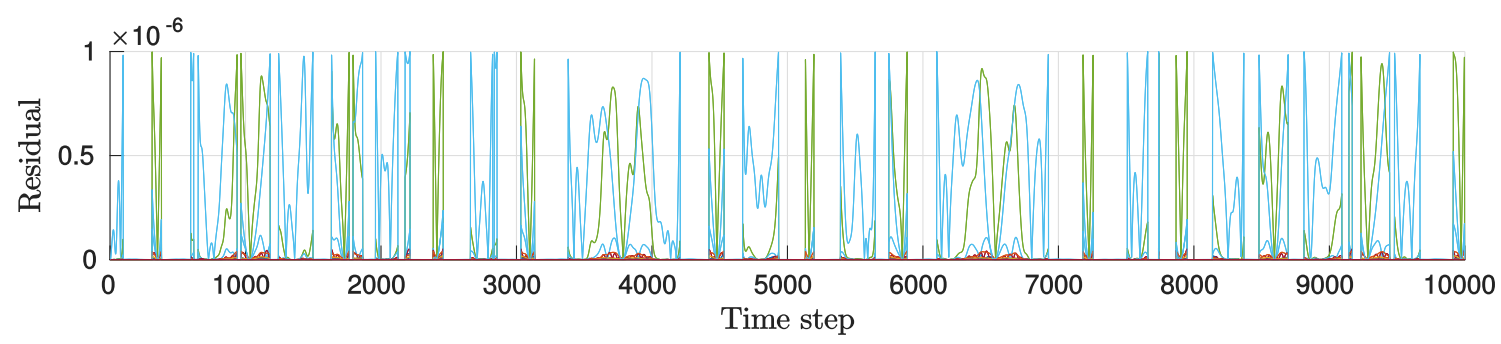

(b) Residual

Figure 3.4: Visualization of the convergence criteria for the Newton-Raphson algorithm. The various lines in the above plots simply show the percent change and the residual of each of each element of the state vector after the convergence criteria has been satisfied. 


\subsection{Numerical Results}

Using the Galerkin method outlined above, the structural equations of motion and the full aeroelastic system are simulated in this section. The results are presented for a number of cases, looking at the time history of the system, as well as looking at phase plane diagrams and the power spectral density (strictly speaking, Fourrier amplitude spectrum) of the response to identify the nonlinear response of the system for various initial conditions. In order to highlight the effect of nonlinearity in the response, the results for the underlying linear system will be presented for various cases to emphasize the importance of incorporating nonlinearity in the structural dynamics. Additionally, other quantities of interest will be presented such as a look at the convergence criteria for the Modified Newton-Raphson algorithm described above, as well as a study of the convergence of the solution with incrementally smaller time steps and increasing number of basis functions.

The flutter speed will be determined for the system, and the system will be studied for free stream air velocities in the proximity of the flutter speed. This will allow for the analysis of the influence of the structural nonlinearities on the post-flutter behaviour of the system.

\begin{tabular}{rlrlll}
\hline \multicolumn{2}{c}{ Stiffness Parameters } & \multicolumn{2}{c}{ Inertial Parameters } & \multicolumn{2}{c}{ Aerodynamic Parameters } \\
\hline \hline$E I_{y}$ & $2.3324 \mathrm{Nm}^{2}$ & $\bar{m}$ & $0.8156 \mathrm{~kg} / \mathrm{m}$ & $\rho$ & $1.225 \mathrm{~kg} / \mathrm{m}^{3}$ \\
$E I_{z}$ & $233.24 \mathrm{Nm}^{2}$ & $\bar{I}_{0}$ & $1.649 \times 10^{-3} \mathrm{kgm}$ & $U$ & $13.89 \mathrm{~m} / \mathrm{s}$ \\
$G J$ & $3.5079 \mathrm{Nm}^{2}$ & $I_{\theta}$ & $1.6 \times 10^{-4} \mathrm{kgm}^{2}$ & $c$ & $0.15 \mathrm{~m}$ \\
$K_{\theta}$ & $0.24 \mathrm{Nm}$ & $D_{\theta}$ & $0.0003 \mathrm{Ns} / \mathrm{m}$ & $S$ & $0.45 \mathrm{~m}$ \\
$E A e_{A}$ & 0 & $e$ & $-0.019 \mathrm{~m}$ & $e_{c}$ & 0 \\
$E C_{1}{ }^{*}$ & 0 & $e_{p}$ & 0 & & \\
$E C_{2}{ }^{*} 0$ & & & & & \\
\hline
\end{tabular}

Table 3.2: Parameter values used in numerical simulations 


\subsubsection{Structural Free Vibration Simulation}

For the purpose of comparing the free vibration results obtained from the nonlinear structural model and the underlying linear model, we consider the case where the wing has a large imposed initial displacement. The results are shown in Figures 3.5 3.6 and 3.7 for initial conditions, $\theta\left(t_{0}\right)=30^{\circ}, \mathcal{W}\left(S, t_{0}\right)=0.1$, while all other initial conditions are equal to zero. Figure 3.5 shows a comparison of the time history of the tip displacements for the first 5 seconds. Figure 3.6 shows the associated phase plane diagram, plotting the tip velocity against the tip displacement between 2.5 seconds and 5 seconds so as to allow the solution to reach a steady state. Figure 3.7 shows the power spectral density of the time trace, revealing the frequency content of the time traces.

In the following figures, the blue lines indicate the nonlinear response, while the green lines show the response of the underlying linear system to the same initial conditions. Clearly, the larger initial displacement induces a stronger nonlinear response as the difference between the two trends is distinct. 


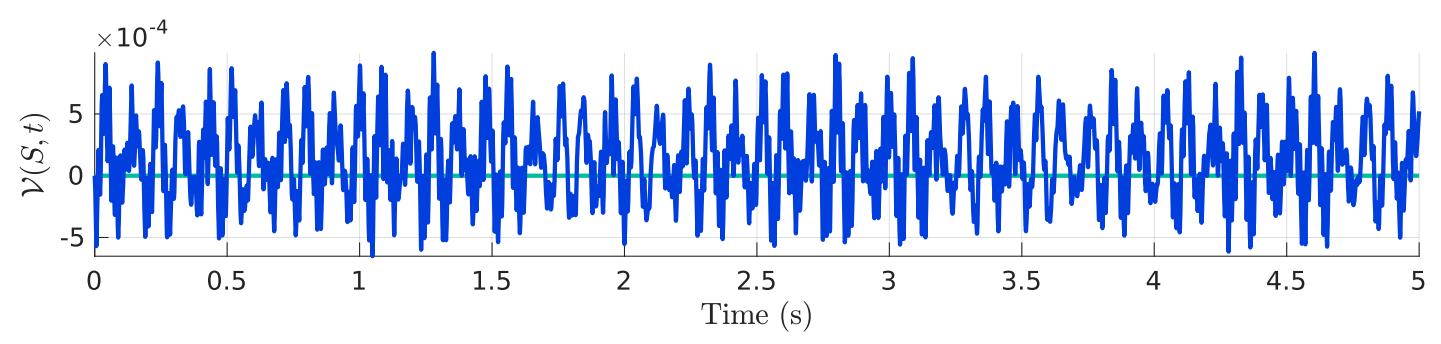

(a) Edgewise bending tip displacement normalized by span length $(\mathrm{m} / \mathrm{m})$

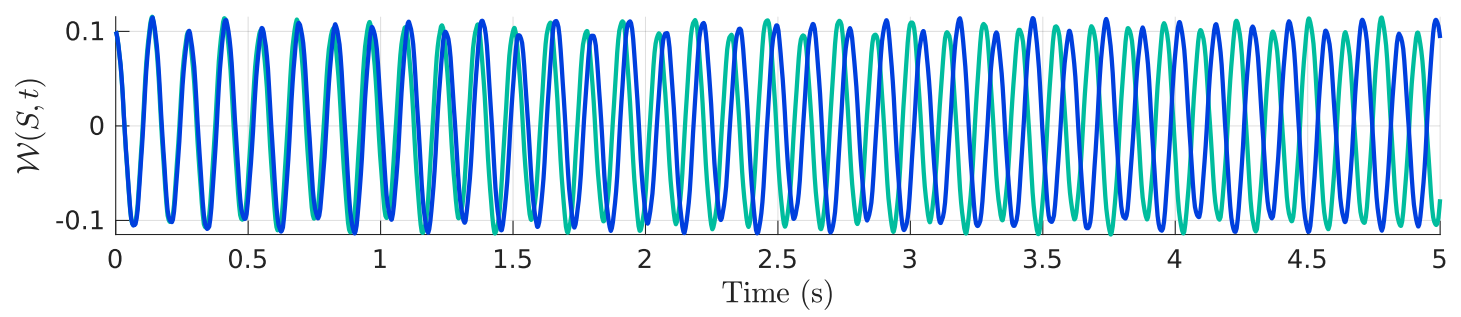

(b) Flapwise bending tip displacement normalized by span length $(\mathrm{m} / \mathrm{m})$

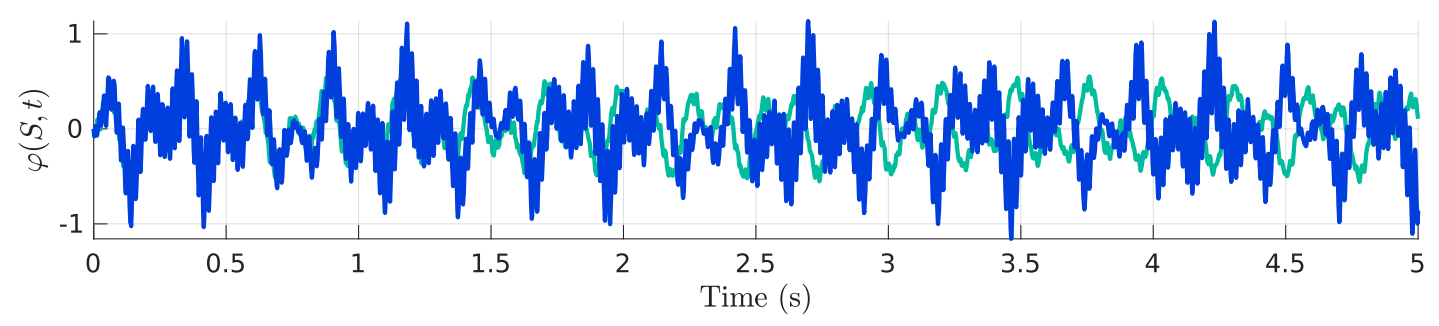

(c) Flexible twist angle tip displacement (in degrees)

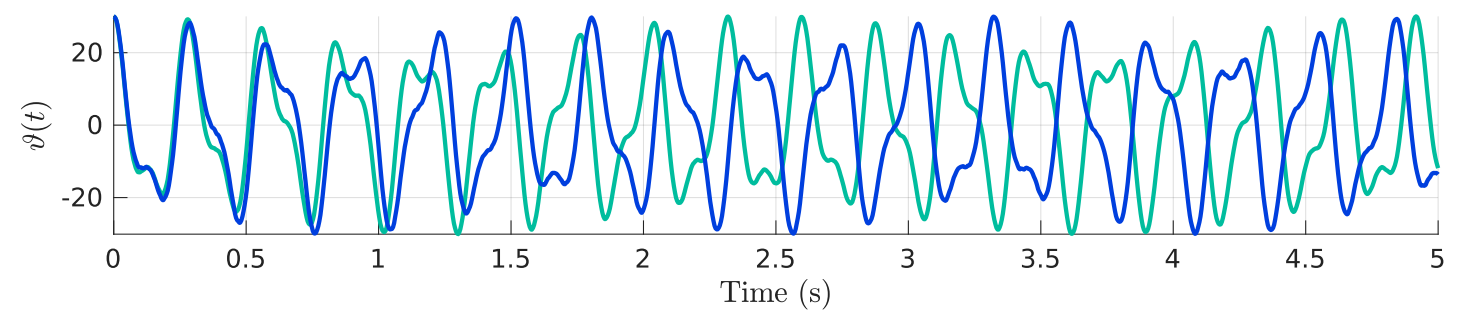

(d) Rigid body pitch rotation (in degrees)

Figure 3.5: Free vibration time trace comparing the linear and nonlinear structural models, with initial conditions of $\theta\left(t_{0}\right)=30^{\circ}, \mathcal{W}\left(S, t_{0}\right)=0.1$, and all other states initially equal to zero.

Linear model $(\square)$, Nonlinear model $(\square)$ 


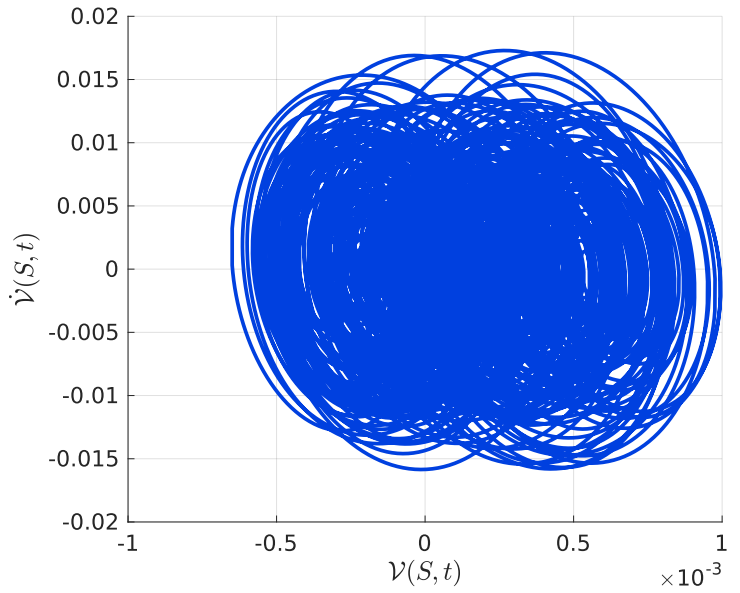

(a) Edgewise bending tip displacement

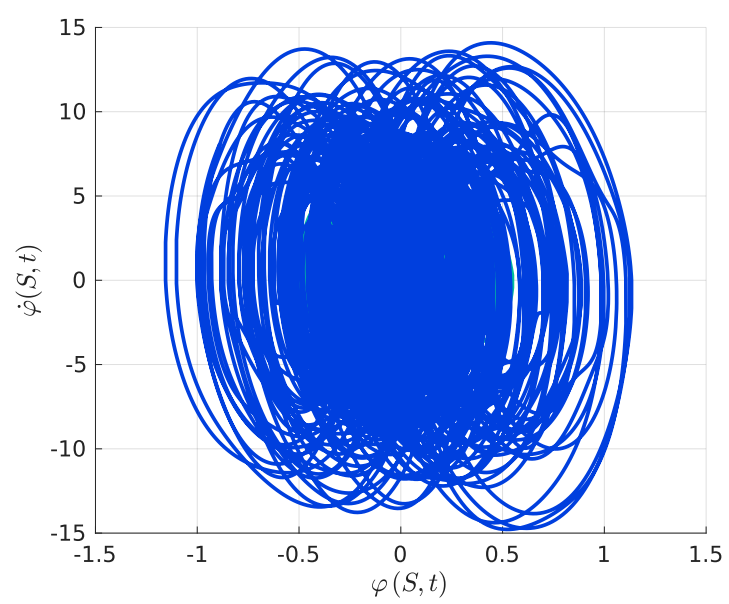

(c) Flexible twist angle tip displacement

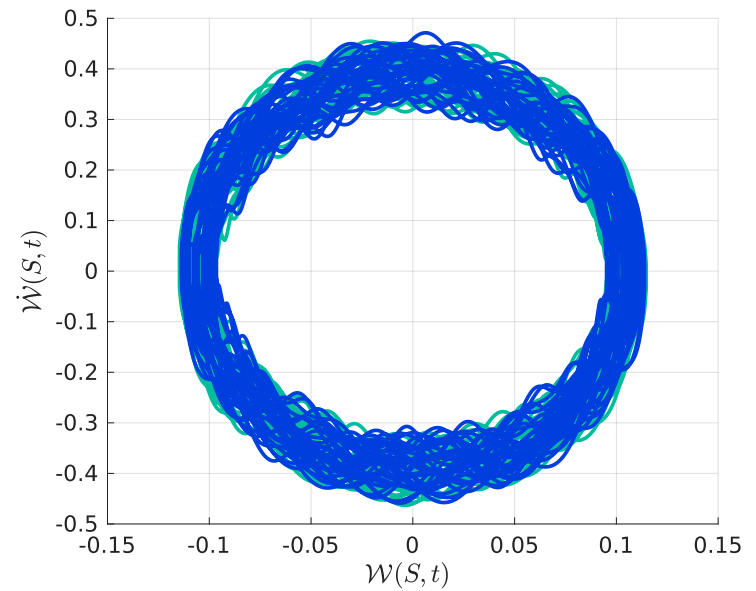

(b) Flapwise bending tip displacement

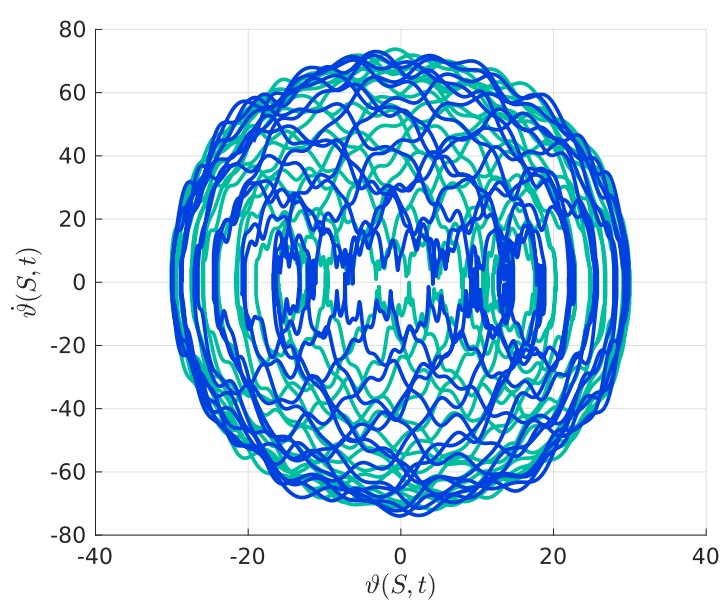

(d) Rigid body base rotation

Figure 3.6: Phase plane diagrams of the tip displacements from Figure 3.5 between $2.5 \mathrm{~s}$ and $5 \mathrm{~s}$

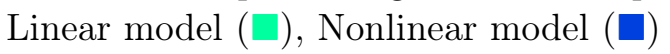




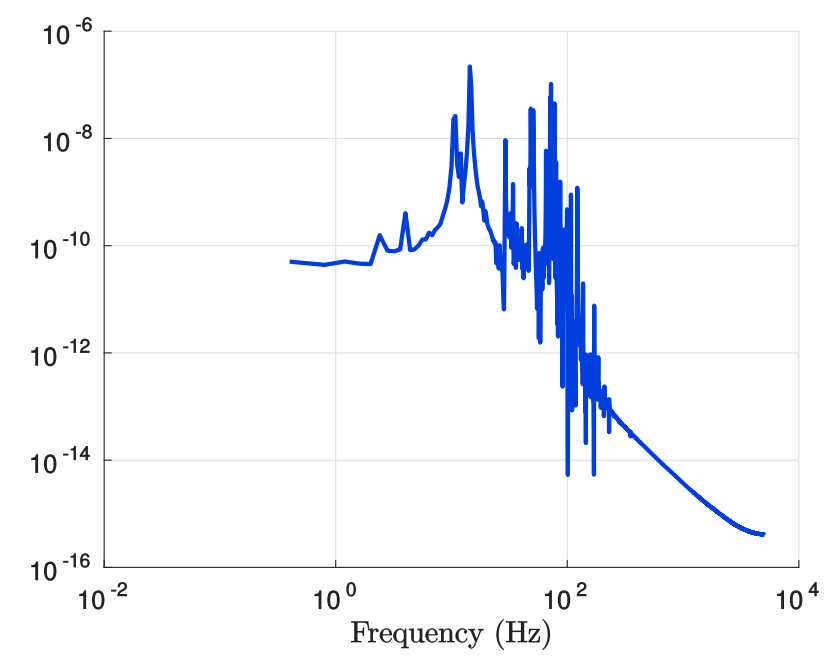

(a) Edgewise bending tip displacement

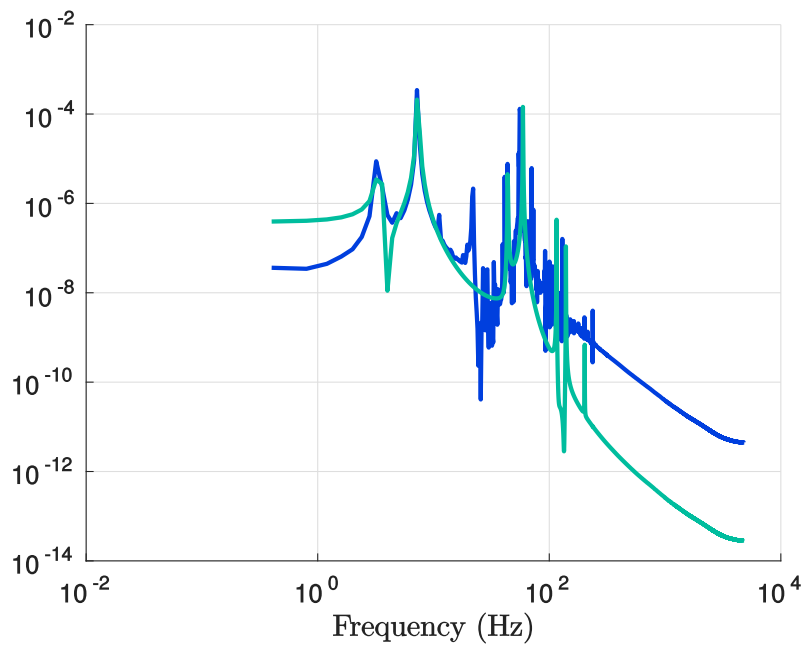

(c) Flexible twist angle tip displacement

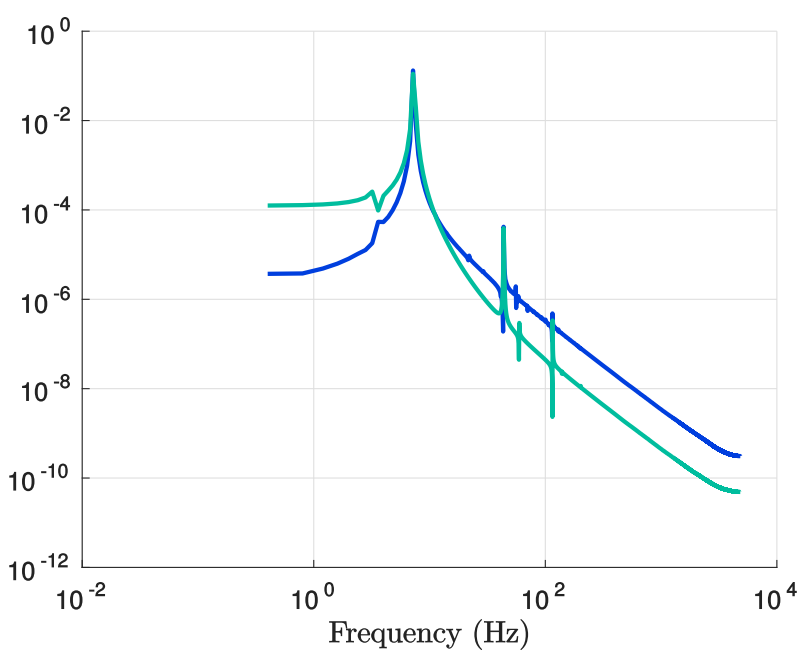

(b) Flapwise bending tip displacement

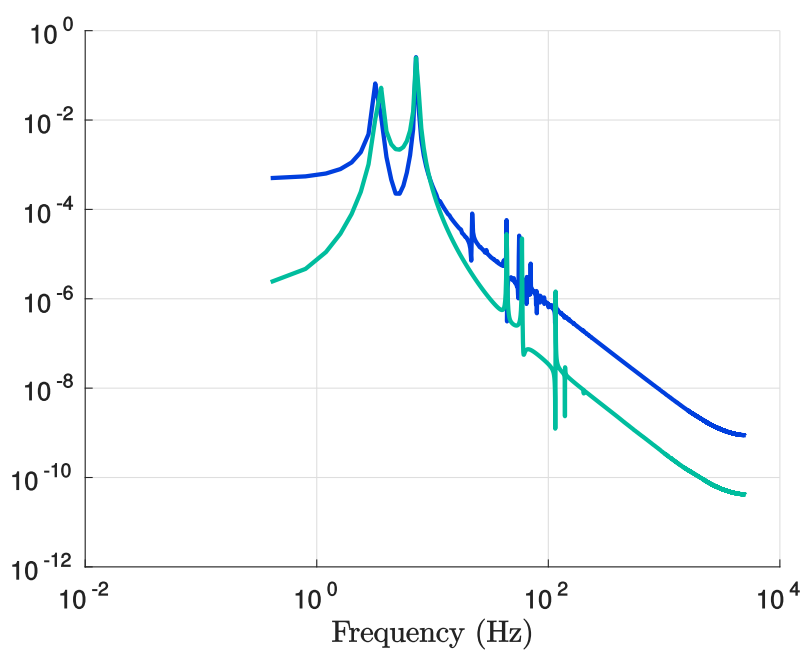

(d) Rigid body base rotation

Figure 3.7: Power spectral density of the tip displacements from Figure 3.5 between 2.5s and 5s

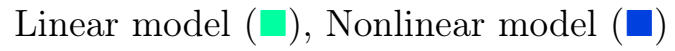




\subsubsection{Full Aeroelastic Simulation}

\section{Eigenvalue analysis}

The structural and aerodynamic loads can be expressed in matricial form as in Eq. 3.46 and Eq. 3.47 as

$$
M_{s} \ddot{\mathbf{x}}+C_{s} \dot{\mathbf{x}}+K_{s} \mathbf{x}+f_{n l}(\mathbf{x}, \dot{\mathbf{x}}, \ddot{\mathbf{x}})=\bar{W} .
$$

The above equation can be written as

$$
M_{a} \ddot{\mathbf{x}}+C_{a} \dot{\mathbf{x}}+K_{a} \mathbf{x}=\bar{W} .
$$

Combining the left hand side of both Eq. 3.46) and Eq.(3.47), an expression of the form of Eq.(3.48) is obtained, where $\left(M-M_{a}\right)$, is the aeroelastic inertia, $\left(C-C_{a}\right)$, is the aeroelastic damping, and $\left(K-K_{a}\right)$, is the aeroelastic stiffness. The structural and aerodynamic mass, damping and stiffness matrices are defined in Appendix B.3.

$$
\left(M_{s}-M_{a}\right) \ddot{\mathbf{x}}+\left(C_{s}-C_{a}\right) \dot{\mathbf{x}}+\left(K_{s}-K_{a}\right) \mathbf{x}+f_{n l}(\mathbf{x}, \dot{\mathbf{x}}, \ddot{\mathbf{x}})=0 .
$$

Though the nonlinear system cannot be conveniently expressed as an explicit set of coupled differential equations, in the absence of the nonlinear terms, we obtain a system of coupled linear differential equations which can be expressed in an explicit form as in Eq. 3.36). Furthermore, we can formulate the equations of motion of the underlying linear system into state space form

$$
\dot{\mathbf{x}}=\mathbf{A x}
$$

where 


$$
\left\{\begin{array}{c}
\dot{x} \\
\ddot{x}
\end{array}\right\}=\left[\begin{array}{cc}
0 & 1 \\
-\mathbf{K}_{a e} / \mathbf{M}_{a e} & -\mathbf{C}_{a e} / \mathbf{M}_{a e}
\end{array}\right]\left\{\begin{array}{c}
x \\
\dot{x}
\end{array}\right\},
$$

the eigenvectors and eigenvalues of the state matrix, A, reveal important information about the system. The real part of the eigenvalue provides an indication of the damping in each of the modes of vibration. Similarly, the imaginary part of the eigenvalues indicates the frequency of that mode. Recognizing these properties of the eigenvalues, are key to understanding so-called V-g-f (velocity-damping-frequency) plots. These plots show the real and imaginary parts of the eigenvalues as a function of the airspeed (varying the airspeed will affect the aerodynamic stiffness and damping matrices). These plots allow us to visually identify at what airspeed the system will become unstable, which is characterized by the airspeed at which the damping (real part of the eigenvalue) of one of the modes goes to zero. This is termed the flutter speed. Accordingly, the imaginary part of that eigenvalue at the flutter speed is the flutter frequency [14]. For the current system, the V-g-f plots are presented in Figure 3.8 Figure 3.8a reveals that the first mode in bending becomes unstable at an airspeed of $12.67 \mathrm{~m} / \mathrm{s}$ (as indicated by the dashed line). Note that the slope of the flapwise bending curve at the flutter speed is relatively steep, indicating a moderate flutter instability. Figure 3.8b shows that at the flutter speed, the first bending mode has a frequency of $5.59 \mathrm{~Hz}$, which corresponds to the flutter frequency. Furthermore, this plot reveals to us that the first bending mode and the rigid body pitch mode coalesce in the vicinity of the flutter speed. The coupling between the first bending mode and the rigid body pitch rotation is the anticipated flutter mode based on similar previous experiments. However, the observation that the first bending mode is becoming unstable rather than the rigid body pitch mode is interesting as it is more subtle, and perhaps less easily deduced from experiments alone. 


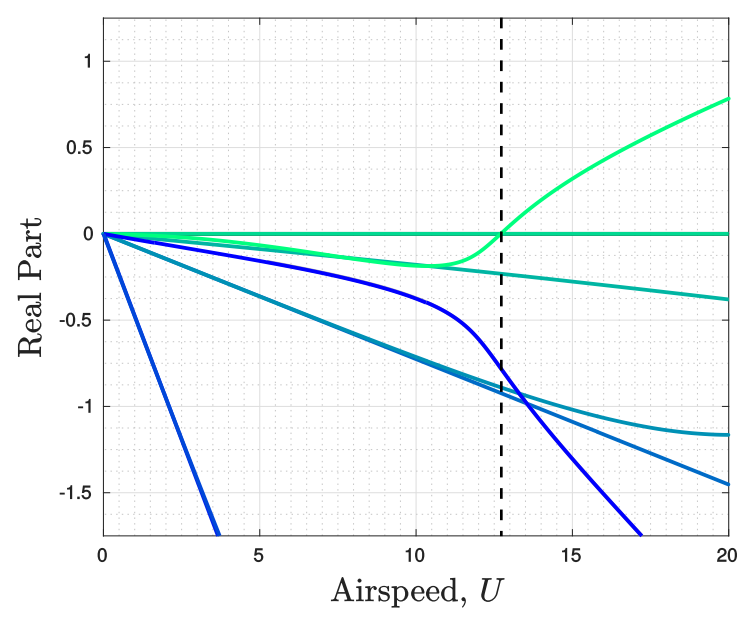

(a) Flutter speed identified as $12.67 \mathrm{~m} / \mathrm{s}$

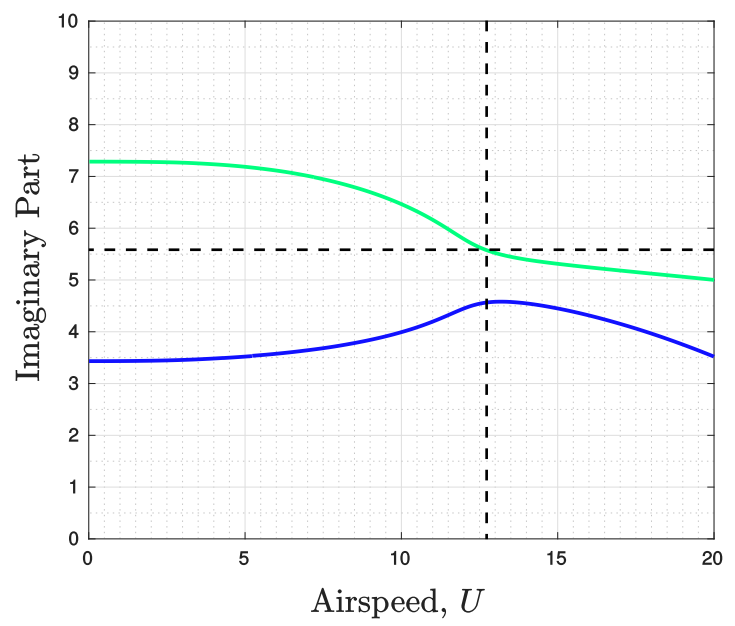

(b) Flutter frequency identified as $5.59 \mathrm{~Hz}$

Figure 3.8: Real and imaginary parts of the eigenvalues of the state matrix as a function of airspeed. First flapwise bending mode $(\square)$, Rigid body pitch rotation

\section{System behaviour near the flutter speed}

Figures 3.9, 3.10, and 3.11, compare the linear and nonlinear structural models behaviour at airspeed of $10 \%$ below the linear flutter speed, at the linear flutter speed, and $10 \%$ above the linear flutter speed. For all the cases, initial deflections of $\theta\left(t_{0}\right)=12^{\circ}, \mathcal{W}\left(S, t_{0}\right)=0.1$ are imposed with all other initial conditions being equal to zero. The purpose of comparing the behaviour of the structural models in the vicinity of the flutter speed is to highlight the importance of the structural nonlinearities, particularly with respect to the post-flutter behaviour of the system.

Immediately before the flutter speed (at $11.40 \mathrm{~m} / \mathrm{s}$ ) in Figure 3.9, it is observed that the system is heavily damped, and the amplitude of the solution quickly decays exponentially to zero. As a direct consequence of the associated small amplitudes, the nonlinear effects are for the most part, negligible, hence the linear and nonlinear structural models return very similar results.

In Figure 3.10 which compares the linear and nonlinear structural models at the flutter speed (12.67 $\mathrm{m} / \mathrm{s})$. As in Figure 3.9, the observed behaviour is very similar in amplitude and frequency. This can again be attributed to the fact that all modes but the first bending mode are still positively 


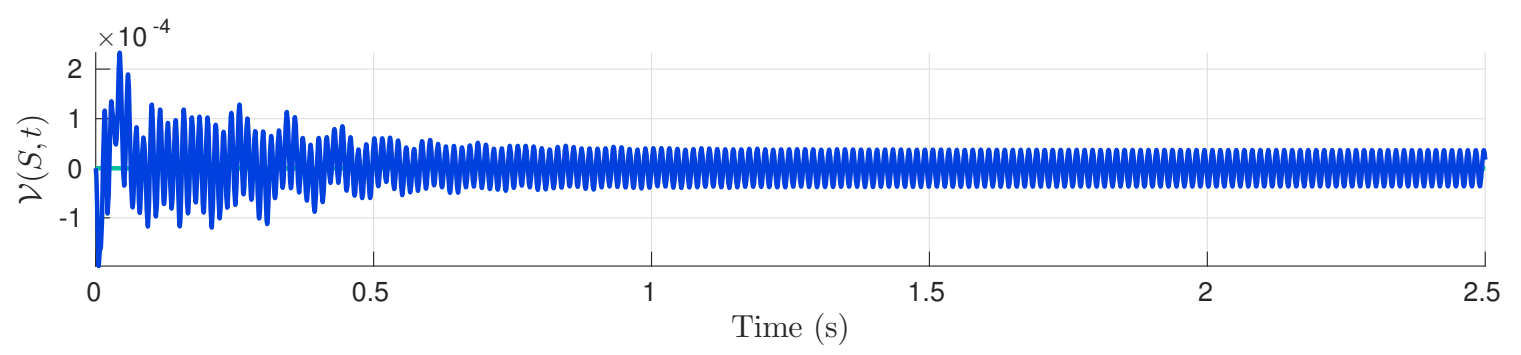

(a) Edgewise bending tip displacement normalized by span length $(\mathrm{m} / \mathrm{m})$

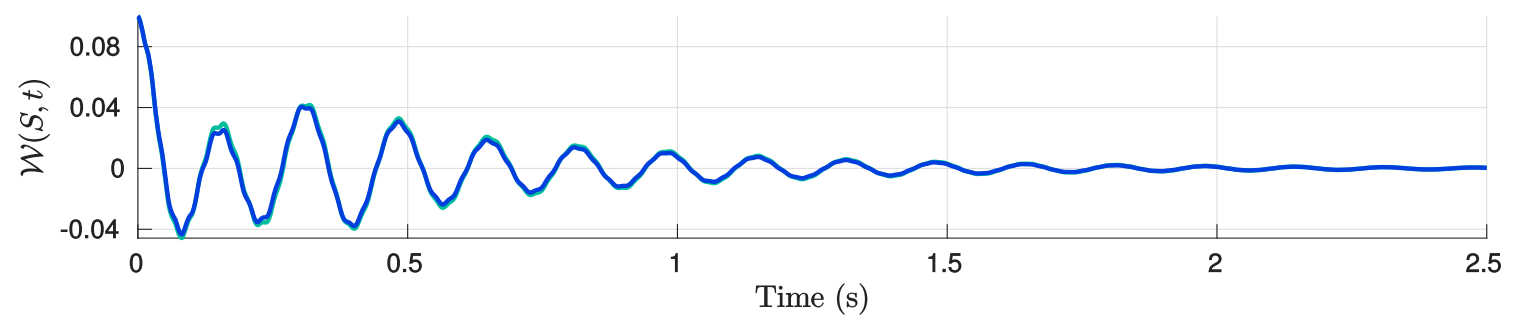

(b) Flapwise bending tip displacement normalized by span length $(\mathrm{m} / \mathrm{m})$

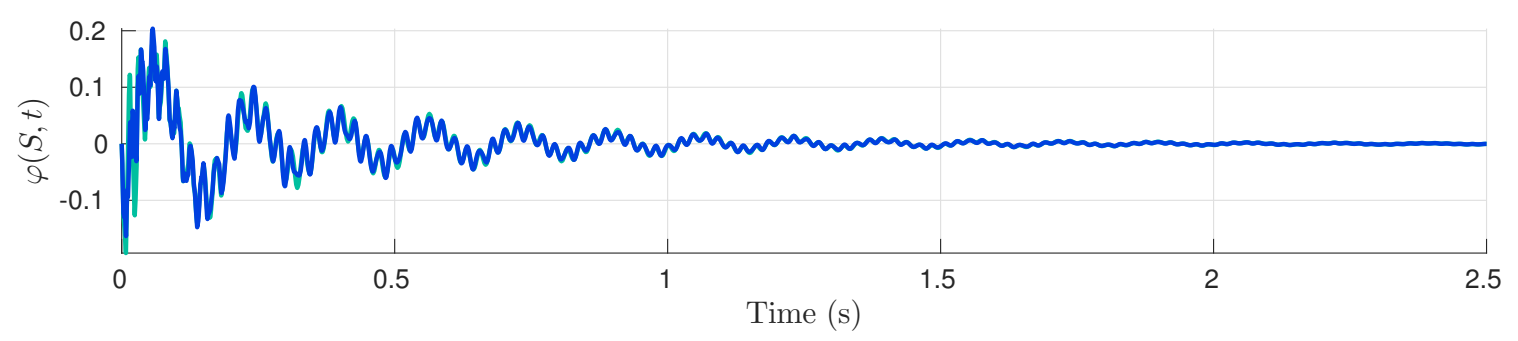

(c) Flexible twist angle tip displacement (in degrees)

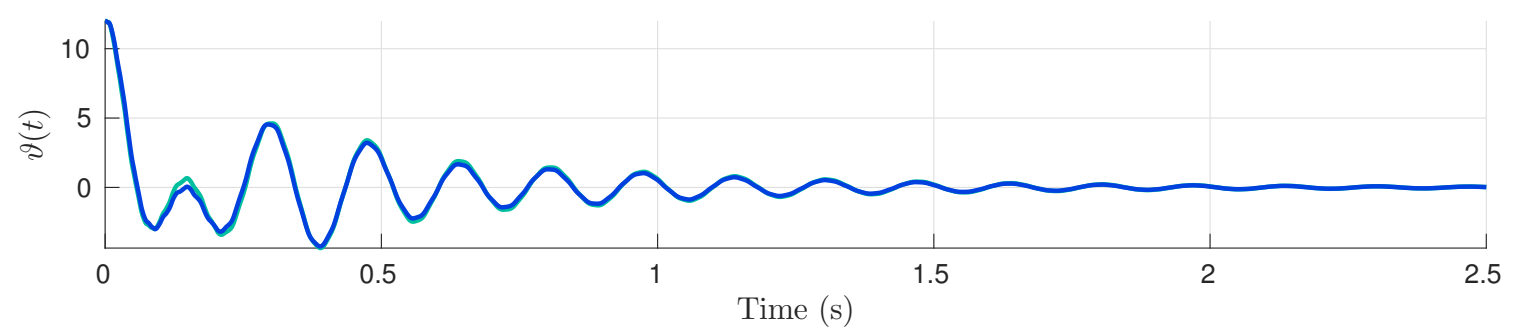

(d) Rigid body pitch rotation (in degrees)

Figure 3.9: Time traces for $10 \%$ below the linear flutter speed

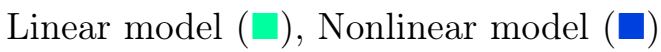


damped, while the first bending mode has zero damping. This is why initially the system decreases in amplitude from the initial conditions before settling into a small amplitude flutter. While the green line is observed to oscillate at a constant amplitude, it can be seen that the amplitudes of the blue curve decrease slightly in time. The structural nonlinearities have delayed the onset of flutter, hence the system undergoes a supercritical bifurcation.

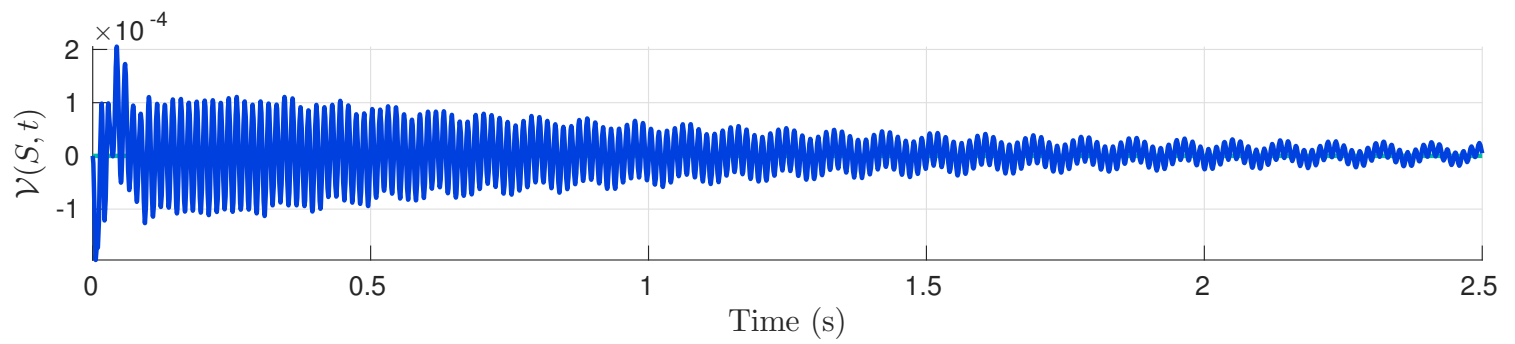

(a) Edgewise bending tip displacement normalized by span length $(\mathrm{m} / \mathrm{m})$

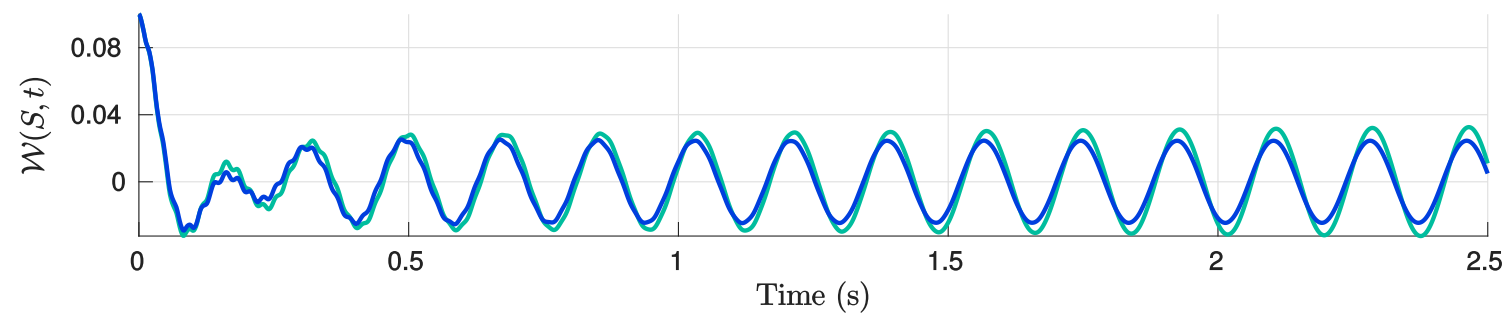

(b) Flapwise bending tip displacement normalized by span length $(\mathrm{m} / \mathrm{m})$

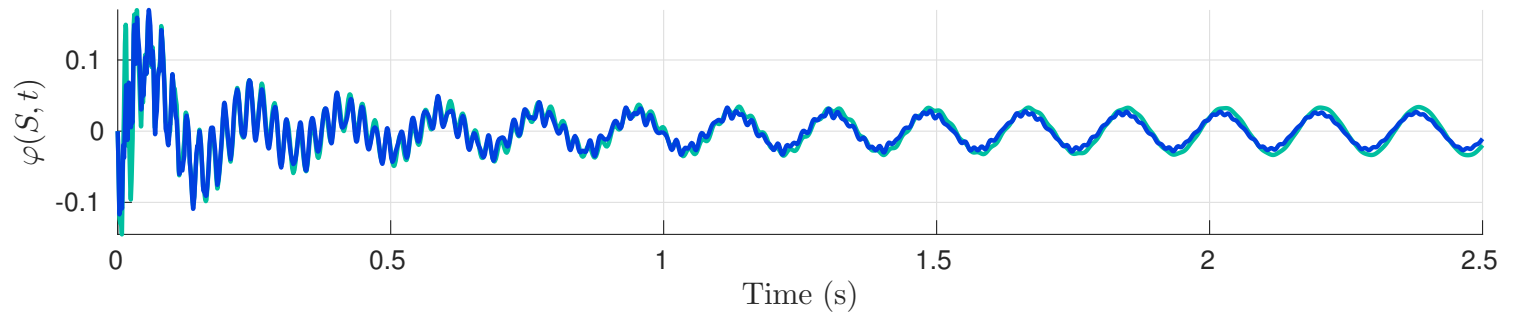

(c) Flexible twist angle tip displacement (in degrees)

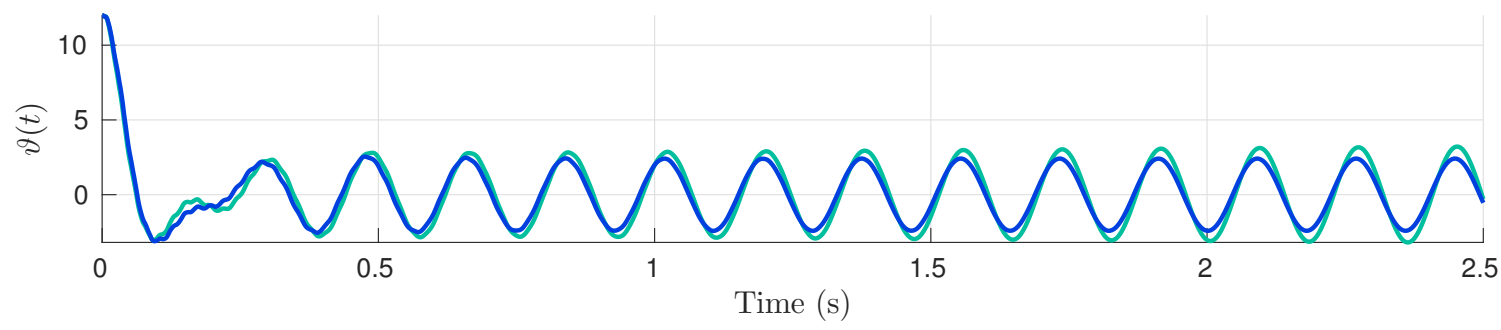

(d) Rigid body pitch rotation (in degrees)

Figure 3.10: Time traces at the linear flutter speed

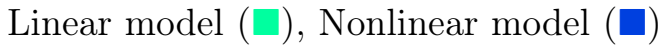


Finally, at $10 \%$ above the flutter speed $(13.94 \mathrm{~m} / \mathrm{s})$ there is a clear distinction between the nonlinear model and the fully linear system. The amplitude of the response of linear system tends to infinity as the overall damping in the system has become negative as evidenced by Figure 3.8a By contrast, the amplitude of the nonlinear model is limited to a limit cycle by the structural nonlinearities.

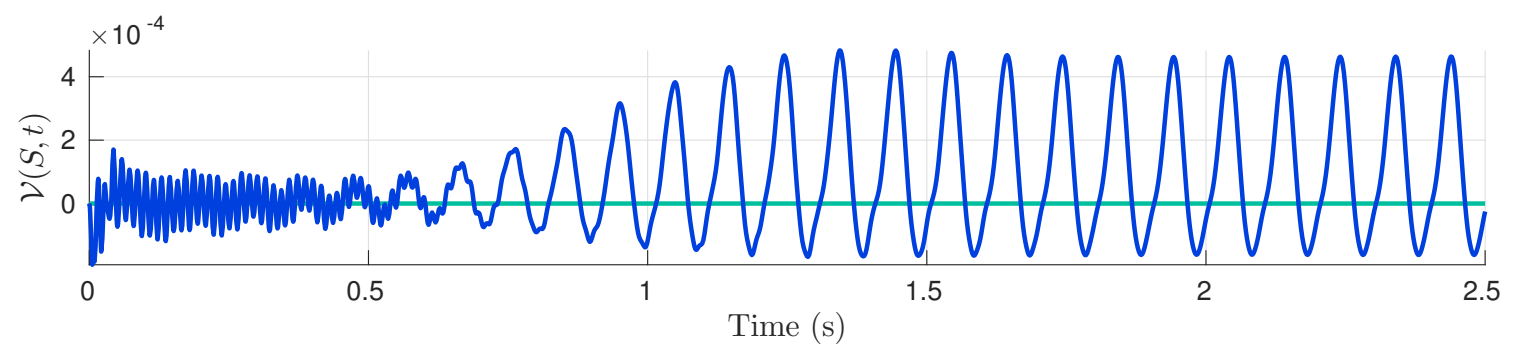

(a) Edgewise bending tip displacement normalized by span length $(\mathrm{m} / \mathrm{m})$

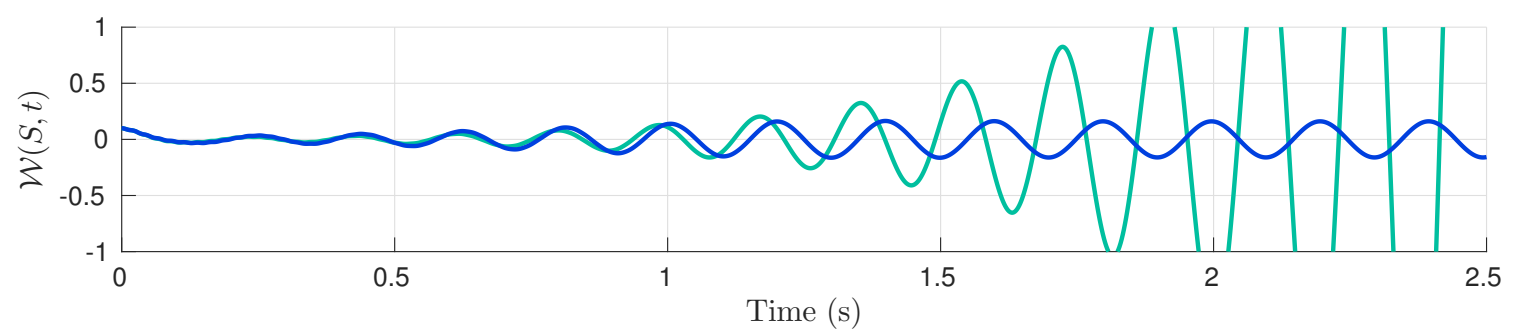

(b) Flapwise bending tip displacement normalized by span length $(\mathrm{m} / \mathrm{m})$

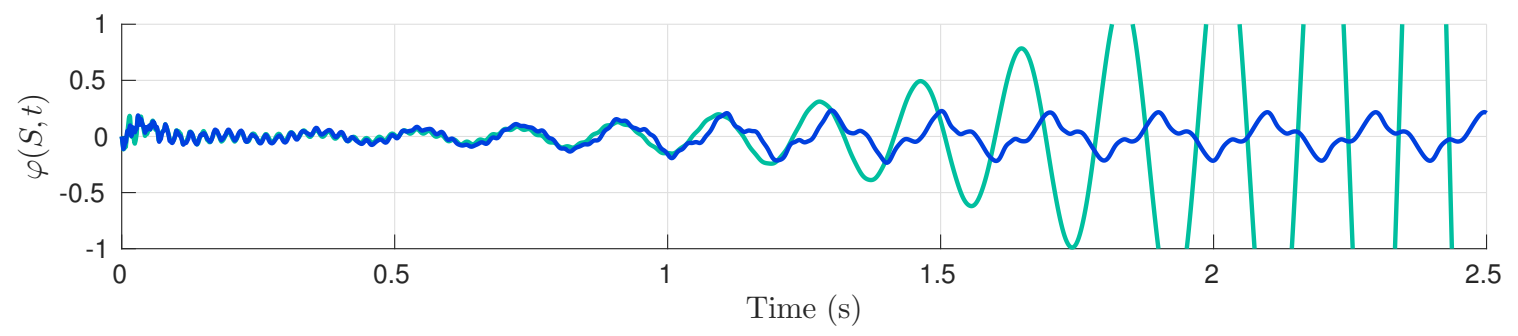

(c) Flexible twist angle tip displacement (in degrees)

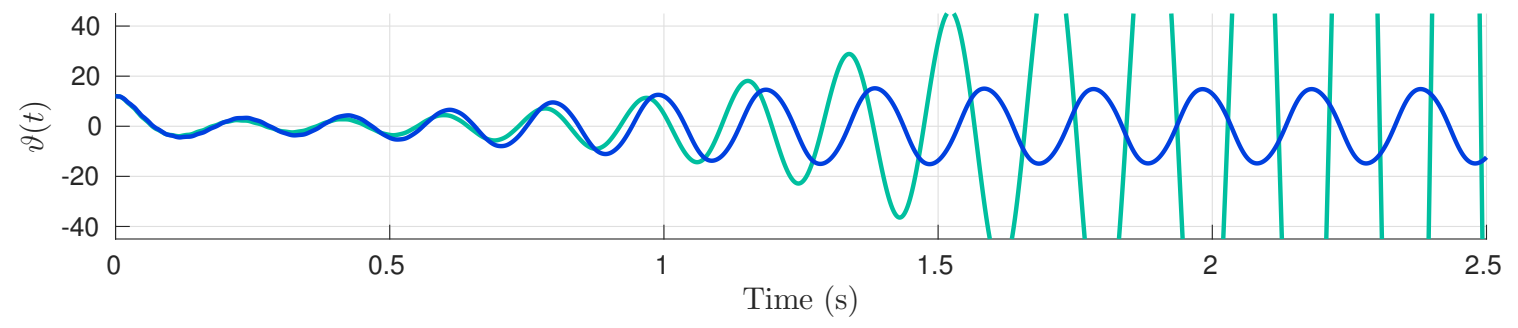

(d) Rigid body pitch rotation (in degrees)

Figure 3.11: Time traces at 10\% above the linear flutter speed

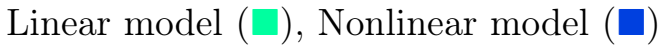


Figure 3.12 highlights another feature of the nonlinear model. For the set of initial conditions that were considered in this investigation, at a given airspeed the response tends to an limit cycle of the same amplitude and frequency (with a phase lag).

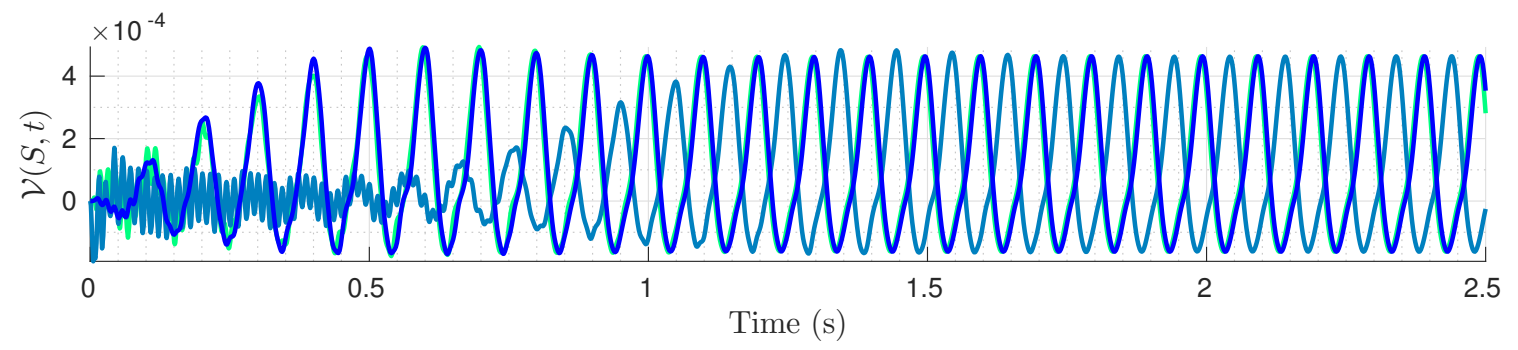

(a) Edgewise bending tip displacement normalized by span length $(\mathrm{m} / \mathrm{m})$

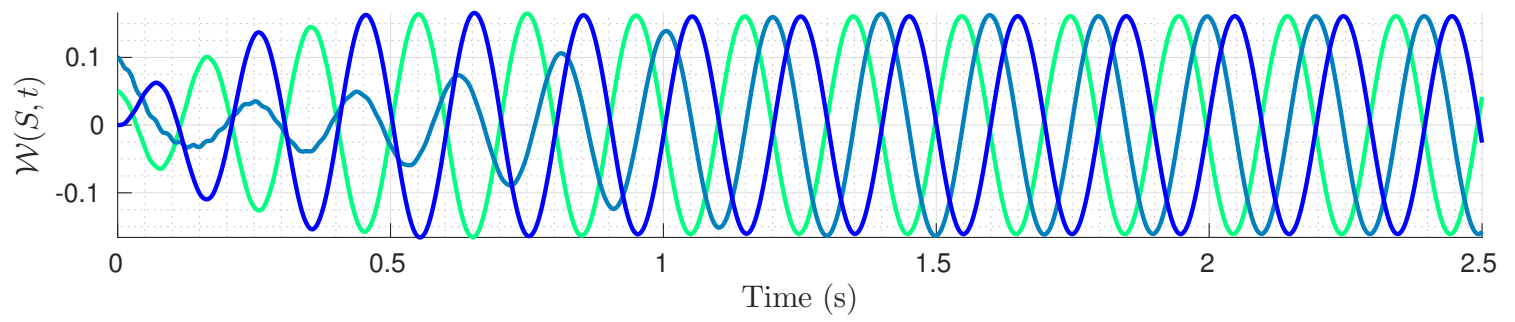

(b) Flapwise bending tip displacement normalized by span length $(\mathrm{m} / \mathrm{m})$

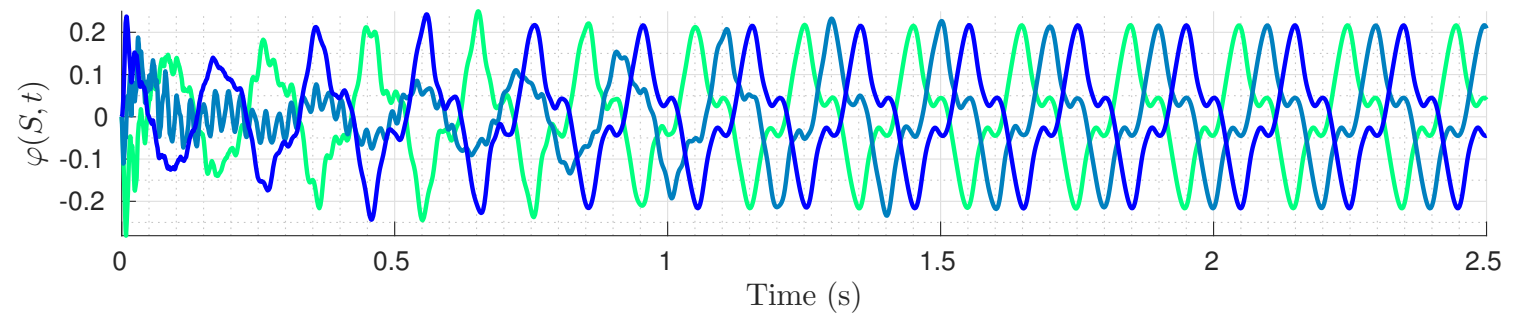

(c) Flexible twist angle tip displacement (in degrees)

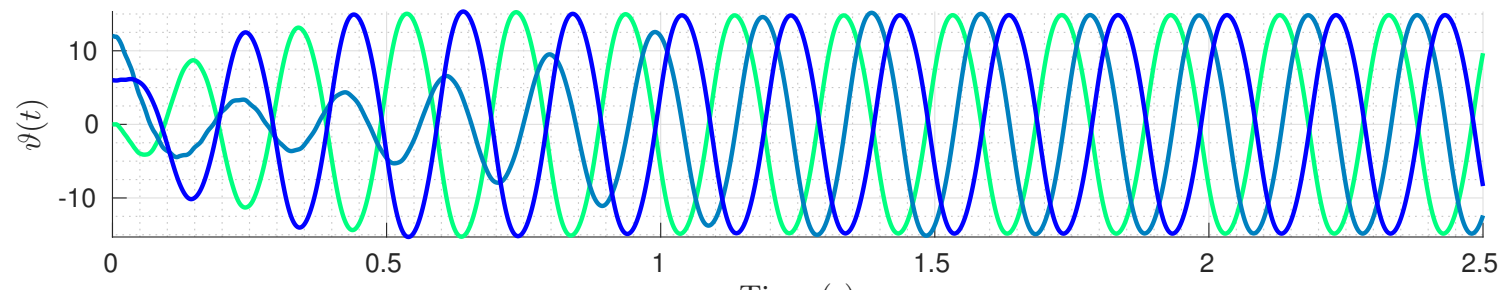

Time (s)

(d) Rigid body pitch rotation (in degrees)

Figure 3.12: Time traces at $10 \%$ above the linear flutter speed for various initial conditions 


\subsection{Verification by Finite Difference Method}

The results that have been presented were obtained by solving a system of algebraic equations that were obtained by discretizing the set of coupled PDEs into a set of coupled ODEs via the so called Galerkin Projection. Subsequently, Houbolt's method was adopted to discretize the set of ODEs in time, to obtain a series of algebraic equations, which were solved iteratively using a Modified Newton-Raphson method. The results that are obtained from adopting this procedure are verified herein by adopting a secondary approach to solve the original set of coupled differential equations.

\subsubsection{Finite Differences}

Using a Taylor Series expansion, we are able to establish finite difference schemes to a desired order of accuracy. For a cantilever beam that is discretized into $L-1$ segments along the span $(i=1,2, \ldots, L)$. We adopt a central difference approximation for the spatial derivatives, and a Houbolt finite difference scheme for the time derivatives. Both difference approximations are accurate to the second order, however the backward difference scheme requires more nodes than the central difference in order to achieve this same level of accuracy 53 .

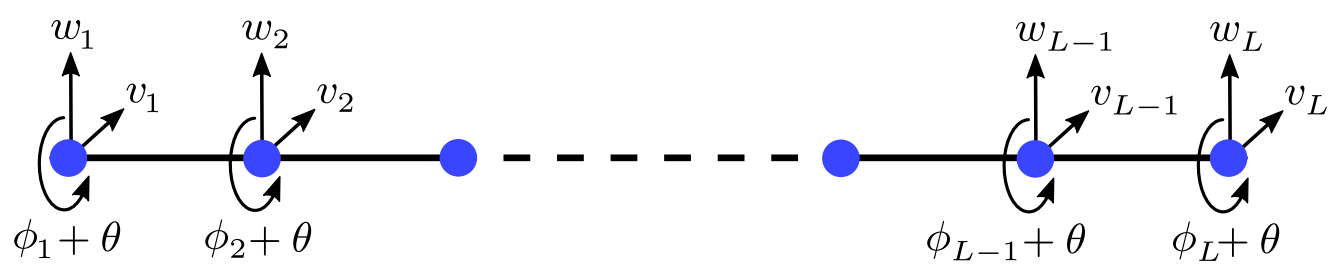

Figure 3.13: Nodes along the span of the wing, discretized by finite difference

The first, second, third and fourth partial derivatives of the state $q_{i}^{n+1}$ with respect to $x$ are given as follows $[53]$ :

$$
\begin{aligned}
\left(\frac{\partial q}{\partial x}\right)_{i}^{n+1} & =\frac{q_{i+1}^{n+1}-q_{i-1}^{n+1}}{2 \Delta x} \\
\left(\frac{\partial^{2} q}{\partial x^{2}}\right)_{i}^{n+1} & =\frac{q_{i+1}^{n+1}-2 q_{i}^{n+1}+q_{i-1}^{n+1}}{\Delta x^{2}}
\end{aligned}
$$




$$
\begin{aligned}
& \left(\frac{\partial^{3} q}{\partial x^{3}}\right)_{i}^{n+1}=\frac{q_{i+2}^{n+1}-2 q_{i+1}^{n+1}+2 q_{i-1}^{n+1}-q_{i-2}^{n+1}}{2 \Delta x^{3}} \\
& \left(\frac{\partial^{4} q}{\partial x^{4}}\right)_{i}^{n+1}=\frac{q_{i+2}^{n+1}-4 q_{i+1}^{n+1}+6 q_{i}^{n+1}-4 q_{i-1}^{n+1}+q_{i-2}^{n+1}}{\Delta x^{4}} .
\end{aligned}
$$

Houbolt's method is used for first and second partial derivatives of the state $q_{i}^{n+1}$ with respect to time as follows $[52$

$$
\begin{aligned}
\left(\frac{\partial^{2} q}{\partial t^{2}}\right)_{i}^{n+1} & =\frac{2 q_{i}^{n+1}-5 q_{i}^{n}+4 q_{i}^{n-1}-q_{i}^{n-2}}{\Delta t^{2}} \\
\left(\frac{\partial q}{\partial t}\right)_{i}^{n+1} & =\frac{11 q_{i}^{n+1}-18 q_{i}^{n}+9 q_{i}^{n-1}-2 q_{i}^{n-2}}{6 \Delta t}
\end{aligned}
$$

In the above expressions, $q_{i}$ is the $i^{\text {th }}$ element of the state vector;

$$
\left\{\begin{array}{c}
q_{1} \\
\vdots \\
q_{L} \\
q_{L+1} \\
\vdots \\
q_{2 L} \\
q_{2 L+1} \\
\vdots \\
q_{3 L} \\
q_{3 L+1}
\end{array}\right\}=\left\{\begin{array}{c}
v_{1} \\
\vdots \\
v_{L} \\
w_{1} \\
\vdots \\
w_{L} \\
\varphi_{1} \\
\vdots \\
\varphi_{L} \\
\vartheta
\end{array}\right\}
$$




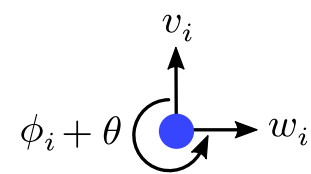

(a) DOFs of node $i$ in the descriptive notation

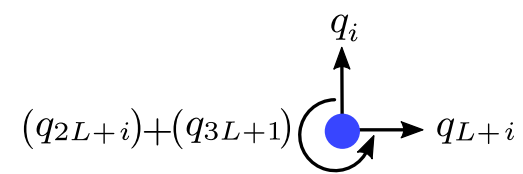

(b) DOFs $i$ in the in the state vector notation

Figure 3.14: The two interchangeable notations for the degrees of freedom at each node, $i$ in the finite difference discretization of the spatial domain (temporal superscript index omitted)

\section{Boundary Conditions}

Using Galerkin projection, the boundary conditions are accounted for in the process of selecting appropriate basis functions. However, in this finite difference method, we need to explicitly enforce the fixed and free end boundary conditions in the equations. For all instances of time $n=1,2, \ldots, N$, the following relations exist,

$$
\begin{aligned}
v(0) & =0 \rightarrow v_{1}=0, \\
v^{\prime}(0) & =0 \rightarrow v_{0}=v_{2}, \\
v^{\prime \prime}(L) & =0 \rightarrow v_{L+1}=2 v_{L}-v_{L-1}, \\
v^{\prime \prime \prime}(L) & =0 \rightarrow v_{L+2}=4 v_{L}-4 v_{L-1}+v_{L-2}, \\
w(0) & =0 \rightarrow w_{1}=0, \\
w^{\prime}(0) & =0 \rightarrow w_{0}=w_{2}, \\
w^{\prime \prime}(L) & =0 \rightarrow w_{L+1}=2 w_{L}-w_{L-1}, \\
w^{\prime \prime \prime}(L) & =0 \rightarrow w_{L+2}=4 w_{L}-4 w_{L-1}+w_{L-2}, \\
\varphi(0) & =0 \rightarrow \varphi_{1}=0, \\
\varphi^{\prime}(L) & =0 \rightarrow \varphi_{L+1}=\varphi_{L-1} .
\end{aligned}
$$

Note that as mentioned before, the nonlinear boundary conditions from Chapter 2 are not accounted for in this model. 


\subsubsection{Imposing Initial Conditions}

Unlike with the Galerkin method, imposing initial conditions using finite difference method is a trivial task. The $i^{\text {th }}$ node is simply prescribed the value of the deflected shape at the spanwise location of that node.

As with the Galerkin method, the first 3 time increments must be prescribed, in order to prescribe the initial velocity of the system, as in the case for the Galerkin method.

\subsubsection{Comparison of the Performance}

For a fixed time step of $\Delta t=10^{-4} \mathrm{~s}$, the convergence of the time history solution of the tip deflections obtained by Galerkin projection with an increasing number of basis functions is shown in Figure 3.15. The number of basis functions used to represent each per state in the projection is incrementally increased from 1 to 5. While an approximation consisting of a single basis function per state $(L=M=N=1)$ provides reasonable results for the flapwise bending and the rigid body pitch rotation response, the solution does not truly converge for all of the plots until at least 4 modes are used per state. This fact is evidenced by the significant changes in the response for the edgewise bending and the flexible twist angle as increasingly more modes are added. The convergence is illustrated over the time range of 0.45 seconds to 0.5 seconds as this shorter time scale allows for the convergence to be assessed visually.

While the time history of the tip displacements are a good indicator of convergence with the addition of a certain number of basis functions, it is important to ensure that for this number of modes, that the solution throughout the domain has converged as well in order to properly truncate the approximation. Figure 3.16 shows equally spaced snapshots in time of the spanwise solution (between 0.45 seconds and 0.5 seconds as in Figure 3.15.

Similarly, with the finite difference numerical scheme, the solution should be expected to converge 


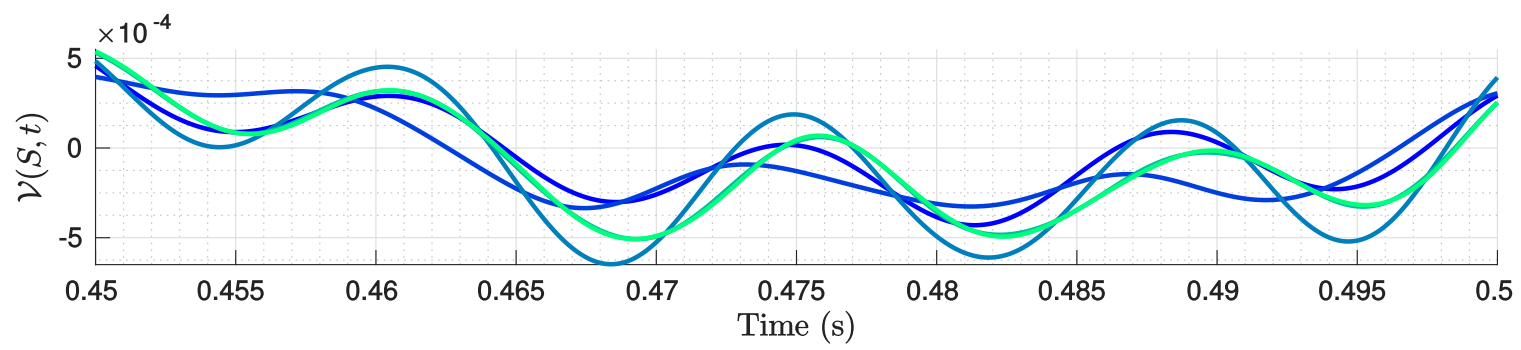

(a) Edgewise bending tip displacement normalized by span length $(\mathrm{m} / \mathrm{m})$

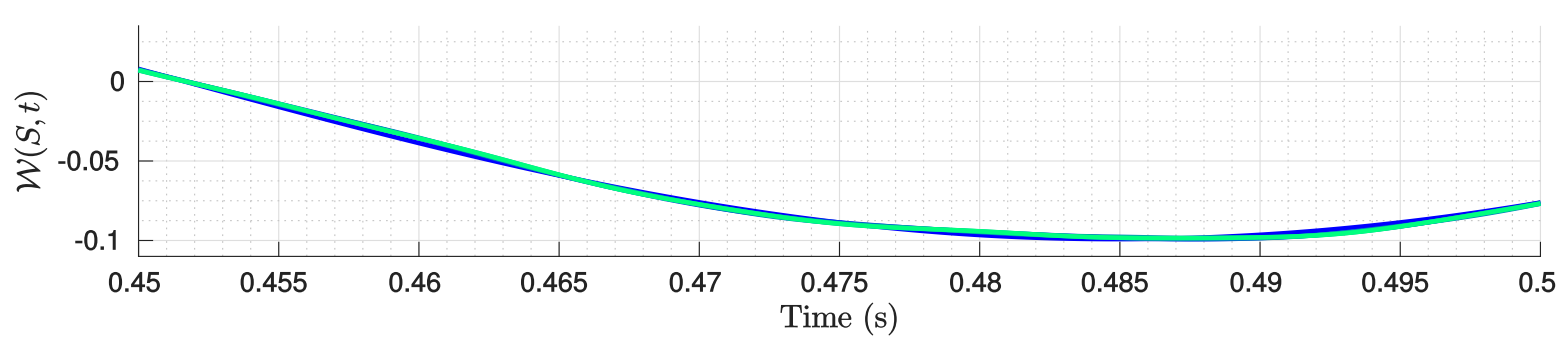

(b) Flapwise bending tip displacement normalized by span length $(\mathrm{m} / \mathrm{m})$

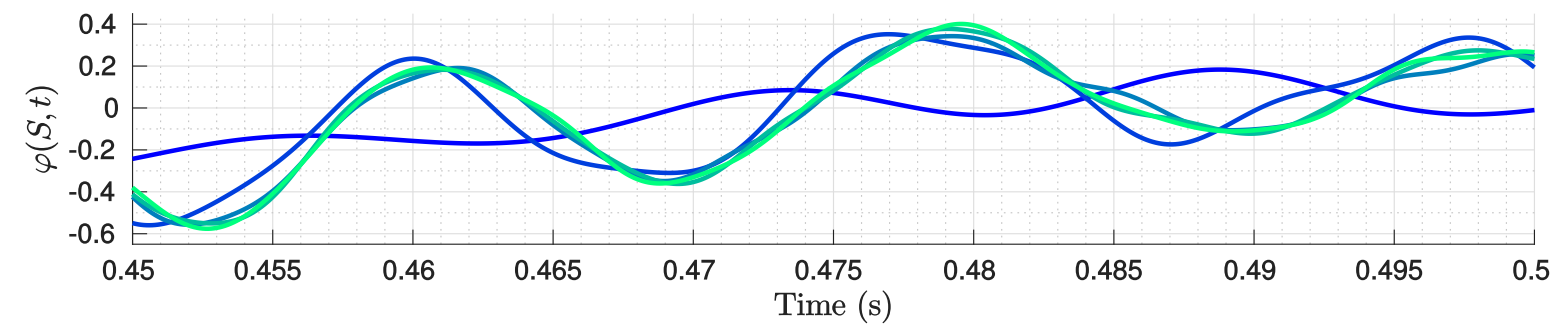

(c) Flexible twist angle tip displacement (in degrees)

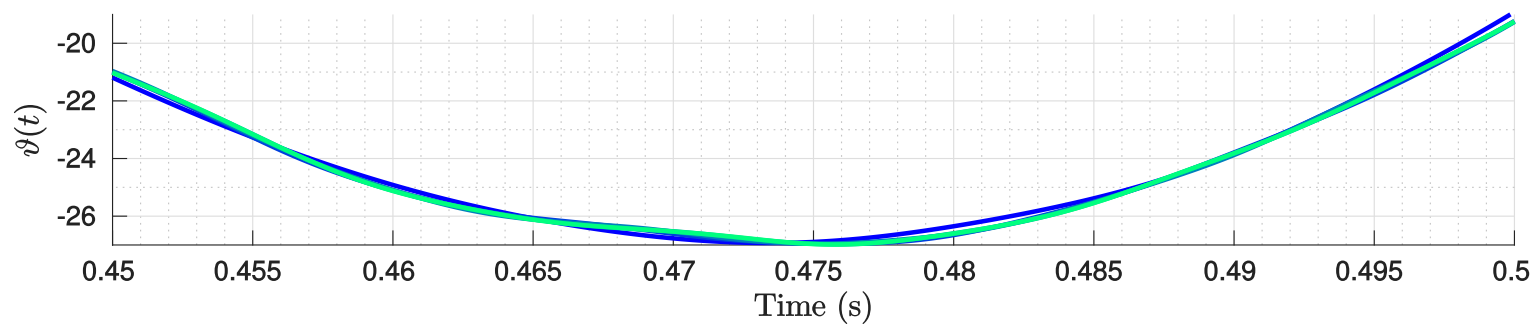

(d) Rigid body pitch rotation (in degrees)

Figure 3.15: Time traces for $\theta\left(t_{0}\right)=30^{\circ}, \mathcal{W}\left(S, t_{0}\right)=0.1$, with all other initial conditions euqual to zero. One mode approximation $(\mathbf{\square})$, two mode approximation $(\mathbf{\square})$, three mode approximation $(\mathbf{\square})$, four mode approximation $(\square)$ and five mode approximation $(\square)$ 


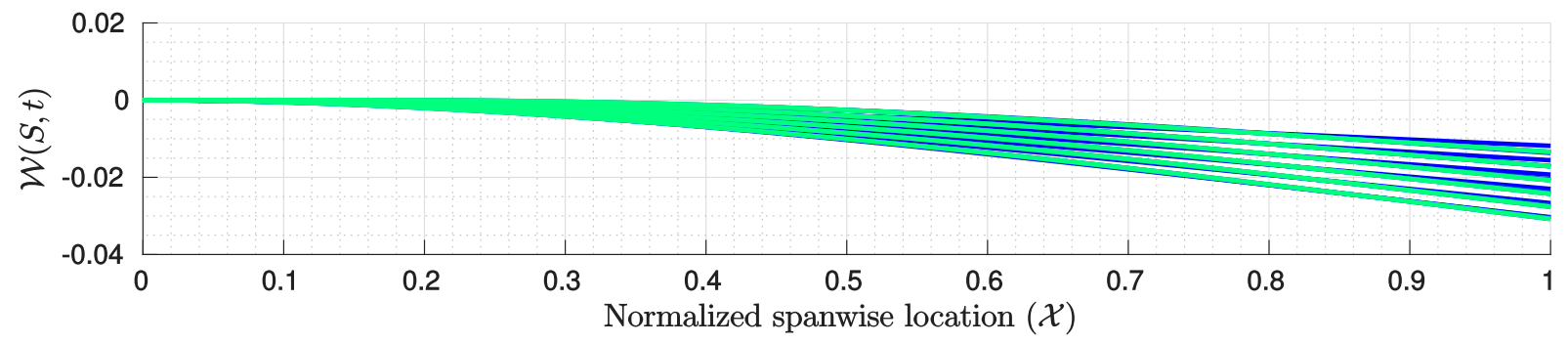

(a) Edgewise bending tip displacement normalized by span length $(\mathrm{m} / \mathrm{m})$

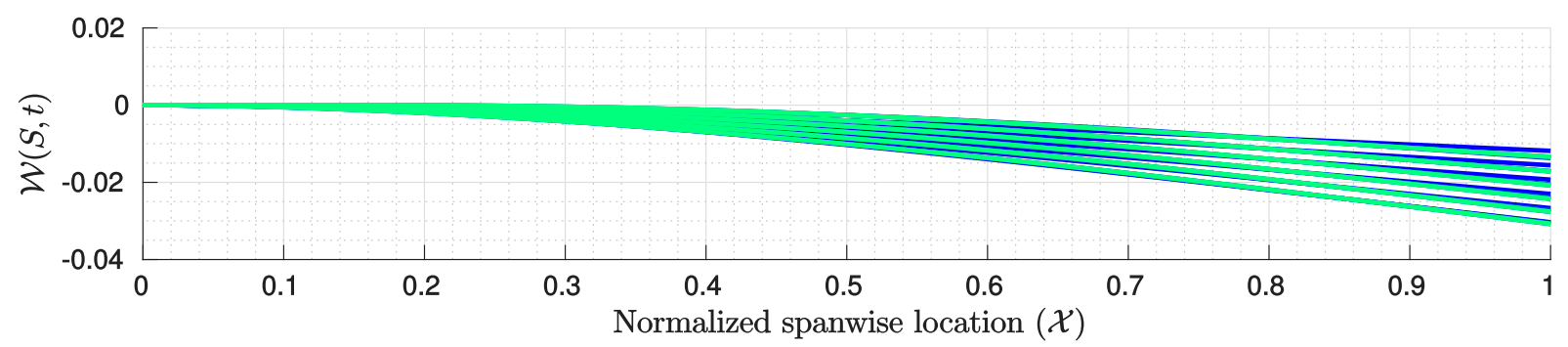

(b) Flapwise bending tip displacement normalized by span length $(\mathrm{m} / \mathrm{m})$

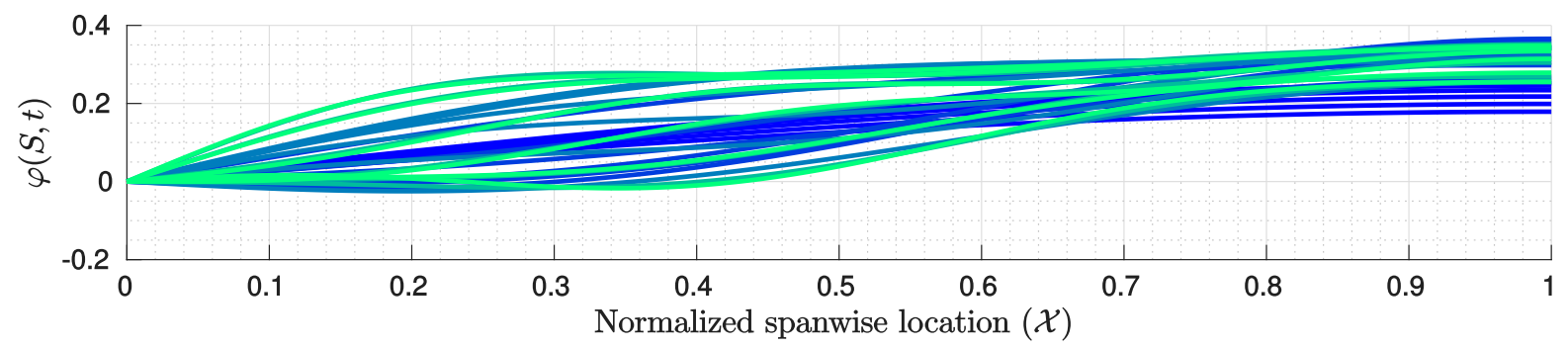

(c) Flexible twist angle tip displacement (in degrees)

Figure 3.16: Snapshots in time taken at equal intervals between $0.45 \mathrm{~s}$ and $0.5 \mathrm{~s}$

One mode approximation ( $\mathbf{\square})$, two mode approximation ( $\mathbf{\square})$, three mode approximation ( $\mathbf{\square})$, four mode approximation $(\square)$ and five mode approximation $(\square)$ 
with a refinement in the spatial discretization. In Figure 3.17 it is shown that a spatial resolution of 100 elements along the span were required to obtain a convergent solution. A noticeable difference between the time trace was observed as the number of spanwise elements was increased from 50 elements to 100 , but no difference was observed after doubling the number of elements again to 200. Again for illustrative purposes this is shown over the time span of 0.45 seconds to 0.5 seconds.

Again, we will check for convergence throughout the spatial domain by plotting snapshots in time as the spatial resolution is made increasingly finer. Figure 3.18 suggests that the solution has converged along the entire spatial domain with a discretization of 100 nodes.

Next we compare the structure of the systems of simultaneous equations for the Galerkin and finite difference method. The Galerkin method required 4 global basis functions per mode before convergence was observed. With the system of equations having a dimenisonality given by $(L+M+N+1)$, the system consists of 13 coupled ordinary differential equations. By contrast, the finite difference method required approximately 100 nodes along the span to obtain a converged solution. With 3 degrees of freedom per node plus an additional mode for the rigid body pitch rotation, a system of 301 equations is obtained.

With the relative dimensionality of the problems in mind, the Modified Newton-Raphson algorithm employed for the numerical integration in time requires that the function evaluated at the current values of the state, be multiplied by the inverse of the Jacobian matrix evaluated at the current system state. Because of this operation, there is some merit to understanding the form of the Jacobian matrices used (which would be of dimensions $13 \times 13$ and $301 \times 301$ for the Galerkin method and the finite difference method respectively). Figures 3.193 .20 and 3.21 compare the sparsity structures of the Jacobians of the structural model, where the nonzero entries are indicated by blue dots.

Figure 3.19 considers the case where the nonlinear structural terms are omitted (see Appendix 


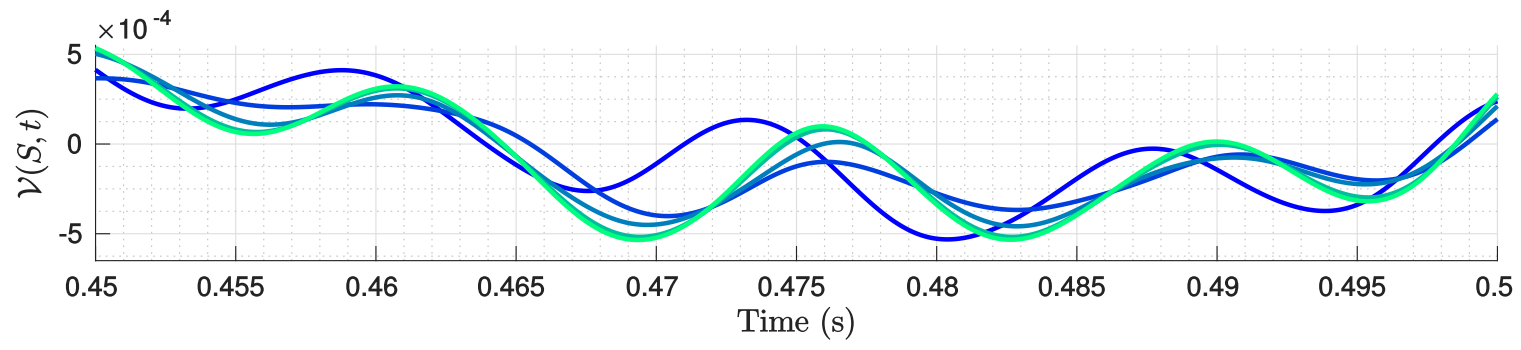

(a) Edgewise bending tip displacement normalized by span length $(\mathrm{m} / \mathrm{m})$

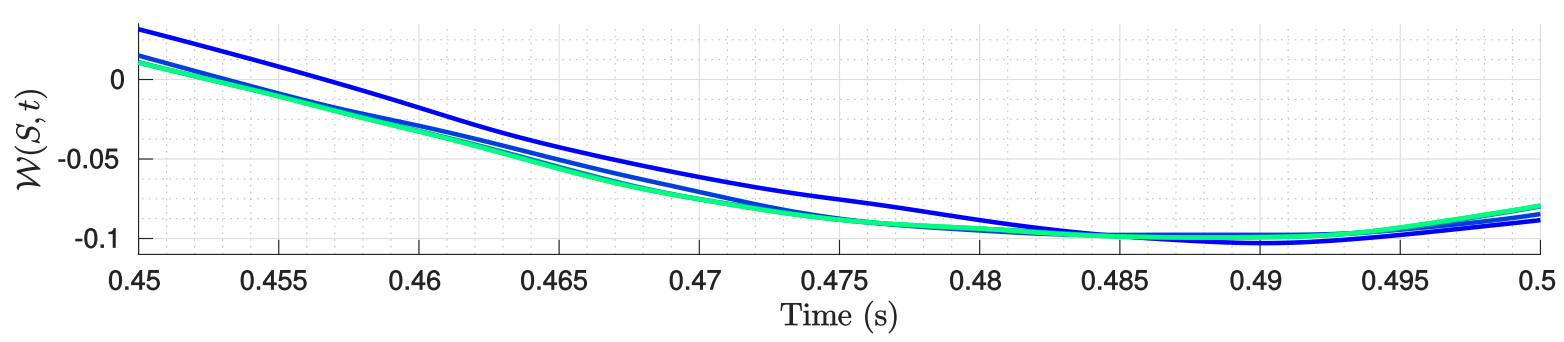

(b) Flapwise bending tip displacement normalized by span length $(\mathrm{m} / \mathrm{m})$

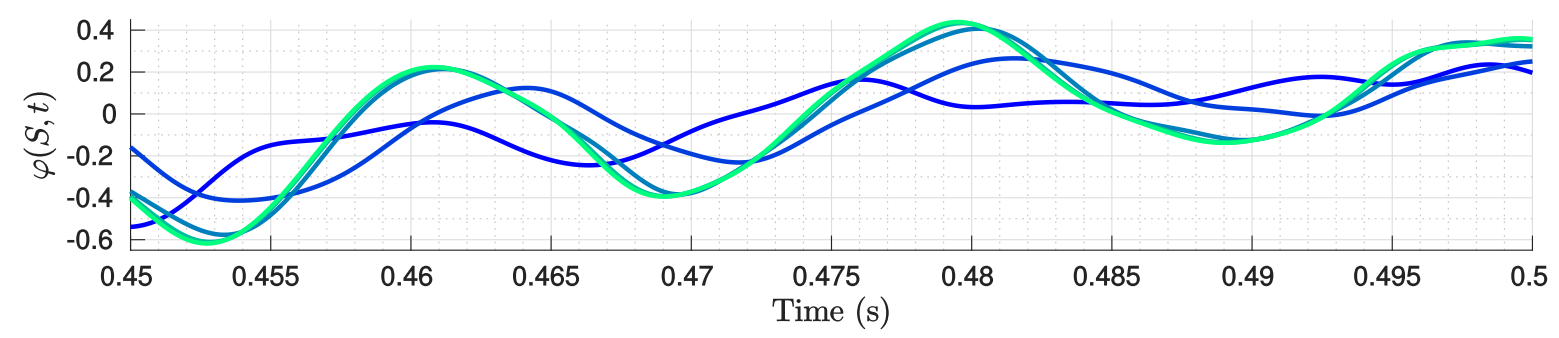

(c) Flexible twist angle tip displacement (in degrees)

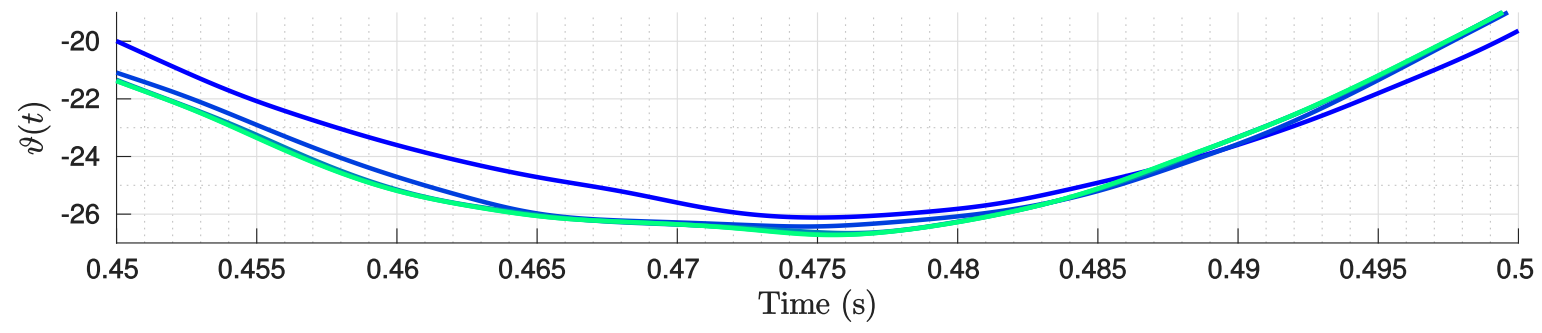

(d) Rigid body pitch rotation (in degrees)

Figure 3.17: Time traces for $\theta\left(t_{0}\right)=30^{\circ}, \mathcal{W}\left(S, t_{0}\right)=0.1$, with all other initial conditions equal to zero. One mode approximation $(\boldsymbol{\square})$, two mode approximation $(\boldsymbol{\square})$, three mode approximation $(\boldsymbol{\square})$, four mode approximation $(\square)$ and five mode approximation ( $\square$ ) 


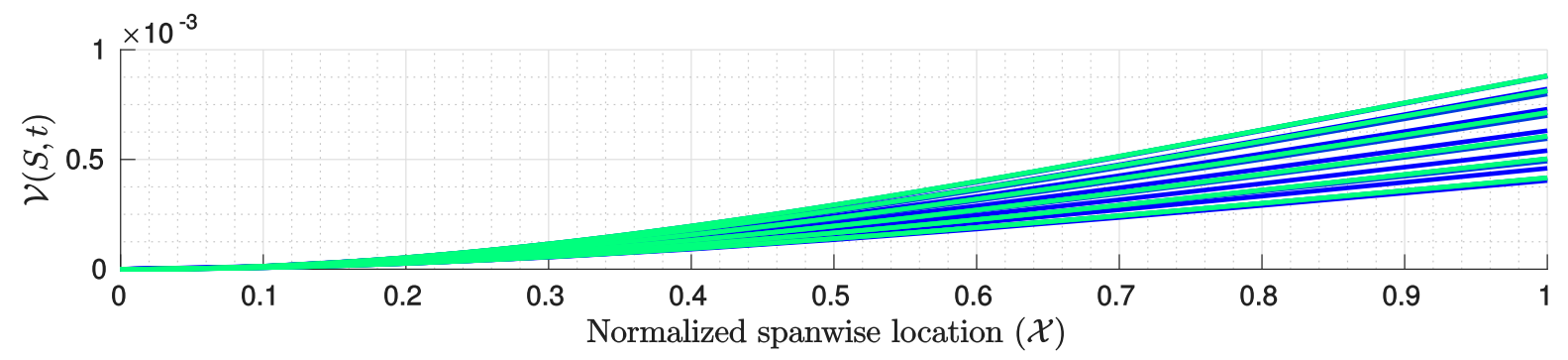

(a) Edgewise bending tip displacement normalized by span length $(\mathrm{m} / \mathrm{m})$

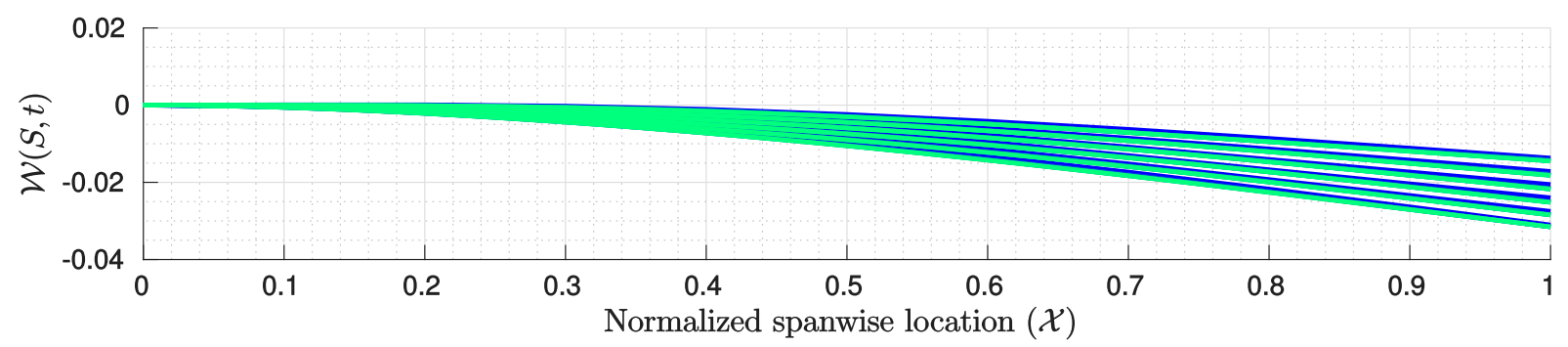

(b) Flapwise bending tip displacement normalized by span length $(\mathrm{m} / \mathrm{m})$

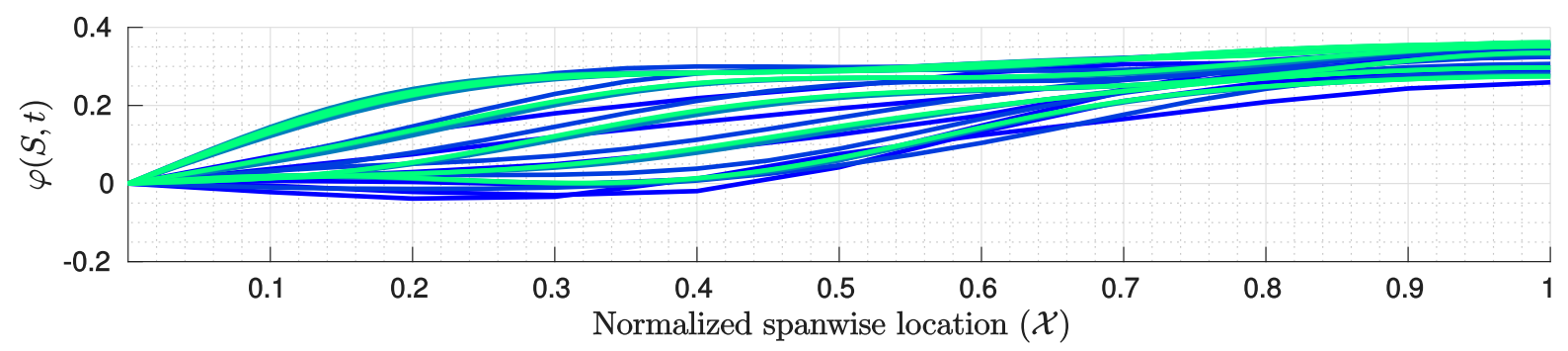

(c) Flexible twist angle tip displacement (in degrees)

Figure 3.18: Snapshots in time taken at equal intervals between $0.45 \mathrm{~s}$ and $0.5 \mathrm{~s}$

One mode approximation ( $\mathbf{\square})$, two mode approximation ( $\mathbf{\square})$, three mode approximation ( $\mathbf{\square})$, four mode approximation $(\square)$ and five mode approximation $(\square)$ 
B.5.1). Figure 3.19a shows the form of the Jacobian matrix for the Galerkin implementation, while Figure $3.19 \mathrm{~b}$ shows the Jacobian for finite difference. The coupling mechanisms are fundamentally different in the two methods, which can help explain the differences observed between the two spy plots. The Galerkin method has a single diagonal of nonzero entries, which is attributed to the orthonormality of the basis functions used in the projection. The blocks can be attributed to the coupling between the flapwise bending and the flexible twist, whose basis functions are not orthonormal with respect to each other. The finite difference Jacobian has a banded structure, where the linear inertial terms are concentrated along the main diagonal and the coupling between the flapwise bending and flexible twist are simply diagonals, where they were previously dense blocks for the Galerkin method. The thick bands around the main diagonal are due to the finite difference representations that were used for the linear stiffness terms. $\partial^{4} v / \partial x^{4}$ and $\partial^{4} w / \partial x^{4}$ are approximated using 5 points, similarly, $\partial^{2} \phi / \partial x^{2}$ is approximated using 3 points.

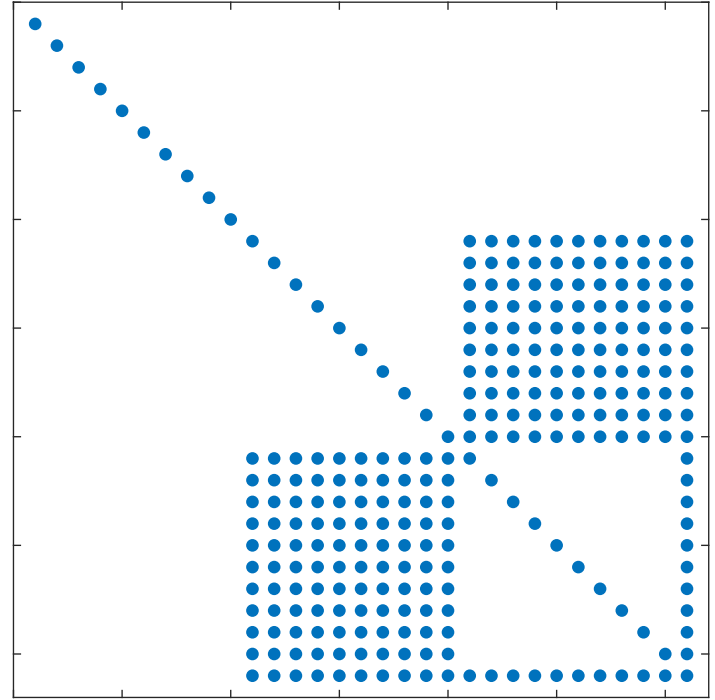

(a) Spy plots of the Jacobian matrix for Galerkin Projection

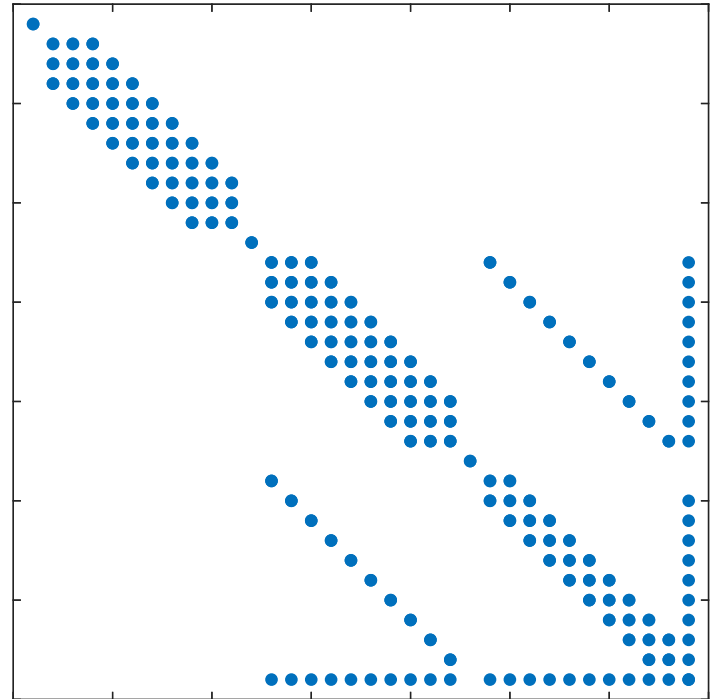

(b) Spy plots of the Jacobian matrix for Finite Difference

Figure 3.19: Sparsity of the Jacobian matrices used in both the Galerkin projection and in the finite difference method for the linear system

The nonlinear terms can be classified into two groups, inertial nonlinearities (see Appendix B.5.2 and stiffness nonlinarities (see Appendix B.5.3). The structure of the Jacobian matrix containing only the terms associated with the inertial nonlinearities are shown in Figures 3.20 


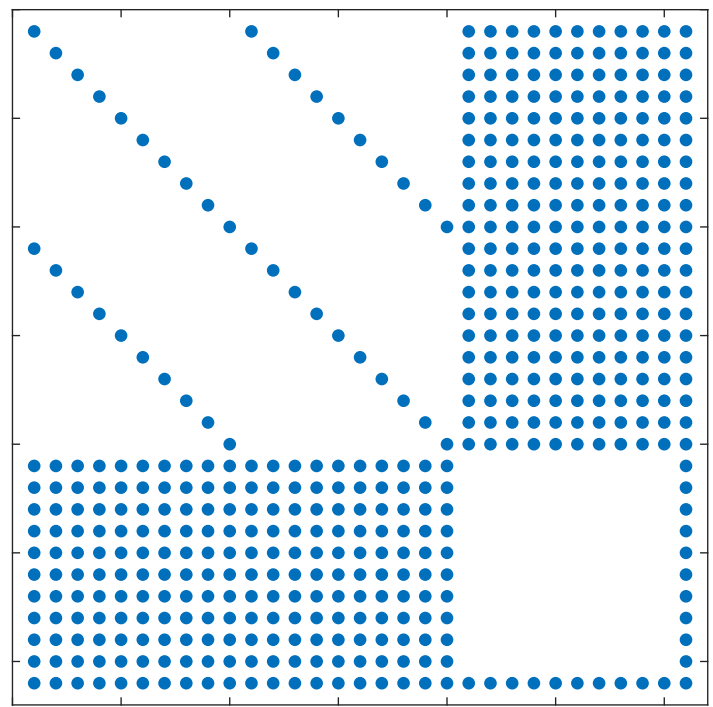

(a) Spy plots of the linear Jacobian matrix for Galerkin Projection

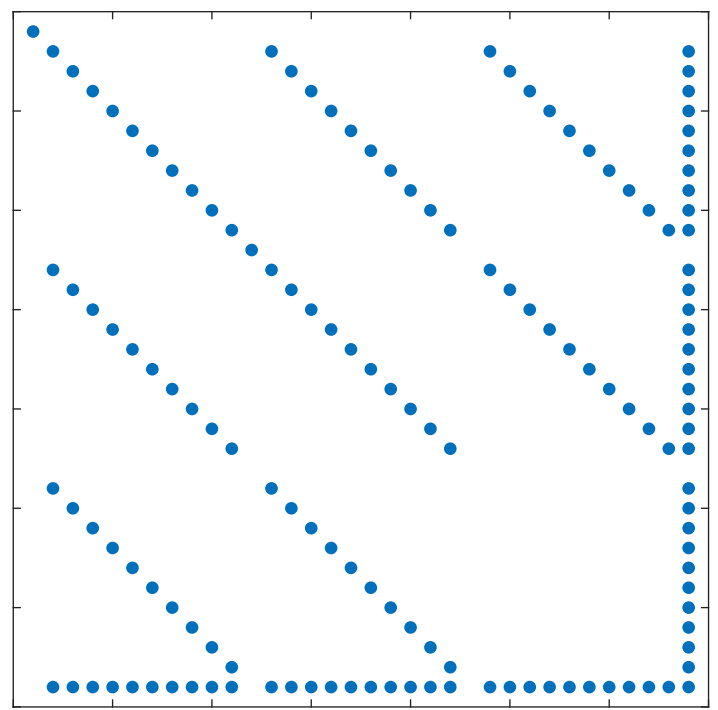

(b) Spy plots of the linear Jacobian matrix for Finite Difference

Figure 3.20: Sparsity of the Jacobian matrices used in both the Galerkin projection and in the finite difference method for the underlying linear system and the inclusion of inertial nonlinearities

Analysing the structure of the Jacobian matrix for the nonlinear stiffness terms, shown in Figures 3.21 reveal the couplings that occur. Note that there are no diagonal terms, as there are no quadratic coupling terms.

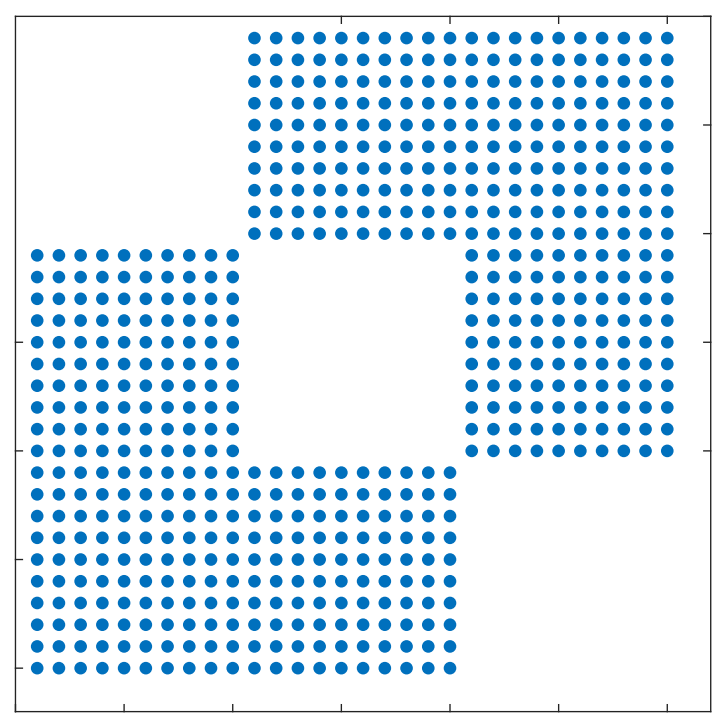

(a) Spy plots of the linear Jacobian matrix for Galerkin Projection

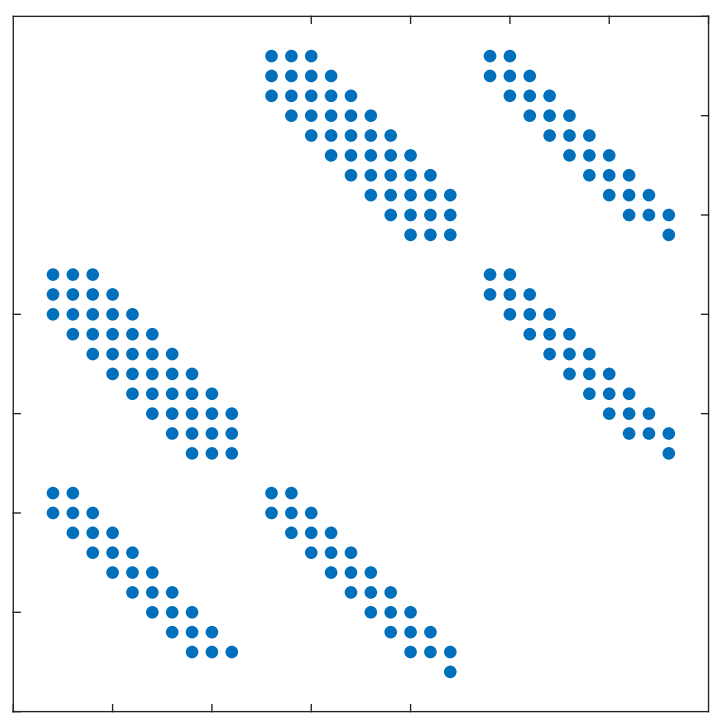

(b) Spy plots of the linear Jacobian matrix for Finite Difference

Figure 3.21: Sparsity of the Jacobian matrices used in both the Galerkin projection and in the finite difference method for the underlying linear system and the inclusion of stiffness nonlinearities 


\subsubsection{Comparison of the Solutions}

This section will compare the results for the undamped free vibration of the nonlinear structural model using two independent numerical schemes. In Figure 3.22 a five second time trace of the tip displacement of a converged Galerkin projection scheme is implemented and shown in blue. This solution is compared to the tip displacement as obtained by a converged finite difference scheme, in green. Qualitatively, the solutions appear to be similar, while some differences can be observed at the graphical scale, the trends are largely consistent across the two numerical schemes, in terms of the amplitudes and frequencies.

In addition to comparing the time history of the tip displacement, and the plots derived from that quantity (phase plane diagrams and power spectral density), we will also compare the solution produced by the two methods in space. We superimpose the converged solution shown in Figure 3.16 and Figure 3.18 for the respective numerical schemes, and present the outcome in Figure 3.23 This shows agreement between the two methods not only for the tip displacement, but also along the entire spatial domain of the cantilever. Furthermore, Figure 3.24 compares the frequency content of the time traces obtained by both numerical methods. 


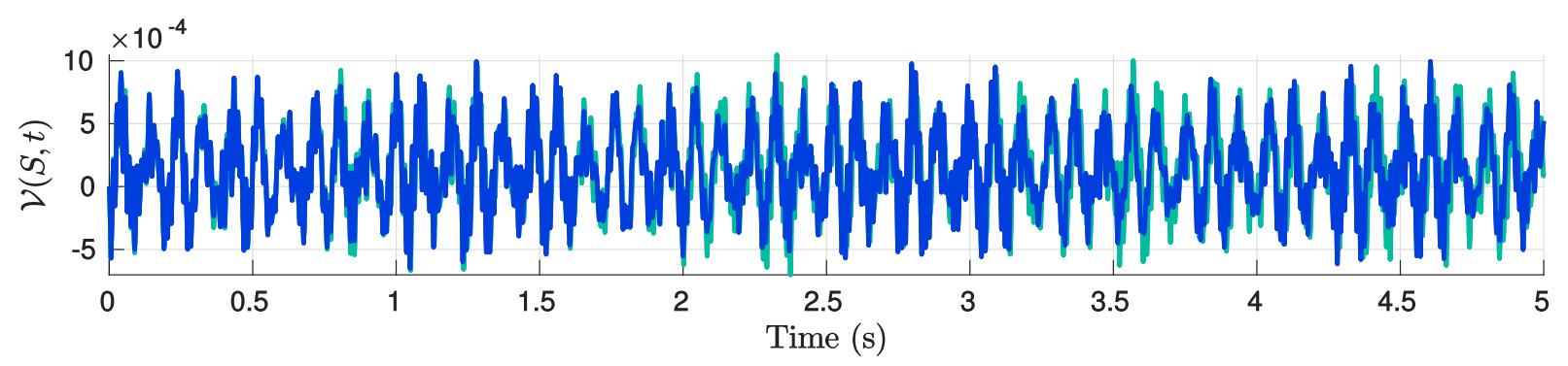

(a) Edgewise bending tip displacement normalized by span length $(\mathrm{m} / \mathrm{m})$

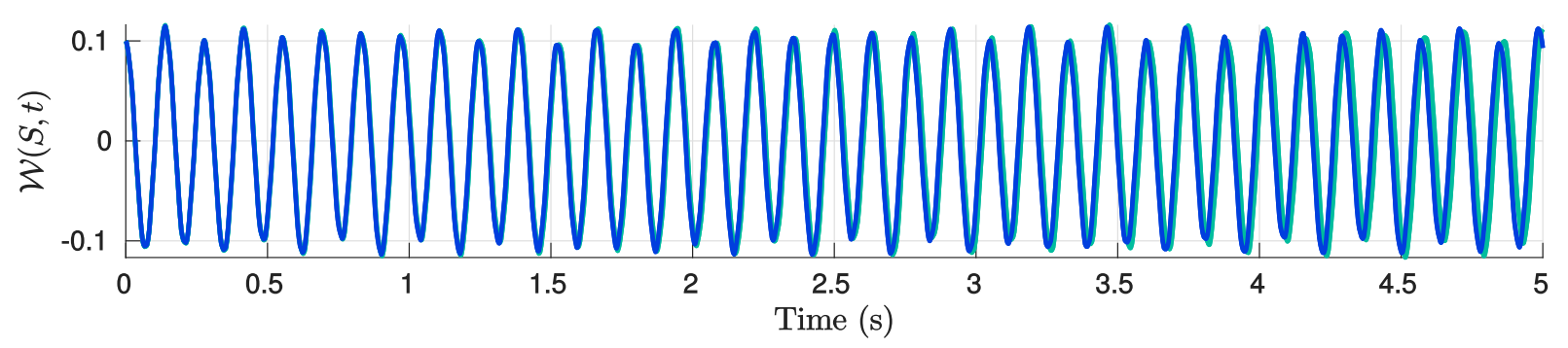

(b) Flapwise bending tip displacement normalized by span length $(\mathrm{m} / \mathrm{m})$

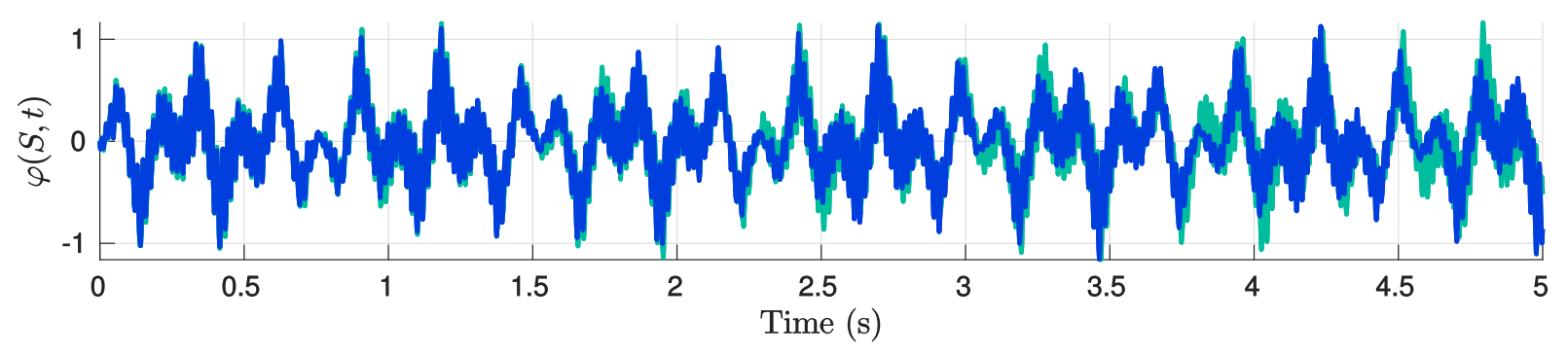

(c) Flexible twist angle tip displacement (in degrees)

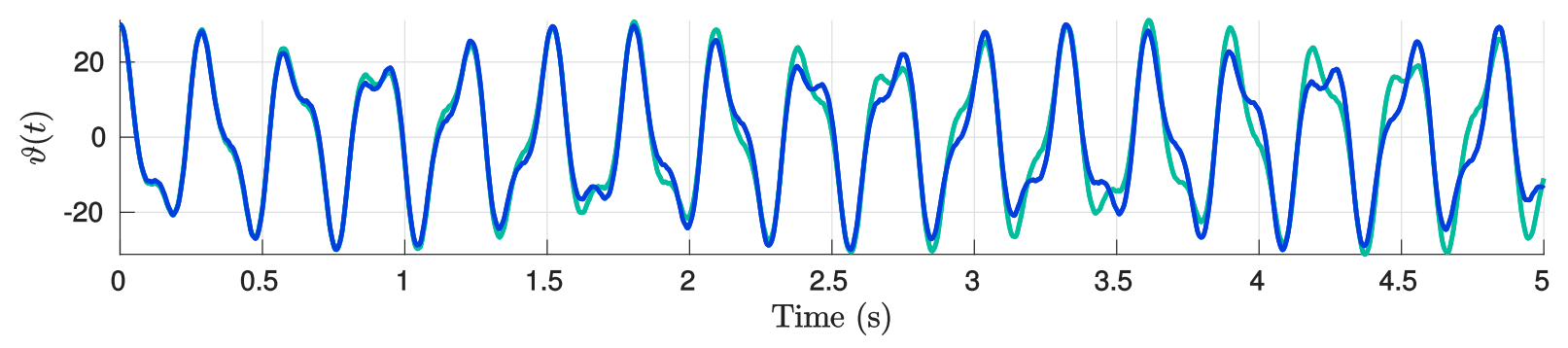

(d) Rigid body pitch rotation (in degrees)

Figure 3.22: Time traces for $\theta\left(t_{0}\right)=30^{\circ}, \mathcal{W}\left(S, t_{0}\right)=0.1$, with all other initial conditions equal to zero. Galerkin projection $(\square)$, finite difference scheme $(\square)$ 


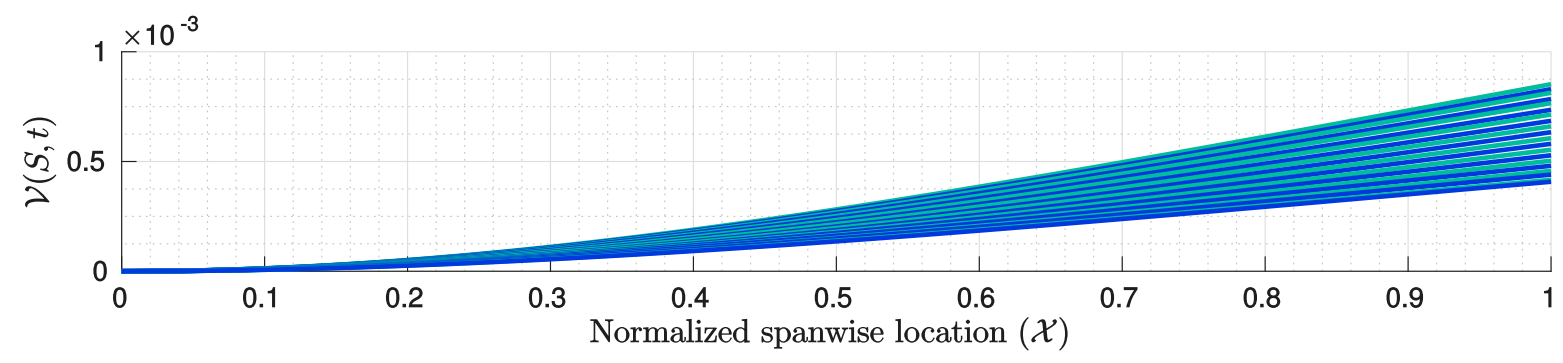

(a) Edgewise bending tip displacement normalized by span length $(\mathrm{m} / \mathrm{m})$

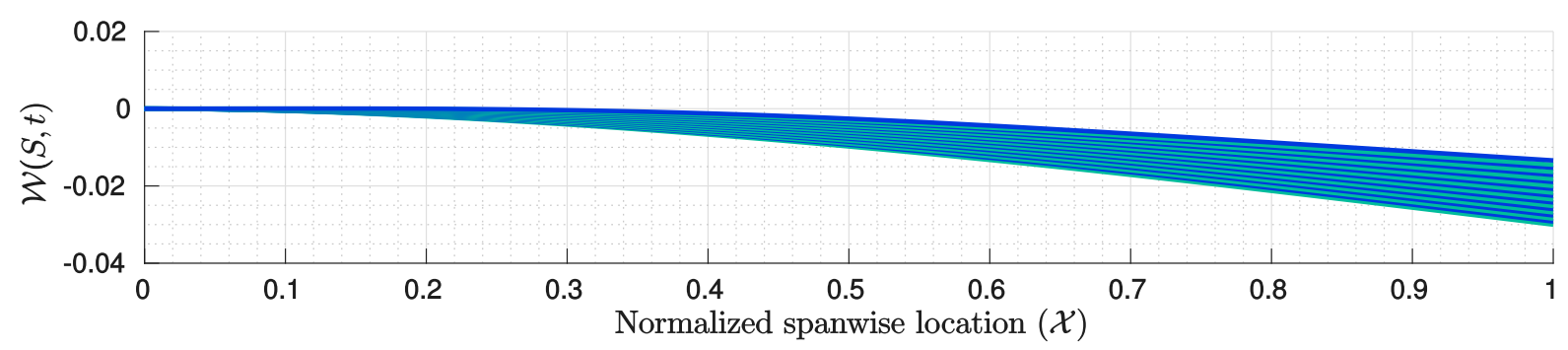

(b) Flapwise bending tip displacement normalized by span length $(\mathrm{m} / \mathrm{m})$

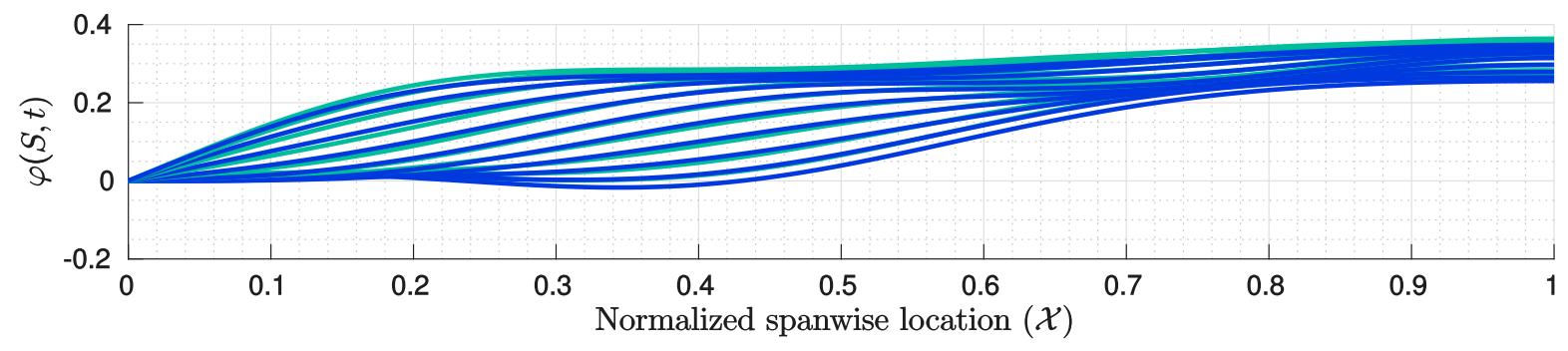

(c) Flexible twist angle tip displacement (in degrees)

Figure 3.23: Snapshots in time taken at equal intervals between $0.45 \mathrm{~s}$ and $0.5 \mathrm{~s}$

Galerkin projection $(\mathbf{\square})$, finite difference scheme $(\square)$ 


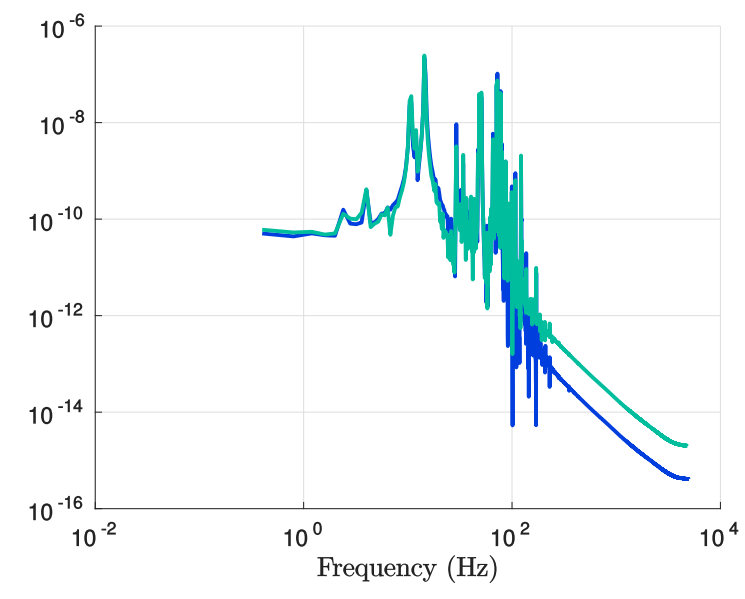

(a) Edgewise bending tip displacement

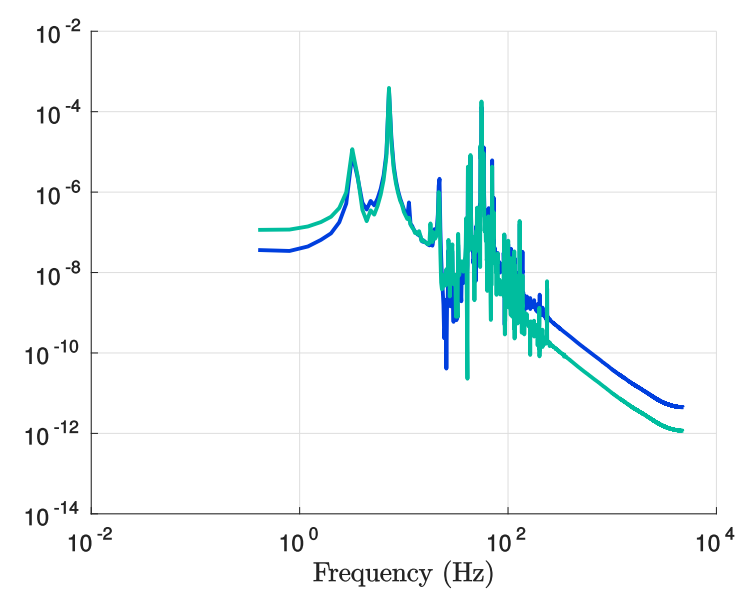

(c) Flexible twist angle tip displacement

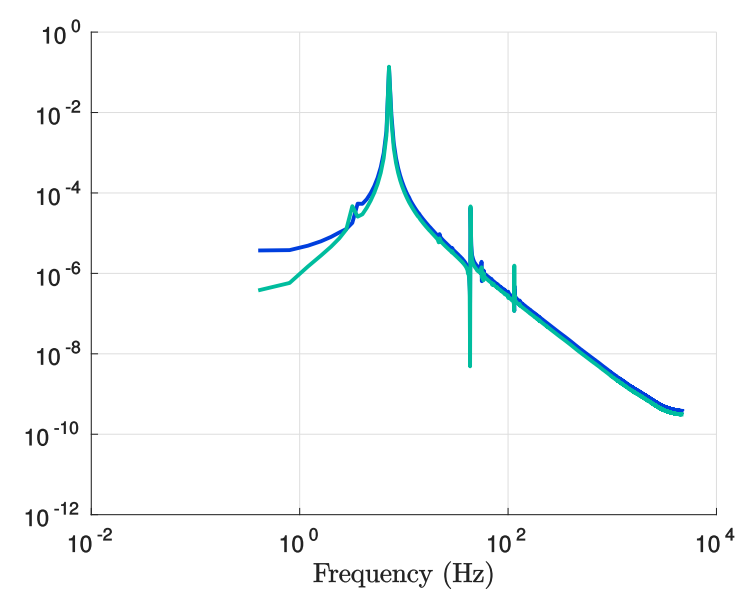

(b) Flapwise bending tip displacement

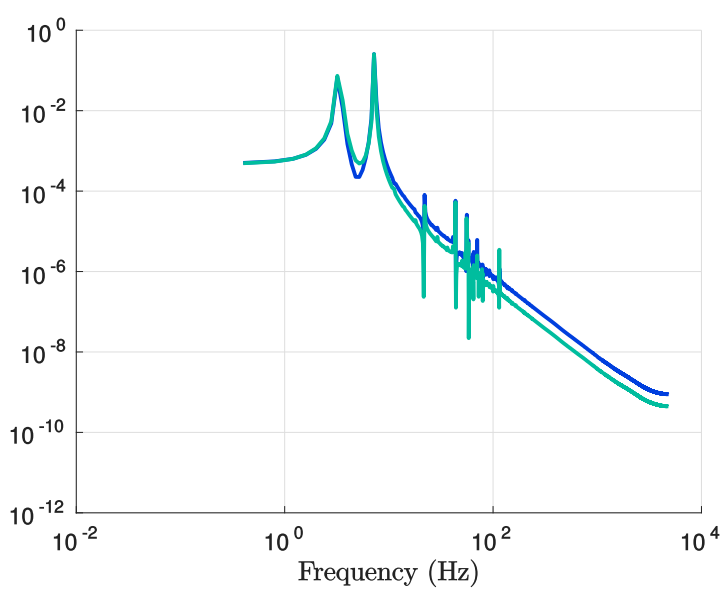

(d) Rigid body base rotation

Figure 3.24: Power spectral density of the tip displacements from Figure 3.5 between 2.5s and 5s Galerkin projection $(\square)$, finite difference scheme $(\square)$ 


\section{Chapter 4}

\section{Variance-Based Methods for Global Sensitivity Anal- ysis}

Global Sensitivity Analysis (GSA) is a statistical framework which allows modellers to apportion the uncertainty in the system output to its various uncertain inputs. There are many benefits to performing GSA. The motivation for performing GSA is to identify which uncertain input parameters have the largest influence on the overall uncertainty of the system output. Such insight can help target efforts in parameter estimation. Identifying the parameters which have negligible influence on a specific output quantity of interest allows the modeller to assign fixed values to these parameters rather than being defined by probability distributions, hence leading to a reduction in the stochastic dimensionality of the model.

In this chapter, global sensitivity analysis will be employed to study the new parameters that are introduced into the system as the the flexible cantilever is extended to include a rigid body pitch rotation. As discussed later, other parameters could be included in the investigation, and future studies may consider larger combinations of uncertain input parameters. However, the current goal of this investigation is to study the effects of the rigid body pitch rotation and its potential to induce an interesting flutter mechanism. 


\subsection{Methods for Global Sensitivity Analysis}

There are two principal types of problem that fall under the general umbrella of uncertainty quantification, being the forward propagation of uncertainty and inverse problems 31 . While inverse uncertainty quantification is beyond the scope of this thesis, it is important to be mindful that it serves as a motivation for much of the content of this body of work.

The most basic methods for sensitivity analysis are based on derivatives. These methods involve taking the derivative of the function with respect to each of the uncertain input factors. Intuitively, for nonlinear models these derivatives only have meaning at the point where the derivative is taken. Because of this, these methods do not allow for a full exploration of the space of the uncertain inputs, and thus are limited to local sensitivity analysis. A step up in complexity from derivative-based methods are regression-based methods. In these methods, a linear regression is fitted to a set out model outputs for a series of Monte Carlo Simulations with some uncertain input parameters which are sampled from their probability distributions. The regression coefficients provide a metric by which to quantify the sensitivity of the output to each input. While this method allows for an exploration of the space of the uncertain input factors, its reliability is still limited by the degree of nonlinearity in the model. For a linear model, these regression-based methods and derivative-based methods are the same. However, regression-based methods are more robust as they can still be applied to nonlinear models for global sensitivity analysis, with the understanding that the accuracy is dependent on to the degree to which the output is nonlinearly dependent on the inputs $[12$. Where there is strong nonlinearity, these methods will also fail to provide meaningful results. Finally there are variance-based methods, which are considered to be model-independent as they are equally applicable to models regardless of their form or degree of nonlinearity [12]. Because of this, and the fact that they provide a framework by which we are able to explore the entire space of the uncertain inputs, we will pursue these variance-based methods in this chapter. Variance-based methods use Sobol' Indices as a metric by which the relative importance of the parameteric uncertainty in the input parameters can be assessed [10]. 


\subsection{Sobol' Indices}

For a given model, the output, $Y$ is given as some function, $f$ of a set of uncertain input factors $X$,

$$
Y=f\left(X_{1}, X_{2}, \ldots, X_{k}\right) .
$$

The input factors in the set $X=\left\{X_{1}, X_{2}, \ldots, X_{k}\right\}$ all have some nonzero variance (i.e. parameters

with deterministic values are not included in this set). For this generic model, we can calculate first order sensitivity measures as [12],

$$
S_{i}=\frac{V_{X_{i}}\left(E_{X_{\sim i}}\left(Y \mid X_{i}\right)\right)}{V(Y)} .
$$

where the inner expectation, $E_{X_{\sim i}}\left(Y \mid X_{i}\right)$ is evaluated for the output, $Y$ conditioned on the input factor $X_{i}$ fixed to some value $x_{i}{ }^{*}$ while the elements of the set $X_{\sim i}=\left\{X_{1}, X_{2}, \ldots, X_{i-1}, X_{i+1}, \ldots, X_{k}\right\}$, are varied. The outer variance is calculated as the inner expectation is evaluated for all the values of $X_{i}=x_{i}{ }^{*}$. The variance $V(Y)$ in the denominator is simply the variance of the output when all factors $X$ are varied.

These indices $S_{i},(i=1,2, \ldots, k)$ are termed first order sensitivity or Sobol indices. These indices measure the independent contribution of the variance of each of the $k$ uncertain inputs, $X_{i}$ to the variance of the output $Y$. However, the first order sensitivity indices fail to account for uncertainty in the output that arises from the interaction between multiple factors (for instance the second order effects of $X_{i}$ and $X_{j}$ for $i \neq j$ ). If we wish to account for the second order effects, the inner expectation is now evaluated for the output conditioned on both of the input parameters, while varying the subset of $X$ that contains neither $X_{i}$ nor $X_{j}$. The outer variance is then evaluated as $X_{i}=x_{i}{ }^{*}$ and $X_{j}=x_{j}{ }^{*}$ are varied. This gives a measure of the variance of the output that can be attributed to the independent effects of varying $X_{i}$ and $X_{j}\left(S_{i}\right.$ and $S_{j}$ as described above), as well as a measure of the interaction effects of varying these parameters simultaneously, $S_{i j}[12$, 


$$
\begin{aligned}
S_{i}+S_{j}+S_{i j} & =\frac{V_{X_{i} X_{j}}\left(E_{X_{\sim i} X_{\sim j}}\left(Y \mid X_{i}, X_{j}\right)\right)}{V(Y)}, \\
S_{i j} & =\frac{V_{X_{i} X_{j}}\left(E_{X_{\sim i} X_{\sim j}}\left(Y \mid X_{i}, X_{j}\right)\right)}{V(Y)}-S_{i}-S_{j}, \\
S_{i j} & =\frac{V_{X_{i} X_{j}}\left(E_{X_{\sim i} X_{\sim j}}\left(Y \mid X_{i}, X_{j}\right)\right)}{V(Y)}-\frac{V_{X_{i}}\left(E_{X_{\sim i}}\left(Y \mid X_{i}\right)\right.}{V(Y)}-\frac{V_{X_{j}}\left(E_{X_{\sim j}}\left(Y \mid X_{j}\right)\right.}{V(Y)} .
\end{aligned}
$$

To reliably account for $100 \%$ of the uncertainty in the ouput due to $k$ input factors, we would be required to account for even higher order interactions (up to the $k^{\text {th }}$ order). For $k$ inputs, there are a total of $2^{k}-1$ sensitivity indices, the sum of which are equal to unity [12, i.e.,

$$
\sum_{i=1}^{k} S_{i}+\sum_{i=1}^{k} \sum_{j>i}^{k} S_{i j}+\sum_{i=1}^{k} \sum_{j>i}^{k} \sum_{l>j}^{k} S_{i j l}+\ldots+S_{123 \ldots k}=1 .
$$

For systems with small numbers of random inputs (ie. low values of $k$ ), the total number of sensitivity indices is manageable. For a system with two input factors, $X_{1}$ and $X_{2}$, we have three indices (namely $S_{1}, S_{2}$ and $S_{12}$ ). If we start looking at a system with a large number of input factors the number of sensitivity indices increases exponentially. In our system, there are a significant number of parameters which could be uncertain. For instance, in analyzing the structural free vibration response of the cantilever described in Chapter 2 we could have uncertainty in our stiffness and mass properties: $E A, E I_{y}, E I_{z}, G J, K_{\theta}, \bar{m}, \bar{I}_{0}, I_{\theta}$. To fully account for the variance of the output of the system by apportioning it to the above input factors, and the interaction between them, we would need $\left(2^{8}-1\right)$ or 255 sensitivity indices, which is excessive to the point where no physical interpretation can reasonably be made of these measures.

To circumvent this issue, the notion of total effect sentitivity intidces, which will be denoted $S_{T_{i}}$, have benn introduced. If we choose to apportion the variance to the uncertain input factors using this approach, we now require only $2 \times k$ Sobol' indices to do so, namely the main effect $S_{i}$ and $S_{T_{i}}$ for $i=1, \ldots, k$. These total effect Sobol' indices account for the variance that can be traced back to input parameter $X_{i}$ and its interaction with all other input factors. The total effect $S_{T_{i}}$ is given 
by [12],

$$
S_{T_{i}}=S_{i}+\sum_{j \neq i}^{k} S_{i j}+\sum_{j \neq i}^{k} \sum_{l>j}^{k} S_{i j l}+\ldots+S_{123 \ldots k} .
$$

While the interpretation of the total effect Sobol' index of parameter $X_{i}$ is relatively straight forward, a direct calculation of $S_{T_{i}}$ is not conveniently available. This can be understood by considering that in Eq.4.3), in order to capture the effects of the interaction of $X_{i}$ with $X_{j}$, we obtained $S_{i}$ and $S_{i j}$ which both contribute to $S_{T_{i}}$, however, we also obtained $S_{j}$. Analogously, in the case where we are trying to capture the interaction of $X_{i}$ with all other inputs $X_{j}$ for $j=1, \ldots, k$, all of the desired terms from Eq.(4.7) are obtained, but we also obtain all of the other terms which account for the interaction of the input factors $X_{\sim i}$ with each other leading instead to Eq.(4.6). This can be understood through Eq. (4.8), which shows that the inner expectation in the numerator is conditioned on all of the elements of $X$, which are assigned fixed values $x^{*}$. The outer variance is then computed, varying all of the elements of $X=x^{*}$. In doing so, one can intuitively see that this operation is identical to calculating the unconditional variance $V(Y)$ as in the numerator below,

$$
1 \equiv \frac{V_{X_{1}, \ldots, X_{i}, \ldots, X_{k}}\left(E_{\varnothing}\left(Y \mid X_{1}, \ldots, X_{i}, \ldots, X_{k}\right)\right)}{V(Y)} .
$$

Hence, in order to obtain the total effect Sobol' index for $X_{i}$, it will be necessary to instead subtract the terms which do not account for any interaction with parameter $X_{i}$ from unity [12], i.e.,

$$
S_{T_{i}}=1-\frac{V_{X_{1}, \ldots, X_{i-1}, X_{i+1}, \ldots, X_{k}}\left(E_{X_{i}}\left(Y \mid X_{1}, \ldots, X_{i-1}, X_{i+1}, \ldots, X_{k}\right)\right)}{V(Y)} .
$$

It means that the total effect Sobol' index for $X_{i}$ is equal to one minus the total effect $S_{T_{\sim i}}$ of the set of parameters $X_{\sim i}[12]$,

$$
S_{T_{i}}=1-\frac{V_{X_{\sim i}}\left(E_{X_{i}}\left(Y \mid X_{\sim i}\right)\right)}{V(Y)} .
$$




\subsection{Sensitivity Indices by Monte-Carlo Sampling}

Having defined the sensitivity indices of interest, namely the first order sensitivity indices $S_{i}$ and the total effect indices $S_{T_{i}}$, we will now discuss how to compute them numerically using Monte-Carlo Sampling [11]. Although the Monte-Carlo Sampling method is not used in calculating the sensitivity indices in this thesis, the author believes that the process explained herein provides an intuitive framework for grasping the mathematical expression for the Sobol' indices in Eq. 4.6.

In a sampling based approach, for each uncertain input parameter $X_{i}(i=1,2, \ldots, k)$, a set of $N$ samples, $x_{i}^{(n)}(n=1, \ldots, N)$ are generated from their marginal distributions using Monte Carlo (MC) sampling. The use of marginal distributions is justified by the assumption that the inputs factors are independent [12]. Arranging these samples into a matrix, we get

$$
X=\left[\begin{array}{llll}
x_{1}^{(1)} & x_{2}^{(1)} & \ldots & x_{k}^{(1)} \\
x_{1}^{(2)} & x_{2}^{(2)} & \ldots & x_{k}^{(2)} \\
\vdots & \vdots & \ddots & \vdots \\
x_{1}^{(N)} & x_{2}^{(N)} & \ldots & x_{k}^{(N)}
\end{array}\right]
$$

Evaluating the model $f$ for input $X^{(n)}=\left[x_{1}^{(n)}, \ldots, x_{k}^{(n)}\right], n=1,2, \ldots, N$, produces a realization of the output $y^{(n)}$. Passing all $X^{(n)}$ for $n=1, \ldots, N$, through the model gives an $N \times 1$ vector of realizations of the model output $Y[12$,

$$
Y=\left[\begin{array}{c}
y^{(1)} \\
y^{(2)} \\
\vdots \\
y^{(N)}
\end{array}\right]
$$

This requires $N$ function evaluations, where $N$ must be sufficiently large in order to obtain reliable results. This is sufficient to allow us to calculate the variance of the output $V(Y)$, which is simply 


$$
V \approx \frac{1}{N} \sum_{n=1}^{N} f\left(X^{(n)}\right)^{2}-f_{0}^{2},
$$

where $f_{0}$ is simply the mean of the output $Y=f\left(X^{(n)}\right)$ given by

$$
f_{0} \approx \frac{1}{N} \sum_{n=1}^{N} f\left(X^{(n)}\right) .
$$

While the calculation of the variance of the output (the denominator in the Sobol' indices) is straight forward, requiring $N$ function evaluations, the numerators are not so straight forward. This is complicated due to the inner expectation of the output $Y$ being conditioned on a fixed value of $X_{i}=x_{i}{ }^{*}$ from the set of samples generated for parameter $X_{i}$ (i.e. $x_{i}{ }^{*} \in x_{i}$ ), while the other input factors $X_{\sim i}$ are varied as noted from Eq.4.6. $N$ function evaluations are required to obtain the output vector of $f\left(X_{1}, \ldots, X_{i}=x_{i}{ }^{*}, \ldots, X_{k}\right)$,

$$
\left(X_{1}, \ldots, X_{i}=x_{i}{ }^{*}, \ldots, X_{k}\right)=\left[\begin{array}{ccccc}
x_{1}^{(1)} & \ldots & x_{i}=x_{i}{ }^{*} & \ldots & x_{k}^{(1)} \\
x_{1}^{(2)} & \ldots & x_{i}=x_{i}{ }^{*} & \ldots & x_{k}^{(2)} \\
\vdots & \ddots & \vdots & \ddots & \vdots \\
x_{1}^{(N)} & \ldots & x_{i}=x_{i}{ }^{*} & \ldots & x_{k}^{(N)}
\end{array}\right]
$$

Thus, we can write,

$$
\begin{aligned}
E_{X_{\sim i}}\left(Y \mid X_{i}=x_{i}{ }^{*}\right) & =E_{X_{\sim i}}\left(f\left(x_{1}, \ldots, x_{i}=x_{i}{ }^{*}, \ldots, x_{k}\right)^{(n)}\right) \\
& \approx \frac{1}{N} \sum_{n=1}^{N} f\left(x_{1}, \ldots, x_{i}=x_{i}{ }^{*}, \ldots, x_{k}\right)^{(n)} .
\end{aligned}
$$

The outer variance is calculated as the inner expectation is evaluated for each value of $x_{i}{ }^{*}=x_{i}^{(n)}$ where $n=1, \ldots, N$. Hence, for $N$ evaluations per inner expectation, and $N$ inner expectations per outer variance calculation, we require $N \times N$ function evaluations to generate the full ensemble, 


$$
\begin{aligned}
E_{X_{\sim i}}\left(Y \mid X_{i}=x_{i}^{(1)}\right) & =E_{X_{\sim i}}\left(f\left(x_{1}, \ldots, X_{i}=x_{i}^{(1)}, \ldots, x_{k}\right)^{(n)}\right), \\
E_{X_{\sim i}}\left(Y \mid X_{i}=x_{i}^{(2)}\right) & =E_{X_{\sim i}}\left(f\left(x_{1}, \ldots, X_{i}=x_{i}^{(2)}, \ldots, x_{k}\right)^{(n)}\right), \\
& \vdots \\
E_{X_{\sim i}}\left(Y \mid X_{i}=x_{i}^{(N)}\right) & =E_{X_{\sim i}}\left(f\left(x_{1}, \ldots, X_{i}=x_{i}^{(N)}, \ldots, x_{k}\right)^{(n)}\right) .
\end{aligned}
$$

Finally, we calculate the variance of these $N$ inner expectations

$$
V_{X_{i}}\left(E_{X_{\sim i}}\left(Y \mid X_{i}=x_{i}{ }^{*}\right)\right) \approx \frac{1}{N} \sum_{n=1}^{N} E_{X_{\sim i}}\left(Y \mid X_{i}=x_{i}^{(n)}\right)^{2}-E_{X_{i}}\left(E_{X_{\sim i}}\left(Y \mid X_{i}=x_{i}^{(n)}\right)\right)^{2},
$$

where,

$$
E_{X_{i}}\left(E_{X_{\sim i}}\left(Y \mid X_{i}=x_{i}{ }^{*}\right)\right) \approx \frac{1}{N} \sum_{n=1}^{N} E_{X_{\sim i}}\left(Y \mid X_{i}=x_{i}^{(n)}\right) .
$$

This is a cumbersome process, and for complex models, the computational cost of this brute force method may be prohibitive.

Saltelli has proposed a more efficient sampling method which reduces the cost of calculating the sensitivity indices to $N \times(k+2)[54]$. In Saltelli's method, 2 matrices, $\mathbf{A}$ and $\mathbf{B}$ are created from $2 \times N$ samples of the $k$ uncertain inputs defined by [54,

$$
\mathbf{A}=\left[\begin{array}{ccccc}
x_{1}^{(1)} & \ldots & x_{i}^{(1)} & \ldots & x_{k}^{(1)} \\
x_{1}^{(2)} & \ldots & x_{i}^{(2)} & \ldots & x_{k}^{(2)} \\
\vdots & \ddots & \vdots & \ddots & \vdots \\
x_{1}^{(N)} & \ldots & x_{i}^{(N)} & \ldots & x_{k}^{(N)}
\end{array}\right], \quad \mathbf{B}=\left[\begin{array}{ccccc}
x_{1}^{(N+1)} & \ldots & x_{i}^{(N+1)} & \ldots & x_{k}^{(N+1)} \\
x_{1}^{(N+2)} & \ldots & x_{i}^{(N+2)} & \ldots & x_{k}^{(N+2)} \\
\vdots & \ddots & \vdots & \ddots & \vdots \\
x_{1}^{(2 N)} & \ldots & x_{i}^{(2 N)} & \ldots & x_{k}^{(2 N)}
\end{array}\right] .
$$


Subsequently, we create $2 k$ matrices, $\mathbf{C}_{i}$ and $\mathbf{D}_{i}$, for $i=1, \ldots, k$. We generate these matrices without requiring any further sampling. Matrix $\mathbf{C}_{i}$ is simply the $\mathbf{B}$ matrix, with the $i^{\text {th }}$ column being replaced by the $i^{t h}$ column from the matrix $\mathbf{A}$. In the same manner, we construct matrices $\mathbf{D}_{i}$ as the $\mathbf{A}$ matrix with the $i^{\text {th }}$ column being replaced by the $i^{\text {th }}$ column from the matrix $\mathbf{B}$ as given by [54,

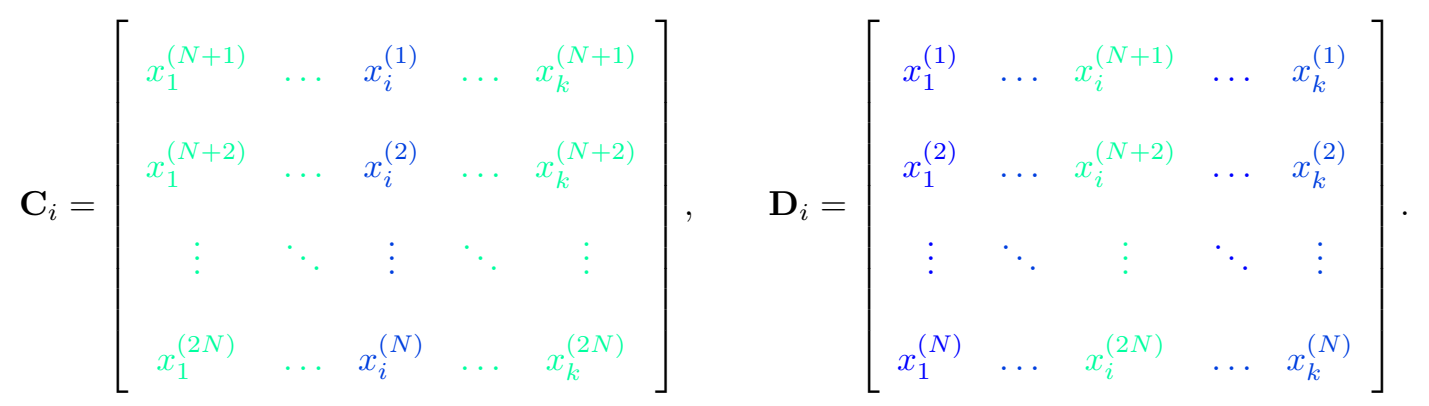

Let the realizations of the model for the matrix inputs be $Y_{A}=f(\mathbf{A}), Y_{B}=f(\mathbf{B}), Y_{C_{i}}=f\left(\mathbf{C}_{i}\right)$ and $Y_{D_{i}}=f\left(\mathbf{D}_{i}\right)$. Define Sobol' indices as [54,

$$
S_{i} \approx \frac{Y_{A} \cdot Y_{C_{i}}-E(Y)^{2}}{Y_{A} \cdot Y_{A}-E(Y)^{2}}=\frac{\frac{1}{N} \sum_{n=1}^{N}\left(Y_{A}^{(n)} Y_{C_{i}}^{(n)}-\left(\frac{1}{N} \sum_{n=1}^{N} Y_{A}^{(n)}\right)^{2}\right)}{\frac{1}{N} \sum_{n=1}^{N}\left(\left(Y_{A}^{(n)}\right)^{2}-\left(\frac{1}{N} \sum_{n=1}^{N} Y_{A}^{(n)}\right)^{2}\right)} .
$$

Likewise the total Sobol' indices are [54],

$$
S_{T_{i}} \approx \frac{Y_{A} \cdot Y_{D_{i}}-E(Y)^{2}}{Y_{A} \cdot Y_{A}-E(Y)^{2}}=\frac{\frac{1}{N} \sum_{n=1}^{N}\left(Y_{A}^{(n)} Y_{D_{i}}^{(n)}-\left(\frac{1}{N} \sum_{n=1}^{N} Y_{A}^{(n)}\right)^{2}\right)}{\frac{1}{N} \sum_{n=1}^{N}\left(\left(Y_{A}^{(n)}\right)^{2}-\left(\frac{1}{N} \sum_{n=1}^{N} Y_{A}^{(n)}\right)^{2}\right)}
$$

Further refinements to the above method using the $\mathbf{A}, \mathbf{B}, \mathbf{C}_{i}$, and $\mathbf{D}_{i}$ matrices are proposed in Tarantola et al. (2006) [55]. These refinements suggest that the accuracy of the estimate of the Sobol' indices can be improved by changing the way we calculate the mean squared term, $E(Y)^{2}$, and in general how we calculate the variance $V(Y)$ (i.e. the denominator of Eq.(4.23) and Eq.(4.24)). Further discussions on this topic are beyond the scope of this thesis, but readers can refer to Lilburne 
and Tarantola (2009) 56.

\subsection{Sensitivity Indices by Analytical Calculation}

Sobol [10] considered the expansion of a generic nonlinear function, $Y=f(X)$ shown in Eq.4.25. $X=\left\{X_{1}, X_{2}, \ldots, X_{k}\right\} \in \Omega \subset \mathbb{R}^{k}$ are uniform random variables on $\left[\begin{array}{ll}0 & 1\end{array}\right]$, such that $\Omega=\left[\begin{array}{ll}0 & 1\end{array}\right]^{k}$, and $X \sim \mathcal{U}(0,1)$. This function, $f$ is decomposed into a finite series of terms, ordered hierarchically such that [10],

$$
\begin{aligned}
f=f_{0} & +\sum_{i} f_{i}\left(X_{i}\right)+\sum_{1 \leq i<j \leq k} f_{i j}\left(X_{i}, X_{j}\right)+\ldots \\
& +\sum_{1 \leq i_{1}<\ldots<i_{s} \leq k} f_{i_{1}, \ldots, i_{s}}\left(X_{i_{1}}, \ldots, X_{i_{s}}\right)+\ldots+f_{12 \ldots k}\left(X_{1}, X_{2}, \ldots, X_{k}\right) .
\end{aligned}
$$

Understanding the function of the summation operator for the higher order terms, $\sum_{1 \leq i_{1}<\ldots<i_{s} \leq k}$, as one can more compactly write the above in a more familiar form as 32 ,

$$
f=f_{0}+\sum_{i} f_{i}\left(X_{i}\right)+\sum_{i} \sum_{j>i} f_{i j}\left(X_{i}, X_{j}\right)+\ldots+f_{1,2, \ldots, k}\left(X_{1}, X_{2}, \ldots, X_{k}\right)
$$

In the context of GSA, this representation has been referred to as a Sobol' decomposition [13, Sobol' expansion [32], or Sobol's variance decomposition [12]. This thesis will adopt the use of the term Sobol' decomposition, however, it is important to acknowledge that this representation of a function $f$ has the same form as the Analysis of Variance High Dimensional Model Representation (ANOVA-HDMR) [32], [12]. There are other variants of high dimensional model representations besides the ANOVA-HDMR, which circumvent the computational burden of evaluating highdimensional integrals which must be evaluated to obtain the expression in Eq.4.25. Variants such as the Random sampling, RS-HDMR, and the cut-HDMR adopt approximate methods to obtain similar expressions to Eq. 4.25). 


\section{Example 4.1}

The Sobol' decomposition (or ANOVA-HDMR) of a function of 3 uncertain inputs $f(X)$, where $X=$ $\left\{X_{1}, X_{2}, X_{3}\right\}$ is,

$$
\begin{aligned}
f & =f_{0}+\sum_{i} f_{i}\left(X_{i}\right)+\sum_{i} \sum_{j>i} f_{i j}\left(X_{i}, X_{j}\right)+f_{1,2,3}\left(X_{1}, X_{2}, X_{3}\right) \\
& =f_{0}+f_{1}\left(X_{1}\right)+f_{2}\left(X_{2}\right)+f_{3}\left(X_{3}\right)+f_{12}\left(X_{1}, X_{2}\right)+f_{13}\left(X_{1}, X_{3}\right)+f_{23}\left(X_{2}, X_{3}\right)+f_{123}\left(X_{1}, X_{2}, X_{3}\right) .
\end{aligned}
$$

It is important to note that this representation of the model is not an approximation by a truncated infinite series, but rather an exact expansion containing $2^{k}$ terms $[32] . \quad f_{0}$ is a constant term representing the mean of $f$, while $f_{i}$ are first order terms, representing the contributions of each individual parameter $X_{i}$ to the output of $f . f_{i j}$ are second-order bivariate terms, representing the contribution of the interaction of parameters $X_{i}$ and $X_{j}$ on $f$. Similarly, the higher order terms $f_{i_{1}, \ldots, i_{s}}\left(X_{i_{1}}, \ldots, X_{i_{s}}\right)$, are functions of proper subsets of the uncertain input vector $X$, representing higher-order interaction effects. Finally, the last term, $f_{1,2, \ldots, k}\left(X_{1}, X_{2}, \ldots, X_{k}\right)$ accounts for any residual higher order effects, lending to the fact that this expansion is an exact representation of the function, $f$, rather than an approximation.

In general, this expansion of $f$ is not unique, however, it is shown in $[10$ that there exists a unique representation for the case where all terms but $f_{0}$ are zero mean, and where all of the terms in the expansion are statistically orthogonal in pairs.

The first of the above conditions, requires that the integrals of the terms $f_{i_{1}, \ldots, i_{s}}\left(X_{i_{1}}, \ldots, X_{i_{s}}\right)$ (i.e. all terms but the mean term, $f_{0}$ ), be equal to zero when taken with respect to variable $X_{i_{p}}$, where $X_{i_{p}} \in\left\{X_{i_{1}}, \ldots, X_{i_{s}}\right\} \subset X$

$$
\int_{0}^{1} f_{i_{1}, \ldots, i_{s}}\left(X_{i_{1}}, \ldots, X_{i_{s}}\right) d X_{i_{p}}=0, \quad i_{1} \leq i_{p} \leq i_{s}
$$


This first condition further implies that 10

$$
\int_{\Omega} f(X) d X=f_{0}
$$

The latter condition, requires that the inner product of any two terms, $f_{i_{1}, \ldots, i_{s}}\left(X_{i_{1}}, \ldots, X_{i_{s}}\right)$ and $f_{j_{1}, \ldots, j_{q}}\left(X_{j_{1}}, \ldots, X_{j_{q}}\right)$, where $\left(i_{1}, \ldots, i_{s}\right) \neq\left(j_{1}, \ldots, j_{q}\right)$, be zero over the domain $\Omega$. Note that the indices are such that the orders of $f_{i_{1}, \ldots, i_{s}}$ and $f_{j_{1}, \ldots, j_{q}}$ need not be the same (although they may be); the only requirement is that there be some element in the set $\left(i_{1}, \ldots, i_{s}\right)$ that is not in $\left(j_{1}, \ldots, j_{q}\right)$ or vise versa, i.e.

$$
\int_{\Omega} f_{i_{1}, \ldots, i_{s}}\left(X_{i_{1}}, \ldots, X_{i_{s}}\right) f_{j_{1}, \ldots, j_{q}}\left(X_{j_{1}}, \ldots, X_{j_{q}}\right) d X=0
$$

Because of these two conditions in Eq.4.27) and Eq.4.29, one can show that 32

$$
\begin{aligned}
f_{0} & =E(Y) \\
f_{i} & =E\left(Y \mid X_{i}\right)-E(Y), \\
f_{i j} & =E\left(Y \mid X_{i}, X_{j}\right)-E\left(Y \mid X_{i}\right)-E\left(Y \mid X_{j}\right)-E(Y) .
\end{aligned}
$$

The above holds for higher order terms as well, however only the zeroth, first and second order terms of the Sobol' decomposition are shown for illustrative purposes. One can arrive at the above expressions by writing the following integral expressions for the unconditioned and conditional expected values of the output $Y$. The expected value of the output $Y$ is simply the multi-dimensional integral of $f(X)$ over all elements of $X$ over the domain $\Omega$ (the $k$-dimensional hypercube on $\left[\begin{array}{ll}0 & 1\end{array}\right]$ ) as in Eq.4.33). Likewise the expected value of $Y$ conditioned on some element(s) of $X$ is given by the integral of $f(X)$ over all elements of $X$ but those upon which the output is conditioned as in Eq.4.34 and Eq.4.35. The condition expressed in Eq.4.27 ensures that the functions (the terms of the Sobol' decomposition) are orthonormal such that the following expressions can be obtained 32 : 


$$
\begin{aligned}
& E(Y)=\int_{0}^{1} f(X) d X \\
& =\int_{0}^{1} f_{0}+\sum_{i} f_{i}\left(X_{i}\right)+\sum_{i} \sum_{j>i} f_{i j}\left(X_{i}, X_{j}\right)+\ldots+f_{1,2, \ldots, k}\left(X_{1}, X_{2}, \ldots, X_{k}\right) d X \\
& =\int_{0}^{1} f_{0} d X+\sum_{i} \int_{0}^{1} f_{i}\left(X_{i}\right) d X+\sum_{i} \sum_{j>i} \int_{0}^{1} f_{i j}\left(X_{i}, X_{j}\right) d X \\
& +\ldots+\int_{0}^{1} f_{1,2, \ldots, k}\left(X_{1}, X_{2}, \ldots, X_{k}\right) d X \\
& =f_{0} \int_{0}^{1} d X \\
& =f_{0} \\
& E\left(Y \mid X_{i}\right)=\int_{0}^{1} f(X) d X_{\sim i} \\
& =\int_{0}^{1} f_{0}+\sum_{i} f_{i}\left(X_{i}\right)+\sum_{i} \sum_{j>i} f_{i j}\left(X_{i}, X_{j}\right)+\ldots+f_{1,2, \ldots, k}\left(X_{1}, X_{2}, \ldots, X_{k}\right) d X_{\sim i} \\
& =\int_{0}^{1} f_{0} d X_{\sim i}+\sum_{i} \int_{0}^{1} f_{i}\left(X_{i}\right) d X_{\sim i}+\sum_{i} \sum_{j>i} \int_{0}^{1} f_{i j}\left(X_{i}, X_{j}\right) d X_{\sim i} \\
& +\ldots+\int_{0}^{1} f_{1,2, \ldots, k}\left(X_{1}, X_{2}, \ldots, X_{k}\right) d X_{\sim i} \\
& =f_{0} \int_{0}^{1} d X_{\sim i}+f_{i}\left(X_{i}\right) \int_{0}^{1} d X_{\sim i}+\sum_{j \neq i} \int_{0}^{1} f_{j}\left(X_{j}\right) d X_{\sim i} \\
& =f_{0}+f_{i}\left(X_{i}\right) \\
& E\left(Y \mid X_{i}, X_{j}\right)=\int_{0}^{1} f(X) d X_{\sim i j} \\
& =\int_{0}^{1} f_{0}+\sum_{i} f_{i}\left(X_{i}\right)+\sum_{i} \sum_{j>i} f_{i j}\left(X_{i}, X_{j}\right)+\ldots+f_{1,2, \ldots, k}\left(X_{1}, X_{2}, \ldots, X_{k}\right) d X_{\sim i j} \\
& =\int_{0}^{1} f_{0} d X_{\sim i j}+\sum_{i} \int_{0}^{1} f_{i}\left(X_{i}\right) d X_{\sim i j}+\sum_{i} \sum_{j>i} \int_{0}^{1} f_{i j}\left(X_{i}, X_{j}\right) d X_{\sim i j} \\
& +\ldots+\int_{0}^{1} f_{1,2, \ldots, k}\left(X_{1}, X_{2}, \ldots, X_{k}\right) d X_{\sim i j} \\
& =f_{0} \int_{0}^{1} d X_{\sim i}+f_{i}\left(X_{i}\right) \int_{0}^{1} d X_{\sim i j}+f_{j}\left(X_{j}\right) \int_{0}^{1} d X_{\sim i j}+\sum_{l \neq i, l \neq j} \int_{0}^{1} f_{l}\left(X_{l}\right) d X_{\sim i j} \\
& +f_{i j}\left(X_{i}, X_{j}\right) \int_{0}^{1} d X_{\sim i j}+\sum_{l \neq i} \sum_{m>l} \int_{0}^{1} f_{l m}\left(X_{l}, X_{m}\right) d X_{\sim i j}+\sum_{m>i, m \neq j} \int_{0}^{1} f_{i m}\left(X_{i}, X_{m}\right) d X_{\sim i j} \\
& =f_{0}+f_{i}\left(X_{i}\right)+f_{j}\left(X_{j}\right)+f_{i j}\left(X_{i}, X_{j}\right)
\end{aligned}
$$


Eq.4.33 shows how one would arrive at the statement in Eq.4.30). Rearranging Eq.(4.34) and substituting the expression for $f_{0}$ from Eq.4.33 yields Eq.4.31. Similarly, Eq.4.32 can be obtained from Eq.4.35, by substituting $f_{0}$ from Eq.4.33) and substituting Eq.4.34 for the case of $X_{i}$, and $X_{j}$.

Furthermore, we can demonstrate how the orthogonality condition in Eq. (4.29) allows us to obtain analytical expressions for the variances, leading directly to the sensitivity indices. Through a similar (although more mathematically complex) approach, we will derive the variances of the expected values in Eq.4.33, Eq.4.34 and Eq.4.35, and rearrange to show the following,

$$
\begin{aligned}
V\left(f_{0}\right) & =0 \\
V\left(f_{i}\right) & =V\left(E\left(Y \mid X_{i}\right)\right), \\
V\left(f_{i j}\right) & =V\left(E\left(Y \mid X_{i}, X_{j}\right)\right)-V\left(E\left(Y \mid X_{i}\right)\right)-V\left(E\left(Y \mid X_{j}\right)\right) .
\end{aligned}
$$

$$
\begin{aligned}
V(E(Y)) & =\int_{0}^{1}(E(Y)-E(E(Y)))^{2} d X \\
& =\int_{0}^{1}\left(\left(f_{0}\right)-E\left(f_{0}\right)\right)^{2} d X \\
& =\int_{0}^{1}\left(f_{0}-f_{0}\right)^{2} d X \\
& =0
\end{aligned}
$$

$$
\begin{aligned}
V\left(E\left(Y \mid X_{i}\right)\right) & =\int_{0}^{1}\left(E\left(Y \mid X_{i}\right)-E\left(E\left(Y \mid X_{i}\right)\right)\right)^{2} d X_{i} \\
& =\int_{0}^{1}\left(\left(f_{0}+f_{i}\left(X_{i}\right)\right)-E\left(f_{0}+f_{i}\left(X_{i}\right)\right)\right)^{2} d X_{i} \\
& =\int_{0}^{1}\left(\left(f_{0}+f_{i}\left(X_{i}\right)\right)-f_{0}\right)^{2} d X_{i} \\
& =\int_{0}^{1}\left(f_{i}\left(X_{i}\right)\right)^{2} d X_{i} \\
& =V\left(f_{i}\left(X_{i}\right)\right)
\end{aligned}
$$




$$
\begin{aligned}
V\left(E\left(Y \mid X_{i}, X_{j}\right)\right)= & \int_{0}^{1}\left(E\left(Y \mid X_{i}, X_{j}\right)-E\left(E\left(Y \mid X_{i}, X_{j}\right)\right)^{2} d X_{i j}\right. \\
= & \int_{0}^{1}\left(\left(f_{0}+f_{i}\left(X_{i}\right)+f_{j}\left(X_{j}\right)+f_{i j}\left(X_{i}, X_{j}\right)\right)-E\left(f_{0}+f_{i}\left(X_{i}\right)+f_{j}\left(X_{j}\right)+f_{i j}\left(X_{i}, X_{j}\right)\right)^{2} d X_{i j}\right. \\
= & \int_{0}^{1}\left(\left(f_{0}+f_{i}\left(X_{i}\right)+f_{j}\left(X_{j}\right)+f_{i j}\left(X_{i}, X_{j}\right)\right)-f_{0}\right)^{2} d X_{i j} \\
= & \int_{0}^{1}\left(f_{i}\left(X_{i}\right)+f_{j}\left(X_{j}\right)+f_{i j}\left(X_{i}, X_{j}\right)\right)^{2} d X_{i j} \\
= & \int_{0}^{1} f_{i}\left(X_{i}\right)^{2}+2 f_{i}\left(X_{i}\right) f_{j}\left(X_{j}\right)+2 f_{i}\left(X_{i}\right) f_{i j}\left(X_{i}, X_{j}\right)+f_{j}\left(X_{j}\right)^{2}+2 f_{j}\left(X_{j}\right) f_{i j}\left(X_{i}, X_{j}\right) \\
& \quad+f_{i j}\left(X_{i}, X_{j}\right)^{2} d X_{i j} \\
= & \int_{0}^{1} f_{i}\left(X_{i}\right)^{2} d X+2 \int_{0}^{1} f_{i}\left(X_{i}\right) f_{j}\left(X_{j}\right) d X+2 \int_{0}^{1} f_{i}\left(X_{i}\right) f_{i j}\left(X_{i}, X_{j}\right) d X_{i j} \\
& \quad+\int_{0}^{1} f_{j}\left(X_{j}\right)^{2} d X+2 \int_{0}^{1} f_{j}\left(X_{j}\right) f_{i j}\left(X_{i}, X_{j}\right) d X+\int_{0}^{1} f_{i j}\left(X_{i}, X_{j}\right)^{2} d X_{i j} \\
= & V\left(f_{i}\left(X_{i}\right)\right)+V\left(f_{j}\left(X_{j}\right)\right)+V\left(f_{i j}\left(X_{i}, X_{j}\right)\right)
\end{aligned}
$$

We can also calculate the unconditional variance of the output $Y$ as follows:

$$
\begin{aligned}
V(Y)= & \int_{0}^{1}(f(X)-E(Y))^{2} d X \\
= & \int_{0}^{1} f(X)^{2}-2 f(X) E(Y)+E(Y)^{2} d X \\
= & \int_{0}^{1} f(X)^{2} d X-2 E(Y) \int_{0}^{1} f(X) d X+E(Y)^{2} \int_{0}^{1} d X \\
= & \int_{0}^{1} f(X)^{2} d X-E(Y)^{2} \\
= & \int_{0}^{1}\left(f_{0}+\sum_{i} f_{i}\left(X_{i}\right)+\sum_{i} \sum_{j>i} f_{i j}\left(X_{i}, X_{j}\right)+\ldots+f_{1,2, \ldots, k}\left(X_{1}, X_{2}, \ldots, X_{k}\right)\right)^{2} d X-E(Y)^{2} \\
= & \int_{0}^{1}\left(f_{0}^{2}+2 f_{0} \sum_{i} f_{i}\left(X_{i}\right)+2 f_{0} \sum_{i} \sum_{j>i} f_{i j}\left(X_{i}, X_{j}\right)+\ldots+2 f_{0} f_{1,2, \ldots, k}\left(X_{1}, X_{2}, \ldots, X_{k}\right)\right. \\
& \quad+\sum_{i} f_{i}\left(X_{i}\right)^{2}+2 \sum_{i} \sum_{j>i} f_{i}\left(X_{i}\right) f_{i j}\left(X_{i}, X_{j}\right)+\ldots+2 \sum_{i} f_{i}\left(X_{i}\right) f_{1,2, \ldots, k}\left(X_{1}, X_{2}, \ldots, X_{k}\right) \\
& \quad+\sum_{i} \sum_{j>i} f_{i j}\left(X_{i}, X_{j}\right)^{2}+\ldots+2 \sum_{i} \sum_{j>i} f_{i j}\left(X_{i}, X_{j}\right) f_{1,2, \ldots, k}\left(X_{1}, X_{2}, \ldots, X_{k}\right) \\
& \left.+f_{1,2, \ldots, k}\left(X_{1}, X_{2}, \ldots, X_{k}\right)^{2}\right) d X-E(Y)^{2} \\
= & \int_{0}^{1}\left(f_{0}^{2}+\sum_{i} f_{i}\left(X_{i}\right)^{2}+\sum_{i} \sum_{j>i} f_{i j}\left(X_{i}, X_{j}\right)^{2}+\ldots+f_{1,2, \ldots, k}\left(X_{1}, X_{2}, \ldots, X_{k}\right)^{2}\right) d X-E(Y)^{2} \\
= & f_{0}^{2} \int_{0}^{1} d X+\sum_{i} \int_{0}^{1} f_{i}\left(X_{i}\right)^{2} d X+\sum_{i} \sum_{j>i} \int_{0}^{1} f_{i j}\left(X_{i}, X_{j}\right)^{2} d X
\end{aligned}
$$




$$
\begin{aligned}
& +\ldots+\int_{0}^{1} f_{1,2, \ldots, k}\left(X_{1}, X_{2}, \ldots, X_{k}\right)^{2} d X-E(Y)^{2} \\
= & \sum_{i} \int_{0}^{1} f_{i}\left(X_{i}\right)^{2} d X+\sum_{i} \sum_{j>i} \int_{0}^{1} f_{i j}\left(X_{i}, X_{j}\right)^{2} d X+\ldots+\int_{0}^{1} f_{1,2, \ldots, k}\left(X_{1}, X_{2}, \ldots, X_{k}\right)^{2} d X \\
= & \sum_{i} V\left(f_{i}\left(X_{i}\right)\right)+\sum_{i} \sum_{j>i} V\left(f_{i j}\left(X_{i}, X_{j}\right)\right)+\ldots+V\left(f_{1,2, \ldots, k}\left(X_{1}, X_{2}, \ldots, X_{k}\right)\right)
\end{aligned}
$$

Should we divide both sides of the equation by $V(Y)$, we obtain a summation of normalized variances adding up to unity. Recalling Eq.4.6), one obtains the analytical expressions of the Sobol' indices as

$$
\begin{aligned}
\frac{V(Y)}{V(Y)}=\sum_{i} \frac{V\left(f_{i}\left(X_{i}\right)\right)}{V(Y)} & +\sum_{i} \sum_{j>i} \frac{V\left(f_{i j}\left(X_{i}, X_{j}\right)\right)}{V(Y)}+\ldots+\frac{V\left(f_{1,2, \ldots, k}\left(X_{1}, X_{2}, \ldots, X_{k}\right)\right)}{V(Y)}, \\
1 & =\sum_{i} S_{i}+\sum_{i} \sum_{j>i} S_{i j}+\ldots+S_{1,2, \ldots, k} .
\end{aligned}
$$

Note that, while the above procedure describes the variance decomposition for the uniform density $\mathcal{U}(0,1)$, similar procedures exist for general probability densities as outlined by 32 .

\section{Example 4.2}

For a function of 3 uncertain inputs $Y=f(X), X=\left\{X_{1}, X_{2}, X_{3}\right\}$ we have

$$
f=f_{0}+\sum_{i} f_{i}\left(X_{i}\right)+\sum_{i} \sum_{j>i} f_{i j}\left(X_{i}, X_{j}\right)+f_{123}\left(X_{1}, X_{2}, X_{3}\right)
$$

with variance $V(Y)$

$$
\begin{aligned}
V(Y)= & \sum_{i} V\left(f_{i}\left(X_{i}\right)\right)+\sum_{i} \sum_{j>i} V\left(f_{i j}\left(X_{i}, X_{j}\right)\right)+V\left(f_{1,2,3}\left(X_{1}, X_{2}, X_{3}\right)\right) \\
= & V\left(f_{1}\left(X_{1}\right)\right)+V\left(f_{2}\left(X_{2}\right)\right)+V\left(f_{3}\left(X_{3}\right)\right)+V\left(f_{12}\left(X_{1}, X_{2}\right)\right)+V\left(f_{13}\left(X_{1}, X_{3}\right)\right)+V\left(f_{23}\left(X_{2}, X_{3}\right)\right) \\
& \quad+V\left(f_{123}\left(X_{1}, X_{2}, X_{3}\right)\right) .
\end{aligned}
$$

Where, 


$$
\begin{aligned}
& V\left(f_{0}\right)= \\
& V\left(f_{1}\right)=V\left(E\left(Y \mid X_{1}\right)\right) \\
& V\left(f_{2}\right)=V\left(E\left(Y \mid X_{2}\right)\right) \\
& V\left(f_{3}\right)=V\left(E\left(Y \mid X_{3}\right)\right) \\
& V\left(f_{12}\right)=V\left(E\left(Y \mid X_{1}, X_{2}\right)\right)-V\left(E\left(Y \mid X_{1}\right)\right)-V\left(E\left(Y \mid X_{2}\right)\right), \\
& V\left(f_{13}\right)=V\left(E\left(Y \mid X_{1}, X_{3}\right)\right)-V\left(E\left(Y \mid X_{1}\right)\right)-V\left(E\left(Y \mid X_{3}\right)\right), \\
& V\left(f_{23}\right)=V\left(E\left(Y \mid X_{2}, X_{3}\right)\right)-V\left(E\left(Y \mid X_{2}\right)\right)-V\left(E\left(Y \mid X_{3}\right)\right), \\
& V\left(f_{123}\right)=V\left(E\left(Y \mid X_{1}, X_{2}, X_{3}\right)\right)-\left(V\left(E\left(Y \mid X_{1}, X_{2}\right)\right)-V\left(E\left(Y \mid X_{1}\right)\right)-V\left(E\left(Y \mid X_{2}\right)\right)\right) \\
& \quad-\quad\left(V\left(E\left(Y \mid X_{1}, X_{3}\right)\right)-V\left(E\left(Y \mid X_{1}\right)\right)-V\left(E\left(Y \mid X_{3}\right)\right)\right) \\
& \quad-\quad\left(V\left(E\left(Y \mid X_{2}, X_{3}\right)\right)-V\left(E\left(Y \mid X_{2}\right)\right)-V\left(E\left(Y \mid X_{3}\right)\right)\right) \\
& \quad-V\left(E\left(Y \mid X_{1}\right)\right)-V\left(E\left(Y \mid X_{2}\right)-V\left(E\left(Y \mid X_{3}\right)\right) .\right.
\end{aligned}
$$

Such that if one were to sum up all of the terms in the above equation for $V\left(f_{123}\right)$, they would arrive at the logical conclusion that the unconditional variance is equal to the variance of $Y$ conditioned upon all elements of $X$,

$$
V(Y)=V\left(E\left(Y \mid X_{1}, X_{2}, X_{3}\right)\right)
$$

\subsubsection{Polynomial Chaos Representation}

The approach that will be explored in this body of work is in creating a polynomial chaos expansion (PCE) of the output space. While a PCE of the output is not formally an HDMR since it is an approximation (requiring infinite terms to be an exact representation), the procedure outlined previously for the analytical calculation of sensitivity indices will apply here by analogy [32]. The PCE surrogate model and the resulting sensitivity indices are obtained using Sandia National Laboratories' Uncertainty Quantification Toolkit (UQTk) [57], however the requisite theoretical background for understanding the results is discussed below. 
There are a number of benefits to such a construction, firstly, once the surrogate model is constructed, sensitivity indices can be calculated analytically (rather than Monte Carlo sampling of the full model). Secondly, once the surrogate model is obtained, inexpensive Monte Carlo sampling can be performed to efficiently obtain marginal and joint probability density functions of quantities of interest. In this case, the function evaluations are very inexpensive, as we are evaluating the polynomial surrogate at randomly generated points, rather than evaluating the full model at these points.

Note that the number of terms in the Polynomial Chaos Expansion, $A$ increases exponentially as the dimensionality of the problem ( $k$ from before) and the order of expansion $(p)$ increase, according to the following expression,

$$
A=\frac{(k+p) !}{(k ! p !)}
$$

To illustrate the above, Table 4.1 below shows the size of the problem in terms of the number of terms in the expansion, as it increases exponentially for dimensionality ranging from 1 to 8 unknowns, and order of expansion increasing from $0^{\text {th }}$ order to $3^{\text {rd }}$ order.

The dimensionality of the problem, simply stated, is given by the cardinality of the set $X=\left\{X_{1}, X_{2}, \ldots, X_{k}\right\}$ (i.e. the dimensionality is $k$ ). Given that the point of GSA is to apportion the uncertainty in an output quantity of interest to various uncertain input factors, it is understood that there must be at least 2 uncertain input factors $(k \geq 2)$ in order to gain any useful insight.

For a given function $f$, which may be a function of space, time and uncertain input parameters, is approximated by the polynomial chaos expansion of the output space, $g$ (whose dependency on space and time is dictated by $f$ ). We model the vector of uncertain inputs $X_{i}, i=1, \ldots, k$ as a vector of random variables $\xi_{i}, i=1, \ldots, k$ hence $g$ is a function of random variables. The 


\begin{tabular}{cllll}
\hline \multirow{2}{*}{ Dimension } & \multicolumn{4}{c}{ Order } \\
\cline { 2 - 5 } & 0 & 1 & 2 & 3 \\
\hline \hline 1 & 1 & 2 & 3 & 4 \\
2 & 1 & 3 & 6 & 10 \\
3 & 1 & 4 & 10 & 20 \\
4 & 1 & 5 & 15 & 35 \\
5 & 1 & 6 & 21 & 56 \\
6 & 1 & 7 & 28 & 84 \\
7 & 1 & 8 & 36 & 120 \\
8 & 1 & 9 & 45 & 165 \\
\hline
\end{tabular}

Table 4.1: Number of terms in a PCE with increasing dimension and order

polynomial chaos expansion is given by the projection of deterministic polynomial chaos coefficients $c_{\alpha}$ (who, again, can vary in space and time per the nature $f$ ) onto a basis of orthogonal polynomials of random variables, $\Psi_{\alpha}(\xi) 32$,

$$
f(x, t, X) \approx g(x, t, \xi)=\sum_{\alpha=0}^{A-1} c_{\alpha}(x, t) \Psi_{\alpha}(\xi)
$$

\subsubsection{Polynomial Basis}

We will construct a $k$-dimensional basis using multivariate polynomials $\Psi(\xi)$. These multivariate polynomials will be the products of univariate polynomials $\psi_{\alpha_{i}}\left(\xi_{i}\right), i=1,2, \ldots, k$, where $k$ is the dimensionality of the problem (i.e. the number of uncertain input factors),

$$
\Psi_{\alpha}(\xi)=\prod_{i=1}^{k} \psi_{\alpha_{i}}\left(\xi_{i}\right)
$$

where $\alpha$ simply counts the multi-indices, $\alpha_{i}$. 
Table 4.2 summarizes the multi-indices and multivariate polynomials $\left\{\Psi_{\alpha}\left(\xi_{i}\right)\right\}_{\alpha=1}^{A}$ up to the third order with dimensionality $k=3$.

\begin{tabular}{|c|c|c|c|}
\hline $\mathrm{P}$ & $\alpha$ & $\left(\alpha_{1}, \alpha_{2}, \alpha_{3}\right)$ & $\Psi_{\alpha}$ \\
\hline \multirow[t]{2}{*}{0} & 0 & $(0,0,0)$ & $\Psi_{0}=\psi_{0}\left(\xi_{1}\right) \psi_{0}\left(\xi_{2}\right) \psi_{0}\left(\xi_{3}\right)$ \\
\hline & 1 & $(1,0,0)$ & $\Psi_{1}=\psi_{1}\left(\xi_{1}\right) \psi_{0}\left(\xi_{2}\right) \psi_{0}\left(\xi_{3}\right)$ \\
\hline \multirow[t]{5}{*}{1} & 2 & $(0,1,0)$ & $\Psi_{2}=\psi_{0}\left(\xi_{1}\right) \psi_{1}\left(\xi_{2}\right) \psi_{0}\left(\xi_{3}\right)$ \\
\hline & 3 & $(0,0,1)$ & $\Psi_{3}=\psi_{0}\left(\xi_{1}\right) \psi_{0}\left(\xi_{2}\right) \psi_{1}\left(\xi_{3}\right)$ \\
\hline & 4 & $(2,0,0)$ & $\Psi_{4}=\psi_{2}\left(\xi_{1}\right) \psi_{0}\left(\xi_{2}\right) \psi_{0}\left(\xi_{3}\right)$ \\
\hline & 5 & $(1,1,0)$ & $\Psi_{5}=\psi_{1}\left(\xi_{1}\right) \psi_{1}\left(\xi_{2}\right) \psi_{0}\left(\xi_{3}\right)$ \\
\hline & 6 & $(1,0,1)$ & $\Psi_{6}=\psi_{1}\left(\xi_{1}\right) \psi_{0}\left(\xi_{2}\right) \psi_{1}\left(\xi_{3}\right)$ \\
\hline \multirow[t]{7}{*}{2} & 7 & $(0,2,0)$ & $\Psi_{7}=\psi_{0}\left(\xi_{1}\right) \psi_{2}\left(\xi_{2}\right) \psi_{0}\left(\xi_{3}\right)$ \\
\hline & 8 & $(0,1,1)$ & $\Psi_{8}=\psi_{0}\left(\xi_{1}\right) \psi_{1}\left(\xi_{2}\right) \psi_{1}\left(\xi_{3}\right)$ \\
\hline & 9 & $(0,0,2)$ & $\Psi_{9}=\psi_{0}\left(\xi_{1}\right) \psi_{0}\left(\xi_{2}\right) \psi_{2}\left(\xi_{3}\right)$ \\
\hline & 10 & $(3,0,0)$ & $\Psi_{10}=\psi_{3}\left(\xi_{1}\right) \psi_{0}\left(\xi_{2}\right) \psi_{0}\left(\xi_{3}\right)$ \\
\hline & 11 & $(2,1,0)$ & $\Psi_{11}=\psi_{2}\left(\xi_{1}\right) \psi_{1}\left(\xi_{2}\right) \psi_{0}\left(\xi_{3}\right)$ \\
\hline & 12 & $(2,0,1)$ & $\Psi_{12}=\psi_{2}\left(\xi_{1}\right) \psi_{0}\left(\xi_{2}\right) \psi_{1}\left(\xi_{3}\right)$ \\
\hline & 13 & $(1,2,0)$ & $\Psi_{13}=\psi_{1}\left(\xi_{1}\right) \psi_{2}\left(\xi_{2}\right) \psi_{0}\left(\xi_{3}\right)$ \\
\hline \multirow[t]{6}{*}{3} & 14 & $(1,1,1)$ & $\Psi_{14}=\psi_{1}\left(\xi_{1}\right) \psi_{1}\left(\xi_{2}\right) \psi_{1}\left(\xi_{3}\right)$ \\
\hline & 15 & $(1,0,2)$ & $\Psi_{15}=\psi_{1}\left(\xi_{1}\right) \psi_{0}\left(\xi_{2}\right) \psi_{2}\left(\xi_{3}\right)$ \\
\hline & 16 & $(0,3,0)$ & $\Psi_{16}=\psi_{0}\left(\xi_{1}\right) \psi_{3}\left(\xi_{2}\right) \psi_{0}\left(\xi_{3}\right)$ \\
\hline & 17 & $(0,2,1)$ & $\Psi_{17}=\psi_{0}\left(\xi_{1}\right) \psi_{2}\left(\xi_{2}\right) \psi_{1}\left(\xi_{3}\right)$ \\
\hline & 18 & $(0,1,2)$ & $\Psi_{18}=\psi_{0}\left(\xi_{1}\right) \psi_{1}\left(\xi_{2}\right) \psi_{2}\left(\xi_{3}\right)$ \\
\hline & 19 & $(0,0,3)$ & $\Psi_{19}=\psi_{0}\left(\xi_{1}\right) \psi_{0}\left(\xi_{2}\right) \psi_{3}\left(\xi_{3}\right)$ \\
\hline
\end{tabular}

Table 4.2: Multi-indices and PC basis functions for $d=3, P=3$ (extended from 32 ) 


\section{Families of Orthogonal Polynomials}

For normalized random variable with a uniform distribution $\mathcal{U}(-1,1)$, univariate Legendre polynomials are used to generate an orthogonal basis [32. The first 6 Legendre polynomials are [58]:

$$
\begin{aligned}
& P_{0}(x)=1, \\
& P_{1}(x)=x \\
& P_{2}(x)=\frac{1}{2}\left(3 x^{2}-1\right), \\
& P_{3}(x)=\frac{1}{2}\left(5 x^{3}-3 x\right), \\
& P_{4}(x)=\frac{1}{8}\left(35 x^{4}-30 x^{2}+3\right), \\
& P_{5}(x)=\frac{1}{8}\left(63 x^{5}-70 x^{3}+15 x\right) .
\end{aligned}
$$

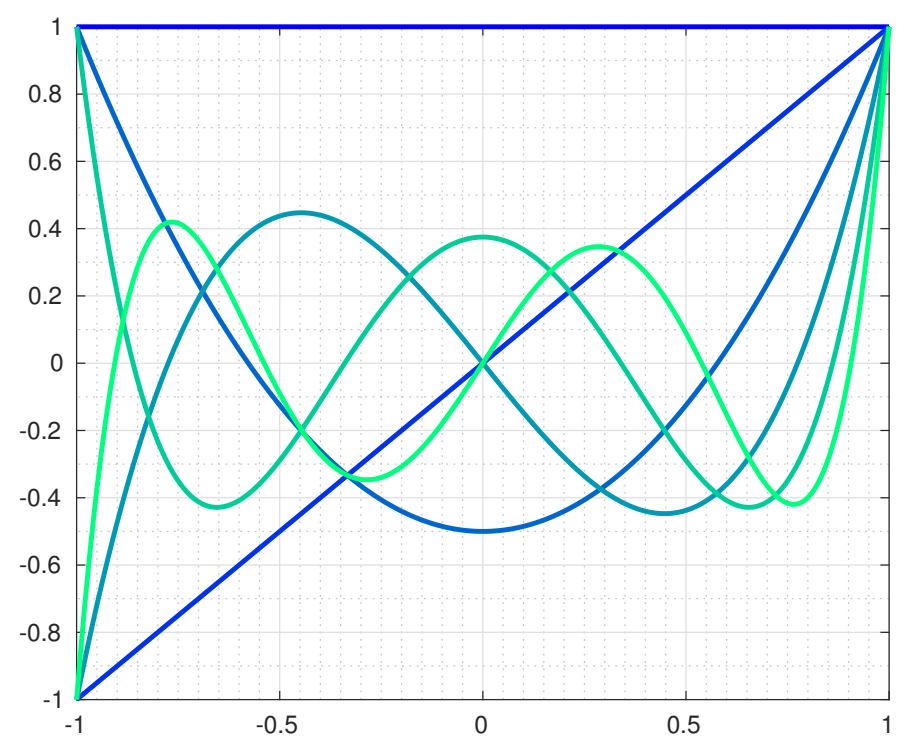

Figure 4.1: Unnormalized Legendre polynomials, $P_{n}(x)$ on $-1 \leq x \leq 1$, for $n=0, \ldots, 5$

It can be shown that these Legendre polynomials are orthogonal [58]

$$
\int_{\Omega} P_{i}(x) P_{j}(x) d x=\frac{2^{k}}{2 j+1} \delta_{i j} .
$$

where $\Omega$ is the $k$ dimensional hypercube $-1 \leq x_{i} \leq 1$, and $\delta_{i j}$ is the Kronecker delta function, 
equal to 1 when $i=j$ and equal to zero otherwise 32 .

If we weight the inner product of $P_{i}(x)$ and $P_{j}(x)$ by its probability density function $p(x)=2^{-k}$, we get the following

$$
\int_{\Omega} P_{i}(x) P_{j}(x) p(x) d x=\frac{1}{2 j+1} \delta_{i j}=\delta_{i j} \gamma_{i}
$$

Taking the univariate polynomials, $\left\{\psi_{\alpha_{i}}\left(\xi_{i}\right)\right\}_{\alpha=1}^{A}$ as the above Legendre polynomials of random variables, $\left\{\xi_{i}\right\}_{i=1}^{k}$, where the inner product with respect to a uniform $\operatorname{pdf}\left\langle\psi_{j}(x)^{2}\right\rangle$ is equal to $\gamma_{\alpha_{i}}$, we say that as in Eq. 4.50 ,

$$
\int_{\Omega} \psi_{\alpha_{i}}\left(\xi_{i}\right) \psi_{\alpha_{j}}\left(\xi_{j}\right) p(x) d \xi=\delta_{i j} \gamma_{\alpha_{i}}
$$

Likewise, the multivariate polynomials, $\left\{\Psi_{\alpha}(\xi)\right\}_{\alpha=1}^{A}$ exhibit a similar orthogonality property 32 :

$$
\int_{\Omega} \Psi_{\alpha}(\xi) \Psi_{\beta}(\xi) p(\xi) d \xi=\delta_{\alpha \beta} \gamma_{\alpha}
$$

where $\gamma_{\alpha}$ is given by the inner product of the multivariate polynomials

$$
\left\langle\Psi_{\alpha}(\xi)^{2}\right\rangle=\gamma_{\alpha}=\prod_{i=1}^{k} \gamma_{\alpha_{i}}
$$

and similarly,

$$
\delta_{\alpha \beta}=\prod_{i=1}^{k} \delta_{\alpha_{i} \beta_{i}} .
$$

Likewise, for the common case of a normalized random variable with a Gaussian distribution with zero mean, and unit variance, $\mathcal{N}(0,1)$, univariate Hermite polynomials are used to create an orthogonal basis 32$]$. There exist other well known orthogonal polynomial bases for standard families 
of distributions, for instance a gamma distribution is represented by Laguerre polynomials, while a Beta distribution would be represented by a Jacobi polynomial basis $[59$. Furthermore, generalized polynomial chaos (gPC) provides a framework for obtaining a basis for an arbitrary probability density function [60].

\subsubsection{Obtaining Polynomial Chaos Coefficients}

There are a number of ways one could obtain the polynomial chaos coefficients ranging from a simple regression to more sophisticated methods. In this investigation we will employ projection as was done in Chapter 3 for imposing initial conditions using the Galerkin method. In the same way, we will project $f$ onto the basis $\Psi(\xi)$ recalling its orthogonality with respect to the probability density function of the random variable, $p(\xi)$. Hence, the PCE coefficients can be computed as follows 32,61

$$
c_{\alpha} \gamma_{\alpha}=\int_{\Omega} f(x, t, X(\xi)) \Psi_{\alpha}(\xi) p(\xi) d \xi
$$

where, $\xi$ is $\left\{\xi_{i}\right\}_{i=1}^{k}$ is the independent random variable associated with the set uncertain inputs $X$. The uncertain input parameter, $X_{i}$ with with a lower bound of $a_{i}$ and upper bound $b_{i}$, is converted to a random variable $\xi_{i}$ between negative and positive one by,

$$
X_{i}=\frac{b_{i}+a_{i}}{2}+\frac{b_{i}-a_{i}}{2} \xi_{i} \quad i=1, \ldots, k
$$

The integral in Eq.4.55 can be evaluated conveniently using quadrature, as follows 32

$$
c_{\alpha} \gamma_{\alpha} \approx \sum_{q=1}^{N} w_{q} f\left(x, t, X\left(\xi_{q}\right)\right) \Psi\left(\xi_{q}\right) .
$$

where $\xi_{q}$ are the quadrature points, and $w_{q}$ are the associated weights. 
The level of quadrature dictates the number of quadrature points that will be taken in the normalized domain of the input probability space. This normalized probability space is a $k$-dimensional hypercube $\Omega^{k} \subset \mathbb{R}^{k}$,

$$
\Omega^{k}=\left\{X \mid-1 \leq \xi_{i} \leq 1 ; i=1,2, \ldots, k\right\} .
$$

The coordinates and weights of the points are dictated by the quadrature method being used. The example below shows the quadrature points in two dimensions for Gauss-Legendre quadrature. In this case, the quadrature points, are the roots of a Legendre polynomial (as shown in Figure 4.2 for a one dimensional case). Hence, Gauss-Legendre quadrature is optimal for the approximation of the integral in Eq.4.55 by Eq.4.57) for the case where $\Psi(\xi)$ are Legendre polynomials, and $p(\xi)$ is a uniform pdf 62 . In view of this, it is worth mentioning that other quadrature methods may be used (Gauss-Hermite, Newton-Cotes, Clenshaw Curtis, etc) 32.

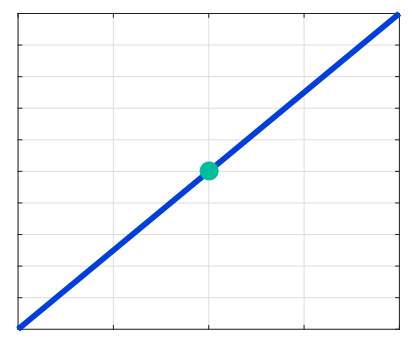

(a) $1^{\text {st }}$ order

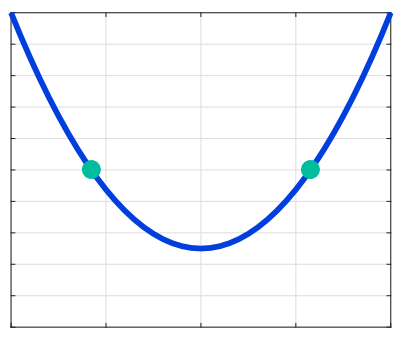

(b) $2^{\text {nd }}$ order

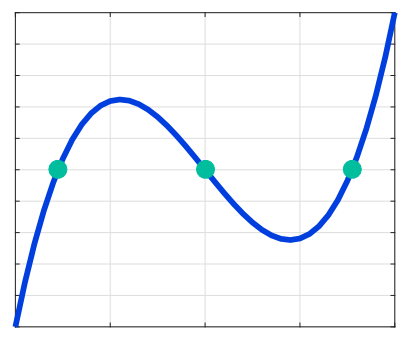

(c) $3^{r d}$ order

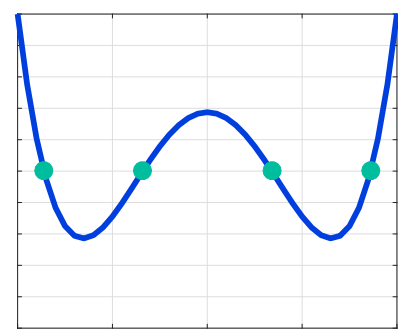

(d) $4^{\text {th }}$ order

Figure 4.2: Full tensor grid weighted quadrature points in 1-D

Figure 4.3 shows the quadrature points in two dimensions using a full tensor grid. Alternatively, a sparse grid could be employed, which would require fewer quadrature points, hence requiring fewer function evaluations to obtain the same level of accuracy 63. For the current problem, the implementation of a sparse grid for quadrature was not necessary. However in cases with a larger number of uncertain input parameters and/or where a higher order of expansion is required, the time savings could become substanital 32,61 . 


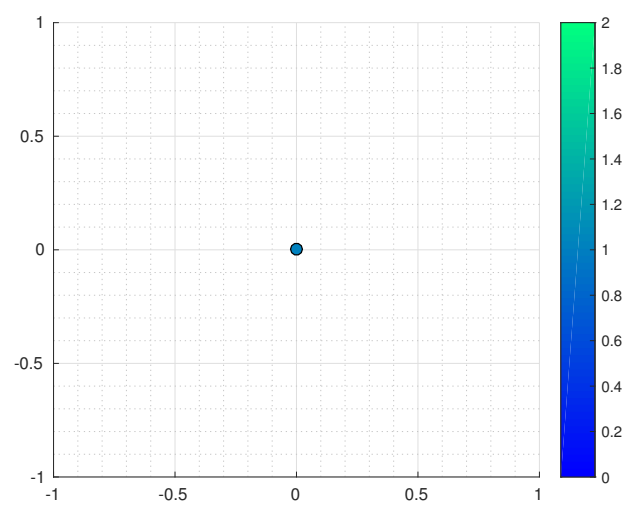

(a) $1^{\text {st }}$ order quadrature points

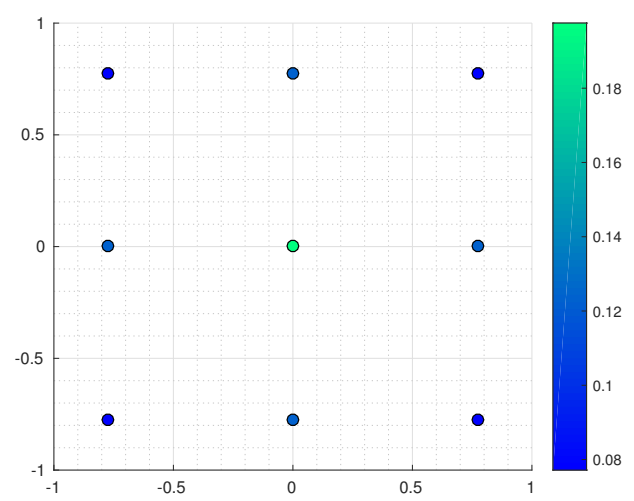

(c) $3^{\text {rd }}$ order quadrature points

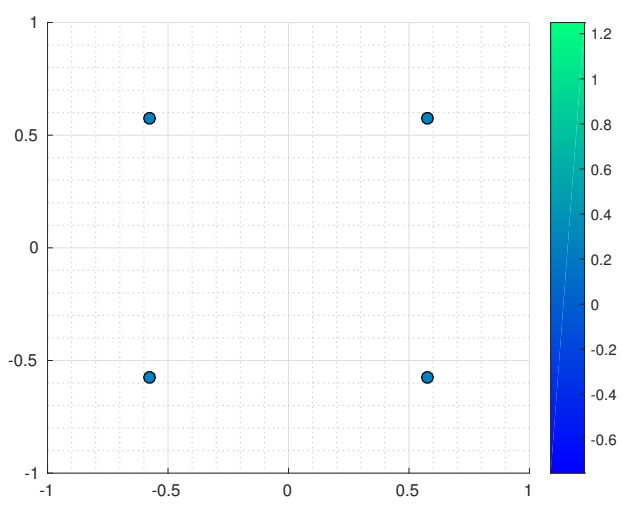

(b) $2^{\text {nd }}$ order quadrature points

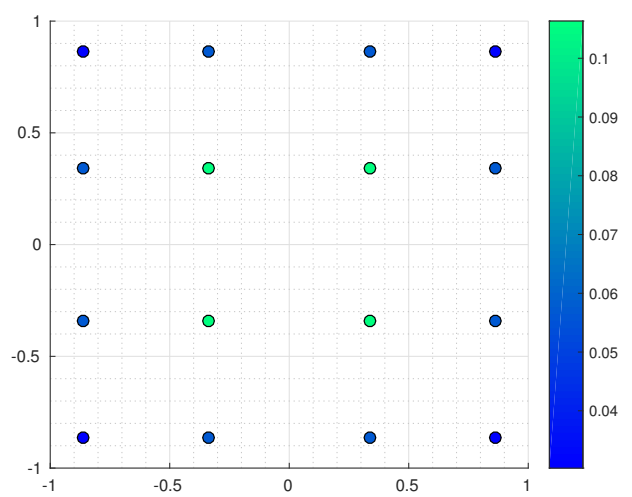

(d) $4^{\text {th }}$ order quadrature points

Figure 4.3: Full tensor grid weighted quadrature points in 2-D, based on [32, generated using UQTk 57

\subsubsection{Statistical Moments}

The goal of this non-intrusive polynomial chaos expansion of the output, $Y$ is to perform sensitivity analysis on the uncertain input factors, $X$. We opted to take a variance-based approach to this problem. Hence we will need to define the variance $Y$ as calculated using the PCE.

Leveraging the pairwise orthogonality between basis functions shown in Eq.(4.51), and noting that the zeroth Legendre polynomial is equal to 1 on $\Omega$ as in Eq.4.48 a, we can say

$$
\begin{gathered}
\int_{\Omega} \Psi_{0}(\xi) p(\xi) d \xi=\gamma_{0} \equiv 1 \\
\int_{\Omega} \Psi_{\alpha}(\xi) p(\xi) d \xi=0
\end{gathered}
$$


Applying these in the derivation of the unconditional mean yields an expression for the mean. Similarly to the ANOVA-HDMR, upon performing the Sobol' decomposition of the PCE, we find that the expected value of $Y$ is the zeroth order coefficient, $c_{0}$.

$$
\begin{aligned}
E(Y) & =\int_{\Omega} g(\xi) \\
& =\int_{\Omega} \sum_{k=0}^{K-1} c_{\alpha} \Psi_{\alpha}(\xi) d \xi \\
& =\int_{\Omega}\left(c_{0} \Psi_{0}(\xi)+c_{1} \Psi_{1}(\xi)+\ldots+c_{A-1} \Psi_{A-1}(\xi)\right) p(\xi) d \xi \\
& =c_{0} \int_{\Omega} \Psi_{0}(\xi) p(\xi) d \xi+c_{1} \int_{\Omega} \Psi_{1}(\xi) p(\xi) d \xi+\ldots+c_{K-1} \int_{\Omega} \Psi_{K-1}(\xi) p(\xi) d \xi \\
& =c_{0}
\end{aligned}
$$

Using the above expression for the mean of $Y$, we can subsequently obtain an expression for the variance. It will be shown that the variance of the output can be obtained as the sum of the squares of the PC coefficients, omitting the mean term (i.e. the PC coefficient with index $\alpha=0$ ).

$$
\begin{aligned}
V(Y)= & \int_{\Omega}(g(\xi)-E(g(\xi)))^{2} d \xi \\
= & \int_{\Omega}\left(\sum_{\alpha=0}^{A-1} c_{\alpha} \Psi_{\alpha}(\xi)-c_{0}\right)^{2} d \xi \\
= & \int_{\Omega}\left(\left(\sum_{\alpha=0}^{A-1} c_{\alpha} \Psi_{\alpha}(\xi)\right)^{2}-2 c_{0} \Psi_{0}(\xi) \sum_{\alpha=0}^{A-1} c_{\alpha} \Psi_{\alpha}(\xi)+c_{0}^{2}\right) d \xi \\
= & \sum_{\alpha=0}^{A-1} \sum_{\beta=0}^{A-1} \int_{\Omega} c_{\alpha} \Psi_{\alpha}(\xi) c_{\beta} \Psi_{\beta}(\xi) d \xi-2 c_{0} \Psi_{0}(\xi) \sum_{\alpha=0}^{A-1} \int_{\Omega} c_{\alpha} \Psi_{\alpha}(\xi) d \xi+c_{0}^{2} \int_{\Omega} d \xi \\
= & \left(\sum_{\alpha=0}^{A-1} c_{\alpha} c_{\alpha} \int_{\Omega} \Psi_{\alpha}(\xi) \Psi_{\alpha}(\xi) d \xi+\sum_{\alpha=0}^{A-1} \sum_{\beta \neq \alpha}^{A-1} c_{\alpha} c_{\beta} \int_{\Omega} \Psi_{\alpha}(\xi) \Psi_{\beta}(\xi) d \xi\right) \\
& -2 c_{0}\left(\int_{\Omega} c_{0} \Psi_{0}(\xi) d \xi+\sum_{\alpha=1}^{A-1} \int_{\Omega} c_{\alpha} \Psi_{\alpha}(\xi) d \xi\right)+c_{0}^{2} \int_{\Omega} d \xi \\
= & \left(\sum_{\alpha=0}^{A-1} c_{\alpha}^{2} \gamma_{\alpha}\right)-2 c_{0}^{2}+c_{0}^{2} \\
= & \sum_{\alpha=1}^{A-1} c_{\alpha}^{2} \gamma_{\alpha}
\end{aligned}
$$


Similarly, the conditional variances are also the same as those derived for the Sobol' decomposition [13],

$$
V\left(E\left(Y \mid X_{i}\right)\right)=V\left(f_{i}\left(X_{i}\right)\right) .
$$

where $f_{i}\left(X_{i}\right)$ is any PCE term for which the basis functions are functions of $\xi_{i}$ only, meaning polynomials for which the only nonzero multi-indices are $\alpha_{i}$,

$$
V\left(E\left(Y \mid X_{i}, X_{j}\right)\right)=V\left(f_{i}\left(X_{i}\right)\right)+V\left(f_{j}\left(X_{j}\right)\right)+V\left(f_{i j}\left(X_{i}, X_{j}\right)\right) .
$$

where the definition above holds for the variances of the first order terms $f_{i}\left(X_{i}\right)$ and $f_{j}\left(X_{j}\right)$, while $f_{i j}\left(X_{i}, X_{j}\right)$ is any PCE term for which the basis functions are functions of both $\xi_{i}$ and $\xi_{j}$ and no other element of $\xi$. In terms of multi-indices, this means the multi-indices $\alpha_{i}$ and $\alpha_{j}$ are both nonzero, while all other multi-indices are zero.

\subsubsection{Sensitivity Indices}

We have defined the sensitivity index $S_{i}$ as the conditional variance of the expected value the ouput $V\left(E\left(Y \mid X_{i}\right)\right)$ normalized by the unconditioned variance $V(Y)$. Hence using PCE, we will define the sensitivity index as the ratio of the variance of all terms whose multi-indices $(\alpha)$ only involve the random variable $\xi_{i}$, to the variance of $Y$ defined in Eq. (4.62) [13],

$$
S_{i}=\frac{V\left(Y \mid X_{i}\right)}{V(Y)}=\frac{\sum_{\alpha=\mathbb{I}_{i}} c_{\alpha}^{2} \gamma_{\alpha}}{\sum_{\alpha=1}^{A-1} c_{\alpha}^{2} \gamma_{\alpha}},
$$

where $\mathbb{I}_{i} \subset \alpha$ is the subset of multi-indices associated with the basis terms that involve only the variable $\xi_{i}$. In terms of multi-indices, $\mathbb{I}_{i}$ corresponds to the values of $\alpha$ where $\alpha_{i}$ is nonzero, while 
$\alpha_{j}$ is equal to zero for all $j \neq i$.

We can define higher order sensitivity indices in a similar manner,

$$
S_{i j}=\frac{V\left(Y \mid X_{i}, X_{j}\right)}{V(Y)}-S_{i}-S_{j}=\frac{\sum_{\alpha=\mathbb{I}_{i j}} c_{\alpha}^{2} \gamma_{\alpha}}{\sum_{\alpha=1}^{A-1} c_{\alpha}^{2} \gamma_{\alpha}},
$$

where $\mathbb{I}_{i j} \subset \alpha$ is the indices of all basis terms that involve both variable $\xi_{i}$ and $\xi_{j}$. In terms of multi-indices, $\mathbb{I}_{i j}$ corresponds to the values of $\alpha$ where $\alpha_{i}$ and $\alpha_{j}$ are both nonzero, and all other multi-indices are zero valued.

The total sensitivity index $S_{T_{i}}$ was defined as unity less the variance of the expected value of $Y$ conditioned on all input factors but $X_{i}$ normalized by the unconditional variance. This translates to one minus the sum of PCE coefficients for which the basis functions are independent of $\xi_{i}$ (i.e. where multi-index $\left.\alpha_{i}=0\right)$. From the opposite perceptive, this can be interpreted simply as the sensitivity indices of all orders that are associated with input factor $X_{i}$ (i.e. any terms where multi-index $\left.\alpha_{i} \neq 0\right) 13$,

$$
S_{T_{i}}=1-\frac{V_{X_{\sim i}}\left(E_{X_{i}}\left(Y \mid X_{\sim i}\right)\right.}{V(Y)}=\frac{\sum_{\alpha=\mathbb{I}_{i}} c_{\alpha}^{2} \gamma_{\alpha}}{\sum_{\alpha=1}^{A-1} c_{\alpha}^{2} \gamma_{\alpha}}
$$

where $\mathbb{I}_{i^{T}} \subset \alpha$ is the indices of all basis terms that involve the variable $\xi_{i}$. In terms of multi-indices, this refers to all basis functions where $\alpha_{i}$ is nonzero.

This is further clarified by a simple example below.

\section{Example 4.3}

For a problem with two uncertain parameters, we chose to create a second order polynomial chaos expansion $Y=f\left(X_{1}, X_{2}\right) \approx g\left(\xi_{1}, \xi_{2}\right)$.

Recalling the truncation scheme in Eq. 4.45, the expansion will consist of $(2+2) ! /(2 ! 2 !)=6$ terms as given by

$$
Y \approx y_{0} \Psi_{0}(\xi)+y_{1} \Psi_{1}(\xi)+y_{2} \Psi_{2}(\xi)+y_{3} \Psi_{3}(\xi)+y_{4} \Psi_{4}(\xi)+y_{5} \Psi_{5}(\xi)
$$


In accordance with the multi-index notation, the multivariate polynomials $\Psi_{\alpha}(\xi)$ are given as the following products of univariate polynomials,

$$
\begin{aligned}
& \Psi_{0}(\xi)=\psi_{0}\left(\xi_{1}\right) \times \psi_{0}\left(\xi_{2}\right)=1 \\
& \Psi_{1}(\xi)=\psi_{1}\left(\xi_{1}\right) \times \psi_{0}\left(\xi_{2}\right)=\xi_{1} \\
& \Psi_{2}(\xi)=\psi_{0}\left(\xi_{1}\right) \times \psi_{1}\left(\xi_{2}\right)=\xi_{2} \\
& \Psi_{3}(\xi)=\psi_{2}\left(\xi_{1}\right) \times \psi_{0}\left(\xi_{2}\right)=\frac{1}{2}\left(3 \xi_{1}-1\right), \\
& \Psi_{4}(\xi)=\psi_{1}\left(\xi_{1}\right) \times \psi_{1}\left(\xi_{2}\right)=\xi_{1} \xi_{2} \\
& \Psi_{5}(\xi)=\psi_{0}\left(\xi_{1}\right) \times \psi_{2}\left(\xi_{2}\right)=\frac{1}{2}\left(3 \xi_{2}-1\right) .
\end{aligned}
$$

Hence, we can write

$$
Y \approx c_{0}+c_{1} \xi_{1}+c_{2} \xi_{2}+c_{3} \frac{1}{2}\left(3 \xi_{1}-1\right)+c_{4} \xi_{1} \xi_{2}+c_{5} \frac{1}{2}\left(3 \xi_{2}-1\right)
$$

We can analytically write the main effect terms, $S_{1}$ and $S_{2}$ as

$$
\begin{aligned}
& S_{1}=\frac{c_{1}^{2} \gamma_{1}+c_{3}^{2} \gamma_{3}}{c_{1}^{2} \gamma_{1}+c_{2}^{2} \gamma_{2}+c_{3}^{2} \gamma_{3}+c_{4}^{2} \gamma_{4}+c_{5}^{2} \gamma_{5}}, \\
& S_{2}=\frac{c_{2}^{2} \gamma_{2}+c_{5}^{2} \gamma_{5}}{c_{1}^{2} \gamma_{1}+c_{2}^{2} \gamma_{2}+c_{3}^{2} \gamma_{3}+c_{4}^{2} \gamma_{4}+c_{5}^{2} \gamma_{5}} .
\end{aligned}
$$

To quantify the interaction effects between the uncertain inputs, we can write $S_{12}$ as

$$
S_{12}=\frac{c_{4}^{2} \gamma_{4}}{c_{1}^{2} \gamma_{1}+c_{2}^{2} \gamma_{2}+c_{3}^{2} \gamma_{3}+c_{4}^{2} \gamma_{4}+c_{5}^{2} \gamma_{5}} .
$$

Or if we prefer to use total effect terms, we can write, $S_{T_{1}}$ and $S_{T_{2}}$ as 


$$
\begin{aligned}
& S_{T_{1}}=\frac{c_{1}^{2} \gamma_{1}+c_{3}^{2} \gamma_{3}+c_{4}^{2} \gamma_{4}}{c_{1}^{2} \gamma_{1}+c_{2}^{2} \gamma_{2}+c_{3}^{2} \gamma_{3}+c_{4}^{2} \gamma_{4}+c_{5}^{2} \gamma_{5}}, \\
& S_{T_{2}}=\frac{c_{2}^{2} \gamma_{2}+c_{4}^{2} \gamma_{4}+c_{5}^{2} \gamma_{5}}{c_{1}^{2} \gamma_{1}+c_{2}^{2} \gamma_{2}+c_{3}^{2} \gamma_{3}+c_{4}^{2} \gamma_{4}+c_{5}^{2} \gamma_{5}} .
\end{aligned}
$$

\subsection{Sensitivity of Flutter Speed and Frequency to Pitch Pa-}

\section{rameters}

The principal contribution of this body of work is in introducing an aeroelastic system in which a flexible wing is coupled with a rigid body pitch rotation. This rigid body pitch rotation introduces a pitch stiffness, $K_{\theta}$, due to a torsional spring at the base, pitch damping, $D_{\theta}$, a pitch moment of inertia, $I_{\theta}$ due to the nonzero mass support mechanism. In view of these new terms that arise as a direct result of this additional degree of freedom, in this section we will investigate the sensitivity of the classical flutter speed and the associated flutter frequency to the variation of these three parameters.

It is known from Chapter 3 that the first mode in bending becomes unstable, coalescing with the rigid body pitch mode to induce flutter. Because of this, it is important to acknowledge that a higher dimensional global sensitivity analysis which considers a broader spectrum of uncertain input parameters would be more telling of which input parameters have the largest influence on the quantities of interest. Other parameters that are good candidates for uncertain inputs are those pertaining to the eccentricities $\left(e_{c}, e, e_{p}\right.$, etc) which relate to the mounting of the wing to the support mechanism, which introduces the possibility of these quantities being imprecisely known.

As described in the theory above, we will be performing the global sensitivity analysis by first creating a polynomial chaos expansion surrogate model of the output space. This is created by evaluating the model to obtain the flutter speed and flutter frequency at the predetermined quadrature points. 
Here, as shown in Figure 4.4 , the model is evaluated along a $5 \times 5 \times 5$ grid. Then the by projection, the polynomial chaos coefficients are obtained, as outlined previously. From these polynomial chaos coefficients, we analytically obtain the sensitivity indices.

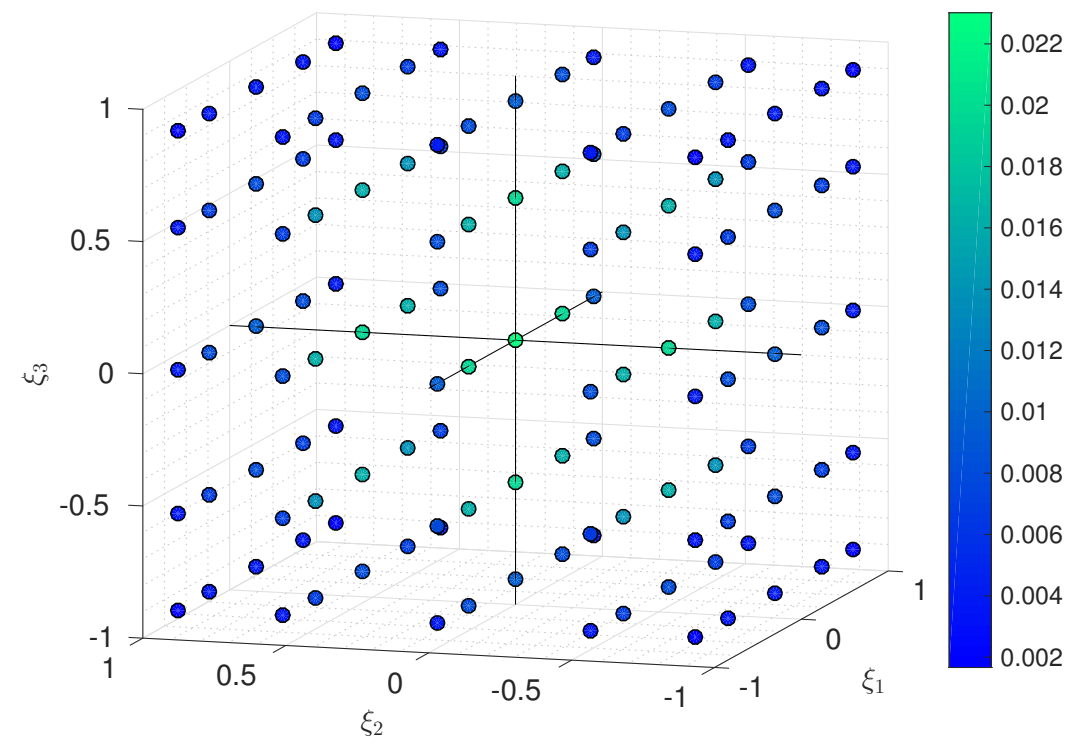

Figure 4.4: Weighted quadrature points in 3-D, generated using UQTk 57

\subsubsection{Sensitivity Indices}

The main and total sensitivity indices are presented here for both the flutter speed and the flutter frequency. In Chapter 4 , values were given for the pitch stiffness and moment of inertia as $K_{\theta}=0.24$ $\mathrm{Nm}$ and $I_{\theta}=1.6 \times 10^{-4} \mathrm{kgm}^{2}$. These values are taken as the mean of the input distributions, as uniform input probability density functions are assigned as follows:

$$
\begin{aligned}
& p\left(K_{\theta}\right)= \begin{cases}\frac{1}{2 \times 0.24} & 0 \leq x \leq 2 \times 0.24 \\
0 & \text { elsewhere }\end{cases} \\
& p\left(I_{\theta}\right)= \begin{cases}\frac{1}{2 \times 1.6 \times 10^{-4}} & 0 \leq x \leq 2 \times 1.6 \times 10^{-4} \\
0 & \text { elsewhere }\end{cases}
\end{aligned}
$$

Similarly, we allow the damping value to vary between $0 \%$ and $10 \%$ of the critical damping value given by 


$$
\zeta=\frac{D_{\theta}}{D_{c r}}=\frac{D_{\theta}}{2 \sqrt{K_{\theta} \times I_{\theta}}} \Longrightarrow D_{\theta}=\zeta 2 \sqrt{K_{\theta} \times I_{\theta}}
$$

with

$$
p(\zeta)= \begin{cases}\frac{1}{0.1} & 0 \leq x \leq 0.1 \\ 0 & \text { elsewhere }\end{cases}
$$

The immediate conclusions that can be drawn from the sensitivity indices in Table 4.3 are consistent with the prevailing knowledge regarding classical flutter. The sensitivity index (main effect and total effect) for the damping accounts for 1.31 percent of the variance both output quantities of interest, despite varying uniformly between values of zero and ten percent of the critical damping. Figure $3.8 \mathrm{a}$ revealed that the system undergoes moderate flutter, as the slope of the unstable mode is steep at the flutter speed. In view of this, it makes sense that the stuctural damping (which essentially shifts the curves in Figure $3.8 \mathrm{a}$ vertically) would have little influence on the flutter speed. In a system where the flutter mechanism is more mild, and the slope is more gentle, this vertical shift could potentially have more adverse effects on the flutter speed. From the perspective of designing future wind tunnel experiments, we now know that changing the stiffness of the torsional spring will have a more significant effect on the flutter characteristics than changing the mass properties of the support.

\begin{tabular}{clllll}
\hline \multirow{2}{*}{ Input Parameter } & \multicolumn{2}{c}{ Flutter Speed } & & \multicolumn{2}{c}{ Flutter Frequency } \\
& Main effect & Total effect & & Main effect & Total effect \\
\hline \hline$I_{\theta}$ & 0.395 & 0.400 & 0.322 & 0.326 \\
$D_{\theta}$ & 0.000 & 0.000 & & 0.011 & 0.013 \\
$K_{\theta}$ & 0.600 & 0.605 & 0.663 & 0.663 \\
\hline
\end{tabular}

Table 4.3: Sobol' indices (main effect and total effect) for the current investigation 
Additionally, one can note that very little of the variance of the flutter speed and frequency that can be attributed to the interaction of any combination of the three uncertain inputs. This is evidenced by the fact that the total effect indices are nearly identical to the main effect indices for the three parameters in both cases.

\subsubsection{PCE Surrogate Model}

Having identified the pitch damping, $D_{\theta}$ as a non-influential parameter, we have fixed it to its mean value and proceed with the investigation using only the remaining two parameters. Conveniently, this allows us to visually analyze how the flutter speed and frequency vary as a function of the pitch inertia and stiffness. Table 4.4 presents the PCE coefficients obtained for a $5^{\text {th }}$ order polynomial chaos expansion of the two quantities of interest (flutter speed and frequency). This surrogate model is constructed using the same range of values for pitch moment of inertia, $I_{\theta}$ and pitch stiffness $K_{\theta}$ as were used previously.

Table 4.4 tabulates the PCE coefficients which characterize the $5^{\text {th }}$ order surrogate model. Note that the first term $c_{0}$ is the mean term. Referring back to Chapter 3 the flutter speed was found to be $12.71 \mathrm{~m} / \mathrm{s}$, with a corresponding flutter frequency of $5.58 \mathrm{~Hz}$. Seeing as uniform distributions centred at the measured values of pitch stiffness and inertial parameters were assigned to the uncertain input parameters, it should be no surprise that the values from Chapter 3 are close to those reported in the first row of Table 4.4 Another observation that should be that the terms $c_{1}$ and $c_{2}$ are the largest terms by an order of magnitude, which would tend to suggest that the relationship between the inputs and outputs are predominantly linear over the range of the inputs.

These PCE bases from Table 4.4 are plotted in Figure 4.5 These functions, $\Psi_{\alpha}$, form a basis in two dimensions (as in this case they are functions of two random variables). As in Table 4.4 we consider a $5^{\text {th }}$ order polynomial chaos expansion, which, for two random variables, requires 21 basis functions according to Eq. 4.45 . 
PC Coefficients $c_{\alpha}$

PC Basis $\Psi_{\alpha}$

Flutter Speed Flutter Frequency

\begin{tabular}{|c|c|c|c|c|c|c|c|}
\hline$c_{0}$ & $=$ & 12.971 & $c_{0}$ & $=$ & 5.586 & $\Psi_{0}=$ & 1 \\
\hline$c_{1}$ & $=$ & 3.273 & $c_{1}$ & $=$ & -0.579 & $\Psi_{1}=$ & $\xi_{1}$ \\
\hline$c_{2}$ & $=$ & -4.063 & $c_{2}$ & $=$ & 0.706 & $\Psi_{2}=$ & $\xi_{2}$ \\
\hline$c_{3}$ & $=$ & -0.041 & $c_{3}$ & $=$ & 0.050 & $\Psi_{3}=$ & $\frac{1}{2}\left(3 \xi_{1}^{2}-1\right)$ \\
\hline$c_{4}$ & $=$ & 0.641 & $c_{4}$ & $=$ & -0.040 & $\Psi_{4}=$ & $\xi_{1} \xi_{2}$ \\
\hline$c_{5}$ & $=$ & -0.609 & $c_{5}$ & $=$ & -0.044 & $\Psi_{5}=$ & $\frac{1}{2}\left(3 \xi_{2}^{2}-1\right)$ \\
\hline$c_{6}$ & $=$ & -0.034 & $c_{6}$ & $=$ & -0.002 & $\Psi_{6}=$ & $\frac{1}{2}\left(5 \xi_{1}^{3}-3 \xi_{1}\right)$ \\
\hline$c_{7}$ & $=$ & 0.190 & $c_{7}$ & $=$ & -0.007 & $\Psi_{7}=$ & $\frac{1}{2}\left(3 \xi_{1}^{2}-1\right) \xi_{2}$ \\
\hline$c_{8}$ & $=$ & -0.106 & $c_{8}$ & $=$ & 0.013 & $\Psi_{8}=$ & $\frac{1}{2} \xi_{1}\left(3 \xi_{2}^{2}-1\right)$ \\
\hline$c_{9}$ & $=$ & -0.038 & $c_{9}$ & $=$ & 0.002 & $\Psi_{9}=$ & $\frac{1}{2}\left(5 \xi_{2}^{3}-3 \xi_{2}\right)$ \\
\hline$c_{10}$ & $=$ & 0.007 & $c_{10}$ & $=$ & -0.000 & $\Psi_{10}=$ & $\frac{1}{8}\left(35 \xi_{1}^{4}-30 \xi_{1}^{2}+3\right)$ \\
\hline$c_{11}$ & $=$ & -0.050 & $c_{11}$ & $=$ & 0.005 & $\Psi_{11}=$ & $\frac{1}{2}\left(5 \xi_{1}^{3}-3 \xi_{1}\right) \xi_{2}$ \\
\hline$c_{12}$ & $=$ & 0.055 & $c_{12}$ & $=$ & -0.008 & $\Psi_{12}=$ & $\frac{1}{4}\left(3 \xi_{1}^{2}-1\right)\left(3 \xi_{2}^{2}-1\right)$ \\
\hline$c_{13}$ & $=$ & -0.023 & $c_{13}$ & $=$ & 0.003 & $\Psi_{13}=$ & $\frac{1}{2} \xi_{1}\left(5 \xi_{2}^{3}-3 \xi_{2}\right)$ \\
\hline$c_{14}$ & $=$ & 0.009 & $c_{14}$ & $=$ & -0.001 & $\Psi_{14}=$ & $\frac{1}{8}\left(35 \xi_{2}^{4}-30 \xi_{2}^{2}+3\right)$ \\
\hline$c_{15}$ & $=$ & 0.000 & $c_{15}$ & $=$ & -0.000 & $\Psi_{15}=$ & $\frac{1}{8}\left(63 \xi_{1}^{5}-70 \xi_{1}^{3}+15 \xi_{1}\right)$ \\
\hline$c_{16}$ & $=$ & 0.009 & $c_{16}$ & $=$ & -0.002 & $\Psi_{16}=$ & $\frac{1}{8}\left(35 \xi_{1}^{4}-30 \xi_{1}^{2}+3\right) \xi_{2}$ \\
\hline$c_{17}$ & $=$ & -0.020 & $c_{17}$ & $=$ & 0.005 & $\Psi_{17}=$ & $\frac{1}{4}\left(5 \xi_{1}^{3}-3 \xi_{1}\right)\left(3 \xi_{2}^{2}-1\right)$ \\
\hline$c_{18}$ & $=$ & 0.006 & $c_{18}$ & $=$ & -0.002 & $\Psi_{18}=$ & $\frac{1}{4}\left(3 \xi_{1}^{2}-1\right)\left(5 \xi_{2}^{3}-3 \xi_{2}\right)$ \\
\hline$c_{19}$ & $=$ & -0.005 & $c_{19}$ & $=$ & 0.002 & $\Psi_{19}=$ & $\frac{1}{8} \xi_{1}\left(35 \xi_{2}^{4}-30 \xi_{2}^{2}+3\right)$ \\
\hline$c_{20}$ & $=$ & 0.000 & $c_{20}$ & $=$ & 0.000 & $\Psi_{20}=$ & $\frac{1}{8}\left(63 \xi_{2}^{5}-70 \xi_{2}^{3}+15 \xi_{2}\right)$ \\
\hline
\end{tabular}

Table 4.4: PCE coefficients for the current surrogate model 


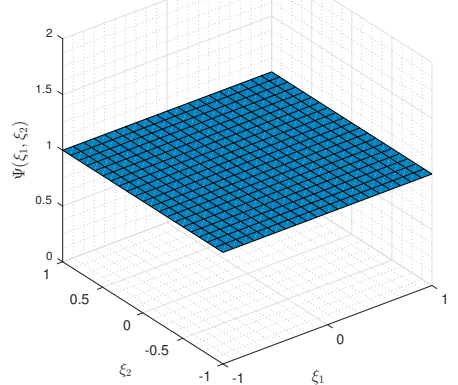

(a) $\Psi_{1}\left(\xi_{1}, \xi_{2}\right)$

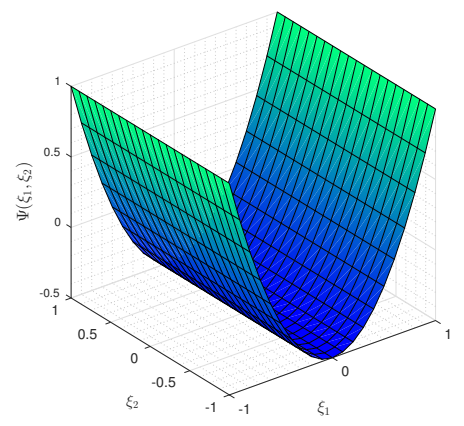

(d) $\Psi_{4}\left(\xi_{1}, \xi_{2}\right)$

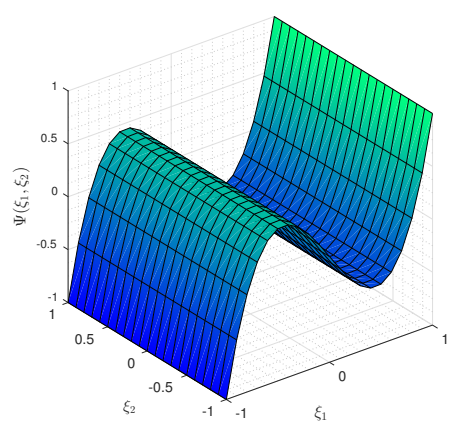

(g) $\Psi_{7}\left(\xi_{1}, \xi_{2}\right)$

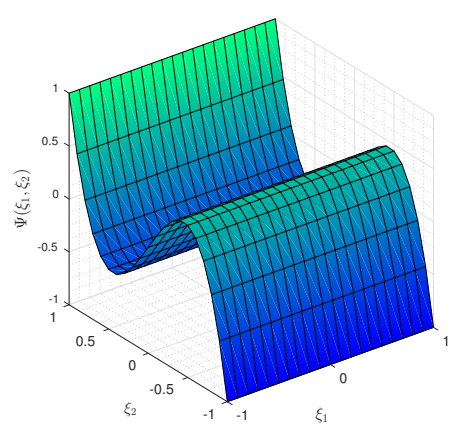

(j) $\Psi_{10}\left(\xi_{1}, \xi_{2}\right)$

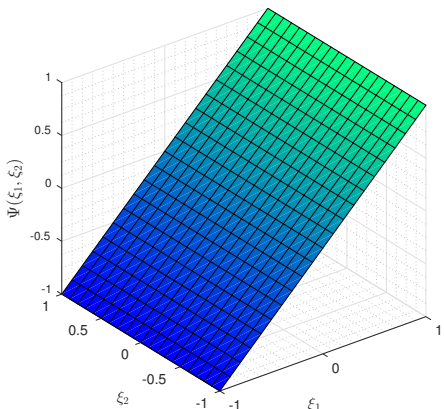

(b) $\Psi_{2}\left(\xi_{1}, \xi_{2}\right)$

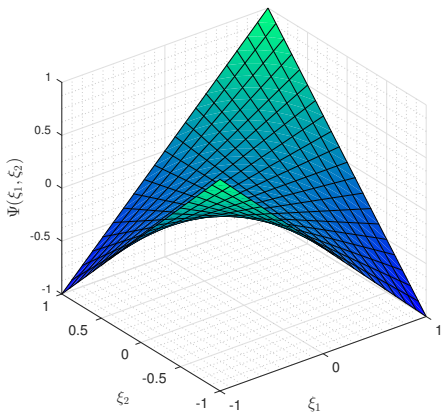

(e) $\Psi_{5}\left(\xi_{1}, \xi_{2}\right)$

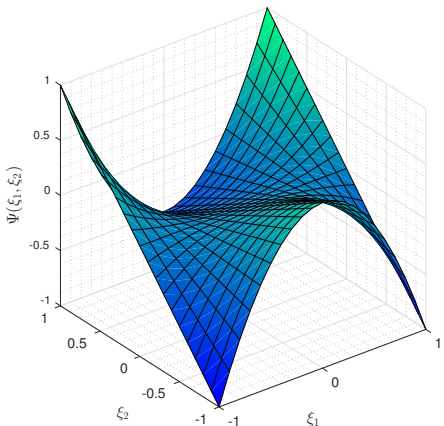

(h) $\Psi_{8}\left(\xi_{1}, \xi_{2}\right)$

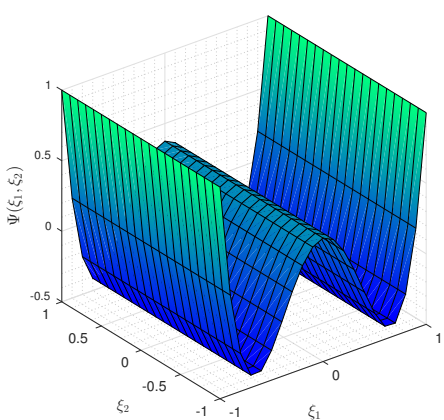

(k) $\Psi_{11}\left(\xi_{1}, \xi_{2}\right)$

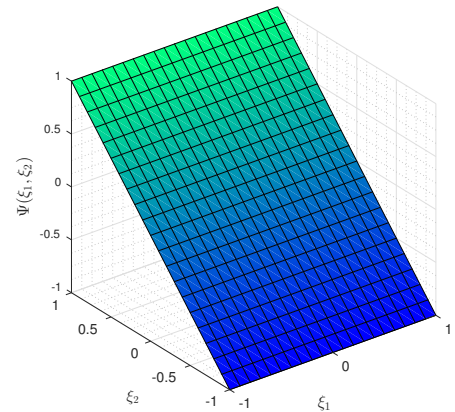

(c) $\Psi_{3}\left(\xi_{1}, \xi_{2}\right)$

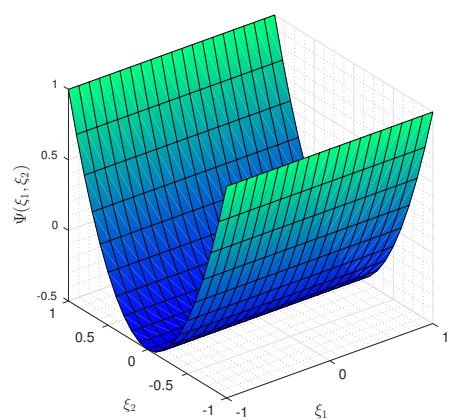

(f) $\Psi_{6}\left(\xi_{1}, \xi_{2}\right)$

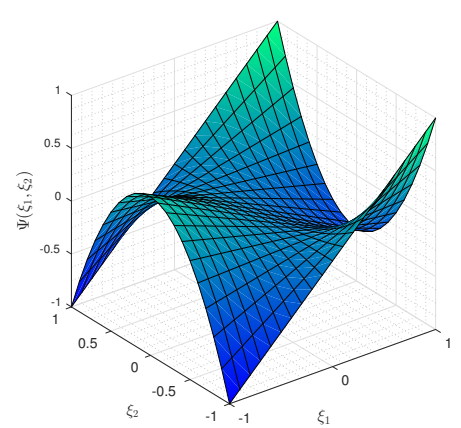

(i) $\Psi_{9}\left(\xi_{1}, \xi_{2}\right)$

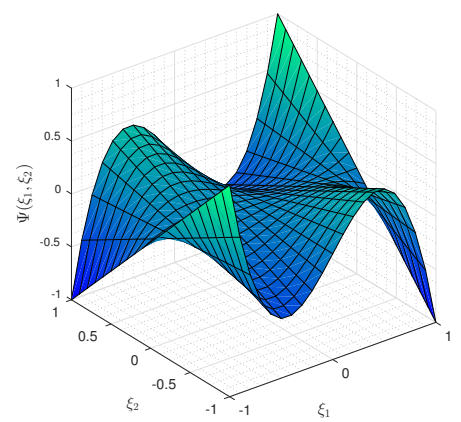

(l) $\Psi_{12}\left(\xi_{1}, \xi_{2}\right)$ 


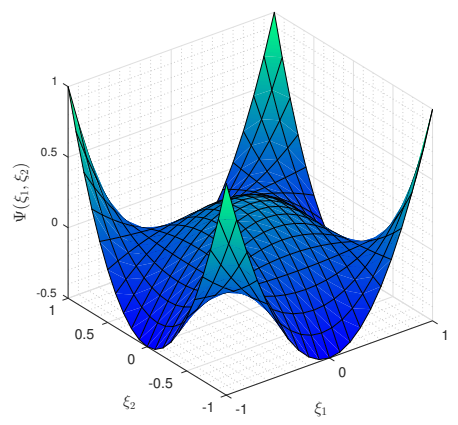

(m) $\Psi_{13}\left(\xi_{1}, \xi_{2}\right)$

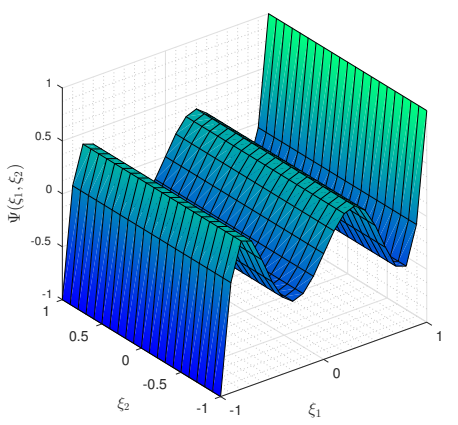

(p) $\Psi_{16}\left(\xi_{1}, \xi_{2}\right)$

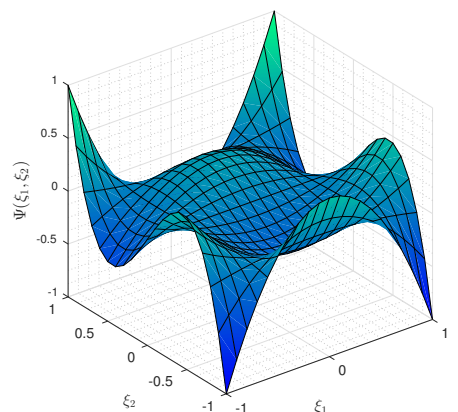

(s) $\Psi_{19}\left(\xi_{1}, \xi_{2}\right)$

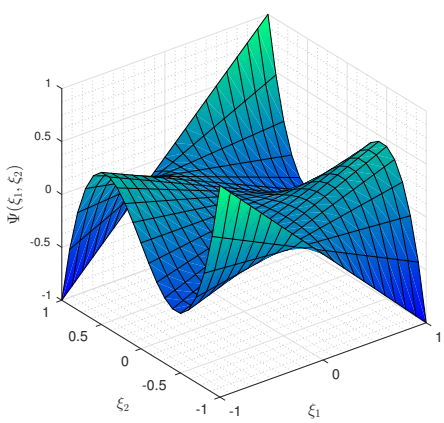

(n) $\Psi_{14}\left(\xi_{1}, \xi_{2}\right)$

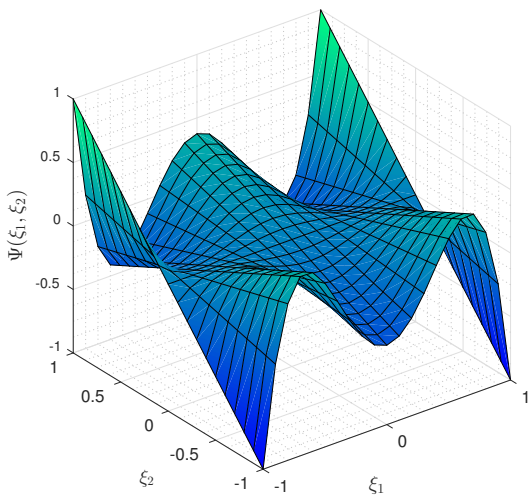

(q) $\Psi_{17}\left(\xi_{1}, \xi_{2}\right)$

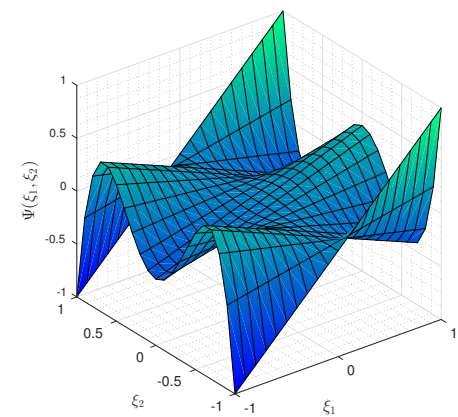

(t) $\Psi_{20}\left(\xi_{1}, \xi_{2}\right)$

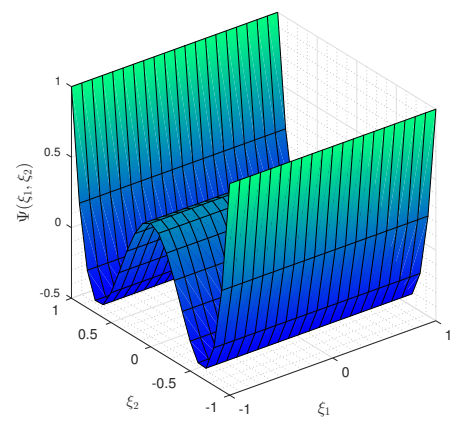

(o) $\Psi_{15}\left(\xi_{1}, \xi_{2}\right)$

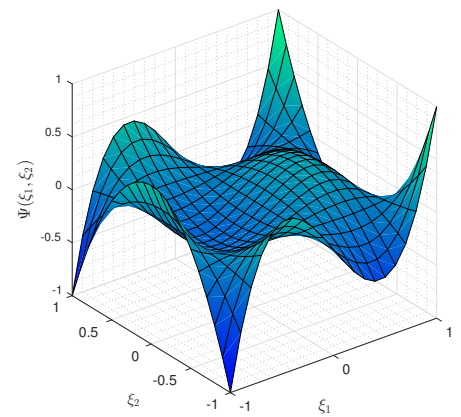

(r) $\Psi_{18}\left(\xi_{1}, \xi_{2}\right)$

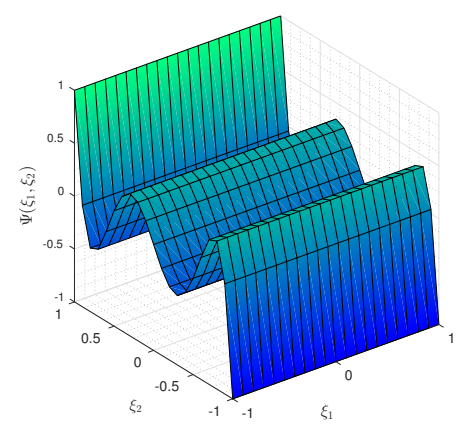

(u) $\Psi_{21}\left(\xi_{1}, \xi_{2}\right)$

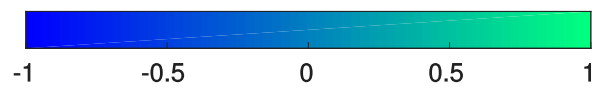

Figure 4.5: PCE basis $\left(\Psi_{\alpha}, \alpha=1, \ldots, 21\right)$ for a $5^{\text {th }}$ order expansion in two dimensions, as tabulated in Table 4.4

Figures 4.6 and 4.7 are the response surfaces of the PC surrogates for the flutter speed and frequency respectively. These response surfaces confirm that the solution is predominantly linear (i.e. could be adequately captured using a $1^{\text {st }}$ order PCE expansion. However this conclusion is only necessarily valid over the current range of inputs. While not pictured, if the range of the pitch stiffness and 
moment of inertia were increased sufficiently, a distinctly nonlinear relationship is observed between the input parameters and the quantities of interest.

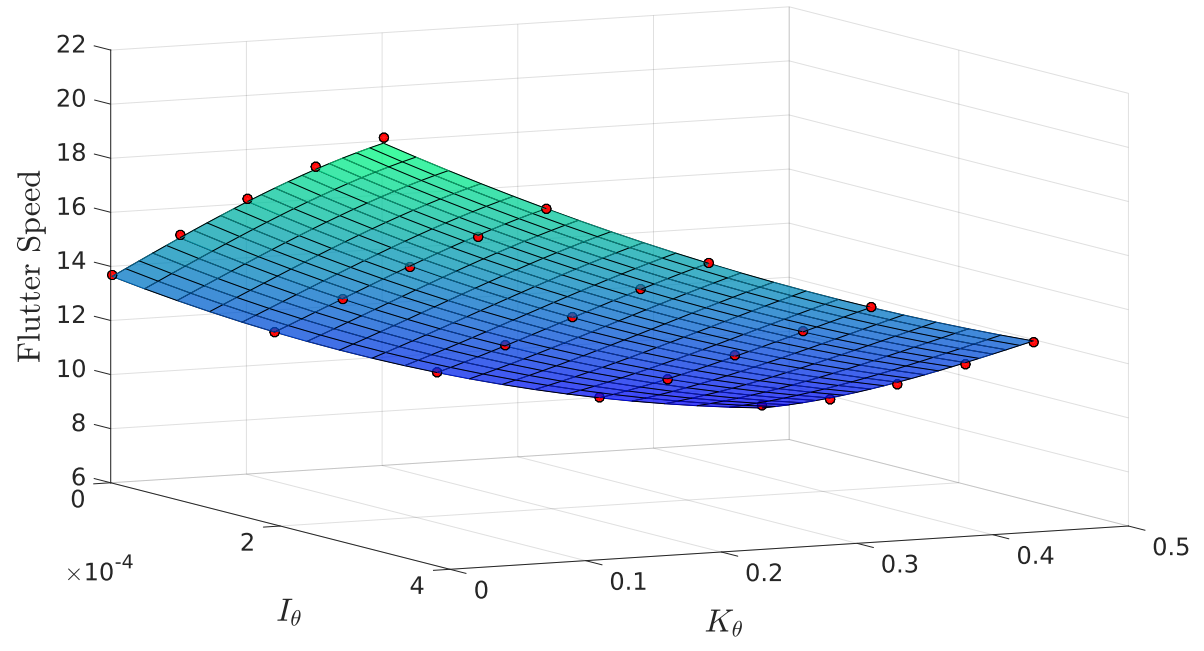

Figure 4.6: Flutter speed response surface

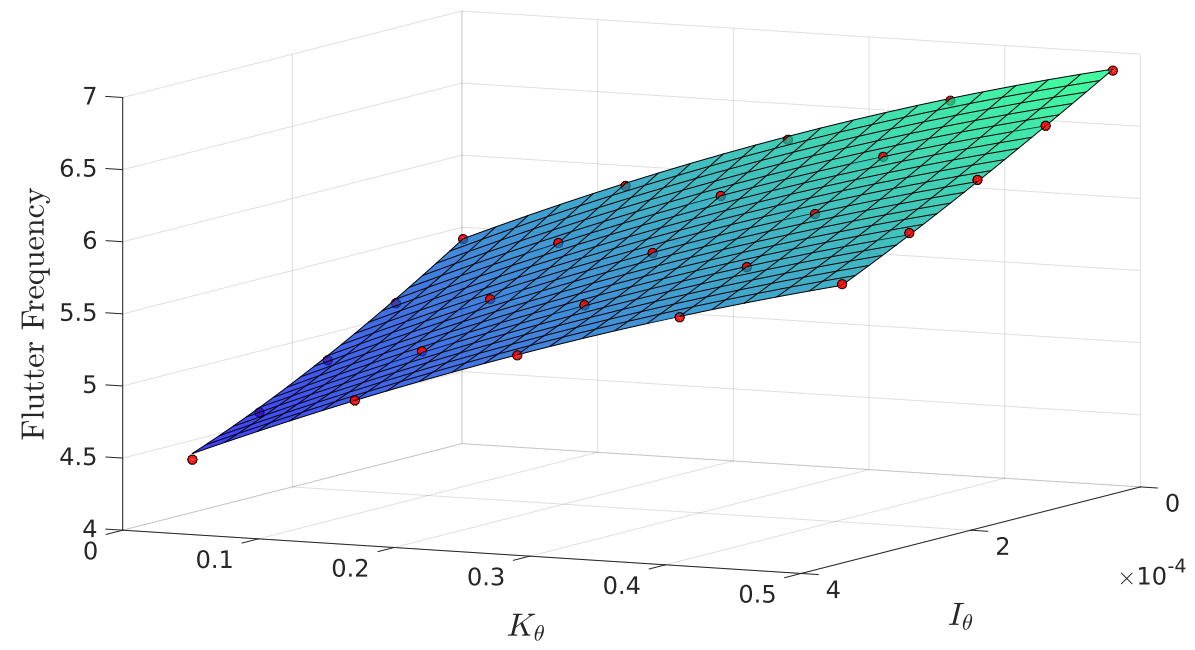

Figure 4.7: Flutter frequency response surface

Furthermore, now that the PCE surrogate model is constructed, we are able to make use of this surrogate to perform very computationally efficient Monte Carlo sampling to obtain marginal and joint probability density functions of the output quantities of interest. The model which calculates the flutter speed and frequency for a given set of input parameters takes in the order of one second to calculate these quantities. 100,000 sets of random inputs are generated from a uniform distribution and shown in Figure 4.8. In order to generate the output probability density functions shown in 
Figure 4.9, the computational time required would be in the order of an entire day. By contrast, is possible to generate 100,000 samples, and obtain the output probability density functions in less than 2.5 seconds using the surrogate model, since it is simply a polynomial function in two dimensions.
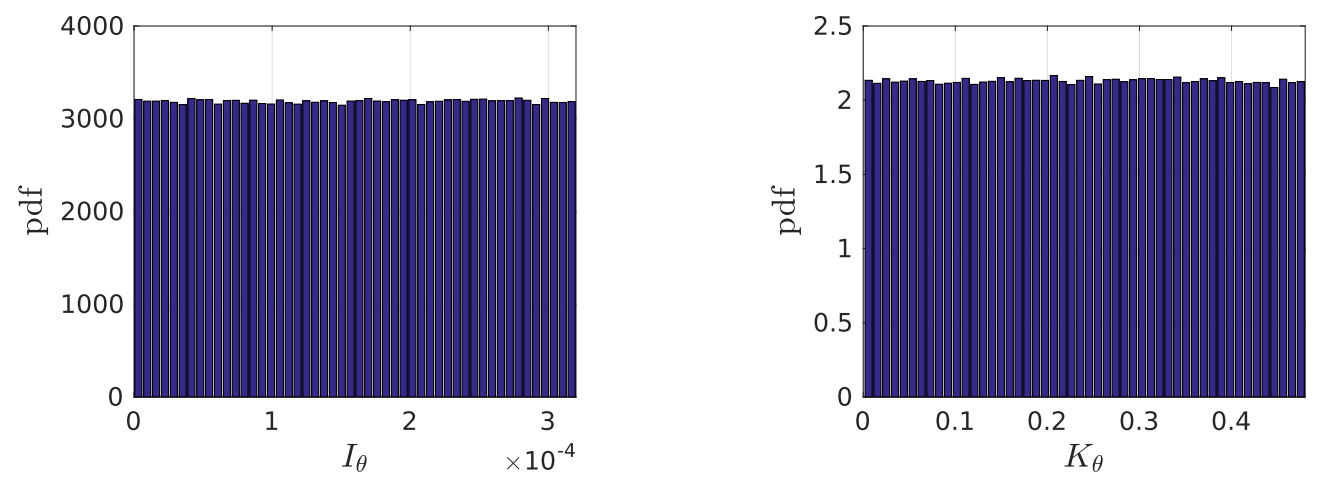

Figure 4.8: Probability density functions for the random inputs, generated from a uniform probability distribution

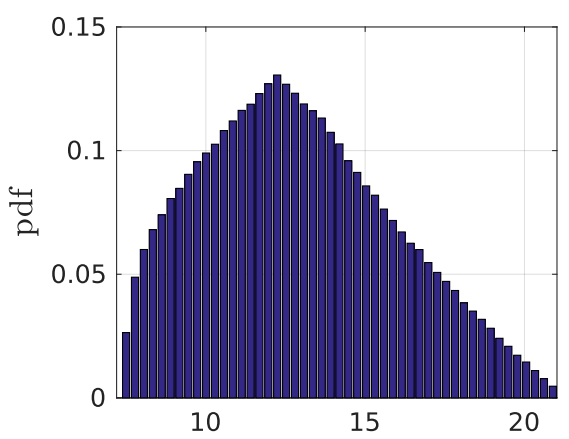

Flutter Speed $(\mathrm{m} / \mathrm{s})$
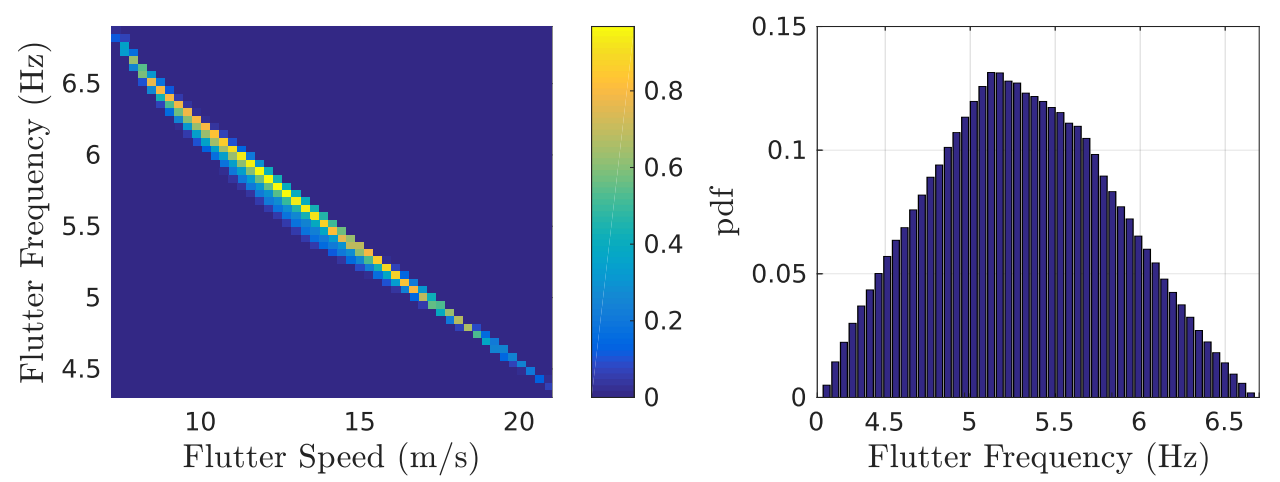

Figure 4.9: Marginal and joint probability density functions of the flutter speed and flutter frequency

Note that it is possible to use the surrogate model to calculate the output probability density functions for other classes of input parameter distributions regardless of the fact that the surrogate model was constructed using uniform random variables. The restriction to this is that the results 
obtained are generally only reliable for the case where the range of the input probability density

functions used are within the range that was used to create the surrogate model, as it is much more well suited for interpolation than for extrapolation. 


\section{Chapter 5}

\section{Conclusion}

\subsection{Concluding Remarks}

In this body of work, a comprehensive derivation of the nonlinear equations of motion for a flexible cantilever coupled with a rigid body pitch rotation at the base is presented. A detailed account of the derivation by [1] is provided. This existing derivation is subsequently adapted to suit the kinematics of the current system, which are in contrast to the kinematics of a rotor blade as in 2 . This thesis presents improvements to the initial effort by da Costa to extend the equations of motion. Among the changes to the formulation include the introduction of the concept of a pitch axis which is not coincident with the elastic axis, as well as relaxing the imposed condition that the axial displacement is equal to zero and retaining more of the terms presented in the works of Hodges and Dowell. An unsteady aerodynamic model was adopted as in 2 . The implementation of this aerodynamic model was refined in order to account for the three dimensional motion of the cantilever wing. The rigid body pitch rotation results in couplings in the aerodynamic forces which were not accounted for previously.

The numerical implementation additionally focused on improvements to the initiatives of $d a$ Costa [2]. Previously, the equations of motion were programmed using a number of built-in MATLAB tools including the numerical integrator ode15i for solving the system of implicit 
differential equations. This original implementation also made use of Sandia National Laboratories' MATLAB add-on, Tensor Toolbox to perform the multiplication of multi-dimensional arrays 64. The dependence on these tools inhibited the implementation of the code in other languages such as Python or $\mathrm{C}++$ which may allow for faster computation. Calling the Tensor Toolbox for every tensor multiplication introduced a bottleneck in the code. The current nonlinear solver is implemented in MATLAB, but is written in such a way that it is essentially independent of built in MATLAB functions, and could reasonably be implemented in other coding languages with minimal modifications. Performing the tensor multiplication using the loop structure discussed in Appendix B.4 removes the aforementioned bottleneck and resulted in simulations that were orders of magnitude faster than the previous solver.

Two numerical schemes were employed to simulate the equations of motion; the first method was a projection based Galerkin method which is desirable due to the computational efficiency that can be achieved. A finite difference scheme was also devised such that the results obtained by the Galerkin method could be verified against the direct numerical integration of the discretized equations of motion. The results obtained from both of these numerical schemes are presented, showing reasonably good agreement for the case of undamped free vibration of the structure (i.e. with no airflow). A brief discussion is presented on the convergence of the two numerical schemes. The results obtained by Galerkin projection converged as the number of basis functions used in the approximation were increased sufficiently. Likewise, the results obtained by finite difference converged as the spatial resolution was refined sufficiently. Comparing the dimensionality of the problems based on each method reveals the benefits of the Galerkin method. Using the Galerkin method, a system of 13 simultaneous ordinary differential equations were needed (4 basis functions required for each of the 3 flexible displacements, plus an additional differential equation for the rigid body pitch rotation). The converged solution by finite difference comprised of 301 equations (100 nodes, 3 degrees of freedom per node, and the rigid body pitch rotation). The vast difference in the size of the system results in the Galerkin method outperforming the finite difference scheme. Furthermore, the dimensionality of the system obtained by the Galerkin method could potentially be reduced further by selecting an optimal set of basis functions for use in the 
projection. This optimal basis could be obtained by performing a proper orthogonal decomposition of the spatio-temporal results of the numerical simulation.

The behaviour of the system is studied using the Galerkin method. Studying the free vibration of the wing with and without flow, reveals the influence of nonlinear terms on the behaviour structural model and the coupled aeroelastic model. The effects of the structural nonlinearities are most pronounced when analyzing the post flutter behaviour of the aeroelastic system. Below the flutter speed, the amplitude of linear aeroelastic oscillator will decay to a stable equilibrium. At the flutter point the same oscillator will settle into a regime of simple harmonic motion, oscillating with a constant amplitude which is dictated by the initial conditions. For the aforementioned scenarios, nearly identical behaviour was observed with a linear and nonlinear structural model. However, operating at an airspeed above the flutter speed, a linear system will oscillate with an exponentially growing amplitude, as the system damping is negative. Meanwhile, with a nonlinear structure, the system is able to settle into a limit cycle, whereby the amplitude does not grow to infinity with time, but rather oscillates at a constant amplitude about an unstable equilibrium point, where the amplitude of the system's oscillations are limited by the nonlinearity in the structure. It is important that the nonlinear structural model was able to capture low amplitude limit cycle oscillations, as this is the same behaviour that was observed in wind tunnel tests performed on similar set-ups. Additionally, the flutter speed, the frequencies and the amplitudes are all qualitatively consistent with what is expected of the system given these previous similar experiments, within the declared constraints of the model's physical limitations. It is also worth noting that the amplitude of the nonlinear system's limit cycle oscillations are not strictly dictated by the initial conditions.

Finally, when extending the equations of motion to account for the rigid body pitch rotation, new terms for the pitch stiffness, pitch damping and pitch moment of inertia are introduced to the system of equations. To study the effect of these new parameters on the classical flutter speed and frequency, a global sensitivity analysis is performed. Allowing these three aforementioned parameters to vary uniformly between zero and two times their reported values, it is found that the uncertainty in the 
pitch stiffness is the primary contributor to the variance in the flutter speed and frequency, while the effect of uncertainty in the pitch damping was non-influential in the variances of flutter speed and frequencies. For future endeavours in parameter estimation, this initial study has demonstrated that the stochastic dimensionality of the problem can be reduced, as the parametric uncertainty in two of the three parameters are primarily responsible for the variation of the quantities of interest.

\section{$5.2 \quad$ Future Research}

The work performed herein was an essential precursor to performing state and parameter estimation of the described aeroelastic system using noisy data coming from wind tunnel experiments. Hence, the Bayesian inference aspect encompasses in itself the majority of the future avenues of research stemming off of the current body of work. There are additional avenues which may be followed, which further pursue the current research objectives of model development and efficient numerical implementation. Some of these such possibilities are presented below:

I Further model reduction by employing proper orthogonal decomposition to select an optimal basis for use in the Galerkin projection numerical scheme.

II Expanding the verification by finite difference scheme to the case of the full aeroelastic system, particularly to validate the modal approximation of the unsteady terms used in the unsteady aerodynamic model in Chapter 2

III Employ a nonlinear aerodynamic model, which is capable of capturing the effects of dynamic stall, hence allowing for the study of aeroelastic simulation at larger limit cycle amplitudes.

IV Employ a higher fidelity model for the aerodynamics, which is versatile enough to account for additional effects such as flow separation, turbulence and other phenomena relating to the Reynolds number.

V Perform global sensitivity analysis on a broader spectrum of input parameters, to obtain a greater insight into which parameters are the most influential on the classical flutter mechanism. 
VI Numerically implement a global sensitivity analysis of a quantity of interest pertaining to the aeroelastic response of the nonlinear system. 


\section{List of References}

[1] D. H. Hodges and E. H. Dowell, "Nonlinear equations of motion for the elastic bending and torsion of twisted nonuniform rotor blades," NASA TN D-7818, 1974.

[2] L. Jose Rocha da Costa, "Nonlinear aeroelastic modeling of a flexible wing and comparison with experiments," Master's thesis, Carleton University, 2016.

[3] B. Robinson, L. J. Rocha da Costa, D. Poirel, C. Pettit, M. Khalil, and A. Sarkar, "Aeroelastic oscillations of a cantilever with structural nonlinearities: theory and numerical simulation.,"

[4] D. Poirel, Y. Harris, and A. Benaissa, "Self-sustained aeroelastic oscillations of a naca0012 airfoil at low-to-moderate reynolds numbers," Journal of Fluids and Structures, vol. 24, no. 5, pp. 700-719, 2008.

[5] W. Yuan, D. Poirel, and B. Wang, "Simulations of pitch-heave limit-cycle oscillations at a transitional reynolds number," AIAA journal, vol. 51, no. 7, pp. 1716-1732, 2013.

[6] W. Yuan, D. Poirel, B. Wang, and A. Benaissa, "Effect of freestream turbulence on airfoil limit-cycle oscillations at transitional reynolds numbers," Journal of Aircraft, vol. 52, no. 4, pp. 1214-1225, 2014.

[7] R. L. Bisplinghoff, H. Ashley, and R. L. Halfman, Aeroelasticity. Courier Corporation, 2013.

[8] D. H. Hodges and G. A. Pierce, Introduction to structural dynamics and aeroelasticity, vol. 15. cambridge university press, 2011.

[9] Ü. Gülįat, "Fundamentals of modern unsteady aerodynamics," 2010.

[10] I. M. Sobol, "Sensitivity estimates for nonlinear mathematical models," Mathematical modelling and computational experiments, vol. 1, no. 4, pp. 407-414, 1993.

[11] I. M. Sobol, "Global sensitivity indices for nonlinear mathematical models and their monte carlo estimates," Mathematics and computers in simulation, vol. 55, no. 1-3, pp. 271-280, 2001.

[12] A. Saltelli, M. Ratto, T. Andres, F. Campolongo, J. Cariboni, D. Gatelli, M. Saisana, and

S. Tarantola, Global sensitivity analysis: the primer. John Wiley \& Sons, 2008. 
[13] B. Sudret, "Global sensitivity analysis using polynomial chaos expansions," Reliability Engineering 8 System Safety, vol. 93, no. 7, pp. 964-979, 2008.

[14] E. H. Dowell, H. C. Curtiss, R. H. Scanlan, and F. Sisto, A modern course in aeroelasticity, vol. 3. Springer, 1989.

[15] H. Liu, Wind engineering: a handbook for structural engineering. Pearson Education, 1990.

[16] I. H. Abbott and A. E. Von Doenhoff, Theory of wing sections, including a summary of airfoil data. Courier Corporation, 1959.

[17] K. W. McAlister, L. W. Carr, and W. J. McCroskey, "Dynamic stall experiments on the naca 0012 airfoil," 1978.

[18] E. H. Dowell, "Nonlinear aeroelasticity," in A Modern Course in Aeroelasticity, pp. 487-529, Springer, 2015.

[19] D. Poirel and S. Price, "Random binary (coalescence) flutter of a two-dimensional linear airfoil," Journal of Fluids and Structures, vol. 18, no. 1, pp. 23-42, 2003.

[20] J. C. Houbolt and G. W. Brooks, "Differential equations of motion for combined flapwise bending, chordwise bending, and torsion of twisted nonuniform rotor blades," NACA-TN-3905, 1957.

[21] D. M. Tang and E. H. Dowell, "Experimental and theoretical study for nonlinear aeroelastic behavior of a flexible rotor blade," AIAA journal, vol. 31, no. 6, pp. 1133-1142, 1993.

[22] D. Tang and E. H. Dowell, "Experimental and theoretical study on aeroelastic response of high-aspect-ratio wings," AIAA journal, vol. 39, no. 8, pp. 1430-1441, 2001.

[23] E. H. Dowell and D. Tang, "Nonlinear aeroelasticity and unsteady aerodynamics," AIAA journal, vol. 40, no. 9, pp. 1697-1707, 2002.

[24] T. O’Neil and T. W. Strganac, "Aeroelastic response of a rigid wing supported by nonlinear springs," Journal of Aircraft, vol. 35, no. 4, pp. 616-622, 1998.

[25] D. M. Tang and E. H. Dowell, "Effects of geometric structural nonlinearity on flutter and limit cycle oscillations of high-aspect-ratio wings," Journal of fluids and structures, vol. 19, no. 3, pp. 291-306, 2004.

[26] J. W. Jaworski and E. H. Dowell, "Comparison of theoretical structural models with experiment for a high-aspect-ratio aeroelastic wing," Journal of Aircraft, vol. 46, no. 2, p. 708, 2009.

[27] D. Poirel and W. Yuan, "Aerodynamics of laminar separation flutter at a transitional reynolds number," Journal of Fluids and Structures, vol. 26, no. 7, pp. 1174-1194, 2010. 
[28] R. Sandhu, D. Poirel, C. Pettit, M. Khalil, and A. Sarkar, "Bayesian inference of nonlinear unsteady aerodynamics from aeroelastic limit cycle oscillations," Journal of Computational Physics, vol. 316, pp. 534-557, 2016.

[29] A. Rosen and P. Friedmann, "The nonlinear behavior of elastic slender straight beams undergoing small strains and moderate rotations," Journal of Applied Mechanics, vol. 46, no. 1, pp. 161-168, 1979 .

[30] K. Kim, Nonlinear aeroelastic analysis of aircraft wing-with-store configurations. PhD thesis, Texas A\&M University, 2004.

[31] T. J. Sullivan, Introduction to uncertainty quantification, vol. 63. Springer, 2015.

[32] R. C. Smith, Uncertainty quantification: theory, implementation, and applications, vol. 12. Siam, 2013.

[33] M. Khalil, D. Poirel, and A. Sarkar, "Probabilistic parameter estimation of a fluttering aeroelastic system in the transitional reynolds number regime," Journal of Sound and Vibration, vol. 332, no. 15, pp. 3670-3691, 2013.

[34] R. Sandhu, M. Khalil, A. Sarkar, and D. Poirel, "Bayesian model selection for nonlinear aeroelastic systems using wind-tunnel data," Computer Methods in Applied Mechanics and Engineering, vol. 282, pp. 161-183, 2014.

[35] R. Sandhu, C. Pettit, M. Khalil, D. Poirel, and A. Sarkar, "Bayesian model selection using automatic relevance determination for nonlinear dynamical systems," Computer Methods in Applied Mechanics and Engineering, vol. 320, pp. 237-260, 2017.

[36] A. Desai and S. Sarkar, "Analysis of a nonlinear aeroelastic system with parametric uncertainties using polynomial chaos expansion," Mathematical Problems in Engineering, vol. 2010, 2010.

[37] C. L. Pettit and P. S. Beran, "Effects of parametric uncertainty on airfoil limit cycle oscillation," Journal of Aircraft, vol. 40, no. 5, pp. 1004-1006, 2003.

[38] P. S. Beran, C. L. Pettit, and D. R. Millman, "Uncertainty quantification of limit-cycle oscillations," Journal of Computational Physics, vol. 217, no. 1, pp. 217-247, 2006.

[39] J. Jaworski, Nonlinear aeroelastic analysis of flexible high aspect ratio wings including correlation with experiment. PhD thesis, 2009.

[40] J. R. Wright and J. E. Cooper, Introduction to aircraft aeroelasticity and loads, vol. 20. John Wiley \& Sons, 2008.

[41] T. Theodorsen, "General theory of aerodynamic instability and the mechanism of flutter," 1949. 
[42] E. Dowell, J. Traybar, and D. H. Hodges, "An experimental-theoretical correlation study of non-linear bending and torsion deformations of a cantilever beam," Journal of Sound and Vibration, vol. 50, no. 4, pp. 533-544, 1977.

[43] D. C. M. Poirel, Random dynamics of a structurally nonlinear airfoil in turbulent flow. 2001.

[44] K. W. McAlister, O. Lambert, and D. Petot, "Application of the onera model of dynamic stall," tech. rep., NATIONAL AERONAUTICS AND SPACE ADMINISTRATION MOFFETT FIELD CA AMES RESEARCH CENTER, 1984.

[45] J. W. Larsen, S. R. Nielsen, and S. Krenk, "Dynamic stall model for wind turbine airfoils," Journal of Fluids and Structures, vol. 23, no. 7, pp. 959-982, 2007.

[46] K.-J. Bathe, Finite element procedures. Klaus-Jurgen Bathe, 2006.

[47] M. P. Paidoussis, Fluid-structure interactions: slender structures and axial flow, vol. 1. Academic press, 1998.

[48] L. Meirovitch, Analytical methods in vibrations, vol. 438. Macmillan New York, 1967.

[49] J. N. Reddy, An Introduction to Nonlinear Finite Element Analysis: with applications to heat transfer, fluid mechanics, and solid mechanics. OUP Oxford, 2014.

[50] A. Matachniouk, Parametric Uncertainty Quantification in Coalescene Flutter. PhD thesis, Carleton University, 2013.

[51] C. Semler, W. C. Gentleman, and M. P. Paidoussis, "Numerical solutions of second order implicit non-linear ordinary differential equations," Journal of Sound and Vibration, vol. 195, no. 4, pp. 553-574, 1996.

[52] R. D. Cook, D. S. Malkus, M. E. Plesha, et al., Concepts and applications of finite element analysis, vol. 4. Wiley New York, 1974.

[53] G. Forsythe and W. R. Wasow, Finite-Difference Methods for Partial Differential Equations, Applied Mathematical Series. Wiley, New York, 1960.

[54] A. Saltelli, "Making best use of model evaluations to compute sensitivity indices," Computer physics communications, vol. 145, no. 2, pp. 280-297, 2002.

[55] S. Tarantola, M. Nardo, M. Saisana, and D. Gatelli, "A new estimator for sensitivity analysis of model output: An application to the e-business readiness composite indicator," Reliability Engineering \& System Safety, vol. 91, no. 10-11, pp. 1135-1141, 2006.

[56] L. Lilburne and S. Tarantola, "Sensitivity analysis of spatial models," International Journal of Geographical Information Science, vol. 23, no. 2, pp. 151-168, 2009.

[57] B. Debusschere, K. Sargsyan, C. Safta, and K. Chowdhary, "Uncertainty quantification toolkit (uqtk)," Handbook of Uncertainty Quantification, pp. 1-21, 2016. 
[58] L. C. Andrews and L. C. Andrews, Special functions of mathematics for engineers. McGraw-Hill New York, 1992.

[59] W. Schoutens, Stochastic processes and orthogonal polynomials, vol. 146. Springer Science \& Business Media, 2012.

[60] D. Xiu and G. E. Karniadakis, "The wiener-askey polynomial chaos for stochastic differential equations," SIAM journal on scientific computing, vol. 24, no. 2, pp. 619-644, 2002.

[61] R. G. Ghanem and P. D. Spanos, "Stochastic finite element method: Response statistics," in Stochastic Finite Elements: A Spectral Approach, pp. 101-119, Springer, 1991.

[62] J. Stoer and R. Bulirsch, Introduction to numerical analysis, vol. 12. Springer Science \& Business Media, 2013.

[63] S. A. Smolyak, "Quadrature and interpolation formulas for tensor products of certain classes of functions," in Doklady Akademii Nauk, vol. 148, pp. 1042-1045, Russian Academy of Sciences, 1963.

[64] B. W. Bader, T. G. Kolda, et al., "Matlab tensor toolbox version 2.6, available online, february 2015," URL http://www. sandia. gov/ tgkolda/TensorToolbox/index-2.6. html, 2015. 


\section{Appendix A}

\section{Derivation of the Nonlinear Equations of Motion}

The equations of motion have been derived using Hamilton's Principle, and presented in Section 2.4 The contents of this appendix outline the expansion of the strain energy components of the PDEs, ODE and the BCs via the substitution of the stress and moment resultants as derived previously. Subsequently, for both the strain energy components and the kinetic energy components, a small angle approximation will be introduced for the sines and cosines of the angle of twist, $\phi$, whereby a first order Taylor Series expansion of these trigonometric functions will be applied. Following this, a final second order approximation will be made to yield the final equations of motion. This approximation takes into account the relative order of magnitude of the axial displacement $u\left(\mathcal{O}\left(\epsilon^{2}\right)\right)$, which is an order smaller than the displacements $v, w$, and $\phi(\mathcal{O}(\epsilon))$, and two orders smaller than $\theta$ $(\mathcal{O}(1))$. It will also take into account the fundamental difference between the physical parameters involved in the strain and kinetic energy components, accounting for an appropriate scaling of the order of the terms considered in the approximation.

\section{A.1 Small Angle Assumption}

The small angle assumption will be imposed for the angle of twist, $\phi$, by linearizing $\sin \phi$, and $\cos \phi$ about zero. To avoid complicating the equations of motion with unnecessary negligibly small terms, taking the first order series expansion of the respective functions. 


$$
\begin{aligned}
& \sin \phi=\phi-\frac{\phi^{3}}{6}+\frac{\phi^{5}}{120}+\mathcal{O}\left(\phi^{7}\right) \approx \phi \quad \sin ^{2} \phi=\phi^{2}-\frac{\phi^{4}}{3}+\frac{2 \phi^{6}}{45}+\mathcal{O}\left(\phi^{8}\right) \approx 0 \\
& \cos \phi=1-\frac{\phi^{2}}{2}+\frac{\phi^{4}}{24}-\frac{2 \phi^{6}}{720}+\mathcal{O}\left(\phi^{7}\right) \approx 1 \quad \cos ^{2} \phi=1-\phi^{2}+\frac{\phi^{4}}{3}-\frac{2 \phi^{6}}{45}+\mathcal{O}\left(\phi^{8}\right) \approx 1 \quad(\text { A. } 1 \quad \text { c, d })
\end{aligned}
$$

\section{A.2 Strain energy components}

The underlined terms will not be retained in the second order approximation. Note that the order of terms retained in the approximation of $\bar{U}_{u}$ will be $\mathcal{O}\left(\epsilon^{4}\right)$ to $\mathcal{O}\left(\epsilon^{5}\right)$. For $\bar{U}_{v}, \bar{U}_{w}$, and $\bar{U}_{\phi}$ terms of order $\mathcal{O}\left(\epsilon^{5}\right)$ to $\mathcal{O}\left(\epsilon^{6}\right)$ will be retained. The single strain energy term $\bar{U}_{\theta}$ which contributes to the ODE associated with $\delta \theta$, is also retained according to this approximation scheme.

In view of the fact that it can be shown that $N_{x}^{\prime}$ (and $N_{x}$ by extension) are equal to zero to the second order, this will be exploited to simplify the equation of motion before beginning to expanding the expressions for the strain components below.

$$
\begin{aligned}
\left\{N_{\mathbf{x}}\right\}^{\prime} & =E A\left(u^{\prime \prime}+\left(v^{\prime \prime} v^{\prime}\right)+\left(w^{\prime \prime} w^{\prime}\right)\right)+E A k_{a}^{2}\left(\left(\phi^{\prime \prime} \phi^{\prime}\right)\right)-E A e_{A}\left(\left(v^{\prime \prime \prime}+w^{\prime \prime} \phi^{\prime}\right) \cos \phi+\left(w^{\prime \prime \prime}-v^{\prime \prime} \phi^{\prime}\right) \sin \phi\right) \\
& =E A\left(u^{\prime \prime}+\left(v^{\prime \prime} v^{\prime}\right)+\left(w^{\prime \prime} w^{\prime}\right)\right)+E A k_{a}{ }^{2}\left(\underline{\phi^{\prime \prime} \phi^{\prime}}\right)-E A e_{A}\left(v^{\prime \prime \prime}+w^{\prime \prime} \phi^{\prime}+w^{\prime \prime \prime} \phi-\underline{v^{\prime \prime} \phi^{\prime} \phi}\right) \\
& =E A\left(u^{\prime \prime}+\left(v^{\prime \prime} v^{\prime}\right)+\left(w^{\prime \prime} w^{\prime}\right)\right)-E A e_{A}\left(v^{\prime \prime \prime}+w^{\prime \prime} \phi^{\prime}+w^{\prime \prime \prime} \phi\right) \\
& \equiv 0
\end{aligned}
$$

Hence, one can write

$$
\left(u^{\prime \prime}+\left(v^{\prime \prime} v^{\prime}\right)+\left(w^{\prime \prime} w^{\prime}\right)\right)=e_{A}\left(v^{\prime \prime \prime}+w^{\prime \prime} \phi^{\prime}+w^{\prime \prime \prime} \phi\right)
$$

Integrating Eq.A.3, one obtains 


$$
\left(u^{\prime}+\frac{v^{\prime 2}}{2}+\frac{w^{\prime 2}}{2}\right)=e_{A}\left(v^{\prime \prime}+w^{\prime \prime} \phi\right)
$$

Likewise, diferentiating Eq.(A.3) gives

$$
\left(u^{\prime \prime \prime}+\left(v^{\prime \prime \prime} v^{\prime}+v^{\prime 2}\right)+\left(w^{\prime \prime \prime} w^{\prime}+w^{\prime \prime 2}\right)\right)=e_{A}\left(v^{\prime \prime \prime \prime}+w^{\prime \prime \prime \prime} \phi+2 w^{\prime \prime \prime} \phi^{\prime}+w^{\prime \prime} \phi^{\prime \prime}\right)
$$

Making these above substitutions (indicated by a double underline), where applicable to remove the instances of $u$ and its derivatives from the remaining differential equations prior to imposing the small angle assumption for $\phi$ and removing higher order terms (indicated by single underline

$$
\begin{aligned}
& \bar{U}_{v}=-\left\{N_{\mathbf{x}} v^{\prime}\right\}^{\prime}+\left\{M_{\mathbf{y}} \sin \phi-M_{\mathbf{z}} \cos \phi\right\}^{\prime \prime} \\
& =-\left(\underline{\underline{N_{\mathbf{x}}^{\prime}}} v^{\prime}+\underline{\underline{N_{\mathbf{x}}}} v^{\prime \prime}\right)+\left(\left(M_{\mathbf{y}}^{\prime \prime}-M_{\mathbf{y}} \phi^{\prime 2}+2 M_{\mathbf{z}}^{\prime} \phi^{\prime}+M_{\mathbf{z}} \phi^{\prime \prime}\right) \sin \phi+\left(-M_{\mathbf{z}}^{\prime \prime}+M_{\mathbf{z}} \phi^{2}+2 M_{\mathbf{y}}^{\prime} \phi^{\prime}+M_{\mathbf{y}} \phi^{\prime \prime}\right) \cos \phi\right) \\
& =\left(\left\{E I_{\mathbf{y}}\left(\left(v^{\prime \prime \prime \prime}-v^{\prime \prime} \phi^{2}+2 w^{\prime \prime} \phi^{\prime}+w^{\prime \prime} \phi^{\prime \prime}\right) \sin \phi+\left(-w^{\prime \prime \prime \prime}+w^{\prime \prime} \phi^{2}+2 v^{\prime \prime \prime} \phi^{\prime}+v^{\prime \prime} \phi^{\prime \prime}\right) \cos \phi\right)-E C_{1}{ }^{*} \phi^{\prime \prime \prime \prime}\right\}\right. \\
& -\left\{E I_{\mathbf{y}}\left(v^{\prime \prime} \sin \phi-w^{\prime \prime} \cos \phi\right)-E C_{1}{ }^{*} \phi^{\prime \prime}\right\} \phi^{\prime 2}+2\left\{E A e_{A}\left(\underline{\underline{u^{\prime \prime}+\left(v^{\prime \prime} v^{\prime}\right)+\left(w^{\prime \prime} w^{\prime}\right)}}\right)+E B_{2} *\left(\left(\phi^{\prime \prime} \phi^{\prime}\right)\right)\right. \\
& \left.+E I_{\mathbf{z}}\left(-\left(v^{\prime \prime \prime}+w^{\prime \prime} \phi^{\prime}\right) \cos \phi-\left(w^{\prime \prime \prime}-v^{\prime \prime} \phi^{\prime}\right) \sin \phi\right)\right\} \phi^{\prime}+\left\{E A e_{A}\left(\underline{\underline{\left(u^{\prime}+\frac{v^{\prime 2}}{2}+\frac{w^{2}}{2}\right.}}\right)+E B_{2} *\left(\frac{\phi^{\prime 2}}{2}\right)\right. \\
& \left.\left.+E I_{\mathbf{z}}\left(-v^{\prime \prime} \cos \phi-w^{\prime \prime} \sin \phi\right)\right\} \phi^{\prime \prime}\right) \sin \phi \\
& +\left(-\left\{E A e_{A}\left(\underline{\underline{u^{\prime \prime \prime}+\left(v^{\prime \prime \prime} v^{\prime}+v^{\prime \prime 2}\right)+\left(w^{\prime \prime \prime} w^{\prime}+w^{\prime \prime 2}\right)}}\right)+E B_{2} *\left(\left(\phi^{\prime \prime \prime} \phi^{\prime}+\phi^{\prime 2}\right)\right)\right.\right. \\
& \left.+E I_{\mathbf{z}}\left(-\left(v^{\prime \prime \prime \prime}+v^{\prime \prime} \phi^{2}-2 w^{\prime \prime \prime} \phi^{\prime}-w^{\prime \prime} \phi^{\prime \prime}\right) \cos \phi-\left(w^{\prime \prime \prime \prime}-w^{\prime \prime} \phi^{2}-2 v^{\prime \prime \prime} \phi^{\prime}-v^{\prime \prime} \phi^{\prime \prime}\right) \sin \phi\right)\right\} \\
& +\left\{E A e_{A}\left(\underline{\underline{u^{\prime}+\frac{v^{\prime 2}}{2}+\frac{w^{\prime 2}}{2}}}\right)+E B_{2} *\left(\frac{\phi^{\prime 2}}{2}\right)+E I_{\mathbf{z}}\left(-v^{\prime \prime} \cos \phi-w^{\prime \prime} \sin \phi\right)\right\} \phi^{\prime 2} \\
& +2\left\{E I_{\mathbf{y}}\left(\left(v^{\prime \prime \prime}+w^{\prime \prime} \phi^{\prime}\right) \sin \phi-\left(w^{\prime \prime \prime}-v^{\prime \prime} \phi^{\prime}\right) \cos \phi\right)-E C_{1}{ }^{*} \phi^{\prime \prime \prime}\right\} \phi^{\prime} \\
& \left.+\left\{E I_{\mathbf{y}}\left(v^{\prime \prime} \sin \phi-w^{\prime \prime} \cos \phi\right)-E C_{1}{ }^{*} \phi^{\prime \prime}\right\} \phi^{\prime \prime}\right) \cos \phi \\
& =E A e_{A}^{2}\left\{2\left(v^{\prime \prime \prime}+w^{\prime \prime} \phi^{\prime}+w^{\prime \prime \prime} \phi\right) \phi^{\prime} \sin \phi+\left(v^{\prime \prime}+w^{\prime \prime} \phi\right) \phi^{\prime \prime} \sin \phi\right. \\
& \left.-\left(v^{\prime \prime \prime \prime}+w^{\prime \prime \prime \prime} \phi+2 w^{\prime \prime \prime} \phi^{\prime}+w^{\prime \prime} \phi^{\prime \prime}\right) \cos \phi+\left(v^{\prime \prime}+w^{\prime \prime} \phi\right) \phi^{\prime 2} \cos \phi\right\} \\
& +E I_{\mathbf{y}}\left\{\left(\left(v^{\prime \prime \prime \prime}-v^{\prime \prime} \phi^{2}+2 w^{\prime \prime} \phi^{\prime}+w^{\prime \prime} \phi^{\prime \prime}\right) \sin ^{2} \phi+\left(-w^{\prime \prime \prime \prime}+w^{\prime \prime} \phi^{2}+2 v^{\prime \prime \prime} \phi^{\prime}+v^{\prime \prime} \phi^{\prime \prime}\right) \sin \phi \cos \phi\right)\right. \\
& -\left(v^{\prime \prime} \sin ^{2} \phi-w^{\prime \prime} \sin \phi \cos \phi\right) \phi^{2}+2\left(\left(v^{\prime \prime \prime}+w^{\prime \prime} \phi^{\prime}\right) \sin \phi \cos \phi-\left(w^{\prime \prime \prime}-v^{\prime \prime} \phi^{\prime}\right) \cos ^{2} \phi\right) \phi^{\prime} \\
& \left.+\left(v^{\prime \prime} \cos \phi \sin \phi-w^{\prime \prime} \cos ^{2} \phi\right) \phi^{\prime \prime}\right\}
\end{aligned}
$$




$$
\begin{aligned}
& +E I_{\mathbf{z}}\left\{2\left(-\left(v^{\prime \prime \prime}+w^{\prime \prime} \phi^{\prime}\right) \sin \phi \cos \phi-\left(w^{\prime \prime \prime}-v^{\prime \prime} \phi^{\prime}\right) \sin ^{2} \phi\right) \phi^{\prime}+\left(-v^{\prime \prime} \sin \phi \cos \phi-w^{\prime \prime} \sin ^{2} \phi\right) \phi^{\prime \prime}\right. \\
& +\left(\left(v^{\prime \prime \prime \prime}+v^{\prime \prime} \phi^{2}-2 w^{\prime \prime \prime} \phi^{\prime}-w^{\prime \prime} \phi^{\prime \prime}\right) \cos ^{2} \phi+\left(w^{\prime \prime \prime \prime}-w^{\prime \prime} \phi^{2}-2 v^{\prime \prime \prime} \phi^{\prime}-v^{\prime \prime} \phi^{\prime \prime}\right) \sin \phi \cos \phi\right) \\
& \left.+\left(-v^{\prime \prime} \cos ^{2} \phi-w^{\prime \prime} \sin \phi \cos \phi\right) \phi^{2}\right\} \\
& +E B_{2}{ }^{*}\left\{2\left(\phi^{\prime \prime} \phi^{\prime}\right) \phi^{\prime} \sin \phi+\left(\frac{\phi^{2}}{2}\right) \phi^{\prime \prime} \sin \phi-\left(\phi^{\prime \prime \prime} \phi^{\prime}+\phi^{\prime \prime 2}\right) \cos \phi+\left(\frac{\phi^{\prime 2}}{2}\right) \phi^{\prime 2} \cos \phi\right\} \\
& +E C_{1}^{*}\left\{-\left(\phi^{\prime \prime \prime \prime}\right) \sin \phi+\left(\phi^{\prime \prime}\right) \phi^{\prime 2} \sin \phi-2\left(\phi^{\prime \prime \prime}\right) \phi^{\prime} \cos \phi-\left(\phi^{\prime \prime}\right) \phi^{\prime \prime} \cos \phi\right\} \\
& =E A e_{A}^{2}\left\{\underline{2 v^{\prime \prime \prime} \phi^{\prime} \phi}+\underline{2 w^{\prime \prime} \phi^{2} \phi}+\underline{2 w^{\prime \prime \prime} \phi^{\prime} \phi^{2}}+\underline{v^{\prime \prime} \phi^{\prime \prime} \phi}+\underline{w^{\prime \prime} \phi^{\prime \prime} \phi^{2}}-v^{\prime \prime \prime \prime}-w^{\prime \prime \prime \prime} \phi-2 w^{\prime \prime \prime} \phi^{\prime}-w^{\prime \prime} \phi^{\prime \prime}+\underline{v^{\prime \prime} \phi^{\prime 2}}+\underline{w^{\prime \prime} \phi^{\prime 2}}\right\} \\
& +E I_{\mathbf{y}}\left\{-w^{\prime \prime \prime \prime} \phi+\underline{w^{\prime \prime} \phi^{2} \phi}+\underline{2 v^{\prime \prime \prime} \phi^{\prime} \phi}+\underline{v^{\prime \prime} \phi^{\prime \prime} \phi}+\underline{w^{\prime \prime} \phi^{2} \phi}+\underline{2 v^{\prime \prime \prime} \phi^{\prime} \phi}+\underline{2 w^{\prime \prime} \phi^{2} \phi}-2 w^{\prime \prime \prime} \phi^{\prime}+\underline{2 v^{\prime \prime} \phi^{\prime 2}}+\underline{v^{\prime \prime} \phi^{\prime \prime} \phi}-w^{\prime \prime} \phi^{\prime \prime}\right\} \\
& +E I_{\mathbf{z}}\left\{-\underline{2 v^{\prime \prime \prime} \phi^{\prime} \phi}-\underline{2 w^{\prime \prime} \phi^{\prime 2} \phi}-\underline{v^{\prime \prime} \phi^{\prime \prime} \phi}+v^{\prime \prime \prime \prime}+\underline{v^{\prime \prime} \phi^{\prime 2}}-2 w^{\prime \prime \prime} \phi^{\prime}-w^{\prime \prime} \phi^{\prime \prime}+w^{\prime \prime \prime \prime} \phi-\underline{w^{\prime \prime} \phi^{2} \phi}-\underline{2 v^{\prime \prime \prime} \phi^{\prime} \phi}-\underline{v^{\prime \prime} \phi^{\prime \prime} \phi}\right. \\
& \left.-\underline{v^{\prime \prime} \phi^{\prime 2}}-\underline{w^{\prime \prime} \phi^{\prime 2} \phi}\right\}+E B_{2}^{*}\left\{\underline{2 \phi^{\prime \prime} \phi^{\prime 2} \phi}+\frac{1}{2} \underline{\phi^{\prime 2} \phi^{\prime \prime} \phi}-\underline{\phi^{\prime \prime \prime} \phi^{\prime}-\phi^{\prime \prime 2}}+\frac{1}{2} \underline{\phi^{\prime 4}}\right\} \\
& +E C_{1}^{*}\left\{-\underline{\phi^{\prime \prime \prime \prime} \phi}+\underline{\phi^{\prime \prime} \phi^{\prime 2} \phi}-\underline{2 \phi^{\prime \prime \prime} \phi^{\prime}}-\underline{\phi^{\prime \prime 2}}\right\} \\
& =-E A e_{A}\left\{v^{\prime \prime \prime \prime}+w^{\prime \prime \prime \prime} \phi+2 w^{\prime \prime \prime} \phi^{\prime}+w^{\prime \prime} \phi^{\prime \prime}\right\}+\left(E I_{\mathbf{z}}-E I_{\mathbf{y}}\right)\left\{w^{\prime \prime} \phi^{\prime \prime}+2 w^{\prime \prime \prime} \phi^{\prime}+w^{\prime \prime \prime \prime} \phi\right\}+E I_{\mathbf{z}}\left\{v^{\prime \prime \prime \prime}\right\} \\
& \bar{U}_{w}=-\left\{N_{x} w^{\prime}\right\}^{\prime}-\left\{M_{z} \sin \phi+M_{y} \cos \phi\right\}^{\prime \prime} \\
& =-\left(\underline{\underline{N_{x}^{\prime}}} w^{\prime}+\underline{\underline{N_{x}}} w^{\prime \prime}\right)-\left(\left(M_{z}^{\prime \prime}-M_{z} \phi^{2}-2 M_{y}^{\prime} \phi^{\prime}-M_{y} \phi^{\prime \prime}\right) \sin \phi+\left(M_{y}^{\prime \prime}-M_{y} \phi^{2}+2 M_{z}^{\prime} \phi^{\prime}+M_{z} \phi^{\prime \prime}\right) \cos \phi\right) \\
& =-\left(\left\{E A e_{A}\left(\underline{\underline{u^{\prime \prime \prime}+\left(v^{\prime \prime \prime} v^{\prime}+v^{\prime \prime 2}\right)+\left(w^{\prime \prime \prime} w^{\prime}+w^{\prime \prime 2}\right)}}\right)+E B_{2} *\left(\left(\phi^{\prime \prime \prime} \phi^{\prime}+\phi^{\prime \prime 2}\right)\right)\right.\right. \\
& \left.+E I_{\mathbf{z}}\left(-\left(v^{\prime \prime \prime \prime}+v^{\prime \prime} \phi^{2}-2 w^{\prime \prime \prime} \phi^{\prime}-w^{\prime \prime} \phi^{\prime \prime}\right) \cos \phi-\left(w^{\prime \prime \prime \prime}-w^{\prime \prime} \phi^{2}-2 v^{\prime \prime \prime} \phi^{\prime}-v^{\prime \prime} \phi^{\prime \prime}\right) \sin \phi\right)\right\} \\
& -\left\{E A e_{A}\left(\underline{\underline{u^{\prime}+\frac{v^{\prime 2}}{2}+\frac{w^{\prime 2}}{2}}}\right)+E B_{2} *\left(\frac{\phi^{\prime 2}}{2}\right)+E I_{\mathbf{z}}\left(-v^{\prime \prime} \cos \phi-w^{\prime \prime} \sin \phi\right)\right\} \phi^{\prime 2} \\
& -2\left\{E I_{\mathbf{y}}\left(\left(v^{\prime \prime \prime}+w^{\prime \prime} \phi^{\prime}\right) \sin \phi-\left(w^{\prime \prime \prime}-v^{\prime \prime} \phi^{\prime}\right) \cos \phi\right)-E C_{1}{ }^{*} \phi^{\prime \prime \prime}\right\} \phi^{\prime} \\
& \left.-\left\{E I_{\mathbf{y}}\left(v^{\prime \prime} \sin \phi-w^{\prime \prime} \cos \phi\right)-E C_{1}{ }^{*} \phi^{\prime \prime}\right\} \phi^{\prime \prime}\right) \sin \phi \\
& -\left(\left\{E I_{\mathbf{y}}\left(\left(v^{\prime \prime \prime \prime}-v^{\prime \prime} \phi^{2}+2 w^{\prime \prime \prime} \phi^{\prime}+w^{\prime \prime} \phi^{\prime \prime}\right) \sin \phi+\left(-w^{\prime \prime \prime \prime}+w^{\prime \prime} \phi^{2}+2 v^{\prime \prime \prime} \phi^{\prime}+v^{\prime \prime} \phi^{\prime \prime}\right) \cos \phi\right)-E C_{1}{ }^{*} \phi^{\prime \prime \prime \prime}\right\}\right. \\
& -\left\{E I_{\mathbf{y}}\left(v^{\prime \prime} \sin \phi-w^{\prime \prime} \cos \phi\right)-E C_{1}{ }^{*} \phi^{\prime \prime}\right\} \phi^{2}+2\left\{E A e_{A}\left(\underline{\underline{u^{\prime \prime}+\left(v^{\prime \prime} v^{\prime}\right)+\left(w^{\prime \prime} w^{\prime}\right)}}\right)+E B_{2} *\left(\left(\phi^{\prime \prime} \phi^{\prime}\right)\right)\right. \\
& \left.+E I_{\mathbf{z}}\left(-\left(v^{\prime \prime \prime}+w^{\prime \prime} \phi^{\prime}\right) \cos \phi-\left(w^{\prime \prime \prime}-v^{\prime \prime} \phi^{\prime}\right) \sin \phi\right)\right\} \phi^{\prime} \\
& \left.+\left\{E A e_{A}\left(\underline{\underline{u^{\prime}+\frac{v^{\prime 2}}{2}+\frac{w^{\prime 2}}{2}}}\right)+E B_{2} *\left(\frac{\phi^{\prime 2}}{2}\right)+E I_{\mathbf{z}}\left(-v^{\prime \prime} \cos \phi-w^{\prime \prime} \sin \phi\right)\right\} \phi^{\prime \prime}\right) \cos \phi \\
& =E A e_{A}^{2}\left\{-\left(v^{\prime \prime \prime \prime}+w^{\prime \prime \prime \prime} \phi+2 w^{\prime \prime \prime} \phi^{\prime}+w^{\prime \prime} \phi^{\prime \prime}\right) \sin \phi+\left(v^{\prime \prime}+w^{\prime \prime} \phi\right) \phi^{2} \sin \phi\right. \\
& \left.-2\left(v^{\prime \prime \prime}+w^{\prime \prime} \phi^{\prime}+w^{\prime \prime \prime} \phi\right) \phi^{\prime} \cos \phi-\left(v^{\prime \prime}+w^{\prime \prime} \phi\right) \phi^{\prime \prime} \cos \phi\right\} \\
& +E I_{\mathbf{y}}\left\{\left(-\left(v^{\prime \prime \prime \prime}-v^{\prime \prime} \phi^{2}+2 w^{\prime \prime \prime} \phi^{\prime}+w^{\prime \prime} \phi^{\prime \prime}\right) \sin \phi \cos \phi-\left(-w^{\prime \prime \prime \prime}+w^{\prime \prime} \phi^{2}+2 v^{\prime \prime \prime} \phi^{\prime}+v^{\prime \prime} \phi^{\prime \prime}\right) \cos ^{2} \phi\right)\right. \\
& +2\left(\left(v^{\prime \prime \prime}+w^{\prime \prime} \phi^{\prime}\right) \sin ^{2} \phi-\left(w^{\prime \prime \prime}-v^{\prime \prime} \phi^{\prime}\right) \cos \phi \sin \phi\right) \phi^{\prime}+\left(v^{\prime \prime} \sin ^{2} \phi-w^{\prime \prime} \cos \phi \sin \phi\right) \phi^{\prime \prime} \\
& \left.+\left(v^{\prime \prime} \sin \phi \cos \phi-w^{\prime \prime} \cos ^{2} \phi\right) \phi^{\prime 2}\right\}
\end{aligned}
$$




$$
\begin{aligned}
& +E I_{\mathbf{z}}\left\{\left(\left(v^{\prime \prime \prime \prime}+v^{\prime \prime} \phi^{2}-2 w^{\prime \prime \prime} \phi^{\prime}-w^{\prime \prime} \phi^{\prime \prime}\right) \cos \phi \sin \phi+\left(w^{\prime \prime \prime \prime}-w^{\prime \prime} \phi^{2}-2 v^{\prime \prime \prime} \phi^{\prime}-v^{\prime \prime} \phi^{\prime \prime}\right) \sin ^{2} \phi\right)\right. \\
& +\left(-v^{\prime \prime} \cos \phi \sin \phi-w^{\prime \prime} \sin ^{2} \phi\right) \phi^{2}+2\left(\left(v^{\prime \prime \prime}+w^{\prime \prime} \phi^{\prime}\right) \cos ^{2} \phi+\left(w^{\prime \prime \prime}-v^{\prime \prime} \phi^{\prime}\right) \sin \phi \cos \phi\right) \phi^{\prime} \\
& \left.+\left(v^{\prime \prime} \cos ^{2} \phi+w^{\prime \prime} \sin \phi \cos \phi\right) \phi^{\prime \prime}\right\} \\
& +E B_{2}^{*}\left\{-\left(\phi^{\prime \prime \prime} \phi^{\prime}+\phi^{\prime \prime 2}\right) \sin \phi+\left(\frac{\phi^{2}}{2}\right) \phi^{\prime 2} \sin \phi-2\left(\phi^{\prime \prime} \phi^{\prime}\right) \phi^{\prime} \cos \phi-\left(\frac{\phi^{2}}{2}\right) \phi^{\prime \prime} \cos \phi\right\} \\
& +E C_{1}^{*}\left\{-2\left(\phi^{\prime \prime \prime}\right) \phi^{\prime} \sin \phi-\left(\phi^{\prime \prime}\right) \phi^{\prime \prime} \sin \phi+\left(\phi^{\prime \prime \prime \prime}\right) \cos \phi-\left(\phi^{\prime \prime}\right) \phi^{\prime 2} \cos \phi\right\} \\
& =E A e_{A}{ }^{2}\left\{-v^{\prime \prime \prime \prime} \phi-\underline{w^{\prime \prime \prime \prime} \phi^{2}}-\underline{2 w^{\prime \prime \prime} \phi^{\prime} \phi}-\underline{w^{\prime \prime} \phi^{\prime \prime} \phi}+\underline{v^{\prime \prime} \phi^{2} \phi}+\underline{w^{\prime \prime} \phi^{\prime 2} \phi^{2}}-2 v^{\prime \prime \prime} \phi^{\prime}-\underline{2 w^{\prime \prime} \phi^{\prime 2}}-\underline{2 w^{\prime \prime \prime} \phi^{\prime} \phi}-v^{\prime \prime} \phi^{\prime \prime}-\underline{w^{\prime \prime} \phi^{\prime \prime} \phi}\right\} \\
& +E I_{\mathbf{y}}\left\{-v^{\prime \prime \prime \prime} \phi-\underline{v^{\prime \prime} \phi^{\prime 2} \phi}+\underline{2 w^{\prime \prime \prime} \phi^{\prime} \phi}+\underline{w^{\prime \prime} \phi^{\prime \prime} \phi}+w^{\prime \prime \prime \prime}-\underline{w^{\prime \prime} \phi^{\prime 2}}-2 v^{\prime \prime \prime} \phi^{\prime}-v^{\prime \prime} \phi^{\prime \prime}-\underline{2 w^{\prime \prime \prime} \phi^{\prime} \phi}+\underline{2 v^{\prime \prime} \phi^{2} \phi}-\underline{w^{\prime \prime} \phi^{\prime \prime} \phi}\right. \\
& \left.+\underline{v^{\prime \prime} \phi^{\prime 2} \phi}-\underline{w^{\prime \prime} \phi^{\prime 2}}\right\} \\
& +E I_{\mathbf{z}}\left\{v^{\prime \prime \prime \prime} \phi+\underline{v^{\prime \prime} \phi^{2} \phi}-\underline{2 w^{\prime \prime \prime} \phi^{\prime} \phi}-\underline{w^{\prime \prime} \phi^{\prime \prime} \phi}-\underline{v^{\prime \prime} \phi^{2} \phi}+2 v^{\prime \prime \prime} \phi^{\prime}+\underline{2 w^{\prime \prime} \phi^{\prime 2}}+\underline{2 w^{\prime \prime \prime} \phi^{\prime} \phi}-\underline{2 v^{\prime \prime} \phi^{\prime 2} \phi}+v^{\prime \prime} \phi^{\prime \prime}+\underline{w^{\prime \prime} \phi^{\prime \prime} \phi}\right\} \\
& +E B_{2}^{*}\left\{-\underline{\phi^{\prime \prime \prime} \phi^{\prime} \phi}-\underline{\phi^{\prime \prime 2} \phi}+\frac{1}{2} \underline{\phi^{\prime 4} \phi}-\underline{2 \phi^{\prime \prime} \phi^{\prime 2}}-\frac{1}{2} \underline{\phi^{\prime 2} \phi^{\prime \prime}}\right\}+E C_{1}^{*}\left\{-\underline{2 \phi^{\prime \prime \prime} \phi^{\prime} \phi}-\underline{\phi^{\prime \prime 2} \phi}+\phi^{\prime \prime \prime \prime}-\underline{\phi^{\prime \prime} \phi^{\prime 2}}\right\} \\
& =-E A e_{A}\left\{v^{\prime \prime \prime \prime} \phi+2 v^{\prime \prime \prime} \phi^{\prime}+v^{\prime \prime} \phi^{\prime \prime}\right\}+E I_{\mathbf{y}}\left\{w^{\prime \prime \prime \prime}\right\}+\left(E I_{\mathbf{z}}-E I_{\mathbf{y}}\right)\left\{v^{\prime \prime \prime \prime} \phi+2 v^{\prime \prime \prime} \phi^{\prime}+v^{\prime \prime} \phi^{\prime \prime}\right\}+E C_{1}^{*}\left\{\phi^{\prime \prime \prime \prime}\right\}
\end{aligned}
$$

$$
\begin{aligned}
& \bar{U}_{\phi}=\left\{P_{x}\right\}^{\prime \prime}-\left\{S_{x}\right\}^{\prime}-\left\{T_{x}\right\}^{\prime}+\left\{M_{y}\right\}\left(v^{\prime \prime} \cos \phi+w^{\prime \prime} \sin \phi\right)+\left\{M_{z}\right\}\left(v^{\prime \prime} \sin \phi-w^{\prime \prime} \cos \phi\right) \\
& =\left\{E C_{1}^{*} \phi^{\prime \prime \prime \prime}+E C_{2}^{*}\left(\left(w^{\prime \prime \prime \prime}-2 v^{\prime \prime \prime}-w^{\prime \prime}\right) \cos \phi-\left(v^{\prime \prime \prime \prime}+2 w^{\prime \prime \prime}-v^{\prime \prime}\right) \sin \phi\right)\right\}-\left\{G J \phi^{\prime \prime}\right\} \\
& -\left\{E A k_{A}^{2}\left(\underline{\underline{\left(u^{\prime}+\frac{v^{\prime 2}}{2}+\frac{w^{\prime 2}}{2}\right.}}\right) \phi^{\prime \prime}+\left(\underline{\underline{u^{\prime \prime}+\left(v^{\prime \prime} v^{\prime}\right)+\left(w^{\prime \prime} w^{\prime}\right)}}\right) \phi^{\prime}\right)+E B_{1}^{*}\left(\frac{3}{2} \phi^{\prime \prime} \phi^{\prime 2}\right) \\
& \left.+E B_{2}^{*}\left(\left(-v^{\prime \prime} \cos \phi-w^{\prime \prime} \sin \phi\right) \phi^{\prime \prime}+\left(-\left(v^{\prime \prime \prime}+w^{\prime \prime} \phi^{\prime}\right) \cos \phi-\left(w^{\prime \prime \prime}-v^{\prime \prime} \phi^{\prime}\right) \sin \phi\right) \phi^{\prime}\right)\right\} \\
& +\left\{E I_{\mathbf{y}}\left(v^{\prime \prime} \sin \phi-w^{\prime \prime} \cos \phi\right)-E C_{1}^{*} \phi^{\prime \prime}\right\}\left(v^{\prime \prime} \cos \phi+w^{\prime \prime} \sin \phi\right) \\
& +\left\{E A e_{A}\left(u^{\prime}+\frac{v^{\prime 2}}{2}+\frac{w^{\prime 2}}{2}\right)+E B_{2} *\left(\frac{\phi^{\prime 2}}{2}\right)+E I_{\mathbf{z}}\left(-v^{\prime \prime} \cos \phi-w^{\prime \prime} \sin \phi\right)\right\}\left(v^{\prime \prime} \sin \phi-w^{\prime \prime} \cos \phi\right) \\
& =E I_{\mathbf{y}}\left\{\left(v^{\prime \prime} \sin \phi-w^{\prime \prime} \cos \phi\right)\left(v^{\prime \prime} \cos \phi+w^{\prime \prime} \sin \phi\right)\right\}+E I_{\mathbf{z}}\left\{\left(-v^{\prime \prime} \cos \phi-w^{\prime \prime} \sin \phi\right)\left(v^{\prime \prime} \sin \phi-w^{\prime \prime} \cos \phi\right)\right\} \\
& +E A e_{A}^{2}\left\{\left(v^{\prime \prime}+w^{\prime \prime} \phi\right)\left(v^{\prime \prime} \sin \phi-w^{\prime \prime} \cos \phi\right)\right\}-E A k_{A}^{2}\left\{e_{A}\left(v^{\prime \prime}+w^{\prime \prime} \phi\right) \phi^{\prime \prime}+e_{A}\left(v^{\prime \prime \prime}+w^{\prime \prime} \phi^{\prime}+w^{\prime \prime \prime} \phi\right) \phi^{\prime}\right\} \\
& -E B_{1}^{*}\left\{\frac{3}{2} \phi^{\prime \prime} \phi^{2}\right\}+E B_{2}^{*}\left\{\left(v^{\prime \prime} \cos \phi+w^{\prime \prime} \sin \phi\right) \phi^{\prime \prime}+\left(\left(v^{\prime \prime \prime}+w^{\prime \prime} \phi^{\prime}\right) \cos \phi+\left(w^{\prime \prime \prime}-v^{\prime \prime} \phi^{\prime}\right) \sin \phi\right) \phi^{\prime}\right. \\
& \left.+\left(\frac{\phi^{\prime 2}}{2}\right)\left(v^{\prime \prime} \sin \phi-w^{\prime \prime} \cos \phi\right)\right\} \\
& +E C_{1}^{*}\left\{\phi^{\prime \prime \prime \prime}-\left(\phi^{\prime \prime}\right)\left(v^{\prime \prime} \cos \phi+w^{\prime \prime} \sin \phi\right)\right\}+E C_{2}^{*}\left\{\left(w^{\prime \prime \prime \prime}-2 v^{\prime \prime \prime}-w^{\prime \prime}\right) \cos \phi-\left(v^{\prime \prime \prime \prime}+2 w^{\prime \prime \prime}-v^{\prime \prime}\right) \sin \phi\right\}-G J\left\{\phi^{\prime \prime}\right\} \\
& =E I_{\mathbf{y}}\left\{\underline{v^{\prime \prime 2} \phi}-v^{\prime \prime} w^{\prime \prime}-\underline{w^{\prime \prime 2} \phi}\right\}+E I_{\mathbf{z}}\left\{-\underline{v^{\prime \prime 2} \phi}+v^{\prime \prime} w^{\prime \prime}-\underline{v^{\prime \prime} w^{\prime \prime} \phi^{2}}+\underline{w^{\prime \prime} \phi^{2}}\right\}-G J\left\{\phi^{\prime \prime}\right\} \\
& +E A e_{A}^{2}\left\{\left(\underline{v^{\prime \prime 2} \phi}-v^{\prime \prime} w^{\prime \prime}+\underline{w^{\prime \prime} v^{\prime \prime} \phi}-\underline{w^{\prime \prime 2} \phi}\right)\right\} \\
& -E A k_{A}^{2}\left\{e_{A}\left(\underline{v^{\prime \prime} \phi^{\prime}}+\underline{w^{\prime \prime} \phi^{\prime} \phi^{\prime}}+\underline{v^{\prime \prime \prime} \phi^{\prime}}+\underline{w^{\prime \prime} \phi^{2}}+\underline{w^{\prime \prime \prime} \phi^{\prime} \phi}\right\}\right. \\
& -E B_{1}^{*}\left\{\underline{\left.\frac{3}{2} \phi^{\prime \prime} \phi^{\prime 2}\right\}}+E B_{2}^{*}\left\{-\underline{v^{\prime \prime} \phi^{\prime \prime}}-\underline{w^{\prime \prime} \phi^{\prime \prime} \phi}-\underline{v^{\prime \prime \prime} \phi^{\prime}}-\underline{w^{\prime \prime} \phi^{\prime 2}}-\underline{w^{\prime \prime \prime} \phi^{\prime} \phi}+\underline{v^{\prime \prime} \phi^{2} \phi}+\frac{1}{2} \underline{v^{\prime \prime} \phi^{\prime 2} \phi}-\frac{1}{2} \underline{w^{\prime \prime} \phi^{\prime 2}}\right\}\right.
\end{aligned}
$$




$$
\begin{aligned}
& +E C_{1}{ }^{*}\left\{\phi^{\prime \prime \prime \prime}-\underline{v^{\prime \prime} \phi^{\prime \prime}}-\underline{w^{\prime \prime} \phi^{\prime \prime} \phi}\right\}+E C_{2}{ }^{*}\left\{w^{\prime \prime \prime \prime}-\underline{w^{\prime \prime} \phi^{2}}-\underline{2 v^{\prime \prime \prime} \phi^{\prime}}-\underline{v^{\prime \prime} \phi^{\prime \prime}}-\underline{v^{\prime \prime \prime \prime} \phi}-\underline{v^{\prime \prime} \phi^{2} \phi}+\underline{2 w^{\prime \prime \prime} \phi^{\prime} \phi}+\underline{w^{\prime \prime} \phi^{\prime \prime} \phi}\right\} \\
& =\left(E I_{\mathbf{z}}-E I_{\mathbf{y}}\right)\left\{v^{\prime \prime} w^{\prime \prime}\right\}-G J\left\{\phi^{\prime \prime}\right\}-E A e_{A}\left\{v^{\prime \prime} w^{\prime \prime}\right\}+E C_{1}^{*}\left\{\phi^{\prime \prime \prime \prime}\right\}+E C_{2}{ }^{*}\left\{w^{\prime \prime \prime \prime}\right\} \\
& \bar{U}_{\theta}=K_{\theta} \theta \\
& b(U)=\left.\left\{N_{\mathbf{x}}\right\} \delta u\right|_{0} ^{S}+\left.\left(\left\{N_{\mathbf{x}^{\prime}}\right\} v^{\prime}+\left\{M_{\mathbf{z}} \cos \phi+M_{\mathbf{y}} \sin \phi\right\}\right) \delta v\right|_{0} ^{S}-\left.\left\{M_{\mathbf{z}} \cos \phi+M_{\mathbf{y}} \sin \phi\right\} \delta v^{\prime}\right|_{0} ^{S} \\
& +\left.\left(\left\{N_{\mathbf{x}^{\prime}}\right\} w^{\prime}-\left\{M_{\mathbf{z}^{\prime}} \sin \phi+M_{y^{\prime}} \cos \phi\right\}\right) \delta w\right|_{0} ^{S}+\left.\left\{M_{z^{\prime}} \sin \phi+M_{y^{\prime}} \cos \phi\right\} \delta w^{\prime}\right|_{0} ^{S} \\
& +\left.\left\{P_{x}\right\} \delta \phi^{\prime}\right|_{0} ^{S}+\left.\left(-\left\{P_{x}\right\}^{\prime}+\left\{S_{x^{\prime}}\right\}+\left\{T_{x^{\prime}}\right\}\right) \delta \phi\right|_{0} ^{S} \\
& =\left(\left\{E A e_{A}\left(\underline{\underline{u^{\prime}+\frac{v^{\prime 2}}{2}+\frac{w^{\prime 2}}{2}}}\right)+E B_{2} *\left(\frac{\phi^{\prime 2}}{2}\right)+E I_{\mathbf{z}}\left(-v^{\prime \prime} \cos \phi-w^{\prime \prime} \sin \phi\right)\right\} \cos \phi\right. \\
& \left.+\left\{E I_{\mathbf{y}}\left(v^{\prime \prime} \sin \phi-w^{\prime \prime} \cos \phi\right)-E C_{1}{ }^{*} \phi^{\prime \prime}\right\} \sin \phi\right)\left.\delta v\right|_{0} ^{S} \\
& +\left(-\left\{E A e_{A}\left(\underline{\underline{\left(u^{\prime}+\frac{v^{\prime 2}}{2}+\frac{w^{\prime 2}}{2}\right.}}\right)+E B_{2} *\left(\frac{\phi^{\prime 2}}{2}\right)+E I_{\mathbf{z}}\left(-v^{\prime \prime} \cos \phi-w^{\prime \prime} \sin \phi\right)\right\} \cos \phi\right. \\
& \left.-\left\{E I_{\mathbf{y}}\left(v^{\prime \prime} \sin \phi-w^{\prime \prime} \cos \phi\right)-E C_{1}{ }^{*} \phi^{\prime \prime}\right\} \sin \phi\right)\left.\delta v^{\prime}\right|_{0} ^{S} \\
& +\left(-\left\{E A e_{A}\left(\underline{\underline{u^{\prime}+\frac{v^{\prime 2}}{2}+\frac{w^{\prime 2}}{2}}}\right)+E B_{2} *\left(\frac{\phi^{\prime 2}}{2}\right)+E I_{\mathbf{z}}\left(-v^{\prime \prime} \cos \phi-w^{\prime \prime} \sin \phi\right)\right\} \sin \phi\right. \\
& \left.-\left\{E I_{\mathbf{y}}\left(v^{\prime \prime} \sin \phi-w^{\prime \prime} \cos \phi\right)-E C_{1}{ }^{*} \phi^{\prime \prime}\right\} \cos \phi\right)\left.\delta w\right|_{0} ^{S} \\
& +\left(\left\{E A e_{A}\left(\underline{\underline{u^{\prime}+\frac{v^{\prime 2}}{2}+\frac{w^{\prime 2}}{2}}}\right)+E B_{2} *\left(\frac{\phi^{\prime 2}}{2}\right)+E I_{\mathbf{z}}\left(-v^{\prime \prime} \cos \phi-w^{\prime \prime} \sin \phi\right)\right\} \sin \phi\right. \\
& \left.+\left\{E I_{\mathbf{y}}\left(v^{\prime \prime} \sin \phi-w^{\prime \prime} \cos \phi\right)-E C_{1}^{*} \phi^{\prime \prime}\right\} \cos \phi\right)\left.\delta w^{\prime}\right|_{0} ^{S} \\
& +\left.\left(\left\{E C_{1}{ }^{*} \phi^{\prime \prime}+E C_{2}{ }^{*}\left(w^{\prime \prime} \cos \phi-v^{\prime \prime} \sin \phi\right)\right\}\right) \delta \phi^{\prime}\right|_{0} ^{S} \\
& +\left(-\left\{E C_{1}^{*} \phi^{\prime \prime \prime}+E C_{2}{ }^{*}\left(\left(w^{\prime \prime \prime}-v^{\prime \prime} \phi^{\prime}\right) \cos \phi-\left(v^{\prime \prime \prime}+w^{\prime \prime} \phi^{\prime}\right) \sin \phi\right)\right\}+\left\{G J \phi^{\prime}\right\}\right. \\
& \left.+\left\{E A k_{A}^{2} \phi^{\prime}\left(\underline{\underline{u^{\prime}+\frac{v^{\prime 2}}{2}+\frac{w^{\prime 2}}{2}}}\right)+E B_{1}^{*}\left(\frac{\phi^{\prime 3}}{2}\right)+E B_{2}^{*} \phi^{\prime}\left(-v^{\prime \prime} \cos \phi-w^{\prime \prime} \sin \phi\right)\right\}\right)\left.\delta \phi\right|_{0} ^{S} \\
& =\left.\left(E A e_{A}^{2}\left(v^{\prime \prime}+w^{\prime \prime} \phi\right)+E I_{\mathbf{z}}\left(-v^{\prime \prime}-w^{\prime \prime} \phi\right)+E I_{\mathbf{y}}\left(\underline{v^{\prime \prime} \phi^{2}}-w^{\prime \prime} \phi\right)+E B_{2} *\left(\frac{1}{2} \frac{\phi^{\prime 2}}{}\right)-E C_{1}^{*}\left(\underline{\phi^{\prime \prime} \phi}\right)\right) \delta v\right|_{0} ^{S} \\
& +\left.\left(-E A e_{A}^{2}\left(v^{\prime \prime}+w^{\prime \prime} \phi\right)-E I_{\mathbf{z}}\left(-v^{\prime \prime}-w^{\prime \prime} \phi\right)-E I_{\mathbf{y}}\left(\underline{v^{\prime \prime} \phi^{2}}-w^{\prime \prime} \phi\right)-E B_{2} *\left(\frac{1}{2} \underline{\phi^{\prime 2}}\right)+E C_{1}{ }^{*}\left(\underline{\phi^{\prime \prime} \phi}\right)\right) \delta v^{\prime}\right|_{0} ^{S} \\
& +\left.\left(-E A e_{A}^{2}\left(v^{\prime \prime} \phi+\underline{w^{\prime \prime} \phi^{2}}\right)-E I_{\mathbf{z}}\left(-v^{\prime \prime} \phi-\underline{w^{\prime \prime} \phi^{2}}\right)-E I_{\mathbf{y}}\left(v^{\prime \prime} \phi-w^{\prime \prime}\right)-E B_{2} *\left(\frac{1}{2} \underline{\phi^{\prime 2} \phi}\right)+E C_{1}^{*}\left(\underline{\phi^{\prime \prime}}\right)\right) \delta w\right|_{0} ^{S} \\
& +\left.\left(E A e_{A}^{2}\left(v^{\prime \prime} \phi+\underline{w^{\prime \prime} \phi^{2}}\right)+E B_{2} *\left(\frac{1}{2} \underline{\phi^{\prime 2} \phi}\right)+E I_{\mathbf{z}}\left(-v^{\prime \prime} \phi-\underline{w^{\prime \prime} \phi^{2}}\right)+E I_{\mathbf{y}}\left(v^{\prime \prime} \phi-w^{\prime \prime}\right)-E C_{1} *\left(\underline{\phi^{\prime \prime}}\right)\right) \delta w^{\prime}\right|_{0} ^{S} \\
& +\left.\left(E C_{1}^{*}\left(\underline{\phi^{\prime \prime}}\right)+E C_{2}^{*}\left(w^{\prime \prime}-\underline{v^{\prime \prime} \phi}\right)\right) \delta \phi^{\prime}\right|_{0} ^{S}
\end{aligned}
$$




$$
\begin{aligned}
& +\left(G J\left(\phi^{\prime}\right)+E A k_{A}{ }^{2}\left(e_{A}\left(\underline{v^{\prime \prime}}+\underline{w^{\prime \prime} \phi}\right)\right)+E B_{1}^{*}\left(\frac{1}{2} \underline{\phi^{\prime 3}}\right)+E B_{2}^{*}\left(\underline{-v^{\prime \prime} \phi^{\prime}}-\underline{w^{\prime \prime} \phi^{\prime} \phi}\right)\right. \\
& \left.-E C_{1}^{*}\left(\underline{\phi^{\prime \prime \prime}}\right)-E C_{2}^{*}\left(w^{\prime \prime \prime}-\underline{v^{\prime \prime} \phi^{\prime}}-\underline{v^{\prime \prime \prime} \phi}-\underline{w^{\prime \prime} \phi^{\prime} \phi}\right)\right)\left.\delta \phi\right|_{0} ^{S} \\
=( & \left.E A e_{A}{ }^{2}\left(v^{\prime \prime}+w^{\prime \prime} \phi\right)+E I_{\mathbf{z}}\left(-v^{\prime \prime}-w^{\prime \prime} \phi\right)+E I_{\mathbf{y}}\left(-w^{\prime \prime} \phi\right)\right)\left.\delta v\right|_{0} ^{S} \\
& +\left.\left(-E A e_{A}^{2}\left(v^{\prime \prime}+w^{\prime \prime} \phi\right)-E I_{\mathbf{z}}\left(-v^{\prime \prime}-w^{\prime \prime} \phi\right)-E I_{\mathbf{y}}\left(-w^{\prime \prime} \phi\right)\right) \delta v^{\prime}\right|_{0} ^{S} \\
& +\left.\left(-E A e_{A}{ }^{2}\left(v^{\prime \prime} \phi\right)-E I_{\mathbf{z}}\left(-v^{\prime \prime} \phi\right)-E I_{\mathbf{y}}\left(v^{\prime \prime} \phi-w^{\prime \prime}\right)\right) \delta w\right|_{0} ^{S} \\
& +\left.\left(E A e_{A}{ }^{2}\left(v^{\prime \prime} \phi+\right)+E I_{\mathbf{z}}\left(-v^{\prime \prime} \phi\right)+E I_{\mathbf{y}}\left(v^{\prime \prime} \phi-w^{\prime \prime}\right)\right) \delta w^{\prime}\right|_{0} ^{S} \\
& +\left.\left(E C_{2}^{*}\left(w^{\prime \prime}\right)\right) \delta \phi^{\prime}\right|_{0} ^{S}+\left.\left(G J\left(\phi^{\prime}\right)-E C_{2}{ }^{*}\left(w^{\prime \prime \prime}\right)\right) \delta \phi\right|_{0} ^{S}
\end{aligned}
$$

\section{A.3 Kinetic energy components}

The terms retained arising from the kinetic energy of the system will be of order 2 lower than those retained from the strain energy components. $\bar{T}_{u}$ will be $\mathcal{O}\left(\epsilon^{2}\right)$ to $\mathcal{O}\left(\epsilon^{3}\right)$. For $\bar{T}_{v}, \bar{T}_{w}$, and $\bar{T}_{\phi}$ terms of order $\mathcal{O}\left(\epsilon^{3}\right)$ to $\mathcal{O}\left(\epsilon^{4}\right)$ will be retained, while $\bar{T}_{\theta}$ will have terms retained up to $\mathcal{O}\left(\epsilon^{5}\right)$.

Note that while the linear inertial term, $I_{0} \ddot{\phi}$ in Eq.A.14 is actually formally negligible according to the ordering scheme, it will be retained so as not to introduce a zero entry on the diagonal of the linear inertial matrix. Here it will also be shown that the kinetic energy terms associated with the axial displacement is formally negligible consistent to the second order approximation of the equations of motion.

$$
\begin{aligned}
\bar{T}_{u} & =m \ddot{u}-m e\left(\left(\ddot{v}^{\prime}+w^{\prime} \ddot{\phi}+2 \dot{w}^{\prime} \dot{\phi}\right) \cos \phi+\left(\ddot{w}^{\prime}-v^{\prime} \ddot{\phi}-2 \dot{v}^{\prime} \dot{\phi}\right) \sin \phi\right) \\
& =\underline{-m \ddot{u}+m e\left(\left(\ddot{v}^{\prime}+w^{\prime} \ddot{\phi}+2 \dot{w}^{\prime} \dot{\phi}\right)+\left(\ddot{w}^{\prime}-v^{\prime} \ddot{\phi}-2 \dot{v}^{\prime} \dot{\phi}\right) \phi\right)} \\
\bar{T}_{v} & =-m \ddot{v}+m e \ddot{\phi} \sin \phi+m \ddot{\theta}(e \sin \phi+w)+m \dot{\theta}^{2}\left(\left(v+e_{p}\right)+e \cos \phi\right)+2 m \dot{\theta}(e \dot{\phi} \cos \phi+\dot{w}) \\
& =-m \ddot{v}+\underline{m e \ddot{\phi} \phi}+m \ddot{\theta}(e \phi+w)+m \dot{\theta}^{2}\left(\left(v+e_{p}\right)+e\right)+2 m \dot{\theta}(e \dot{\phi}+\dot{w}) \\
\bar{T}_{w} & =-m \ddot{w}-m e \ddot{\phi} \cos \phi-m \ddot{\theta}\left(e \cos \phi+\left(v+e_{p}\right)\right)+m \dot{\theta}^{2}(e \sin \phi+w)+2 m \dot{\theta}(e \dot{\phi} \sin \phi-\dot{v})
\end{aligned}
$$




$$
\begin{aligned}
& =-m \ddot{w}-m e \ddot{\phi}-m \ddot{\theta}\left(e+\left(v+e_{p}\right)\right)+m \dot{\theta}^{2}(e \phi+w)+2 m \dot{\theta}(\underline{e \dot{\phi} \phi}-\dot{v}) \\
& \bar{T}_{\phi}=m e \ddot{v} \sin \phi-m e \ddot{w} \cos \phi-I_{0}(\ddot{\phi}+\ddot{\theta})-m e \ddot{\theta}\left(\left(v+e_{p}\right) \cos \phi+w \sin \phi\right)+m e \dot{\theta}^{2}\left(w \cos \phi-\left(v+e_{p}\right) \sin \phi\right) \\
& +2 m e \dot{\theta}(-\dot{v} \cos \phi-\dot{w} \sin \phi) \\
& =\underline{m e \ddot{v} \phi}-m e \ddot{w}-I_{0}(\ddot{\phi}+\ddot{\theta})-m e \ddot{\theta}\left(\left(v+e_{p}\right)-\underline{w \phi}\right)+m e \dot{\theta}^{2}\left(w-\underline{\left(v+e_{p}\right)} \phi\right)+2 m e \dot{\theta}(-\dot{v}-\underline{\dot{w} \phi}) \\
& \bar{T}_{\theta}=\int_{0}^{S}\left\{m \ddot{v}(e \sin \phi+w)+m \ddot{w}\left(-e \cos \phi-\left(v+e_{p}\right)\right)+\ddot{\phi}\left(-I_{0}-m e\left(\left(v+e_{p}\right) \cos \phi+w \sin \phi\right)\right)\right. \\
& +\ddot{\theta}\left(-I_{0}-2 m e\left(\left(v+e_{p}\right) \cos \phi+w \sin \phi\right)-m w^{2}-m\left(v+e_{p}\right)^{2}\right) \\
& \left.+2 m \dot{\theta} \dot{v}\left(-e \cos \phi-\left(v+e_{p}\right)\right)+2 m \dot{\theta} \dot{w}(-e \sin \phi-w)+2 m e \dot{\theta} \dot{\phi}\left(-w \cos \phi-\left(v+e_{p}\right) \sin \phi\right)\right\} d x-I_{b} \ddot{\theta} \\
& =\int_{0}^{S}\left\{m \ddot{v}(e \phi+w)+m \ddot{w}\left(-e-\left(v+e_{p}\right)\right)+\ddot{\phi}\left(-I_{0}-m e\left(\left(v+e_{p}\right)+\underline{w \phi}\right)\right)\right. \\
& +\ddot{\theta}\left(-I_{0}-2 m e\left(\left(v+e_{p}\right)+w \phi\right)-m w^{2}-m\left(v+e_{p}\right)^{2}\right) \\
& -2 m \dot{\theta}\left(\dot{v}\left(e+\left(v+e_{p}\right)\right)+\dot{w}(e \phi+w)+e \dot{\phi}\left(w-\underline{\left.\left(v+e_{p}\right) \phi\right)}\right)\right\} d x-I_{b} \ddot{\theta}
\end{aligned}
$$

Considering the order of the section integrals $m K_{m_{1}}, m K_{m_{2}}$ and $m D_{1}{ }^{*}$, one will notice that they are several orders of magnitude larger than the terms retained according to the approximation scheme, thus it can be asserted that to the second order, the kinetic energy terms' contribution to the boundary conditions are formally negligible.

$$
\begin{aligned}
b(T)= & -\left.\left(\left(\ddot{v}^{\prime}+\ddot{\phi} w^{\prime}+2 \dot{\phi} \dot{w}^{\prime}\right)\left(m K_{m_{1}} \sin ^{2} \phi+m K_{m_{2}} \cos ^{2} \phi\right)+\left(\ddot{w}^{\prime}-\ddot{\phi} v^{\prime}-2 \dot{\phi} \dot{v}^{\prime}\right)\left(m K_{m_{1}}+m K_{m_{2}}\right) \sin \phi \cos \phi\right) \delta v\right|_{0} ^{S} \\
& -\left.\left(\left(\ddot{v}^{\prime}+\ddot{\phi} w^{\prime}+2 \dot{\phi} \dot{w}^{\prime}\right)\left(m K_{m_{1}}+m K_{m_{2}}\right) \sin \phi \cos \phi+\left(\ddot{w}^{\prime}-\ddot{\phi} v^{\prime}-2 \dot{\phi} \dot{v}^{\prime}\right)\left(m K_{m_{1}} \sin ^{2} \phi+m K_{m_{2}} \cos ^{2} \phi\right)\right) \delta w\right|_{0} ^{S} \\
& +\left.\left(m D_{1}^{*}\left(-\left(\ddot{v}^{\prime}+\ddot{\phi} w^{\prime}+2 \dot{\phi} \dot{w}^{\prime}\right) \cos \phi+\left(\ddot{w}^{\prime}-\ddot{\phi} v^{\prime}-2 \dot{\phi} \dot{v}^{\prime}\right) \sin \phi\right)\right) \delta \phi\right|_{0} ^{S} \\
= & -\left.\left(\left(\ddot{v}^{\prime}+\ddot{\phi} w^{\prime}+2 \dot{\phi} \dot{w}^{\prime}\right)\left(m K_{m_{2}}\right)+\left(\ddot{w}^{\prime}-\ddot{\phi} v^{\prime}-2 \dot{\phi} \dot{v}^{\prime}\right)\left(m K_{m_{1}}+m K_{m_{2}}\right) \phi\right) \delta v\right|_{0} ^{S} \\
& -\left.\left(\left(\ddot{v}^{\prime}+\ddot{\phi} w^{\prime}+2 \dot{\phi} \dot{w}^{\prime}\right)\left(m K_{m_{1}}+m K_{m_{2}}\right) \phi+\left(\ddot{w}^{\prime}-\ddot{\phi} v^{\prime}-2 \dot{\phi} \dot{v}^{\prime}\right)\left(m K_{m_{2}}\right)\right) \delta w\right|_{0} ^{S} \\
& +\left.\left(m D_{1}^{*}\left(-\left(\ddot{v}^{\prime}+\ddot{\phi} w^{\prime}+2 \dot{\phi} \dot{w}^{\prime}\right)+\left(\ddot{w}^{\prime}-\ddot{\phi} v^{\prime}-2 \dot{\phi} \dot{v}^{\prime}\right) \phi\right)\right) \delta \phi\right|_{0} ^{S}
\end{aligned}
$$




\section{Appendix B}

\section{Numerical Solution of the Nonlinear Equations of Motion}

A finite dimensional representation of the equations of motion is required for the numerical im-

plementation of the equations of motion. A Galerkin projection and a finite difference scheme are employed independently. This appendix presents the Galerkin projection of the structural and aerodynamic loads in Section B.1, and summarizes the Galerkin projection coefficients in Section B.2. The aeroelastic stiffness, damping and inertial matrices for the underlying linear system are presented in Section B.3. These matrices are central to the eigenvalue analysis which reveals the flutter speed and frequency of the system. Finally, the numerical implementation of both numerical schemes is presented, referencing back to the Modified Newton-Raphson algorithm introduced in Chapter 3 .

\section{B.1 Galerkin Projection}

The coupled differential equations presented in Chapter 2 are discretized in space using a Galerkin projection scheme. By doing so, a set of three coupled partial differential equations and an ordinary differential equation are converted into $L+M+N+1$ coupled ordinary differential equations. The long form of these discretized equations are presented below: 


\section{B.1.1 Finite Dimensional Representation of the Structural Equations}

$$
\begin{aligned}
& \delta v \\
& \left\{\mathcal{K}_{z}\left(\mathcal{V}^{\prime \prime \prime \prime}\right)+\left(\mathcal{K}_{z}-\mathcal{K}_{y}\right)\left(\mathcal{W}^{\prime \prime} \varphi^{\prime \prime}+2 \mathcal{W}^{\prime \prime \prime} \varphi^{\prime}+\mathcal{W}^{\prime \prime \prime \prime} \varphi\right)-\mathcal{E}_{A}{ }^{2}\left(\mathcal{V}^{\prime \prime \prime \prime}+\mathcal{W}^{\prime \prime} \varphi^{\prime \prime}+2 \mathcal{W}^{\prime \prime \prime} \varphi^{\prime}+\mathcal{W}^{\prime \prime \prime \prime} \varphi\right)\right\} \\
& +\left\{\ddot{\mathcal{V}}-\ddot{\vartheta}(\mathcal{E} \varphi+\mathcal{W})-\dot{\vartheta}^{2}\left(\left(\mathcal{V}+\mathcal{E}_{p}\right)+\mathcal{E}\right)-2 \dot{\vartheta}(\mathcal{E} \dot{\varphi}+\dot{\mathcal{W}})\right\}=\overline{\mathcal{W}}_{v} \\
& \mathcal{K}_{z}\left\{\int_{0}^{1}\left(\sum_{i=1}^{L} \sum_{j=1}^{L} \Upsilon_{i}(x) \Upsilon_{j}^{\prime \prime \prime \prime}(x)\right) d \mathcal{X} V_{j}(t)\right\} \\
& +\left(\mathcal{K}_{z}-\mathcal{K}_{y}\right)\left\{\int_{0}^{1}\left(\sum_{i=1}^{L} \sum_{j=1}^{M} \sum_{k=1}^{N} \Upsilon_{i}(x) \Psi_{j}^{\prime \prime \prime \prime}(x) \Omega_{k}(x)\right) d \mathcal{X} W_{j}(t) \Phi_{k}(t)\right. \\
& +2 \int_{0}^{1}\left(\sum_{i=1}^{L} \sum_{j=1}^{M} \sum_{k=1}^{N} \Upsilon_{i}(x) \Psi_{j}^{\prime \prime \prime}(x) \Omega_{k}^{\prime}(x)\right) d \mathcal{X} W_{j}(t) \Phi_{k}(t) \\
& \left.+\int_{0}^{1}\left(\sum_{i=1}^{L} \sum_{j=1}^{M} \sum_{k=1}^{N} \Upsilon_{i}(x) \Psi_{j}^{\prime \prime}(x) \Omega_{k}^{\prime \prime}(x)\right) d \mathcal{X} W_{j}(t) \Phi_{k}(t)\right\} \\
& -\mathcal{E}_{A}^{2}\left\{\int_{0}^{1}\left(\sum_{i=1}^{L} \sum_{j=1}^{L} \Upsilon_{i}(x) \Upsilon_{j}^{\prime \prime \prime \prime}(x)\right) d \mathcal{X} V_{j}(t) V_{k}(t)\right. \\
& +\int_{0}^{1}\left(\sum_{i=1}^{L} \sum_{j=1}^{M} \sum_{k=1}^{N} \Upsilon_{i}(x) \Psi_{j}^{\prime \prime \prime \prime}(x) \Omega_{k}(x)\right) d \mathcal{X} W_{j}(t) \Phi_{k}(t) \\
& +2 \int_{0}^{1}\left(\sum_{i=1}^{L} \sum_{j=1}^{M} \sum_{k=1}^{N} \Upsilon_{i}(x) \Psi_{j}^{\prime \prime \prime}(x) \Omega_{k}^{\prime}(x)\right) d \mathcal{X} W_{j}(t) \Phi_{k}(t) \\
& \left.+\int_{0}^{1}\left(\sum_{i=1}^{L} \sum_{j=1}^{M} \sum_{k=1}^{N} \Upsilon_{i}(x) \Psi_{j}^{\prime \prime}(x)^{\prime \prime} \Omega_{k}(x)\right) d \mathcal{X} W_{j}(t) \Phi_{k}(t)\right\} \\
& +\int_{0}^{1}\left(\sum_{i=1}^{L} \sum_{j=1}^{L} \Upsilon_{i}(x) \Upsilon_{j}(x)\right) d \mathcal{X} \ddot{V}_{j}(t) \\
& -\ddot{\Theta}(t)\left\{\mathcal{E} \int_{0}^{1}\left(\sum_{i=1}^{L} \sum_{j=1}^{N} \Upsilon_{i}(x) \Omega_{j}(x)\right) d \mathcal{X} \Phi_{j}(t)+\int_{0}^{1}\left(\sum_{i=1}^{L} \sum_{j=1}^{M} \Upsilon_{i}(x) \Psi_{j}(x)\right) d \mathcal{X} W_{j}(t)\right\} \\
& -\dot{\Theta}^{2}(t)\left\{\left(\int_{0}^{1}\left(\sum_{i=1}^{L} \sum_{j=1}^{L} \Upsilon_{i}(x) \Upsilon_{j}(x)\right) d \mathcal{X} V_{j}(t)+\mathcal{E}_{P} \int_{0}^{1}\left(\sum_{i=1}^{L} \Upsilon_{i}(x)\right) d \mathcal{X}\right)+\mathcal{E} \int_{0}^{1}\left(\sum_{i=1}^{L} \Upsilon_{i}(x)\right) d \mathcal{X}\right\} \\
& -2 \dot{\Theta}(t)\left\{\mathcal{E} \int_{0}^{1}\left(\sum_{i=1}^{L} \sum_{j=1}^{N} \Upsilon_{i}(x) \Omega_{j}(x)\right) d \mathcal{X} \dot{\Phi}_{j}(t)+\int_{0}^{1}\left(\sum_{i=1}^{L} \sum_{j=1}^{M} \Upsilon_{i}(x) \Psi_{j}(x)\right) d \mathcal{X} \dot{W}_{j}(t)\right\}=\mathcal{W}_{v}
\end{aligned}
$$


$\delta w$

$$
\begin{aligned}
& \left\{\mathcal{K}_{y}\left(\mathcal{W}^{\prime \prime \prime \prime}\right)+\left(\mathcal{K}_{z}-\mathcal{K}_{y}\right)\left(\mathcal{V}^{\prime \prime \prime \prime} \varphi+2 \mathcal{V}^{\prime \prime \prime} \varphi^{\prime}+\mathcal{V}^{\prime \prime} \varphi^{\prime \prime}\right)-\mathcal{E}_{A}^{2}\left(\mathcal{V}^{\prime \prime \prime \prime} \varphi+2 \mathcal{V}^{\prime \prime \prime} \varphi^{\prime}+\mathcal{V}^{\prime \prime} \varphi^{\prime \prime}\right)+\mathcal{K}_{\lambda 1}\left(\varphi^{\prime \prime \prime \prime}\right)\right\} \\
& +\left\{\ddot{\mathcal{W}}+\mathcal{E} \ddot{\varphi}+\ddot{\vartheta}\left(\left(\mathcal{V}+\mathcal{E}_{p}\right)+\mathcal{E}\right)-\dot{\vartheta}^{2}(\mathcal{E} \varphi+\mathcal{W})+2 \dot{\vartheta}(\dot{\mathcal{V}})\right\}=\overline{\mathcal{W}}_{w}
\end{aligned}
$$

$$
\begin{aligned}
& \mathcal{K}_{y}\left\{\int_{0}^{1}\left(\sum_{i=1}^{M} \sum_{j=1}^{M} \Psi_{i}(x) \Psi_{j}^{\prime \prime \prime \prime}(x)\right) d \mathcal{X} W_{j}(t)\right\} \\
& +\left(\mathcal{K}_{z}-\mathcal{K}_{y}\right)\left\{\int_{0}^{1}\left(\sum_{i=1}^{M} \sum_{j=1}^{L} \sum_{k=1}^{N} \Psi_{i}(x) \Upsilon_{j}^{\prime \prime \prime \prime}(x) \Omega_{k}(x)\right) d \mathcal{X} V_{j}(t) \Phi_{k}(t)\right. \\
& +2 \int_{0}^{1}\left(\sum_{i=1}^{M} \sum_{j=1}^{L} \sum_{k=1}^{N} \Psi_{i}(x) \Upsilon_{j}^{\prime \prime \prime}(x) \Omega_{k}^{\prime}(x)\right) d \mathcal{X} V_{j}(t) \Phi_{k}(t) \\
& \left.+\int_{0}^{1}\left(\sum_{i=1}^{M} \sum_{j=1}^{L} \sum_{k=1}^{N} \Psi_{i}(x) \Upsilon_{j}^{\prime \prime}(x) \Omega_{k}^{\prime \prime}(x)\right) d \mathcal{X} V_{j}(t) \Phi_{k}(t)\right\} \\
& -\mathcal{E}_{A}^{2}\left\{\int_{0}^{1}\left(\sum_{i=1}^{M} \sum_{j=1}^{L} \sum_{k=1}^{N} \Psi_{i}(x) \Upsilon_{j}^{\prime \prime \prime \prime}(x) \Omega_{k}(x)\right) d \mathcal{X} V_{j}(t) \Phi_{k}(t)\right. \\
& +2 \int_{0}^{1}\left(\sum_{i=1}^{M} \sum_{j=1}^{L} \sum_{k=1}^{N} \Psi_{i}(x) \Upsilon_{j}^{\prime \prime \prime}(x) \Omega_{k}^{\prime}(x)\right) d \mathcal{X} V_{j}(t) \Phi_{k}(t) \\
& \left.+\int_{0}^{1}\left(\sum_{i=1}^{M} \sum_{j=1}^{L} \sum_{k=1}^{N} \Psi_{i}(x) \Upsilon_{j}^{\prime \prime}(x) \Omega_{k}^{\prime \prime}(x)\right) d \mathcal{X} V_{j}(t) \Phi_{k}(t)\right\} \\
& +\mathcal{K}_{\lambda 1}\left\{\int_{0}^{1}\left(\sum_{i=1}^{M} \sum_{j=1}^{N} \Psi_{i}(x) \Omega_{j}^{\prime \prime \prime \prime}(x)\right) d \mathcal{X} \Phi_{j}(t)\right\} \\
& +\int_{0}^{1}\left(\sum_{i=1}^{M} \sum_{j=1}^{M} \Psi_{i}(x) \Psi_{j}(x)\right) d \mathcal{X} \ddot{W}_{i}(t)+\mathcal{E} \int_{0}^{1}\left(\sum_{i=1}^{M} \sum_{j=1}^{N} \Psi_{i}(x) \Omega_{j}(x)\right) d \mathcal{X} \ddot{\Phi}_{i}(t) \\
& +\ddot{\Theta}(t)\left\{\mathcal{E} \int_{0}^{1}\left(\sum_{i=1}^{M} \Psi_{i}(x)\right) d \mathcal{X}+\int_{0}^{1}\left(\sum_{i=1}^{M} \sum_{i=1}^{L} \Psi_{i}(x) \Upsilon_{i}(x)\right) d \mathcal{X} V_{i}(t)+\mathcal{E}_{P} \int_{0}^{1}\left(\sum_{i=1}^{M} \Psi_{i}(x)\right) d \mathcal{X}\right\} \\
& -\dot{\Theta}^{2}(t)\left\{\mathcal{E} \int_{0}^{1}\left(\sum_{i=1}^{M} \sum_{i=1}^{N} \Psi_{i}(x) \Omega_{i}(x)\right) d \mathcal{X} \Phi_{i}(t)+\int_{0}^{1}\left(\sum_{i=1}^{M} \sum_{i=1}^{M} \Psi_{i}(x) \Psi_{i}(x)\right) d \mathcal{X} W_{i}(t)\right\} \\
& +2 \dot{\Theta}(t)\left\{\int_{0}^{1}\left(\sum_{i=1}^{M} \sum_{i=1}^{L} \Psi_{i}(x) \Upsilon_{i}(x)\right) d \mathcal{X} \dot{V}_{i}(t)\right\}=\mathcal{W}_{w}
\end{aligned}
$$




$$
\begin{aligned}
& \delta \phi \\
\{- & \left.\mathcal{K}_{x}\left(\varphi^{\prime \prime}\right)+\left(\mathcal{K}_{z}-\mathcal{K}_{y}\right)\left(\mathcal{V}^{\prime \prime} \mathcal{W}^{\prime \prime}\right)-\mathcal{E}_{A}^{2}\left(\mathcal{V}^{\prime \prime} \mathcal{W}^{\prime \prime}\right)+\mathcal{K}_{\lambda 1}\left(\varphi^{\prime \prime \prime \prime}\right)+\mathcal{K}_{\lambda 2}\left(\mathcal{W}^{\prime \prime \prime \prime}\right)\right\} \\
& +\left\{\mathcal{M}_{0}(\ddot{\varphi}+\ddot{\vartheta})+\mathcal{E} \ddot{\mathcal{V}}+\ddot{\vartheta}\left(\mathcal{E}\left(\mathcal{V}+\mathcal{E}_{p}\right)\right)-\dot{\vartheta}^{2}(\mathcal{E} \mathcal{W})+2 \mathcal{E} \dot{\vartheta}(\dot{\mathcal{V}})\right\}=\overline{\mathcal{W}}_{\phi} \\
- & \mathcal{K}_{x}\left\{\int_{0}^{1}\left(\sum_{i=1}^{N} \sum_{j=1}^{N} \Omega_{i}(x) \Omega_{j}^{\prime \prime}(x)\right) d \mathcal{X} \Phi_{j}(t)\right\} \\
& +\left(\mathcal{K}_{z}-\mathcal{K}_{y}\right)\left\{\int_{0}^{1}\left(\sum_{i=1}^{N} \sum_{j=1}^{L} \sum_{k=1}^{M} \Omega_{i}(x) \Upsilon_{j}^{\prime \prime}(x) \Psi_{k}^{\prime \prime}(x)\right) d \mathcal{X} V_{j}(t) W_{k}(t)\right\} \\
& -\mathcal{E}_{A}^{2}\left\{\int_{0}^{1}\left(\sum_{i=1}^{N} \sum_{j=1}^{L} \sum_{k=1}^{M} \Omega_{i}(x) \Upsilon_{j}^{\prime \prime}(x) \Psi_{k}^{\prime \prime}(x)\right) d \mathcal{X} V_{j}(t) W_{k}(t)\right\} \\
& +\mathcal{K}_{\lambda 1}\left\{\int_{0}^{1}\left(\sum_{i=1}^{N} \sum_{j=1}^{M} \Omega_{i}(x) \Omega_{j}^{\prime \prime \prime \prime}(x)\right) d \mathcal{X} \Phi_{j}(t)\right\}+\mathcal{K}_{\lambda 2}\left\{\int_{0}^{1}\left(\sum_{i=1}^{N} \sum_{j=1}^{M} \Omega_{i}(x) \Psi_{j}^{\prime \prime \prime \prime}(x)\right) d \mathcal{X} W_{j}(t)\right\} \\
& +\mathcal{M}_{0} \int_{0}^{1}\left(\sum_{i=1}^{N} \sum_{j=1}^{N} \Omega_{i}(x) \Omega_{j}(x)\right) d \mathcal{X} \ddot{\Phi}_{j}(t) \\
& +\ddot{\Theta}(t)\left\{\mathcal{M}_{0} \int_{0}^{1}\left(\Omega_{i}(x)\right) d \mathcal{X}+\mathcal{E}\left(\int_{0}^{1}\left(\sum_{i=1}^{N} \sum_{j=1}^{L} \Omega_{i}(x) \Upsilon_{j}(x)\right) d \mathcal{X} V_{j}(t)+\mathcal{E}_{P} \int_{0}^{1}\left(\Omega_{i}(x)\right) d \mathcal{X}\right)\right\} \\
& +\mathcal{E} \int_{0}^{1}\left(\sum_{i=1}^{N} \sum_{j=1}^{M} \Omega_{i}(x) \Psi_{j}(x)\right) d \mathcal{X} \ddot{W}_{j}(t)-\mathcal{E} \dot{\Theta}^{2}(t)\left\{\int_{0}^{1}\left(\sum_{i=1}^{N} \sum_{j=1}^{M} \Omega_{i}(x) \Psi_{j}(x)\right) d \mathcal{X} W_{j}(t)\right\} \\
+ & 2 \dot{\Theta}(t)\left\{\int_{0}^{1}\left(\sum_{i=1}^{N} \sum_{j=1}^{L} \Omega_{i}(x) \Upsilon_{j}(x)\right) d \mathcal{X} \dot{V}_{j}(t)\right\}=\mathcal{W}_{\phi}
\end{aligned}
$$


$\delta \theta$

$$
\begin{aligned}
\mathcal{M}_{\theta} \ddot{\vartheta} & +\mathcal{D}_{\theta} \dot{\vartheta}+\mathcal{K}_{\vartheta} \vartheta+\int_{0}^{1}\left\{\mathcal{M}_{0}(\ddot{\varphi}+\ddot{\vartheta})-\ddot{\mathcal{V}}(\mathcal{E} \varphi+\mathcal{W})+\ddot{\mathcal{W}}\left(\left(\mathcal{V}+\mathcal{E}_{p}\right)+\mathcal{E}\right)+\mathcal{E} \ddot{\varphi}\left(\left(\mathcal{V}+\mathcal{E}_{p}\right)\right)\right. \\
& +2 \mathcal{E} \ddot{\vartheta}\left(\left(\mathcal{V}+\mathcal{E}_{p}\right)+\mathcal{W} \varphi\right)+\ddot{\vartheta}\left(\mathcal{W}^{2}+\left(\mathcal{V}+\mathcal{E}_{p}\right)^{2}\right) \\
& \left.+2 \dot{\vartheta}\left(\dot{\mathcal{V}}\left(\left(\mathcal{V}+\mathcal{E}_{p}\right)+\mathcal{E}\right)+\dot{\mathcal{V}}(\mathcal{E} \varphi+\mathcal{W})+\mathcal{E} \dot{\varphi}(\mathcal{W})\right)\right\} d \mathcal{X}=\overline{\mathcal{W}}_{\theta}
\end{aligned}
$$

$$
\begin{aligned}
\mathcal{M}_{\theta} \ddot{\Theta}(t) \int_{0}^{1} d \mathcal{X}+\mathcal{D}_{\theta} \dot{\Theta}(t) \int_{0}^{1} d \mathcal{X}+\mathcal{K}_{\vartheta} \Theta(t) \int_{0}^{1} d \mathcal{X}+\mathcal{M}_{0} \int_{0}^{1}\left(\sum_{j=1}^{N} \Omega_{j}(x)\right) d \mathcal{X} \ddot{\Phi}_{j}(t)+\mathcal{M}_{0} \ddot{\Theta}(t) \int_{0}^{1} d \mathcal{X} \\
-\mathcal{E} \int_{0}^{1}\left(\sum_{j=1}^{L} \sum_{k=1}^{N} \Upsilon_{j}(x) \Omega_{k}(x)\right) d \mathcal{X} \ddot{V}_{j}(t) \Phi_{k}(t)-\int_{0}^{1}\left(\sum_{j=1}^{L} \sum_{k=1}^{M} \Upsilon_{j}(x) \Psi_{k}(x)\right) d \mathcal{X} \ddot{V}_{j}(t) W_{k}(t) \\
+\int_{0}^{1}\left(\sum_{j=1}^{M} \sum_{k=1}^{L} \Psi_{j}(x) \Upsilon_{k}(x)\right) d \mathcal{X} \ddot{W}_{j}(t) V_{k}(t)+\mathcal{E} \int_{0}^{1}\left(\sum_{j=1}^{M} \Psi_{j}(x)\right) d \mathcal{X} \ddot{W}_{j}(t)+\mathcal{E}_{P} \int_{0}^{1}\left(\sum_{j=1}^{M} \Psi_{j}(x)\right) d \mathcal{X} \ddot{W}_{j}(t) \\
+\mathcal{E} \int_{0}^{1}\left(\sum_{j=1}^{N} \sum_{k=1}^{L} \Omega_{j}(x) \Upsilon_{k}(x)\right) d \mathcal{X} \ddot{\Phi}_{j}(t) V_{k}(t)+\mathcal{E} \mathcal{E}_{P} \int_{0}^{1}\left(\sum_{j=1}^{N} \Omega_{j}(x)\right) d \mathcal{X} \ddot{\Phi}_{j}(t) \\
+\ddot{\Theta}\left\{2 \mathcal{E} \int_{0}^{1}\left(\sum_{j=1}^{L} \Upsilon_{j}(x)\right) d \mathcal{X} V_{j}(t)+2 \mathcal{E} \mathcal{E}_{P} \int_{0}^{1} d \mathcal{X}+2 \mathcal{E} \int_{0}^{1}\left(\sum_{j=1}^{M} \sum_{k=1}^{N} \Psi_{j}(x) \Omega_{k}(x)\right) d \mathcal{X} W_{j}(t) \Phi_{k}(t)\right. \\
+\int_{0}^{1}\left(\sum_{j=1}^{M} \sum_{k=1}^{M} \Psi_{j}(x) \Psi_{k}(x)\right) d \mathcal{X} W_{j}(t) W_{k}(t)+\int_{0}^{1}\left(\sum_{j=1}^{L} \sum_{k=1}^{L} \Upsilon_{j}(x) \Upsilon_{k}(x)\right) d \mathcal{X} V_{j}(t) V_{k}(t) \\
\left.+2 \mathcal{E}_{P} \int_{0}^{1}\left(\sum_{j=1}^{L} \Upsilon_{j}(x)\right) d \mathcal{X} V_{j}(t)+\mathcal{E}_{P}^{2} \int_{0}^{1} d \mathcal{X}\right\}_{j} \\
\left.+\int_{0}^{1}\left(\sum_{j=1}^{M} \sum_{k=1}^{M} \Psi_{j}(x) \Psi_{k}(x)\right) d \mathcal{X} \dot{W}_{j}(t) W_{k}(t)+\mathcal{E} \int_{0}^{1}\left(\sum_{j=1}^{M} \sum_{k=1}^{N} \Psi_{j}(x) \Omega_{k}(x)\right) d \mathcal{X} W_{j}(t) \dot{\Phi}_{k}(t)\right\}=\mathcal{W}_{\theta} \\
+\mathcal{\Theta}(t)\left\{\int_{0}^{1}\left(\sum_{j=1}^{L} \sum_{k=1}^{L} \Upsilon_{j}(x) \Upsilon_{k}(x)\right) d \mathcal{X} V_{k}(t) \dot{V}_{j}(t)+\mathcal{E}_{P} \int_{0}^{1}\left(\sum_{j=1}^{L} \Upsilon_{j}(x)\right) d \mathcal{X} \dot{V}_{j}(t)\right. \\
\left.+\sum_{j=1}^{L} \Upsilon_{j}(x)\right) d \mathcal{X} \dot{V}_{j}(t)+\mathcal{E} \int_{0}^{1}\left(\sum_{j=1}^{M} \sum_{k=1}^{N} \Psi_{j}(x) \Omega_{k}(x)\right) d \mathcal{X} \dot{W}_{j}(t) \Phi_{k}(t) \\
+\int_{0}(x)
\end{aligned}
$$




\section{B.1.2 Finite Dimensional Representation of the Aerodynamic Loads}

As with the structural equations, a finite dimensional representation of the aerodynamic loads is also sought. As discussed in Chapter 2 , modelling the unsteady aerodynamic loads requires that an additional differential equation be introduced. As a result of this additional equation and the selected basis functions, the Galerkin projection consists of $L+2 M+2 N+1$ coupled ordinary differential equations.

$$
\begin{aligned}
& \delta v \\
& \overline{\mathcal{W}}_{v}=\sqrt{1-\mathcal{X}^{2}}\left\{\frac{1}{4} \mathcal{P} \mathcal{C}^{2} \pi\left(-(\ddot{\mathcal{V}} \sin \vartheta+\ddot{\mathcal{W}} \cos \vartheta)+\mathcal{U}(\dot{\varphi}+\dot{\vartheta})-\left(\mathcal{E}_{c}-\frac{1}{4} \mathcal{C}\right) \ddot{\varphi}-\left(\left(\mathcal{E}_{c}+\mathcal{E}_{p}\right)-\frac{1}{4} \mathcal{C}\right) \ddot{\vartheta}\right)\right. \\
& \left.+\frac{1}{2} \mathcal{P U C} 2 \pi\left(\left(1-\tilde{A}_{1}-\tilde{A}_{2}\right) \tilde{w}_{3 c / 4}+\left(\tilde{A}_{1} \tilde{b}_{1}+\tilde{A}_{2} \tilde{b}_{2}\right) \dot{\mathcal{Z}}+\tilde{b}_{1} \tilde{b}_{2}\left(\tilde{A}_{1}+\tilde{A}_{2}\right) \mathcal{Z}\right)\right\} \times \sin \vartheta \\
& =\left\{\frac { 1 } { 4 } \mathcal { P C } ^ { 2 } \pi \left(-\int_{0}^{1}\left(\sum_{i=1}^{L} \sum_{j=1}^{L} \sqrt{\left(1-\mathcal{X}^{2}\right)} \Upsilon_{i}(\mathcal{X}) \Upsilon_{j}(\mathcal{X})\right) d \mathcal{X} \ddot{V}_{j}(\mathcal{T}) \sin \Theta(\mathcal{T})\right.\right. \\
& -\int_{0}^{1}\left(\sum_{i=1}^{L} \sum_{j=1}^{M} \sqrt{\left(1-\mathcal{X}^{2}\right)} \Upsilon_{i}(\mathcal{X}) \Psi_{j}(\mathcal{X})\right) d \mathcal{X} \ddot{W}_{j}(\mathcal{T}) \cos \Theta(\mathcal{T}) \\
& +\mathcal{U}\left(\int_{0}^{1}\left(\sum_{i=1}^{L} \sum_{j=1}^{N} \sqrt{\left(1-\mathcal{X}^{2}\right)} \Upsilon_{i}(\mathcal{X}) \Omega_{j}(\mathcal{X})\right) d \mathcal{X} \dot{\Phi}_{j}(\mathcal{T})\right. \\
& \left.+\int_{0}^{1}\left(\sum_{i=1}^{L} \sqrt{\left(1-\mathcal{X}^{2}\right)} \Upsilon_{i}(\mathcal{X})\right) d \mathcal{X} \dot{\Theta}(\mathcal{T})\right) \\
& -\left(\mathcal{E}_{c}-\frac{1}{4} \mathcal{C}\right) \int_{0}^{1}\left(\sum_{i=1}^{L} \sum_{j=1}^{N} \sqrt{\left(1-\mathcal{X}^{2}\right)} \Upsilon_{i}(\mathcal{X}) \Omega_{j}(\mathcal{X})\right) d \mathcal{X} \ddot{\Phi}_{j}(\mathcal{T}) \\
& \left.-\left(\left(\mathcal{E}_{c}+\mathcal{E}_{p}\right)-\frac{1}{4} \mathcal{C}\right) \int_{0}^{1}\left(\sum_{i=1}^{L} \sqrt{\left(1-\mathcal{X}^{2}\right)} \Upsilon_{i}(\mathcal{X})\right) d \mathcal{X} \ddot{\Theta}(\mathcal{T})\right) \\
& +\frac{1}{2} \mathcal{P U C} 2 \pi\left(\left(1-\tilde{A}_{1}-\tilde{A}_{2}\right) \int_{0}^{1}\left(\sum_{i=1}^{L} \sqrt{\left(1-\mathcal{X}^{2}\right)} \Upsilon_{i}(\mathcal{X}) \tilde{w}_{3 c / 4}(\mathcal{X}, \mathcal{T})\right) d \mathcal{X}\right. \\
& +\left(\tilde{A}_{1} \tilde{b}_{1}+\tilde{A}_{2} \tilde{b}_{2}\right)\left(\int_{0}^{1}\left(\sum_{i=1}^{L} \sum_{j=1}^{M} \sqrt{\left(1-\mathcal{X}^{2}\right)} \Upsilon_{i}(\mathcal{X}) \Psi_{j}(\mathcal{X})\right) d \mathcal{X} \dot{Z}_{j}^{\Psi}(\mathcal{T})\right. \\
& \left.+\int_{0}^{1}\left(\sum_{i=1}^{L} \sum_{j=1}^{N} \sqrt{\left(1-\mathcal{X}^{2}\right)} \Upsilon_{i}(\mathcal{X}) \Omega_{j}(\mathcal{X})\right) d \mathcal{X} \dot{Z}_{j}^{\Omega}(\mathcal{T})\right) \\
& +\tilde{b}_{1} \tilde{b}_{2}\left(\tilde{A}_{1}+\tilde{A}_{2}\right)\left(\int_{0}^{1}\left(\sum_{i=1}^{L} \sum_{j=1}^{M} \sqrt{\left(1-\mathcal{X}^{2}\right)} \Upsilon_{i}(\mathcal{X}) \Psi_{j}(\mathcal{X})\right) d \mathcal{X} Z_{j}^{\Psi}(\mathcal{T})\right. \\
& \left.\left.\left.+\int_{0}^{1}\left(\sum_{i=1}^{L} \sum_{j=1}^{N} \sqrt{\left(1-\mathcal{X}^{2}\right)} \Upsilon_{i}(\mathcal{X}) \Omega_{j}(\mathcal{X})\right) d \mathcal{X} Z_{j}^{\Omega}(\mathcal{T})\right)\right)\right\} \times \sin \Theta(\mathcal{T})
\end{aligned}
$$


$\delta w$

$$
\begin{aligned}
& \overline{\mathcal{W}}_{w}=\sqrt{1-\mathcal{X}^{2}}\left\{\frac{1}{4} \mathcal{P} \mathcal{C}^{2} \pi\left(-(\ddot{\mathcal{V}} \sin \vartheta+\ddot{\mathcal{W}} \cos \vartheta)+\mathcal{U}(\dot{\varphi}+\dot{\vartheta})-\left(\mathcal{E}_{c}-\frac{1}{4} \mathcal{C}\right) \ddot{\varphi}-\left(\left(\mathcal{E}_{c}+\mathcal{E}_{p}\right)-\frac{1}{4} \mathcal{C}\right) \ddot{\vartheta}\right)\right. \\
& \left.+\frac{1}{2} \mathcal{P U C} 2 \pi\left(\left(1-\tilde{A}_{1}-\tilde{A}_{2}\right) \tilde{w}_{3 c / 4}+\dot{\mathcal{Z}}\left(\tilde{A}_{1} \tilde{b}_{1}+\tilde{A}_{2} \tilde{b}_{2}\right)+\mathcal{Z} \tilde{b}_{1} \tilde{b}_{2}\left(\tilde{A}_{1}+\tilde{A}_{2}\right)\right)\right\} \times \cos \vartheta \\
& =\left\{\frac { 1 } { 4 } \mathcal { P C } ^ { 2 } \pi \left(-\int_{0}^{1}\left(\sum_{i=1}^{M} \sum_{j=1}^{L} \sqrt{\left(1-\mathcal{X}^{2}\right)} \Psi_{i}(\mathcal{X}) \Upsilon_{j}(\mathcal{X})\right) d \mathcal{X} \ddot{V}_{j}(\mathcal{T}) \sin \Theta(\mathcal{T})\right.\right. \\
& -\int_{0}^{1}\left(\sum_{i=1}^{M} \sum_{j=1}^{M} \sqrt{\left(1-\mathcal{X}^{2}\right)} \Psi_{i}(\mathcal{X}) \Psi_{j}(\mathcal{X})\right) d \mathcal{X} \ddot{W}_{j}(\mathcal{T}) \cos \Theta(\mathcal{T}) \\
& +\mathcal{U}\left(\int_{0}^{1}\left(\sum_{i=1}^{M} \sum_{j=1}^{N} \sqrt{\left(1-\mathcal{X}^{2}\right)} \Psi_{i}(\mathcal{X}) \Omega_{j}(\mathcal{X})\right) d \mathcal{X} \dot{\Phi}_{j}(\mathcal{T})\right. \\
& \left.+\int_{0}^{1}\left(\sum_{i=1}^{M} \sqrt{\left(1-\mathcal{X}^{2}\right)} \Psi_{i}(\mathcal{X})\right) d \mathcal{X} \dot{\Theta}(\mathcal{T})\right) \\
& -\left(\mathcal{E}_{c}-\frac{1}{4} \mathcal{C}\right) \int_{0}^{1}\left(\sum_{i=1}^{M} \sum_{j=1}^{N} \sqrt{\left(1-\mathcal{X}^{2}\right)} \Psi_{i}(\mathcal{X}) \Omega_{j}(\mathcal{X})\right) d \mathcal{X} \ddot{\Phi}_{j}(\mathcal{T}) \\
& \left.-\left(\left(\mathcal{E}_{c}+\mathcal{E}_{p}\right)-\frac{1}{4} \mathcal{C}\right) \int_{0}^{1}\left(\sum_{i=1}^{M} \sqrt{\left(1-\mathcal{X}^{2}\right)} \Psi_{i}(\mathcal{X})\right) d \mathcal{X} \ddot{\Theta}(\mathcal{T})\right) \\
& +\frac{1}{2} \mathcal{P U C} 2 \pi\left(\left(1-\tilde{A}_{1}-\tilde{A}_{2}\right) \int_{0}^{1}\left(\sum_{i=1}^{M} \sqrt{\left(1-\mathcal{X}^{2}\right)} \Psi_{i}(\mathcal{X}) \tilde{w}_{3 c / 4}(\mathcal{X}, \mathcal{T})\right) d \mathcal{X}\right. \\
& +\left(\tilde{A}_{1} \tilde{b}_{1}+\tilde{A}_{2} \tilde{b}_{2}\right)\left(\int_{0}^{1}\left(\sum_{i=1}^{M} \sum_{j=1}^{M} \sqrt{\left(1-\mathcal{X}^{2}\right)} \Psi_{i}(\mathcal{X}) \Psi_{j}(\mathcal{X})\right) d \mathcal{X} \dot{Z}_{j}^{\Psi}(\mathcal{T})\right. \\
& \left.+\int_{0}^{1}\left(\sum_{i=1}^{M} \sum_{j=1}^{N} \sqrt{\left(1-\mathcal{X}^{2}\right)} \Psi_{i}(\mathcal{X}) \Omega_{j}(\mathcal{X})\right) d \mathcal{X} \dot{Z}_{j}^{\Omega}(\mathcal{T})\right) \\
& +\tilde{b}_{1} \tilde{b}_{2}\left(\tilde{A}_{1}+\tilde{A}_{2}\right)\left(\int_{0}^{1}\left(\sum_{i=1}^{M} \sum_{j=1}^{M} \sqrt{\left(1-\mathcal{X}^{2}\right)} \Psi_{i}(\mathcal{X}) \Psi_{j}(\mathcal{X})\right) d \mathcal{X} Z_{j}^{\Psi}(\mathcal{T})\right. \\
& \left.\left.\left.+\int_{0}^{1}\left(\sum_{i=1}^{M} \sum_{j=1}^{N} \sqrt{\left(1-\mathcal{X}^{2}\right)} \Psi_{i}(\mathcal{X}) \Omega_{j}(\mathcal{X})\right) d \mathcal{X} Z_{j}^{\Omega}(\mathcal{T})\right)\right)\right\} \times \sin \Theta(\mathcal{T})
\end{aligned}
$$




$$
\begin{aligned}
& \delta \phi \\
& \overline{\mathcal{W}}_{\varphi}=\sqrt{1-\mathcal{X}^{2}}\left\{\frac { 1 } { 4 } \mathcal { P } \mathcal { C } ^ { 2 } \pi \left(-\left(\mathcal{E}_{c}-\frac{1}{4} \mathcal{C}\right)(\ddot{\mathcal{V}} \sin \vartheta+\ddot{\mathcal{W}} \cos \vartheta)-\mathcal{U}\left(\frac{1}{2} \mathcal{C}-\mathcal{E}_{c}\right) \dot{\varphi}-\mathcal{U}\left(\frac{1}{2} \mathcal{C}-\left(\mathcal{E}_{c}+\mathcal{E}_{p}\right)\right) \dot{\vartheta}\right.\right. \\
& \left.-\left(\left(\mathcal{E}_{c}-\frac{1}{4} \mathcal{C}\right)^{2}+\frac{1}{32} \mathcal{C}^{2}\right) \ddot{\varphi}-\left(\left(\left(\mathcal{E}_{c}+\mathcal{E}_{p}\right)-\frac{1}{4} \mathcal{C}\right)\left(\mathcal{E}_{c}-\frac{1}{4} \mathcal{C}\right)+\frac{1}{32} \mathcal{C}^{2}\right) \ddot{\vartheta}\right) \\
& \left.+\frac{1}{2} \mathcal{P U C} 2 \pi \mathcal{E}_{c}\left(\left(1-\tilde{A}_{1}-\tilde{A}_{2}\right) \tilde{w}_{3 c / 4}+\dot{\mathcal{Z}}\left(\tilde{A}_{1} \tilde{b}_{1}+\tilde{A}_{2} \tilde{b}_{2}\right)+\mathcal{Z} \tilde{b}_{1} \tilde{b}_{2}\left(\tilde{A}_{1}+\tilde{A}_{2}\right)\right)\right\} \\
& =\left\{\frac { 1 } { 4 } \mathcal { P } \mathcal { C } ^ { 2 } \pi \left(-\left(\mathcal{E}_{c}-\frac{1}{4} \mathcal{C}\right)\left(\int_{0}^{1}\left(\sum_{i=1}^{N} \sum_{j=1}^{L} \sqrt{\left(1-\mathcal{X}^{2}\right)} \Omega_{i}(\mathcal{X}) \Upsilon_{j}(\mathcal{X})\right) d \mathcal{X} \ddot{V}_{j}(\mathcal{T}) \sin \Theta(\mathcal{T})\right.\right.\right. \\
& \left.+\int_{0}^{1}\left(\sum_{i=1}^{N} \sum_{j=1}^{M} \sqrt{\left(1-\mathcal{X}^{2}\right)} \Omega_{i}(\mathcal{X}) \Psi_{j}(\mathcal{X})\right) d \mathcal{X} \ddot{W}_{j}(\mathcal{T}) \cos \Theta(\mathcal{T})\right) \\
& -\mathcal{U}\left(\frac{1}{2} \mathcal{C}-\mathcal{E}_{c}\right) \int_{0}^{1}\left(\sum_{i=1}^{N} \sum_{j=1}^{N} \sqrt{\left(1-\mathcal{X}^{2}\right)} \Omega_{i}(\mathcal{X}) \Psi_{j}(\mathcal{X})\right) d \mathcal{X} \dot{\Phi}_{j}(\mathcal{T}) \\
& -\mathcal{U}\left(\frac{1}{2} \mathcal{C}-\left(\mathcal{E}_{c}+\mathcal{E}_{p}\right)\right) \int_{0}^{1}\left(\sum_{i=1}^{N} \sqrt{\left(1-\mathcal{X}^{2}\right)} \Omega_{i}(\mathcal{X})\right) d \mathcal{X} \dot{\Theta}(\mathcal{T}) \\
& -\left(\left(\mathcal{E}_{c}-\frac{1}{4} \mathcal{C}\right)^{2}+\frac{1}{32} \mathcal{C}^{2}\right) \int_{0}^{1}\left(\sum_{i=1}^{N} \sum_{j=1}^{N} \sqrt{\left(1-\mathcal{X}^{2}\right)} \Omega_{i}(\mathcal{X}) \Psi_{j}(\mathcal{X})\right) d \mathcal{X} \ddot{\Phi}_{j}(\mathcal{T}) \\
& \left.-\left(\left(\left(\mathcal{E}_{c}+\mathcal{E}_{p}\right)-\frac{1}{4} \mathcal{C}\right)\left(\mathcal{E}_{c}-\frac{1}{4} \mathcal{C}\right)+\frac{1}{32} \mathcal{C}^{2}\right) \int_{0}^{1}\left(\sum_{i=1}^{N} \sum_{j=1}^{M} \sqrt{\left(1-\mathcal{X}^{2}\right)} \Omega_{i}(\mathcal{X})(\mathcal{X})\right) d \mathcal{X} \ddot{\Theta}(\mathcal{T})\right) \\
& +\frac{1}{2} \mathcal{P U C} 2 \pi \mathcal{E}_{c}\left(\left(1-\tilde{A}_{1}-\tilde{A}_{2}\right) \int_{0}^{1}\left(\sum_{i=1}^{N} \sqrt{\left(1-\mathcal{X}^{2}\right)} \Omega_{i}(\mathcal{X}) \tilde{w}_{3 c / 4}(\mathcal{X}, \mathcal{T})\right) d \mathcal{X}\right. \\
& +\left(\tilde{A}_{1} \tilde{b}_{1}+\tilde{A}_{2} \tilde{b}_{2}\right)\left(\int_{0}^{1}\left(\sum_{i=1}^{N} \sum_{j=1}^{M} \sqrt{\left(1-\mathcal{X}^{2}\right)} \Omega_{i}(\mathcal{X}) \Psi_{j}(\mathcal{X})\right) d \mathcal{X} \dot{Z}_{j}^{\Psi}(\mathcal{T})\right. \\
& \left.+\int_{0}^{1}\left(\sum_{i=1}^{N} \sum_{j=1}^{N} \sqrt{\left(1-\mathcal{X}^{2}\right)} \Omega_{i}(\mathcal{X}) \Omega_{j}(\mathcal{X})\right) d \mathcal{X} \dot{Z}_{j}^{\Omega}(\mathcal{T})\right) \\
& +\tilde{b}_{1} \tilde{b}_{2}\left(\tilde{A}_{1}+\tilde{A}_{2}\right)\left(\int_{0}^{1}\left(\sum_{i=1}^{N} \sum_{j=1}^{M} \sqrt{\left(1-\mathcal{X}^{2}\right)} \Omega_{i}(\mathcal{X}) \Psi_{j}(\mathcal{X})\right) d \mathcal{X} Z_{j}^{\Psi}(\mathcal{T})\right. \\
& \left.\left.\left.+\int_{0}^{1}\left(\sum_{i=1}^{N} \sum_{j=1}^{N} \sqrt{\left(1-\mathcal{X}^{2}\right)} \Omega_{i}(\mathcal{X}) \Omega_{j}(\mathcal{X})\right) d \mathcal{X} Z_{j}^{\Omega}(\mathcal{T})\right)\right)\right\}
\end{aligned}
$$




$$
\begin{aligned}
& \delta \theta \\
& \overline{\mathcal{W}}_{\vartheta}=\int_{0}^{S} \sqrt{1-\mathcal{X}^{2}}\left\{\frac { 1 } { 4 } \mathcal { P } \mathcal { C } ^ { 2 } \pi \left(-\left(\left(\mathcal{E}_{c}+\mathcal{E}_{p}\right)-\frac{1}{4} \mathcal{C}\right)(\ddot{\mathcal{V}} \sin \vartheta+\ddot{\mathcal{W}} \cos \vartheta)-\mathcal{U}\left(\frac{1}{2} \mathcal{C}-\mathcal{E}_{c}\right) \dot{\varphi}-\mathcal{U}\left(\frac{1}{2} \mathcal{C}-\left(\mathcal{E}_{c}+\mathcal{E}_{p}\right)\right) \dot{\vartheta}\right.\right. \\
& \left.-\left(\left(\left(\mathcal{E}_{c}+\mathcal{E}_{p}\right)-\frac{1}{4} \mathcal{C}\right)\left(\mathcal{E}_{c}-\frac{1}{4} \mathcal{C}\right)+\frac{1}{32} \mathcal{C}^{2}\right) \ddot{\varphi}-\left(\left(\left(\mathcal{E}_{c}+\mathcal{E}_{p}\right)-\frac{1}{4} \mathcal{C}\right)^{2}+\frac{1}{32} \mathcal{C}^{2}\right) \ddot{\vartheta}\right) \\
& \left.+\frac{1}{2} \mathcal{P U C} 2 \pi\left(\mathcal{E}_{c}+\mathcal{E}_{p}\right)\left(\left(1-\tilde{A}_{1}-\tilde{A}_{2}\right) \tilde{w}_{3 c / 4}+\dot{\mathcal{Z}}\left(\tilde{A}_{1} \tilde{b}_{1}+\tilde{A}_{2} \tilde{b}_{2}\right)+\mathcal{Z} \tilde{b}_{1} \tilde{b}_{2}\left(\tilde{A}_{1}+\tilde{A}_{2}\right)\right)\right\} d x \\
& =\left\{\frac { 1 } { 4 } \mathcal { P } \mathcal { C } ^ { 2 } \pi \left(-\left(\left(\mathcal{E}_{c}+\mathcal{E}_{p}\right)-\frac{1}{4} \mathcal{C}\right)\left(\int_{0}^{1}\left(\sum_{j=1}^{L} \sqrt{\left(1-\mathcal{X}^{2}\right)} \Upsilon_{j}(\mathcal{X})\right) d \mathcal{X} \ddot{V}_{j}(\mathcal{T}) \sin \Theta(\mathcal{T})\right.\right.\right. \\
& \left.+\int_{0}^{1}\left(\sum_{j=1}^{m} \sqrt{\left(1-\mathcal{X}^{2}\right)} \Psi_{j}(\mathcal{X})\right) d \mathcal{X} \ddot{W}_{j}(\mathcal{T}) \cos \Theta(\mathcal{T})\right) \\
& -\mathcal{U}\left(\frac{1}{2} \mathcal{C}-\mathcal{E}_{c}\right) \int_{0}^{1}\left(\sum_{j=1}^{N} \sqrt{\left(1-\mathcal{X}^{2}\right)} \Omega_{j}(\mathcal{X})\right) d \mathcal{X} \dot{\Phi}_{j}(\mathcal{T}) \\
& -\mathcal{U}\left(\frac{1}{2} \mathcal{C}-\left(\mathcal{E}_{c}+\mathcal{E}_{p}\right)\right) \int_{0}^{1}\left(\sqrt{\left(1-\mathcal{X}^{2}\right)}(\mathcal{X})\right) d \mathcal{X} \dot{\Theta}(\mathcal{T}) \\
& -\left(\left(\left(\mathcal{E}_{c}+\mathcal{E}_{p}\right)-\frac{1}{4} \mathcal{C}\right)\left(\mathcal{E}_{c}-\frac{1}{4} \mathcal{C}\right)+\frac{1}{32} \mathcal{C}^{2}\right) \int_{0}^{1}\left(\sum_{j=1}^{N} \sqrt{\left(1-\mathcal{X}^{2}\right)} \Omega_{j}(\mathcal{X})\right) d \mathcal{X} \ddot{\Phi}_{j}(\mathcal{T}) \\
& \left.-\left(\left(\left(\mathcal{E}_{c}+\mathcal{E}_{p}\right)-\frac{1}{4} \mathcal{C}\right)^{2}+\frac{1}{32} \mathcal{C}^{2}\right) \int_{0}^{1}\left(\sqrt{\left(1-\mathcal{X}^{2}\right)}(\mathcal{X})\right) d \mathcal{X} \ddot{\Theta}(\mathcal{T})\right) \\
& +\frac{1}{2} \mathcal{P U C} 2 \pi\left(\mathcal{E}_{c}+\mathcal{E}_{p}\right)\left(\left(1-\tilde{A}_{1}-\tilde{A}_{2}\right) \int_{0}^{1}\left(\sqrt{\left(1-\mathcal{X}^{2}\right)} \tilde{w}_{3 c / 4}(\mathcal{X}, \mathcal{T})\right) d \mathcal{X}\right. \\
& +\left(\tilde{A}_{1} \tilde{b}_{1}+\tilde{A}_{2} \tilde{b}_{2}\right)\left(\int_{0}^{1}\left(\sum_{j=1}^{M} \sqrt{\left(1-\mathcal{X}^{2}\right)} \Psi_{j}(\mathcal{X})\right) d \mathcal{X} \dot{Z}_{j}^{\Psi}(\mathcal{T})\right. \\
& \left.+\int_{0}^{1}\left(\sum_{j=1}^{N} \sqrt{\left(1-\mathcal{X}^{2}\right)} \Omega_{j}(\mathcal{X})\right) d \mathcal{X} \dot{Z}_{j}^{\Omega}(\mathcal{T})\right) \\
& +\tilde{b}_{1} \tilde{b}_{2}\left(\tilde{A}_{1}+\tilde{A}_{2}\right)\left(\int_{0}^{1}\left(\sum_{j=1}^{M} \sqrt{\left(1-\mathcal{X}^{2}\right)} \Psi_{j}(\mathcal{X})\right) d \mathcal{X} Z_{j}^{\Psi}(\mathcal{T})\right. \\
& \left.\left.\left.+\int_{0}^{1}\left(\sum_{j=1}^{N} \sqrt{\left(1-\mathcal{X}^{2}\right)} \Omega_{j}(\mathcal{X})\right) d \mathcal{X} Z_{j}^{\Omega}(\mathcal{T})\right)\right)\right\}
\end{aligned}
$$




$$
\begin{aligned}
& \tilde{w}_{3 c / 4}=\left(\int_{0}^{1}(\right.\left.\left.\sum_{i=1}^{M} \sum_{j=1}^{M} \Psi_{i}(\mathcal{X}) \Psi_{j}(\mathcal{X})\right) d \mathcal{X} \ddot{Z}_{j}^{\Psi}+\int_{0}^{1}\left(\sum_{i=1}^{M} \sum_{j=1}^{M} \Psi_{i}(\mathcal{X}) \Omega_{j}(\mathcal{X})\right) d \mathcal{X} \ddot{Z}_{j}^{\Omega}\right) \\
&+\left(\tilde{b}_{1}+\tilde{b}_{2}\right)\left(\int_{0}^{1}\left(\sum_{i=1}^{M} \sum_{j=1}^{M} \Psi_{i}(\mathcal{X}) \Psi_{j}(\mathcal{X})\right) d \mathcal{X} \dot{Z}_{j}^{\Psi}+\int_{0}^{1}\left(\sum_{i=1}^{M} \sum_{j=1}^{M} \Psi_{i}(\mathcal{X}) \Omega_{j}(\mathcal{X})\right) d \mathcal{X} \dot{Z}_{j}^{\Omega}\right) \\
&+\left(\tilde{b}_{1} \tilde{b}_{2}\right)\left(\int_{0}^{1}\left(\sum_{i=1}^{M} \sum_{j=1}^{M} \Psi_{i}(\mathcal{X}) \Psi_{j}(\mathcal{X})\right) d \mathcal{X} Z_{j}^{\Psi}+\int_{0}^{1}\left(\sum_{i=1}^{M} \sum_{j=1}^{M} \Psi_{i}(\mathcal{X}) \Omega_{j}(\mathcal{X})\right) d \mathcal{X} Z_{j}^{\Omega}\right) \\
& \tilde{w}_{3 c / 4}=\left(\int_{0}^{1}\left(\sum_{i=1}^{N} \sum_{j=1}^{M} \Omega_{i}(\mathcal{X}) \Psi_{j}(\mathcal{X})\right) d \mathcal{X} \ddot{Z}_{j}^{\Psi}+\int_{0}^{1}\left(\sum_{i=1}^{N} \sum_{j=1}^{N} \Omega_{i}(\mathcal{X}) \Omega_{j}(\mathcal{X})\right) d \mathcal{X} \ddot{Z}_{j}^{\Omega}\right) \\
&+\left(\tilde{b}_{1}+\tilde{b}_{2}\right)\left(\int_{0}^{1}\left(\sum_{i=1}^{N} \sum_{j=1}^{M} \Omega_{i}(\mathcal{X}) \Psi_{j}(\mathcal{X})\right) d \mathcal{X} \dot{Z}_{j}^{\Psi}+\int_{0}^{1}\left(\sum_{i=1}^{N} \sum_{j=1}^{N} \Omega_{i}(\mathcal{X}) \Omega_{j}(\mathcal{X})\right) d \mathcal{X} \dot{Z}_{j}^{\Omega}\right) \\
&+\left(\tilde{b}_{1} \tilde{b}_{2}\right)\left(\int_{0}^{1}\left(\sum_{i=1}^{N} \sum_{j=1}^{M} \Omega_{i}(\mathcal{X}) \Psi_{j}(\mathcal{X})\right) d \mathcal{X} Z_{j}^{\Psi}+\int_{0}^{1}\left(\sum_{i=1}^{N} \sum_{j=1}^{N} \Omega_{i}(\mathcal{X}) \Omega_{j}(\mathcal{X})\right) d \mathcal{X} Z_{j}^{\Omega}\right)
\end{aligned}
$$

\section{B.2 Galerkin Projection Coefficients}

The Galerkin projection coefficients for the structural equations and the aerodynamic loads are summarized below in Table B.1 and Table B.2 respectively. 


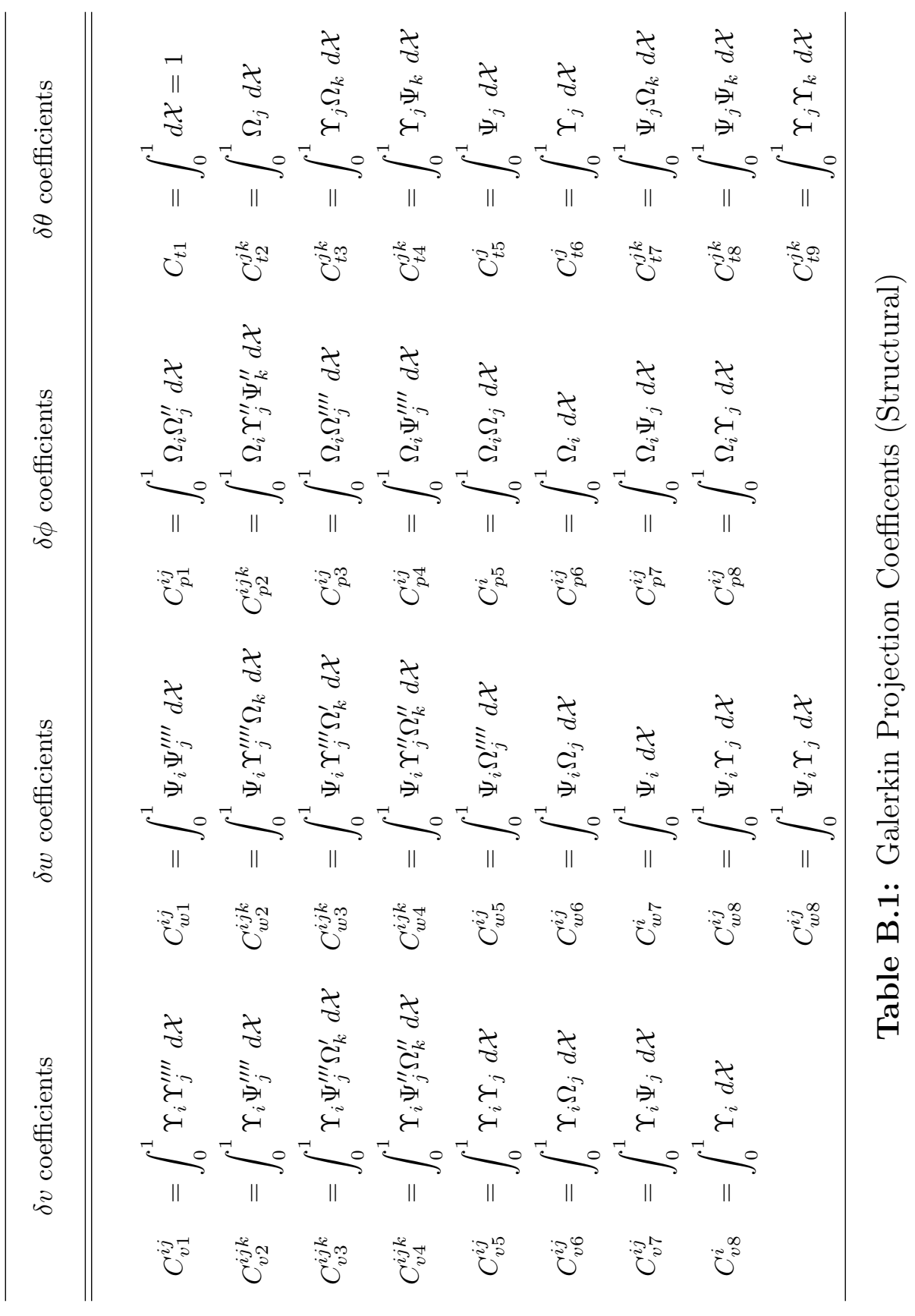




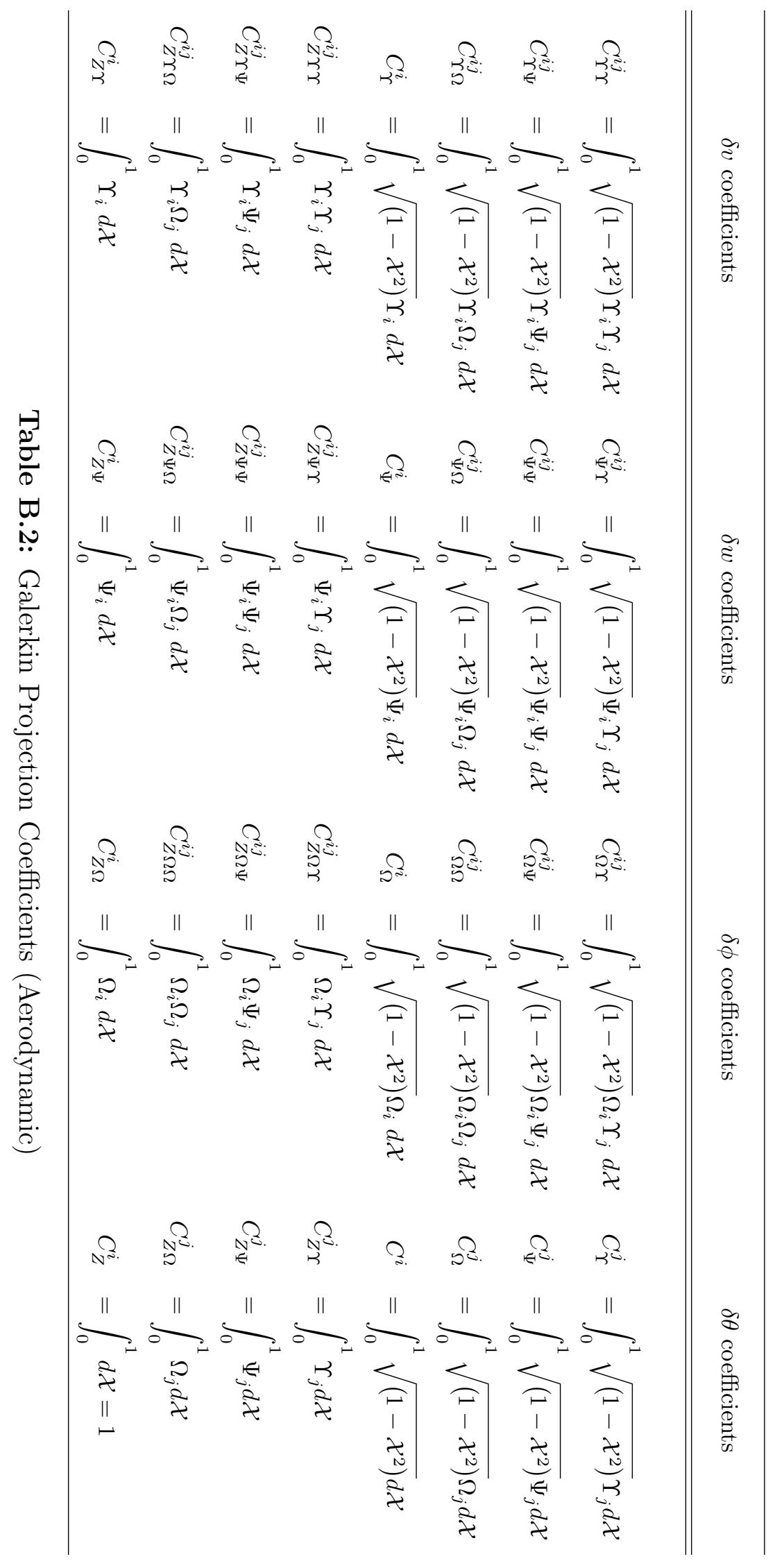




\section{B.3 Linear Structural and Aerodynamic M, C, K matrices}

The structural mass, damping and stiffness matrices are given below

$$
\mathbf{M}_{s}=\left[\begin{array}{cccccc}
C v_{5}^{i j} & 0 & 0 & 0 & 0 & 0 \\
0 & C w_{6}^{i j} & \mathcal{E} C w_{7}^{i j} & \left(\mathcal{E}+\mathcal{E}_{P}\right) C w_{9}^{i j} & 0 & 0 \\
0 & \mathcal{E} C p_{7}^{i j} & \mathcal{M}_{0} C p_{5}^{i j} & \left(\mathcal{M}_{0}+\mathcal{E} \mathcal{E}_{P}\right) C p_{6}^{i j} & 0 & 0 \\
0 & \left(\mathcal{E}+\mathcal{E}_{P}\right) C t_{5}^{i j} & \left(\mathcal{M}_{0}+\mathcal{E} \mathcal{E}_{P}\right) C t_{2} & \left(\mathcal{M}_{0}+\mathcal{M}_{b}+2 \mathcal{E} \mathcal{E}_{P}+\mathcal{E}_{P}^{2}\right) C t_{1} & 0 & 0 \\
0 & 0 & 0 & 0 & 0 & 0 \\
0 & 0 & 0 & 0 & 0 & 0
\end{array}\right]
$$

$$
\mathbf{C}_{s}=\left[\begin{array}{cccccc}
0 & 0 & 0 & 0 & 0 & 0 \\
0 & 0 & 0 & 0 & 0 & 0 \\
0 & 0 & 0 & 0 & 0 & 0 \\
0 & 0 & 0 & \mathcal{D}_{\theta} C t_{1} & 0 & 0 \\
0 & 0 & 0 & 0 & 0 & 0 \\
\mathbf{K}_{s}= & 0 & 0 & 0 & 0
\end{array}\right]
$$


The aerodynamic mass, damping and stiffness matrices are given below.

$$
\mathbf{M}_{a}=\frac{1}{4} \mathcal{P C} \pi\left[\begin{array}{cccccc}
0 & 0 & 0 & 0 & 0 & 0 \\
0 & m_{22} & m_{23} & m_{24} & 0 & 0 \\
0 & m_{32} & m_{33} & m_{34} & 0 & 0 \\
0 & m_{42} & m_{43} & m_{44} & 0 & 0 \\
0 & 0 & 0 & 0 & m_{55} & m_{56} \\
0 & 0 & 0 & 0 & m_{65} & m_{66}
\end{array}\right]
$$

$$
\begin{aligned}
& m_{22}=-\frac{1}{4} \mathcal{P C} \pi C_{\Psi \Psi}^{i j} \rho b^{2} \pi \\
& m_{23}=-\frac{1}{4} \mathcal{P C} \pi C_{\Psi \Omega}^{i j}\left(\mathcal{E}_{c}-\frac{1}{4} \mathcal{C}\right) \\
& m_{24}=-\frac{1}{4} \mathcal{P} \mathcal{C} \pi C_{\Psi}^{i}\left(\mathcal{E}_{c}-\frac{1}{4} \mathcal{C}\right) \\
& m_{32}=-\frac{1}{4} \mathcal{P} \mathcal{C} \pi C_{\Omega \Psi}^{i j}\left(\mathcal{E}_{c}-\frac{1}{4} \mathcal{C}\right) \\
& m_{33}=-\frac{1}{4} \mathcal{P} \mathcal{C} \pi C_{\Omega \Omega}^{i j}\left(\left(\mathcal{E}_{c}-\frac{1}{4} \mathcal{C}\right)^{2}+\frac{1}{32} \mathcal{C}^{2}\right) \\
& m_{34}=-\frac{1}{4} \mathcal{P} \mathcal{C} \pi C_{\Omega}^{i}\left(\left(\left(\mathcal{E}_{c}+\mathcal{E}_{c}\right)-\frac{1}{4} \mathcal{C}\right)\left(\mathcal{E}_{c}-\frac{1}{4} \mathcal{C}\right)+\frac{1}{32} \mathcal{C}^{2}\right) \\
& m_{42}=-\frac{1}{4} \mathcal{P C} \pi C_{\Psi}^{j}\left(\mathcal{E}_{c}-\frac{1}{4} \mathcal{C}\right) \\
& m_{43}=-\frac{1}{4} \mathcal{P C} \pi C_{\Omega}^{j}\left(\left(\mathcal{E}_{c}-\frac{1}{4} \mathcal{C}\right)^{2}+\frac{1}{32} \mathcal{C}^{2}\right) \\
& m_{44}=-\frac{1}{4} \mathcal{P C} \pi C^{i}\left(\left(\left(\mathcal{E}_{c}+\mathcal{E}_{c}\right)-\frac{1}{4} \mathcal{C}\right)\left(\mathcal{E}_{c}-\frac{1}{4} \mathcal{C}\right)+\frac{1}{32} \mathcal{C}^{2}\right) \\
& m_{55}=-C_{Z \Psi \Psi}^{i j} \\
& m_{56}=-C_{Z \Psi \Omega}^{i j} \\
& m_{65}=-C_{Z \Omega \Psi}^{i j} \\
& m_{66}=-C_{Z \Omega \Omega}^{i j}
\end{aligned}
$$




$$
\mathbf{C}_{a}=\left[\begin{array}{cccccc}
0 & 0 & 0 & 0 & 0 & 0 \\
0 & c_{22} & c_{23} & c_{24} & c_{25} & c_{26} \\
0 & c_{32} & c_{33} & c_{34} & c_{35} & c_{36} \\
0 & c_{42} & c_{43} & c_{44} & c_{45} & c_{46} \\
0 & c_{52} & c_{53} & c_{54} & c_{55} & c_{56} \\
0 & c_{62} & c_{63} & c_{64} & c_{65} & c_{66}
\end{array}\right]
$$

$$
\begin{aligned}
& c_{22}=-\frac{1}{2} \mathcal{P} \mathcal{U C} 2 \pi C_{\Psi \Psi}^{i j}(1-A 1-A 2) \\
& c_{23}=\frac{1}{4} \mathcal{P} \mathcal{U C} \pi C_{\Psi \Omega}^{i j}\left(\mathcal{C}+4(1-A 1-A 2)\left(\frac{1}{2} \mathcal{C}-\mathcal{E}_{c}\right)\right) \\
& c_{24}=\frac{1}{4} \mathcal{P U C} \pi C_{\Psi \Omega}^{i j}\left(\mathcal{C}+4(1-A 1-A 2)\left(\frac{1}{2} \mathcal{C}-\left(\mathcal{E}_{c}+\mathcal{E}_{p}\right)\right)\right) \\
& c_{25}=\frac{1}{2} \mathcal{P U C} 2 \pi C_{\Psi \Psi}^{i j}\left(A_{1} b_{1}+A_{2} b_{2}\right) \\
& c_{26}=\frac{1}{2} \mathcal{P U C} 2 \pi C_{\Psi \Omega}^{i j}\left(A_{1} b_{1}+A_{2} b_{2}\right) \\
& c_{32}=\frac{1}{2} \mathcal{P U C} 2 \pi \mathcal{E}_{c} C_{\Omega \Psi}^{i j}\left(1-A_{1}-A_{2}\right) \\
& c_{33}=\frac{1}{4} \mathcal{P U C} \pi C_{\Omega \Omega}^{i j}\left(\frac{1}{2} \mathcal{C}-\mathcal{E}_{c}\right)\left(\mathcal{C}+4 \mathcal{E}_{c}\left(1-A_{1}-A_{2}\right)\right) \\
& c_{34}=\frac{1}{4} \mathcal{P U C} \pi C_{\Omega}^{i}\left(\frac{1}{2} \mathcal{C}-\left(\mathcal{E}_{c}-\mathcal{E}_{p}\right)\right)\left(\mathcal{C}+4 \mathcal{E}_{c}\left(1-A_{1}-A_{2}\right)\right) \\
& c_{35}=\frac{1}{2} \mathcal{P U C} 2 \pi \mathcal{E}_{c} C_{\Omega \Psi}^{i j}\left(A_{1} b_{1}+A_{2} b_{2}\right) \\
& c_{36}=\frac{1}{2} \mathcal{P U C} 2 \pi \mathcal{E}_{c} C_{\Omega \Omega}^{i j}\left(A_{1} b_{1}+A_{2} b_{2}\right) \\
& c_{42}=\frac{1}{2} \mathcal{P U C} 2 \pi \mathcal{E}_{c} C_{\Psi}^{j}\left(1-A_{1}-A_{2}\right) \\
& c_{43}=\frac{1}{4} \mathcal{P U C} \pi C_{\Omega}^{j}\left(\frac{1}{2} \mathcal{C}-\mathcal{E}_{c}\right)\left(\mathcal{C}+4\left(\mathcal{E}_{c}+\mathcal{E}_{p}\right)\left(1-A_{1}-A_{2}\right)\right) \\
& c_{44}=\frac{1}{4} \mathcal{P} \mathcal{U C} \pi C^{i}\left(\frac{1}{2} \mathcal{C}-\left(\mathcal{E}_{c}-\mathcal{E}_{p}\right)\right)\left(\mathcal{C}+4\left(\mathcal{E}_{c}+\mathcal{E}_{p}\right)\left(1-A_{1}-A_{2}\right)\right) \\
& c_{45}=\frac{1}{2} \mathcal{P} \mathcal{U C} 2 \pi C_{\Psi}^{j}\left(\mathcal{E}_{c}+\mathcal{E}_{p}\right)\left(A_{1} b_{1}+A_{2} b_{2}\right) \\
& c_{46}=\frac{1}{2} \mathcal{P} \mathcal{U C} 2 \pi C_{\Omega}^{j}\left(\mathcal{E}_{c}+\mathcal{E}_{p}\right)\left(A_{1} b_{1}+A_{2} b_{2}\right) \\
& c_{52}=-C_{Z \Psi \Psi}^{i j} \\
& \left.c_{53}=C_{Z \Psi \Omega}^{i j}\left(\frac{1}{2} \mathcal{C}-\mathcal{E}_{c}\right)\right) \\
& c_{54}=C_{Z \Psi}^{i}\left(\frac{1}{2} \mathcal{C}-\left(\mathcal{E}_{c}+\mathcal{E}_{p}\right)\right)
\end{aligned}
$$




$$
\begin{aligned}
& c_{55}=-C_{Z \Psi \Psi}^{i j}\left(b_{1}+b_{2}\right) \\
& c_{56}=-C_{Z \Psi \Omega}^{i j}\left(b_{1}+b_{2}\right) \\
& c_{62}=-C_{Z \Omega \Psi}^{i j} \\
& \left.c_{63}=C_{Z \Omega \Omega}^{i j}\left(\frac{1}{2} \mathcal{C}-\mathcal{E}_{c}\right)\right) \\
& c_{64}=C_{Z \Omega}^{i}\left(\frac{1}{2} \mathcal{C}-\left(\mathcal{E}_{c}+\mathcal{E}_{p}\right)\right) \\
& c_{65}=-C_{Z \Omega \Psi}^{i j}\left(b_{1}+b_{2}\right) \\
& c_{66}=-C_{Z \Omega \Omega}^{i j}\left(b_{1}+b_{2}\right)
\end{aligned}
$$

$$
\begin{aligned}
& \mathbf{K}_{a}=\left[\begin{array}{cccccc}
0 & 0 & 0 & 0 & 0 & 0 \\
0 & 0 & k_{23} & k_{24} & k_{25} & k_{26} \\
0 & 0 & k_{23} & k_{24} & k_{25} & k_{26} \\
0 & 0 & k_{23} & k_{24} & k_{25} & k_{26} \\
0 & 0 & k_{23} & k_{24} & k_{25} & k_{26} \\
0 & 0 & k_{23} & k_{24} & k_{25} & k_{26}
\end{array}\right] \\
& k_{23}=\frac{1}{2} \mathcal{P} \mathcal{U}^{2} \mathcal{C} 2 \pi\left(1-A_{1}-A_{2}\right) C_{\Psi \Omega}^{i j} \\
& k_{24}=\frac{1}{2} \mathcal{P} \mathcal{U}^{2} \mathcal{C} 2 \pi\left(1-A_{1}-A_{2}\right) C_{\Psi}^{i} \\
& k_{25}=\frac{1}{2} \mathcal{P U C} 2 \pi C_{\Psi \Psi}^{i j}\left(A_{1}+A_{2}\right) b_{1} b_{2} \\
& k_{26}=\frac{1}{2} \mathcal{P U C} 2 \pi C_{\Psi \Omega}^{i j}\left(A_{1}+A_{2}\right) b_{1} b_{2} \\
& k_{33}=\frac{1}{2} \mathcal{P} \mathcal{U}^{2} \mathcal{C} 2 \pi \mathcal{E}_{c}\left(1-A_{1}-A_{2}\right) C_{\Omega \Omega}^{i j} \\
& k_{34}=\frac{1}{2} \mathcal{P} \mathcal{U}^{2} \mathcal{C} 2 \pi \mathcal{E}_{c}\left(1-A_{1}-A_{2}\right) C_{\Omega}^{i} \\
& k_{35}=\frac{1}{2} \mathcal{P U C} 2 \pi \mathcal{E}_{c} C_{\Omega \Psi}^{i j}\left(A_{1}+A_{2}\right) b_{1} b_{2} \\
& k_{36}=\frac{1}{2} \mathcal{P U C} 2 \pi \mathcal{E}_{c} C_{\Omega \Omega}^{i j}\left(A_{1}+A_{2}\right) b_{1} b_{2} \\
& k_{33}=\frac{1}{2} \mathcal{P} \mathcal{U}^{2} \mathcal{C} 2 \pi\left(\mathcal{E}_{c}+\mathcal{E}_{p}\right)\left(1-A_{1}-A_{2}\right) C_{\Omega \Omega}^{i j} \\
& k_{34}=\frac{1}{2} \mathcal{P} \mathcal{U}^{2} \mathcal{C} 2 \pi\left(\mathcal{E}_{c}+\mathcal{E}_{p}\right)\left(1-A_{1}-A_{2}\right) C_{\Omega}^{i} \\
& k_{45}=\frac{1}{2} \mathcal{P U C} 2 \pi\left(\mathcal{E}_{c}+\mathcal{E}_{p}\right) C_{\Psi}^{j}\left(A_{1}+A_{2}\right) b_{1} b_{2}
\end{aligned}
$$




$$
\begin{aligned}
& k_{46}=\frac{1}{2} \mathcal{P} \mathcal{U C} 2 \pi\left(\mathcal{E}_{c}+\mathcal{E}_{p}\right) C_{\Omega}^{j}\left(A_{1}+A_{2}\right) b_{1} b_{2} \\
& k_{53}=C_{Z \Psi \Omega}^{i j} \mathcal{U} \\
& k_{54}=C_{Z \Psi}^{i} \mathcal{U} \\
& k_{55}=-C_{Z \Psi \Psi}^{i j}\left(b_{1} b_{2}\right) \\
& k_{56}=-C_{Z \Psi \Omega}^{i j}\left(b_{1} b_{2}\right) \\
& k_{63}=C_{Z \Omega \Omega}^{i j} \mathcal{U} \\
& k_{64}=C_{Z \Omega}^{i} \mathcal{U} \\
& k_{65}=C_{Z \Omega \Psi}^{i j}\left(b_{1} b_{2}\right) \\
& k_{66}=C_{Z \Omega \Omega}^{i j}\left(b_{1} b_{2}\right)
\end{aligned}
$$




\section{B.4 Numerical Implementation: Galerkin Projection}

The first row of equations presents the equations as shown in the main body of the text. For the sake of presenting the equations in a concise form, the $(t)$ notation was dropped for $V(t)$, $W(t), \Phi(t)$ and $\Theta(t)$, as well as the summation signs in Eq.(B.9a), Eq.(B.10a), Eq.(B.11a) and Eq. B.12a. However it is implied that $V, W, \Phi$ and $\Theta$ are functions of time, and that on is to sum over the indices given in the superscripts of the coefficients $C_{x x}^{x x x}$.

Collecting the like functions of time, and explicitly reintroduce the summations in the equations, the simplified from seen in Eq. B.9b), Eq.(B.10b), Eq.(B.11b) and Eq.(B.12b) is obtained. Finally these terms can be ordered such that the summations are nested as in in Eq.(B.9c), Eq.(B.10c), Eq. B.11c and Eq. B.12c). This allows us to obtain an efficient loop structure for the numerical implementation of these equations such that it would minimize the number of computations (if coded in this form).

Referring to the Modified Newton-Raphson algorithm, (see Eq.(3.41), for the structural equations, the elements of the function vector $\mathbf{f}(\dot{x}, x, t)$ are given by Eq.(B.9d), Eq. B.10d), Eq.(B.11d) and Eq.B.12d). Similarly, the Jacobian matrix, $\mathbf{J}(\dot{x}, x, t)$ is constructed as shown in Eq. B.9e), Eq. B.10e), Eq. B.11e and Eq.(B.12e respectively.

$\delta v$

$$
\begin{aligned}
& \mathcal{K}_{z}\{\left.C_{v 1}^{i j} V_{j}\right\}+\left(\mathcal{K}_{z}-\mathcal{K}_{y}\right)\left\{C_{v 2}^{i j k} W_{j} \Phi_{k}+2 C_{v 3}^{i j k} W_{j} \Phi_{k}+C_{v 4}^{i j k} W_{j} \Phi_{k}\right\} \\
&-\mathcal{E}_{A}^{2}\left\{C_{v 1}^{i j} V_{j}+C_{v 2}^{i j k} W_{j} \Phi_{k}-2 C_{v 3}^{i j k} W_{j} \Phi_{k}+C_{v 4}^{i j k} W_{j} \Phi_{k}\right\} \\
&+C_{v 5}^{i j} \ddot{V}_{j}-\ddot{\Theta}\left\{\mathcal{E} C_{v 6}^{i j} \Phi_{j}+C_{v 7}^{i j} W_{j}\right\}-\dot{\Theta}^{2}\left\{C_{v 5}^{i j} V_{j}+\mathcal{E}_{p} C_{v 8}^{i}+\mathcal{E} C_{v 8}^{i}\right\}-2 \dot{\Theta}\left\{\mathcal{E} C_{v 6}^{i j k} \dot{\Phi}_{j}+C_{v 7}^{i j} \dot{W}_{j}\right\}=\mathcal{W}_{v} \\
& \mathcal{K}_{z}\left\{\sum_{i=1}^{L} \sum_{j=1}^{L} C_{v 1}^{i j} V_{j}\right\}+\left(\mathcal{K}_{z}-\mathcal{K}_{y}\right)\left\{\sum_{i=1}^{L} \sum_{j=1}^{M} \sum_{k=1}^{N}\left(C_{v 2}^{i j k}+2 C_{v 3}^{i j k}+C_{v 4}^{i j k}\right) W_{j} \Phi_{k}\right\} \\
& \quad-\mathcal{E}_{A}^{2}\left\{\sum_{i=1}^{L} \sum_{j=1}^{L} C_{v 1}^{i j} V_{j}+\sum_{i=1}^{L} \sum_{j=1}^{M} \sum_{k=1}^{N}\left(C_{v 2}^{i j k}-2 C_{v 3}^{i j k}+C_{v 4}^{i j k}\right) W_{j} \Phi_{k}\right\}
\end{aligned}
$$




$$
\begin{aligned}
& +\sum_{i=1}^{L} \sum_{j=1}^{L} C_{v 5}^{i j} \ddot{V}_{j}-\ddot{\Theta}\left\{\mathcal{E} \sum_{i=1}^{L} \sum_{j=1}^{N} C_{v 6}^{i j} \Phi_{j}+\sum_{i=1}^{L} \sum_{j=1}^{M} C_{v 7}^{i j} W_{j}\right\}-\dot{\Theta}^{2}\left\{\sum_{i=1}^{L} \sum_{j=1}^{L} C_{v 5}^{i j} V_{j}+\mathcal{E}_{p} \sum_{i=1}^{L} C_{v 8}^{i}+\mathcal{E} \sum_{i=1}^{L} C_{v 8}^{i}\right\} \\
& -2 \dot{\Theta}\left\{\mathcal{E} \sum_{i=1}^{L} \sum_{j=1}^{N} C_{v 6}^{i j k} \dot{\Phi}_{j}+\sum_{i=1}^{L} \sum_{j=1}^{M} C_{v 7}^{i j} \dot{W}_{j}\right\}=\mathcal{W}_{v}
\end{aligned}
$$

$$
\begin{aligned}
\sum_{i=1}^{L}[ & -C_{v 8}^{i} \dot{\Theta}^{2}\left(\mathcal{E}_{P}+\mathcal{E}\right) \\
& +\sum_{j=1}^{L}\left[C_{v 1}^{i j}\left(\ddot{V}_{j}-\dot{\Theta}^{2} V_{j}\right)+\left(\mathcal{K}_{z}-\mathcal{E}_{A}{ }^{2}\right) C_{v 5}^{i j} V_{j}\right] \\
& +\sum_{j=1}^{M}\left[-C_{v 6}^{i j}\left(\ddot{\Theta} W_{j}+2 \dot{\Theta} \dot{W}_{j}\right)\right. \\
& \left.+\sum_{k=1}^{N}\left[\left(\mathcal{K}_{z}-\mathcal{K}_{y}-\mathcal{E}_{A}^{2}\right)\left(C_{v 2}^{i j k}+2 C_{v 3}^{i j k}+C_{v 4}^{i j k}\right) W_{j} \Phi_{k}\right]\right] \\
& \left.+\sum_{j=1}^{N}\left[-C_{v 18}^{i j} \mathcal{E}\left(\ddot{\Theta} \Phi_{j}+2 \dot{\Theta}_{j}\right)\right]\right]=\mathcal{W}_{v}
\end{aligned}
$$

for $i=1: L$

$$
\mathbf{f}(i)=-C_{v 8}^{i}\left(\frac{1}{36 \Delta \mathcal{T}^{2}}\left(11 \Theta^{n+1}-18 \Theta^{n}+9 \Theta^{n-1}-2 \Theta^{n-2}\right)^{2}\left(\mathcal{E}_{P}+\mathcal{E}\right)\right)
$$

for $j=1: L$

$$
\begin{aligned}
\mathbf{f}(i)=\mathbf{f}(i)+C_{v 1}^{i j}\left(\frac{1}{\Delta \mathcal{T}^{2}}\right. & \left(2 V_{j}^{n+1}-5 V_{j}^{n}+4 V_{j}^{n-1}-V_{j}^{n-2}\right) \\
& \left.-\frac{1}{36 \Delta \mathcal{T}^{2}}\left(11 \Theta^{n+1}-18 \Theta^{n}+9 \Theta^{n-1}-2 \Theta^{n-2}\right)^{2} V_{j}\right)+C_{v 5}^{i j}\left(\mathcal{K}_{z}-\mathcal{E}_{A}{ }^{2}\right) V_{j}
\end{aligned}
$$

end

for $j=1: M$

$$
\begin{aligned}
\mathbf{f}(i)=\mathbf{f}(i)-C_{v 6}^{i j}\left(\frac{1}{\Delta \mathcal{T}^{2}}\right. & \left(2 \Theta^{n+1}-5 \Theta^{n}+4 \Theta^{n-1}-\Theta^{n-2}\right) W_{j} \\
& \left.+\frac{2}{36 \Delta \mathcal{T}^{2}}\left(11 \Theta^{n+1}-18 \Theta^{n}+9 \Theta^{n-1}-2 \Theta^{n-2}\right)\left(11 W_{j}^{n+1}-18 W_{j}^{n}+9 W_{j}^{n-1}-2 W_{j}^{n-2}\right)\right)
\end{aligned}
$$

for $k=1: N$

$$
\mathbf{f}(i)=\mathbf{f}(i)+\left(C_{v 2}^{i j k}+2 C_{v 3}^{i j k}+C_{v 4}^{i j k}\right)\left(\mathcal{K}_{z}-\mathcal{K}_{y}-\mathcal{E}_{A}^{2}\right) W_{j} \Phi_{k}
$$

end

end

for $j=1: N$

$\mathbf{f}(i)=\mathbf{f}(i)-C_{v 6}^{i j} \mathcal{E}\left(\frac{1}{\Delta \mathcal{T}^{2}}\left(2 \Theta^{n+1}-5 \Theta^{n}+4 \Theta^{n-1}-\Theta^{n-2}\right) \Phi_{j}\right.$ 


$$
\left.+\frac{2}{36 \Delta \mathcal{T}^{2}}\left(11 \Theta^{n+1}-18 \Theta^{n}+9 \Theta^{n-1}-2 \Theta^{n-2}\right)\left(11 \Phi_{j}^{n+1}-18 \Phi_{j}^{n}+9 \Phi_{j}^{n-1}-2 \Phi_{j}^{n-2}\right)\right)
$$

end

end

for $n=1: L+M+N+1$

for $i=1: L$

$$
\begin{aligned}
& \text { if } n=L+M+N+1 \\
& \mathbf{J}(i, n)=-C_{v 8}^{i}\left(\frac{22}{36 \Delta \mathcal{T}^{2}}\left(11 \Theta^{n+1}-18 \Theta^{n}+9 \Theta^{n-1}-2 \Theta^{n-2}\right)\left(\mathcal{E}_{P}+\mathcal{E}\right)\right)
\end{aligned}
$$

else

$\mathbf{J}(i, n)=0$

end

for $j=1: L$

$$
\begin{aligned}
& \text { if } n=j \\
& \mathbf{J}(i, n)=\mathbf{J}(i, n)+C_{v 1}^{i j}\left(\frac{2}{\Delta \mathcal{T}^{2}}-\frac{1}{36 \Delta \mathcal{T}^{2}}\left(11 \Theta^{n+1}-18 \Theta^{n}+9 \Theta^{n-1}-2 \Theta^{n-2}\right)^{2}\right)+C_{v 5}^{i j}\left(\mathcal{K}_{z}-\mathcal{E}_{A}{ }^{2}\right) \\
& \text { else if } n=L+M+N+1 \\
& \mathbf{J}(i, n)=\mathbf{J}(i, n)+C_{v 1}^{i j}\left(-\frac{22}{36 \Delta \mathcal{T}^{2}}\left(11 \Theta^{n+1}-18 \Theta^{n}+9 \Theta^{n-1}-2 \Theta^{n-2}\right) V_{j}^{n+1}\right)
\end{aligned}
$$

end

end

for $j=1: M$

if $n=j$

$$
\begin{aligned}
\mathbf{J}(i, n)=\mathbf{J}(i, n)- & C_{v 6}^{i j}\left(\frac{1}{\Delta \mathcal{T}^{2}}\left(2 \Theta^{n+1}-5 \Theta^{n}+4 \Theta^{n-1}-\Theta^{n-2}\right)\right. \\
& \left.+\frac{22}{36 \Delta \mathcal{T}^{2}}\left(11 \Theta^{n+1}-18 \Theta^{n}+9 \Theta^{n-1}-2 \Theta^{n-2}\right)\right)
\end{aligned}
$$

else if $n=L+M+N+1$

$$
\begin{aligned}
\mathbf{J}(i, n)=\mathbf{J}(i, n)- & C_{v 6}^{i j}\left(\frac{1}{\Delta \mathcal{T}^{2}}\left(2 \Theta^{n+1}-5 \Theta^{n}+4 \Theta^{n-1}-\Theta^{n-2}\right) W_{j}\right. \\
& \left.+\frac{22}{36 \Delta \mathcal{T}^{2}}\left(11 W_{j}^{n+1}-18 W_{j}^{n}+9 W_{j}^{n-1}-2 W_{j}^{n-2}\right)\right)
\end{aligned}
$$

end

for $k=1: N$

$$
\text { if } n=L+j
$$




$$
\begin{aligned}
& \mathbf{J}(i, n)=\mathbf{J}(i, n)+\left(C_{v 2}^{i j k}+2 C_{v 3}^{i j k}+C_{v 4}^{i j k}\right)\left(\mathcal{K}_{z}-\mathcal{K}_{y}-\mathcal{E}_{A}^{2}\right) \Phi_{k} \\
& \text { if } n=L+M+k \\
& \mathbf{J}(i, n)=\mathbf{J}(i, n)+\left(C_{v 2}^{i j k}+2 C_{v 3}^{i j k}+C_{v 4}^{i j k}\right)\left(\mathcal{K}_{z}-\mathcal{K}_{y}-\mathcal{E}_{A}^{2}\right) W_{j} \\
& \text { end }
\end{aligned}
$$

end

end

for $j=1: N$

$$
\begin{aligned}
& \text { if } n=L+M+j \\
& \mathbf{J}(i, n)=\mathbf{J}(i, n)-C_{v 6}^{i j} \mathcal{E}\left(\frac{1}{\Delta \mathcal{T}^{2}}\left(2 \Theta^{n+1}-5 \Theta^{n}+4 \Theta^{n-1}-\Theta^{n-2}\right)+\frac{22}{36 \Delta \mathcal{T}^{2}}\left(11 \Theta^{n+1}-18 \Theta^{n}+9 \Theta^{n-1}-2 \Theta^{n-2}\right)\right)
\end{aligned}
$$$$
\text { else if } n=L+M+N+1
$$$$
\mathbf{J}(i, n)=\mathbf{J}(i, n)-C_{v 6}^{i j} \mathcal{E}\left(\frac{2}{\Delta \mathcal{T}^{2}} \Phi_{j}+\frac{22}{36 \Delta \mathcal{T}^{2}}\left(11 \Phi_{j}^{n+1}-18 \Phi_{j}^{n}+9 \Phi_{j}^{n-1}-2 \Phi_{j}^{n-2}\right)\right)
$$

end

end

end

end 
$\delta w$

$$
\begin{aligned}
& \mathcal{K}_{y}\{\left.C_{w 1}^{i j} W_{j}\right\}+\left(\mathcal{K}_{z}-\mathcal{K}_{y}\right)\left\{C_{w 2}^{i j k} V_{j} \Phi_{k}+2 C_{w 3}^{i j k} V_{j} \Phi_{k}+C_{w 4}^{i j k} V_{j} \Phi_{k}\right\} \\
&-\mathcal{E}_{A}{ }^{2}\left\{C_{w 2}^{i j k} V_{j} \Phi_{k}+2 C_{w 3}^{i j k} V_{j} \Phi_{k}+C_{w 4}^{i j k} V_{j} \Phi_{k}\right\}+\mathcal{K}_{\lambda 1}\left\{C_{w 5}^{i j k l} \Phi_{j}\right\} \\
&+C_{w 6}^{i j} \ddot{W}_{i}+\mathcal{E} C_{w 7}^{i j} \ddot{\Phi}_{i}+\ddot{\Theta}\left\{C_{w 8}^{i j} V_{j}+\mathcal{E}_{p} C_{w 9}^{i}+\mathcal{E} C_{w 9}^{i}\right\}-\dot{\Theta}^{2}\left\{\mathcal{E} C_{w 7}^{i j} \Phi_{i}+C_{w 6}^{i j} W_{i}\right\}+2 \dot{\Theta}\left\{C_{w 8}^{i j} \dot{V}_{j}\right\}=\mathcal{W}_{w} \\
& \mathcal{K}_{y}\left\{\sum_{i=1}^{M} \sum_{j=1}^{M} C_{w 1}^{i j} W_{j}\right\}+\left(\mathcal{K}_{z}-\mathcal{K}_{y}\right)\left\{\sum_{i=1}^{M} \sum_{j=1}^{L} \sum_{k=1}^{N}\left(C_{w 2}^{i j k}+2 C_{w 3}^{i j k}+C_{w 4}^{i j k}\right) V_{j} \Phi_{k}\right\} \\
&-\mathcal{E}_{A}{ }^{2}\left\{\sum_{i=1}^{M} \sum_{j=1}^{L} \sum_{k=1}^{N}\left(C_{w 2}^{i j k}+2 C_{w 3}^{i j k}+C_{w 4}^{i j k}\right) V_{j} \Phi_{k}\right\}+\mathcal{K}_{\lambda 1}\left\{\sum_{i=1}^{M} \sum_{j=1}^{N} C_{w 5}^{i j k l} \Phi_{j}\right\} \\
&+\sum_{i=1}^{M} \sum_{j=1}^{M} C_{w 6}^{i j} \ddot{W}_{i}+\mathcal{E} \sum_{i=1}^{M} \sum_{j=1}^{N} C_{w 7}^{i j} \ddot{\Phi}_{i}+\ddot{\Theta}\left\{\sum_{i=1}^{M} \sum_{j=1}^{L} C_{w 8}^{i j} V_{j}+\mathcal{E}_{p} \sum_{i=1}^{M} C_{w 9}^{i}+\mathcal{E} \sum_{i=1}^{M} C_{w 9}^{i}\right\} \\
&-\dot{\Theta}^{2}\left\{\mathcal{E} \sum_{i=1}^{M} \sum_{j=1}^{N} C_{w 7}^{i j} \Phi_{i}+\sum_{i=1}^{M} \sum_{j=1}^{M} C_{w 6}^{i j} W_{i}\right\}+2 \dot{\Theta}\left\{\sum_{i=1}^{M} \sum_{j=1}^{L} C_{w 8}^{i j} \dot{V}_{j}\right\}=\mathcal{W}_{w}
\end{aligned}
$$

$$
\begin{aligned}
\sum_{i=1}^{M}[ & C_{w 9}^{i} \ddot{\Theta}\left(\mathcal{E}+\mathcal{E}_{P}\right) \\
& +\sum_{j=1}^{L}\left[C_{w 8}^{i j}\left(\ddot{\Theta} V_{j}+2 \dot{\Theta} \dot{V}_{j}\right)\right. \\
& \left.+\sum_{k=1}^{N}\left[\left(C_{w 2}^{i j k}+2 C_{w 3}^{i j k}+C_{w 4}^{i j k}\right)\left(\mathcal{K}_{z}-\mathcal{K}_{y}-\mathcal{E}_{A}^{2}\right) V_{j} \Phi_{k}\right]\right] \\
& +\sum_{j=1}^{M}\left[C_{w 1}^{i j} \mathcal{K}_{y} W_{j}+C_{w 6}^{i j}\left(\ddot{W}_{j}-\dot{\Theta}^{2} W_{j}\right)\right] \\
& \left.+\sum_{j=1}^{N}\left[C_{w 7}^{i j}\left(\mathcal{E} \ddot{\Phi}_{j}-\mathcal{E} \dot{\Theta}^{2} \Phi_{j}\right)+C_{w 5}^{i j} K_{\lambda 1} \Phi_{j}\right]\right]=\mathcal{W}_{w}
\end{aligned}
$$

for $i=1: M$

$$
\begin{aligned}
& \mathbf{f}(L+i)=C_{w 9}^{i}\left(\frac{1}{\Delta \mathcal{T}^{2}}\left(2 \Theta^{n+1}-5 \Theta^{n}+4 \Theta^{n-1}-\Theta^{n-2}\right)\left(\mathcal{E}+\mathcal{E}_{P}\right)\right) \\
& \qquad \begin{array}{r}
\text { for } j=1: L \\
\mathbf{f}(L+i)=\mathbf{f}(L+i)+C_{w 8}^{i j}\left(\frac{1}{\Delta \mathcal{T}^{2}}\left(2 \Theta^{n+1}-5 \Theta^{n}+4 \Theta^{n-1}-\Theta^{n-2}\right) V_{j}\right. \\
\left.\quad+\frac{2}{36 \Delta \mathcal{T}^{2}}\left(11 \Theta^{n+1}-18 \Theta^{n}+9 \Theta^{n-1}-2 \Theta^{n-2}\right)\left(11 V_{j}^{n+1}-18 V_{j}^{n}+9 V_{j}^{n-1}-2 V_{j}^{n-2}\right)\right)
\end{array}
\end{aligned}
$$

for $k=1: N$ 
$\mathbf{f}(L+i)=\mathbf{f}(L+i)+\left(C_{w 2}^{i j k}+2 C_{w 3}^{i j k}+C_{w 4}^{i j k}\right)\left(\mathcal{K}_{z}-\mathcal{K}_{y}-\mathcal{E}_{A}^{2}\right) V_{j} \Phi_{k}$

end

end

for $j=1: M$

$$
\begin{array}{r}
\mathbf{f}(L+i)=\mathbf{f}(L+i)+C_{w 1}^{i j} \mathcal{K}_{y} W_{j}+C_{w 6}^{i j}\left(\frac{1}{\Delta \mathcal{T}^{2}}\left(2 W_{j}^{n+1}-5 W_{j}^{n}+4 W_{j}^{n-1}-W_{j}^{n-2}\right)\right. \\
\left.-\frac{1}{36 \Delta \mathcal{T}^{2}}\left(11 \Theta^{n+1}-18 \Theta^{n}+9 \Theta^{n-1}-2 \Theta^{n-2}\right)^{2} W_{j}\right)
\end{array}
$$

end

for $j=1: N$

$$
\begin{aligned}
\mathbf{f}(L+i)=\mathbf{f}(L+i)+ & C_{w 7}^{i j}\left(\mathcal{E} \frac{1}{\Delta \mathcal{T}^{2}}\left(2 \Phi_{j}^{n+1}-5 \Phi_{j}^{n}+4 \Phi_{j}^{n-1}-\Phi_{j}^{n-2}\right)\right. \\
& \left.-\mathcal{E} \frac{1}{36 \Delta \mathcal{T}^{2}}\left(11 \Theta^{n+1}-18 \Theta^{n}+9 \Theta^{n-1}-2 \Theta^{n-2}\right)^{2} \Phi_{j}\right)+C_{w 5}^{i j} K_{\lambda 1} \Phi_{j}
\end{aligned}
$$

end

end

for $n=1: L+M+N+1$

for $i=1: M$

$$
\begin{aligned}
& \text { if } n=L+M+N+1 \\
& \mathbf{J}(L+i, n)=C_{w 9}^{i}\left(\frac{2}{\Delta \mathcal{T}^{2}}\left(\mathcal{E}+\mathcal{E}_{P}\right)\right)
\end{aligned}
$$

else

$$
\mathbf{J}(L+i, n)=0
$$

end

for $j=1: L$

$$
\begin{aligned}
& \text { if } n=j \\
& \begin{aligned}
\mathbf{J}(L+i, n)=\mathbf{J}(L+i, n)+C_{w 8}^{i j}\left(\frac{1}{\Delta \mathcal{T}^{2}}\left(2 \Theta^{n+1}-5 \Theta^{n}+4 \Theta^{n-1}-\Theta^{n-2}\right)\right. \\
\left.\quad+\frac{22}{36 \Delta \mathcal{T}^{2}}\left(11 \Theta^{n+1}-18 \Theta^{n}+9 \Theta^{n-1}-2 \Theta^{n-2}\right)\right)
\end{aligned}
\end{aligned}
$$

else if $n=L+M+N+1$

$$
\begin{aligned}
\mathbf{J}(L+i, n)=\mathbf{J}(L+ & +i, n)+C_{w 8}^{i j}\left(\frac{1}{\Delta \mathcal{T}^{2}}\left(2 \Theta^{n+1}-5 \Theta^{n}+4 \Theta^{n-1}-\Theta^{n-2}\right) V_{j}\right. \\
& \left.+\frac{2}{36 \Delta \mathcal{T}^{2}}\left(11 \Theta^{n+1}-18 \Theta^{n}+9 \Theta^{n-1}-2 \Theta^{n-2}\right)\left(11 V_{j}^{n+1}-18 V_{j}^{n}+9 V_{j}^{n-1}-2 V_{j}^{n-2}\right)\right)
\end{aligned}
$$

for $k=1: N$ 
if $n=j$

$\mathbf{J}(L+i, n)=\mathbf{J}(L+i, n)+\left(C_{w 2}^{i j k}+2 C_{w 3}^{i j k}+C_{w 4}^{i j k}\right)\left(\mathcal{K}_{z}-\mathcal{K}_{y}-\mathcal{E}_{A}^{2}\right) \Phi_{k}$

else if $n=L+M+k$

$\mathbf{J}(L+i, n)=\mathbf{J}(L+i, n)+\left(C_{w 2}^{i j k}+2 C_{w 3}^{i j k}+C_{w 4}^{i j k}\right)\left(\mathcal{K}_{z}-\mathcal{K}_{y}-\mathcal{E}_{A}^{2}\right) V_{j}$

end

end

end

for $j=1: M$

if $n=L+j$

$\mathbf{J}(L+i, n)=\mathbf{J}(L+i, n)+C_{w 1}^{i j} \mathcal{K}_{y}+C_{w 6}^{i j}\left(\frac{2}{\Delta \mathcal{T}^{2}}-\frac{1}{36 \Delta \mathcal{T}^{2}}\left(11 \Theta^{n+1}-18 \Theta^{n}+9 \Theta^{n-1}-2 \Theta^{n-2}\right)^{2}\right)$

else if $n=L+M+N+1$

$\mathbf{J}(L+i, n)=\mathbf{J}(L+i, n)+C_{w 6}^{i j}\left(\frac{1}{\Delta \mathcal{T}^{2}}\left(2 W_{j}^{n+1}-5 W_{j}^{n}+4 W_{j}^{n-1}-W_{j}^{n-2}\right)\right.$

$$
\left.-\frac{22}{36 \Delta \mathcal{T}^{2}}\left(11 \Theta^{n+1}-18 \Theta^{n}+9 \Theta^{n-1}-2 \Theta^{n-2}\right) W_{j}\right)
$$

end

end

for $j=1: N$

if $n=L+M+j$

$\mathbf{J}(L+i, n)=\mathbf{J}(L+i, n)+C_{w 7}^{i j}\left(\mathcal{E} \frac{2}{\Delta \mathcal{T}^{2}}-\mathcal{E} \frac{1}{36 \Delta \mathcal{T}^{2}}\left(11 \Theta^{n+1}-18 \Theta^{n}+9 \Theta^{n-1}-2 \Theta^{n-2}\right)^{2}\right)+C_{w 5}^{i j} K_{\lambda 1}$

else if $n=L+M+N+1$

$\mathbf{J}(L+i, n)=\mathbf{J}(L+i, n)+C_{w 7}^{i j}\left(-\mathcal{E} \frac{22}{36 \Delta \mathcal{T}^{2}}\left(11 \Theta^{n+1}-18 \Theta^{n}+9 \Theta^{n-1}-2 \Theta^{n-2}\right) \Phi_{j}\right)$

end

end

end

end 


$$
\begin{aligned}
& \delta \phi \\
& -\mathcal{K}_{x}\left\{C_{p 1}^{i j} \Phi_{j}\right\}+\left(\mathcal{K}_{z}-\mathcal{K}_{y}\right)\left\{C_{p 2}^{i j k} V_{j} W_{k}\right\}-\mathcal{E}_{A}^{2}\left\{C_{p 2}^{i j k l} V_{j} W_{k}\right\}+\mathcal{K}_{\lambda 1}\left\{C_{p 3}^{i j} \Phi_{j}\right\}+\mathcal{K}_{\lambda 2}\left\{C_{p 4}^{i j} W_{j}\right\} \\
& +\mathcal{M}_{0}\left\{C_{p 5}^{i j} \ddot{\Phi}+C_{p 6}^{i} \ddot{\Theta}\right\}+\mathcal{E} C_{p 7}^{i j} \ddot{W}_{j}+\ddot{\Theta}\left\{\mathcal{E} C_{p 8}^{i j} V_{j}+\mathcal{E} \mathcal{E}_{p} C_{p 6}^{i}\right\}-\mathcal{E}^{2}\left\{C_{p 7}^{i j} W_{j}\right\}+2 \mathcal{E} \dot{\Theta}\left\{C_{p 8}^{i j} \dot{V}_{j}\right\}=\mathcal{W}_{\phi} \\
& -\mathcal{K}_{x}\left\{\sum_{i=1}^{N} \sum_{j=1}^{N} C_{p 1}^{i j} \Phi_{j}\right\}+\left(\mathcal{K}_{z}-\mathcal{K}_{y}\right)\left\{\sum_{i=1}^{N} \sum_{j=1}^{L} \sum_{k=1}^{M} C_{p 2}^{i j k} V_{j} W_{k}\right\}-\mathcal{E}_{A}^{2}\left\{\sum_{i=1}^{N} \sum_{j=1}^{L} \sum_{k=1}^{M} C_{p 2}^{i j k l} V_{j} W_{k}\right\} \\
& +\mathcal{K}_{\lambda 1}\left\{\sum_{i=1}^{N} \sum_{j=1}^{N} C_{p 3}^{i j} \Phi_{j}\right\}+\mathcal{K}_{\lambda 2}\left\{\sum_{i=1}^{N} \sum_{j=1}^{M} C_{p 4}^{i j} W_{j}\right\}+\mathcal{M}_{0}\left\{\sum_{i=1}^{N} \sum_{j=1}^{N} C_{p 5}^{i j} \ddot{\Phi}+\sum_{i=1}^{N} C_{p 6}^{i} \ddot{\Theta}\right\}+\mathcal{E} \sum_{i=1}^{N} \sum_{j=1}^{M} C_{p 7}^{i j} \ddot{W}_{j} \\
& +\ddot{\Theta}\left\{\mathcal{E} \sum_{i=1}^{N} \sum_{j=1}^{L} C_{p 8}^{i j} V_{j}+\mathcal{E} \mathcal{E}_{p} \sum_{i=1}^{N} C_{p 6}^{i}\right\}-\mathcal{E} \dot{\Theta}^{2}\left\{\sum_{i=1}^{N} \sum_{j=1}^{M} C_{p 7}^{i j} W_{j}\right\}+2 \mathcal{E} \dot{\Theta}\left\{\sum_{i=1}^{N} \sum_{j=1}^{L} C_{p 8}^{i j} \dot{V}_{j}\right\}=\mathcal{W}_{\phi} \\
& \sum_{i=1}^{N}\left[C_{p 6}^{i}\left(\mathcal{M}_{0}+\mathcal{E} \mathcal{E}_{P}\right) \ddot{\Theta}\right. \\
& +\sum_{j=1}^{L}\left[C_{p 8}^{i j}\left(\mathcal{E} \ddot{\Theta} V_{j}+2 \dot{\Theta} \dot{V}_{j}\right)\right. \\
& \left.+\sum_{k=1}^{M}\left[C_{p 2}^{i j k}\left(\mathcal{K}_{z}-\mathcal{K}_{y}-\mathcal{E}_{A}^{2}\right) V_{j} W_{k}\right]\right] \\
& +\sum_{j=1}^{M}\left[C_{p 4}^{i j} \mathcal{K}_{\lambda 2} W_{j}+\mathcal{E} C_{p 7}^{i j}\left(\ddot{W}_{j}-\dot{\Theta}^{2} W_{j}\right)\right] \\
& \left.+\sum_{j=1}^{N}\left[\left(-C_{p 1}^{i j} \mathcal{K}_{x}+C_{p 3}^{i j} \mathcal{K}_{\lambda 1}\right) \Phi_{j}+\mathcal{M}_{0} C_{p 5}^{i j} \ddot{\Phi}_{j}\right]\right]=\mathcal{W}_{\phi}
\end{aligned}
$$

for $i=1: N$

$$
\mathbf{f}(L+M+i)=C_{p 6}^{i}\left(\mathcal{M}_{0}+\mathcal{E} \mathcal{E}_{P}\right) \frac{1}{\Delta \mathcal{T}^{2}}\left(2 \Theta^{n+1}-5 \Theta^{n}+4 \Theta^{n-1}-\Theta^{n-2}\right)
$$$$
\text { for } j=1: L
$$$$
\begin{aligned}
\mathbf{f}(L+M+i)=\mathbf{f}(L+ & M+i)+C_{p 8}^{i j}\left(\mathcal{E} \frac{1}{\Delta \mathcal{T}^{2}}\left(2 \Theta^{n+1}-5 \Theta^{n}+4 \Theta^{n-1}-\Theta^{n-2}\right) V_{j}\right. \\
& \left.+\frac{2}{36 \Delta \mathcal{T}^{2}}\left(11 \Theta^{n+1}-18 \Theta^{n}+9 \Theta^{n-1}-2 \Theta^{n-2}\right)\left(11 V_{j}^{n+1}-18 V_{j}^{n}+9 V_{j}^{n-1}-2 V_{j}^{n-2}\right)\right)
\end{aligned}
$$

for $k=1: M$

$\mathbf{f}(L+M+i)=\mathbf{f}(L+M+i)+C_{p 2}^{i j k}\left(\mathcal{K}_{z}-\mathcal{K}_{y}-\mathcal{E}_{A}^{2}\right) V_{j} W_{k}$

end

end 
for $j=1: M$

$$
\begin{gathered}
\mathbf{f}(L+M+i)=\mathbf{f}(L+M+i)+C_{p 4}^{i j} \mathcal{K}_{\lambda 2} W_{j}+\mathcal{E} C_{p 6}^{i j}\left(\frac{1}{\Delta \mathcal{T}^{2}}\left(2 W_{j}^{n+1}-5 W_{j}^{n}+4 W_{j}^{n-1}-W_{j}^{n-2}\right)\right. \\
\left.-\frac{1}{36 \Delta \mathcal{T}^{2}}\left(11 \Theta^{n+1}-18 \Theta^{n}+9 \Theta^{n-1}-2 \Theta^{n-2}\right)^{2} W_{j}\right)
\end{gathered}
$$

end

for $j=1: N$

$\mathbf{f}(L+M+i)=\mathbf{f}(L+M+i)\left(-C_{p 1}^{i j} \mathcal{K}_{x}+C_{p 3}^{i j} \mathcal{K}_{\lambda 1}\right) \Phi_{j}+C_{p 5}^{i j} \mathcal{M}_{0} \frac{1}{\Delta \mathcal{T}^{2}}\left(2 \Phi_{j}^{n+1}-5 \Phi_{j}^{n}+4 \Phi_{j}^{n-1}-\Phi_{j}^{n-2}\right)$

end

end

for $n=1: L+M+N+1$

for $i=1: N$

if $n=L+M+N+1$

$\mathbf{J}(L+M+i, n)=C_{p 6}^{i}\left(\mathcal{M}_{0}+\mathcal{E} \mathcal{E}_{P}\right) \frac{2}{\Delta \mathcal{T}^{2}}$

else

$\mathbf{J}(L+M+i, n)=0$

end

for $j=1: L$

$$
\begin{aligned}
& \text { if } n=j \\
& \begin{aligned}
\mathbf{J}(L+M+i, n)=\mathbf{J}(L+M+i, n)+C_{p 8}^{i j} & \left(\mathcal{E} \frac{1}{\Delta \mathcal{T}^{2}}\left(2 \Theta^{n+1}-5 \Theta^{n}+4 \Theta^{n-1}-\Theta^{n-2}\right)\right. \\
& \left.+\frac{22}{36 \Delta \mathcal{T}^{2}}\left(11 \Theta^{n+1}-18 \Theta^{n}+9 \Theta^{n-1}-2 \Theta^{n-2}\right)\right)
\end{aligned}
\end{aligned}
$$

else if $n=L+M+N+1$

$\mathbf{J}(L+M+i, n)=\mathbf{J}(L+M+i, n)+C_{p 8}^{i j}\left(\mathcal{E} \frac{2}{\Delta \mathcal{T}^{2}} V_{j}+\frac{22}{36 \Delta \mathcal{T}^{2}}\left(11 V_{j}^{n+1}-18 V_{j}^{n}+9 V_{j}^{n-1}-2 V_{j}^{n-2}\right)\right)$

end

for $k=1: M$

if $n=j$

$\mathbf{J}(L+M+i, n)=\mathbf{J}(L+M+i, n)+C_{p 2}^{i j k}\left(\mathcal{K}_{z}-\mathcal{K}_{y}-\mathcal{E}_{A}^{2}\right) W_{k}$

else if $n=L+k$

$\mathbf{J}(L+M+i, n)=\mathbf{J}(L+M+i, n)+C_{p 2}^{i j k}\left(\mathcal{K}_{z}-\mathcal{K}_{y}-\mathcal{E}_{A}^{2}\right) V_{j}$ 
end

end

end

for $j=1: M$

else if $n=L+j$

$\mathbf{J}(L+M+i, n)=\mathbf{J}(L+M+i, n)+C_{p 4}^{i j} \mathcal{K}_{\lambda 2}+\mathcal{E} C_{p 6}^{i j}\left(\frac{1}{\Delta \mathcal{T}^{2}}-\frac{1}{36 \mathcal{T}^{2}}\left(11 \Theta^{n+1}-18 \Theta^{n}+9 \Theta^{n-1}-2 \Theta^{n-2}\right)^{2}\right)$

else if $n=L+M+N+1$

$\mathbf{J}(L+M+i, n)=\mathbf{J}(L+M+i, n)+\mathcal{E} C_{p 6}^{i j}\left(-\frac{22}{36 \Delta \mathcal{T}^{2}}\left(11 \Theta^{n+1}-18 \Theta^{n}+9 \Theta^{n-1}-2 \Theta^{n-2}\right) W_{j}\right)$

end

end

for $j=1: N$

if $n=L+M+j$

$\mathbf{J}(L+M+i, n)=\mathbf{J}(L+M+i, n)\left(-C_{p 1}^{i j} \mathcal{K}_{x}+C_{p 3}^{i j} \mathcal{K}_{\lambda 1}\right) \Phi_{j}+C_{p 5}^{i j} \mathcal{M}_{0} \frac{2}{\Delta \mathcal{T}^{2}}$

end

end

end

end 
$\delta \theta$

$$
\begin{aligned}
\mathcal{M}_{\theta} & C_{t 1} \ddot{\Theta}+\mathcal{D}_{\theta} C_{t 1} \dot{\Theta}+\mathcal{K}_{\vartheta} C_{t 1} \Theta+\mathcal{M}_{0}\left\{C_{t 2}^{j} \ddot{\Phi}_{j}+C_{t 1} \ddot{\Theta}\right\}-\mathcal{E} C_{t 3}^{j k} \ddot{V}_{j} \Phi_{k}-C_{t 4}^{j k} \ddot{V}_{j} W_{k} \\
& +C_{t 3}^{j k} \ddot{W}_{j} V_{k}+\mathcal{E}_{p} C_{t 5}^{j} \ddot{W}_{j}+\mathcal{E} C_{t 5}^{j} \ddot{W}_{j}+\mathcal{E} C_{t 3}^{j k} \ddot{\Phi}_{j} V_{k}+\mathcal{E} \mathcal{E}_{p} C_{t 2}^{j} \ddot{\Phi}_{j} \\
& +\ddot{\Theta}\left\{2 \mathcal{E} C_{t 6}^{j} V_{j}+2 \mathcal{E} \mathcal{E}_{p} C_{t 1}+2 \mathcal{E} C_{t 7}^{j k} W_{j} \Phi_{k}+C_{t 8}^{j k} W_{j} W_{k}+C_{t 9}^{j k} V_{j} V_{k}+2 \mathcal{E}_{p} C_{t 6}^{j} V_{j}+\mathcal{E}_{p}{ }^{2} C_{t 1}\right\} \\
& +2 \dot{\Theta}\left\{C_{t 9}^{j k} V_{k} \dot{V}_{j}+\mathcal{E}_{p} C_{t 6}^{j} \dot{V}_{j}+\mathcal{E} C_{t 6}^{j} \dot{V}_{j}+\mathcal{E} C_{t 7}^{j k} \dot{W}_{j} \Phi_{k}+C_{t 8}^{j k} \dot{W}_{j} W_{k}+\mathcal{E} C_{t 7}^{j k} W_{j} \dot{\Phi}_{k}\right\}=\mathcal{W}_{\theta}
\end{aligned}
$$

$$
\begin{aligned}
\mathcal{M}_{\theta} C_{t 1} \ddot{\Theta}+ & \mathcal{D}_{\theta} C_{t 1} \dot{\Theta}+\mathcal{K}_{\vartheta} C_{t 1} \Theta+\mathcal{M}_{0}\left\{\sum_{j=1}^{N} C_{t 2}^{j} \ddot{\Phi}_{j}+C_{t 1} \ddot{\Theta}\right\}-\mathcal{E} \sum_{j=1}^{L} \sum_{k=1}^{N} C_{t 3}^{j k} \ddot{V}_{j} \Phi_{k}-\sum_{j=1}^{L} \sum_{k=1}^{M} C_{t 4}^{j k} \ddot{V}_{j} W_{k} \\
+ & \sum_{j=1}^{L} \sum_{j=1}^{M} C_{t 3}^{j k} V_{j} \ddot{W}_{k}+\mathcal{E}_{p} \sum_{j=1}^{M} C_{t 5}^{j} \ddot{W}_{j}+\mathcal{E} \sum_{j=1}^{M} C_{t 5}^{j} \ddot{W}_{j}+\mathcal{E} \sum_{j=1}^{L} \sum_{k=1}^{N} C_{t 3}^{j k} V_{j} \ddot{\Phi}_{k}+\mathcal{E} \mathcal{E}_{p} \sum_{j=1}^{N} C_{t 2}^{j} \ddot{\Phi}_{j} \\
+ & \ddot{\Theta}\left\{2 \mathcal{E} \sum_{j=1}^{L} C_{t 6}^{j} V_{j}+2 \mathcal{E} \mathcal{E}_{p} C_{t 1}+2 \mathcal{E} \sum_{j=1}^{M} \sum_{k=1}^{N} C_{t 7}^{j k} W_{j} \Phi_{k}+\sum_{j=1}^{M} \sum_{k=1}^{M} C_{t 8}^{j k} W_{j} W_{k}+\sum_{j=1}^{L} \sum_{k=1}^{L} C_{t 9}^{j k} V_{j} V_{k}\right. \\
& \left.+2 \mathcal{E}_{p} \sum_{j=1}^{L} C_{t 6}^{j} V_{j}+\mathcal{E}_{p}{ }^{2} C_{t 1}\right\} \\
+ & \dot{\Theta}\left\{\sum_{j=1}^{L} \sum_{k=1}^{L} C_{t 9}^{j k} V_{k} \dot{V}_{j}+\mathcal{E}_{p} \sum_{j=1}^{L} C_{t 6}^{j} \dot{V}_{j}+\mathcal{E} \sum_{j=1}^{L} C_{t 6}^{j} \dot{V}_{j}+\mathcal{E} \sum_{j=1}^{M} \sum_{k=1}^{N} C_{t 7}^{j k} \dot{W}_{j} \Phi_{k}+\sum_{j=1}^{M} \sum_{k=1}^{M} C_{t 8}^{j k} \dot{W}_{j} W_{k}\right. \\
& \left.+\mathcal{E} \sum_{j=1}^{M} \sum_{k=1}^{N} C_{t 7}^{j k} W_{j} \dot{\Phi}_{k}\right\}=\mathcal{W}_{\theta}
\end{aligned}
$$$$
C_{t 1}\left(\mathcal{K}_{\vartheta} \Theta+\ddot{\Theta}\left(\mathcal{M}_{0}+\mathcal{M}_{b}+2 \mathcal{E}_{P}+\mathcal{E}_{P}{ }^{2}\right)\right)
$$$$
+\sum_{j=1}^{L}\left[2 C_{t 6}^{j}\left(\mathcal{E}+\mathcal{E}_{P}\right)\left(\ddot{\Theta} V_{j}+\dot{\Theta} \dot{V}_{j}\right)\right.
$$$$
+\sum_{k=1}^{L}\left[C_{t 9}^{j k}\left(\ddot{\Theta} V_{j} V_{k}+2 \dot{\Theta} \dot{V}_{j} V_{k}\right)\right]
$$$$
+\sum_{k=1}^{M}\left[C_{t 4}^{j k}\left(-\ddot{V}_{j} W_{k}+V_{j} \ddot{W}_{k}\right)\right]
$$$$
\left.+\sum_{k=1}^{N}\left[C_{t 3}^{j k}\left(-\mathcal{E} \ddot{V}_{j} \Phi_{k}+\mathcal{E} V_{j} \ddot{\Phi}_{k}\right)\right]\right]
$$$$
+\sum_{j=1}^{M}\left[C_{t 5}^{j}\left(\mathcal{E}+\mathcal{E}_{P}\right) \ddot{W}_{j}\right.
$$$$
+\sum_{k=1}^{M}\left[C_{t 8}^{j k}\left(\ddot{\Theta} W_{j} W_{k}+2 \dot{\Theta} \dot{W}_{j} W_{k}\right)\right]
$$ 


$$
\begin{aligned}
\left.+\sum_{k=1}^{N}\left[C_{t 8}^{j k}\left(2 \mathcal{E} \ddot{\Theta} W_{j} \Phi_{k}+2 \mathcal{E} \dot{\Theta}\left(\dot{W}_{j} \Phi_{k}+W_{j} \dot{\Phi}_{k}\right)\right)\right]\right] \\
+\sum_{j=1}^{N}\left[C_{t 2}^{j}\left(\mathcal{M}_{0}+\mathcal{E} \mathcal{E}_{P}\right) \ddot{\Phi}_{j}\right]=\mathcal{W}_{\theta}
\end{aligned}
$$

$$
\mathbf{f}(L+M+N+1)=C_{t 1}\left(\mathcal{K}_{\vartheta} \Theta+\frac{1}{\Delta \mathcal{T}^{2}}\left(2 \Theta^{n+1}-5 \Theta^{n}+4 \Theta^{n-1}-\Theta^{n-2}\right)\left(\mathcal{M}_{0}+\mathcal{M}_{b}+2 \mathcal{E} \mathcal{E}_{P}+\mathcal{E}_{P}{ }^{2}\right)\right)
$$

for $j=1: L$

$$
\begin{aligned}
\mathbf{f}(L+M+N+1)= & \mathbf{f}(L+M+N+1)+2 C_{t 6}^{j}\left(\mathcal{E}+\mathcal{E}_{P}\right)\left(\frac{1}{\Delta \mathcal{T}^{2}}\left(2 \Theta^{n+1}-5 \Theta^{n}+4 \Theta^{n-1}-\Theta^{n-2}\right) V_{j}\right. \\
& \left.+\frac{1}{36 \Delta \mathcal{T}^{2}}\left(11 \Theta^{n+1}-18 \Theta^{n}+9 \Theta^{n-1}-2 \Theta^{n-2}\right)\left(11 V_{j}^{n+1}-18 V_{j}^{n}+9 V_{j}^{n-1}-2 V_{j}^{n-2}\right)\right)
\end{aligned}
$$

for $k=1: M$

$$
\begin{aligned}
\mathbf{f}(L+M+N+1) & =\mathbf{f}(L+M+N+1)+C_{t 9}^{j k}\left(\frac{1}{\Delta \mathcal{T}^{2}}\left(2 \Theta^{n+1}-5 \Theta^{n}+4 \Theta^{n-1}-\Theta^{n-2}\right) V_{j} V_{k}\right. \\
& \left.+\frac{2}{6 \Delta \mathcal{T}^{2}}\left(11 \Theta^{n+1}-18 \Theta^{n}+9 \Theta^{n-1}-2 \Theta^{n-2}\right)\left(11 V_{j}^{n+1}-18 V_{j}^{n}+9 V_{j}^{n-1}-2 V_{j}^{n-2}\right) V_{k}\right)
\end{aligned}
$$

end

for $k=1: M$

$$
\begin{aligned}
\mathbf{f}(L+M+N+1) & =\mathbf{f}(L+M+N+1)+C_{t 4}^{j k}\left(-\frac{1}{\Delta \mathcal{T}^{2}}\left(2 V_{j}^{n+1}-5 V_{j}^{n}+4 V_{j}^{n-1}-V_{j}^{n-2}\right) W_{k}\right. \\
& \left.+\frac{1}{\Delta \mathcal{T}^{2}} V_{j}\left(2 W_{j}^{n+1}-5 W_{j}^{n}+4 W_{j}^{n-1}-W_{j}^{n-2}\right)\right)
\end{aligned}
$$

end

for $k=1: N$

$$
\begin{aligned}
\mathbf{f}(L+M+N+1) & =\mathbf{f}(L+M+N+1)+C_{t 3}^{j k}\left(-\mathcal{E} \frac{1}{\Delta \mathcal{T}^{2}}\left(2 V_{j}^{n+1}-5 V_{j}^{n}+4 V_{j}^{n-1}-V_{j}^{n-2}\right) \Phi_{k}\right. \\
& \left.+\mathcal{E} \frac{1}{\Delta \mathcal{T}^{2}} V_{j}\left(2 \Phi_{k}^{n+1}-5 \Phi_{k}^{n}+4 \Phi_{k}^{n-1}-\Phi_{k}^{n-2}\right)\right)
\end{aligned}
$$

end

end

for $j=1: M$

$$
\begin{aligned}
& \mathbf{f}(L+M+N+1)=\mathbf{f}(L+M+N+1)+C_{t 5}^{j}\left(\mathcal{E}+\mathcal{E}_{P}\right) \frac{1}{\Delta \mathcal{T}^{2}}\left(2 W_{j}^{n+1}-5 W_{j}^{n}+4 W_{j}^{n-1}-W_{j}^{n-2}\right) \\
& \qquad \begin{array}{r}
\text { for } k=1: M \\
\mathbf{f}(L+M+N+1)=\mathbf{f}(L+M+N+1)+C_{t 8}^{j k}\left(\frac{1}{\Delta \mathcal{T}^{2}}\left(2 \Theta^{n+1}-5 \Theta^{n}+4 \Theta^{n-1}-\Theta^{n-2}\right) W_{j} W_{k}\right. \\
\left.\quad+\frac{2}{36 \Delta \mathcal{T}^{2}}\left(11 \Theta^{n+1}-18 \Theta^{n}+9 \Theta^{n-1}-2 \Theta^{n-2}\right)\left(11 W_{j}^{n+1}-18 W_{j}^{n}+9 W_{j}^{n-1}-2 W_{j}^{n-2}\right) W_{k}\right)
\end{array}
\end{aligned}
$$

end 
for $k=1: N$

$$
\begin{aligned}
\mathbf{f}(L+M+N+1)=\mathbf{f}(L+M+N+1)+C_{t 7}^{j k}\left(2 \mathcal{E} \frac{1}{\Delta \mathcal{T}^{2}}\left(2 \Theta^{n+1}-5 \Theta^{n}+4 \Theta^{n-1}-\Theta^{n-2}\right) W_{j} \Phi_{k}\right. \\
+2 \mathcal{E} \frac{1}{6 \Delta \mathcal{T}}\left(11 \Theta^{n+1}-18 \Theta^{n}+9 \Theta^{n-1}-2 \Theta^{n-2}\right) \\
\quad \times\left(\frac{1}{6 \Delta \mathcal{T}}\left(11 W_{j}^{n+1}-18 W_{j}^{n}+9 W_{j}^{n-1}-2 W_{j}^{n-2}\right) \Phi_{k}\right. \\
\left.\left.\quad+W_{j} \frac{1}{6 \Delta \mathcal{T}}\left(11 \Phi_{k}^{n+1}-18 \Phi_{k}^{n}+9 \Phi_{k}^{n-1}-2 \Phi_{k}^{n-2}\right)\right)\right)
\end{aligned}
$$

end

end

for $j=1: N$

$\mathbf{f}(L+M+N+1)=\mathbf{f}(L+M+N+1)+C_{t 2}^{j}\left(\mathcal{M}_{0}+\mathcal{E} \mathcal{E}_{P}\right) \frac{1}{\Delta \mathcal{T}^{2}}\left(2 \Phi_{j}^{n+1}-5 \Phi_{j}^{n}+4 \Phi_{j}^{n-1}-\Phi_{j}^{n-2}\right)$

end

for $j=1: L+M+N+1$

if $n=L+M+N+1$

$\mathbf{J}(L+M+N+1, n)=C_{t 1}\left(\mathcal{K}_{\vartheta}+\frac{2}{\Delta \mathcal{T}^{2}}\left(\mathcal{M}_{0}+\mathcal{M}_{b}+2 \mathcal{E E}_{P}+\mathcal{E}_{P}{ }^{2}\right)\right)$

else

$\mathbf{J}(L+M+N+1, n)=0$

end

for $j=1: L$

if $n=j$

$$
\begin{gathered}
\mathbf{J}(L+M+N+1, n)=\mathbf{J}(L+M+N+1, n)+2 C_{t 6}^{j}\left(\mathcal{E}+\mathcal{E}_{P}\right)\left(\frac{1}{\Delta \mathcal{T}^{2}}\left(2 \Theta^{n+1}-5 \Theta^{n}+4 \Theta^{n-1}-\Theta^{n-2}\right)\right. \\
\left.+\frac{11}{36 \Delta \mathcal{T}^{2}}\left(11 \Theta^{n+1}-18 \Theta^{n}+9 \Theta^{n-1}-2 \Theta^{n-2}\right)\right)
\end{gathered}
$$

else if $n=L+M+N+1$

$$
\begin{aligned}
\mathbf{J}(L+M+N+1, n)= & \mathbf{J}(L+M+N+1, n)+2 C_{t 6}^{j}\left(\mathcal{E}+\mathcal{E}_{P}\right)\left(\frac{2}{\Delta \mathcal{T}^{2}} V_{j}\right. \\
+ & \left.\frac{11}{36 \Delta \mathcal{T}^{2}}\left(11 V_{j}^{n+1}-18 V_{j}^{n}+9 V_{j}^{n-1}-2 V_{j}^{n-2}\right)\right)
\end{aligned}
$$

end

for $k=1: M$

$$
\begin{aligned}
& \text { if } n=j=k \\
& \mathbf{J}(L+M+N+1, n)=\mathbf{J}(L+M+N+1, n)+C_{t 9}^{j k}\left(\frac{1}{\Delta \mathcal{T}^{2}}\left(2 \Theta^{n+1}-5 \Theta^{n}+4 \Theta^{n-1}-\Theta^{n-2}\right) 2 V_{j}\right.
\end{aligned}
$$




$$
\left.+\frac{2}{6 \Delta \mathcal{T}^{2}}\left(22 \Theta^{n+1}-18 \Theta^{n}+9 \Theta^{n-1}-2 \Theta^{n-2}\right)\left(11 V_{j}^{n+1}-18 V_{j}^{n}+9 V_{j}^{n-1}-2 V_{j}^{n-2}\right)\right)
$$

else if $n=j$

$$
\begin{gathered}
\mathbf{J}(L+M+N+1, n)=\mathbf{J}(L+M+N+1, n)+C_{t 9}^{j k}\left(\frac{1}{\Delta \mathcal{T}^{2}}\left(2 \Theta^{n+1}-5 \Theta^{n}+4 \Theta^{n-1}-\Theta^{n-2}\right) V_{k}\right. \\
\left.+\frac{22}{6 \Delta \mathcal{T}^{2}}\left(11 \Theta^{n+1}-18 \Theta^{n}+9 \Theta^{n-1}-2 \Theta^{n-2}\right) V_{k}\right)
\end{gathered}
$$

else if $n=k$

$$
\begin{aligned}
\mathbf{J}(L+M+N & +1, n)=\mathbf{J}(L+M+N+1, n)+C_{t 9}^{j k}\left(\frac{1}{\Delta \mathcal{T}^{2}}\left(2 \Theta^{n+1}-5 \Theta^{n}+4 \Theta^{n-1}-\Theta^{n-2}\right) V_{j}\right. \\
& \left.+\frac{2}{6 \Delta \mathcal{T}^{2}}\left(11 \Theta^{n+1}-18 \Theta^{n}+9 \Theta^{n-1}-2 \Theta^{n-2}\right)\left(11 V_{j}^{n+1}-18 V_{j}^{n}+9 V_{j}^{n-1}-2 V_{j}^{n-2}\right)\right)
\end{aligned}
$$

else if $n=L+M+N+1$

$$
\begin{array}{r}
\mathbf{J}(L+M+N+1, n)=\mathbf{J}(L+M+N+1, n)+C_{t 9}^{j k}\left(\frac{2}{\Delta \mathcal{T}^{2}} V_{j} V_{k}\right. \\
\left.+\frac{22}{36 \Delta \mathcal{T}^{2}}\left(11 V_{j}^{n+1}-18 V_{j}^{n}+9 V_{j}^{n-1}-2 V_{j}^{n-2}\right) V_{k}\right)
\end{array}
$$

end

end

for $k=1: M$

if $n=j$

$$
\begin{gathered}
\mathbf{J}(L+M+N+1, n)=\mathbf{J}(L+M+N+1, n)+C_{t 4}^{j k}\left(-\frac{2}{\Delta \mathcal{T}^{2}} W_{k}\right. \\
\left.+\frac{1}{\Delta \mathcal{T}^{2}}\left(2 W_{j}^{n+1}-5 W_{j}^{n}+4 W_{j}^{n-1}-W_{j}^{n-2}\right)\right)
\end{gathered}
$$

else if $n=L+k$

$\mathbf{J}(L+M+N+1, n)=\mathbf{J}(L+M+N+1, n)+C_{t 4}^{j k}\left(-\frac{1}{\Delta \mathcal{T}^{2}}\left(2 V_{j}^{n+1}-5 V_{j}^{n}+4 V_{j}^{n-1}-V_{j}^{n-2}\right)+\frac{2}{\Delta \mathcal{T}^{2}} V_{j}\right)$

end

end

for $k=1: N$

if $n=j$

$$
\begin{aligned}
\mathbf{J}(L+M+N & +1, n)=\mathbf{J}(L+M+N+1, n)+C_{t 3}^{j k}\left(-\mathcal{E} \frac{2}{\Delta \mathcal{T}^{2}} \Phi_{k}\right. \\
& \left.+\mathcal{E} \frac{1}{\Delta \mathcal{T}^{2}}\left(2 \Phi_{k}^{n+1}-5 \Phi_{k}^{n}+4 \Phi_{k}^{n-1}-\Phi_{k}^{n-2}\right)\right)
\end{aligned}
$$

else if $n=L+M+k$

$$
\begin{aligned}
\mathbf{J}(L+M+N & +1, n)=\mathbf{J}(L+M+N+1, n)+C_{t 3}^{j k}\left(-\mathcal{E} \frac{1}{\Delta \mathcal{T}^{2}}\left(2 V_{j}^{n+1}-5 V_{j}^{n}+4 V_{j}^{n-1}-V_{j}^{n-2}\right)\right. \\
& \left.+\mathcal{E} \frac{2}{\Delta \mathcal{T}^{2}} V_{j}\right)
\end{aligned}
$$


end

end

end

for $j=1: M$

if $n=L+j$

$\mathbf{J}(L+M+N+1, n)=\mathbf{J}(L+M+N+1, n)+C_{t 5}^{j}\left(\mathcal{E}+\mathcal{E}_{P}\right) \frac{2}{\Delta \mathcal{T}^{2}}$

end

for $k=1: M$

if $n=j=k$

$\mathbf{J}(L+M+N+1, n)=\mathbf{J}(L+M+N+1, n)+C_{t 8}^{j k}\left(\frac{1}{\Delta \mathcal{T}^{2}}\left(2 \Theta^{n+1}-5 \Theta^{n}+4 \Theta^{n-1}-\Theta^{n-2}\right) 2 W_{j}\right.$

$\left.+\frac{2}{36 \Delta \mathcal{T}^{2}}\left(11 \Theta^{n+1}-18 \Theta^{n}+9 \Theta^{n-1}-2 \Theta^{n-2}\right)\left(22 W_{j}^{n+1}-18 W_{j}^{n}+9 W_{j}^{n-1}-2 W_{j}^{n-2}\right) W_{k}\right)$

else if $n=j$

$$
\begin{aligned}
\mathbf{J}(L+M+N & +1, n)=\mathbf{J}(L+M+N+1, n)+C_{t 8}^{j k}\left(\frac{1}{\Delta \mathcal{T}^{2}}\left(2 \Theta^{n+1}-5 \Theta^{n}+4 \Theta^{n-1}-\Theta^{n-2}\right) W_{k}\right. \\
& \left.+\frac{22}{36 \Delta \mathcal{T}^{2}}\left(11 \Theta^{n+1}-18 \Theta^{n}+9 \Theta^{n-1}-2 \Theta^{n-2}\right) W_{k}\right)
\end{aligned}
$$

else if $n=k$

$$
\begin{aligned}
\mathbf{J}(L+M+N & +1, n)=\mathbf{J}(L+M+N+1, n)+C_{t 8}^{j k}\left(\frac{1}{\Delta \mathcal{T}^{2}}\left(2 \Theta^{n+1}-5 \Theta^{n}+4 \Theta^{n-1}-\Theta^{n-2}\right) W_{j}\right. \\
& \left.+\frac{2}{36 \Delta \mathcal{T}^{2}}\left(11 \Theta^{n+1}-18 \Theta^{n}+9 \Theta^{n-1}-2 \Theta^{n-2}\right)\left(11 W_{j}^{n+1}-18 W_{j}^{n}+9 W_{j}^{n-1}-2 W_{j}^{n-2}\right)\right)
\end{aligned}
$$

else if $n=L+M+N+1$

$$
\begin{aligned}
\mathbf{J}(L+M+N & +1, n)=\mathbf{J}(L+M+N+1, n)+C_{t 8}^{j k}\left(\frac{2}{\Delta \mathcal{T}^{2}} W_{j} W_{k}\right. \\
& \left.+\frac{22}{36 \Delta \mathcal{T}^{2}}\left(11 W_{j}^{n+1}-18 W_{j}^{n}+9 W_{j}^{n-1}-2 W_{j}^{n-2}\right) W_{k}\right)
\end{aligned}
$$

end

end

for $k=1: N$

if $n=L+j$

$$
\begin{aligned}
\mathbf{J}(L+M+N & +1, n)=\mathbf{J}(L+M+N+1, n)+C_{t 7}^{j k}\left(2 \mathcal{E} \frac{1}{\Delta \mathcal{T}^{2}}\left(2 \Theta^{n+1}-5 \Theta^{n}+4 \Theta^{n-1}-\Theta^{n-2}\right) \Phi_{k}\right. \\
& +2 \mathcal{E} \frac{1}{6 \Delta \mathcal{T}}\left(11 \Theta^{n+1}-18 \Theta^{n}+9 \Theta^{n-1}-2 \Theta^{n-2}\right)
\end{aligned}
$$




$$
\left.\times\left(\frac{11}{6 \Delta \mathcal{T}} \Phi_{k}+\frac{1}{6 \Delta \mathcal{T}}\left(11 \Phi_{j}^{n+1}-18 \Phi_{j}^{n}+9 \Phi_{j}^{n-1}-2 \Phi_{j}^{n-2}\right)\right)\right)
$$

else if $n=L+M+k$

$$
\begin{aligned}
\mathbf{J}(L+M+N+1, n)=\mathbf{J}(L+M+N+1, n)+C_{t 7}^{j k}\left(2 \mathcal{E} \frac{1}{\Delta \mathcal{T}^{2}}\left(2 \Theta^{n+1}-5 \Theta^{n}+4 \Theta^{n-1}-\Theta^{n-2}\right) W_{j}\right. \\
+2 \mathcal{E} \frac{1}{6 \Delta \mathcal{T}}\left(11 \Theta^{n+1}-18 \Theta^{n}+9 \Theta^{n-1}-2 \Theta^{n-2}\right) \\
\left.\quad \times\left(\frac{1}{6 \Delta \mathcal{T}}\left(11 W_{j}^{n+1}-18 W_{j}^{n}+9 W_{j}^{n-1}-2 W_{j}^{n-2}\right)+W_{j} \frac{11}{6 \Delta \mathcal{T}}\right)\right)
\end{aligned}
$$

else if $n=L+M+N+1$

$$
\begin{aligned}
& \mathbf{J}(L+M+N+1, n)=\mathbf{J}(L+M+N+1, n)+C_{t 7}^{j k}\left(2 \mathcal{E} \frac{2}{\Delta \mathcal{T}^{2}} W_{j} \Phi_{k}\right. \\
&+\mathcal{E} \frac{22}{6 \Delta \mathcal{T}}\left(\frac{1}{6 \Delta \mathcal{T}}\left(11 W_{j}^{n+1}-18 W_{j}^{n}+9 W_{j}^{n-1}-2 W_{j}^{n-2}\right) \Phi_{k}\right. \\
&\left.\left.\quad+W_{j} \frac{1}{6 \Delta \mathcal{T}}\left(11 \Phi_{j}^{n+1}-18 \Phi_{j}^{n}+9 \Phi_{j}^{n-1}-2 \Phi_{j}^{n-2}\right)\right)\right)
\end{aligned}
$$

end

end

end

for $j=1: N$

if $n=L+M+j$

$\mathbf{J}(L+M+N+1, n)=\mathbf{J}(L+M+N+1, n)+C_{t 2}^{j}\left(\mathcal{M}_{0}+\mathcal{E} \mathcal{E}_{P}\right) \frac{2}{\Delta \mathcal{T}^{2}}$

end

end

end 


\section{B.5 Numerical Implementation: Finite Difference scheme}

As in Section B.4, the vector $\mathbf{f}$ and Jacobian matrix $\mathbf{J}$ are constructed per the below. Initially, the finite difference scheme for the underlying linear system of equations will be presented in subsection B.5.1. Subsequently, building off of the linear equations, the inertial nonlinearities will be added in subsection B.5.2, and finally the stiffness nonlinearities will be presented in subsection B.5.3 Note that the purpose of this segmentation is in order to present the discretization in a digestible manner and additionally to enable the reader to relate back to the spy plots presented in Figures $3.19 \mathrm{~b}, 3.20 \mathrm{~b}$ and $3.21 \mathrm{~b}$ which showed the sparsity structures of the Jacobian matrices presented in subsection B.5.1, B.5.2 and B.5.3 respectively.

\section{B.5.1 Linear Bending-Bending-Torsion-Pitch of a Cantilever}

The linear equations of motion for the flexible bending-torsion vibration of a beam are given by:

$\delta v$

$$
\begin{aligned}
& \left\{\mathcal{K}_{z}\left(\mathcal{V}^{\prime \prime \prime \prime}\right)+\left(\mathcal{K}_{z}-\mathcal{K}_{y}\right)\left(\mathcal{W}^{\prime \prime} \varphi^{\prime \prime}+2 \mathcal{W}^{\prime \prime \prime} \varphi^{\prime}+\mathcal{W}^{\prime \prime \prime \prime} \varphi\right)-\mathcal{E}_{A}^{2}\left(\mathcal{V}^{\prime \prime \prime \prime}+\mathcal{W}^{\prime \prime} \varphi^{\prime \prime}+2 \mathcal{W}^{\prime \prime \prime} \varphi^{\prime}+\mathcal{W}^{\prime \prime \prime \prime} \varphi\right)\right\} \\
& \quad+\left\{\ddot{\mathcal{V}}-\ddot{\vartheta}(\mathcal{E} \varphi+\mathcal{W})-\dot{\vartheta}^{2}\left(\left(\mathcal{V}+\mathcal{E}_{p}\right)+\mathcal{E}\right)-2 \dot{\vartheta}(\mathcal{E} \dot{\varphi}+\dot{\mathcal{V}})\right\}=\overline{\mathcal{W}}_{v}
\end{aligned}
$$

$\delta w$

$$
\begin{aligned}
& \left\{\mathcal{K}_{y}\left(\mathcal{W}^{\prime \prime \prime \prime}\right)+\left(\mathcal{K}_{z}-\mathcal{K}_{y}\right)\left(\mathcal{V}^{\prime \prime \prime \prime} \varphi+2 \mathcal{V}^{\prime \prime \prime} \varphi^{\prime}+\mathcal{V}^{\prime \prime} \varphi^{\prime \prime}\right)-\mathcal{E}_{A}^{2}\left(\mathcal{V}^{\prime \prime \prime \prime} \varphi+2 \mathcal{V}^{\prime \prime \prime} \varphi^{\prime}+\mathcal{V}^{\prime \prime} \varphi^{\prime \prime}\right)+\mathcal{K}_{\lambda 1}\left(\varphi^{\prime \prime \prime \prime}\right)\right\} \\
& \quad+\left\{\ddot{\mathcal{V}}+\mathcal{E} \ddot{\varphi}+\ddot{\vartheta}\left(\left(\mathcal{V}+\mathcal{E}_{p}\right)+\mathcal{E}\right)-\dot{\vartheta}^{2}(\mathcal{E} \varphi+\mathcal{W})+2 \dot{\vartheta}(\dot{\mathcal{V}})\right\}=\overline{\mathcal{W}}_{w}
\end{aligned}
$$

$\delta \phi$

$$
\begin{gathered}
\left\{-\mathcal{K}_{x}\left(\varphi^{\prime \prime}\right)+\left(\mathcal{K}_{z}-\mathcal{K}_{y}\right)\left(\mathcal{V}^{\prime \prime} \mathcal{W}^{\prime \prime}\right)-\mathcal{E}_{A}^{2}\left(\mathcal{V}^{\prime \prime} \mathcal{W}^{\prime \prime}\right)+\mathcal{K}_{\lambda 1}\left(\varphi^{\prime \prime \prime \prime}\right)+\mathcal{K}_{\lambda 2}\left(\mathcal{W}^{\prime \prime \prime \prime}\right)\right\} \\
+\left\{\mathcal{M}_{0}(\ddot{\varphi}+\ddot{\vartheta})+\mathcal{E} \ddot{\mathcal{V}}+\ddot{\vartheta}\left(\mathcal{E}\left(\mathcal{V}+\mathcal{E}_{p}\right)\right)-\dot{\vartheta}^{2}(\mathcal{E} \mathcal{W})+2 \mathcal{E} \dot{\vartheta}(\dot{\mathcal{V}})\right\}=\overline{\mathcal{W}}_{\phi}
\end{gathered}
$$




$$
\begin{aligned}
\mathcal{M}_{\theta} \ddot{\vartheta} & +\mathcal{D}_{\theta} \dot{\vartheta}+\mathcal{K}_{\vartheta} \vartheta+\int_{0}^{1}\left\{\mathcal{M}_{0}(\ddot{\varphi}+\ddot{\vartheta})-\ddot{\mathcal{V}}(\mathcal{E} \varphi+\mathcal{W})+\ddot{\mathcal{W}}\left(\left(\mathcal{V}+\mathcal{E}_{p}\right)+\mathcal{E}\right)+\mathcal{E} \ddot{\varphi}\left(\left(\mathcal{V}+\mathcal{E}_{p}\right)\right)\right. \\
& +2 \mathcal{E} \ddot{\vartheta}\left(\left(\mathcal{V}+\mathcal{E}_{p}\right)+\mathcal{W} \varphi\right)+\ddot{\vartheta}\left(\mathcal{W}^{2}+\left(\mathcal{V}^{2}+2 \mathcal{E}_{p} \mathcal{V}+\mathcal{E}_{p}{ }^{2}\right)\right) \\
& \left.+2 \dot{\vartheta}\left(\dot{\mathcal{V}}\left(\left(\mathcal{V}+\mathcal{E}_{p}\right)+\mathcal{E}\right)+\dot{\mathcal{W}}(\mathcal{E} \varphi+\mathcal{W})+\mathcal{E} \dot{\varphi}(\mathcal{W})\right)\right\} d \mathcal{X}=\overline{\mathcal{W}}_{\theta}
\end{aligned}
$$

Equations 1 to $L$ relate to Eq.(B.13); the PDE governing the edgewise bending.

$\delta v$

Fixed End Boundary Conditions $(i=1,2,3)$

$$
\begin{aligned}
& f_{1}^{n+1}=v_{1}^{n+1} \\
& f_{2}^{n+1}=\frac{\mathcal{K}_{z}-\mathcal{E}_{A}^{2}}{\Delta \mathcal{X}^{4}}\left\{\left(v_{4}^{n+1}-4 v_{3}^{n+1}+7 v_{2}^{n+1}\right)\right\}+\frac{1}{\Delta \mathcal{T}^{2}}\left\{\left(2 v_{2}^{n+1}-5 v_{2}^{n}+4 v_{2}^{n-1}-v_{2}^{n-2}\right)\right\} \\
& f_{3}^{n+1}=\frac{\mathcal{K}_{z}-\mathcal{E}_{A}^{2}}{\Delta \mathcal{X}^{4}}\left\{\left(v_{5}^{n+1}-4 v_{4}^{n+1}+6 v_{3}^{n+1}-4 v_{2}^{n+1}\right)\right\}+\frac{1}{\Delta \mathcal{T}^{2}}\left\{\left(2 v_{3}^{n+1}-5 v_{3}^{n}+4 v_{3}^{n-1}-v_{3}^{n-2}\right)\right\}
\end{aligned}
$$

Intermediate Nodes $(i=4,5, \ldots, L-2)$

$f_{i}^{n+1}=\frac{\mathcal{K}_{z}-\mathcal{E}_{A}^{2}}{\Delta \mathcal{X}^{4}}\left\{\left(v_{i+2}^{n+1}-4 v_{i+1}^{n+1}+6 v_{i}^{n+1}-4 v_{i-1}^{n+1}+v_{i-2}^{n+1}\right)\right\}+\frac{1}{\Delta \mathcal{T}^{2}}\left\{\left(2 v_{i}^{n+1}-5 v_{i}^{n}+4 v_{i}^{n-1}-v_{i}^{n-2}\right)\right\}$

Free end Boundary Conditions $(i=L-1, L)$

$$
\begin{aligned}
f_{L-1}^{n+1} & =\frac{\mathcal{K}_{z}-\mathcal{E}_{A}^{2}}{\Delta \mathcal{X}^{4}}\left\{\left(-2 v_{3}^{n+1}+5 v_{L-1}^{n+1}-4 v_{L-2}+v_{L-3}\right)\right\}+\frac{1}{\Delta \mathcal{T}^{2}}\left\{\left(2 v_{L-1}^{n+1}-5 v_{L-1}^{n}+4 v_{L-1}^{n-1}-v_{L-1}^{n-2}\right)\right\} \\
f_{L}^{n+1} & =\frac{\mathcal{K}_{z}-\mathcal{E}_{A}^{2}}{\Delta \mathcal{X}^{4}}\left\{\left(2 v_{L}^{n+1}-4 v_{L-1}^{n+1}+2 v_{L-2}^{n+1}\right)\right\}+\frac{1}{\Delta \mathcal{T}^{2}}\left\{\left(2 v_{L}^{n+1}-5 v_{L}^{n}+4 v_{L}^{n-1}-v_{L}^{n-2}\right)\right\}
\end{aligned}
$$

Fixed End Boundary Conditions $(i=1,2,3)$

$$
J_{1,1}^{n+1}=1
$$




$$
\begin{aligned}
J_{2,2}^{n+1} & =\frac{\mathcal{K}_{z}-\mathcal{E}_{A}^{2}}{\Delta \mathcal{X}^{4}}\{7\}+\frac{1}{\Delta \mathcal{T}^{2}}\{2\} \\
J_{2,3}^{n+1} & =\frac{\mathcal{K}_{z}-\mathcal{E}_{A}^{2}}{\Delta \mathcal{X}^{4}}\{-4\} \\
J_{2,4}^{n+1} & =\frac{\mathcal{K}_{z}-\mathcal{E}_{A}^{2}}{\Delta \mathcal{X}^{4}}\{1\} \\
J_{3,2}^{n+1} & =\frac{\mathcal{K}_{z}-\mathcal{E}_{A}{ }^{2}}{\Delta \mathcal{X}^{4}}\{-4\} \\
J_{3,3}^{n+1} & =\frac{\mathcal{K}_{z}-\mathcal{E}_{A}^{2}}{\Delta \mathcal{X}^{4}}\{6\}+\frac{1}{\Delta \mathcal{T}^{2}}\{2\} \\
J_{3,4}^{n+1} & =\frac{\mathcal{K}_{z}-\mathcal{E}_{A}^{2}}{\Delta \mathcal{X}^{4}}\{-4\} \\
J_{3,5}^{n+1} & =\frac{\mathcal{K}_{z}-\mathcal{E}_{A}^{2}}{\Delta \mathcal{X}^{4}}\{1\}
\end{aligned}
$$

Intermediate Nodes $(i=4,5, \ldots, L-2)$

$$
\begin{aligned}
J_{i, i-2}^{n+1} & =\frac{\mathcal{K}_{z}-\mathcal{E}_{A}{ }^{2}}{\Delta \mathcal{X}^{4}}\{1\} \\
J_{i, i-1}^{n+1} & =\frac{\mathcal{K}_{z}-\mathcal{E}_{A}^{2}}{\Delta \mathcal{X}^{4}}\{-4\} \\
J_{i, i}^{n+1} & =\frac{\mathcal{K}_{z}-\mathcal{E}_{A}^{2}}{\Delta \mathcal{X}^{4}}\{6\}+\frac{1}{\Delta \mathcal{T}^{2}}\{2\} \\
J_{i, i+1}^{n+1} & =\frac{\mathcal{K}_{z}-\mathcal{E}_{A}{ }^{2}}{\Delta \mathcal{X}^{4}}\{-4\} \\
J_{i, i+2}^{n+1} & =\frac{\mathcal{K}_{z}-\mathcal{E}_{A}{ }^{2}}{\Delta \mathcal{X}^{4}}\{1\}
\end{aligned}
$$

Free end Boundary Conditions $(i=L-1, L)$

$$
\begin{aligned}
J_{L-1, L-3}^{n+1} & =\frac{\mathcal{K}_{z}-\mathcal{E}_{A}{ }^{2}}{\Delta \mathcal{X}^{4}}\{1\} \\
J_{L-1, L-2}^{n+1} & =\frac{\mathcal{K}_{z}-\mathcal{E}_{A}{ }^{2}}{\Delta \mathcal{X}^{4}}\{-4\} \\
J_{L-1, L-1}^{n+1} & =\frac{\mathcal{K}_{z}-\mathcal{E}_{A}{ }^{2}}{\Delta \mathcal{X}^{4}}\{5\}+\frac{1}{\Delta \mathcal{T}^{2}}\{2\} \\
J_{L-1, L}^{n+1} & =\frac{\mathcal{K}_{z}-\mathcal{E}_{A}{ }^{2}}{\Delta \mathcal{X}^{4}}\{-2\} \\
J_{L, L-2}^{n+1} & =\frac{\mathcal{K}_{z}-\mathcal{E}_{A}{ }^{2}}{\Delta \mathcal{X}^{4}}\{2\} \\
J_{L, L-1}^{n+1} & =\frac{\mathcal{K}_{z}-\mathcal{E}_{A}{ }^{2}}{\Delta \mathcal{X}^{4}}\{-4\} \\
J_{L, L}^{n+1} & =\frac{\mathcal{K}_{z}-\mathcal{E}_{A}{ }^{2}}{\Delta \mathcal{X}^{4}}\{2\}+\frac{1}{\Delta \mathcal{T}^{2}}\{2\}
\end{aligned}
$$


Equations $L+1$ to $2 L$ relate to Eq.B.14; the PDE governing the flapwise bending.

$\delta w$

Fixed End Boundary Conditions $(i=1,2,3)$

$f_{L+1}^{n+1}=w_{1}^{n+1}$

$$
\begin{aligned}
f_{L+2}^{n+1}=\frac{\mathcal{K}_{z}}{\Delta \mathcal{X}^{4}}\{ & \left.\left(w_{4}^{n+1}-4 w_{3}^{n+1}+7 w_{2}^{n+1}\right)\right\}+\frac{\mathcal{K}_{\lambda 1}}{\Delta \mathcal{X}^{4}}\left\{\left(\phi_{4}^{n+1}-4 \phi_{3}^{n+1}+7 \phi_{2}^{n+1}\right)\right\} \\
& +\frac{1}{\Delta \mathcal{T}^{2}}\left\{\left(2 w_{2}^{n+1}-5 w_{2}^{n}+4 w_{2}^{n-1}-w_{2}^{n-2}\right)+\mathcal{E}\left(2 \phi_{2}^{n+1}-5 \phi_{2}^{n}+4 \phi_{2}^{n-1}-\phi_{2}^{n-2}\right)\right\} \\
f_{L+3}^{n+1}=\frac{\mathcal{K}_{z}}{\Delta \mathcal{X}^{4}}\{ & \left.\left(w_{5}^{n+1}-4 w_{4}^{n+1}+6 w_{3}^{n+1}-4 w_{2}^{n+1}\right)\right\}+\frac{\mathcal{K}_{\lambda 1}}{\Delta \mathcal{X}^{4}}\left\{\left(\phi_{5}^{n+1}-4 \phi_{4}^{n+1}+6 \phi_{3}^{n+1}-4 \phi_{2}^{n+1}\right)\right\} \\
& +\frac{1}{\Delta \mathcal{T}^{2}}\left\{\left(2 w_{3}^{n+1}-5 w_{3}^{n}+4 w_{3}^{n-1}-w_{3}^{n-2}\right)+\mathcal{E}\left(2 \phi_{3}^{n+1}-5 \phi_{3}^{n}+4 \phi_{3}^{n-1}-\phi_{3}^{n-2}\right)\right\}
\end{aligned}
$$

Intermediate Nodes $(i=4,5, \ldots, L-2)$

$f_{L+i}^{n+1}=\frac{\mathcal{K}_{z}}{\Delta \mathcal{X}^{4}}\left\{\left(w_{i+2}^{n+1}-4 w_{i+1}^{n+1}+6 w_{i}^{n+1}-4 w_{i-1}^{n+1}+w_{i-2}^{n+1}\right)\right\}+\frac{\mathcal{K}_{\lambda 1}}{\Delta \mathcal{X}^{4}}\left\{\left(\phi_{i+2}^{n+1}-4 \phi_{i+1}^{n+1}+6 \phi_{i}^{n+1}-4 \phi_{i-1}^{n+1}+\phi_{i-2}^{n+1}\right)\right\}$

$$
+\frac{1}{\Delta \mathcal{T}^{2}}\left\{\left(2 w_{i}^{n+1}-5 w_{i}^{n}+4 w_{i}^{n-1}-w_{i}^{n-2}\right)+\mathcal{E}\left(2 \phi_{i}^{n+1}-5 \phi_{i}^{n}+4 \phi_{i}^{n-1}-\phi_{i}^{n-2}\right)\right\}
$$

Free end Boundary Conditions $(i=L-1, L)$

$$
\begin{aligned}
& f_{2 L-1}^{n+1}=\frac{\mathcal{K}_{z}}{\Delta \mathcal{X}^{4}}\{(\left.\left.-2 w_{L}^{n+1}+5 w_{L-1}^{n+1}-4 w_{L-2}+w_{L-3}\right)\right\}+\frac{\mathcal{K}_{\lambda 1}}{\Delta \mathcal{X}^{4}}\left\{\left(-2 \phi_{L}^{n+1}+5 \phi_{L-1}^{n+1}-4 \phi_{L-2}+\phi_{L-3}\right)\right\} \\
&+\frac{1}{\Delta \mathcal{T}^{2}}\left\{\left(2 w_{L-1}^{n+1}-5 w_{L-1}^{n}+4 w_{L-1}^{n-1}-w_{L-1}^{n-2}\right)+\mathcal{E}\left(2 \phi_{L-1}^{n+1}-5 \phi_{L-1}^{n}+4 \phi_{L-1}^{n-1}-\phi_{L-1}^{n-2}\right)\right\} \\
& f_{2 L}^{n+1}=\frac{\mathcal{K}_{z}}{\Delta \mathcal{X}^{4}}\left\{\left(2 w_{L}^{n+1}-4 w_{L-1}^{n+1}+2 w_{L-2}^{n+1}\right)\right\}+\frac{\mathcal{K}_{\lambda 1}}{\Delta \mathcal{X}^{4}}\left\{\left(2 \phi_{L}^{n+1}-4 \phi_{L-1}^{n+1}+2 \phi_{L-2}^{n+1}\right)\right\} \\
&+\frac{1}{\Delta \mathcal{T}^{2}}\left\{\left(2 w_{L}^{n+1}-5 w_{L}^{n}+4 w_{L}^{n-1}-v_{L}^{n-2}\right)+\mathcal{E}\left(2 \phi_{L}^{n+1}-5 \phi_{L}^{n}+4 \phi_{L}^{n-1}-\phi_{L}^{n-2}\right)\right\}
\end{aligned}
$$

Fixed End Boundary Conditions $(i=1,2,3)$

$$
J_{1,1}^{n+1}=1
$$

$$
J_{L+2, L+2}^{n+1}=\frac{\mathcal{K}_{z}}{\Delta \mathcal{X}^{4}}\{7\}+\frac{1}{\Delta \mathcal{T}^{2}}\{2\}
$$




$$
\begin{aligned}
J_{L+2, L+3}^{n+1} & =\frac{\mathcal{K}_{z}}{\Delta \mathcal{X}^{4}}\{-4\} \\
J_{L+2, L+4}^{n+1} & =\frac{\mathcal{K}_{z}}{\Delta \mathcal{X}^{4}}\{1\} \\
J_{L+2,2 L+2}^{n+1} & =\frac{\mathcal{K}_{\lambda 1}}{\Delta \mathcal{X}^{4}}\{7\}+\frac{1}{\Delta \mathcal{T}^{2}}\{2 \mathcal{E}\} \\
J_{L+2, L+3}^{n+1} & =\frac{\mathcal{K}_{\lambda 1}}{\Delta \mathcal{X}^{4}}\{-4\} \\
J_{L+2, L+4}^{n+1} & =\frac{\mathcal{K}_{\lambda 1}}{\Delta \mathcal{X}^{4}}\{1\} \\
J_{L+2,3 L+1}^{n+1} & =\frac{1}{\Delta \mathcal{T}^{2}}\{2 \mathcal{E}\} \\
J_{L+3, L+2}^{n+1} & =\frac{\mathcal{K}_{z}}{\Delta \mathcal{X}^{4}}\{-4\} \\
J_{L+3, L+3}^{n+1} & =\frac{\mathcal{K}_{z}}{\Delta \mathcal{X}^{4}}\{7\}+\frac{1}{\Delta \mathcal{T}^{2}}\{2\} \\
J_{L+3, L+4}^{n+1} & =\frac{\mathcal{K}_{z}}{\Delta \mathcal{X}^{4}}\{-4\} \\
J_{L+3, L+5}^{n+1} & =\frac{\mathcal{K}_{z}}{\Delta \mathcal{X}^{4}}\{1\} \\
J_{L+3,2 L+2}^{n+1} & =\frac{\mathcal{K}_{\lambda 1}}{\Delta \mathcal{X}^{4}}\{-4\} \\
J_{L+3,2 L+3}^{n+1} & =\frac{\mathcal{K}_{\lambda 1}}{\Delta \mathcal{X}^{4}}\{7\}+\frac{1}{\Delta \mathcal{T}^{2}}\{2 \mathcal{E}\} \\
J_{L+3,2 L+4}^{n+1} & =\frac{\mathcal{K}_{\lambda 1}}{\Delta \mathcal{X}^{4}}\{-4\} \\
J_{L+3,2 L+5}^{n+1} & =\frac{\mathcal{K}_{\lambda 1}}{\Delta \mathcal{X}^{4}}\{1\} \\
J_{L+3,3 L+1}^{n+1} & =\frac{1}{\Delta \mathcal{T}^{2}}\{2 \mathcal{E}\} \\
&
\end{aligned}
$$

Intermediate Nodes $(i=4,5, \ldots, L-2)$

$$
\begin{aligned}
J_{L+i, L+i-2}^{n+1} & =\frac{\mathcal{K}_{z}}{\Delta \mathcal{X}^{4}}\{1\} \\
J_{L+i, L+i-1}^{n+1} & =\frac{\mathcal{K}_{z}}{\Delta \mathcal{X}^{4}}\{-4\} \\
J_{L+i, L+i}^{n+1} & =\frac{\mathcal{K}_{z}}{\Delta \mathcal{X}^{4}}\{6\}+\frac{1}{\Delta \mathcal{T}^{2}}\{2\} \\
J_{L+i, L+i+1}^{n+1} & =\frac{\mathcal{K}_{z}}{\Delta \mathcal{X}^{4}}\{-4\} \\
J_{L+i, L+i+2}^{n+1} & =\frac{\mathcal{K}_{z}}{\Delta \mathcal{X}^{4}}\{1\} \\
J_{L+i, 2 L+2}^{n+1} & =\frac{\mathcal{K}_{\lambda 1}}{\Delta \mathcal{X}^{4}}\{-4\} \\
J_{L+i, 2 L+3}^{n+1} & =\frac{\mathcal{K}_{\lambda 1}}{\Delta \mathcal{X}^{4}}\{7\}+\frac{1}{\Delta \mathcal{T}^{2}}\{2 \mathcal{E}\} \\
J_{L+i, 2 L+4}^{n+1} & =\frac{\mathcal{K}_{\lambda 1}}{\Delta \mathcal{X}^{4}}\{-4\} \\
J_{L+i, 2 L+5}^{n+1} & =\frac{\mathcal{K}_{\lambda 1}}{\Delta \mathcal{X}^{4}}\{1\} \\
J_{L+i, 3 L+1}^{n+1} & =\frac{1}{\Delta \mathcal{T}^{2}}\{2 \mathcal{E}\}
\end{aligned}
$$


Free end Boundary Conditions $(i=L-1, L)$

$$
\begin{aligned}
& J_{2 L-1,2 L-3}^{n+1}=\frac{\mathcal{K}_{z}}{\Delta \mathcal{X}^{4}}\{1\} \\
& J_{2 L-1,2 L-2}^{n+1}=\frac{\mathcal{K}_{z}}{\Delta \mathcal{X}^{4}}\{-4\} \\
& J_{2 L-1,2 L-1}^{n+1}=\frac{\mathcal{K}_{z}}{\Delta \mathcal{X}^{4}}\{5\}+\frac{1}{\Delta \mathcal{T}^{2}}\{2\} \\
& J_{2 L-1,2 L}^{n+1}=\frac{\mathcal{K}_{z}}{\Delta \mathcal{X}^{4}}\{-2\} \\
& J_{2 L-1,3 L-3}^{n+1}=\frac{\mathcal{K}_{\lambda 1}}{\Delta \mathcal{X}^{4}}\{1\} \\
& J_{2 L-1,3 L-2}^{n+1}=\frac{\mathcal{K}_{\lambda 1}}{\Delta \mathcal{X}^{4}}\{-4\} \\
& J_{2 L-1,3 L-1}^{n+1}=\frac{\mathcal{K}_{\lambda 1}}{\Delta \mathcal{X}^{4}}\{5\}+\frac{1}{\Delta \mathcal{T}^{2}}\{2 \mathcal{E}\} \\
& J_{2 L-1,3 L}^{n+1}=\frac{\mathcal{K}_{\lambda 1}}{\Delta \mathcal{X}^{4}}\{-2\} \\
& J_{2 L-1,3 L+1}^{n+1}=\frac{1}{\Delta \mathcal{T}^{2}}\{2 \mathcal{E}\} \\
& J_{2 L, 2 L-2}^{n+1}=\frac{\mathcal{K}_{z}}{\Delta \mathcal{X}^{4}}\{2\} \\
& J_{2 L, 2 L-1}^{n+1}=\frac{\mathcal{K}_{z}}{\Delta \mathcal{X}^{4}}\{-4\} \\
& J_{2 L, 2 L}^{n+1}=\frac{\mathcal{K}_{z}}{\Delta \mathcal{X}^{4}}\{2\}+\frac{1}{\Delta \mathcal{T}^{2}}\{2\} \\
& J_{2 L, 3 L-2}^{n+1}=\frac{\mathcal{K}_{\lambda 1}}{\Delta \mathcal{X}^{4}}\{2\} \\
& J_{2 L, 3 L-1}^{n+1}=\frac{\mathcal{K}_{\lambda 1}}{\Delta \mathcal{X}^{4}}\{-4\} \\
& J_{2 L, 3 L}^{n+1}=\frac{\mathcal{K}_{\lambda 1}}{\Delta \mathcal{X}^{4}}\{2\}+\frac{1}{\Delta \mathcal{T}^{2}}\{2 \mathcal{E}\} \\
& J_{2 L, 3 L+1}^{n+1}=\frac{1}{\Delta \mathcal{T}^{2}}\{2 \mathcal{E}\}
\end{aligned}
$$

Equations $2 L+1$ to $3 L$ relate to Eq. B.15); the PDE governing the flexible twisting.

$$
\delta \phi
$$

Fixed End Boundary Conditions $(i=1,2,3)$

$$
\begin{aligned}
& f_{2 L+1}^{n+1}=\phi_{1}^{n+1} \\
& f_{2 L+2}^{n+1}=\frac{-\mathcal{K}_{x}}{\Delta \mathcal{X}^{2}}\left\{\left(\phi_{3}^{n+1}-2 \phi_{2}^{n+1}\right)\right\}+\frac{\mathcal{K}_{\lambda 1}}{\Delta \mathcal{X}^{4}}\left\{\left(\phi_{4}^{n+1}-4 \phi_{3}^{n+1}+7 \phi_{2}^{n+1}\right)\right\}
\end{aligned}
$$




$$
\begin{aligned}
+ & \frac{\mathcal{K}_{\lambda 2}}{\Delta \mathcal{X}^{4}}\left\{\left(w_{4}^{n+1}-4 w_{3}^{n+1}+7 w_{2}^{n+1}\right)\right\}+\frac{\mathcal{E}}{\Delta \mathcal{T}^{2}}\left\{\left(2 w_{2}^{n+1}-5 w_{2}^{n}+4 w_{2}^{n-1}-w_{2}^{n-2}\right)\right\} \\
+ & \frac{\mathcal{M}_{0}}{\Delta \mathcal{T}^{2}}\left\{\left(2 \phi_{2}^{n+1}-5 \phi_{2}^{n}+4 \phi_{2}^{n-1}-\phi_{2}^{n-2}\right)+\left(2 \theta^{n+1}-5 \theta^{n}+4 \theta^{n-1}-\theta^{n-2}\right)\right\} \\
f_{2 L+3}^{n+1}=\frac{-\mathcal{K}_{x}}{\Delta \mathcal{X}^{2}}\{ & \left.\left(\phi_{4}^{n+1}-2 \phi_{3}^{n+1}+\phi_{2}^{n+1}\right)\right\}+\frac{\mathcal{K}_{\lambda 1}}{\Delta \mathcal{X}^{4}}\left\{\left(\phi_{5}^{n+1}-4 \phi_{4}^{n+1}+6 \phi_{3}^{n+1}-4 \phi_{2}^{n+1}\right)\right\} \\
& +\frac{\mathcal{K}_{\lambda 2}}{\Delta \mathcal{X}^{4}}\left\{\left(w_{5}^{n+1}-4 w_{4}^{n+1}+6 w_{3}^{n+1}-4 w_{2}^{n+1}\right)\right\}+\frac{\mathcal{E}}{\Delta \mathcal{T}^{2}}\left\{\left(2 w_{2}^{n+1}-5 w_{2}^{n}+4 w_{2}^{n-1}-w_{2}^{n-2}\right)\right\} \\
& +\frac{\mathcal{M}_{0}}{\Delta \mathcal{T}^{2}}\left\{\left(2 \phi_{2}^{n+1}-5 \phi_{2}^{n}+4 \phi_{2}^{n-1}-\phi_{2}^{n-2}\right)+\left(2 \theta^{n+1}-5 \theta^{n}+4 \theta^{n-1}-\theta^{n-2}\right)\right\}
\end{aligned}
$$

Intermediate Nodes $(i=4,5, \ldots, L-1)$

$$
\begin{aligned}
f_{2 L+i}^{n+1}=\frac{-\mathcal{K}_{x}}{\Delta \mathcal{X}^{2}}\{ & \left.\left(\phi_{i+1}^{n+1}-2 \phi_{i}^{n+1}+\phi_{i-1}^{n+1}\right)\right\}+\frac{\mathcal{K}_{\lambda 1}}{\Delta \mathcal{X}^{4}}\left\{\left(\phi_{i+2}^{n+1}-4 \phi_{i+1}^{n+1}+6 \phi_{i}^{n+1}-4 \phi_{i-1}^{n+1}+\phi_{i-2}^{n+1}\right)\right\} \\
& +\frac{\mathcal{K}_{\lambda 2}}{\Delta \mathcal{X}^{4}}\left\{\left(w_{i+2}^{n+1}-4 w_{i+1}^{n+1}+6 w_{i}^{n+1}-4 w_{i-1}^{n+1}+w_{i-2}^{n+1}\right)\right\}+\frac{\mathcal{E}}{\Delta \mathcal{T}^{2}}\left\{\left(2 w_{i}^{n+1}-5 w_{i}^{n}+4 w_{i}^{n-1}-w_{i}^{n-2}\right)\right\} \\
& +\frac{\mathcal{M}_{0}}{\Delta \mathcal{T}^{2}}\left\{\left(2 \phi_{i}^{n+1}-5 \phi_{i}^{n}+4 \phi_{i}^{n-1}-\phi_{i}^{n-2}\right)+\left(2 \theta^{n+1}-5 \theta^{n}+4 \theta^{n-1}-\theta^{n-2}\right)\right\}
\end{aligned}
$$

Free end Boundary Conditions $(i=L)$

$$
\begin{aligned}
f_{3 L-1}^{n+1}=\frac{-\mathcal{K}_{x}}{\Delta \mathcal{X}^{2}}\{ & \left.\left(\phi_{L}^{n+1}-2 \phi_{L-1}^{n+1}+\phi_{L-2}^{n+1}\right)\right\}+\frac{\mathcal{K}_{\lambda 1}}{\Delta \mathcal{X}^{4}}\left\{\left(-2 \phi_{L}^{n+1}+5 \phi_{L-1}^{n+1}-4 \phi_{L-2}+\phi_{L-3}\right)\right\} \\
& +\frac{\mathcal{K}_{\lambda 2}}{\Delta \mathcal{X}^{4}}\left\{\left(-2 w_{L}^{n+1}+5 w_{L-1}^{n+1}-4 w_{L-2}+w_{L-3}\right)\right\}+\frac{\mathcal{E}}{\Delta \mathcal{T}^{2}}\left\{\left(2 w_{L}^{n+1}-5 w_{L}^{n}+4 w_{L}^{n-1}-w_{L}^{n-2}\right)\right\} \\
& +\frac{\mathcal{M}_{0}}{\Delta \mathcal{T}^{2}}\left\{\left(2 \phi_{L}^{n+1}-5 \phi_{L}^{n}+4 \phi_{L}^{n-1}-\phi_{L}^{n-2}\right)+\left(2 \theta^{n+1}-5 \theta^{n}+4 \theta^{n-1}-\theta^{n-2}\right)\right\} \\
f_{3 L}^{n+1}=\frac{-\mathcal{K}_{x}}{\Delta \mathcal{X}^{2}}\{ & \left.\left(-2 \phi_{L}^{n+1}+2 \phi_{L-1}^{n+1}\right)\right\}+\frac{\mathcal{K}_{\lambda 1}}{\Delta \mathcal{X}^{4}}\left\{\left(2 \phi_{L}^{n+1}-4 \phi_{L-1}^{n+1}+2 \phi_{L-2}^{n+1}\right)\right\} \\
& +\frac{\mathcal{K}_{\lambda 2}}{\Delta \mathcal{X}^{4}}\left\{\left(2 w_{L}^{n+1}-4 w_{L-1}^{n+1}+2 w_{L-2}^{n+1}\right)\right\}+\frac{\mathcal{E}}{\Delta \mathcal{T}^{2}}\left\{\left(2 w_{L}^{n+1}-5 w_{L}^{n}+4 w_{L}^{n-1}-w_{L}^{n-2}\right)\right\} \\
& +\frac{\mathcal{M}_{0}}{\Delta \mathcal{T}^{2}}\left\{\left(2 \phi_{L}^{n+1}-5 \phi_{L}^{n}+4 \phi_{L}^{n-1}-\phi_{L}^{n-2}\right)+\left(2 \theta^{n+1}-5 \theta^{n}+4 \theta^{n-1}-\theta^{n-2}\right)\right\}
\end{aligned}
$$

Fixed End Boundary Conditions $(i=1,2)$

$$
J_{2 L+1,1}^{n+1}=1
$$

$$
\begin{aligned}
J_{2 L+2, L+2}^{n+1} & =\frac{\mathcal{K}_{\lambda 2}}{\Delta \mathcal{X}^{4}}\{7\}+\frac{1}{\Delta \mathcal{T}^{2}}\{2 \mathcal{E}\} \\
J_{2 L+2, L+3}^{n+1} & =\frac{\mathcal{K}_{\lambda 2}}{\Delta \mathcal{X}^{4}}\{-4\} \\
J_{2 L+2, L+4}^{n+1} & =\frac{\mathcal{K}_{\lambda 2}}{\Delta \mathcal{X}^{4}}\{1\} \\
J_{2 L+2,2 L+2}^{n+1} & =\frac{-\mathcal{K}_{x}}{\Delta \mathcal{X}^{2}}\{-2\}+\frac{\mathcal{K}_{\lambda 1}}{\Delta \mathcal{X}^{4}}\{7\}+\frac{\mathcal{M}_{0}}{\Delta \mathcal{T}^{2}}\{2\}
\end{aligned}
$$




$$
\begin{aligned}
J_{2 L+2,2 L+3}^{n+1} & =\frac{-\mathcal{K}_{x}}{\Delta \mathcal{X}^{2}}\{1\}+\frac{\mathcal{K}_{\lambda 1}}{\Delta \mathcal{X}^{4}}\{-4\} \\
J_{2 L+2,2 L+4}^{n+1} & =\frac{\mathcal{K}_{\lambda 1}}{\Delta \mathcal{X}^{4}}\{1\} \\
J_{2 L+2,3 L+1}^{n+1} & =\frac{\mathcal{M}_{0}}{\Delta \mathcal{T}^{2}}\{2\} \\
J_{2 L+3, L+2}^{n+1} & =\frac{\mathcal{K}_{\lambda 2}}{\Delta \mathcal{X}^{4}}\{-4\} \\
J_{2 L+3, L+3}^{n+1} & =\frac{\mathcal{K}_{\lambda 2}}{\Delta \mathcal{X}^{4}}\{6\}+\frac{1}{\Delta \mathcal{T}^{2}}\{2 \mathcal{E}\} \\
J_{2 L+3, L+4}^{n+1} & =\frac{\mathcal{K}_{\lambda 2}}{\Delta \mathcal{X}^{4}}\{-4\} \\
J_{2 L+3, L+5}^{n+1} & =\frac{\mathcal{K}_{\lambda 2}}{\Delta \mathcal{X}^{4}}\{1\} \\
J_{2 L+3,2 L+2}^{n+1} & =\frac{-\mathcal{K}_{x}}{\Delta \mathcal{X}^{2}}\{1\}+\frac{\mathcal{K}_{\lambda 1}}{\Delta \mathcal{X}^{4}}\{-4\} \\
J_{2 L+3,2 L+3}^{n+1} & =\frac{-\mathcal{K}_{x}}{\Delta \mathcal{X}^{2}}\{-2\}+\frac{\mathcal{K}_{\lambda 1}}{\Delta \mathcal{X}^{4}}\{6\}+\frac{\mathcal{M}_{0}}{\Delta \mathcal{T}^{2}}\{2\} \\
J_{2 L+3,2 L+4}^{n+1} & =\frac{-\mathcal{K}_{x}}{\Delta \mathcal{X}^{2}}\{1\}+\frac{\mathcal{K}_{\lambda 1}}{\Delta \mathcal{X}^{4}}\{-4\} \\
J_{2 L+3,2 L+3}^{n+1} & =\frac{\mathcal{K}_{\lambda 1}}{\Delta \mathcal{X}^{4}}\{1\} \\
J_{2 L+3,3 L+1}^{n+1} & =\frac{\mathcal{M}_{0}}{\Delta \mathcal{T}^{2}}\{2\}
\end{aligned}
$$

$$
\text { Intermediate Nodes }(i=3,4, \ldots, L-2)
$$

$$
\begin{aligned}
J_{2 L+i, L+i-2}^{n+1} & =\frac{\mathcal{K}_{\lambda 2}}{\Delta \mathcal{X}^{4}}\{1\} \\
J_{2 L+i, L+i-1}^{n+1} & =\frac{\mathcal{K}_{\lambda 2}}{\Delta \mathcal{X}^{4}}\{-4\} \\
J_{2 L+i, L+i}^{n+1} & =\frac{\mathcal{K}_{\lambda 2}}{\Delta \mathcal{X}^{4}}\{6\}+\frac{1}{\Delta \mathcal{T}^{2}}\{2 \mathcal{E}\} \\
J_{2 L+i, L+i+1}^{n+1} & =\frac{\mathcal{K}_{\lambda 2}}{\Delta \mathcal{X}^{4}}\{-4\} \\
J_{2 L+i, L+i+2}^{n+1} & =\frac{\mathcal{K}_{\lambda 2}}{\Delta \mathcal{X}^{4}}\{1\} \\
J_{2 L+i, 2 L+i-2}^{n+1} & =\frac{\mathcal{K}_{\lambda 1}}{\Delta \mathcal{X}^{4}}\{1\} \\
J_{2 L+i, 2 L+i-1}^{n+1} & =\frac{-\mathcal{K}_{x}}{\Delta \mathcal{X}^{2}}\{1\}+\frac{\mathcal{K}_{\lambda 1}}{\Delta \mathcal{X}^{4}}\{-4\} \\
J_{2 L+i, 2 L+i}^{n+1} & =\frac{-\mathcal{K}_{x}}{\Delta \mathcal{X}^{2}}\{-2\}+\frac{\mathcal{K}_{\lambda 1}}{\Delta \mathcal{X}^{4}}\{6\}+\frac{\mathcal{M}_{0}}{\Delta \mathcal{T}^{2}}\{2\} \\
J_{2 L+i, 2 L+i+1}^{n+1} & =\frac{-\mathcal{K}_{x}}{\Delta \mathcal{X}^{2}}\{1\}+\frac{\mathcal{K}_{\lambda 1}}{\Delta \mathcal{X}^{4}}\{-4\} \\
J_{2 L+i, 2 L+i+2}^{n+1} & =\frac{\mathcal{K}_{\lambda 1}}{\Delta \mathcal{X}^{4}}\{1\} \\
J_{2 L+i, 3 L+1}^{n+1} & =\frac{\mathcal{M}_{0}}{\Delta \mathcal{T}^{2}}\{2\}
\end{aligned}
$$

Free End Boundary Conditions $(i=L-1, L)$ 


$$
\begin{aligned}
J_{3 L-1,2 L-3}^{n+1} & =\frac{\mathcal{K}_{\lambda 2}}{\Delta \mathcal{X}^{4}}\{1\} \\
J_{3 L-1,2 L-2}^{n+1} & =\frac{\mathcal{K}_{\lambda 2}}{\Delta \mathcal{X}^{4}}\{-4\} \\
J_{3 L-12,2 L-1}^{n+1} & =\frac{\mathcal{K}_{\lambda 2}}{\Delta \mathcal{X}^{4}}\{5\}+\frac{1}{\Delta \mathcal{T}^{2}}\{2 \mathcal{E}\} \\
J_{3 L-1,2 L}^{n+1} & =\frac{\mathcal{K}_{\lambda 2}}{\Delta \mathcal{X}^{4}}\{-2\} \\
J_{3 L-1,3 L-3}^{n+1} & =\frac{\mathcal{K}_{\lambda 1}}{\Delta \mathcal{X}^{4}}\{1\} \\
J_{3 L-1,3 L-2}^{n+1} & =\frac{-\mathcal{K}_{x}}{\Delta \mathcal{X}^{2}}\{1\}+\frac{\mathcal{K}_{\lambda 1}}{\Delta \mathcal{X}^{4}}\{-4\} \\
J_{3 L-1,3 L-1}^{n+1} & =\frac{-\mathcal{K}_{x}}{\Delta \mathcal{X}^{2}}\{-2\}+\frac{\mathcal{K}_{\lambda 1}}{\Delta \mathcal{X}^{4}}\{5\}+\frac{\mathcal{M}_{0}}{\Delta \mathcal{T}^{2}}\{2\} \\
J_{3 L-1,3 L}^{n+1} & =\frac{-\mathcal{K}_{x}}{\Delta \mathcal{X}^{2}}\{1\}+\frac{\mathcal{K}_{\lambda 1}}{\Delta \mathcal{X}^{4}}\{-2\} \\
J_{3 L-1,3 L+1}^{n+1} & =\frac{\mathcal{M}_{0}}{\Delta \mathcal{T}^{2}}\{2\} \\
J_{3 L, 2 L-2}^{n+1} & =\frac{\mathcal{K}_{\lambda 2}}{\Delta \mathcal{X}^{4}}\{2\} \\
J_{3 L, 2 L-1}^{n+1} & =\frac{\mathcal{K}_{\lambda 2}}{\Delta \mathcal{X}^{4}}\{-4\} \\
J_{3 L, 2 L}^{n+1} & =\frac{\mathcal{K}_{\lambda 2}}{\Delta \mathcal{X}^{4}}\{2\}+\frac{1}{\Delta \mathcal{T}^{2}}\{2 \mathcal{E}\} \\
J_{3 L-1,3 L-2}^{n+1} & =\frac{\mathcal{K}_{\lambda 1}}{\Delta \mathcal{X}^{4}}\{2\} \\
J_{3 L, 3 L-1}^{n+1} & =\frac{-\mathcal{K}_{x}}{\Delta \mathcal{X}^{2}}\{2\}+\frac{\mathcal{K}_{\lambda 1}}{\Delta \mathcal{X}^{4}}\{-4\} \\
J_{3 L, 3 L}^{n+1} & =\frac{-\mathcal{K}_{x}}{\Delta \mathcal{X}^{2}}\{-2\}+\frac{\mathcal{K}_{\lambda 1}}{\Delta \mathcal{X}^{4}}\{2\}+\frac{\mathcal{M}_{0}}{\Delta \mathcal{T}^{2}}\{2\} \\
J_{3 L, 3 L+1}^{n+1} & =\frac{\mathcal{M}_{0}}{\Delta \mathcal{T}^{2}}\{2\} \\
&
\end{aligned}
$$

Equation $3 L+1$ relates to Eq. B.16); the ODE governing the rigid body pitch rotation. Here, the integrals of the distributed parameters over the span of the wing are evaluated using the trapezoidal rule.

$$
\int_{0}^{S} z\left(x, t_{n+1}\right) d x \approx \sum_{i=1}^{L-1} \frac{1}{2}\left(z_{i}^{n+1}+z_{i+1}^{n+1}\right)=\frac{1}{2} z_{1}^{n+1}+\sum_{i=2}^{L-1} z_{i}^{n+1}+\frac{1}{2} z_{L}^{n+1}
$$

Hence, combining the trapezoidal rule for numerical integration, Eq. B.142), the zero displacement boundary conditions at the fixed end $\left(w_{1}=\phi_{1}=0\right)$ are reflected in the summation used in the integral expression. 


$$
\begin{aligned}
& \delta \theta \\
& f_{3 L+1}^{n+1}=\mathcal{K}_{\theta}\left\{\theta^{n+1}\right\}+\frac{\mathcal{D}_{\theta}}{\Delta \mathcal{T}}\left\{\frac{1}{6}\left(11 \theta^{n+1}-18 \theta^{n}+9 \theta^{n-1}-2 \theta^{n-2}\right)\right\} \\
& +\frac{\left(\mathcal{M}_{0}+\mathcal{M}_{\theta}\right)}{\Delta \mathcal{T}^{2}}\left\{\left(2 \theta^{n+1}-5 \theta^{n}+4 \theta^{n-1}-\theta^{n-2}\right)\right\} \\
& +\frac{\mathcal{M}_{0} \Delta \mathcal{X}}{\Delta \mathcal{T}^{2}}\left\{\sum_{i=1}^{L-1}\left(2 \phi_{i}^{n+1}-5 \phi_{i}^{n}+4 \phi_{i}^{n-1}-\phi_{i}^{n-2}\right)+\frac{1}{2}\left(2 \phi_{L}^{n+1}-5 \phi_{L}^{n}+4 \phi_{L}^{n-1}-\phi_{L}^{n-2}\right)\right\} \\
& +\frac{\mathcal{E} \Delta \mathcal{X}}{\Delta \mathcal{T}^{2}}\left\{\sum_{i=1}^{L-1}\left(2 w_{i}^{n+1}-5 w_{i}^{n}+4 w_{i}^{n-1}-w_{i}^{n-2}\right)+\frac{1}{2}\left(2 w_{L}^{n+1}-5 w_{L}^{n}+4 w_{L}^{n-1}-w_{L}^{n-2}\right)\right\} \\
& J_{3 L+1, L+i}^{n+1}=\frac{1}{\Delta \mathcal{T}^{2}}\{2 e \Delta \mathcal{X}\} \quad i=2, \ldots, L-1 \\
& J_{3 L+1,2 L}^{n+1}=\frac{1}{\Delta \mathcal{T}^{2}}\{e \Delta \mathcal{X}\} \\
& J_{3 L+1,2 L+i}^{n+1}=\frac{\mathcal{M}_{0}}{\Delta \mathcal{T}^{2}}\{2 \Delta \mathcal{X}\} \quad i=2, \ldots, L-1 \\
& J_{3 L+1,3 L}^{n+1}=\frac{\mathcal{M}_{0}}{\Delta \mathcal{T}^{2}}\{1 \Delta \mathcal{X}\} \\
& J_{3 L+1,3 L+1}^{n+1}=\frac{\mathcal{M}_{0}+\mathcal{M}_{\theta}}{\Delta \mathcal{T}^{2}}\{2\}+\frac{\mathcal{D}_{\theta}}{\Delta \mathcal{T}}\left\{\frac{11}{6}\right\}+\mathcal{K}_{\theta}\{1\}
\end{aligned}
$$

\section{B.5.2 Nonlinear Inertial Terms}

Building on top of the currently defined linear system in the following subsections, the additional terms associated with the inertial nonlinearities will be presented, highlighted below in blue.

$\delta v$

$$
\begin{aligned}
& \left\{\mathcal{K}_{z}\left(\mathcal{V}^{\prime \prime \prime \prime}\right)+\left(\mathcal{K}_{z}-\mathcal{K}_{y}\right)\left(\mathcal{W}^{\prime \prime} \varphi^{\prime \prime}+2 \mathcal{W}^{\prime \prime \prime} \varphi^{\prime}+\mathcal{W}^{\prime \prime \prime \prime} \varphi\right)-\mathcal{E}_{A}{ }^{2}\left(\mathcal{V}^{\prime \prime \prime \prime}+\mathcal{W}^{\prime \prime} \varphi^{\prime \prime}+2 \mathcal{W}^{\prime \prime \prime} \varphi^{\prime}+\mathcal{W}^{\prime \prime \prime \prime} \varphi\right)\right\} \\
& +\left\{\ddot{\mathcal{V}}-\ddot{\vartheta}(\mathcal{E} \varphi+\mathcal{W})-\dot{\vartheta}^{2}\left(\left(\mathcal{V}+\mathcal{E}_{p}\right)+\mathcal{E}\right)-2 \dot{\vartheta}(\mathcal{E} \dot{\varphi}+\dot{\mathcal{W}})\right\}=\overline{\mathcal{W}}_{v}
\end{aligned}
$$

$\delta w$

$$
\begin{aligned}
& \left\{\mathcal{K}_{y}\left(\mathcal{W}^{\prime \prime \prime \prime}\right)+\left(\mathcal{K}_{z}-\mathcal{K}_{y}\right)\left(\mathcal{V}^{\prime \prime \prime \prime} \varphi+2 \mathcal{V}^{\prime \prime \prime} \varphi^{\prime}+\mathcal{V}^{\prime \prime} \varphi^{\prime \prime}\right)-\mathcal{E}_{A}^{2}\left(\mathcal{V}^{\prime \prime \prime \prime} \varphi+2 \mathcal{V}^{\prime \prime \prime} \varphi^{\prime}+\mathcal{V}^{\prime \prime} \varphi^{\prime \prime}\right)+\mathcal{K}_{\lambda}\left(\varphi^{\prime \prime \prime \prime}\right)\right\} \\
& +\left\{\ddot{\mathcal{W}}+\mathcal{E} \ddot{\varphi}+\ddot{\vartheta}\left(\left(\mathcal{V}+\mathcal{E}_{p}\right)+\mathcal{E}\right)-\dot{\vartheta}^{2}(\mathcal{E} \varphi+\mathcal{W})+2 \dot{\vartheta}(\dot{\mathcal{V}})\right\}=\overline{\mathcal{W}}_{w}
\end{aligned}
$$




$$
\begin{aligned}
& \delta \phi \\
& \left\{-\mathcal{K}_{x}\left(\varphi^{\prime \prime}\right)+\left(\mathcal{K}_{z}-\mathcal{K}_{y}\right)\left(\mathcal{V}^{\prime \prime} \mathcal{W}^{\prime \prime}\right)-\mathcal{E}_{A}^{2}\left(\mathcal{V}^{\prime \prime} \mathcal{W}^{\prime \prime}\right)+\mathcal{K}_{\lambda}\left(\mathcal{W}^{\prime \prime \prime \prime}\right)\right\} \\
& +\left\{\mathcal{M}_{0}(\ddot{\varphi}+\ddot{\vartheta})+\mathcal{E} \ddot{\mathcal{W}}+\ddot{\vartheta}\left(\mathcal{E}\left(\mathcal{V}+\mathcal{E}_{p}\right)\right)-\dot{\vartheta}^{2}(\mathcal{E} \mathcal{W})+2 \mathcal{E} \dot{\vartheta}(\dot{\mathcal{V}})\right\}=\overline{\mathcal{W}}_{\phi} \\
& \mathcal{M}_{\theta} \ddot{\vartheta}+\mathcal{D}_{\theta} \dot{\vartheta}+\mathcal{K}_{\vartheta} \vartheta+\int_{0}^{1}\left\{\mathcal{M}_{0}(\ddot{\varphi}+\ddot{\vartheta})-\ddot{\mathcal{V}}(\mathcal{E} \varphi+\mathcal{W})+\ddot{\mathcal{W}}\left(\left(\mathcal{V}+\mathcal{E}_{p}\right)+\mathcal{E}\right)+\mathcal{E} \ddot{\varphi}\left(\left(\mathcal{V}+\mathcal{E}_{p}\right)\right)\right. \\
& \left.+2 \mathcal{E} \ddot{\vartheta}\left(\left(\mathcal{V}+\mathcal{E}_{p}\right)+\mathcal{W} \varphi\right)+\ddot{\vartheta}\left(\mathcal{W}^{2}+\left(\mathcal{V}+\mathcal{E}_{p}\right)^{2}\right)+2 \dot{\vartheta}\left(\dot{\mathcal{V}}\left(\left(\mathcal{V}+\mathcal{E}_{p}\right)+\mathcal{E}\right)+\dot{\mathcal{W}}(\mathcal{E} \varphi+\mathcal{W})+\mathcal{E} \dot{\varphi}(\mathcal{W})\right)\right\} d \mathcal{X}=\overline{\mathcal{W}}_{\theta}
\end{aligned}
$$

Equations $f_{i}^{n+1}$ for $i=2, \ldots, L$ relate to the PDE governing the edgewise bending.

$$
\begin{aligned}
& \delta v \\
& f_{i}^{n+1}=\frac{1}{\Delta \mathcal{T}^{2}}\left\{-\left(2 \theta^{n+1}-5 \theta^{n}+4 \theta^{n-1}-\theta^{n-2}\right)\left(\mathcal{E} \phi_{i}^{n+1}+w_{i}^{n+1}\right)\right. \\
&-\frac{1}{36}\left(11 \theta^{n+1}-18 \theta^{n}+9 \theta^{n-1}-2 \theta^{n-2}\right)^{2}\left(v_{i}^{n+1}+\mathcal{E}\right) \\
&-\frac{2}{36}\left(11 \theta^{n+1}-18 \theta^{n}+9 \theta^{n-1}-2 \theta^{n-2}\right)\left(\mathcal{E}\left(11 \phi_{i}^{n+1}-18 \phi_{i}^{n}+9 \phi_{i}^{n-1}-2 \phi_{i}^{n-2}\right)\right. \\
&\left.\left.+\left(11 w_{i}^{n+1}-18 w_{i}^{n}+9 w_{i}^{n-1}-2 w_{i}^{n-2}\right)\right)\right\} \\
& J_{i, i}^{n+1}=\frac{1}{\Delta \mathcal{T}^{2}}\left\{-\frac{1}{36}\left(11 \theta^{n+1}-18 \theta^{n}+9 \theta^{n-1}-2 \theta^{n-2}\right)^{2}\right\} \\
& J_{i, L+i}^{n+1}=\frac{1}{\Delta \mathcal{T}^{2}}\left\{-\left(2 \theta^{n+1}-5 \theta^{n}+4 \theta^{n-1}-\theta^{n-2}\right)-\frac{2}{36}\left(11 \theta^{n+1}-18 \theta^{n}+9 \theta^{n-1}-2 \theta^{n-2}\right)(11)\right\} \\
& J_{i, 2 L+i}^{n+1}=\frac{1}{\Delta \mathcal{T}^{2}}\left\{-\mathcal{E}\left(2 \theta^{n+1}-5 \theta^{n}+4 \theta^{n-1}-\theta^{n-2}\right)-\frac{2}{36}\left(11 \theta^{n+1}-18 \theta^{n}+9 \theta^{n-1}-2 \theta^{n-2}\right)(11 \mathcal{E})\right\} \\
& J_{i, 3 L+1}^{n+1}=\frac{1}{\Delta \mathcal{T}^{2}}\left\{(-2)\left(\mathcal{E} \phi_{i}^{n+1}+w_{i}^{n+1}\right)-\frac{22}{36}\left(11 \theta^{n+1}-18 \theta^{n}+9 \theta^{n-1}-2 \theta^{n-2}\right)^{2}\left(v_{i}^{n+1}+\mathcal{E}\right)\right. \\
&\left.\quad-\frac{2}{4}(3)\left(\mathcal{E}\left(11 \phi_{i}^{n+1}-18 \phi_{i}^{n}+9 \phi_{i}^{n-1}-2 \phi_{i}^{n-2}\right)+\left(11 w_{i}^{n+1}-18 w_{i}^{n}+9 w_{i}^{n-1}-2 w_{i}^{n-2}\right)\right)\right\}
\end{aligned}
$$

Equations $f_{L+i}^{n+1}$ for $i=1, \ldots, L$ relate to the PDE governing the flapwise bending. 


$$
\begin{aligned}
& \delta w \\
& f_{L+i}^{n+1}=\frac{1}{\Delta \mathcal{T}^{2}}\left\{\left(2 \theta^{n+1}-5 \theta^{n}+4 \theta^{n-1}-\theta^{n-2}\right)\left(v_{i}^{n+1}+\mathcal{E}\right)\right.-\frac{1}{36}\left(11 \theta^{n+1}-18 \theta^{n}+9 \theta^{n-1}-2 \theta^{n-2}\right)^{2}\left(w_{i}^{n+1}+\mathcal{E} \phi_{i}^{n+1}\right) \\
&\left.\quad-\frac{2}{36}\left(11 \theta^{n+1}-18 \theta^{n}+9 \theta^{n-1}-2 \theta^{n-2}\right)\left(11 v_{i}^{n+1}-18 v_{i}^{n}+9 v_{i}^{n-1}-2 v_{i}^{n-1}\right)\right\} \\
& J_{L+i, i}^{n+1}=\frac{1}{\Delta \mathcal{T}^{2}}\left\{\left(2 \theta^{n+1}-5 \theta^{n}+4 \theta^{n-1}-\theta^{n-2}\right)-\frac{2}{36}\left(11 \theta^{n+1}-18 \theta^{n}+9 \theta^{n-1}-2 \theta^{n-2}\right)(11)\right\} \\
& J_{L+i, L+i}^{n+1}=\frac{1}{\Delta \mathcal{T}^{2}}\left\{-\frac{1}{36}\left(11 \theta^{n+1}-18 \theta^{n}+9 \theta^{n-1}-2 \theta^{n-2}\right)^{2}\right\} \\
& J_{L+i, 2 L+i}^{n+1}=\frac{1}{\Delta \mathcal{T}^{2}}\left\{-\frac{2}{36}\left(11 \theta^{n+1}-18 \theta^{n}+9 \theta^{n-1}-2 \theta^{n-2}\right)^{2}(e)\right\} \\
& J_{L+i, 3 L+1}^{n+1}=\frac{1}{\Delta \mathcal{T}^{2}}\left\{2\left(v_{i}^{n+1}\right)-\frac{22}{36}\left(11 \theta^{n+1}-18 \theta^{n}+9 \theta^{n-1}-2 \theta^{n-2}\right)^{2}\left(w_{i}^{n+1}+\mathcal{E} \phi_{i}^{n+1}\right)\right. \\
&\left.-\frac{2}{36}(11)\left(11 v_{i}^{n+1}-18 v_{i}^{n}+9 v_{i}^{n-1}-2 v_{i}^{n-2}\right)\right\}
\end{aligned}
$$

Equations $f_{2 L+i}^{n+1}$ for $i=1, \ldots, L$ relate to the PDE governing the flexible twisting.

$$
\begin{aligned}
& \delta \phi \\
& f_{2 L+i}^{n+1}=\frac{\mathcal{E}}{\Delta \mathcal{X}^{2}}\left\{\left(2 w_{i}^{n+1}-5 w_{i}^{n}+4 w_{i}^{n-1}-w_{i}^{n-2}\right)-\left(2 \theta^{n+1}-5 \theta^{n}+4 \theta^{n-1}-\theta^{n-2}\right)\left(v_{i}^{n+1}\right)\right.-\frac{1}{36}\left(11 \theta^{n+1}-18 \theta^{n}+9 \theta^{n-1}-2 \theta^{n-2}\right)^{2}\left(w_{i}^{n+1}\right) \\
&\left.-\frac{2}{36}\left(11 \theta^{n+1}-18 \theta^{n}+9 \theta^{n-1}-2 \theta^{n-2}\right)\left(11 v_{i}^{n+1}-18 v_{i}^{n}+9 v_{i}^{n-1}-2 v_{i}^{n-2}\right)\right\} \\
& J_{2 L+i, i}^{n+1}=\frac{1}{\Delta \mathcal{T}^{2}}\left\{\left(2 \theta^{n+1}-5 \theta^{n}+4 \theta^{n-1}-\theta^{n-2}\right)(\mathcal{E})-\frac{2}{36}\left(11 \theta^{n+1}-18 \theta^{n}+9 \theta^{n-1}-2 \theta^{n-2}\right)(11 \mathcal{E})\right\} \\
& J_{2 L+i, L+i}^{n+1}=\frac{1}{\Delta \mathcal{T}^{2}}\left\{-\frac{2}{36}\left(11 \theta^{n+1}-18 \theta^{n}+9 \theta^{n-1}-2 \theta^{n-2}\right)^{2}\right\} \\
& J_{2 L+i, 3 L+1}^{n+1}=\frac{1}{\Delta \mathcal{T}^{2}}\left\{2 \mathcal{E}\left(v_{i}^{n+1}\right)-\frac{22}{36}\left(11 \theta^{n+1}-18 \theta^{n}+9 \theta^{n-1}-2 \theta^{n-2}\right)\left(w_{i}^{n+1}\right)\right. \\
&\left.\quad-\frac{2}{36}(11 \mathcal{E})\left(11 v_{i}^{n+1}-18 v_{i}^{n}+9 v_{i}^{n-1}-2 v_{i}^{n-2}\right)\right\}
\end{aligned}
$$

The nonlinear inertial terms in the ODE governing the rigid body pitch rotation are given in equation $f_{3 L+1}^{n+1}$. This simply allows $f_{3 L+1}^{n+1}$ to be expressed more concisely according to the trapezoidal rule in 
Eq. B.142.

$$
\begin{aligned}
& \delta \theta \\
& f_{3 L+1}^{n+1}=\frac{\Delta \mathcal{X}}{2 \Delta \mathcal{T}^{2}} \sum_{i=2}^{L-1} \alpha\left\{2 \mathcal{E}\left\{\left(2 \theta^{n+1}-5 \theta^{n}+4 \theta^{n-1}-\theta^{n-2}\right)\left(v_{i}^{n+1}\right)\right\}\right. \\
& +2 \mathcal{E}\left\{\frac{1}{36}\left(11 \theta^{n+1}-18 \theta^{n}+9 \theta^{n-1}-2 \theta^{n-2}\right)\left(11 v_{i}^{n+1}-18 v_{i}^{n}+9 v_{i}^{n-1}-2 v_{i}^{n-2}\right)\right\} \\
& +\left\{\left(2 \theta^{n+1}-5 \theta^{n}+4 \theta^{n-1}-\theta^{n-2}\right)\left(\left(v_{i}^{n+1}\right)\left(v_{i}^{n+1}\right)\right)\right\} \\
& +2\left\{\frac{1}{36}\left(11 \theta^{n+1}-18 \theta^{n}+9 \theta^{n-1}-2 \theta^{n-2}\right)\left(\left(11 v_{i}^{n+1}-18 v_{i}^{n}+9 v_{i}^{n-1}-2 v_{i}^{n-2}\right)\left(v_{i}^{n+1}\right)\right)\right\} \\
& -\left\{\left(\left(2 v_{i}^{n+1}-5 v_{i}^{n}+4 v_{i}^{n-1}-v_{i}^{n-2}\right)\left(w_{i}^{n+1}\right)\right)\right\} \\
& +\left\{\left(\left(2 w_{i}^{n+1}-5 w_{i}^{n}+4 w_{i}^{n-1}-w_{i}^{n-2}\right)\left(v_{i}^{n+1}\right)\right)\right\} \\
& -\mathcal{E}\left\{\left(\left(2 v_{i}^{n+1}-5 v_{i}^{n}+4 v_{i}^{n-1}-v_{i}^{n-2}\right)\left(\phi_{i}^{n+1}\right)\right)\right\} \\
& +\mathcal{E}\left\{\left(\left(2 \phi_{i}^{n+1}-5 \phi_{i}^{n}+4 \phi_{i}^{n-1}-\phi_{i}^{n-2}\right)\left(v_{i}^{n+1}\right)\right)\right\} \\
& +\left\{\left(2 \theta^{n+1}-5 \theta^{n}+4 \theta^{n-1}-\theta^{n-2}\right)\left(\left(w_{i}^{n+1}\right)\left(w_{i}^{n+1}\right)\right)\right\} \\
& +2\left\{\frac{1}{36}\left(11 \theta^{n+1}-18 \theta^{n}+9 \theta^{n-1}-2 \theta^{n-2}\right)\left(\left(11 w_{i}^{n+1}-18 w_{i}^{n}+9 w_{i}^{n-1}-2 w_{i}^{n-2}\right)\left(w_{i}^{n+1}\right)\right)\right\} \\
& +2 \mathcal{E}\left\{\left(2 \theta^{n+1}-5 \theta^{n}+4 \theta^{n-1}-\theta^{n-2}\right)\left(\left(w_{i}^{n+1}\right)\left(\phi_{i}^{n+1}\right)\right)\right\} \\
& +2 \mathcal{E}\left\{\frac{1}{36}\left(11 \theta^{n+1}-18 \theta^{n}+9 \theta^{n-1}-2 \theta^{n-2}\right)\left(\left(11 w_{i}^{n+1}-18 w_{i}^{n}+9 w_{i}^{n-1}-2 w_{i}^{n-2}\right)\left(\phi_{i}^{n+1}\right)\right)\right\} \\
& \left.+2 \mathcal{E}\left\{\frac{1}{36}\left(11 \theta^{n+1}-18 \theta^{n}+9 \theta^{n-1}-2 \theta^{n-2}\right)\left(\left(11 \phi_{i}^{n+1}-18 \phi_{i}^{n}+9 \phi_{i}^{n-1}-2 \phi_{i}^{n-2}\right)\left(w_{i}^{n+1}\right)\right)\right\}\right\} \\
& J_{3 L+1, i}^{n+1}=\frac{\Delta \mathcal{X}}{\Delta \mathcal{T}^{2}}\left\{2 \mathcal{E}\left\{\left(2 \theta^{n+1}-5 \theta^{n}+4 \theta^{n-1}-\theta^{n-2}\right)(1)\right\}\right. \\
& +2 \mathcal{E}\left\{\frac{1}{36}\left(11 \theta^{n+1}-18 \theta^{n}+9 \theta^{n-1}-2 \theta^{n-2}\right)(11)\right\} \\
& +\left\{\left(2 \theta^{n+1}-5 \theta^{n}+4 \theta^{n-1}-\theta^{n-2}\right)\left(2\left(v_{i}^{n+1}\right)\right)\right\} \\
& \left.+2\left\{\frac{1}{36}\left(3 \theta^{n+1}-4 \theta^{n}+\theta^{n-1}\right)\left(22 v_{i}^{n+1}-18 v_{i}^{n}+9 v_{i}^{n-1}-2 v_{i}^{n-2}\right)\right)\right\} \\
& \left.-\left\{(2)\left(w_{i}^{n+1}\right)\right\}+\left\{\left(2 w_{i}^{n+1}-5 w_{i}^{n}+4 w_{i}^{n-1}-w_{i}^{n-2}\right)\right)(1)\right\} \\
& \left.-\mathcal{E}\left\{(2)\left(\phi_{i}^{n+1}\right)\right\}+\mathcal{E}\left\{\left(2 \phi_{i}^{n+1}-5 \phi_{i}^{n}+4 \phi_{i}^{n-1}-\phi_{i}^{n-2}\right)(1)\right\}\right\} \\
& J_{3 L+1, L}^{n+1}=\frac{\Delta \mathcal{X}}{2 \Delta \mathcal{T}^{2}}\left\{2 \mathcal{E}\left\{\left(2 \theta^{n+1}-5 \theta^{n}+4 \theta^{n-1}-\theta^{n-2}\right)(1)\right\}\right. \\
& +2 \mathcal{E}\left\{\frac{1}{36}\left(11 \theta^{n+1}-18 \theta^{n}+9 \theta^{n-1}-2 \theta^{n-2}\right)(11)\right\} \\
& +\left\{\left(2 \theta^{n+1}-5 \theta^{n}+4 \theta^{n-1}-\theta^{n-2}\right)\left(2\left(v_{L}^{n+1}\right)\right)\right\}
\end{aligned}
$$




$$
\begin{aligned}
& +2\left\{\frac{1}{36}\left(3 \theta^{n+1}-4 \theta^{n}+\theta^{n-1}\right)\left(22 v_{L}^{n+1}-18 v_{L}^{n}+9 v_{L}^{n-1}-2 v_{L}^{n-2}\right)\right\} \\
& -\left\{(2)\left(w_{L}^{n+1}\right)\right\}+\left\{\left(2 w_{L}^{n+1}-5 w_{L}^{n}+4 w_{L}^{n-1}-w_{L}^{n-2}\right)(1)\right\} \\
& \left.-\mathcal{E}\left\{(2)\left(\phi_{L}^{n+1}\right)\right\}+\mathcal{E}\left\{\left(2 \phi_{L}^{n+1}-5 \phi_{L}^{n}+4 \phi_{L}^{n-1}-\phi_{L}^{n-2}\right)(1)\right\}\right\} \\
& J_{3 L+1, L+i}^{n+1}=\frac{\Delta \mathcal{X}}{\Delta \mathcal{T}^{2}}\left\{-\left\{\left(2 v_{i}^{n+1}-5 v_{i}^{n}+4 v_{i}^{n-1}-v_{i}^{n-2}\right)(1)\right\}\right. \\
& +\left\{(2)\left(v_{i}^{n+1}\right)\right\}+\left\{\left(2 \theta^{n+1}-5 \theta^{n}+4 \theta^{n-1}-\theta^{n-2}\right)\left(2\left(w_{i}^{n+1}\right)\right)\right\} \\
& +2\left\{\frac{1}{36}\left(11 \theta^{n+1}-18 \theta^{n}+9 \theta^{n-1}-2 \theta^{n-2}\right)\left(22 w_{i}^{n+1}-18 w_{i}^{n}+9 w_{i}^{n-1}-2 w_{i}^{n-2}\right)\right\} \\
& +2 \mathcal{E}\left\{\left(2 \theta^{n+1}-5 \theta^{n}+4 \theta^{n-1}-\theta^{n-2}\right)(1)\left(\phi_{i}^{n+1}\right)\right\} \\
& +2 \mathcal{E}\left\{\frac{1}{36}\left(11 \theta^{n+1}-18 \theta^{n}+9 \theta^{n-1}-2 \theta^{n-2}\right)(11)\left(\phi_{i}^{n+1}\right)\right\} \\
& \left.+2 \mathcal{E}\left\{\frac{1}{36}\left(11 \theta^{n+1}-18 \theta^{n}+9 \theta^{n-1}-2 \theta^{n-2}\right)\left(11 \phi_{i}^{n+1}-18 \phi_{i}^{n}+9 \phi_{i}^{n-1}-2 \phi_{i}^{n-2}\right)(1)\right\}\right\} \\
& J_{3 L+1,2 L}^{n+1}=\frac{\Delta \mathcal{X}}{2 \Delta \mathcal{T}^{2}}\left\{-\left\{\left(2 v_{L}^{n+1}-5 v_{L}^{n}+4 v_{L}^{n-1}-v_{L}^{n-2}\right)(1)\right\}\right. \\
& +\left\{(2)\left(v_{L}^{n+1}\right)\right\}+\left\{\left(2 \theta^{n+1}-5 \theta^{n}+4 \theta^{n-1}-\theta^{n-2}\right)\left(2\left(w_{L}^{n+1}\right)\right)\right\} \\
& +2\left\{\frac{1}{36}\left(11 \theta^{n+1}-18 \theta^{n}+9 \theta^{n-1}-2 \theta^{n-2}\right)\left(22 w_{L}^{n+1}-18 w_{L}^{n}+9 w_{L}^{n-1}-2 w_{L}^{n-2}\right)\right\} \\
& +2 \mathcal{E}\left\{\left(2 \theta^{n+1}-5 \theta^{n}+4 \theta^{n-1}-\theta^{n-2}\right)(1)\left(\phi_{L}^{n+1}\right)\right\} \\
& +2 \mathcal{E}\left\{\frac{1}{36}\left(11 \theta^{n+1}-18 \theta^{n}+9 \theta^{n-1}-2 \theta^{n-2}\right)(11)\left(\phi_{L}^{n+1}\right)\right\} \\
& \left.+2 \mathcal{E}\left\{\frac{1}{36}\left(11 \theta^{n+1}-18 \theta^{n}+9 \theta^{n-1}-2 \theta^{n-2}\right)\left(11 \phi_{L}^{n+1}-18 \phi_{L}^{n}+9 \phi_{L}^{n-1}-2 \phi_{L}^{n-2}\right)(1)\right\}\right\} \\
& J_{3 L+1,2 L+i}^{n+1}=\frac{\Delta \mathcal{X}}{\Delta \mathcal{T}^{2}}\left\{-\mathcal{E}\left\{\left(2 v_{i}^{n+1}-5 v_{i}^{n}+4 v_{i}^{n-1}-v_{i}^{n-2}\right)(1)\right\}+\mathcal{E}\left\{(2)\left(v_{i}^{n+1}\right)\right\}\right. \\
& +2 \mathcal{E}\left\{\left(2 \theta^{n+1}-5 \theta^{n}+4 \theta^{n-1}-\theta^{n-2}\right)\left(w_{i}^{n+1}\right)(1)\right\} \\
& +2 \mathcal{E}\left\{\frac{1}{36}\left(11 \theta^{n+1}-18 \theta^{n}+9 \theta^{n-1}-2 \theta^{n-2}\right)\left(11 w_{i}^{n+1}-18 w_{i}^{n}+9 w_{i}^{n-1}-2 w_{i}^{n-2}\right)(1)\right\} \\
& \left.+2 \mathcal{E}\left\{\frac{1}{36}\left(11 \theta^{n+1}-18 \theta^{n}+9 \theta^{n-1}-2 \theta^{n-2}\right)(11)\left(w_{i}^{n+1}\right)\right\}\right\} \\
& J_{3 L+1,3 L}^{n+1}=\frac{\Delta \mathcal{X}}{2 \Delta \mathcal{T}^{2}}\left\{-\mathcal{E}\left\{\left(2 v_{L}^{n+1}-5 v_{L}^{n}+4 v_{L}^{n-1}-v_{L}^{n-2}\right)(1)\right\}+\mathcal{E}\left\{(2)\left(v_{L}^{n+1}\right)\right\}\right. \\
& +2 \mathcal{E}\left\{\left(2 \theta^{n+1}-5 \theta^{n}+4 \theta^{n-1}-\theta^{n-2}\right)\left(w_{L}^{n+1}\right)(1)\right\} \\
& +2 \mathcal{E}\left\{\frac{1}{36}\left(11 \theta^{n+1}-18 \theta^{n}+9 \theta^{n-1}-2 \theta^{n-2}\right)\left(11 w_{L}^{n+1}-18 w_{L}^{n}+9 w_{L}^{n-1}-2 w_{L}^{n-2}\right)(1)\right\} \\
& \left.+2 \mathcal{E}\left\{\frac{1}{36}\left(11 \theta^{n+1}-18 \theta^{n}+9 \theta^{n-1}-2 \theta^{n-2}\right)(11)\left(w_{L}^{n+1}\right)\right\}\right\}
\end{aligned}
$$




$$
\begin{aligned}
J_{3 L+1,3 L+1}^{n+1}=\frac{\Delta \mathcal{X}}{2 \Delta \mathcal{T}^{2}} & \sum_{i=2}^{L} \alpha\left\{2 \mathcal{E}\left\{(2)\left(v_{i}^{n+1}\right)\right\}+2 \mathcal{E}\left\{\frac{1}{36}(11)\left(11 v_{i}^{n+1}-18 v_{i}^{n}+9 v_{i}^{n-1}-2 v_{i}^{n-2}\right)\right\}\right. \\
+ & \left\{(2)\left(\left(v_{i}^{n+1}\right)\left(v_{i}^{n+1}\right)\right)\right\}+2\left\{\frac{1}{36}(11)\left(\left(11 v_{i}^{n+1}-18 v_{i}^{n}+9 v_{i}^{n-1}-2 v_{i}^{n-2}\right)\left(v_{i}^{n+1}\right)\right)\right\} \\
+ & \left\{(2)\left(\left(w_{i}^{n+1}\right)\left(w_{i}^{n+1}\right)\right)\right\}+2\left\{\frac{1}{36}(11)\left(\left(11 w_{i}^{n+1}-18 w_{i}^{n}+9 w_{i}^{n-1}-2 w_{i}^{n-2}\right)\left(w_{i}^{n+1}\right)\right)\right\} \\
+ & 2 \mathcal{E}\left\{(2)\left(\left(w_{i}^{n+1}\right)\left(\phi_{i}^{n+1}\right)\right)\right\}+2 \mathcal{E}\left\{\frac{1}{36}(11)\left(\left(11 w_{i}^{n+1}-18 w_{i}^{n}+9 w_{i}^{n-1}-2 w_{i}^{n-2}\right)\left(\phi_{i}^{n+1}\right)\right)\right\} \\
+ & \left.2 \mathcal{E}\left\{\frac{1}{36}(11)\left(\left(11 \phi_{i}^{n+1}-18 \phi_{i}^{n}+9 \phi_{i}^{n-1}-2 \phi_{i}^{n-2}\right)\left(w_{i}^{n+1}\right)\right)\right\}\right\}
\end{aligned}
$$

where the coefficient, $\alpha$ is given as Eq. B.175 for the case where the trapezoidal rule is used

$$
\alpha= \begin{cases}1 & i=1 \\ 2 & i=2, \ldots, L-1 \\ 1 & i=L\end{cases}
$$

\section{B.5.3 Nonlinear Stiffness Terms}

Finally, the terms associated with the stiffness nonlinearities are highlighted in the set of coupled PDEs in blue. Note that there are no stiffness nonlinearities in the ODE which governs the pitch motion, as seen in B.179.

$$
\begin{aligned}
& \delta v \\
& \left\{\mathcal{K}_{z}\left(\mathcal{V}^{\prime \prime \prime \prime}\right)+\left(\mathcal{K}_{z}-\mathcal{K}_{y}\right)\left(\mathcal{W}^{\prime \prime} \varphi^{\prime \prime}+2 \mathcal{W}^{\prime \prime \prime} \varphi^{\prime}+\mathcal{W}^{\prime \prime \prime \prime} \varphi\right)-\mathcal{E}_{A}^{2}\left(\mathcal{V}^{\prime \prime \prime \prime}+\mathcal{W}^{\prime \prime} \varphi^{\prime \prime}+2 \mathcal{W}^{\prime \prime \prime} \varphi^{\prime}+\mathcal{W}^{\prime \prime \prime \prime} \varphi\right)\right\} \\
& \quad+\left\{\ddot{\mathcal{V}}-\ddot{\vartheta}(\mathcal{E} \varphi+\mathcal{W})-\dot{\vartheta}^{2}\left(\left(\mathcal{V}+\mathcal{E}_{p}\right)+\mathcal{E}\right)-2 \dot{\vartheta}(\mathcal{E} \dot{\varphi}+\dot{\mathcal{W}})\right\}=\overline{\mathcal{W}}_{v} \\
& \quad \delta w \\
& \left\{\mathcal{K}_{y}\left(\mathcal{W}^{\prime \prime \prime \prime}\right)+\left(\mathcal{K}_{z}-\mathcal{K}_{y}\right)\left(\mathcal{V}^{\prime \prime \prime \prime} \varphi+2 \mathcal{V}^{\prime \prime \prime} \varphi^{\prime}+\mathcal{V}^{\prime \prime} \varphi^{\prime \prime}\right)-\mathcal{E}_{A}{ }^{2}\left(\mathcal{V}^{\prime \prime \prime \prime} \varphi+2 \mathcal{V}^{\prime \prime \prime} \varphi^{\prime}+\mathcal{V}^{\prime \prime} \varphi^{\prime \prime}\right)+\mathcal{K}_{\lambda}\left(\varphi^{\prime \prime \prime \prime}\right)\right\} \\
& \quad+\left\{\ddot{\mathcal{V}}+\mathcal{E} \ddot{\varphi}+\ddot{\vartheta}\left(\left(\mathcal{V}+\mathcal{E}_{p}\right)+\mathcal{E}\right)-\dot{\vartheta}^{2}(\mathcal{E} \varphi+\mathcal{W})+2 \dot{\vartheta}(\dot{\mathcal{V}})\right\}=\overline{\mathcal{W}}_{w}
\end{aligned}
$$




$$
\begin{aligned}
& \quad \delta \phi \\
& \left\{-\mathcal{K}_{x}\left(\varphi^{\prime \prime}\right)+\left(\mathcal{K}_{z}-\mathcal{K}_{y}\right)\left(\mathcal{V}^{\prime \prime} \mathcal{W}^{\prime \prime}\right)-\mathcal{E}_{A}^{2}\left(\mathcal{V}^{\prime \prime} \mathcal{W}^{\prime \prime}\right)+\mathcal{K}_{\lambda}\left(\mathcal{W}^{\prime \prime \prime \prime}\right)\right\} \\
& \quad+\left\{\mathcal{M}_{0}(\ddot{\varphi}+\ddot{\vartheta})+\mathcal{E} \ddot{\mathcal{V}}+\ddot{\vartheta}\left(\mathcal{E}\left(\mathcal{V}+\mathcal{E}_{p}\right)\right)-\dot{\vartheta}^{2}(\mathcal{E} \mathcal{W})+2 \mathcal{E} \dot{\vartheta}(\dot{\mathcal{V}})\right\}=\overline{\mathcal{W}}_{\phi}
\end{aligned}
$$

$\delta \theta$

$$
\begin{aligned}
\mathcal{M}_{\theta} \ddot{\vartheta} & +\mathcal{D}_{\theta} \dot{\vartheta}+\mathcal{K}_{\vartheta} \vartheta+\int_{0}^{1}\left\{\mathcal{M}_{0}(\ddot{\varphi}+\ddot{\vartheta})-\ddot{\mathcal{V}}(\mathcal{E} \varphi+\mathcal{W})+\ddot{\mathcal{W}}\left(\left(\mathcal{V}+\mathcal{E}_{p}\right)+\mathcal{E}\right)+\mathcal{E} \ddot{\varphi}\left(\left(\mathcal{V}+\mathcal{E}_{p}\right)\right)\right. \\
& \left.+2 \mathcal{E} \ddot{\vartheta}\left(\left(\mathcal{V}+\mathcal{E}_{p}\right)+\mathcal{W} \varphi\right)+\ddot{\vartheta}\left(\mathcal{W}^{2}+\left(\mathcal{V}+\mathcal{E}_{p}\right)^{2}\right)+2 \dot{\vartheta}\left(\dot{\mathcal{V}}\left(\left(\mathcal{V}+\mathcal{E}_{p}\right)+\mathcal{E}\right)+\dot{\mathcal{W}}(\mathcal{E} \varphi+\mathcal{W})+\mathcal{E} \dot{\varphi}(\mathcal{W})\right)\right\} d \mathcal{X}=\overline{\mathcal{W}}_{\theta}
\end{aligned}
$$

The first $L$ equations relate to the PDE governing the edgewise bending.

$\delta v$

Fixed End Boundary Conditions $(i=1,2)$

$$
\begin{aligned}
& f_{2}^{n+1}=\frac{\mathcal{K}_{z}-\mathcal{K}_{y}-\mathcal{E}_{A}^{2}}{\Delta \mathcal{X}^{4}}\left\{\frac{2}{4}\left(w_{4}^{n+1}-2 w_{3}^{n+1}-w_{2}^{n+1}\right)\left(\varphi_{3}^{n+1}\right)+\left(w_{3}^{n+1}-2 w_{2}^{n+1}\right)\left(\varphi_{3}^{n+1}-2 \varphi_{2}^{n+1}\right)\right. \\
&\left.+\left(w_{4}^{n+1}-4 w_{3}^{n+1}+7 w_{2}^{n+1}\right)\left(\varphi_{2}^{n+1}\right)\right\}
\end{aligned}
$$

Intermediate Nodes $(i=3,4, \ldots, L-2)$

$$
\begin{aligned}
f_{i}^{n+1}=\frac{\mathcal{K}_{z}-\mathcal{K}_{y}-\mathcal{E}_{A}^{2}}{\Delta \mathcal{X}^{4}} & \left\{\frac{2}{4}\left(w_{i+2}^{n+1}-2 w_{i+1}^{n+1}+2 w_{i-1}^{n+1}-w_{i-2}^{n+1}\right)\left(\varphi_{i+1}^{n+1}-\varphi_{i-1}^{n+1}\right)\right. \\
& +\left(w_{i+1}^{n+1}-2 w_{i}^{n+1}+w_{i-1}^{n+1}\right)\left(\varphi_{i+1}^{n+1}-2 \varphi_{i}^{n+1}+\varphi_{i-1}^{n+1}\right) \\
& \left.+\left(w_{i+2}^{n+1}-4 w_{i+1}^{n+1}+6 w_{i}^{n+1}-4 w_{i-1}^{n+1}+w_{i-2}^{n+1}\right)\left(\varphi_{i}^{n+1}\right)\right\}
\end{aligned}
$$

Free end Boundary Conditions $(i=L-1, L)$

$$
\begin{aligned}
& f_{L-1}^{n+1}=\frac{\mathcal{K}_{z}-\mathcal{K}_{y}-\mathcal{E}_{A}^{2}}{\Delta \mathcal{X}^{4}}\left\{\frac{2}{4}\left(-w_{L-1}^{n+1}+2 w_{L-2}^{n+1}-w_{L-3}^{n+1}\right)\left(\varphi_{L}^{n+1}-\varphi_{L-2}^{n+1}\right)\right.+\left(w_{L}^{n+1}-2 w_{L-1}^{n+1}+w_{L-2}^{n+1}\right)\left(\varphi_{L}^{n+1}-2 \varphi_{L-1}^{n+1}+\varphi_{L-2}^{n+1}\right) \\
&\left.+\left(-2 w_{L}^{n+1}+5 w_{L-1}^{n+1}-4 w_{L-2}^{n+1}+w_{L-3}^{n+1}\right)\left(\varphi_{L-1}^{n+1}\right)\right\} \\
& f_{L}^{n+1}=\frac{\mathcal{K}_{z}-\mathcal{K}_{y}-\mathcal{E}_{A}{ }^{2}}{\Delta \mathcal{X}^{4}}\left\{\left(2 w_{L}^{n+1}-4 w_{L-1}^{n+1}+2 w_{L-2}^{n+1}\right)\left(\varphi_{L}^{n+1}\right)\right\}
\end{aligned}
$$


Fixed End Boundary Conditions $(i=1,2)$

$$
\begin{aligned}
J_{2, L+2}^{n+1} & =\frac{\mathcal{K}_{z}-\mathcal{K}_{y}-\mathcal{E}_{A}^{2}}{\Delta \mathcal{X}^{4}}\left\{\frac{2}{4}(-1)\left(\varphi_{3}^{n+1}\right)+(-2)\left(\varphi_{3}^{n+1}-2 \varphi_{2}^{n+1}\right)+(7)\left(\varphi_{2}^{n+1}\right)\right\} \\
J_{2, L+3}^{n+1} & =\frac{\mathcal{K}_{z}-\mathcal{K}_{y}-\mathcal{E}_{A}^{2}}{\Delta \mathcal{X}^{4}}\left\{\frac{2}{4}(-2)\left(\varphi_{3}^{n+1}\right)+(1)\left(\varphi_{3}^{n+1}-2 \varphi_{2}^{n+1}\right)+(-4)\left(\varphi_{2}^{n+1}\right)\right\} \\
J_{2, L+4}^{n+1} & =\frac{\mathcal{K}_{z}-\mathcal{K}_{y}-\mathcal{E}_{A}^{2}}{\Delta \mathcal{X}^{4}}\left\{\frac{2}{4}(1)\left(\varphi_{3}^{n+1}\right)+(1)\left(\varphi_{2}^{n+1}\right)\right\} \\
J_{2,2 L+2}^{n+1} & =\frac{\mathcal{K}_{z}-\mathcal{K}_{y}-\mathcal{E}_{A}^{2}}{\Delta \mathcal{X}^{4}}\left\{\left(w_{3}^{n+1}-2 w_{2}^{n+1}\right)(-2)+\left(w_{4}^{n+1}-4 w_{3}^{n+1}+7 w_{2}^{n+1}\right)(1)\right\} \\
J_{2,2 L+3}^{n+1} & =\frac{\mathcal{K}_{z}-\mathcal{K}_{y}-\mathcal{E}_{A}{ }^{2}}{\Delta \mathcal{X}^{4}}\left\{\frac{2}{4}\left(w_{4}^{n+1}-2 w_{3}^{n+1}-w_{2}^{n+1}\right)(1)+\left(w_{3}^{n+1}-2 w_{2}^{n+1}\right)(1)\right\}
\end{aligned}
$$

Intermediate Nodes $(i=3,4, \ldots, L-2)$

$$
\begin{aligned}
J_{i, L+i-2}^{n+1}= & \frac{\mathcal{K}_{z}-\mathcal{K}_{y}-\mathcal{E}_{A}^{2}}{\Delta \mathcal{X}^{4}}\left\{\frac{2}{4}(-1)\left(\varphi_{i+1}^{n+1}-\varphi_{i-1}^{n+1}\right)+(1)\left(\varphi_{i}^{n+1}\right)\right\} \\
J_{i, L+i-1}^{n+1}= & \frac{\mathcal{K}_{z}-\mathcal{K}_{y}-\mathcal{E}_{A}^{2}}{\Delta \mathcal{X}^{4}}\left\{\frac{2}{4}(2)\left(\varphi_{i+1}^{n+1}-\varphi_{i-1}^{n+1}\right)+(1)\left(\varphi_{i+1}^{n+1}-2 \varphi_{i}^{n+1}+\varphi_{i-1}^{n+1}\right)+(-4)\left(\varphi_{i}^{n+1}\right)\right\} \\
J_{i, L+i}^{n+1}= & \frac{\mathcal{K}_{z}-\mathcal{K}_{y}-\mathcal{E}_{A}^{2}}{\Delta \mathcal{X}^{4}}\left\{(-2)\left(\varphi_{i+1}^{n+1}-2 \varphi_{i}^{n+1}+\varphi_{i-1}^{n+1}\right)+(6)\left(\varphi_{i}^{n+1}\right)\right\} \\
J_{i, L+i+1}^{n+1}= & \frac{\mathcal{K}_{z}-\mathcal{K}_{y}-\mathcal{E}_{A}^{2}}{\Delta \mathcal{X}^{4}}\left\{\frac{2}{4}(-2)\left(\varphi_{i+1}^{n+1}-\varphi_{i-1}^{n+1}\right)+(1)\left(\varphi_{i+1}^{n+1}-2 \varphi_{i}^{n+1}+\varphi_{i-1}^{n+1}\right)+(-4)\left(\varphi_{i}^{n+1}\right)\right\} \\
J_{i, L+i+2}^{n+1}= & \frac{\mathcal{K}_{z}-\mathcal{K}_{y}-\mathcal{E}_{A}^{2}}{\Delta \mathcal{X}^{4}}\left\{\frac{2}{4}(1)\left(\varphi_{i+1}^{n+1}-\varphi_{i-1}^{n+1}\right)+(1)\left(\varphi_{i}^{n+1}\right)\right\} \\
J_{i, 2 L+i-1}^{n+1}= & \frac{\mathcal{K}_{z}-\mathcal{K}_{y}-\mathcal{E}_{A}^{2}}{\Delta \mathcal{X}^{4}}\left\{\frac{2}{4}\left(w_{i+2}^{n+1}-2 w_{i+1}^{n+1}+2 w_{i-1}^{n+1}-w_{i-2}^{n+1}\right)(-1)+\left(w_{i+1}^{n+1}-2 w_{i}^{n+1}+w_{i-1}^{n+1}\right)(1)\right\} \\
J_{i, 2 L+i}^{n+1}= & \frac{\mathcal{K}_{z}-\mathcal{K}_{y}-\mathcal{E}_{A}{ }^{2}}{\Delta \mathcal{X}^{4}}\left\{\left(w_{i+1}^{n+1}-2 w_{i}^{n+1}+w_{i-1}^{n+1}\right)(-2)+\left(w_{i+2}^{n+1}-4 w_{i+1}^{n+1}+6 w_{i}^{n+1}-4 w_{i-1}^{n+1}+w_{i-2}^{n+1}\right)\right. \\
J_{i, 2 L+i+1}^{n+1}= & \frac{\mathcal{K}_{z}-\mathcal{K}_{y}-\mathcal{E}_{A}^{2}}{\Delta \mathcal{X}^{4}}\left\{\frac{2}{4}\left(w_{i+2}^{n+1}-2 w_{i+1}^{n+1}+2 w_{i-1}^{n+1}-w_{i-2}^{n+1}\right)(1)+\left(w_{i+1}^{n+1}-2 w_{i}^{n+1}+w_{i-1}^{n+1}\right)(1)\right\}
\end{aligned}
$$

Free end Boundary Conditions $(i=L-1, L)$

$$
\begin{aligned}
J_{L-1,2 L-3}^{n+1}= & \frac{\mathcal{K}_{z}-\mathcal{K}_{y}-\mathcal{E}_{A}^{2}}{\Delta \mathcal{X}^{4}}\left\{\frac{2}{4}(-1)\left(\varphi_{L}^{n+1}-\varphi_{L-2}^{n+1}\right)+(1)\left(\varphi_{L-1}^{n+1}\right)\right\} \\
J_{L-1,2 L-2}^{n+1}= & \frac{\mathcal{K}_{z}-\mathcal{K}_{y}-\mathcal{E}_{A}^{2}}{\Delta \mathcal{X}^{4}}\left\{\frac{2}{4}(2)\left(\varphi_{L}^{n+1}-\varphi_{L-2}^{n+1}\right)+(1)\left(\varphi_{L}^{n+1}-2 \varphi_{L-1}^{n+1}+\varphi_{L-2}^{n+1}\right)+(-4)\left(\varphi_{L-1}^{n+1}\right)\right\} \\
J_{L-1,2 L-1}^{n+1}= & \frac{\mathcal{K}_{z}-\mathcal{K}_{y}-\mathcal{E}_{A}^{2}}{\Delta \mathcal{X}^{4}}\left\{\frac{2}{4}(-1)\left(\varphi_{L}^{n+1}-\varphi_{L-2}^{n+1}\right)+(-2)\left(\varphi_{L}^{n+1}-2 \varphi_{L-1}^{n+1}+\varphi_{L-2}^{n+1}\right)+(5)\left(\varphi_{L-1}^{n+1}\right)\right\} \\
J_{L-1,2 L}^{n+1} & =\frac{\mathcal{K}_{z}-\mathcal{K}_{y}-\mathcal{E}_{A}^{2}}{\Delta \mathcal{X}^{4}}\left\{(1)\left(\varphi_{L}^{n+1}-2 \varphi_{L-1}^{n+1}+\varphi_{L-2}^{n+1}\right)+(-2)\left(\varphi_{L-1}^{n+1}\right)\right\} \\
J_{L-1,3 L-2}^{n+1} & =\frac{\mathcal{K}_{z}-\mathcal{K}_{y}-\mathcal{E}_{A}^{2}}{\Delta \mathcal{X}^{4}}\left\{\frac{2}{4}\left(-w_{L-1}^{n+1}+2 w_{L-2}^{n+1}-w_{L-3}^{n+1}\right)(-1)+\left(w_{L}^{n+1}-2 w_{L-1}^{n+1}+w_{L-2}^{n+1}\right)(1)\right\} \\
J_{L-1,3 L-1}^{n+1} & =\frac{\mathcal{K}_{z}-\mathcal{K}_{y}-\mathcal{E}_{A}^{2}}{\Delta \mathcal{X}^{4}}\left\{\left(w_{L}^{n+1}-2 w_{L-1}^{n+1}+w_{L-2}^{n+1}\right)(-2)+\left(-2 w_{L}^{n+1}+5 w_{L-1}^{n+1}-4 w_{L-2}^{n+1}+w_{L-3}^{n+1}\right)(1)\right\}(\mathrm{B} .2 \\
J_{L-1,3 L}^{n+1} & =\frac{\mathcal{K}_{z}-\mathcal{K}_{y}-\mathcal{E}_{A}^{2}}{\Delta \mathcal{X}^{4}}\left\{\frac{2}{4}\left(-w_{L-1}^{n+1}+2 w_{L-2}^{n+1}-w_{L-3}^{n+1}\right)(1)+\left(w_{L}^{n+1}-2 w_{L-1}^{n+1}+w_{L-2}^{n+1}\right)(1)\right\}
\end{aligned}
$$




$$
\begin{aligned}
J_{L, 2 L-2}^{n+1} & =\frac{\mathcal{K}_{z}-\mathcal{K}_{y}-\mathcal{E}_{A}{ }^{2}}{\Delta \mathcal{X}^{4}}\left\{(2)\left(\varphi_{L}^{n+1}\right)\right\} \\
J_{L, 2 L-1}^{n+1} & =\frac{\mathcal{K}_{z}-\mathcal{K}_{y}-\mathcal{E}_{A}{ }^{2}}{\Delta \mathcal{X}^{4}}\left\{(-4)\left(\varphi_{L}^{n+1}\right)\right\} \\
J_{L, 2 L}^{n+1} & =\frac{\mathcal{K}_{z}-\mathcal{K}_{y}-\mathcal{E}_{A}{ }^{2}}{\Delta \mathcal{X}^{4}}\left\{(2)\left(\varphi_{L}^{n+1}\right)\right\} \\
J_{L, 3 L}^{n+1} & =\frac{\mathcal{K}_{z}-\mathcal{K}_{y}-\mathcal{E}_{A}{ }^{2}}{\Delta \mathcal{X}^{4}}\left\{\left(2 w_{L}^{n+1}-4 w_{L-1}^{n+1}+2 w_{L-2}^{n+1}\right)\left(\varphi_{L}^{n+1}\right)\right\}
\end{aligned}
$$

Equations $L+1$ to $2 L$ relate to the PDE governing the flapwise bending.

$\delta w$

Fixed End Boundary Conditions $(i=1,2)$

$$
\begin{aligned}
& f_{L+2}^{n+1}=\frac{\mathcal{K}_{z}-\mathcal{K}_{y}-\mathcal{E}_{A}{ }^{2}}{\Delta \mathcal{X}^{4}}\left\{\frac{2}{4}\left(v_{4}^{n+1}-2 v_{3}^{n+1}-v_{2}^{n+1}\right)\left(\varphi_{3}^{n+1}\right)\right. \\
&\left.+\left(v_{3}^{n+1}-2 v_{2}^{n+1}\right)\left(\varphi_{3}^{n+1}-2 \varphi_{2}^{n+1}\right)+\left(v_{4}^{n+1}-4 v_{3}^{n+1}+7 v_{2}^{n+1}\right)\left(\varphi_{2}^{n+1}\right)\right\}
\end{aligned}
$$

Intermediate Nodes $(i=3,4, \ldots, L-2)$

$$
\begin{aligned}
f_{L+i}^{n+1}=\frac{\mathcal{K}_{z}-\mathcal{K}_{y}-\mathcal{E}_{A}^{2}}{\Delta \mathcal{X}^{4}} & \left\{\frac{2}{4}\left(v_{i+2}^{n+1}-2 v_{i+1}^{n+1}+2 v_{i-1}^{n+1}-v_{i-2}^{n+1}\right)\left(\varphi_{i+1}^{n+1}-\varphi_{i-1}^{n+1}\right)\right. \\
& +\left(v_{i+1}^{n+1}-2 v_{i}^{n+1}+v_{i-1}^{n+1}\right)\left(\varphi_{i+1}^{n+1}-2 \varphi_{i}^{n+1}+\varphi_{i-1}^{n+1}\right) \\
& \left.+\left(v_{i+2}^{n+1}-4 v_{i+1}^{n+1}+6 v_{i}^{n+1}-4 v_{i-1}^{n+1}+v_{i-2}^{n+1}\right)\left(\varphi_{i}^{n+1}\right)\right\}
\end{aligned}
$$

Free end Boundary Conditions $(i=L-1, L)$

$$
\begin{aligned}
& f_{2 L-1}^{n+1}=\frac{\mathcal{K}_{z}-\mathcal{K}_{y}-\mathcal{E}_{A}^{2}}{\Delta \mathcal{X}^{4}}\left\{\frac{2}{4}\left(-v_{L-1}^{n+1}+2 v_{L-2}^{n+1}-v_{L-3}^{n+1}\right)\left(\varphi_{L}^{n+1}-\varphi_{L-2}^{n+1}\right)\right. \\
&+\left(v_{L}^{n+1}-2 v_{L-1}^{n+1}+v_{L-2}^{n+1}\right)\left(\varphi_{L}^{n+1}-2 \varphi_{L-1}^{n+1}+\varphi_{L-2}^{n+1}\right) \\
&\left.+\left(-2 v_{L}^{n+1}+5 v_{L-1}^{n+1}-4 v_{L-2}^{n+1}+v_{L-3}^{n+1}\right)\left(\varphi_{L-1}^{n+1}\right)\right\} \\
& f_{2 L}^{n+1}=\frac{\mathcal{K}_{z}-\mathcal{K}_{y}-\mathcal{E}_{A}{ }^{2}}{\Delta \mathcal{X}^{4}}\left\{\left(2 v_{L}^{n+1}-4 v_{L-1}^{n+1}+2 v_{L-2}^{n+1}\right)\left(\varphi_{L}^{n+1}\right)\right\}
\end{aligned}
$$

Fixed End Boundary Conditions $(i=1,2)$

$$
J_{L+2,2}^{n+1}=\frac{\mathcal{K}_{z}-\mathcal{K}_{y}-\mathcal{E}_{A}{ }^{2}}{\Delta \mathcal{X}^{4}}\left\{\frac{2}{4}(-1)\left(\varphi_{3}^{n+1}\right)+(-2)\left(\varphi_{3}^{n+1}-2 \varphi_{2}^{n+1}\right)+(7)\left(\varphi_{2}^{n+1}\right)\right\}
$$




$$
\begin{aligned}
J_{L+2,3}^{n+1} & =\frac{\mathcal{K}_{z}-\mathcal{K}_{y}-\mathcal{E}_{A}^{2}}{\Delta \mathcal{X}^{4}}\left\{\frac{2}{4}(-2)\left(\varphi_{3}^{n+1}\right)+(1)\left(\varphi_{3}^{n+1}-2 \varphi_{2}^{n+1}\right)+(-4)\left(\varphi_{2}^{n+1}\right)\right\} \\
J_{L+2,4}^{n+1} & =\frac{\mathcal{K}_{z}-\mathcal{K}_{y}-\mathcal{E}_{A}^{2}}{\Delta \mathcal{X}^{4}}\left\{\frac{2}{4}(1)\left(\varphi_{3}^{n+1}\right)+(1)\left(\varphi_{2}^{n+1}\right)\right\} \\
J_{L+2,2 L+2}^{n+1} & =\frac{\mathcal{K}_{z}-\mathcal{K}_{y}-\mathcal{E}_{A}^{2}}{\Delta \mathcal{X}^{4}}\left\{\left(w_{3}^{n+1}-2 w_{2}^{n+1}\right)(-2)+\left(w_{4}^{n+1}-4 w_{3}^{n+1}+7 w_{2}^{n+1}\right)(1)\right\} \\
J_{L+2,2 L+3}^{n+1} & =\frac{\mathcal{K}_{z}-\mathcal{K}_{y}-\mathcal{E}_{A}^{2}}{\Delta \mathcal{X}^{4}}\left\{\frac{2}{4}\left(w_{4}^{n+1}-2 w_{3}^{n+1}-w_{2}^{n+1}\right)(1)+\left(w_{3}^{n+1}-2 w_{2}^{n+1}\right)(1)\right\}
\end{aligned}
$$

Intermediate Nodes $(i=3,4, \ldots, L-2)$

$$
\begin{aligned}
J_{L+i, i-2}^{n+1}= & \frac{\mathcal{K}_{z}-\mathcal{K}_{y}-\mathcal{E}_{A}{ }^{2}}{\Delta \mathcal{X}^{4}}\left\{\frac{2}{4}(-1)\left(\varphi_{i+1}^{n+1}-\varphi_{i-1}^{n+1}\right)+(1)\left(\varphi_{i}^{n+1}\right)\right\} \\
J_{L+i, i-1}^{n+1} & =\frac{\mathcal{K}_{z}-\mathcal{K}_{y}-\mathcal{E}_{A}{ }^{2}}{\Delta \mathcal{X}^{4}}\left\{\frac{2}{4}(2)\left(\varphi_{i+1}^{n+1}-\varphi_{i-1}^{n+1}\right)+(1)\left(\varphi_{i+1}^{n+1}-2 \varphi_{i}^{n+1}+\varphi_{i-1}^{n+1}\right)+(-4)\left(\varphi_{i}^{n+1}\right)\right\} \\
J_{L+i, i}^{n+1} & =\frac{\mathcal{K}_{z}-\mathcal{K}_{y}-\mathcal{E}_{A}{ }^{2}}{\Delta \mathcal{X}^{4}}\left\{(-2)\left(\varphi_{i+1}^{n+1}-2 \varphi_{i}^{n+1}+\varphi_{i-1}^{n+1}\right)+(6)\left(\varphi_{i}^{n+1}\right)\right\} \\
J_{L+i, i+1}^{n+1} & =\frac{\mathcal{K}_{z}-\mathcal{K}_{y}-\mathcal{E}_{A}{ }^{2}}{\Delta \mathcal{X}^{4}}\left\{\frac{2}{4}(-2)\left(\varphi_{i+1}^{n+1}-\varphi_{i-1}^{n+1}\right)+(1)\left(\varphi_{i+1}^{n+1}-2 \varphi_{i}^{n+1}+\varphi_{i-1}^{n+1}\right)+(-4)\left(\varphi_{i}^{n+1}\right)\right\} \\
J_{L+i, i+2}^{n+1} & =\frac{\mathcal{K}_{z}-\mathcal{K}_{y}-\mathcal{E}_{A}{ }^{2}}{\Delta \mathcal{X}^{4}}\left\{\frac{2}{4}(1)\left(\varphi_{i+1}^{n+1}-\varphi_{i-1}^{n+1}\right)+(1)\left(\varphi_{i}^{n+1}\right)\right\} \\
J_{L+i, 2 L+i-1}^{n+1} & =\frac{\mathcal{K}_{z}-\mathcal{K}_{y}-\mathcal{E}_{A}{ }^{2}}{\Delta \mathcal{X}^{4}}\left\{\frac{2}{4}\left(w_{i+2}^{n+1}-2 w_{i+1}^{n+1}+2 w_{i-1}^{n+1}-w_{i-2}^{n+1}\right)(-1)+\left(w_{i+1}^{n+1}-2 w_{i}^{n+1}+w_{i-1}^{n+1}\right)(1)\right\} \\
J_{L+i, 2 L+i}^{n+1} & =\frac{\mathcal{K}_{z}-\mathcal{K}_{y}-\mathcal{E}_{A}{ }^{2}}{\Delta \mathcal{X}^{4}}\left\{-2\left(w_{i+1}^{n+1}-2 w_{i}^{n+1}+w_{i-1}^{n+1}\right)(-2)+\left(w_{i+2}^{n+1}-4 w_{i+1}^{n+1}+6 w_{i}^{n+1}-4 w_{i-1}^{n+1}+w_{i-2}^{n+1}\right)\right.
\end{aligned}
$$

$$
J_{L+i, 2 L+i+1}^{n+1}=\frac{\mathcal{K}_{z}-\mathcal{K}_{y}-\mathcal{E}_{A}^{2}}{\Delta \mathcal{X}^{4}}\left\{\frac{2}{4}\left(w_{i+2}^{n+1}-2 w_{i+1}^{n+1}+2 w_{i-1}^{n+1}-w_{i-2}^{n+1}\right)(1)+\left(w_{i+1}^{n+1}-2 w_{i}^{n+1}+w_{i-1}^{n+1}\right)(1)\right\}
$$

Free end Boundary Conditions $(i=L-1, L)$

$$
\begin{aligned}
J_{2 L-1, L-3}^{n+1}= & \frac{\mathcal{K}_{z}-\mathcal{K}_{y}-\mathcal{E}_{A}^{2}}{\Delta \mathcal{X}^{4}}\left\{\frac{2}{4}(-1)\left(\varphi_{L}^{n+1}-\varphi_{L-2}^{n+1}\right)+(1)\left(\varphi_{L-1}^{n+1}\right)\right\} \\
J_{2 L-1, L-2}^{n+1}= & \frac{\mathcal{K}_{z}-\mathcal{K}_{y}-\mathcal{E}_{A}^{2}}{\Delta \mathcal{X}^{4}}\left\{\frac{2}{4}(2)\left(\varphi_{L}^{n+1}-\varphi_{L-2}^{n+1}\right)+(1)\left(\varphi_{L}^{n+1}-2 \varphi_{L-1}^{n+1}+\varphi_{L-2}^{n+1}\right)+(-4)\left(\varphi_{L-1}^{n+1}\right)\right\} \\
J_{2 L-1, L-1}^{n+1}= & \frac{\mathcal{K}_{z}-\mathcal{K}_{y}-\mathcal{E}_{A}^{2}}{\Delta \mathcal{X}^{4}}\left\{\frac{2}{4}(-1)\left(\varphi_{L}^{n+1}-\varphi_{L-2}^{n+1}\right)+(-2)\left(\varphi_{L}^{n+1}-2 \varphi_{L-1}^{n+1}+\varphi_{L-2}^{n+1}\right)+(5)\left(\varphi_{L-1}^{n+1}\right)\right\} \\
J_{2 L-1, L}^{n+1} & =\frac{\mathcal{K}_{z}-\mathcal{K}_{y}-\mathcal{E}_{A}^{2}}{\Delta \mathcal{X}^{4}}\left\{(1)\left(\varphi_{L}^{n+1}-2 \varphi_{L-1}^{n+1}+\varphi_{L-2}^{n+1}\right)+(-2)\left(\varphi_{L-1}^{n+1}\right)\right\} \\
J_{2 L-1,3 L-2}^{n+1} & =\frac{\mathcal{K}_{z}-\mathcal{K}_{y}-\mathcal{E}_{A}^{2}}{\Delta \mathcal{X}^{4}}\left\{\frac{2}{4}\left(-w_{L-1}^{n+1}+2 w_{L-2}^{n+1}-w_{L-3}^{n+1}\right)(-1)+\left(w_{L}^{n+1}-2 w_{L-1}^{n+1}+w_{L-2}^{n+1}\right)(1)\right\} \\
J_{2 L-1,3 L-1}^{n+1} & =\frac{\mathcal{K}_{z}-\mathcal{K}_{y}-\mathcal{E}_{A}^{2}}{\Delta \mathcal{X}^{4}}\left\{\left(w_{L}^{n+1}-2 w_{L-1}^{n+1}+w_{L-2}^{n+1}\right)(-2)+\left(-2 w_{L}^{n+1}+5 w_{L-1}^{n+1}-4 w_{L-2}^{n+1}+w_{L-3}^{n+1}\right)(1)\right\} \\
J_{2 L-1,3 L}^{n+1} & =\frac{\mathcal{K}_{z}-\mathcal{K}_{y}-\mathcal{E}_{A}^{2}}{\Delta \mathcal{X}^{4}}\left\{\frac{2}{4}\left(-w_{L-1}^{n+1}+2 w_{L-2}^{n+1}-w_{L-3}^{n+1}\right)(1)+\left(w_{L}^{n+1}-2 w_{L-1}^{n+1}+w_{L-2}^{n+1}\right)(1)\right\} \\
J_{2 L, L-2}^{n+1} & =\frac{\mathcal{K}_{z}-\mathcal{K}_{y}-\mathcal{E}_{A}^{2}}{\Delta \mathcal{X}^{4}}\left\{(2)\left(\varphi_{L}^{n+1}\right)\right\}
\end{aligned}
$$




$$
\begin{aligned}
J_{2 L, L-1}^{n+1} & =\frac{\mathcal{K}_{z}-\mathcal{K}_{y}-\mathcal{E}_{A}{ }^{2}}{\Delta \mathcal{X}^{4}}\left\{(-4)\left(\varphi_{L}^{n+1}\right)\right\} \\
J_{2 L, L}^{n+1} & =\frac{\mathcal{K}_{z}-\mathcal{K}_{y}-\mathcal{E}_{A}^{2}}{\Delta \mathcal{X}^{4}}\left\{(2)\left(\varphi_{L}^{n+1}\right)\right\} \\
J_{2 L, 3 L}^{n+1} & =\frac{\mathcal{K}_{z}-\mathcal{K}_{y}-\mathcal{E}_{A}{ }^{2}}{\Delta \mathcal{X}^{4}}\left\{\left(2 w_{L}^{n+1}-4 w_{L-1}^{n+1}+2 w_{L-2}^{n+1}\right)\left(\varphi_{L}^{n+1}\right)\right\}
\end{aligned}
$$

Equations $2 L+1$ to $3 L$ relate to the PDE governing the flexible twisting.

$$
\begin{aligned}
& \delta \phi \\
& \text { Intermediate Nodes }(i=2,3, \ldots, L-1) \\
& f_{2 L+i}^{n+1}=\frac{\mathcal{K}_{z}-\mathcal{K}_{y}-\mathcal{E}_{A}^{2}}{\Delta \mathcal{X}^{4}}\left\{\left(v_{i+1}^{n+1}-2 v_{i}^{n+1}+v_{i-1}^{n+1}\right)\left(w_{i+1}^{n+1}-2 w_{i}^{n+1}+w_{i-1}^{n+1}\right)\right\} \\
& \text { Intermediate Nodes }(i=3,4, \ldots, L-2) \\
& J_{2 L+i, i-1}^{n+1}=\frac{\mathcal{K}_{z}-\mathcal{K}_{y}-\mathcal{E}_{A}^{2}}{\Delta \mathcal{X}^{4}}\left\{(1)\left(w_{i+1}^{n+1}-2 w_{i}^{n+1}+w_{i-1}^{n+1}\right)\right\} \\
& J_{2 L+i, i}^{n+1}=\frac{\mathcal{K}_{z}-\mathcal{K}_{y}-\mathcal{E}_{A}^{2}}{\Delta \mathcal{X}^{4}}\left\{(-2)\left(w_{i+1}^{n+1}-2 w_{i}^{n+1}+w_{i-1}^{n+1}\right)\right\} \\
& J_{2 L+i, i+1}^{n+1}=\frac{\mathcal{K}_{z}-\mathcal{K}_{y}-\mathcal{E}_{A}^{2}}{\Delta \mathcal{X}^{4}}\left\{(1)\left(w_{i+1}^{n+1}-2 w_{i}^{n+1}+w_{i-1}^{n+1}\right)\right\} \\
& J_{2 L+i, L+i-1}^{n+1}= \frac{\mathcal{K}_{z}-\mathcal{K}_{y}-\mathcal{E}_{A}^{2}}{\Delta \mathcal{X}^{4}}\left\{\left(v_{i+1}^{n+1}-2 v_{i}^{n+1}+v_{i-1}^{n+1}\right)(1)\right\} \\
& J_{2 L+i, L+i}^{n+1}= \frac{\mathcal{K}_{z}-\mathcal{K}_{y}-\mathcal{E}_{A}^{2}}{\Delta \mathcal{X}^{4}}\left\{\left(v_{i+1}^{n+1}-2 v_{i}^{n+1}+v_{i-1}^{n+1}\right)(-2)\right\} \\
& J_{2 L+i, L+i+1}^{n+1}= \frac{\mathcal{K}_{z}-\mathcal{K}_{y}-\mathcal{E}_{A}^{2}}{\Delta \mathcal{X}^{4}}\left\{\left(v_{i+1}^{n+1}-2 v_{i}^{n+1}+v_{i-1}^{n+1}\right)(1)\right\}
\end{aligned}
$$

Equation $3 L+1$ relates to the ODE governing the rigid body pitch rotation, and has no nonlinear stiffness terms. In fact, the only stiffness term in the expression relates to the torsional spring that restrains the rigid body motion. This torsional spring is currently modelled as a linear spring. 